\section{Pacific Northwest}

National Laboratory

Operated by Battelle for the

U.S. Department of Energy

\title{
Uncertainty Analysis Framework - Hanford Site-Wide Groundwater Flow and Transport Model
}

\author{
C. R. Cole \\ P. D. Thorne \\ M. P. Bergeron \\ S. K. Wurstner \\ C. J. Murray \\ P. M. Rogers (Jacobs Engineering)
}

November 2001

Prepared for the U.S. Department of Energy under Contract DE-AC06-76RL01830 


\section{Uncertainty Analysis Framework - Hanford Site-Wide Groundwater Flow and Transport Model}
C. R. Cole
P. D. Thorne
M. P. Bergeron
S. K. Wurstner
C. J. Murray
P. M. Rogers (Jacobs Engineering)

November 2001

Prepared for

the U.S. Department of Energy

under Contract DE-AC06-76RL01830

Pacific Northwest National Laboratory

Richland, Washington 99352 


\section{Summary}

At the request of the U.S. Department of Energy (DOE), Pacific Northwest National Laboratory (PNNL) embarked on a new initiative to strengthen the technical defensibility of the Hanford site-wide groundwater model (SGM) used to make groundwater flow and transport predictions at the U.S. Department of Energy's Hanford Site in southeastern Washington State (Figure 1.1). The initial focus of the initiative is the characterization of major uncertainties in the current conceptual model that would affect model predictions. The long-term goals of the initiative are the development and implementation of a stochastic uncertainty estimation methodology in future assessments and analyses using the site-wide groundwater model. This report focuses on the development and implementation of the uncertainty analysis framework. The overall technical approach for this framework will closely follow the recommendations of the SGM external peer review panel made in 1999 (Gorelick et al. 1999). As suggested by the panel, the framework being developed acknowledges the inherent uncertainty in conceptual model representations and associated model inputs and thus in any predictions. This new framework acknowledges that prescribed processes, physical features, initial and boundary conditions, system stresses, field data, and model parameter values are not known and cannot be known with certainty and, as a result, predictions of heads and concentrations in three dimensions over time will be uncertain as well. The approach will specifically address those areas of special interest that were identified by the expert panel. These include uncertainty related to

- alternative model structures and constructs of processes (e.g., different zonation, different boundary conditions, large-scale features, stresses, and chemical reactions)

- model parameters

- model scale and resolution issues.

This report represents one of the first steps in development of this new SGM uncertainty framework by

- identifying the types of assessments for which the SGM will likely be applied (Section 2)

- discussing the various sources of uncertainty and the issues associated with the assessment of uncertainty as they relate to the development of this new framework for assessment of uncertainty in the Hanford SGM predictions. The sources of uncertainty include the most common high-level categories: uncertainty resulting from natural variability, model structure, and model parameters (Section 3).

- providing a detailed description of the current conceptual model of the Hanford Site unconfined aquifer system that includes an assessment of the uncertainties and issues associated with that model (Section 4), the well picks used in the current interpretation of the hydrostratigraphic structure (Appendix A), and the results of a literature search that developed an initial bibliography and 
assessment regarding the various potential intercommunication mechanisms between the unconfined aquifer system and the uppermost confined aquifer system in the underlying basalts (Appendix B and attachments)

- identifying the technical approach and initial plans and steps to be undertaken to implement the new framework (Section 5), including the current status of implementation effort.

The uncertainty framework being developed is based on a classical approach involving set and probability theory. The approach used for uncertainty assessment will include both stochastic uncertainty (e.g., resulting from or attributable to geologic heterogeneity and natural variability and subjective uncertainty arising from ignorance or imperfect knowledge about processes or other aspects of the conceptual model) and vagueness regarding the future (i.e., scenarios). The general approach addresses most of the issues identified in Beck's (1987) frame of reference diagram for the analysis of uncertainty (Figure 3.11). The emphasis will be on combining the knowledge and assumptions on the external and internal descriptions of the system in order to identify plausible alternative model structures that encompass the uncertainty in the historical and current system state for use in the prediction phase (i.e., the uncertainty propagation phase). Uncertainty related to future conditions and their uncertainty (i.e., scenarios) is generally not addressed by the methodology to be outlined in this report. Scenario uncertainty, because of its complexity, will need to be addressed by a separate effort. In our current assessments of uncertainty, we will treat uncertainty related to future system states through sensitivity analysis since probabilities of occurrence will not be assigned. The uncertain predictions from one future state can be compared with those for another future state (e.g., no future development or climate change results could be compared to results for a scenario with full development and slowly changing climate). The effect of uncertainty related to scenarios is accounted for as part of the subjective uncertainty discussed in Section 3. The exception to treatment of scenario uncertainty would be when the uncertainty can be adequately treated by uncertainty in, for example, a boundary condition parameter or some other type of simple, characterizable parameter uncertainty and thus addressable through standard Monte Carlo methods.

The general approach for addressing uncertainty in groundwater flow and transport predictions will follow that used in probabilistic risk assessment. The basic approach is to use sensitivity analysis for those aspects of the analysis related to vagueness and indefiniteness (e.g., alternative conceptual models [ACMs], model structure, and future scenarios) and uncertainty analysis for those situations where the uncertainty (e.g., for parameters) can and should be represented by a probability density function (pdf) (e.g., as interpreted from measurements using geostatistical methods, or determined from inverse modeling). The approach identified for addressing Hanford SGM uncertainty consists of the following basic steps:

1. Identify Alternative Conceptual Models - This effort consists of identifying and documenting the major features and assumptions associated with ACMs for the Hanford SGM. It will also involve periodically reviewing this list of alternative conceptual models for the Hanford SGM based on any new data or understanding to see if any new Alternative Conceptual Models (ACMs) should be added, developed, and evaluated against existing ones, or if existing ACMs should be eliminated. Totally new ACMs could be developed, but more likely the new ACMs will involve expanding 
and/or modifying existing ACMs (e.g., adding additional components, using different representations of processes, modifications or changes to the model structure based on new data and interpretations, altering/expanding the domain, and/or altering the model boundary conditions).

2. ACM Development - This step involves developing each new or altered ACM. This will generally involve an inverse modeling step where historical site data on parameters, system responses (e.g., head and concentration), and external driving forces will be used in a parameter identification step to develop the best representation of this model and to help determine the associated uncertainty estimates in some of its parameters that is consistent with the historical data available from the start of Hanford operations in 1943. Inverse modeling has already been completed for two ACMs and is documented in Cole et al. (2001) and Vermeul et al. (2001).

3. ACM Evaluation - This third step involves evaluating each ACM that has been developed to identify the plausible conceptual models that will be the subject of subsequent uncertainty assessments. Following the ACM development, some ACMs may drop out without the need for further evaluation or comparison with existing "plausible" ACMs based on their inability to reasonably match historical site data on parameters, and system responses. However, it is envisioned that there will be multiple plausible ACMs or ACM variations and some approach will be required to evaluate or rate them to determine those that are plausible enough to include in order to capture the subjective uncertainty associated with alternative plausible conceptual models. ACM evaluation will consist of examining statistical measures of overall model fit and examining, as appropriate, the model structure identification measures suggested and discussed in Carrera and Neuman (1986a,b,c).

4. ACM Uncertainty Assessment - Steps 1 through 3 of above must be completed for all reasonable ACMs, while this step is carried out only for the ACMs determined to be plausible in step 3. The parameter uncertainty assessment process generally involves

a. Model Complexity Optimization - This step involves sensitivity studies to identify the important or relevant parameters for the uncertainty analysis. The identification will be based on the uncertainty in the parameter value and the sensitivity of model predictions of interest to variations in the parameter. Optimization of model complexity is an issue and process that must be revisited at all steps of the uncertainty assessment because the total number of parameters whose uncertainty could be characterized with a pdf, and thus varied to produce uncertainty estimates, is large compared to the capacity to compute.

b. Characterize Parameter Uncertainty - This step involves developing the pdfs for the important uncertain parameters including the identification of any correlations among parameters.

c. Propagate Uncertainties - This step involves propagating the uncertainties through the model (e.g., by First Order Second Moment (FOSM) methods, if applicable, or by a Monte Carlo approach) to determine the uncertainty in the model predictions of interest.

5. Estimation of Combined ACM and Scenario Uncertainty - This step involves estimating the combined uncertainty by compositing the stochastic (or parameter) uncertainty associated with each particular scenario and plausible ACM. 
The composited uncertainty associated with the plausible ACMs for the various scenarios of interest represents the best estimate of uncertainty that can be developed based on current information and understanding. While the five steps above is a convenient way to describe the approach, there is considerable overlap in some of the items. This is because ACM identification and development requires examining and optimizing model complexity (as measured by the number and complexity of processes and parameters included) to be consistent with availability of information while attempting to honor the antithetical concept of model parsimony that requires the modeler to seek the simplest model parameterization consistent with the evidence. As discussed, while it is important to attempt to improve model accuracy and reliability by including as many relevant processes at the finest possible spatial and temporal discretizations, there is no guarantee that model reliability will continue to increase with model complexity.

The final important part of the uncertainty analysis framework is the identification, enumeration, and documentation of all the assumptions

- made during conceptual model development

- required by the mathematical model

- required by the numerical model

- made during the spatial and temporal descretization process (e.g., Figure 3.20)

- needed to assign the statistical model and associated parameters that describe the uncertainty in the relevant input parameters

- required by the propagation method.

Additionally, any issues or new hypotheses or ACM modifications identified during the development, testing, and uncertainty propagation step for a given ACM must be documented. This comprehensive documentation of the uncertainty analysis performed for each ACM will be the key to achieving transparency. Documentation of the information described above is important because the uncertainties related to these assumptions can only be addressed through additional sensitivity or uncertainty studies and/or alternative conceptual model evaluations. Additional sensitivity or uncertainty studies will also be required to determine the impact of new findings (e.g., new data or new geologic models) that are not included in an uncertainty assessment. 


\section{Acknowledgments}

We want to acknowledge the technical support provided by the following individuals whose ideas and insights in the area of conceptual and numerical model uncertainty helped to shape the ideas and concepts put forth in this report.

Steve Gorelick - Stanford University

Jim Mercer - HIS-Geotrans, Inc.

Charles Andrews - S. S. Papadoupoulos and Associates

Shlomo Neuman - University of Arizona

Jesus Carrera - Barcelona University

Eileen Poeter - Colorado School of Mines

Evan Anderman - Calibra Consulting

Mary C. Hill - U.S. Geological Survey

Mikhail F. Kanevski - Nuclear Safety Institute (IBRAE), Russian Academy of Sciences 


\section{Acronyms}

\begin{tabular}{|c|c|}
\hline $\begin{array}{l}\text { ARRAMIS } \\
\text { ACM }\end{array}$ & $\begin{array}{l}\text { Advanced Risk and Reliability Assessment Model Integrated S/W } \\
\text { alternative conceptual model }\end{array}$ \\
\hline CCDF & Complementary Cumulative Distribution Function \\
\hline $\mathrm{CDF}$ & Cumulative Distribution Function \\
\hline CFEST & Coupled Fluid Energy and Solute Transport code \\
\hline CRWMS M\&O & $\begin{array}{l}\text { Civilian Radioactive Waste Management and System Management and } \\
\text { Operating Contractor }\end{array}$ \\
\hline DOE & U.S. Department of Energy \\
\hline Ecology & Washington State Department of Ecology \\
\hline EIS & Environmental Impact Statement \\
\hline EPA & U.S. Environmental Protection Agency \\
\hline ERC & Environmental Restoration Contractor \\
\hline FEP & features, events, and processes \\
\hline FOSM & First Order Second Moment \\
\hline FY & fiscal year \\
\hline GIS & Geographic Information System \\
\hline $\mathrm{HCP}$ & Hanford Comprehensive Land-Use Plan \\
\hline HGWP & Hanford Groundwater Project \\
\hline HRA & Hanford Remedial Action \\
\hline HSRAM & Hanford Site Risk Assessment Methodology \\
\hline IAEA & International Atomic Energy Agency \\
\hline IBRAE & Nuclear Safety Institute, Russian Academy of Sciences \\
\hline ILAW & Immobilized Low-Activity Waste \\
\hline ISRM & In Situ Redox Manipulation \\
\hline LHS & Latin Hypercube Sampling \\
\hline LLBG & low-level burial grounds \\
\hline LLMW & low-level mixed waste \\
\hline LLW & Low-Level Waste \\
\hline $\begin{array}{l}\text { MEPAS } \\
\text { MODFLOWP }\end{array}$ & $\begin{array}{l}\text { Multimedia Environmental Pollutant Assessment System } \\
\text { Modular Flow Program }\end{array}$ \\
\hline
\end{tabular}




$\begin{array}{ll}\text { NAS } & \text { National Academy of Sciences } \\ \text { NCRP } & \text { National Council on Radiation Protection } \\ \text { NEPA } & \text { National environmental Policy Act } \\ \text { ND } & \text { number of observations } \\ \text { NP } & \text { number of parameters } \\ \text { NRC } & \text { U.S. Nuclear Regulatory Commission } \\ \text { NUREG } & \text { U.S. Nuclear Regulatory Guide (document indicator) } \\ \text { ORP } & \text { Office of River Protection } \\ \text { PA } & \text { performance assessment } \\ \text { pdf } & \text { probability density function } \\ \text { PHMC } & \text { Project Hanford Management Contractor } \\ \text { PNNL } & \text { Pacific Northwest National Laboratory } \\ \text { PRA } & \text { probabilistic risk assessment } \\ & \\ \text { QMC } & \text { Quasi Monte Carlo } \\ \text { REDOX } & \text { reduction and oxidation } \\ \text { RIP } & \text { Repository Integration Program } \\ \text { RL } & \text { Department of Energy, Richland Operations Office } \\ \text { RPP } & \text { River Protection Program } \\ & \text { Waste Isolation Pilot Plant (Carlsbad, New Mexico) } \\ \text { SAC } & \text { System Assessment Capability } \\ \text { SALDS } & \text { State Approved Land Disposal Site } \\ \text { SGM } & \text { site-wide groundwater model } \\ \text { SRS } & \text { Simple Random Sampling } \\ \text { TRU } & \text { transuranic (waste) } \\ \text { WWRS } & \text { Universal inverse modeling code } \\ \text { WIPP } & \text { U.S Neological Survey } \\ \text { USGS } & \end{array}$




\section{Contents}

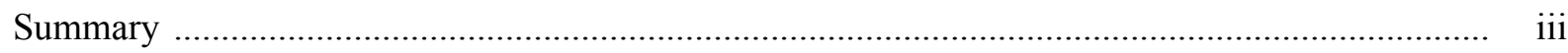

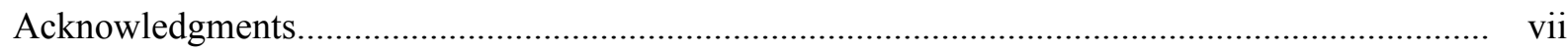

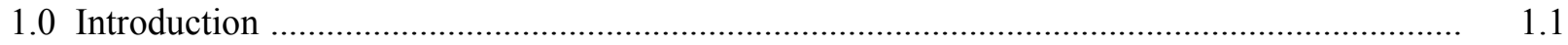

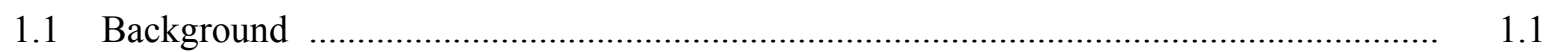

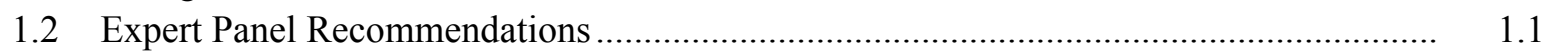

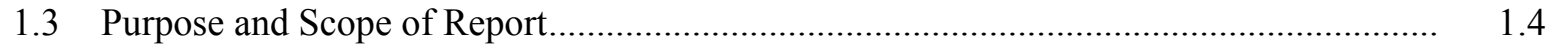

2.0 Current and Anticipated Scope of Site-Wide Groundwater Modeling .................................. 2.1

2.1 Regional-Scale Flow and Transport Analyses and Assessments.................................... 2.2

2.1.1 Tank Waste Remediation System Environmental Impact Statement ..................... 2.3

2.1.2 Hanford Remedial Action and Comprehensive Land-Use
Environmental Impact Statement................................................................. 2.4

2.1.3 Modeling Support to Hanford Site-Wide Groundwater Remediation Strategy ....... $\quad 2.5$

2.1.4 Modeling Support to Hanford Groundwater Monitoring Program ........................ 2.6

2.1.5 Composite Analysis of Radiological Sources in 200 Area Plateau ....................... 2.7

2.1.6 Future Needs for Regional Scale Flow and Transport Analyses ........................... 2.7

2.2 Support to Local-Scale Model Analyses and Assessments ............................................... 2.8

2.2.1 Appropriate Uses and Selected Examples ......................................................... 2.8

2.2.2 Inappropriate Uses and Selected Examples ........................................................ 2.11

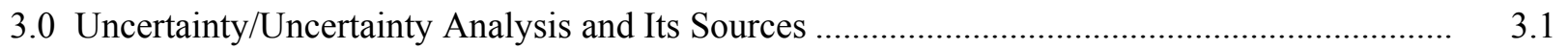

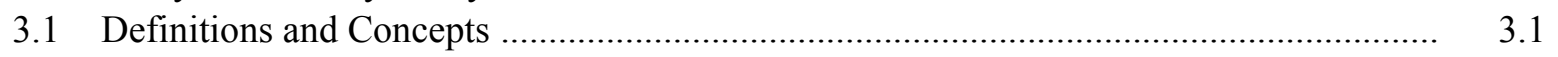

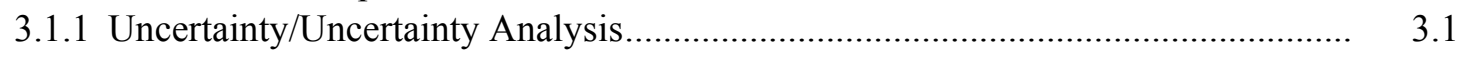

3.1.2 Modeling and Conceptual Models …............................................................. 3.20

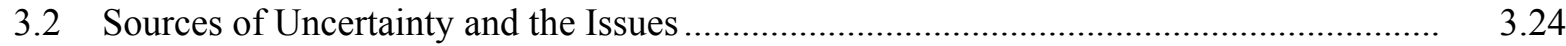

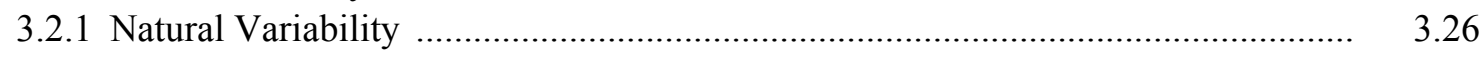

3.2.2 Uncertainty in Model Structure ................................................................... 3.31

3.2.3 Uncertainty in Model Parameters ................................................................... $\quad 3.40$

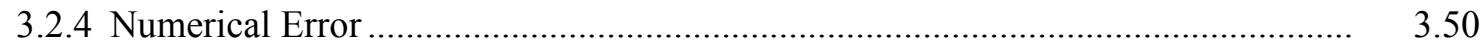

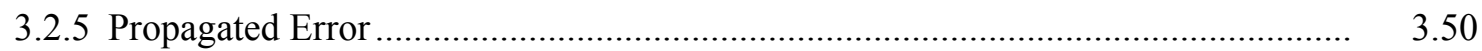

3.3 Approach for Addressing Groundwater Model Uncertainties ...................................... 3.51

3.3.1 Identify Alternative Conceptual Models............................................................. $\quad 3.52$

3.3.2 Develop and Evaluate Alternative Conceptual Models ....................................... $\quad 3.54$

3.3.3 Perform Uncertainty Assessment for Plausible ACMs ...................................... $\quad 3.54$

4.0 Conceptual Model of Aquifer System and Uncertainties ............................................... 4.1

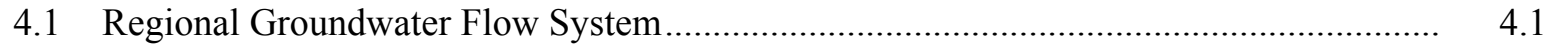

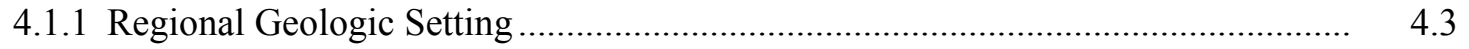

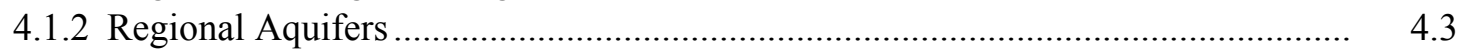


4.2 Local Groundwater Flow System ...............................................................................

4.2.1 Local Geologic Setting .................................................................................. 4.5

4.2.2 Local Unconfined Aquifer System ................................................................ 4.12

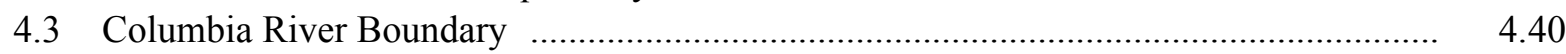

4.3.1 Interaction Between Groundwater and the Columbia River................................... 4.43

4.3.2 Uncertainty in Model Representation of the Columbia River ................................ 4.47

$4.4 \quad$ Yakima River Boundary ...................................................................................... 4.50

4.4.1 Groundwater-Yakima River Interaction ............................................................ 4.51

4.4.2 Uncertainty in Model Representation of the Yakima River ................................... 4.51

4.5 Basalt Above the Water Table .................................................................................... 4.52

4.6 Bottom Boundary of Local Aquifer System ........................................................... 4.52

4.6.1 Upper Basalt-Confined Aquifer System ............................................................... 4.53

4.6.2 Interface Between Basalt-Confined and Unconfined Aquifer Systems ................... $\quad 4.53$

4.6.3 Potential Mechanisms for Aquifer Intercommunication......................................... 4.53

4.7 Top Aquifer Boundary …………………………………………………….. 4.59

4.8 Natural Recharge from Precipitation and Springs …………………………………...... 4.59

4.8.1 Climate ................................................................................................... 4.62

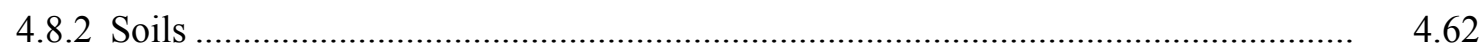

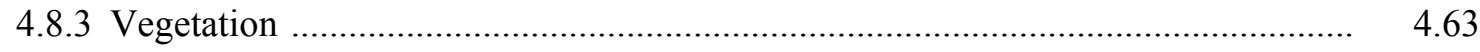

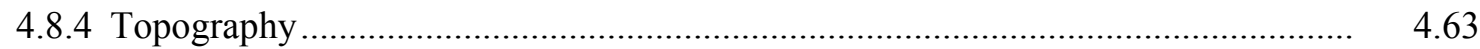

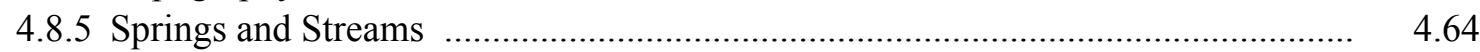

4.8.6 Uncertainties in Natural Recharge Estimates......................................................... $\quad 4.64$

4.9 Artificial Recharge at the Hanford Site …………….................................................. 4.67

4.9.1 Brief History of Waste Disposal at Hanford ........................................................... 4.67

4.9.2 Uncertainty in Artificial Recharge .................................................................... 4.68

4.10 Current Groundwater Use and Pumpage ................................................................... 4.69

4.11 Anticipated Future Flow Conditions ............................................................................... 4.69

4.12 Existing Radiological and Chemical Contamination ..................................................... 4.72

4.12.1 Current Distribution of Radiological and Chemical Contaminants..................... 4.73

4.12.2 Uncertainty in Distributions of Plumes ............................................................... 4.73

4.12.3 Potential Sources of Contaminants .................................................................... 4.76

4.12.4 Uncertainty Issues Related to Transport of Existing Plumes
and Future Contaminant Sources................................................................... 4.77

5.0 Technical Approach and Methods for Assessing Uncertainty in Hanford Site Groundwater Model Predictions ....................................................................................................... 5.1

5.1 Overall Technical Approach for SGM Uncertainty Framework ...................................... 5.1

5.2 Uncertainty Framework Implementation........................................................................ 5.7

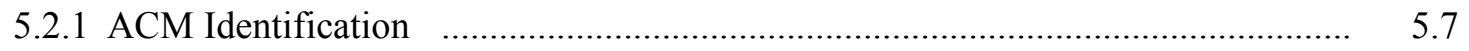

5.2.2 ACM Development..................................................................................... 5.13

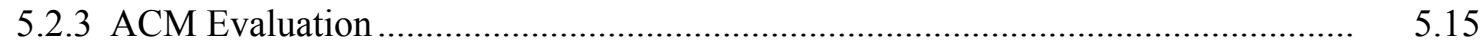

5.2.4 ACM Uncertainty Assessment............................................................................ 5.16

5.2.5 Estimation of Combined ACM and Scenario Uncertainty...................................... 5.20

5.2.6 ACM Assumption and Issue Tracking................................................................... 5.21 
Appendix A - Interpreted Top and Bottom Elevations of Major Hydrogeologic Units

Appendix B - Bibliography and Preliminary Information on Intercommunication Between the Unconfined Aquifer and the Uppermost Confined Aquifer at the Hanford Site

Exhibit 1 -Task 4.2: Effects of Surface Waste Disposal Activity on Ground-Water Levels in the Saddle Mountains Basalt

\section{Figures}

1.1 Location of the Hanford Site

3.1 Illustration of the Difference Between a Deterministic and Probabilistic Model for Concentration at Location $\mathrm{x}=2$.

3.2 Pictorial Representation of the Differences Between the Inputs and Outputs for a Two-Parameter Deterministic Model and a Two-Parameter Probabilistic Model for the Concentration, $\mathrm{C}$, at $\mathrm{x}=2$, Illustrated in Figure 3.1

3.3 Relationship Between Measures of Central Tendency in an Asymmetric Positively Skewed Probability Density Function

3.4 Three Different Graphical Ways to Present a Univariate Probabilistic Input or Result; as a Probability Density Function, as a Cumulative Distribution Function, and as a Complementary Cumulative Distribution Function ....

3.5 Probability Density Function Indicating How Areas Under the pdf Curve Relate to Various Probabilities of Occurrence

3.6 Scatter Diagrams a-f, Illustrate Various Possible Relationships Between Variables $x_{1}$ and $x_{2}$ and the Associated Pearson Correlation Coefficient Values, $\rho$

3.7 Bivariate Normal Densities with Means of 0, Standard Deviations of 1, and Pearson Correlations of $\rho=0, \rho=0.3, \rho=0.6$, and $\rho=0.9$

3.8 Plot of the Normal Frequency Distribution Indicating the Relationship Between the Central Tendency Measures, Mode, Mean, and Median for this Distribution and the Area Enclosed by Successive Standard Deviation Intervals Around the Mean

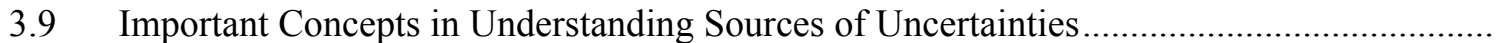

3.10 Accuracy and Precision in the Measurement Process

3.11 Frame of Reference for the Analysis of Uncertainty ........................................................ 3.19

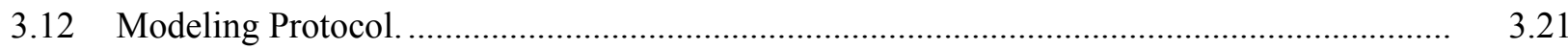

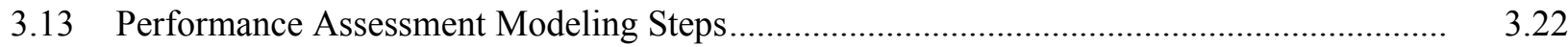

3.14 Scale Dependence of Porous Medium Parameters .............................................................

3.15 Measured River Stage and Well Responses at Various Distances from the Columbia River Near N-Springs on the Hanford Site During March 1995 
3.16 Examples of the Various Types and Scales of Spatial Variability in the Hanford Sediments on the Hanford Site.

3.17 Examples of the Various Types and Scales of Spatial Variability in Unit E of the Ringold Formation Across the River from the Hanford Site.....

3.18 Expected Relationship Between Parameter Variability and Uncertainty in the Average Parameter Value

3.19 Illustration of a Typical Relationship Between a Groundwater System Being Studied and the Rest of the Parts of the Full Hydrologic Cycle

3.20 Representation of a Real System by a Schematic of the Model System Domain Boundaries and the Representation of this System by a Rectangular Grid

3.21 Theoretical Development of Local, Intermediate, and Regional Flow Systems in a Deep Basin

3.22 Illustration of an Equivalent, Anisotropic, Porous Medium Consisting of Alternating Layers of Differing Macroscopic Properties

3.23 Diagram of Various Simple Processes that Could Affect Solute Concentrations in Porous Media...

3.24 Diagram at the Microscopic Scale of Various Type of Media

3.25 Images of a Discrete Model Representation of Point Bar Sediments in the Wabash River and a Continuous Stochastic Representation of the Same Parameter Field, in Which the First and Second Statistical Moments are Preserved Using the Spectral Synthesis Method

3.26 Illustration of Two-Scale Hybrid Model Approach

3.27 Hypothetical Diagram Illustrating the Importance Relationship that Links Parameter Uncertainty and Sensitivity to Parameter Importance in Uncertainty Assessment

3.28 Hypothetical Illustration of the Effect of Support Volume on Hydraulic Conductivity Parameter Uncertainty Related to Natural Variability

3.29 Example of CCDF that Attempts to Account for Both the Stochastic and Subjective Uncertainty Through a Family of CCDFs

3.30 Three CDFs Constructed from Various Numbers of Random Samples of a Simulated Output Developed from a Normal Distribution to Illustrate the Bootstrap Procedure for Determining the Number of Monte Carlo Model Samples to Achieve a Desired Level of Precision in the Estimate of the Mean $(\bar{y})$.

3.31 Number of Monte Carlo Samples to Achieve a Desired Level of Precision in Estimate of the Mean for Two Different Distributions

3.32 Sampling, Gaps and Clusters Associated with SRS for a Two-Dimensional Uncertain Input Vector, $z=\left(z_{1}, z_{2}\right)$, Comparison of Sampling Uniformity for a Uncorrelated and Highly Correlated Two-Dimensional Uncertain Input Vector, $z=\left(z_{1}, z_{2}\right)$, for Three Different Sampling Approaches SRS, LHS, and a Hammersley Sequence.

4.1 Extent and Cross Section of the Regional Columbia Plateau Aquifer System 
4.3 Distributions of the Pre-Missoula Gravel Facies and Fine-Grained Facies of the

Plio-Pleistocene Unit on the Hanford Site..................................................................... 4.8

4.4 Photograph of Hanford Formation Gravel Showing Open Matrix Structure. ....................... 4.9

4.5 Structural Geologic Features of the Pasco Basin................................................................ 4.11

4.6 Map of SGM Hydrogeologic Units Containing the Water Table in March 1999. ................ 4.13

4.7 Interpreted Water Table for March 1999 Measurements. ....................................................

4.8 Comparison of Geologic Stratigraphy and Hydrogeologic Units Defined for the SGM........ 4.19

4.9 North-South Cross Section of Lindsey (1995) Compared with the SGM Model Units........ 4.20

4.10 East-West Cross Section of Lindsey (1995) Compared with the SGM Model Units............. $\quad 4.21$

4.11 Extent of SGM Hydrogeologic Unit 4 (Upper Ringold Muds). .......................................... 4.22

4.12 Extent of SGM Hydrogeologic Unit 5 (Ringold Gravel). ......................................................

4.13 Extent of SGM Hydrogeologic Unit 6 (Ringold Over Bank Muds)....................................

4.14 Extent of SGM Hydrogeologic Unit 7 (Ringold Gravel). ..................................................

4.15 Extent of SGM Hydrogeologic Unit 8 (Lower Ringold Muds)..........................................

4.16 Extent of SGM Hydrogeologic Unit 9 (Basal Ringold Gravel).........................................

4.17 Distribution of Boreholes Used to Determine Hydrogeologic Structure for the SGM.......... 4.29

4.18 Location of the May Junction and Cold Creek Faults. ........................................................

4.19 Distribution of Wells with Hydraulic Conductivity Determined from

Aquifer Pumping Tests............................................................................................ 4.34

4.20 Average Daily Flows of the Columbia River from 1991 Through 2001 ............................. 4.41

4.21 Cross Sections of the Columbia River at Three Locations Showing High and Low River Stages.

4.42

4.22 Location of Cross Sections Shown in Figure 4.21.

4.23 Geologic Cross Section of Lindsey (1995) Across the Columbia River Near the 100 F Area.

4.24 North-South Cross Section of Lindsey (1995) Across the Columbia River East of the $100 \mathrm{~F}$ Area.

4.25 Geologic Cross Section of Lindsey (1995) Across the Columbia River South of the Old Hanford Townsite.

4.26 Generalized Cross Section of the Groundwater/Columbia River Interface at $100 \mathrm{H}$

\subsection{Water-Level Responses at Three Wells Compared to Columbia River} Stage Fluctuations.

4.29 Average Daily Flows for the Yakima River at Kiona from 1991 Through 2001 ................ $\quad 4.50$

4.30 Isopach Map of the Elephant Mountain Basalt on the Hanford Site. .....................................

4.31 Isopach Map of the Elephant Mountain Basalt in the West Lake and B Pond Area............. $\quad 4.55$

4.32 Isopach Map of the Rattlesnake Ridge Interbed in the West Lake and B Pond Area. .......... $\quad 4.56$

4.33 Isopach Map of the Pomona Basalt in the West Lake and B Pond Area..............................

4.34 Photograph of West Lake Looking Northeast Toward Gable Mountain............................... 4.60

4.35 Estimates of Hanford Site Recharge for 1979 Conditions.................................................... 4.61

4.36 Water Supply Wells on the Hanford Site. ..................................................................... 4.70

4.37 Extent of Radiological Contaminant Plumes at the Hanford Site. ....................................... 4.74

4.38 Extent of Hazardous Chemical Contaminant Plumes at the Hanford Site. .......................... $\quad 4.75$ 
5.1 Simulated Versus Observed Heads for All Observations Through Time for Prior Model and Baseline Model....

5.2 Histograms of Predicted Head Residuals for All Observations for Baseline Model and Best-fit Basalt Model.

5.3 Probability of Unit 4 Presence Estimated by Probabilistic Neural Network Methods and by Taking the Average of 50 Sequential Indicator Simulations

5.4 Facies-Based Zonation of Model Unit 1 and Unit 5 as Exposed at the Water Table

\section{Tables}

3.1 Maximum Entropy Probability Distributions ....................................................................

4.1 Sediment Facies Types in Ringold Deposits .................................................................. 4.7

4.2 Summary of Characteristics and Depositional Environments of the

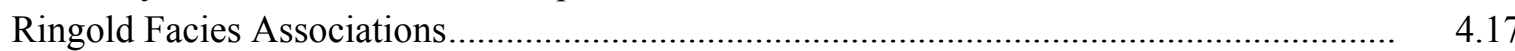

$4.3 \quad$ Hydraulic Test Results for Mud-Dominated Units........................................................ 4.35

4.4 Summary of Current Hydraulic Parameter Estimates Interpreted from Field and Laboratory Tests for the Conceptual Model Hydrogeologic Units

5.1 Principles of Good Practice for Monte Carlo Simulation .................................................. 5.6 


\subsection{Introduction}

At the request of the U.S. Department of Energy, Pacific Northwest National Laboratory (PNNL) embarked on a new initiative to strengthen the technical defensibility of the predictions being made with a site-wide groundwater flow and transport model at the U.S. Department of Energy (DOE) Hanford Site in southeastern Washington State (Figure 1.1). The initial focus of the initiative is on the characterization of major uncertainties in the current conceptual model that would affect model predictions. The long-term goals of the initiative are the development and implementation of an uncertainty estimation methodology in future assessments and analyses using the site-wide groundwater model. This report focuses on the development and implementation of an uncertainty analysis framework.

Since the inception of the Hanford Site in 1943, Hanford activities have discharged large volumes of wastewater to a variety of waste facilities. These operational discharges have raised the water table, created groundwater mounds, and been the source of local and regional-scale contaminant plumes under waste management sites and facilities along the Columbia River and in the central part of the Site. Since 1988, the mission of the Hanford Site has changed from weapons production to environmental restoration. As a result in this primary change in mission, wastewater discharges have declined significantly, which caused the water table to decline significantly over the past decade.

\subsection{Background}

In 1996, the U.S. Department of Energy, Richland Operations Office (RL) initiated a project to consolidate multiple groundwater models at the Hanford Site into a single consolidated site-wide groundwater model. Out of that process, RL selected a three-dimensional groundwater flow and transport model developed by the Hanford Groundwater Project (DOE-RL 2000) as the preferred alternative for the initial phase of the site-wide groundwater-model-consolidation process.

\subsection{Expert Panel Recommendations}

In fall 1998, an external peer review panel was convened to conduct a technical review of the selected Hanford site-wide groundwater model (SGM). The three-member review panel was asked to comment on three specific issues: 1) adequacy of the conceptual model and its technical capabilities to meet the anticipated uses and needs, 2) possible improvements to the modeling framework and implementation, and 3) immediate new data needs. Results of their review are documented in a formal report transmitted to RL on January 14,1999 . $^{\text {(a) }}$

(a) Gorelick, S., C. Andrews, and J. Mercer. 1999. Report of the Peer Review Panel on the Proposed Hanford Site-Wide Groundwater Model, January 14, 1999. Letter Report to U.S. Department of Energy, Richland Operations Office, Richland, Washington. 


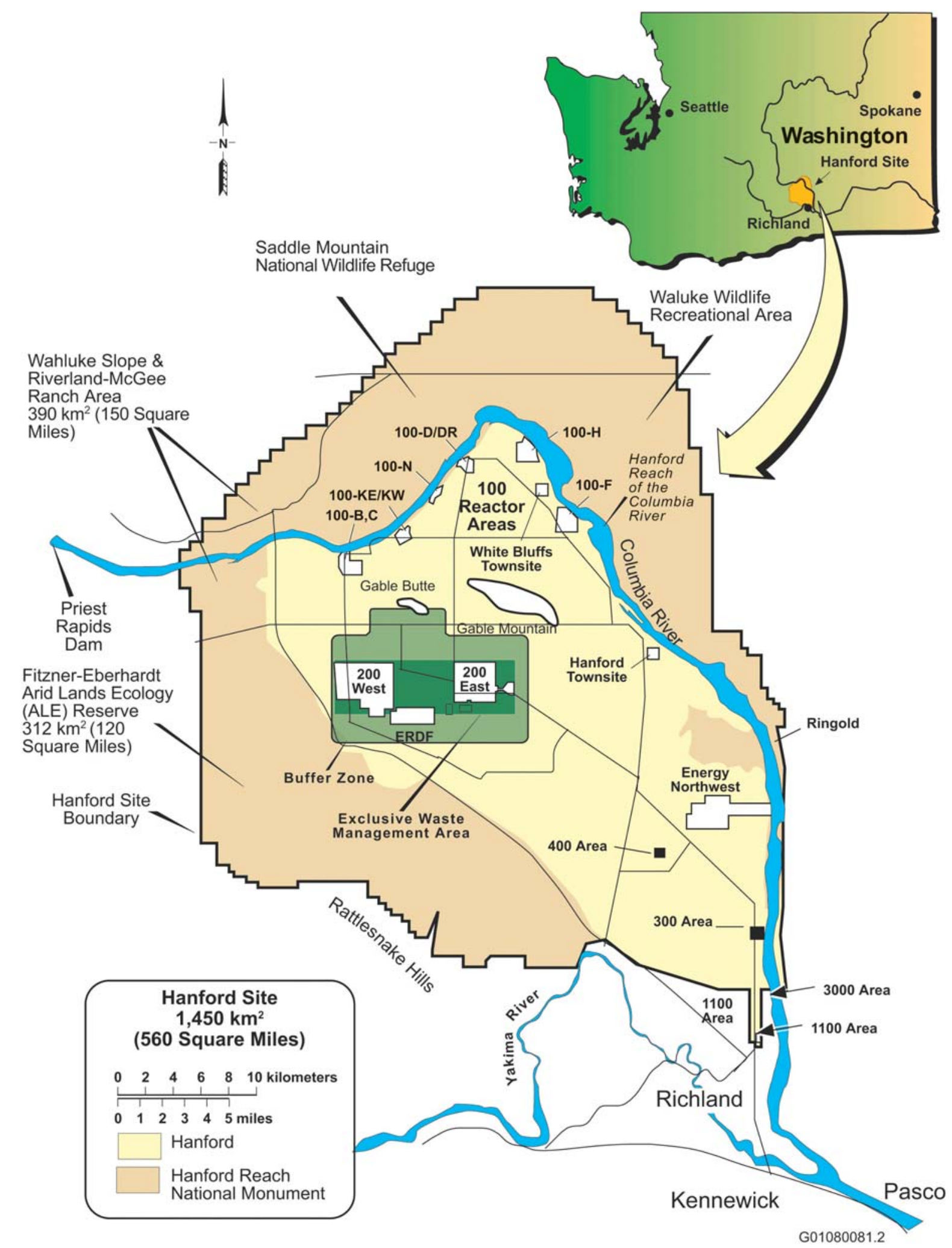

Figure 1.1. Location of the Hanford Site. 
The panel indicated that the spectrum of anticipated uses and needs for the model is so broad, ranging from time scales of less than 1 day to thousands of years and spatial scales of meters to kilometers, that this or any general-use, site-wide model cannot be expected to be adequate for all potential uses. They suggested an initial task be undertaken to specify a narrower, and perhaps more pragmatic, list of model uses that involve less disparate temporal and spatial scales and contaminants whose behavior can be adequately characterized by linear sorption and first-order decay.

Regarding improvements in the modeling framework, the panel made several comments and related recommendations that centered on a broad theme of uncertainty. The key comments and recommendations from their review are as follows:

- The existing deterministic modeling effort has not acknowledged that the prescribed processes, physical features, initial and boundary conditions, system stresses, field data, and model parameter values are not known and cannot be known with certainty. Consequently, predictions of heads and concentrations in three dimensions over time will be uncertain as well.

- A new modeling framework must be established that accepts the inherent uncertainty in model conceptual representations, inputs, and outputs. Given such a framework, the expected values of heads and concentrations, as well as the ranges (or distributions) of predictions, would be products of the site-wide groundwater model.

The panel recommended that the concept of uncertainty be acknowledged and that a new modeling framework be established that is stochastic rather than purely deterministic. Both the expected values of heads and concentrations as well as the spread of predicted values (about these expected values) should be produced by the model. The panel suggested that different conceptual models be considered and their potential impact on predictive uncertainty be assessed.

More specifically, the panel requested an assessment of the relative importance of uncertainties due to alternative model structures and constructs of processes (e.g., different zonation, different boundary conditions, large-scale features, stresses, chemical reactions) and due to variations in parameter values. This includes uncertainties related to model scale and resolution. For cases where significant variations in parameters are known to exist (on grid scale), the panel recommended using Monte Carlo simulations to determine the expected values of predictions and their associated uncertainties.

The panel also suggested that a priority item for the model consolidation effort is to construct a comprehensive list of alternative conceptual model components and assess each of their potential impacts on predictive uncertainty. Tools that will aid in such hypothesis testing include inverse modeling of flow and transport (which includes sensitivity and first-order analyses) followed by model structure identification criteria used by Carrera and Neuman (1986a,b,c) as discussed in Section 3.2.2 and Section 5.2.3.

Regarding the collection of new data, the panel indicated that it is premature to initiate a campaign to collect new data. The highest priority is to adopt a broader modeling framework that accepts conceptual model uncertainty. Within this new framework, the site-wide model would serve as an important tool to help guide new data collection efforts. First, the degree of likely impacts of the various sources of 
uncertainty can be assessed through analysis of all uncertainties including those introduced by alternative conceptual models. Second, the worth of new data for reducing costs and risks can be evaluated. Only then can the issue of additional data collection be logically addressed.

\subsection{Purpose and Scope of Report}

The purpose of this report is to describe the development of an uncertainty analysis framework for the Hanford SGM that will enable the quantification of key uncertainties in model predictions of groundwater flow and transport. Section 2 of this report discusses appropriate uses and limitations of the current sitewide groundwater model to support regional-scale and local-scale flow and transport analysis and assessments. Section 3 presents a variety of definitions of uncertainty/uncertainty analysis to illustrate the complexity of the subject, introduces a variety of statistical concepts important to parameter uncertainty analysis, discusses the sources of uncertainty and taxonomies to categorize these sources, and concludes by describing approaches for identifying, combining, and propagating these uncertainties. Section 4 summarizes the current understanding of the Hanford Site aquifer system (i.e., the conceptual model) and the associated uncertainties with various components of the overall system and conceptual model. Section 5 discusses the overall technical approach and methods to be developed for use in assessing uncertainty in Hanford Site future predictions of groundwater and contaminant transport.

Appendix A provides a summary of top and bottom elevations of each hydrogeologic unit used in the Hanford site-wide groundwater flow and transport model as interpreted from well data that provide the basis for the interpreted distributions and thicknesses of major hydrogeologic units summarized in Section 4.

Appendix B is an initial bibliography and summation of hydrogeologic data relative to the intercommunication between the unconfined aquifer and the uppermost confined aquifer at the Hanford Site. 


\subsection{Current and Anticipated Scope of Site-Wide Groundwater Modeling}

As stated in DOE-RL (2000), site-wide groundwater modeling is an important component of an overall system-assessment capability at the Hanford Site needed to quantify the environmental consequences of past, present, and future DOE activities at impacted compliance boundaries and receptor points at the Site and within the region. Because of the long-term nature of many of the studies and assessments at the Hanford Site, groundwater modeling capabilities are needed to evaluate the past, present, and anticipated future transient behavior of the groundwater system. The planned cessation of past practices of discharging dilute waste liquids to the subsurface has resulted in water table declines of the unconfined aquifer that will cause near- and long-term changes in groundwater flow patterns. These flow patterns may also be impacted by future land uses and water resources impacts both on and outside of the Hanford Site. Changes in on-site land uses may result as lands outside of the exclusive waste management and buffer areas (Figure 1.1) are remediated and released to the general public for alternative land uses.

Groundwater models must be able to assess current and future impacts of the groundwater transport of a broad variety of radioactive and chemical contaminants of varying environmental mobility. On a site-wide scale, the migration of relatively mobile, long-lived radionuclides and chemical contaminants presents the highest potential long-term threats to the environment and to human health and safety and is the major target of the site-wide groundwater-modeling effort.

The specific analyses and assessments that have or will potentially use the groundwater modeling are defined by the diverse locations of waste on the Site in the 100, 200, and 300 areas (Figure 1.1), and a number of miscellaneous waste sites outside these main operating areas. Several hundred individual waste sites within the exclusive waste management area and buffer zone may need to be analyzed using a combination of source-release, vadose-zone, and groundwater models to evaluate their potential impacts. Groundwater models must be able to evaluate the potential impacts of past practices of discharging large volumes of liquid wastes to the subsurface, and accidental and unplanned leaks and releases over the past 50 to 55 years that have already impacted the unconfined aquifer system and may be seen for decades to come. Groundwater models must also be able to predict the potential impacts from past disposal of solid low-level radioactive wastes (LLW) and transuranic (TRU) and mixed wastes and future disposal of solid LLW and mixed wastes that may impact the groundwater system for several hundreds to thousands of years.

The following section of the report provides a discussion of appropriate uses and limitations of the current site-wide groundwater model to support regional-scale and local-scale flow and transport analysis and assessments with selected examples to illustrate the key points of both appropriate and inappropriate uses of the site-wide model. 


\subsection{Regional-Scale Flow and Transport Analyses and Assessments}

While not necessarily applicable to all types of groundwater modeling assessments that will be done at the Hanford Site, the current design of the site-wide groundwater model is a very suitable tool for evaluating flow conditions (i.e., water table elevations and flow directions) in the Hanford Site unconfined aquifer system, which occupies hundreds of square miles (Figure 1.1). Given the scale of the interpretations of the hydrogeologic units used in the site-wide groundwater model (i.e., 9 geohydrologic units with thicknesses to $60 \mathrm{~m}$ ) and the current numerical implementation (i.e., $\sim 375 \mathrm{~m}$ grid) transport predictions would be appropriate only at spatial scales exceeding $1 \mathrm{~km}$. The appropriate temporal scale for the site-wide scale groundwater model would correspond to the time for water to flow over these distances (i.e., years to decades or more).

The current site-wide groundwater model would also be considered generally suitable for evaluation of regional-scale transport of contaminants whose mobility is unaffected by chemical processes other than those that can be represented by the linear equilibrium sorption-desorption process described by a soil/water equilibrium partition coefficient or $\mathrm{K}_{\mathrm{d}}$. Additional modeling capabilities or codes would be required to address transport predictions of contaminant behavior that are potentially affected by more complex chemical processes (e.g., nonlinear sorption created by waste stream composition, $\mathrm{pH}$ effects, and/or presence of organics or complexants; precipitation/dissolution; oxidation-reduction; complexation; abiotic/biotic reduction; biodegradation; colloids transport; diffusive mass transport, or the effect of nonaqueous phase liquids, etc.).

As indicated in Gorelick et al. (1999), the current site-wide model is capable of representing transport of individual noninteracting solutes undergoing first-order decay and linear sorption. First-order decay is appropriate to represent radioactive decay, and may be appropriate for representing simple degradation processes that can be appropriately represented by a first-order degradation reaction. These processes are a small subset of all possible chemical processes, and may not be adequate for some compounds of concern at the Hanford Site. As it stands, the responsibility for the use of the limited chemistry in the SGM to simulate a particular contaminant rests on the model user.

The use of $\mathrm{K}_{d} \mathrm{~S}$ is an engineering approach to represent the retardation of contaminants moving at trace levels through the groundwater system as a result of various sorption-desorption mechanisms. Such an approach does not restrict the use of the model for prediction of the movement of a majority of contaminants of concern at the Hanford Site. For the most part the majority of the contaminants of concern are the relatively mobile, long-lived radionuclides and chemical contaminants because they present the greatest potential long-term threats to the environment and to human health and safety. For applications involving the migration of tritium through the aquifer, the chemical processes in the SGM (decay and no sorption) are adequate. For other contaminants, such as carbon tetrachloride, the model may provide reasonable predictions if volatilization from the dissolved phase is negligible, water quality is nearly constant, and the chemistry can be represented by first-order decay and linear sorption. In any application of the SGM, justification of the engineering approach to retardation is needed. 
For regional-scale transport under Hanford Site conditions, contaminants that would appropriately be evaluated with the site-wide model generally fall into the category of long-lived or persistent and mobile contaminants like those we currently see in the unconfined aquifer system. For current conditions, these contaminants include radiological constituents like tritium, technetium-99, uranium, iodine-129, and hazardous chemicals like carbon tetrachloride, trichlorethylene, chromium, and nitrate. Several other radiological constituents can be found within the aquifer system that may be significant on a local scale, but because of their affinity to sorb onto Hanford sediments they are not important on a regional transport scale. These constituents include radiological contaminants like cesium-137, strontium-90, plutonium, and americium isotopes that are highly sorbed.

Given the limitations of the current site-wide model transport capabilities, careful consideration should be given to the potential geochemical factors affecting the mobility of each contaminant being evaluated in a groundwater assessment (e.g., $\mathrm{pH}$, redox). Transport of contaminants whose mobility is potentially impacted by processes other than simple linear equilibrium sorption should be evaluated using additional appropriate modeling capabilities.

Some excellent examples of modeling studies and investigations involving appropriate uses of a sitewide model and its general capabilities at the Hanford Site include recent modeling studies described in DOE Tank Waste Remediation System Environmental Impact Statement (TWRS EIS) 1994, DOE Hanford Remedial Action Environmental Impact Statement and Comprehensive Land-Use Plan (HRA EIS) 1996, Chiaramonte et al. (1997), Cole et al. (1997), and Kincaid et al. (1998). A brief description of the each of these analyses and how a site-wide model was used is summarized in the following section.

\subsubsection{Tank Waste Remediation System Environmental Impact Statement}

The TWRS EIS addresses actions proposed by DOE to manage and dispose of radioactive, hazardous, and mixed waste within the River Protection Program (RPP) at the Site (DOE 1996b). The waste includes more than 177 million curies in about 212 million liters of waste stored or to be stored in underground tanks in the 200 Area Plateau. This EIS also addresses DOE's plans to manage and dispose of 1930 capsules containing 68 million curies of cesium and strontium.

As part of this EIS, environmental consequence analyses were performed to evaluate the impacts of a number of tank-waste-management alternatives including continued management with no retrieval, minimal-retrieval, partial-retrieval, and extensive-retrieval alternatives. The groundwater part of the consequence analysis evaluated contaminant transport through the saturated unconfined aquifer using a model based on the VAM2D code (Huyakorn et al. 1991) at each of the eight tank-source areas and the Immobilized Low-Activity Waste (ILAW) disposal facility. The spatial scale of the analysis was to evaluate the potential impacts over several kilometers between the waste sites and the Columbia River. The time scale of the analysis was primarily focused on the Hanford Post Closure period (i.e., ten thousand years beyond 2050). 
The first phase of the modeling effort entailed establishing the steady state flow field that was consistent with previous site-wide groundwater flow simulations (Wurstner and Devary 1993). This was accomplished by adopting, as closely as possible, the hydraulic parameters used in the site-wide groundwater model. The steady state results obtained with the VAM2D model matched results previously reported. This effort made use of EarthVision and ARC/INFO software capabilities to translate parameter distributions used for the CFEST (Gupta et al. 1987; Cole et al. 1988) version of the site-wide model into formats suitable for use by VAM2D.

\subsubsection{Hanford Remedial Action and Comprehensive Land-Use Environmental Impact Statement}

As part of the transition from production of nuclear materials for national defense to environmental restoration and long-term management of wastes, DOE needs to determine the optimum use of Hanford Site lands, facilities, and resources and how these lands and facilities should be remediated to allow for beneficial future uses. In response to public comment, DOE changed the name of this environmental impact statement from the HRA EIS (DOE 1996a) to the Hanford Comprehensive Land-Use Plan EIS (HCP EIS) (DOE 1999b). In the Notice of Intent in 1992, establishing future land uses was listed as one of the HRA EIS objectives. Since that time, various considerations have led to the Final HCP EIS in which future land use is the main objective. To reflect this reduction in scope from the 1996 Draft HRA EIS, DOE solicited comments on the proposed name change (as well as the contents), and in response to comments changed the name to the HCP EIS.

Originally, this EIS was intended to provide an environmental review under the National Environmental Policy Act of 1969 (NEPA) for all aspects of the developing Hanford Environmental Restoration Project. The document, however, no longer directly considers remediation issues. Instead, remediation issues are now integrated into specific Tri-Party Agreement remediation decision documents. Remediation decisions are made by the U.S. Environmental Protection Agency and the State of Washington, as lead regulatory agencies, and DOE as lead implementing agency. The DOE expects that the EIS process will assist Hanford remediation efforts by determining reasonably foreseeable land uses and establishing land-use decision-making processes to ensure the viability of any future institutional control that might be required.

In the original HRA EIS (DOE 1996a), the approach used to assess the human-health impacts for the land-use alternatives combined individual waste sites into groups and integrated the effects of potential releases to the environment. This was accomplished by grouping waste sites by medium (e.g., soils, groundwater) and aggregating the waste sites into $1-\mathrm{km}^{2}\left(0.4-\mathrm{mi}^{2}\right)$ cells in a grid overlaid on the Hanford Site. The potential contaminant release and transport through the environment from each $1-\mathrm{km}^{2}\left(0.4-\mathrm{mi}^{2}\right)$ cell were estimated using the Multimedia Environmental Pollutant Assessment System (MEPAS) computer model (Droppo 1991). Modeling results from multiple cells were combined to estimate the contaminant concentrations in the soil, groundwater, surface water, and air to which a human or ecological receptor might be exposed. Source-term data were compiled from the Waste Information Data System, Solid Waste Information Tracking System, and Hanford Environmental Information System databases, and from field investigation reports and other sources, when applicable. 
The risk to a given receptor was determined by estimating the quantity of contaminant transported from a source to that receptor. Risk calculations were simplified by separating the computational process into discrete modules. These modules included the source (waste) terms, contaminant-transport mechanisms, exposure scenarios, and the variables used to calculate the risk or hazard index from a given exposure. The MEPAS model was used to estimate risk.

As stated in DOE (1996a), MEPAS was selected because it was the only multimedia computer model that included all of the required features. MEPAS 1) addresses radioactive and hazardous chemical wastes, 2) provides user flexibility by allowing the use of site-specific data, 3) performs on- and off-site calculations, 4) is largely based on the solutions to the advection-dispersion equations for solute transport, 5) includes the ability to model various atmospheric transport mechanisms, 6) addresses both active and inactive sites and releases, 7) allows for arbitrary time-varying source-term emission rates, and 8) addresses contaminated soils, ponded sites, liquid discharges, injection wells, and point, line, and area sources.

To better represent the distribution of contaminants (and risk) over the Hanford Site, the groundwater transport portion of MEPAS was solved along aquifer flow path lines originating at all $1-\mathrm{km}^{2}$ cells representing waste sites. Straight-line approximations to the path lines were used to accommodate the assumption of one-dimensional advection used in MEPAS. The path lines were based on the predicted flow field from 1992.

To generate path lines for input to MEPAS, the unconfined aquifer at the Hanford Site was simulated with a site-wide groundwater model developed under the Groundwater Surveillance Project (Wurstner and Devary 1993). This two-dimensional groundwater flow model used the finite element code CFEST (Gupta et al. 1987). The spatial scale of the analysis was focused on predicting contaminant concentration levels on a regional scale between the wastes and the Columbia River. The temporal-scale of this analysis ranged from the present to post-Hanford conditions (i.e., from site closure at $\sim 2050$ to thousands of years beyond 2050).

\subsubsection{Modeling Support to Hanford Site-Wide Groundwater Remediation Strategy}

The Hanford Site-Wide Groundwater Remediation Strategy, summarized in Law et al. (1996) and Chiaramonte et al. (1997), describes the approach to remediate the major groundwater contaminant plumes in the 100 and 200 areas of the Hanford Site. As part of the strategy, a site-wide groundwater model was developed to be used in estimating the effectiveness of alternative groundwater cleanup approaches to support planning and implementation of remediation alternatives, to support risk assessments, and to evaluate the impact of changes in the groundwater flow field.

Geologic and hydrogeologic conceptual models were based primarily on a regional synthesis of data and information presented in a number of previous studies. The VAM3D-CG code (Huyakorn and Panday 1994) was used to perform the modeling. Two hydrostratigraphic units were represented in the model, the pre-Missoula/Hanford formation and the Ringold Formation. Calibration of the model was initially carried out by adjusting the assigned hydraulic conductivities, solving for the steady state flow field, and comparing the model results to the average water level measurements from 1976 to 1979. 
Transient flow simulations of 14 years were also carried out during the calibration, with comparisons of the hydraulic head field during 1988 and 1993 used to evaluate the numerical model. Finally, a simulation of tritium transport was carried out for the same 14-year period to further evaluate the calibrated model. Tritium concentrations from 1979 were used as the initial condition.

The calibrated groundwater model was used to predict water table elevations and contaminant transport for several key contaminant plumes (tritium, iodine-129, uranium, technetium-99, nitrate, carbon tetrachloride, trichloroethylene, and chloroform) for 200 years using 1995 data as the initial condition. Initial sources in the 100 and 200 areas were modeled. For those contaminants that contributed to risk, an estimate of cumulative risk was made using the industrial and residential scenarios defined in the Hanford Site Risk Assessment Methodology (HSRAM) (DOE-RL 1995a).

\subsubsection{Modeling Support to Hanford Groundwater Monitoring Program}

In Cole et al. (1997), a three-dimensional site-wide model of groundwater flow and transport was developed under the Hanford Groundwater Project to increase the understanding of contaminant transport on the Site and to better forecast the migration of the contaminant plumes being monitored by the project. The falling water table, resulting from the cessation of Hanford operations, is reducing the saturated extent of the highly conductive Hanford formation and is also causing the water table to move from the Hanford formation into mud units of the underlying Ringold Formation. Consideration of the complicated interactions on future predictions resulting from the rapidly falling water table in the complex hydrostratigraphy of the Hanford Site was a major consideration that predicated development of a three-dimensional model.

The geologic conceptual model for the three-dimensional application was developed from available well logs, which were used to define the lateral and horizontal extent of the major hydrogeologic units of the Ringold and Hanford formations. Interpreted areal distributions and thicknesses for the major units were integrated with EarthVision, a three-dimensional visualization software package, which was then used to construct a database of the three-dimensional site conceptual model. The resulting conceptual model contains nine hydrogeologic units above the uppermost basalt.

The boundary conditions for the three-dimensional model were similar to those used in a previously developed two-dimensional model. To determine the three-dimensional spatial distribution of hydraulic parameters, the steady state, two-dimensional model of the unconfined aquifer system used in Jacobson and Freshley (1990) was recalibrated to 1979 water table conditions using the statistical inverse method implemented in CFEST-INV (Devary 1987). The three-dimensional hydraulic conductivity was set such that it was consistent with the two-dimensional results of the recalibration and also with knowledge of the three-dimensional structure of the aquifer and the estimated properties of the hydrogeologic units. Specific yield of the three-dimensional model was also calibrated to match the observed, transient water table elevations between 1979 and 1996.

The three-dimensional model was applied to predict the future response of the water table to postulated changes in Hanford operations. Modeling activities included three-dimensional model simulations of the existing tritium, iodine-129, technetium-99, uranium, and strontium-90 plumes 
originating from the 200 Area Plateau. Each of the transport simulations was based on the predicted future transient-flow conditions and a high-resolution, finite-element grid designed to resolve transport calculations in the areas of current and future contamination.

\subsubsection{Composite Analysis of Radiological Sources in 200 Area Plateau}

A radiological impacts analysis (Composite Analysis) was performed by Kincaid et al. (1998) for low-level waste disposal sites and other contaminant sources in the 200 Area Plateau. The objective of the analysis was to assess cumulative dose impacts to hypothetical future members of the public in an accessible environment postulated between the 200 Area Plateau and the Columbia River during the 1000 years after projected site closure (2050). Estimating dose for the Composite Analysis was a multistep process involving 1) estimation of radiological inventories and releases for 241 unique source sites to the environment; 2) assessment of contaminant migration through the vadose zone, groundwater, and atmospheric pathways; and 3) estimation of doses for scenarios based on agricultural, residential, industrial, and recreational land use. The radionuclides included in the vadose zone and groundwater pathway analyses of releases were those in existing plumes including iodine-129, strontium-90, technetium-99, tritium, and uranium. They also included those that will be important in future sources over a 1000-year period including carbon-14, chlorine-36, iodine-129, selenium-79, technetium-99, and selected uranium isotopes.

As part of this analysis, site-wide groundwater modeling was carried out to assess dose impacts for the off-site transport of existing plumes and future releases of contaminants from categories of waste sites in the 200 areas. Flow and transport in the unsaturated zone beneath each individual source was modeled in one-dimension using STOMP (White and Oostrom 1996, 1997; Nichols et al. 1997). Contaminant fluxes to the aquifer resulting from the STOMP simulations were used as input to a three-dimensional model of groundwater flow and transport. Flow conditions were simulated from 1996 to the year 4000 using projected operational discharges and estimates of natural recharge. Current and future contaminant

plume transport was simulated from present day conditions to the year 3000. Forecasts of concentrations of key radioactive contaminants provided the basis for final dose calculations using standard dose conversion methodologies and exposure scenarios and parameters identified by the HSRAM (DOE-RL 1995a). Dose impacts from the existing plumes and future releases of contaminants were assessed in the area outside of the waste-management exclusion areas and the surrounding buffer areas established by the Future Site Uses Working Group. Potential dose impacts to the public after site closure in 2050 for four potential exposure scenarios derived from HSRAM (the agricultural, residential, industrial, and recreational exposure scenarios) were evaluated.

\subsubsection{Future Needs for Regional Scale Flow and Transport Analyses}

Activities in which regional-scale flow and transport analyses using the site-wide groundwater model will be required in the next 3 to 5 years include:

- the Solid Waste EIS

- continued modeling support to the HGWP 
- future revisions and updates of the Composite Analysis of the 200 Area Plateau

- future revisions and updates to site-wide assessments using the System Assessment Capability

- future assessments of corrective actions, tank waste retrieval, and tank farm closure being performed by the Tank Farm Vadose Zone and Closure Programs to support

- future revisions to the performance assessment (PA) of the RPP's Immobilized Low-Activity Tank Waste Disposal Facilities.

\subsection{Support to Local-Scale Model Analyses and Assessments}

The following section provides a discussion and specific examples of the appropriate and inappropriate use of the site-wide groundwater flow and transport model in supporting local-scale modeling studies and assessments.

\subsubsection{Appropriate Uses and Selected Examples}

In its current design and numerical implementation, use of the site-wide model in providing a regional hydrogeologic framework for a local-scale model or in prescribing boundary conditions for local-scale analysis is considered very appropriate for many situations. Some examples of local-scale modeling studies and investigations supported by a site-wide model include modeling studies described in Wood et al. (1995, 1996), Barnett et al. (1997), and Bergeron and Wurstner (2000). A brief summary of each of these efforts and how a site-wide model was used in supporting the analysis is provided in the following sections.

\subsubsection{Performance Assessment of Solid Low-Level Waste Burial Grounds}

Since September 26, 1988, performance-assessment analyses have been required by DOE Order 5820.2A and now 435.1 to demonstrate that DOE-operated waste-disposal facilities containing DOEgenerated LLW can comply with the appropriate performance objectives. Two separate performance assessments that included use of groundwater modeling have been completed recently for post-1988 solid LLW disposal facilities located in the 200 East and 200 West Areas (Wood et al. 1995, 1996). The following is a brief description of the scope and groundwater modeling activities carried out to support these analyses.

The performance assessment of the 200 East Area low-level burial grounds (LLBG) examined the long-term impacts of LLW and radioactive constituents of the low-level mixed wastes (LLMW) disposed in waste burial areas in two locations: 1) the active 218-E-10 burial ground and adjacent burial grounds in the northwest corner of the 200 East Area and 2) the active 218-E-12B burial ground and adjacent inactive burial grounds located in the northeast corner of 200 East Area. A separate analysis was included to examine the impacts of reactor compartment wastes disposed in trench 94 of the 218-E-12B disposal facility. LLW disposed in active and inactive burial grounds before September 26, 1988, were not considered in this analysis. 
The performance assessment of the 200 West Area LLW burial grounds examined the long-term impacts of LLW and radioactive constituents of the LLMW disposed in several active waste burial areas situated along the west boundary of 200 West Area. Burial grounds considered in the analysis included 218-W-3A, 218-W-3E, 218-W4C, and 218-W-5. LLW disposed in retired or inactive burial grounds before September 26, 1988 (218-W-2, 218-W-4A, 218-W-4B, and 218-W-11), were not considered in this analysis.

To address the performance objectives related to groundwater contamination, two groundwater exposure scenarios were considered. One scenario consisted of an all-pathways exposure in which 1) radionuclides are leached from the disposal facilities and are subsequently transported by infiltrating water through the vadose zone to the underlying unconfined aquifer, and 2) an individual drills a well that draws contaminated water for drinking, crop irrigation, and livestock production, and a dose is received by ingestion of contaminated water, crops, milk, and beef, direct exposure to gamma-producing radionuclides in soil, and inhalation of contaminated dust. The second exposure scenario involved a drinking water scenario where only ingestion of contaminated water from the unconfined aquifer was considered.

The conceptual model of the analyses by Wood et al. $(1995,1996)$ focused on incorporating two general processes that control projected concentrations of radionuclides released from the LLW disposal facilities in groundwater withdrawn from the unconfined aquifer from a downstream well: 1) the total radionuclide mass flux being leached from the disposal facility per unit time and 2) the dilution that occurs as the radionuclide activity mixes with the volume of groundwater determined by the regional flow characteristics to flow beneath the facilities. To represent these processes, Wood et al. $(1995,1996)$ assumed that the waste volume representative of the total wastes disposed in the LLW facilities could be approximated by a three-dimensional rectangular box projected onto a two-dimensional plane oriented parallel to the general direction of groundwater flow.

The numerical representation of this conceptual model was established in a two-dimensional crosssectional model based on the VAM3D-CG code (Huyakorn and Panday 1994) that extended from the disposal facility to the uppermost $5 \mathrm{~m}$ of the unconfined aquifer. The position of the water table in the cross section was estimated using the site-wide model developed for use in the performance assessment (see Appendix E of Wood et al. 1996). The model was used to estimate steady state post-Hanford Site conditions underlying the various LLBG areas.

\subsubsection{Modeling Support to the State Approved Liquid Disposal Site Permit}

In 1997, groundwater modeling was performed to support ongoing permitting requirements for the State Approved Land Disposal Site (SALDS) disposal site located just north of the 200 West Area (Barnett et al. 1997). The SALDS receives treated effluent containing tritium, which is allowed to infiltrate through the soil column to the water table. The facility-operating permit, promulgated by WAC 173-216 (Ecology 1986), requires groundwater monitoring, reporting of monitoring results, and periodic review of the monitoring network. 
The SALDS began operations in November 1995, and tritium was first detected in groundwater monitoring wells around the facility in July 1996. The SALDS groundwater-monitoring plan requires a reevaluation of the monitoring-well network and a revision of the predictive groundwater model used in the original permit 1 year after the first detection of tritium in groundwater.

The SALDS groundwater model was a modification of the three-dimensional site-wide groundwater model developed for use in the Hanford Groundwater Project. The decision to modify the Hanford Groundwater Project model was made because of the ease in refining the pre-existing model and assigning appropriate parameter values and because of the experience in using that model. The horizontal grid spacing of the SALDS model was 350 m over most of the Hanford Site, but was refined to a 45-m grid in the region around the SALDS. Vertical discretization in this region was refined to a 6-m grid spacing. Boundary conditions and the model parameters were based on the Hanford Groundwater Project model, but were obtained for this model using a separate calibration. Effluent discharge to the SALDS, a portion of which contained tritium, was modeled. Flow and transport in the unsaturated zone were not modeled.

The model was used to simulate transient flow and tritium transport from the SALDS over the next approximately 100 years. Results were presented as plan-view contours of hydraulic head and tritium concentration and as cross-sectional views of tritium concentration.

\subsubsection{Groundwater Modeling Support to Immobilized Low-Activity Waste Disposal Facility Performance Assessment}

The application of the Hanford Site-Wide Groundwater Model and its use in local-scale models to support the ILAW disposal facility PA is described in Bergeron and Wurstner (2000). In this analysis, the site-wide model and supporting local-scale models are used to evaluate impacts from the transport of contaminants at a hypothetical pumping well $100 \mathrm{~m}$ downgradient of the disposal facilities and to evaluate regional flow conditions and transport from the ILAW disposal facilities to the Columbia River. These models were used to compute well-intercept factors (WIFs) or dilution factors from a given areal flux of a hypothetical contaminant released to the unconfined aquifer from the ILAW disposal facilities for two waste-disposal options: 1) a remote-handled trench concept and 2) a concrete-vault concept. The WIF is defined as the ratio of the concentration at a well location in the aquifer to the concentration of infiltrating water entering the aquifer. These WIFs are being used in conjunction with calculations of released contaminant fluxes through the vadose zone to estimate potential impacts from radiological and hazardous chemical contaminants within the ILAW disposal facility at compliance points.

Uncertainties in the following key factors affecting calculated WIFs were investigated with sensitivity analyses:

- source-release area at the water table

- vertical position of the post-closure water table and the associated direction of groundwater flow 
- lateral position of the Hanford-Ringold Formation contact

- hydraulic properties of Hanford and Ringold sediments.

The spatial scale of this analysis was to evaluate the WIF at both $100 \mathrm{~m}$ down gradient of the waste disposal facility and at a hypothetical down-gradient location about $15 \mathrm{~km}$ from the Site along the Columbia River. The temporal scale of the analysis is the post-Hanford period.

In this analysis, we used the regional-scale model interpretation on both the regional- and local-scale models for a variety of reasons including

- the time scale of the analysis required that boundary conditions needed for the local-scale model be developed based on a simulation approach rather than relying on local-scale measurements

- the additional information was available in the vicinity of the ILAW disposal was generally consistent with the regional interpretation of the hydrogeologic framework

- local-scale extrapolation of regional-scale model features was considered appropriate since a key feature of the local-scale model (a high-permeability facies of the Hanford formation found at the water table) is reasonably well represented in the regional-scale model.

\subsubsection{Inappropriate Uses and Selected Examples}

In its current design and numerical implementation, the use of boundary conditions, the hydrogeologic framework, and transport capabilities available for the site-wide model may not always be the most appropriate approach for some local-scale assessments. Examples of these situations include recent modeling studies described in DOE-RL (1999a,b; 2000) and Connelly et al. (1997). A brief summary of each of these efforts and some of the technical issues associated with these analyses that need careful consideration are provided in the following sections.

\subsubsection{Modeling Support to Pump-and-Treat Remediation}

Local-scale models being used to support pump-and-treat remediation efforts at the $100 \mathrm{~K}, \mathrm{~N}, \mathrm{D}$, and $\mathrm{H}$ Areas and 200 West Area of the Hanford Site could also be supported using the site-wide groundwater model. Recent efforts of these studies and their corresponding level of modeling are described in detail in reports by DOE-RL (1999a,b; 2000).

As described in DOE-RL (1999a,b; 2000), groundwater models are being used at a local scale in operable units in the 100 and 200 Areas to assess the performance of groundwater pump-and-treat systems as an interim measure to remediate contamination within the unconfined aquifer system. The models are used to evaluate system performance and overall progress toward remediation objectives and goals, including evaluating different extraction and injection well configurations, predicting effects of different operational and pumping schedules, assessing the extent of hydraulic influence, and evaluating groundwater-travel times and the extent of the capture zone. The evaluation of all the pump-and-treats 
uses a capture zone modeling approach that evaluates flow conditions only in the vicinity of the pumping wells. The spatial scale of the analysis is on the order of a few hundred meters in the immediate vicinity of the pump-and treat systems and the temporal scale of the analyses is for current conditions. Boundary conditions being used to support the pump-and-treat local-scale models are being estimated using available water level measurements in proximity to the model boundaries.

Because of the limited nature and objectives of the capture zone analyses in evaluating and optimizing active pump-and-treat remediation, use of local-scale data and information for development of the hydrogeologic framework, hydraulic properties, and boundary conditions in the local-scale model for this type of analysis is preferable over the regional-scale model. Use of the hydrogeologic framework and hydraulic properties of the regional-scale model would be considered appropriate if the objective of the analysis were to evaluate the long-term regional-scale behavior of local-scale contamination to other forms of remediation such as natural attenuation (e.g., $100 \mathrm{~B} / \mathrm{C}$ and $100 \mathrm{~F}$ ) that may be considered as final remediation measures.

Local-scale modeling predictions at more highly resolved spatial and temporal scales require a careful interpretation of the local data and information to identify any smaller scale spatial and temporal variability that may be important to these more refined local scale predictions. In general, both local-scale phenomena and/or other features that could impact local-scale flow and transport not properly accounted for in the regional-scale site-wide model need to be recognized and evaluated for inclusion in any models used in the local-scale analyses.

\subsubsection{Local Scale Modeling Near the Columbia River}

Modeling studies that examine flow and contaminant transport behavior in areas close to the Columbia River provide good examples of when alternate models may be needed. Local-scale model analyses of flow and transport at locations along the Columbia River may, in some cases, require a coupled vadose-zone and saturated zone model to accurately represent dynamic groundwater-river processes that significantly affect contaminant behavior in the lower part of the vadose and the upper part of the aquifer system. One such situation is when the dynamics of rapidly changing river stages and corresponding water table changes in the aquifer system cannot be adequately represented using the same interpretations and resolution available in the site-wide model.

Some examples of local-scale modeling studies and investigations, using more refined or sophisticated modeling capabilities or approaches to address flow and transport behavior along the Columbia River, include recent modeling studies described in Connelly et al. (1997). The objective of this model analysis was to examine the effect of the daily time varying Columbia River stage changes and its effect on flow conditions and potential transport of contaminants found at the $100 \mathrm{~N}$ Area (primarily strontium-90). Several previous modeling studies conducted at the $100 \mathrm{~N}$ Area (Lu 1990; DOE-RL 1995b, 1996a) had assumed a time-invariant boundary condition for the Columbia River. Connelly et al. (1991) considered only seasonal changes in the river stage. The Columbia River's stage is known to vary, however, on annual, seasonal, and daily cycles. This time varying boundary condition was shown by 
Connelly et al. (1997) to have potentially significant impacts on contaminant transport in the groundwater near the river that could affect the design of a permeable reactive barrier being considered for installation near the river.

In this analysis, the two-dimensional cross-sectional model developed by Connelly et al. (1997) used the STOMP code (White and Oostrom 1996, 1997; Nichols et al. 1997) to simulate the interaction between the rise and fall of the Columbia River, the unconfined, and the capillary fringe directly above the water table in the $100 \mathrm{~N}$ Area. The stratigraphy used in the modeling was based on geologic data from boreholes drilled in the $100 \mathrm{~N}$ Area. The two major hydrogeologic units considered included the Hanford Gravel and the Ringold Unit E, which is a variably cemented pebble to cobble gravel with a fineto coarse-grained sand matrix. The vertical sequence modeled ranged from an elevation of $125 \mathrm{~m}$ to a depth of $107 \mathrm{~m}$, where the base of the model was assumed to be the top of the lower Ringold mud unit.

Estimates of the unsaturated zone hydraulic properties were also made using available information on hydraulic conductivity, particle density, specific storage, porosity, and the assumed van Genuchten curve fitting parameters. The estimates of hydraulic conductivity and porosity were varied to calibrate the model to transient observed water-level measurements in wells between the Columbia River and well 199-N-67.

The lower boundary on the top of the Ringold mud unit was assumed to be a no-flow boundary. The upper boundary was a constant-flux boundary representing natural recharge of $2 \mathrm{~cm} / \mathrm{yr}$. The boundary of the model inland from the river was set at no flow in the vadose zone and to a time-dependent constanthead boundary in the saturated zone. The value of the head in the saturated zone was varied on an hourly basis based on water-level data recorded at a well (well number 199-N-67). Nodes on the riverbed were set to a time-dependent constant-head boundary based on river-stage measurements made at the 100-N Area river-monitoring station. The remaining boundary was set as no flow.

A 125-hour transient simulation was used to develop initial conditions for a 4-week period of simulation. During this period, the model was used to simulate the transient interaction of the Columbia River and the unconfined aquifer in 1-hour time steps. Because of the large volume of data generated by the simulation, the modeling results were summarized in a time-series animation of river stage and aquiferhead fluctuations during the period of simulation. This animation was used to display changes in water travel times in the riverbank and water-flux calculation to and from the Columbia River due to both bank storage and regional groundwater gradients.

Results of the modeling demonstrated that the variation in the Columbia River stage has a significant impact on the unconfined aquifer system close to the river. Particle-tracking analyses showed that consideration of the transient conditions of the river increased water velocities over those calculated for steady state conditions. Water-mass calculations also demonstrated the importance of bank storage in calculating total water movement from the unconfined aquifer and the Columbia River at the 100-N Area. 
Because of the spatial and time-scale and the specific processes involved in performing this analysis, this example analysis provides a clear case where a local-scale model based entirely on local-scale interpretations, measurements, and estimates of hydraulic properties with very limited information and data from the regional-scale model would be the most appropriate analysis.

\subsubsection{Modeling of Complex Chemical Properties}

Other examples where use of the site-wide model would be inappropriate include situations where the modeling analysis must consider more complex chemical processes than represented in a linear equilibrium sorption model. Examples at Hanford are modeling studies that were used to support feasibility studies of various remediation technologies on local-scale contamination issues. Good examples of modeling studies include those used to support feasibility studies of In Situ Redox Manipulation (ISRM) remediation technology on remediation of chromium plumes in $100 \mathrm{D}$ and $100 \mathrm{H}$ areas as described in Williams et al. (1994, 1999), Chilakapati et al. (2000), Williams and Oostrom (2000). These ISRM modeling studies represent supporting analytical and numerical activities performed to examine the feasibility of altering the subsurface oxidation-reduction (REDOX) conditions at Hanford to either destroy or immobilize certain contaminants (e.g., hexavalent chromium, uranium, technetium, and chlorinated solvents) by changes in redox potential.

Model studies by Williams et al. (1994) were used to assist preliminary designs of an initial field experiment of a single-well injection of a chemical agent and nonreactive tracer to induce reduction of in situ solid-phase ferric iron. Model studies were performed to evaluate important design factors including well and borehole placement, reagent concentration, injection and withdrawal rates, and optimal duration of each phase of the experiment (injection, reaction, and withdrawal). Additional models were developed to explore the influence of local-scale heterogeneities on the performance and interpretation of the experiment using high-resolution, synthetic aquifer models.

In Williams et al. (1999) and Williams and Oostrom (2000), experimental and modeling studies were performed to examine the fate of an anoxic groundwater plume created by in situ redox manipulation near the Columbia River in the unconfined aquifer with a fluctuating water table as would be expected close to the Columbia River environment. The modeling work consisted of using a two-dimensional unsaturatedsaturated cross-sectional model in $100 \mathrm{D}$ area to simulate a fluctuating water table induced by the Columbia River, groundwater mixing with river water near the river's edge, along with air entrapment in the zone of fluctuation.

Chilakapati et al. (2000), describes the use of reactive transport models and simple cost models to optimally select the number of wells, the injection rate, and the number of regenerations of a large-scale in situ redox barrier for remediation of a chromium plume in $100 \mathrm{H}$ Area (Williams and Oostrom 2000).

These series of modeling efforts are very good examples of studies where additional modeling capabilities and levels of complexity and detail are needed to achieve the principal objectives of the analysis. Use of the site-wide groundwater flow and transport with its very limited capabilities for 
simulating chemical processes (e.g., linear equilibrium sorption) and important local-scale chemical processes and features (e.g., oxidation-reduction, heterogeneity, air-entrapment in a fluctuating water table, bank storage effects on the capillary fringe) would be inappropriate.

The objectives and resulting analysis needs of all local-scale analysis should be carefully evaluated before choosing to perform the analysis with the site-wide groundwater and transport model because of the limited physical and chemical processes represented in the site-wide groundwater and transport model and because the hydrogeologic interpretation used in the site-wide model is limited to a regional-scale hydrogeologic interpretation and a regional-scale implementation of boundary conditions (e.g., aquiferColumbia River interactions). 


\subsection{Uncertainty/Uncertainty Analysis and Its Sources}

The site-wide groundwater model is being developed for making predictions about contamination states to evaluate potential risk, cost, and consequence given assumptions regarding monitoring well placement, cleanup strategies, waste management decisions, as well as future conditions at the Hanford Site that could affect the migration and fate of contaminants. The desired analysis should estimate the uncertainty in the description of the system, its model, and the model parameters and boundary conditions/driving forces. The analysis also should be able to propagate these uncertainties to calculate future system states and the associated uncertainty in these estimates to understand the effects of these uncertainties on the uncertainty in risk and cost. In this section, we discuss uncertainty concepts and outline the nature and sources of uncertainty as they apply to the Hanford site-wide groundwater modeling activities. This section starts with an examination of various definitions of uncertainty/ uncertainty analysis to illustrate the complexity and importance of the subject, presents a variety of statistical concepts important to parameter uncertainty analysis to provide a basis for subsequent discussion, and then proceeds to a discussion of the sources of uncertainty and the various taxonomies developed to categorize these sources. Finally, we discuss the approaches for identifying, combining, and propagating these uncertainties. The following section discusses the geohydrology of the Hanford Site and surrounding area and our conceptual understanding of the groundwater system and the currently identified sources of uncertainty.

\subsection{Definitions and Concepts}

Data for describing the behavior of wastes disposed in natural systems over long periods of time pose a difficult problem that requires the use of mathematical and numerical models. However, the inability to completely characterize natural systems makes it impossible not only to determine the parameters for these models with certainty, but many times available data are insufficient to determine which of several models apply (HYDROCOIN 1992). Sensitivity and uncertainty analysis techniques provide the means to make predictions of future behavior of wastes disposed in natural systems over long periods of time in light of the uncertainty in the appropriate model and the exact parameters. Definitions and concepts related to uncertainty, uncertainty analysis, sensitivity analysis, and modeling are discussed in the following subsections.

\subsubsection{Uncertainty/Uncertainty Analysis}

Katz (1999) presents well-known quotes ${ }^{(a)}$ that appear in the book: Uncertainty: A Guide to Dealing with Uncertainty in Quantitative Risk and Policy Analysis by Morgan and Henrion (1990). The quotes make it evident that, long ago, eminent scholars had a genuine appreciation of the need to take uncertainty into account.

(a) Pliny the Elder: "The only certainty is uncertainty."

Lao Tzu: "To know one's ignorance is the best part of knowledge."

Cicero: "Probabilities direct the conduct of the wise man."

Socrates: "As for me, all I know is I know nothing." 
The terms uncertainty and uncertainty analysis have been defined in many ways and from many different viewpoints. Several technical definitions of uncertainty and uncertainty analysis taken from a few selected references provide some perspective.

The science and technology definition of uncertainty (McGraw-Hill 1983) is given as "The estimated amount by which an observed or calculated value may depart from the true value."

Uncertainty and the problem it poses is discussed in the National Research Council's book on science and judgment in risk assessment (NRC 1994) as: "Uncertainty can be defined as a lack of precise knowledge as to what the truth is, whether qualitative or quantitative. The lack of knowledge creates an intellectual problem - that we do not know what the scientific truth is; and a practical problemwe need to determine how to assess and deal with risk in light of that uncertainty."

The U.S. Nuclear Regulatory Commission (USNRC) defines uncertainty analysis (USNRC 1990) as "An analysis that estimates the uncertainty in a system's performance resulting from the uncertainty of one or more factors associated with the system. Such an analysis requires a definition of a system, description of the uncertainties in the factors that are to be investigated, and the characteristics of the system that is to be observed."

The USNRC also provides the following description of uncertainty analysis in the Regulatory Analysis Technical Evaluation Handbook (NUREG/BR-0184, Rev. 2 USNRC 1997) as it applies to probabilistic risk assessments (PRAs): "With respect to power reactor facilities, much has been written about uncertainty analysis in risk assessments. The more rigorous assessments typically provide an uncertainty analysis, usually performed via stochastic simulation on a computer. Briefly, the analyst determines probability distributions for as many of his input parameters as deemed necessary and practical. A computer code then samples values from each distribution randomly and propagates these values through the risk equation to yield one result. When repeated a large number of times (at least several hundred), a probability distribution for the result is generated, from which the analyst can extract meaningful statistical values (e.g., mean, standard deviation, median, and upper and lower bounds for given confidence levels)."

The international HYDROCOIN (1992) project for studying groundwater flow modeling in the context of radioactive waste disposal talks about uncertainty as follows: "uncertainty means lack of knowledge, usually a lack of knowledge of the exact state of the natural world. Thus one speaks of uncertainty in a model, meaning lack of knowledge as to exactly which model corresponds to the natural world."

The Environmental Protection Agency (EPA) (1997) in the guidance on Monte Carlo analysis discusses uncertainty as follows: "Uncertainty refers to lack of knowledge about specific factors, parameters, or models. For example, we may be uncertain about the mean concentration of a specific pollutant at a contaminated site or we may be uncertain about a specific measure of uptake (e.g., 95th percentile fish consumption rate among all adult males in the United States). Uncertainty includes parameter uncertainty (measurement errors, sampling errors, systematic errors), model uncertainty (uncertainty due to necessary simplification of real-world processes, mis-specification of 
the model structure, model misuse, use of inappropriate surrogate variables), and scenario uncertainty (descriptive errors, aggregation errors, errors in professional judgment, incomplete analysis)."

The purpose of a systematic and quantitative uncertainty analysis, as indicated in Isukapalli (1999), is to use available information to quantify the degree of confidence in existing data and models and not to reduce uncertainty, because uncertainty reduction can be realized only through the acquisition of additional data and/or understanding that fills gaps in data and/or understanding. He indicates that uncertainty analysis additionally aids in identification of key sources of uncertainty, sources of uncertainty not important to system responses of concern, and in the identification of conclusion robustness given the modeling assumptions and data uncertainties; thus providing guidance in targeting additional data needs and/or model refinement.

\subsubsection{Statistical Concepts Important to Uncertainty Analysis}

This section introduces a variety of statistical concepts important to parameter uncertainty analysis to provide a basis for subsequent discussions. In this section, deterministic and probabilistic modeling are contrasted.

\subsection{Deterministic Versus Probabilistic Modeling Used in Uncertainty Analysis}

Figure 3.1, adapted from Goovaerts (1997), provides a good illustration of the difference between a deterministic and probabilistic modeling approach used in an uncertainty analysis. The probabilistic modeling approach discussed deals only with the uncertainty in the parameter inputs. In this hypothetical example, a deterministic model and a probabilistic model are developed based on the observations illustrated by the points in the concentration versus distance graph in the top part of the figure. Both models are constructed to estimate the concentration at the unsampled location at $\mathrm{x}=2$. As illustrated with the deterministic model, there is only one estimate for the unknown concentration $\mathrm{C}$ at location $\mathrm{x}=2$ (i.e., $\mathrm{C}=0.7 \mathrm{ppm}$ ), while the result from the probabilistic model is a probability density function (pdf) indicating the probability of the concentration being any of a range of values between 0 and $\sim 3.5 \mathrm{ppm}$, and since the area under the pdf above $\mathrm{C}=1.0 \mathrm{ppm}$ is 0.2 , the probability of the concentration being greater than $1.0 \mathrm{ppm}$ is 0.2 . Figure 3.2, adapted from Isukapalli (1999), illustrates pictorially other details regarding the difference between a two-parameter deterministic and a two-parameter probabilistic model. The illustration depicts the two input parameters for both types of models, the model results for both types of models, and the model response surface over the range of probabilistic model inputs. In this probabilistic model, the two input parameters are assumed to be independent (i.e., uncorrelated) so that their uncertainty can be represented by the two univariate pdfs illustrated along the two horizontal axes of Figure 3.2 as the pdfs for input 1 and input 2. The two deterministic model input parameters, typically termed "best parameter estimates," would be either the mean, median, or modal value of their respective non-normal distributions illustrated in Figure 3.2. Figure 3.3, adapted from Davis (1986), illustrates and defines these three different types of statistical measures of central tendency for non-normal distributions. The three-dimensional surface illustrated in Figure 3.2 is the model response surface that illustrates the model predicted value for concentration, $\mathrm{C}$, at $\mathrm{x}=2$ for any of the various two-parameter combinations of inputs shown along the two horizontal axes of the figure. The deterministic model result is the single 
model output near the center of the response surface (i.e., the model result obtained when using the two deterministic inputs shown) and the probabilistic model result is the univariate pdf at the top of Figure 3.2.

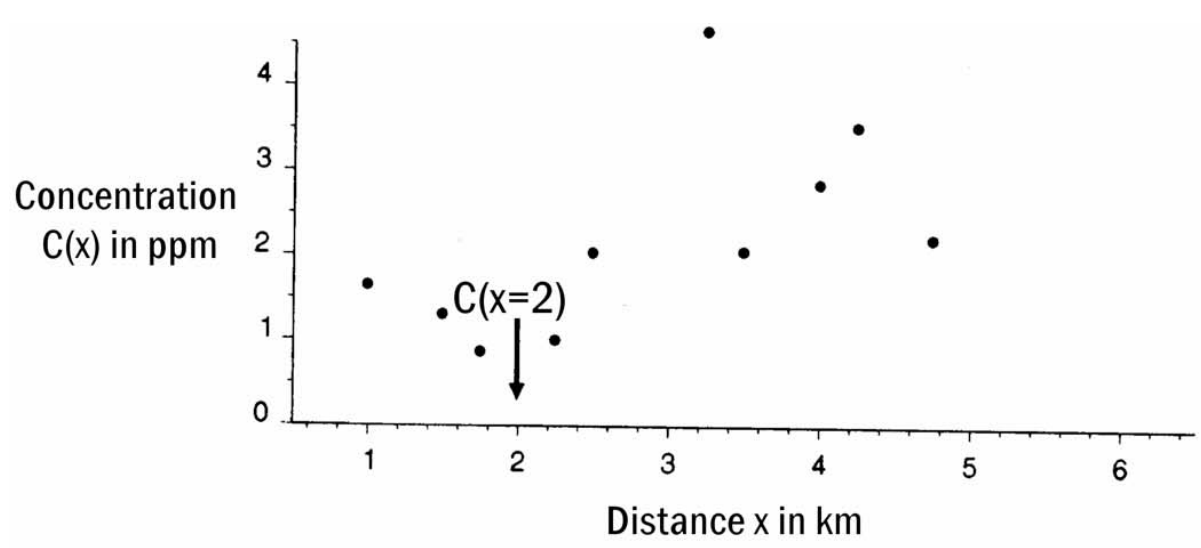

\section{Deterministic model}

$$
C(x=2)=0.7 p p m
$$

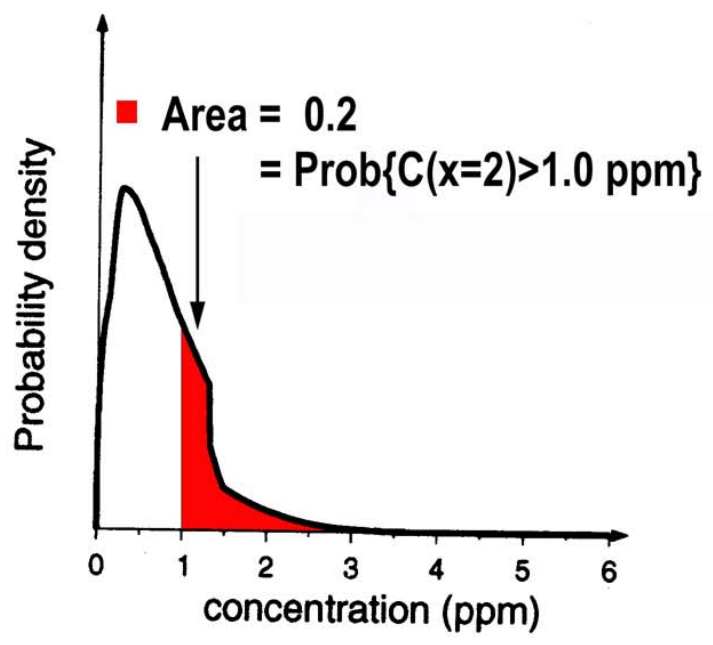

Figure 3.1. Illustration of the Difference Between a Deterministic and Probabilistic Model for Concentration at Location $\mathrm{x}=2$ (adapted from Goovaerts 1997). 


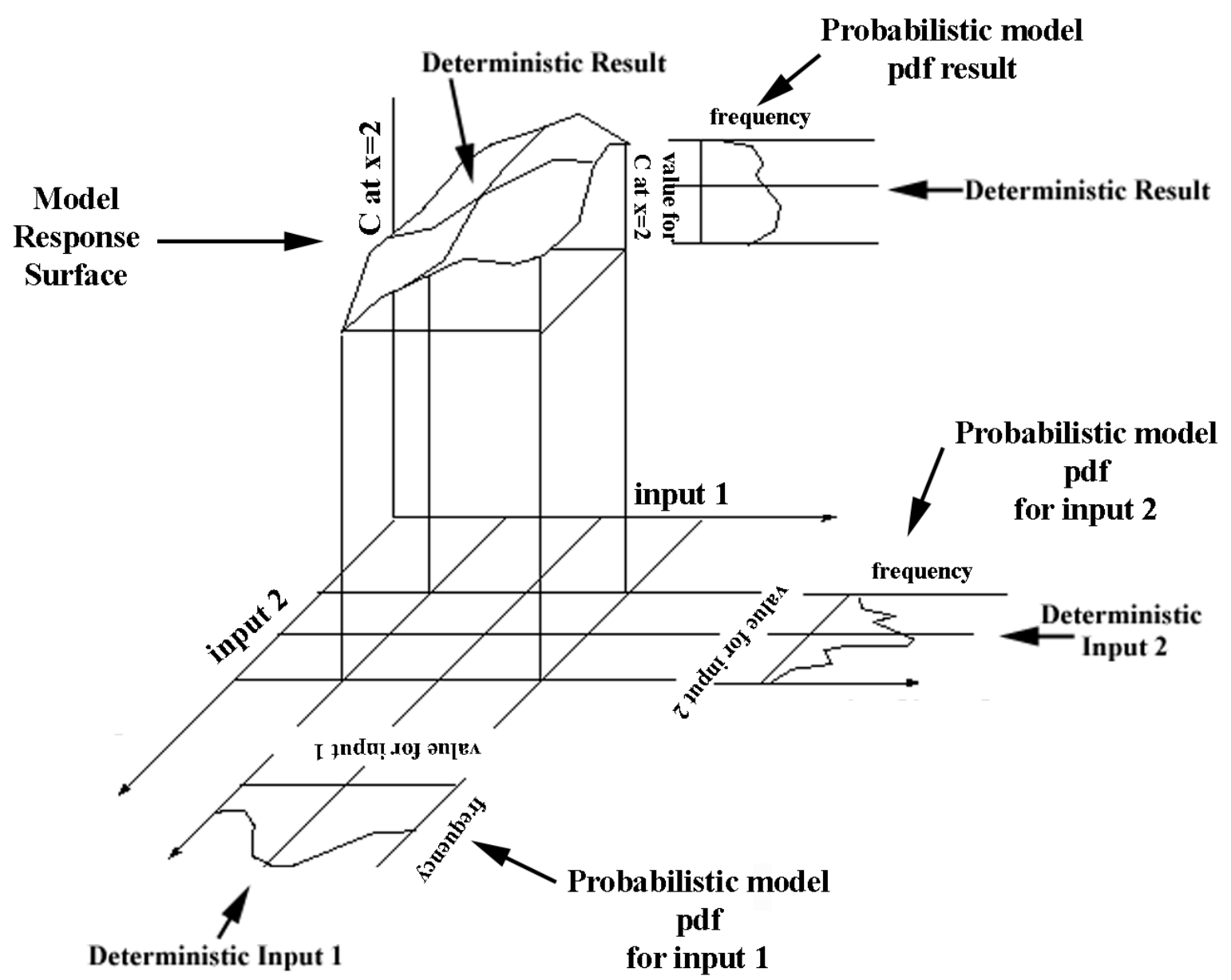

Figure 3.2. Pictorial Representation of the Differences Between the Inputs and Outputs for a Two-Parameter Deterministic Model and a Two-Parameter Probabilistic Model for the Concentration, $\mathrm{C}$, at $\mathrm{x}=2$, Illustrated in Figure 3.1. This figure was adapted from Isukapalli (1999).

\subsection{Univariate Description of Model Inputs/Results and Summary Measures}

Univariate probabilistic model inputs and results can be presented graphically in several different ways (i.e., pdf, cumulative distribution function [CDF], and a complementary cumulative distribution function $[\mathrm{CCDF}]$ ) as illustrated with smooth continuous functions in Figure 3.4 (a-c). In most computational and data-gathering activities, the data and results would be displayed as histograms rather than continuous functions. Presentation as a pdf, illustrated in the familiar form of the bell-shaped curve of the normal distribution in Figure 3.4 (a) or as the skewed and multimodal pdf results for the hypothetical probabilistic model (Figure 3.1 and Figure 3.2, respectively), is useful because a pdf visually conveys information about important features of the distribution. This includes the following: 


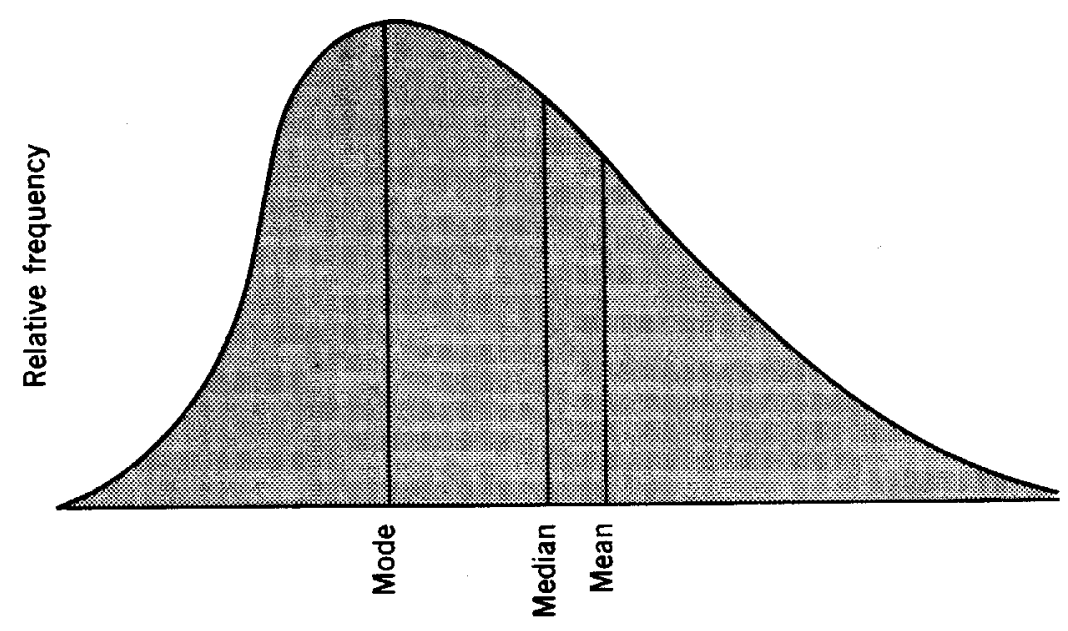

Figure 3.3. Relationship Between Measures of Central Tendency in an Asymmetric Positively Skewed Probability Density Function (adapted from Davis 1986). As discussed in Davis, the median is the mid value in the frequency distribution having half of the population (i.e., area under the curve) below and half of the population above the median value that lies between the mode, which is the value that occurs with the greatest frequency (i.e., the peak value of the pdf), and the mean, which is the arithmetic average.
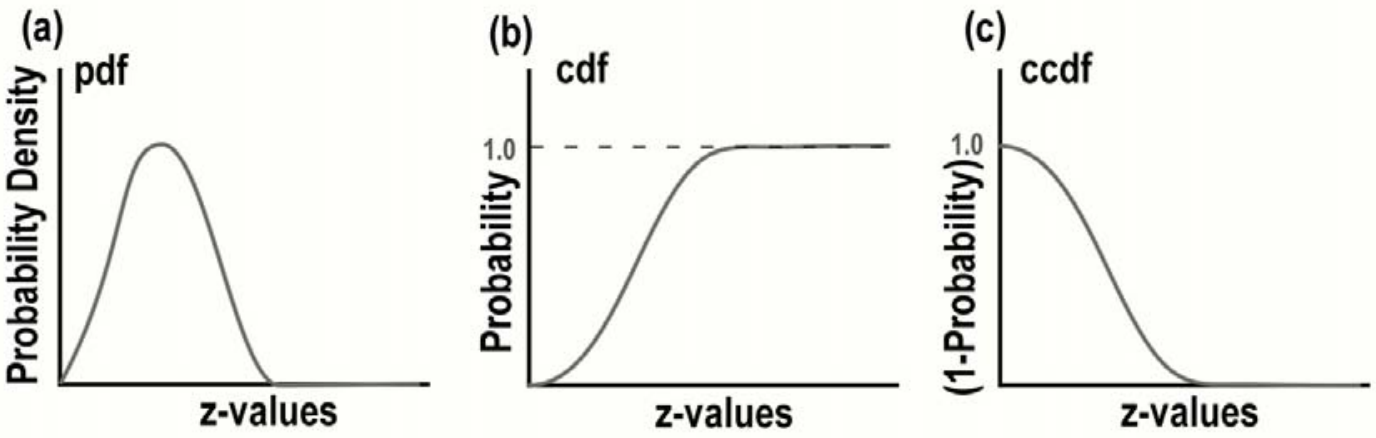

Figure 3.4. Three Different Graphical Ways to Present a Univariate Probabilistic Input or Result; (a) as a Probability Density Function, (b) as a Cumulative Distribution Function (i.e., $C D F=\int p d f$ ), and (c) as a Complementary Cumulative Distribution Function $\{$ i.e., $C C D F=(1-C D F)\}$. 
- Central Tendency - This is usually taken as the arithmetic mean but includes the median and mode as illustrated and defined in Figure 3.3. For a set of $\mathrm{n}$ input parameter measurements, $z_{i}$, the arithmetic mean, $m$ is given by:

$$
m=1 / n \sum_{i=1}^{n} z_{i} .
$$

The central tendency for a normal distribution is unambiguous since the mean=median=mode. However, for nonsymmetric distributions, the median is a better measure of central tendency and is most easily determined from a plot of the CDF or CCDF since the median is the abscissa value that corresponds to the probability ordinate of 0.5 , the point value that divides the population in half. Only the mode of the distribution can be determined directly from a plot of an asymmetric pdf.

- Variation Around the Central Tendency - This is indicated by the spread around the mean (i.e., the variance, $\sigma^{2}$, or its square root, $\sigma$, known as the standard deviation) which for the same set of $n$ input parameter measurements, $z_{i}$, would be given by:

$$
\sigma^{2}=1 / n \sum_{i=1}^{n}\left(z_{i .}-m\right)^{2}
$$

Like the mean, the variance and standard deviation are strongly affected by the presence of outliers in a distribution. For skewed distributions or those containing outliers, a robust measure of deviation is the interquartile range, which is the difference between the upper quartile $\left(75^{\text {th }}\right.$ percentile $)$ and lower quartile $\left(25^{\text {th }}\right.$ percentile) of a distribution. The interquartile range provides a direct measure of the variability of a distribution about the median value. For non-negative parameters, another useful measure of confidence that can be deduced from all three graphical forms of the probability distribution is the unit free measure known as the coefficient of variation, $C V$, which is the ratio of the standard deviation to the mean:

$$
C V=\sigma / m
$$

As discussed in Rice (1988) the coefficient of variation can often be more meaningful because a standard deviation of 10 means one thing if the parameter value is 100 and quite a different thing if the parameter value is 10,000 .

- Asymmetry or Skewness of the Distribution - The asymmetry or skewness of the distribution (i.e., a long tail of large values represents positive skewness and a long tail of small values represents negative skewness) can be simply represented by $\vartheta$, which is the difference between the mean, $m$, and median, $M$, value of the distribution (i.e., $\vartheta^{\prime}=m-M$ ); or more fully represented as the coefficient of skewness for the same set of $n$ input parameter measurements, $z_{i}$, as: 


$$
\vartheta=1 / n \sum_{i=1}^{n}\left(z_{i .}-m\right)^{3} / \sigma^{3},
$$

Goovaerts (1997).

While the pdf representation is useful because it provides the visual information discussed above, a pdf also does not provide an easy means to determine the probability of a parameter or result being in a given range (e.g., between $a$ and $b$ ), or the probability of a result being below, for example, some compliance value $a$, or the probability of exceeding, for example, some compliance requirement, $b$. Probabilities for continuous distributions are always associated with an interval because for continuous distributions the probability of a result or input taking on any particular value (e.g., $z=a$ ) is zero. To obtain information on probabilities from a pdf representation, one has to estimate the various areas under the pdf curve, as illustrated in Figure 3.5, adapted from Haan (1977). The CDF, which is the integral of the pdf shown in Figure 3.4 (b) provides a straightforward means to determine the probability of a result being below some compliance value $a$, since the ordinate value at $z=a$ provides a direct visual estimate of the probability of a result or input parameter, $z$, being at or below $a$. In a similar manner the ordinate value at location $z=b$ of a CCDF, which is 1-CDF, as shown in Figure 3.4 (c) provides the desired exceedance probability (i.e., the probability of a result or input parameter, $z$, being at or above a value of $b$ ). Both the $\mathrm{CDF}$ and CCDF representation provide a straightforward means to determine the probability of a result or parameter being in a given range (e.g., between $a$ and $b$ ) as this estimate is simply the difference in the ordinate value at $z=b$ and $z=a$.

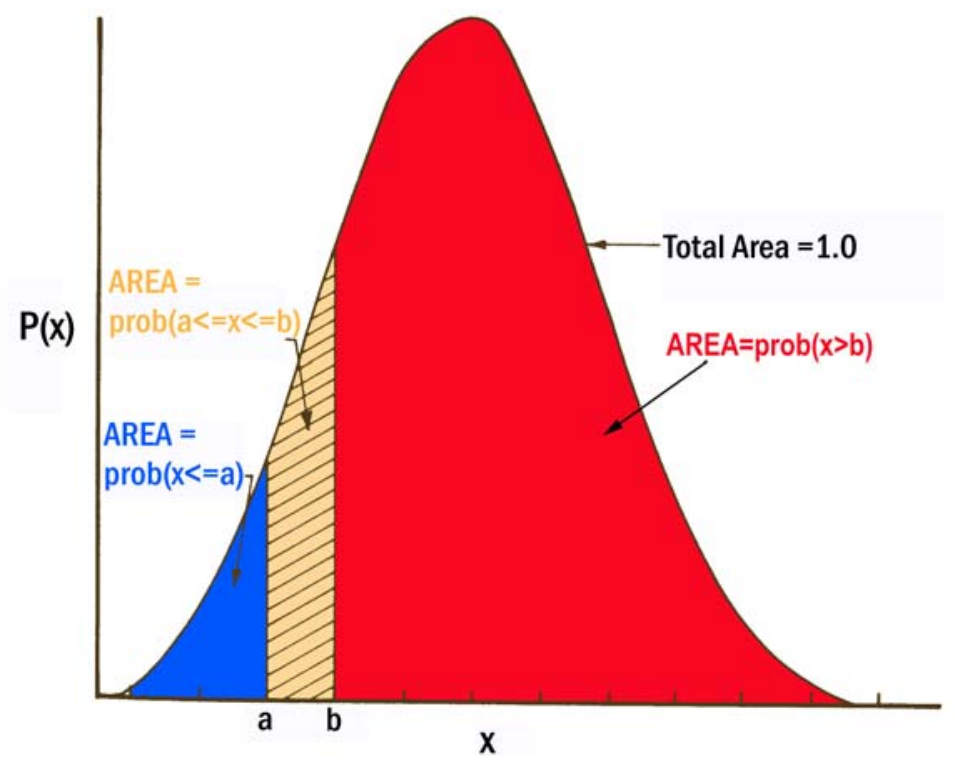

Figure 3.5. Probability Density Function (after Haan 1977) Indicating How Areas Under the pdf Curve Relate to Various Probabilities of Occurrence (probability that the result $x$ is less than or equal to $a$, probability that the result $x$ is between $a$ and $b$, and probability that the result $x$ is greater than $b$ ). 
The CDF and CCDF representations also provide a simple means to split the data, parameter, or result distributions in smaller parts than halves, as is done with the median. This includes fractional based quantiles (e.g., $q_{f}$, where the subscript $f$ represents the fraction of the input or result distribution that lies below the input or result value $q_{f}$ ) or other named "-tiles" representing different splits (e.g., quartiles for quarter splits, deciles for tenth splits, and percentiles for percentage splits). This is easily accomplished with CDF and CCDF representations because the abscissa value that corresponds to a given probability ordinate (e.g., 0.25 ) represents the $q_{0.25}$ quantile, which is equivalent to the first quartile, and the $25^{\text {th }}$ percentile.

\subsection{Multivariate Description of Model Inputs}

When probabilistic model inputs are correlated, then multivariate pdfs can be used to describe the sets of correlated inputs. This is because correlated model inputs can no longer be selected randomly and independently from probability distribution functions. Critics of formal uncertainty analysis often point out that limitations of knowledge about the nature and extent of correlation among variables fundamentally limit our ability to make meaningful statements about the degree of uncertainty in dose assessments (USNRC 2000, Smith et al. 1992). USNRC (2000) notes that many of the parameters in a probabilistic assessment are correlated because some input pdfs are derived from other input distributions. Correlation among inputs must be properly accounted for to avoid the situation where two correlated quantities treated as uncorrelated leads to unlikely combinations of parameters. Proper accounting of correlation between parameters is more important when correlations are strong among the model's most sensitive parameters. Discussions in USNRC (2000) also indicate that failing to account for weak correlations between sensitive parameters and strong correlations among insensitive parameters will generally have very little impact on the overall calculated dose (NCRP 1996a). The correlation coefficient (or Pearson correlation coefficient as it is known in the statistical literature), $\rho_{i j}$, is the statistic commonly used to measure the linear relationship between two variables, $z_{i}$ and $z_{j}$, which can be either model input parameters or model results. This correlation coefficient,

$$
\rho_{i j}=\sigma_{i j} /\left(\sigma_{i} \sigma_{j}\right)
$$

is a unit free normalized measure of this linear relationship derived by normalizing the covariance,

$$
\sigma_{i j}=\frac{1}{n} \sum_{\alpha=1}^{n}\left(z_{i}(\alpha)-m_{i}\right)^{2}\left(z_{j}(\alpha)-m_{j}\right)^{2}
$$

which is a measure of the joint variation of the variables $z_{i}$ and $z_{j}$ around their means $m_{i}$ and $m_{j}$, by their standard deviations, $\sigma_{i}$ and $\sigma_{j}$. The nature of the covariance relationship is such that when variables $z_{i}$ and $z_{j}$ are positively associated (i.e., $\sigma_{i j}>0$ ), it means that when $z_{i}$ is greater than its mean, $z_{j}$ will likely be greater than its mean. A negative association means that when $z_{i}$ is greater than its mean, $z_{j}$ will likely be less than its mean. Because the correlation coefficient is simply a measure of how close the $z_{i}$ and $z_{j}$ values come to falling on a straight line on a scatter plot (e.g., for $\rho_{i j}=1$ they create a straight line) and because variables may be highly dependent on each other while having a zero linear correlation (e.g., the 
classic example where $z_{i}=\left[z_{j}\right]^{2}$ and $z_{j}$ has a symmetric distribution, Goovaerts [1997]) the Pearson correlation coefficient may be a poor summary statistic (Isaaks and Srivastava 1989). This is illustrated in Figure 3.6, which shows the Pearson correlation coefficient and scatter plots for various related variables, some of which are not linear. In addition to insensitivity to nonlinear relationships, the correlation coefficient, like the mean and variance, is strongly affected by extreme values and therefore it is useful to supplement the correlation coefficient with the more robust rank correlation coefficient or Spearman
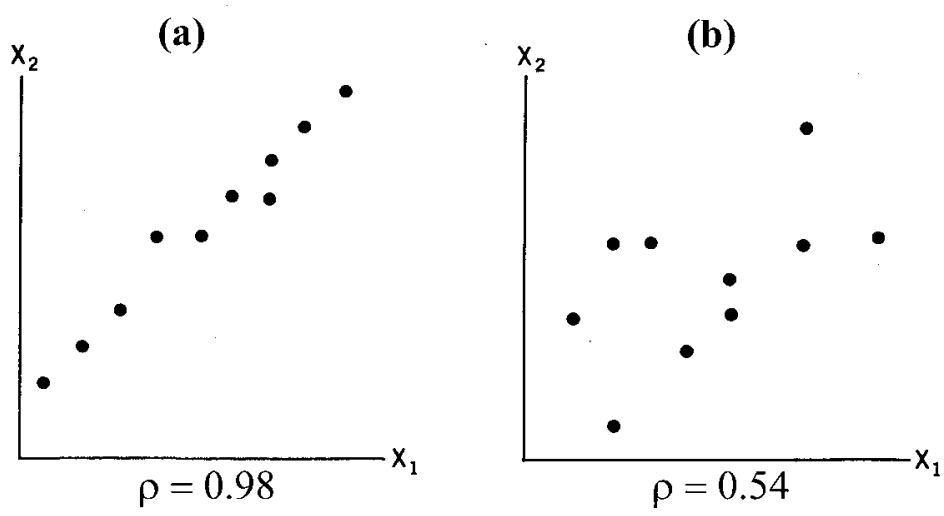

(c)

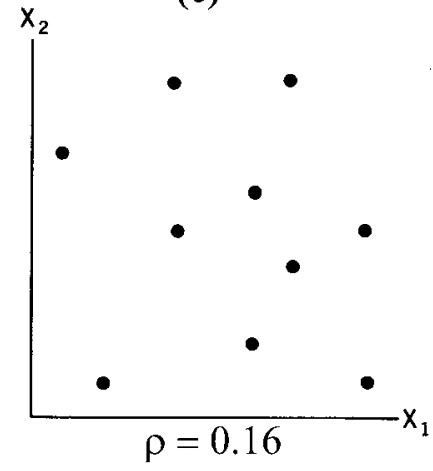

(d)

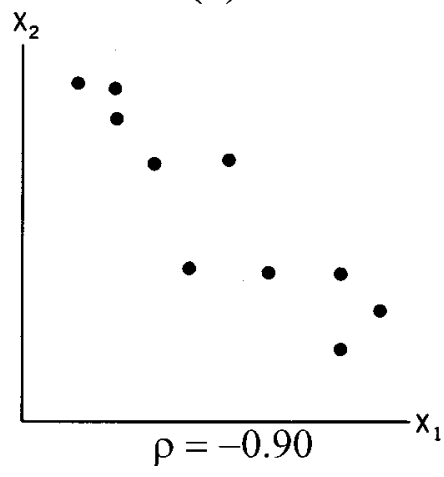

(e)

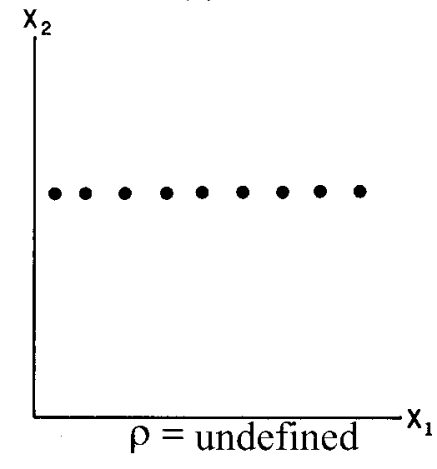

(f)

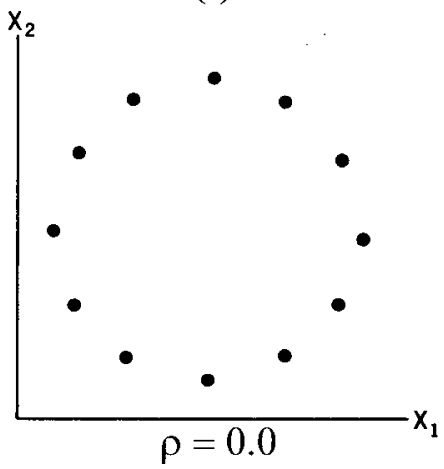

Figure 3.6. Scatter Diagrams a-f (after Davis 1986), Illustrate Various Possible Relationships Between Variables $x_{1}$ and $x_{2}$ and the Associated Pearson Correlation Coefficient Values, $\rho$. Note that scatter diagram f illustrates that the Pearson correlation coefficient fails to identify an obvious nonlinear relationship. 
correlation coefficient as it is known in the statistical literature, $\rho_{i j}{ }^{R}$, that is not strongly influenced by extreme pairs, requires no distributional assumptions, and can indicate nonlinear relationships when there is a large deviation between $\rho_{i j}$ and $\rho_{i j}{ }^{R}$ (Goovaerts 1997; Isaaks and Srivastava 1989; Iman and Conover 1982b). The equation for the Spearman (i.e., rank) correlation coefficient, $\rho_{\mathrm{ij}}{ }^{\mathrm{R}}$, is exactly the same as for the Pearson correlation except it is posed in terms of the rank transformed variables $R z_{i}$ and $R z_{j}$ corresponding to the untransformed variables $z_{i}$ and $z_{j}$ and their associated rank means $m_{R i}$ and $m_{R j}$, and rank standard deviations, $\sigma_{\mathrm{Ri}}$ and $\sigma_{\mathrm{Rj}}$ or

$$
\rho_{\mathrm{ij}}^{\mathrm{R}}=\left[\frac{1}{\mathrm{n}} \sum_{\alpha=1}^{\mathrm{n}}\left(\mathrm{R} \mathrm{z}_{\mathrm{i}}(\alpha)-\mathrm{m}_{\mathrm{Ri}}\right)^{2}\left(\mathrm{R} \mathrm{z}_{\mathrm{j}}(\alpha)-\mathrm{m}_{\mathrm{Rj}}\right)^{2}\right] /\left(\sigma_{\mathrm{Ri}} \sigma_{\mathrm{Rj}}\right)
$$

The rank, $R z_{i}$, of variable $z_{i}$ is simply the index, $i$, assigned to $z_{i}$ when all the $n$ values have been sorted in ascending order. From Equation 3.1, for the mean it is obvious that the rank means $m_{R i}$ and $m_{R j}$ are given by $(n+1) / 2$ so that replacing the rank standard deviations, $\sigma_{R i}$ and $\sigma_{R j}$, by the square root of their variances (Equation 3.2) the simpler Equation (3.8) for the Spearman correlation coefficient presented in Iman and Conover (1982b) is obtained.

$$
\rho_{i j}^{R}=\frac{\sum_{\alpha=1}^{\mathrm{n}}\left\{R z_{i}(\alpha)-(n+1) / 2\right\}\left\{R z_{j}(\alpha)-(n+1) / 2\right\}}{\sqrt{\sum_{\alpha=1}^{\mathrm{n}}\left\{R z_{i}(\alpha)-(n+1) / 2\right\}^{2} \sum_{\alpha=1}^{\mathrm{n}}\left\{R z_{j}(\alpha)-(n+1) / 2\right\}^{2}}}
$$

Section 3.2.3 discusses in detail the description/representation of uncertainty in spatially varying geohydrologic parameters and the effects of correlation between these parameters.

For the probabilistic model shown in Figure 3.2, a single bivariate pdf would be required if both model inputs were correlated. Figure 3.7, adapted from Rice (1987), illustrates four different bivariate normal pdfs depicted both as a three-dimensional surface and in the form of equally spaced isoprobability density contours. The four bivariate distributions shown (a-d) represent correlation coefficients of 0.0 , 0.3, 0.6, and 0.9, respectively. Figure 3.7 (c) uses the same color coding as used in Figure 3.5 to illustrate the integration limits needed to determine the bivariate probabilities that are equivalent to the univariate probabilities illustrated in Figure 3.5. To determine the desired probabilities, the volume under the bivariate probability density function must be determined by integration within the limits shown. The blue areas extend off the graph where they intersect the edge to negative infinity for both $x$ and $y$ and the red areas extend off the graph where they intersect the edge to positive and negative infinity for both $x$ and $y$. 

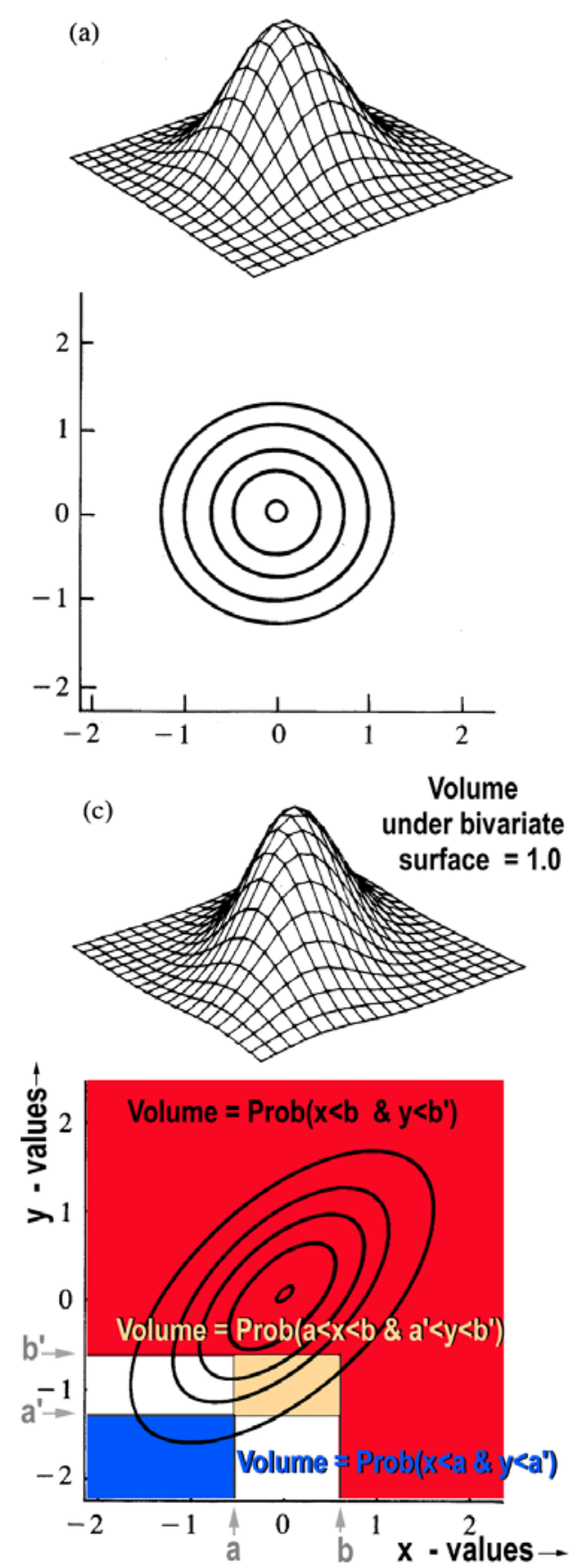

(b)
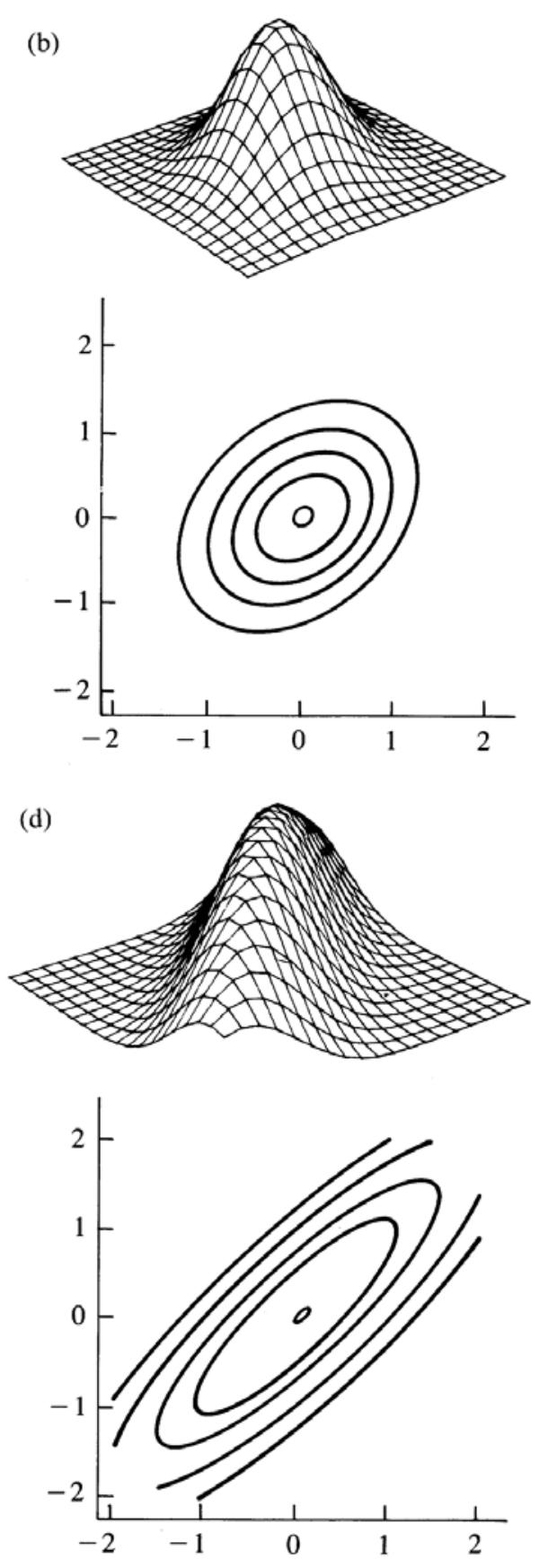

Figure 3.7. Bivariate Normal Densities with Means of 0 , Standard Deviations of 1 , and Pearson Correlations of (a) $\rho=0$, (b) $\rho=0.3$, (c) $\rho=0.6$, and (d) $\rho=0.9$ (adapted from Rice 1987). Two views are shown for each of the four cases: The upper view is the three-dimensional surface, and the lower view displays the equally spaced probability density contours.

(c) Also illustrates the integration limits for determining the bivariate equivalent of the univariate probabilities shown in Figure 3.5. 


\subsection{Representation of Uncertainty in Model Results}

This section discusses the various ways of representing the uncertainty in "model result(s)." The term "model result(s)" is used to emphasize that it is the uncertainty in a result (or results) that has been estimated (i.e., predicted) by a model. While the dependent variables in the groundwater flow and transport equations are head and concentration, the model result(s) of interest for which an "uncertainty description" is desired can be more complicated than just the uncertainty in head or concentration at a specific location and at a specific point in time. The model result(s) of interest is typically one or more management or decision measures or criteria. For example, it might be the maximum concentration through time at a compliance boundary or the maximum contaminant flux through time to a specific reach of river. Borrowing from Hill (1994), the model result for which an uncertainty description is desired might include an even more complicated set of criteria. For example, given the parameter uncertainty, what confidence do we have that in a certain area (i.e., at some group of model nodes) the drawdown will not exceed 2 feet and the flow from the groundwater system to a reach of river will not be decreased by more than $20 \%$ over the values predicted before granting a right to pump a well at some specified rate. Model result(s) of interest can include any quantity that is a simple function of the dependent variables (e.g., cost or dose) as long as there are no uncertainties in the additional parameters of these functions, otherwise those uncertainties must be part of the propagation process.

The various ways that uncertainty in model result(s) can be represented, at least theoretically, was discussed in Peck et al. (1988). From the most complex (most information) to the simplest, they include

- Full multivariate probability distribution (also described as joint probability distribution). For model results this could be a $n+1$ dimensional surface whose $\mathrm{n}$ axes would represent each possible model result (e.g., head $_{i}$ where $i$ ranges from 1 to the number of model nodes, concentration $_{i}$ for $i$ from 1 to the number of model nodes, flux to river "A," flux to river "B," etc.) and whose surface height (the $n+1^{s t}$ dimension) would have been normalized so that the volume under $n+1^{s t}$ dimensional surface is 1.0. As discussed in Peck et al. (1988), construction of a full multivariate distribution for all results, while theoretically possible, is impractical.

- Limited multivariate probability distribution. A bivariate probability distribution such as illustrated in Figure 3.7 would provide the most complete description for Hill's management criteria above. The $x$-values axis would represent the area drawdown axis and the $y$-values axis would represent the reduction in river flow axis.

- Univariate (also monovariate) probability distribution function. A univariate pdf (CDF or CCDF) for each result of interest as discussed in Section 3.1.1.1.1. Through Monte Carlo analysis, a univariate pdf can be constructed by evaluating the desired model result(s) for each random realization generated.

- Moments. The uncertainty in model results can be limited to estimating the first moment, the expected value (i.e., most probable value), and the second moment, the variance or covariance of the desired model results. 


\subsection{Normal Distribution}

The normal distribution plays an important part in uncertainty analysis because of its central role in probability and statistics for the various reasons discussed in Rice (1988) and Davis (1986). These include

- its role as a model for measurement errors as proposed by Carl Friedrich Gauss and hence its alternate name, the Gaussian distribution (e.g., the assumption of a normal error vector is required to apply maximum likelihood estimation methods as discussed in Beck and Arnold [1977])

- its applicability as a good description of the variability in parameters describing some natural populations

- the frequent assumption that random variables are normally distributed, probably due to their natural origins, that has resulted in many statistical tests being based on this assumption

- the central limit theorem that justifies the use of the normal distribution in many applications.

As discussed in Davis (1986), repeated measurements on large samples drawn from natural populations produce the characteristic bell-shaped normal distribution which, as illustrated in Figure 3.8, has the unique characteristic that most values are clustered around a uniform central tendency that is the same regardless of the type of central tendency measure (i.e., mode $=$ median=mean as defined in Figure 3.3). As with all probability density functions, the area under the pdf is 1.0 and, for continuous distributions, probabilities are only associated with a non-zero interval since the probability of an individual specific value is zero. Another useful property of the normal distribution is that the area under the curve within any specified range can be precisely calculated as is illustrated for standard deviation intervals of 2, 4, and 6 centered around the mean (Figure 3.8). The 95\% confidence interval for a normally distributed quantity of mean, $m$, and standard deviation $\sigma$, lies between $(m-1.96 \sigma, m+1.96 \sigma)$ since $2.5 \%$ of the values lie below $(m-1.96 \sigma)$ and $2.5 \%$ of the values lie above $(m+1.96 \sigma)$.

Davis (1986) indicates that the reason the variation measured in most naturally occurring phenomena can be described by the normal distribution is a reflection of the central limit theorem, which states that the sums of many independently operating processes tend to be normally distributed as the number of effects becomes large. The central limits theorem also provides a means to determine the population mean and variance even if the shape of the distribution is unknown, since as discussed in Davis (1986), sample means calculated from random samples taken from any population will tend to be normally distributed and the normality will increase with larger sample sizes, $n$. As $n$ gets large, the sample means will converge to the population mean and the variance of the sample means, $s_{m}{ }^{2}$, is equal to $1 / n$ times the population variance, $\sigma^{2}$, or

$$
s_{m}{ }^{2}=\sigma^{2} / n
$$




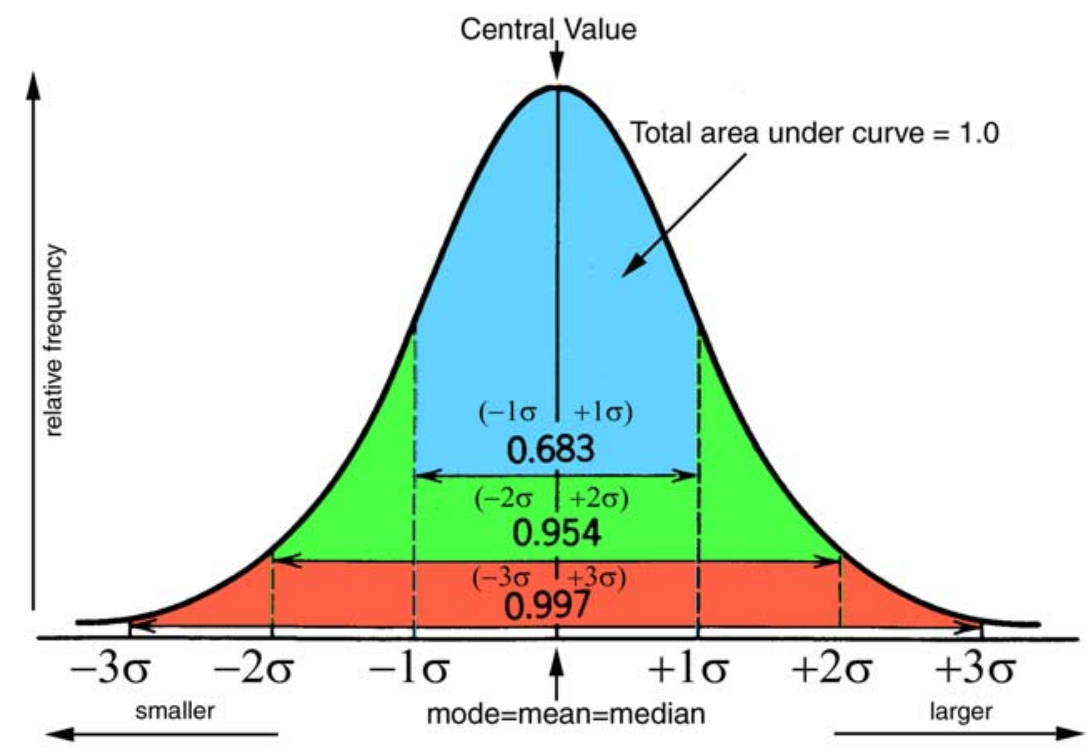

Figure 3.8. Plot of the Normal Frequency Distribution Indicating the Relationship Between the Central Tendency Measures, Mode, Mean, and Median for this Distribution and the Area Enclosed by Successive Standard Deviation Intervals Around the Mean (after Davis 1986).

The standard deviation of the sample means (i.e., the standard error of the estimate of the mean) or simply the standard error, $s_{e}$, is given by (3.10).

$$
s_{e}=\sqrt{\frac{\sigma^{2}}{n}}=\frac{\sigma}{\sqrt{n}}
$$

\subsubsection{Sensitivity Versus Uncertainty Analysis}

In a section on Treatment of Uncertainty in NUREG/BR-0184, Rev. 2 (USNRC 1997) the USNRC describes the similarities and differences between the related subjects of sensitivity analysis and uncertainty analysis as they apply to PRAs.

"As defined by Vesely and Rasmuson (1984), uncertainty and sensitivity analyses are similar in that both strive to evaluate the variation in results arising from the variations in the assumptions, models, and data. However, they differ in approach, scope, and the information they provide.

Uncertainty analysis attempts to describe the likelihood for different size variations and tends to be more formalized than sensitivity analysis. An uncertainty analysis explicitly quantifies the uncertainties and their relative magnitudes, but requires probability distributions for each of the random variables. The assignment of these distributions often involves as much uncertainty as that to be quantified.

Sensitivity analysis is generally more straightforward than uncertainty analysis, requiring only the separate (simpler) or simultaneous (more complex) changing of one or more of the inputs. Expert judgment is involved to the extent that the analyst decides which inputs to change, and how much to change them." 
Frey (1998) indicates that the most common approach for addressing uncertainty is to ignore it or to use simple sensitivity analysis with most model parameters held at nominal values, while others are varied from high to low values and the effect on the important measures is observed. However, as Frey points out, the combinatorial explosion in possible sensitivity scenarios becomes unmanageable and the results not only become uninterpretable, but sensitivity analysis "provides no insight into the likelihood of obtaining any particular result." This contrast between sensitivity and uncertainty analysis will be important in subsequent discussions related to which analysis method may be the most appropriate for addressing specific types of uncertainties.

As discussed in USNRC (2000), uncertainty and sensitivity analyses are closely linked, and ideally, they should be considered together. Sensitivity analysis, when performed in conjunction with uncertainty analysis, is used to identify parameters and assumptions that have the largest effect on the model result and thus should be included as part of the uncertainty assessment. In this manner, sensitivity analysis provides a tool for understanding and explaining the influence of key assumptions and parameter values on the variability of the predicted results. In contrast, uncertainty analysis provides more information to the decision-maker than deterministic analysis by characterizing a range of possible outcomes and their associated probabilities. However, results of an uncertainty assessment must always be viewed and evaluated based on the understanding that not all sources of uncertainty can be addressed and that any key assumptions made as part of the analysis are assumed to be substantially correct.

\subsubsection{Measurement/Observation and Reality}

Most of the definitions of uncertainty presented in the introductory paragraphs of Section 3.1.1 above link uncertainty with a modeling activity for predicting future conditions and the need to understand the uncertainty in these predictions for the purpose of decision making. This applies directly to the uncertainty needs of the site-wide groundwater modeling activity. Figure 3.9 (adapted from various figures in the textbook on parameter estimation by Beck and Arnold [1977]) illustrates some important concepts in understanding some of the basic sources of uncertainty. The key uncertainty concept illustrated in Figure 3.9 is that reality (or the actual system state) and observed system state are likely to be different because of uncertainties related to the measurement and interpretation process. This is similar to the well-known Heisenberg uncertainty principle of quantum mechanics that measurements do not reflect reality because the measurement process itself alters the system.

Uncertainty arises because devices and methods used to make measurements have errors (e.g., related to imprecision and/or bias as illustrated in Figure 3.10 adapted from Frey [1998]), can alter the system, and are incomplete in terms of their spatial and/or temporal resolutions. As discussed in Frey (1998) and illustrated in Figure 3.10, measurements can contain both random and systematic errors. Random errors arise from imperfections in the measurement techniques (e.g., quality of the measurement instrument) or from processes that are random or statistically independent of each other, while systematic errors may be related to device calibration errors and thus would affect all measurements in a similar manner. While, as discussed in Frey (1998), random measurement error is inversely related to precision (i.e., the agreement among repeated measurements of the same quantity), these random deviations provide no insight into the presence of systematic error. 


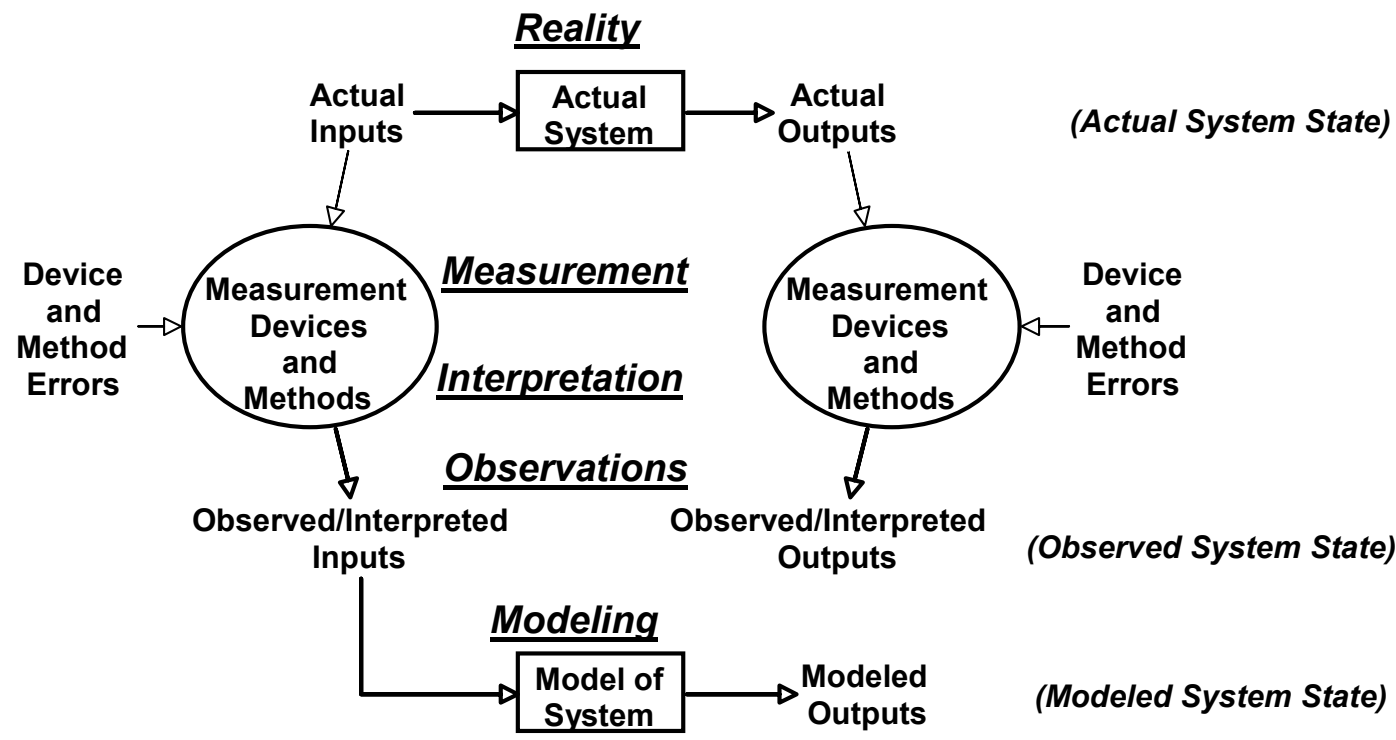

Figure 3.9. Important Concepts in Understanding Sources of Uncertainties (adapted from various diagrams in Beck and Arnold 1977).
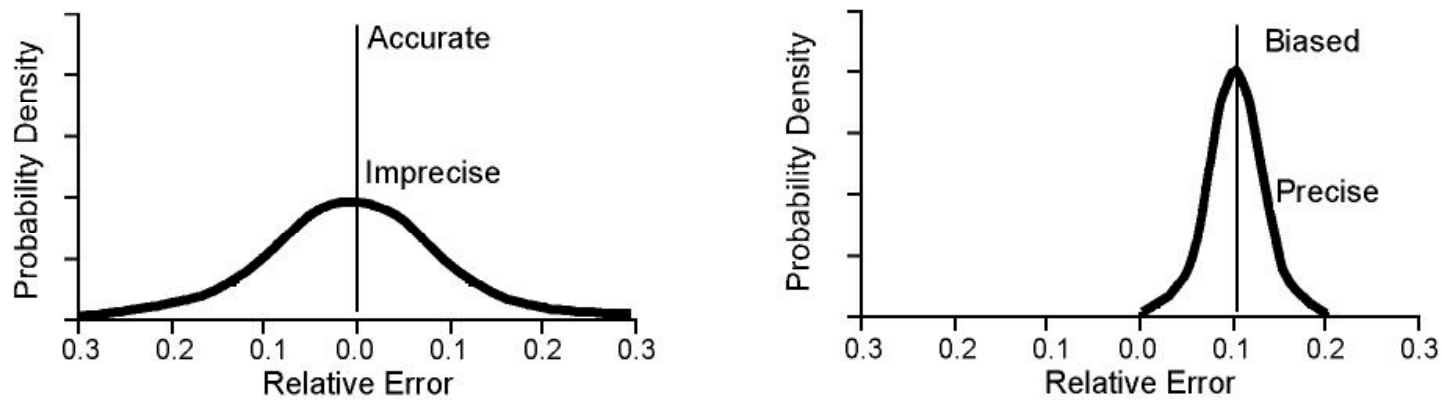

Figure 3.10. Accuracy and Precision in the Measurement Process (adapted from Frey 1998).

Other uncertainties arise in the process of interpreting and extrapolating a limited number of observations in space and time into the full spatial and temporal distributions of input and outputs needed to select, calibrate, and use an appropriate model(s) of the system to predict future-system state. At issue in this step is the important Nyquist sampling frequency law, which states that spatial and temporal frequencies with periods less than one-half the smallest spatial or temporal sample spacing cannot be resolved (Kanasewich 1981).

Not illustrated in Figure 3.9 is the uncertainty in future predictions related to what will later be described as scenario uncertainty, i.e., uncertainty in assumptions about future boundary conditions and driving forces not part of the system being modeled (e.g., future weather patterns, disposal alternatives and their effects, and land use). 


\subsubsection{Basic Steps in an Uncertainty Analysis}

A final important aspect of uncertainty analysis, which was clearly identified in the USNRC (1997) definition above, is that uncertainty analysis involves

- an identification step, where the probability distributions for the uncertain input quantities are developed

- a propagation step, where these input quantity uncertainties are appropriately propagated through the "risk equation" to generate output probability distributions for the measures of concern.

Data, expert opinion, inverse methods, and combinations of these sources can drive the identification process. The uncertainty propagation step, according to Wilson and Shlyakhter (1995), is described as error propagation in formal statistical theory that has its origin in the nearly two-centuries-old mathematical theory of measurement error attributed to Gauss. An important issue in this propagation step is the existence of correlation between measurements since only when each of the measurements is statistically independent or uncorrelated with other measurements (Wilson and Shlyakhter 1995) can the errors in the measurements be added in quadrature (i.e., the square of the combined error is the sum of the squares of the component errors). Wilson and Shlyakhter (1995) also indicate that because the word "error" used in formal statistical theory has the liability connotation of "mistake" in discussions of public health, the words "uncertainty analysis" have replaced "theory of error."

Beck's (1987) extensive review of the analysis of uncertainty identifies more details of the steps involved in the analysis of uncertainty. These include

1. Generating preliminary hypotheses based on sparse uncertain observations to identify plausible model structure(s); where model structure identification is defined by Beck (1987) to be

"The unambiguous determination, by reference to in situ field data $\left\{\boldsymbol{u}\left(t_{k}\right), \boldsymbol{y}\left(t_{k}\right)\right\}$, of how measured input disturbances $\boldsymbol{u}$ are related to state variables $\boldsymbol{x}$ and how these latter are in turn related both among themselves and to the measured output responses $\boldsymbol{y}$ of the system under study."

2. Selection and evaluation of model structure(s).

3. Estimation of parameters (or model calibration) to determine for the selected and evaluated model structure(s) what Beck (1987) describes as

"Uncertainty about the values of the parameters (coefficients) appearing in the identified structure of the dynamic model for the system's behavior."

4. Checks and balances on the identified model(s) (i.e., verification through analysis of residuals for example, and model discrimination). Beck (1987) suggests that a pragmatic set of stopping criterion to decide when a particular model structure is adequate might include: a) model parameters be 
invariant with time; b) estimation error variances and covariances be low indicating good parameter identifiability; and c) residual errors (model versus observations) should be small and not have any significantly nonrandom character.

5. Propagation of prediction error, which Beck (1987) defines as follows:

"Given the model structure and parameter estimates, subject to uncertainty, determine future behavior under different (assumed) uncertain input conditions."

Figure 3.11, from Beck (1987), illustrates the many sources of uncertainty. This figure also illustrates what Beck describes as "the logical connections between the cyclical triplet of (prior assumptions $\rightarrow$ identification $\rightarrow$ prediction)" and the difference between the internal description of the system (e.g., its parameters) and the external description of the system (i.e., the system's environment).

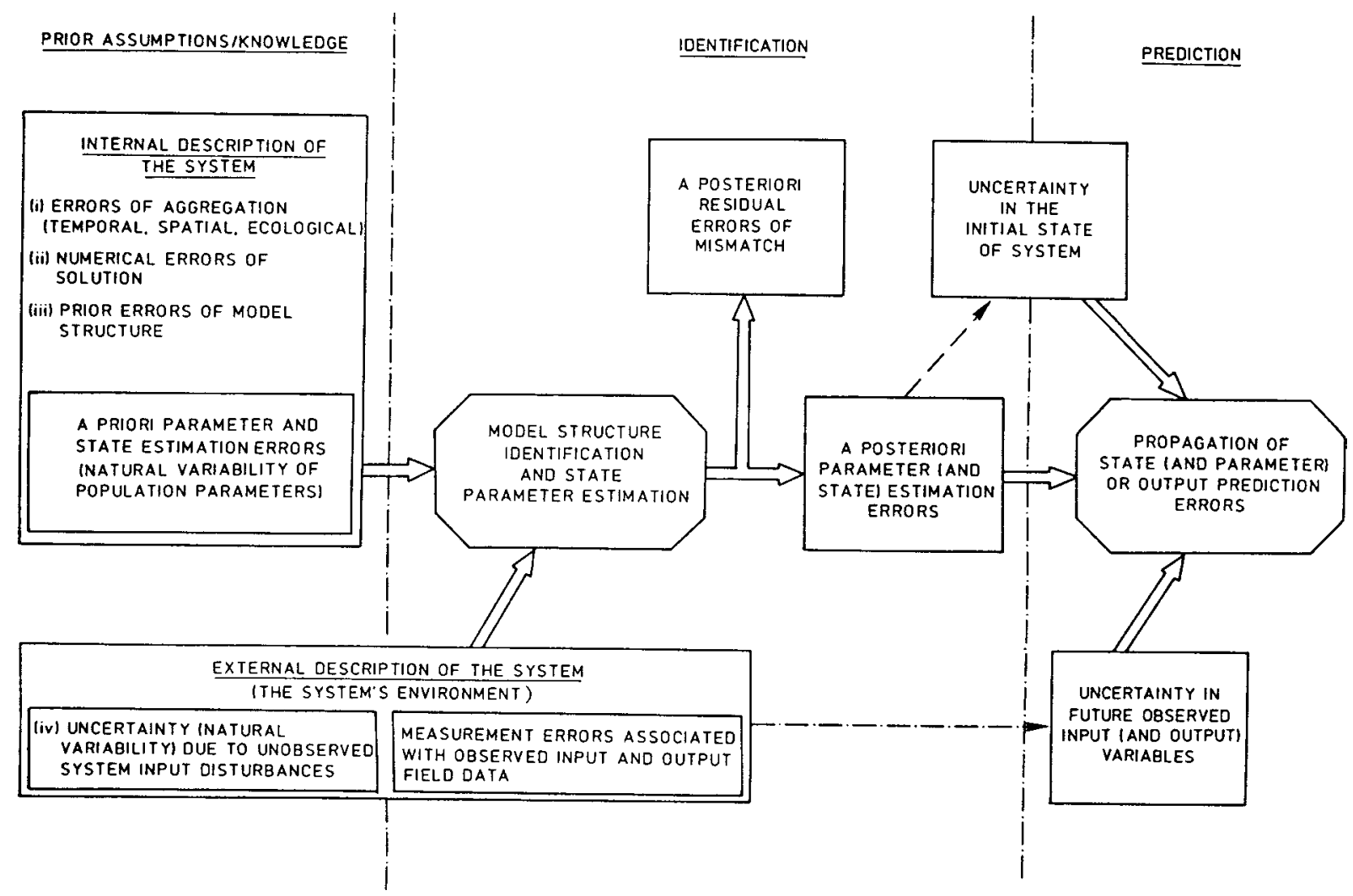

Figure 3.11. Frame of Reference for the Analysis of Uncertainty (from Beck 1987). 


\subsubsection{Modeling and Conceptual Models}

Model uncertainty and reliability concepts similar to those discussed in Chapter 6 (Issues in the Development and Use of Models) of the National Resource Council book, Ground Water Models Scientific and Regulatory Applications (NRC 1990, pp. 216-217) are also evident in Figure 3.9. In this chapter, modeling is defined as the art and science of collecting a set of discrete observations (our incomplete knowledge of the real world) and producing predictions of the behavior of the system. According to Meyer and Gee (1999) the conventional definition of a groundwater conceptual model is a mostly qualitative and often pictorial description of the groundwater system, including a delineation of the hydrogeologic units, the system boundaries, inputs/outputs, and a description of the soils and sediments and their properties. NAS (1996) characterizes a groundwater conceptual model as a hypothesis that describes the main features of the geology, hydrology, and geochemistry of a site, as well as the relationships between these components and the patterns of flow and contaminant transport. USNRC (2000), in its discussion of conceptual models, expands the definition by including the mathematical equations. According to USNRC (2000), the simplified representation of the site for the purpose of analyzing the release and migration of radionuclides through the natural environment and/or engineered systems, at a specific site, including the associated mathematical equations (i.e., mathematical models) is commonly referred to as the conceptual model of the site. Conceptual model development, according to USNRC (2000), involves making simplifying assumptions, including simplification of the appropriate governing equations, to reflect the physical setting and simplifying assumptions related to the geometry of the system, the spatial and temporal variability of parameters, the isotropy of the system, and the influence of the surrounding environment.

Since models are the source of the uncertain predictions used in decision making with the Hanford site-wide model, it is important to understand the modeling process in order to identify all the sources of uncertainty. Other definitions of models ranging from the simple to complex include

“Cartoons of Reality,” Chow (1970).

"a simplified version of the real system that approximately simulates the excitationresponse relations of the latter," - Bear (1985).

"A model is any device that represents an approximation of a field situation. Physical models such as laboratory sand tanks simulate flow directly. A mathematical model simulates groundwater flow indirectly by means of a governing equation thought to represent the physical processes that occur in the system, together with the equations that describe heads or flows along the boundaries of the model (boundary conditions). For time-dependent problems an equation describing the initial distribution of heads in the system is also needed (initial conditions)." - Anderson and Woessner (1992). 
Because modeling involves simplification, the first and most important step in any modeling protocol is to define the purpose of the modeling as illustrated in Figure 3.12 (after Anderson and Woessner [1992]) and Figure 3.13 (after Simmons and Cole [1985]). In both of these modeling protocols, conceptual model development is one of the most important steps. Bear (1985) indicates that selection of the appropriate conceptual model is dependent on the objectives of the modeling effort, which dictate the features to be represented as well as the accuracy required. Simmons and Cole (1985) describe the conceptual model as the hub in a wheel of steps required to perform a groundwater modeling performance assessment (Figure 3.13). A conceptual model is the set of assumptions needed to describe an approximation of the real system sufficient to model those aspects of the real system important to the intended use of the model.

Conceptual modeling (i.e., step 1 of the sequence of steps involved in the analysis of uncertainty described above) is the complex process of data interpretation and issue identification required to arrive at an appropriate set of conceptual model assumptions that integrates model purpose with available data and understanding. The technical issues are simply questions regarding what constitutes the correct way to model the system under study given the purpose of the effort. For example what is the

- appropriate model domain

- controlling and interacting processes at the desired space and time scales of interest

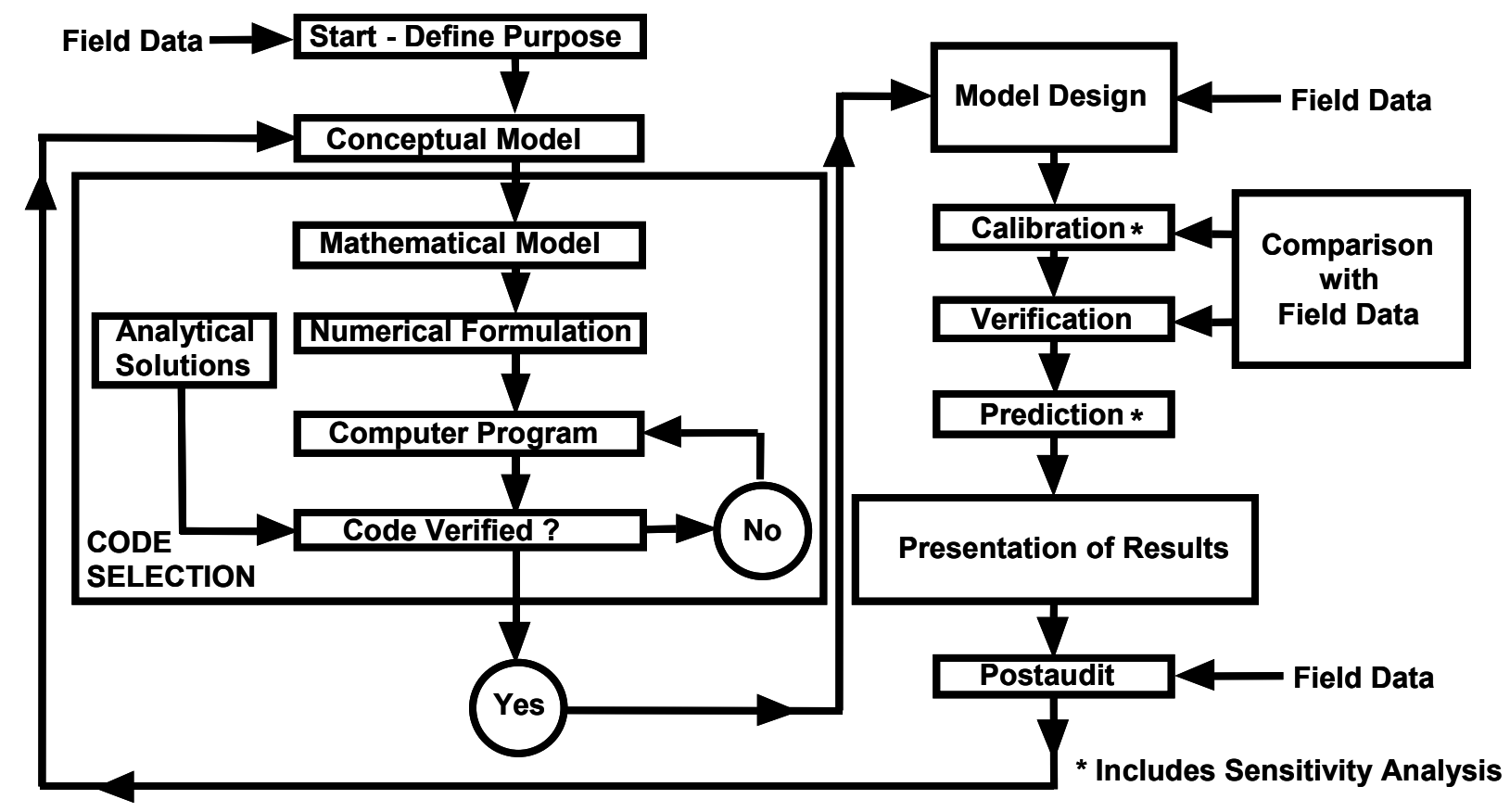

Figure 3.12. Modeling Protocol (after Anderson and Woessner 1992). 


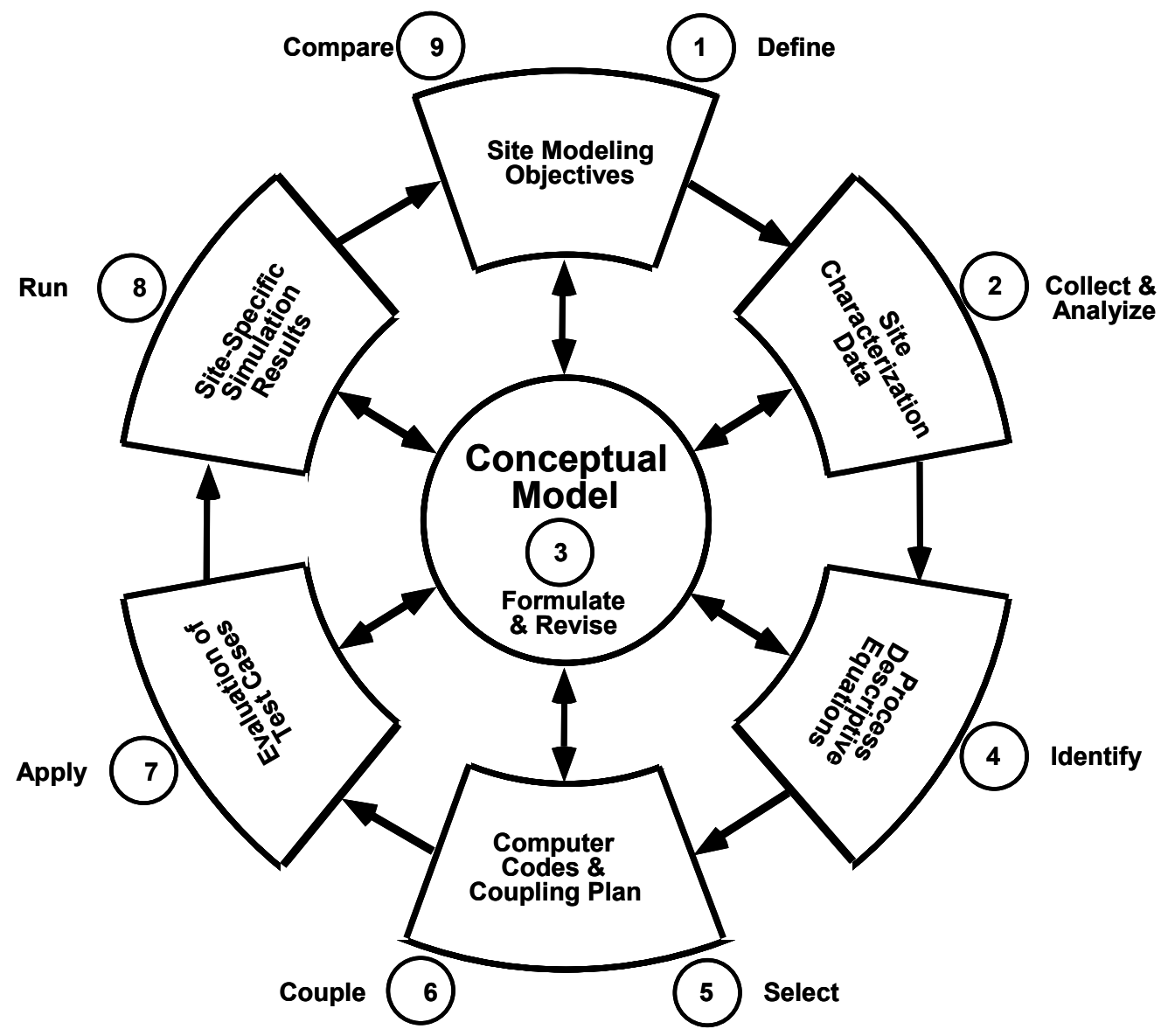

Figure 3.13. Performance Assessment Modeling Steps (after Simmons and Cole 1985).

- appropriate way to parameterize the system (e.g., number of layers or geohydrologic structure, heterogeneity within the layers, areal extent, relevant boundary and initial conditions)

- appropriate way to extrapolate measurements and observation into the spatial and temporal distributions of parameters, driving forces (past, present, and future), initial conditions, and responses.

One major difficulty is related to the reality that groundwater systems are hidden and nonhomogeneous (i.e., spatially variable) and subject to temporal driving forces and changes that cannot be fully identified and characterized. Wells can be thought of as small windows that reveal system responses to disturbances (e.g., pumping) as well as information on system parameters, but they are expensive to construct and provide varying degrees of clarity. Wells typically provide reasonable information on stratigraphic detail and hydraulic head but are of limited use in revealing information on parameters like permeability, effective porosity, and dispersivity. This is because, as will be discussed in subsequent paragraphs, the values and scale associated with these parameters are a function of the model, the test scale (i.e., averaging volume), the method of testing, and the sampling or observation strategy as illustrated in Figure 3.14. 
Scale Dependence of Porous Medium (microscopic to macroscopic)
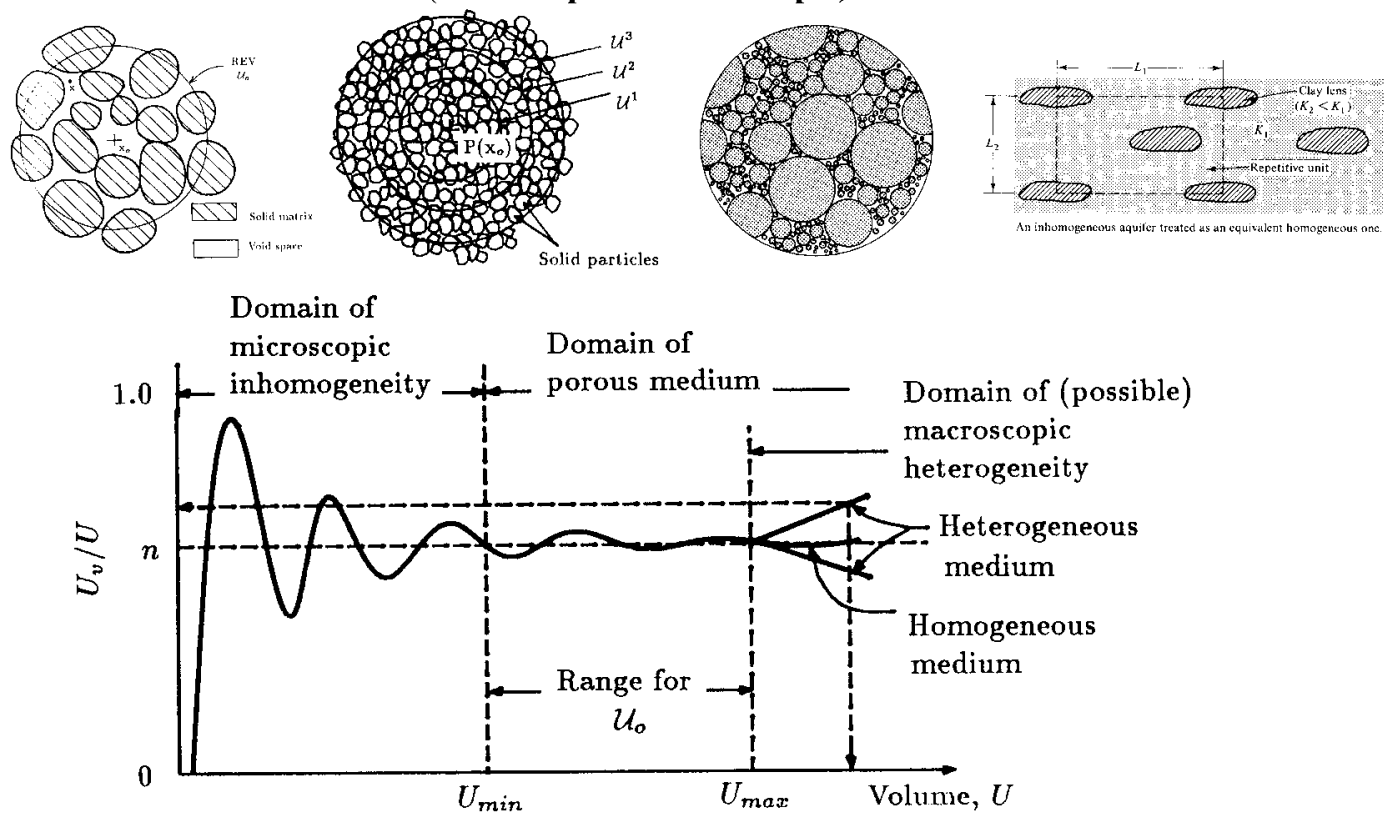

Scale Dependence of Dispersivity

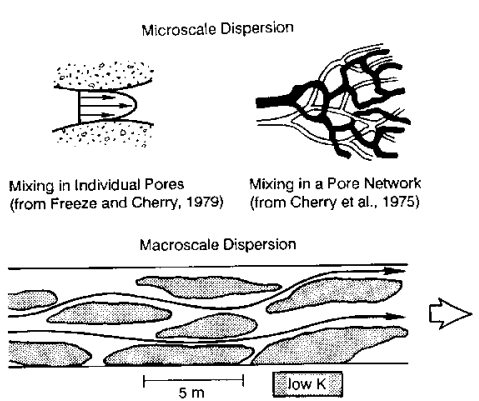

Dependence of Dispersivity on Sampling Strategy
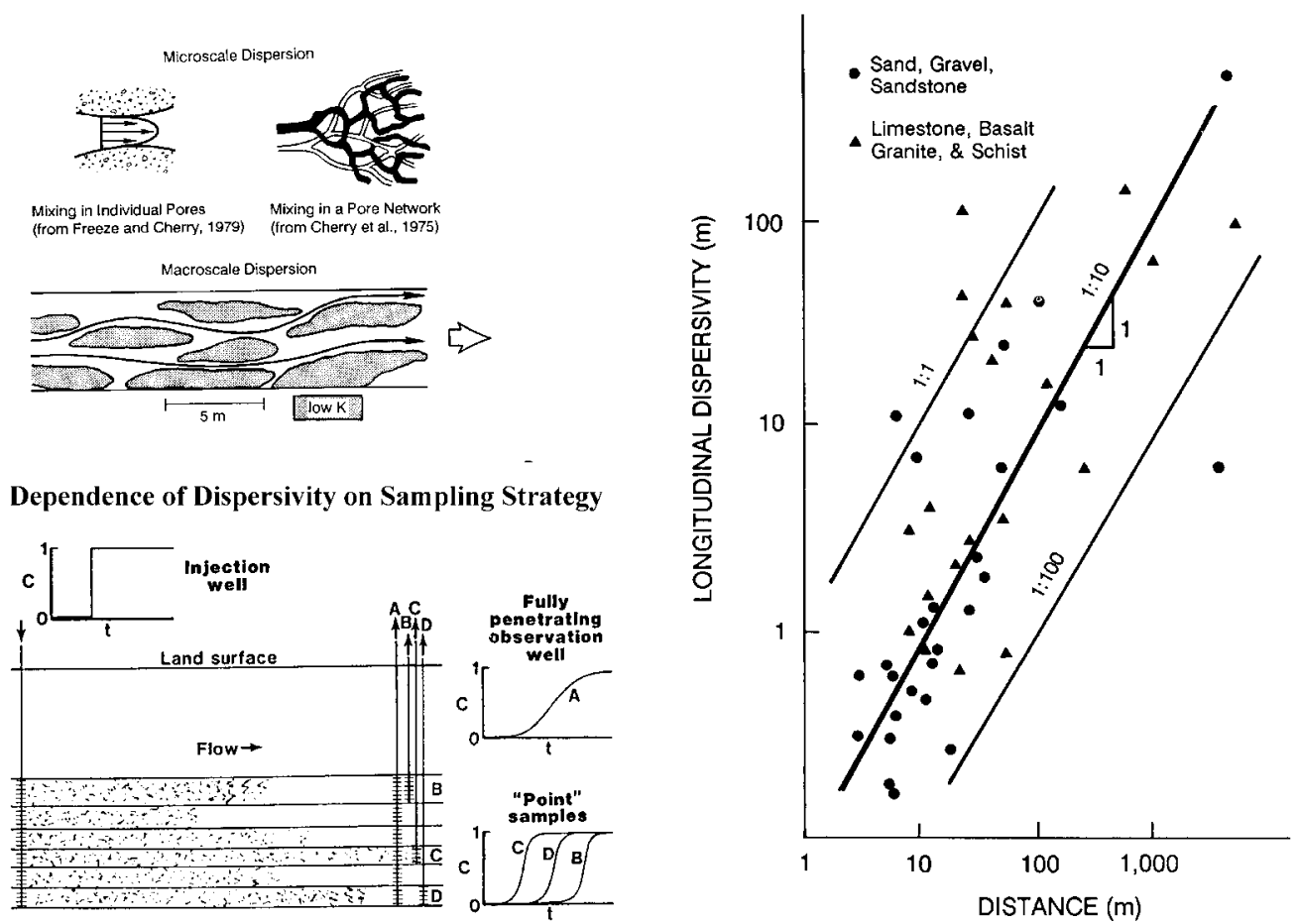

Figure 3.14. Scale Dependence of Porous Medium Parameters (adapted from various figures in NRC 1990; Bear and Bachmat 1990; and Bear 1972; with references to Freeze and Cherry 1979; and Cherry et al. 1975). 
Neuman (1999), in his draft report on "Methodology to Identify and Evaluate Conceptual Models and Uncertainty Related to Groundwater Transport at Nuclear Facilities and Sites" for the USNRC contrasts engineering and hydrologic systems as follows:

"Contrary to engineering systems which are generally closed, relatively simple and well defined, hydrogeologic systems are open, complex and only partially defined. The open nature of hydrogeologic systems means that they are not amenable to fully controlled experimentation; as they are additionally complex, their description must remain forever incomplete and imprecise."

As discussed in Neuman (1999), Anderson and Woessner (1992), Simmons and Cole (1985), and illustrated in Figures 3.12 and 3.13, the conceptual modeling step must be revisited continually to test if conceptual models are still plausible or need to be altered in light of new information and understanding. For example, the revised hydrogeology of the $200 \mathrm{E}$ Area (Williams et al. 2000) is being incorporated into the SGM.

\subsection{Sources of Uncertainty and the Issues}

In this section, the various sources of uncertainty and the associated issues that contribute to uncertainty will be identified and discussed. While there are many hierarchical classifications of the sources of uncertainty in hydrologic modeling, the most common high-level categories discussed include the following:

- uncertainty resulting from natural variability

- uncertainty in the model structure or conceptual model uncertainty

- uncertainty in the model parameters.

The above high-level categorization of uncertainty in hydrologic systems is attributed to Vincens et al. (1975) by Lence and Ruszczynski (1996).

Another common high-level categorization of uncertainty common in discussions of total system performance assessment is presented in Civilian Radioactive Waste Management and System Management and Operating Contractor (CRWMS M\&O 1999), which discusses the treatment of uncertainty in the assessment of the long-term performance of a potential geologic repository at Yucca Mountain. This performance assessment involves modeling various coupled thermal, mechanical, geochemical, and/or hydrologic processes taking place within engineered and natural barriers over extended periods of time. USNRC (2000), in Appendix C of its guidance document for preparation of Nuclear Material Safety and Safeguards Decommissioning Standard Review Plan, presents the same categorization that includes the following types of uncertainties: 
- parameter uncertainty, which arises because definitive values for relevant parameters, as well as the descriptive measures of their spatial and temporal variability, are difficult to obtain due to limited characterization of the natural system

- conceptual model uncertainty, which arises because alternative process models for various components of the disposal system may be equally likely or defensible because of incomplete understanding, limited information, or paucity of data

- scenario uncertainty, which arises because the future evolution of the geologic and natural environment surrounding the disposal facility is unpredictable.

A key difference between this latter categorization of uncertainty and the first categorization presented is the emphasis on the role of scenarios because of the need to consider very long time frames when developing a performance assessment and the need to examine release and migration of radionuclides through both engineered systems as well as the natural environment. The first categorization, attributed to hydrologists, places a major emphasis on natural variability. The main inputs to the conceptual model presented in USNRC (2000) further emphasize this difference as only site data, engineering designs, and scenarios are included.

USNRC (2000) points out that an important issue in uncertainty and sensitivity analysis is that not all sources of uncertainty can be easily quantified, and that of the three primary sources of uncertainty, the most mature methods address only parameter uncertainty. Scenario uncertainty is one of the most important sources of uncertainty related to making predictions about future outcomes (USNRC 2000), but scenario uncertainty, like conceptual model uncertainty, is much less developed than parameter uncertainty and there are no methods for quantifying these types of uncertainties. This is because of the difficulty in predicting the characteristics of future driving forces and conditions both natural (e.g., rainfall, river stage) and human-induced (e.g., pumping and waste discharge). An approach for identifying and quantifying scenario uncertainty is being developed as part of the full probabilistic analyses (e.g., CRWMS M\&O 1998) for licensing of nuclear waste repositories. This approach examines the expected features, events, and processes (FEPs) that can represent the evolution of the natural system and any engineered components, including any interactions in what is described as a FEPs approach to scenario uncertainty (Section 2.2 of CRWMS M\&O 1999). No formal approaches for identifying and quantifying conceptual model uncertainty have been identified at this time.

There are additional important sources of uncertainty left out by this classification that will be discussed (e.g., numerical error - identified with the numerical mathematics used in the code, and propagated error - related to erroneously estimated values of input parameters). However, the following sources of error or uncertainty, not dealt with by most texts according to James (1994), also are not discussed in this report.

"In practical applications, the most common errors may be traced to wrong data in the input files, caused by blunders, data entry errors, and user/modeller misconceptions. But the most serious errors are probably those that are made well after the model runs are 
completed: poor interpretation of the results, their inherent error, and reliability, by model builders, users and decision-makers alike."

Freeze et al. (1987) discuss some of the problems with using the results from uncertainty analyses without understanding all conceptual strengths and operational weaknesses, especially in systems with sparse data sets. Konikow and Ewing (1999) question whether reliable and meaningful assessments can be generated by applying probabilistic methods to complex natural hydrogeologic and geochemical systems. This is because they believe the degree and nature of the uncertainties are themselves highly uncertain and difficult to characterize, which means the uncertainty in these estimates of the uncertainty may be significant enough to render the uncertainty estimates themselves useless.

\subsubsection{Natural Variability}

Natural geohydrologic systems are inherently variable in both space and through time. System state variables (e.g., observations such as head, contaminant concentrations, and temperature) at any given location are changing through time as are the driving forces acting on these systems (e.g., river stage and precipitation-related-recharge), as illustrated in Figure 3.15. Even system properties (e.g., porosity, permeability, and $\mathrm{Kd}$ ) can change through time (albeit slowly). While some quantities can be and are

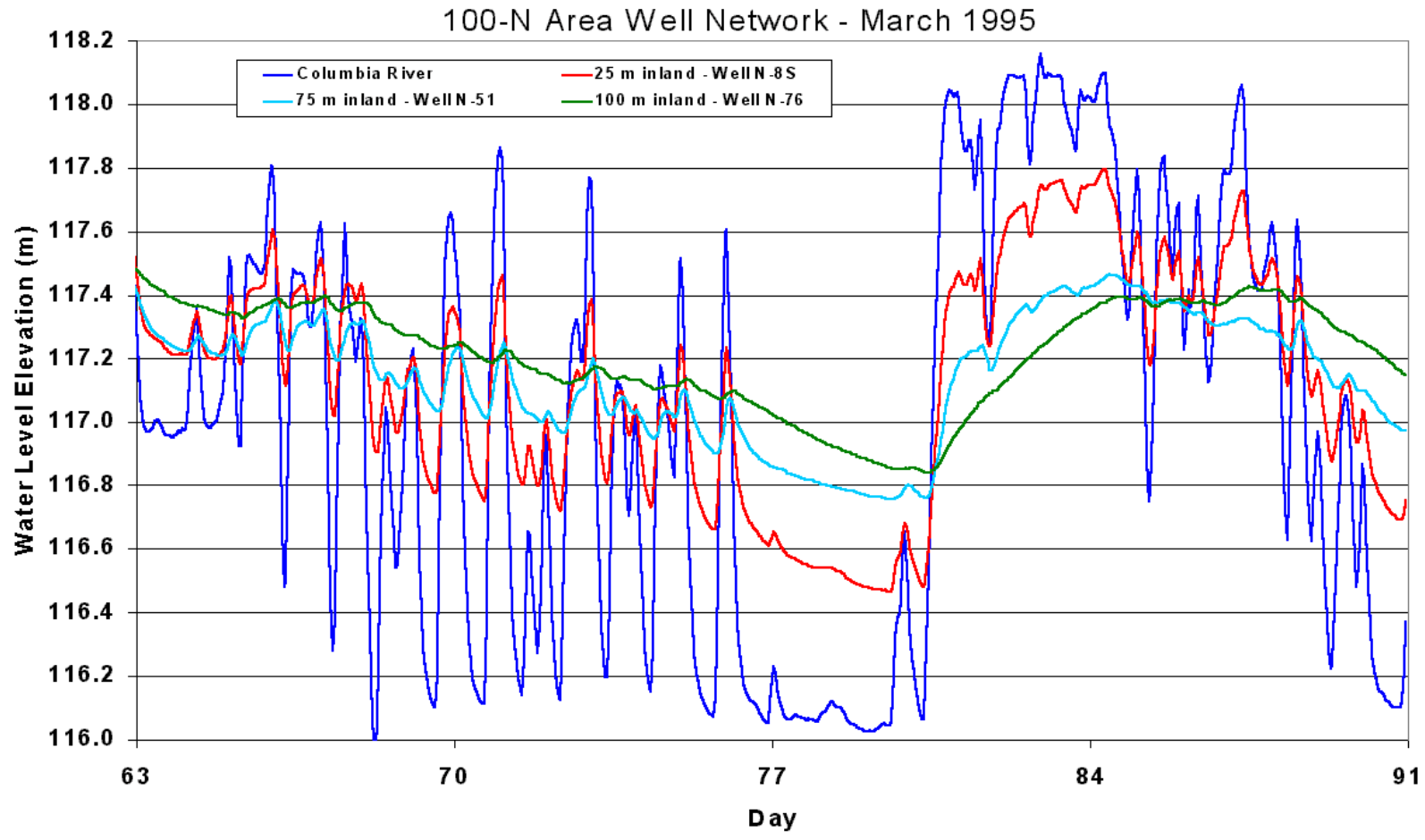

Figure 3.15. Measured River Stage and Well Responses at Various Distances from the Columbia River Near N-Springs (100 N Area) on the Hanford Site During March 1995. 
considered random in principle (e.g., precipitation), others are viewed as random due to the impracticability of continuous measurements both through time (e.g., river stage as illustrated in Figure 3.15) and over space (Isukapalli 1999).

The sparsity of available data to characterize groundwater systems is discussed by Eggleston et al. (1996). In their article, they evaluate the relative value of various geostatistical methods to predict unsampled hydrologic parameter values. They indicate, for example, that for a typical groundwater modeling effort, the parameter distribution in an aquifer volume of $\sim 10 \mathrm{~km}^{3}$ must be deduced from a data set consisting of $\sim 40$ point measurements. They also indicate that even for the most heavily sampled aquifers, such as the Macrodispersion Experiment Site in Mississippi, the 2200 available hydraulic conductivity measurements provide data on no more than $1 \%$ of the total aquifer volume.

Assume that all the measurements needed to characterize and observe the state of the real geohydrologic system were directly measurable. This real, spatial, and temporal variable system can be observed only using a finite number of observations associated with a sampling process that must choose what parameters and state variables to measure, what instruments to use, as well as where and when to measure them (James 1994; NRC 1990; McLaughlin and Wood 1988). The sampling process itself introduces uncertainty (James 1994; NRC 1990; McLaughlin and Wood 1988) in the form of

- two components of observational errors or uncertainty arising from the measurement devices: 1) a random component and 2) a systematic or bias component (Figure 3.10 after Frey 1998)

- sampling errors associated with the timing, location of the sampling (e.g., where convenient, available for other reasons, attainable because the measurement can be made), and disparate scales associated with the samples that result in measurements at only a small number of points and uncertainty at other locations in space/time or at equivalent scales. Sampling errors also can impart both: 1) a random component and 2) a bias component (e.g., pump test data are typically poor or not carried out in low permeability zones) to the uncertainty.

A consequence of the limited sampling is that the conditions between the sampled points in both space and time are not known with certainty, especially in systems that have significant spatial and temporal variability (NRC 1990). Instead, they must be interpreted/extrapolated from the available observations (e.g., through geostatistical techniques, Deutsch and Journel 1992) in an attempt to account for spatial and temporal correlations between the measurements and the associated uncertainties. Frey (1998) discusses a related issue of representativeness or the "law of small numbers" and warns that one should be cautious in inferring patterns from small data sets because actual patterns may not be completely characterized until substantially more data are collected. James (1994) makes the related point that observation and sampling errors, and the structural error due to discretization, arise because of the inherent variability of natural hydrological (e.g., Figures 3.16 and 3.17) and ecological systems. The inability to fully characterize as well as represent this variability in a practical model means that model inputs will require spatial (and temporal) averaging, even where the scales of spatial (or temporal) heterogeneity in nature may be very large (e.g., the effects of the layering and sand lenses of the Middle Ringold shown in Figure 3.17 and the uncertainty they impose need to be factored into the average or upscaled flow and transport parameters needed as input to a model at the scale of the Hanford SGM). 


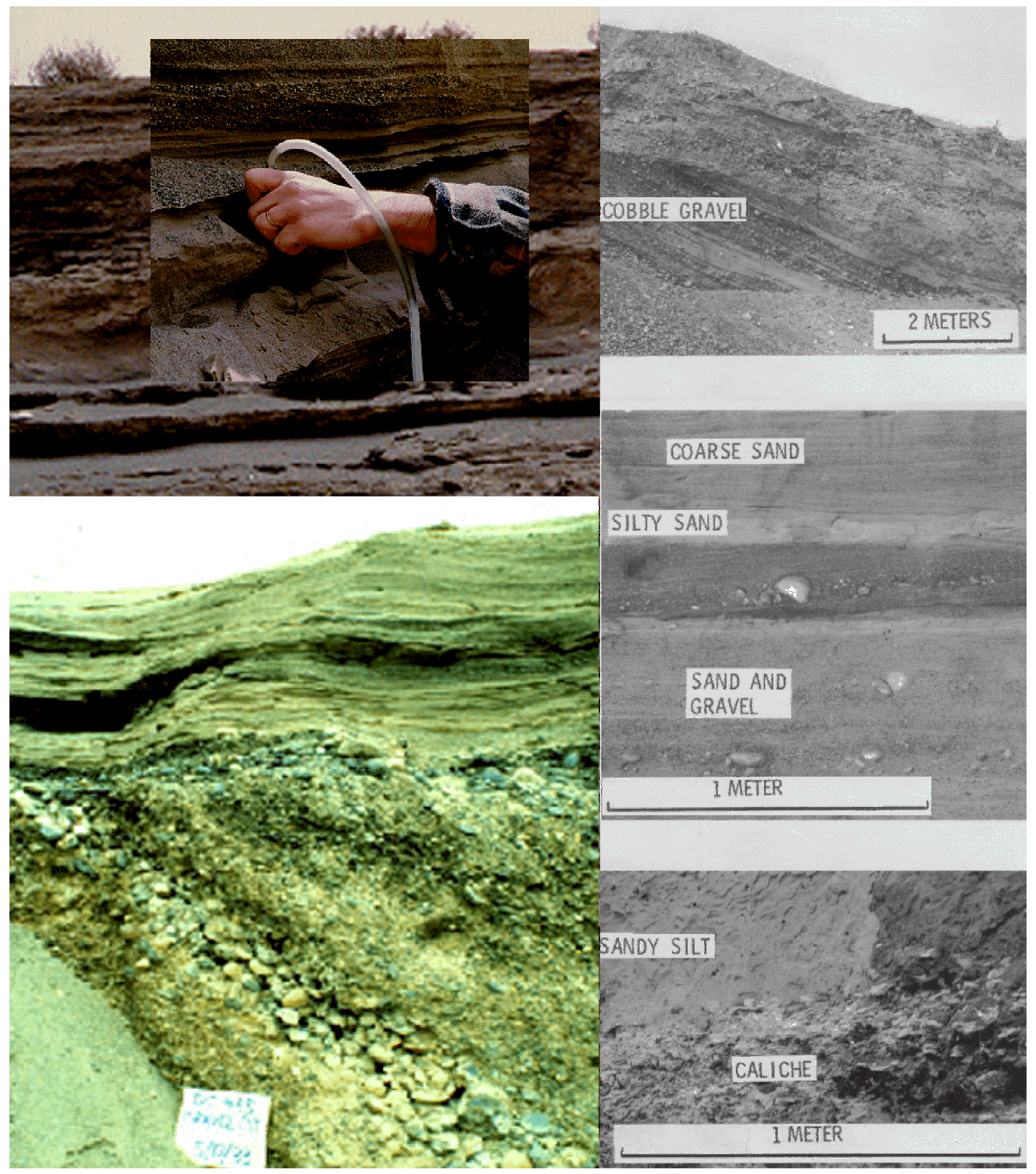

Figure 3.16. Examples of the Various Types and Scales of Spatial Variability (heterogeneity) in the Hanford Sediments on the Hanford Site. 


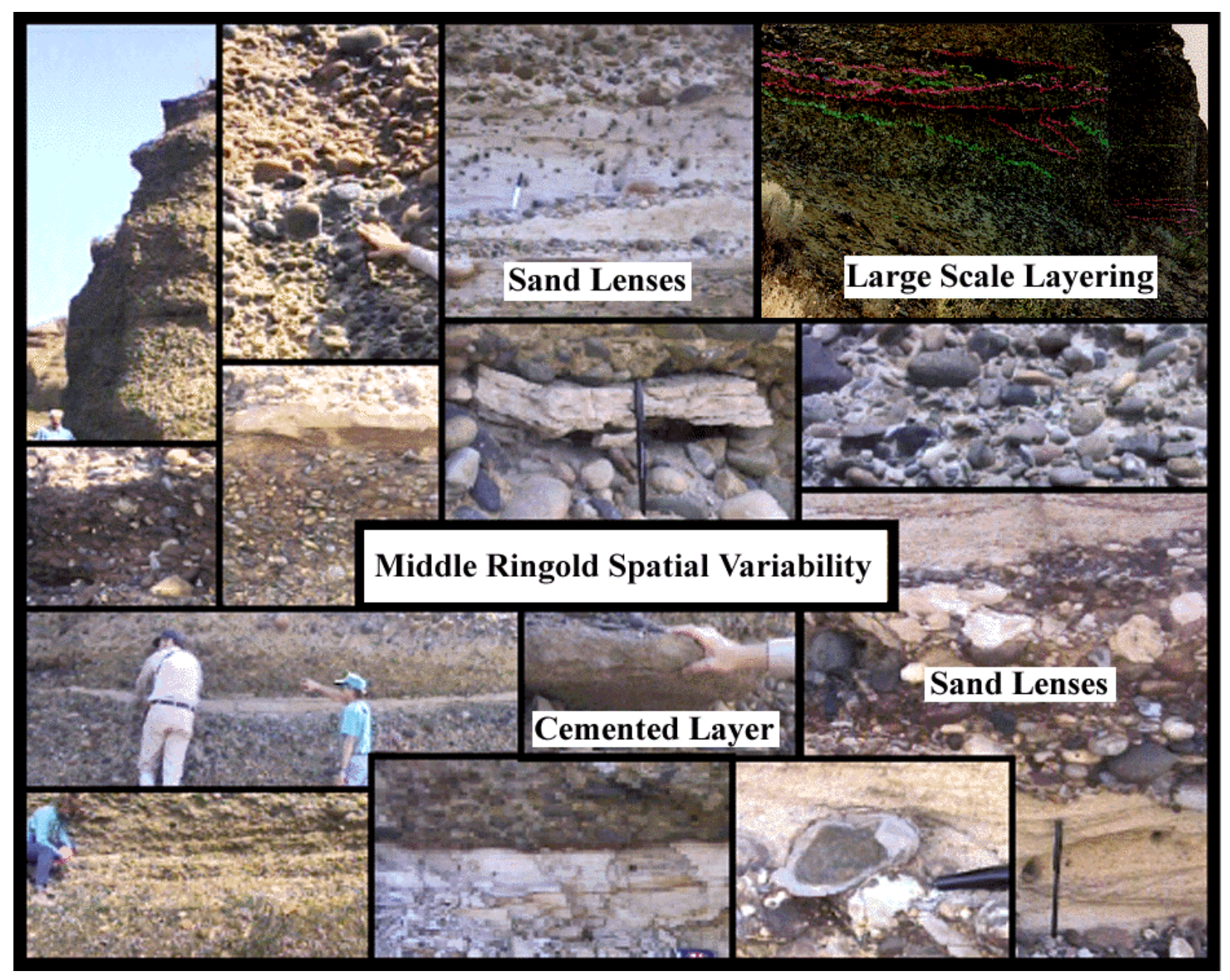

Figure 3.17. Examples of the Various Types and Scales of Spatial Variability (heterogeneity) in Unit E of the Ringold Formation (i.e., Middle Ringold) Across the River from the Hanford Site.

Other major issues arise related to the natural variability of geohydrologic systems and the fact that most models of groundwater systems are distributed parameter models in which the parameters are not directly measurable, but in fact must be interpreted from historical observations of state variables and the associated driving forces (Yeh 1986). NRC (1990), in discussing groundwater model parameters that are not directly observable, describes them as constitutive parameters (e.g., hydraulic conductivity, dispersion coefficients, and partition coefficients) and indicates they arise when models are formulated from basic principles (e.g., conservation of mass) and quasi-empirical laws (e.g., Darcy's law). Bear (1985), in his discussion of model coefficients and their estimation, makes the argument that permeability and dispersivity, for example, are coefficients that express the "macroscopic effects of the microscopic configuration of the solid-fluid interfaces within a porous medium." They are "coefficients of the models" that represent the passage from real system to the specific mathematical one and that "in spite of the similarity in their names in different models, their interpretation and actual values may be different from one model (of the same porous medium domain) to the next." Bear (1985) notes that "following 
common practice we refer to these as aquifer coefficients and not as coefficients of the aquifer's model," realizing the coefficients are derived to correspond to a specific model. Both Bear (1985) and Yeh (1986) discuss the inappropriate but standard practice of determining aquifer parameters derived from field data at one scale interpreted, for example, using a homogeneous isotropic radial flow model (Theis 1935) in more regional models that describe flow in finite, nonradial flow systems, which contain heterogeneities at ever-increasing scales from the microscopic to macroscopic as illustrated in Figures 3.14, 3.16, and 3.17. While the issues of measurement scale and aggregation of measurements apply to all models, the use of constitutive parameter models introduces additional scale and upscaling issues (e.g., how to combine parameters measured at different, usually smaller scales and/or for different constitutive models for use in deterministic and or stochastic models) that result in additional sources of uncertainty (Farmer 1986; Desbarats 1998; and Schulze-Makuch et al. 1999).

Meyer and Gee (1999) discuss the effect of the averaging process, related to upscaling both spatially variable and temporally varying parameter estimates, on the appropriate parameter variability of these averaged or upscaled parameters. They indicate that while the variability in meteorological processes such as rainfall can be characterized on the scale of hours to years, the precipitation required as an input parameter to relatively simple codes for the purpose of modeling assessments over long time periods and larger spatial scales is the long-term average value. This is similar to choosing the appropriate time interval for averaging river stage for input to the SGM (Figure 3.15). Meyer and Gee point out that when conducting an uncertainty analysis, it is not the small time scale uncertainties that must be characterized but the uncertainties in the averaged or upscaled values that must be characterized. In a similar manner, it is not the total uncertainty in geohydrologic properties at the smallest scale of natural variability that must be characterized, but the uncertainty in upscaled or averaged hydrologic parameters appropriate for use in the simpler model, with its larger spatial scales, that is being used in the assessment. As they indicate, the issue is a matter of differences in scale and requires the correct interpretation of both model parameters and the uncertainty in these upscaled parameters including the uncertainty introduced by the upscaling process. According to Meyer and Gee (1999), the expected form of the relationship between natural parameter variability and uncertainty in average parameter value appropriate for a larger space/time scale model is illustrated in Figure 3.18 (adapted from Meyer and Gee 1999). Figure 3.18 shows the relative form of the two probability density functions that illustrate the Meyer and Gee intuitive concept that the uncertainty in an average parameter value is less than the variability of that parameter, a fact which they attribute to the central limit theorem property discussed in Section 3.1.1.1.5, which shows that variance in the sample mean is less than the variance of the parameter in the population, as given in Equation 3.9. It should be noted that the above argument assumes that the averaging process for upscaling the parameter is well defined. Peck et al. (1988) discuss this same subject and indicate that in Monte Carlo simulations it must be realized that it is the statistical properties of the parameters averaged over the elements or blocks of the model that must be used. They indicate that for a stationary case, the mean is unchanged, but the variance and covariance are changed by the spatial averaging. EPA (1997) also indicates that the variability depends on the intervals selected for time and space averaging or on the ways in which the data are aggregated, but they also note that standard data analysis tends to understate variability by focusing solely on random error while overstating variability on the other hand by implicitly including measurement error. 


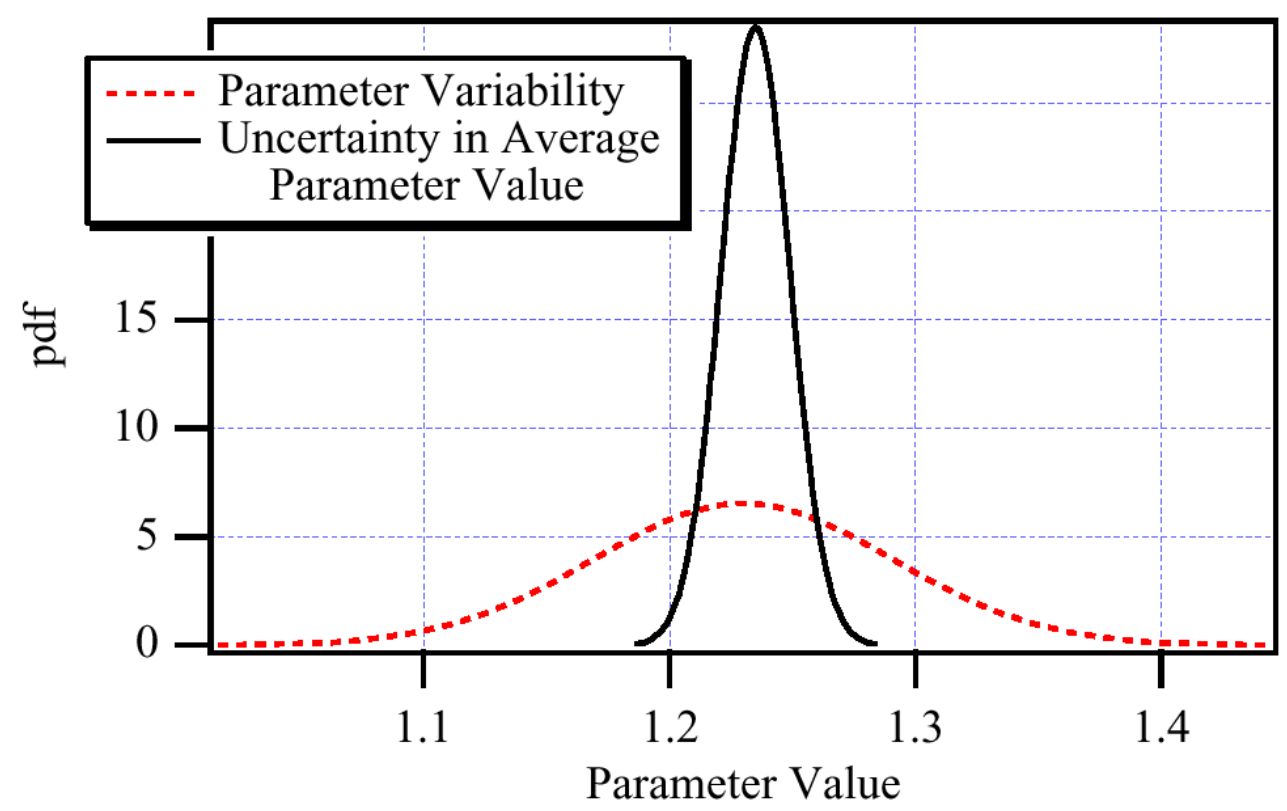

Figure 3.18. Expected Relationship Between Parameter Variability and Uncertainty in the Average Parameter Value (adapted from Meyer and Gee 1999). ( $\mathrm{pdf}=$ probability density function)

\subsubsection{Uncertainty in Model Structure}

Beck's (1987) frame of reference diagram (Figure 3.11) clearly identifies model structure identification as an explicit part of the identification process that involves determination of the internal description of the system as well as its external description. Model structure identification for the distributed parameter models of geohydrologic systems under study, as a practical matter, involve a reduction in the number of processes included, decoupling of processes, or a simplification of the mathematical representations of included processes (when necessary), and decisions about the necessary spatial and temporal requirements (e.g., spatial grid and time step size). See the quote from Isukapalli (1999) on this subject:

"Mathematical models are necessarily simplified representations of the phenomena being studied and a key aspect of the modeling process is the judicious choice of model assumptions. The optimal mechanistic model will provide the greatest simplifications, while providing an adequately accurate representation of the processes affecting the phenomena of interest. Hence, the structure of mathematical models employed to represent transport-transformation systems is often a key source of uncertainty."

Selecting the model with the greatest simplifications that adequately represents the processes affecting the phenomena of interest is consistent with the principle of parsimony discussed in Hill's 1998 methods and guidelines for effective model calibration. 
As discussed in NRC (1990), the parameter estimation effort is "critically dependent on the validity of the underlying model formulation." Model formulation includes, for example identification of: 1) the geometry of the model domain, 2) the dominant physical and chemical processes, 3) geological heterogeneities and their geometry and nature, 4) source term information (e.g., lack of information on source locations, poorly known history of contaminant releases, unknown variability in mass or concentration distributions of contaminants, complexity in the chemical composition of contaminants [NRC 1999]), 6) scenarios (e.g., future driving forces and other conditions), and 7) initial conditions. Uncertainties arise from each of these components of a specific model formulation; however, there may also be alternative model formulations (i.e., sets of scientific or technical assumptions for developing a model [Isukapalli 1999]) that must be evaluated. There are very few objective measures for evaluating the uncertainty associated with the alternative models beyond statistical measures of overall model fit discussed in Hill (1998) and the four measures suggested and discussed in Carrera and Neuman (1986a,b,c) whose use in the estimation of hydraulic parameters is illustrated in Hyun and Lee (1998). Beyond the maximum likelihood objective function, the four additional measures include: 1) Akaike's First Information Criterion (1974), 2) Akaike's Second Information Criterion (1977), 3) Hannan's Criterion (1980), and 4) Kashyap's Criterion (1977). These four additional measures were developed to address the criticism that calculated error variance and standard error do not sufficiently represent the drawbacks associated with increasing the number of estimated parameters (i.e., the issue of model complexity). All four criteria use $-2 \ln$ (maximum likelihood) as a base value and add penalty terms (e.g., 2 NP or NP $\ln$ (ND), where NP is the number of parameters and ND is the number of observations) to reflect the fact that adding too many parameters produces a poorer model with unreliable parameter estimates. Although these statistics were developed for time-series problems, Carrera and Neuman (1986b) successfully used them to discriminate between different parameterizations of a test case of groundwater flow. For each of these statistics, given randomly distributed residuals, smaller values indicate a more accurate model. However, as discussed in Hill (1998), if the statistics for a model with fewer parameters are only slightly larger than the statistics of another model, it may be better to select the model with fewer parameters unless the investigator has sufficient information to indicate the validity of the more complicated model. Hyun and Lee (1998), through their study of the usefulness of these four additional measures, which included testing with synthetic data sets, showed that there are resolution difficulties and that noisy data present problems with the use and interpretation of these measures for realistic problems. An additional difficulty posed by alternative models is that a probability cannot be assigned to each of these alternative models from the statistical measures of overall model fit (Hill 1998) or as a result of evaluating these four different criteria. This means model structure uncertainty must be dealt with through sensitivity analysis. Isukapalli (1999) states "if the results from competing models result in similar conclusions, then one can be confident that the decision is robust in the face of uncertainty. If, however, alternative model formulations lead to different conclusions, further model evaluation might be required."

The uncertainties in model structure arise from the assumptions and data interpretations involved at each of the steps in the model structure identification process. The steps are interdependent with assumptions at one step affecting decisions and assumptions at another. Sources of model structure uncertainty are best elucidated through examination of the major assumptions and interpretations made at each step in the model structure identification process. This process could be illustrated as a series of steps with various feedback loops as illustrated in Figure 3.12 or as a wheel of steps with feedback between all of the steps as illustrated in Figure 3.13. As illustrated in Beck's (1987) frame of reference 
diagram for uncertainty analysis, part of the structure identification process involves developing the external description of the system and the associated uncertainty (e.g., model domain and the associated boundary conditions that describe the interactions with those parts of the system not modeled) and the internal structure of the system being modeled (e.g., the internal geometry and relevant processes). The model structure uncertainty arises due to the uncertainty associated with each of the assumptions and interpretations required in the model structure identification steps discussed below.

\subsubsection{Spatial Domain and Associated Boundary Conditions}

As illustrated in Figure 3.19, the natural groundwater system being modeled is always only some small part of the full hydrologic system. Thus, spatial domain identification involves using available data and understanding to select the appropriate physical extent for the model given its purpose and desired spatial resolution for the state variables and resolution requirements for the processes to be modeled. A model's spatial domain (i.e., top, bottom, and lateral external boundaries) is chosen to

- include all the important types of pathways of interest from sources to receptor locations or to external boundaries to other systems (e.g., rivers)

- facilitate the description of the boundary conditions along the interface between the modeled and the unmodeled or external portion of the hydrologic system.

Conditions along these boundaries must be identified or assumed for the past (i.e., during the history matching or calibration phase) as well as for the present and future for the purpose of making predictions. Uncertainties associated with these assumed conditions must be included as appropriate (i.e., as a pdf or through sensitivity studies). The selection of the model boundary is also related to and dependent on any model process or geometrical simplifications (e.g., two-dimensional) and is influenced by the internal geometry of the system (e.g., hydrostratigraphy and facies/parameter zones).

Figure 3.20 (from NRC 1990, after Freeze 1969), illustrates the spatial domain identification process for a simplified two-dimensional model of a real system. When possible, natural boundaries (e.g., rivers, lakes, drainage basin divides) should be used (Bear 1979), where, for example, assumptions of prescribed head (based on observations) or zero flow can be made. Truncated domains that do not extend to natural boundaries give rise to additional uncertainties and may involve using prescribed flux boundaries or headdependent flux boundaries, which are difficult to measure and characterize. Uncertainties are associated with the exact physical location and presumed boundary conditions along each type of boundary. As illustrated in Figure 3.21, a theoretical diagram from Toth (1963), natural boundaries are not free of uncertainty because the zero flux divides between local drainage basin divides and beneath large rivers and lakes are not always vertical as commonly assumed and, additionally, their location may vary through time as a result of external and internal influences. 


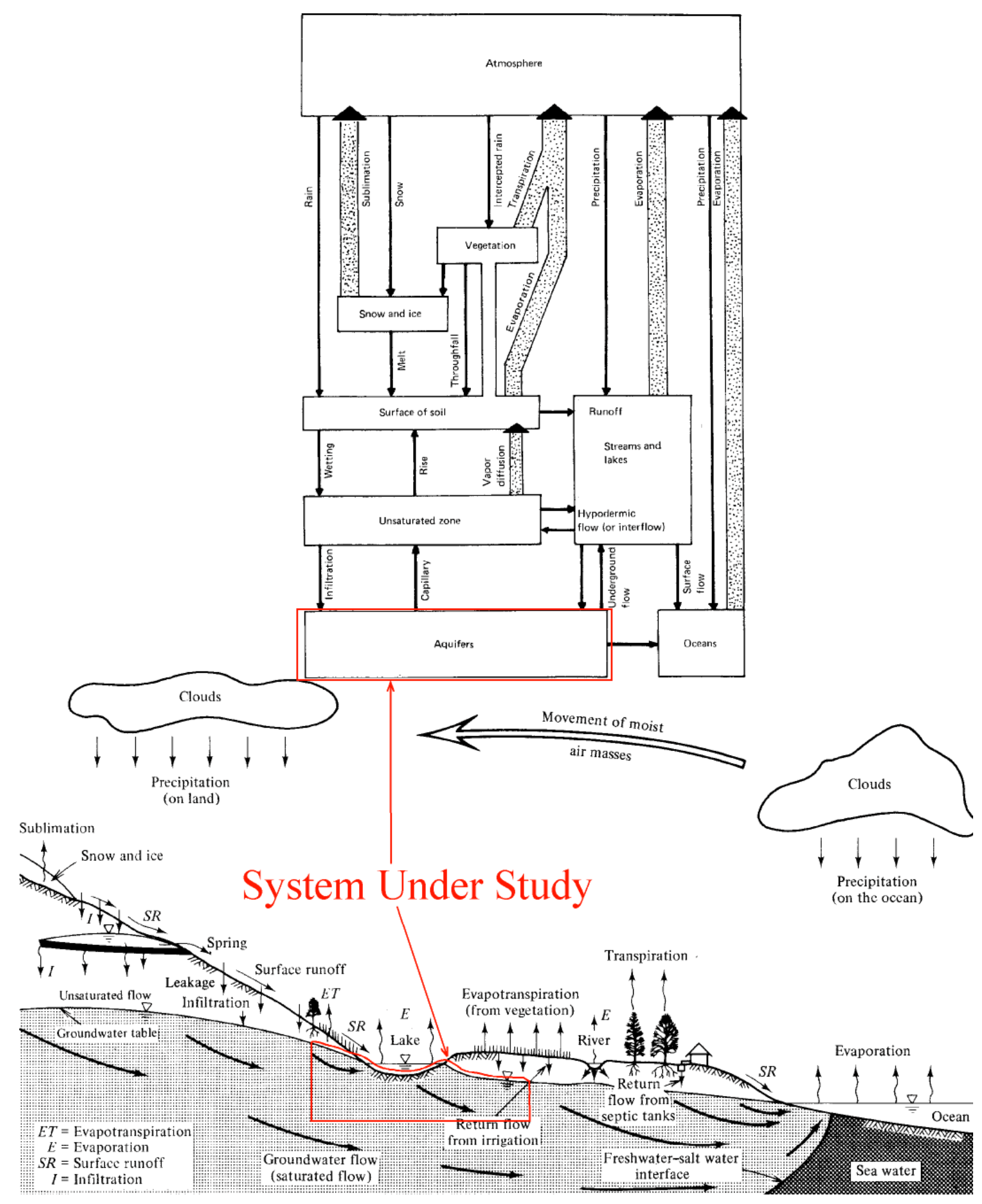

Figure 3.19. Illustration of a Typical Relationship Between a Groundwater System Being Studied and the Rest of the Parts of the Full Hydrologic Cycle. Shown are both a diagram of the full hydrologic cycle illustrating general processes and interactions from de Marsily (1986) after Eagleson (1970) and a pictorial diagram of the hydrologic cycle from Bear (1979). 

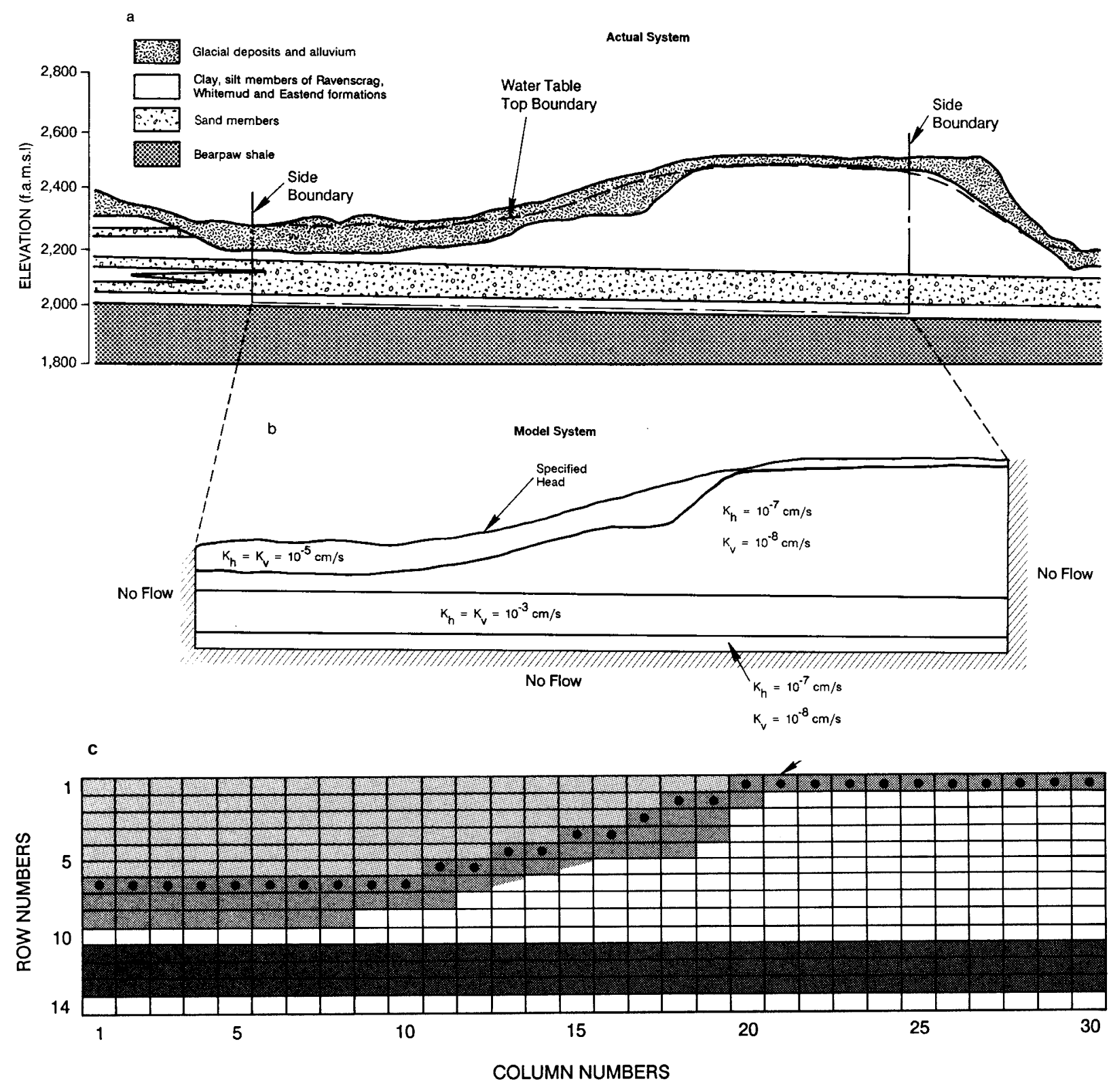

Figure 3.20. Representation of (a) a Real System by a (b) Schematic of the Model System Domain Boundaries (complete with boundary conditions along these boundaries and internal geometry of the geohydrologic layers) and (c) the Representation of this System by a Rectangular Grid. This figure was adapted from NRC 1990, which was based on work by Freeze 1969. 


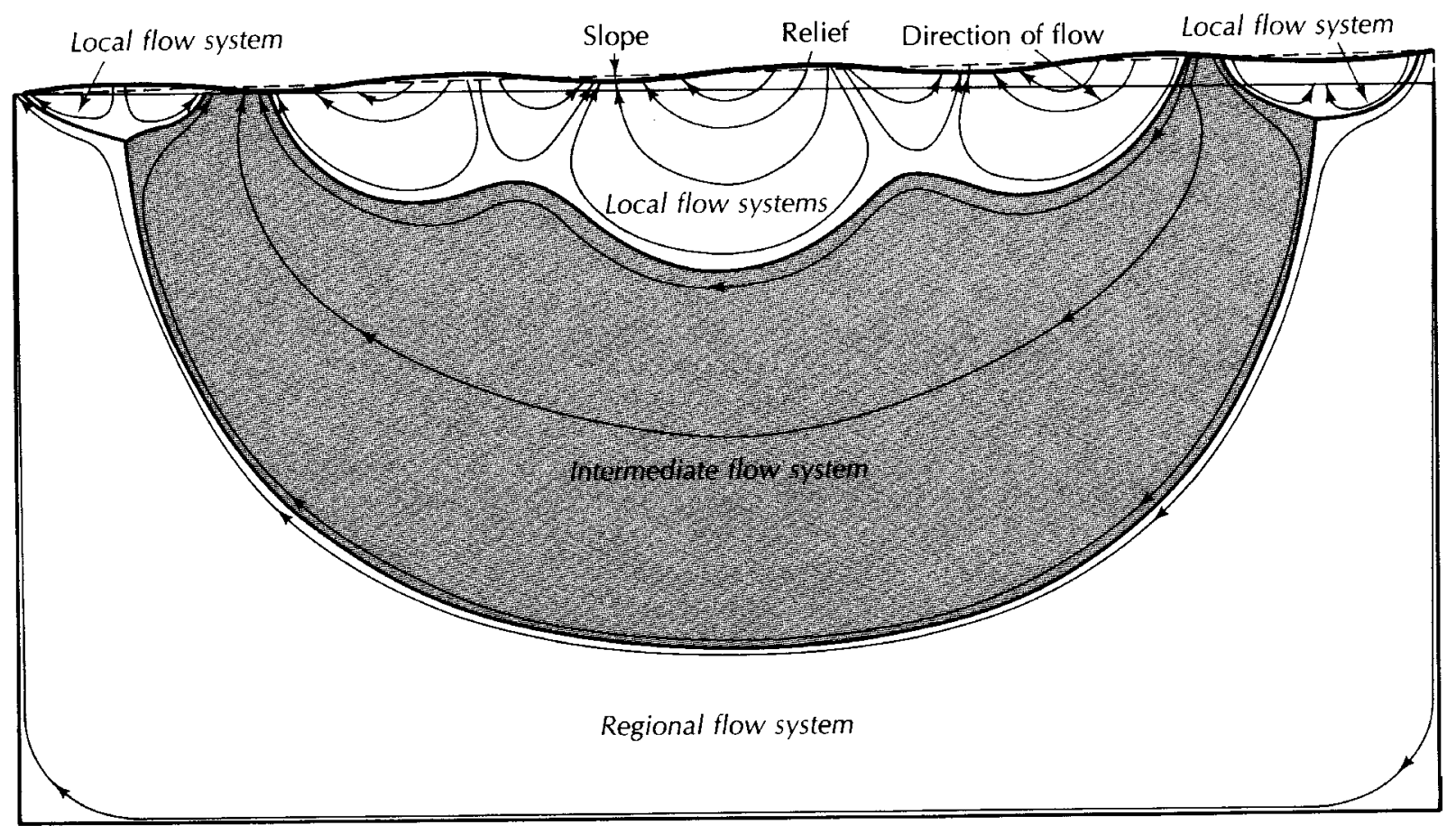

Figure 3.21. Theoretical Development of Local, Intermediate, and Regional Flow Systems in a Deep Basin (from Toth 1963).

The spatial domain for groundwater problems can range from a few meters to tens, hundreds, or thousands of meters. However, the desired spatial resolution scale for groundwater systems, even for local problems, does not attempt to resolve macroscale variability at the scales illustrated in Figures 3.14 and 3.16, although some well-planned field experiments have been designed to address these scales (e.g., Moltyaner 1987; Freyberg 1986; LeBlanc et al. 1991; Adams and Gelhar 1992; Hills and Wierenga 1994) through appropriate measurement techniques, sampling and observation scales, and modeling. Generally, groundwater models are based on some equivalent porous media concept (e.g., as illustrated in Figure 3.22 and the upper right corner of Figure 3.14) with spatial resolutions varying from meters to tens and even hundreds of meters, depending on the overall domain scale of interest, processes involved, and computational restrictions such as the grid Peclet and Courant conditions. The spatial resolution selected for the modeling will introduce additional uncertainty related to upscaling and averaging of observations and parameters at one spatial and temporal scale for use in a model that involves a different scale, although the total uncertainty, as discussed in Meyer and Gee (1999), may decrease.

\subsubsection{Temporal Domain}

The temporal domain (e.g., years or tens, hundreds, or thousands of years) and the desired temporal resolution scale (e.g., hourly, daily, monthly as illustrated in Figure 3.15, semiannual) of interest must be consistent with the model's purpose and desired spatial and temporal resolution for the state variables and the temporal resolution requirements for the processes to be modeled. Large temporal domain requirements needed by many risk assessment models (e.g., hundreds to thousands of years) should be 


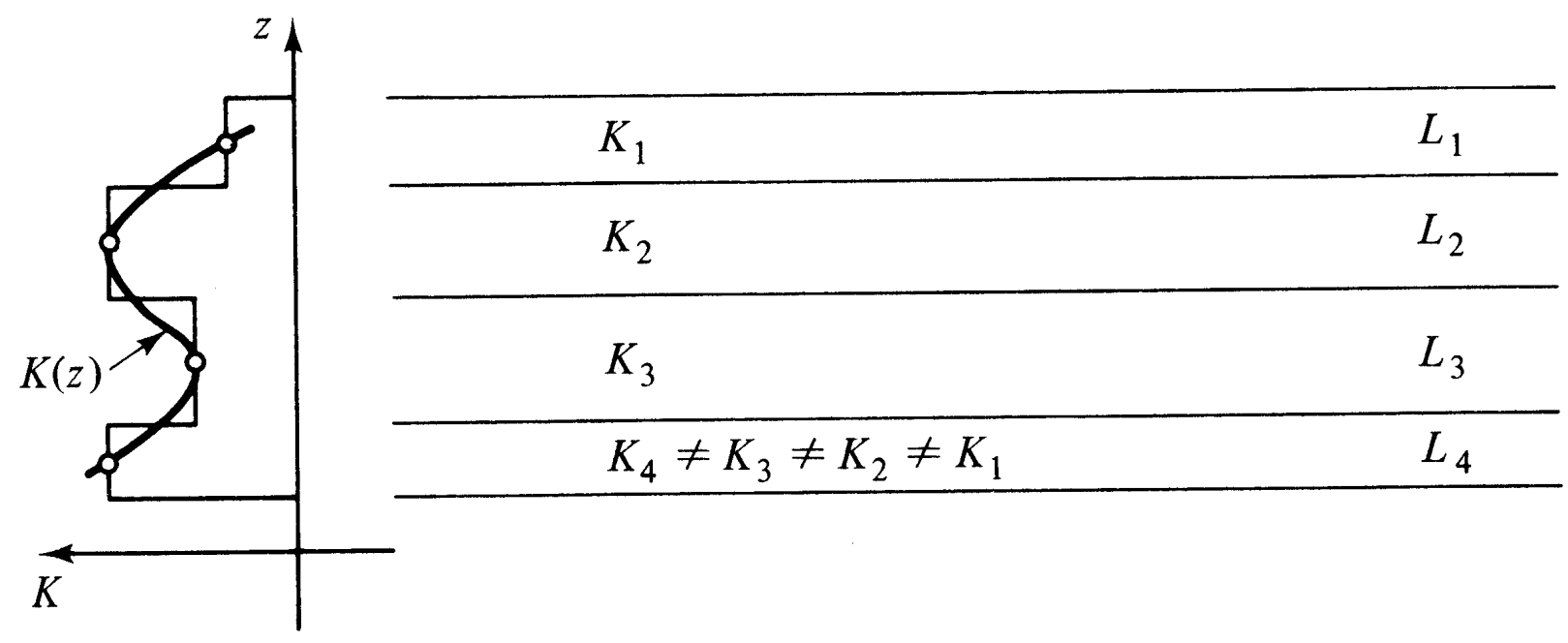

Figure 2-8 A layered aquifer.

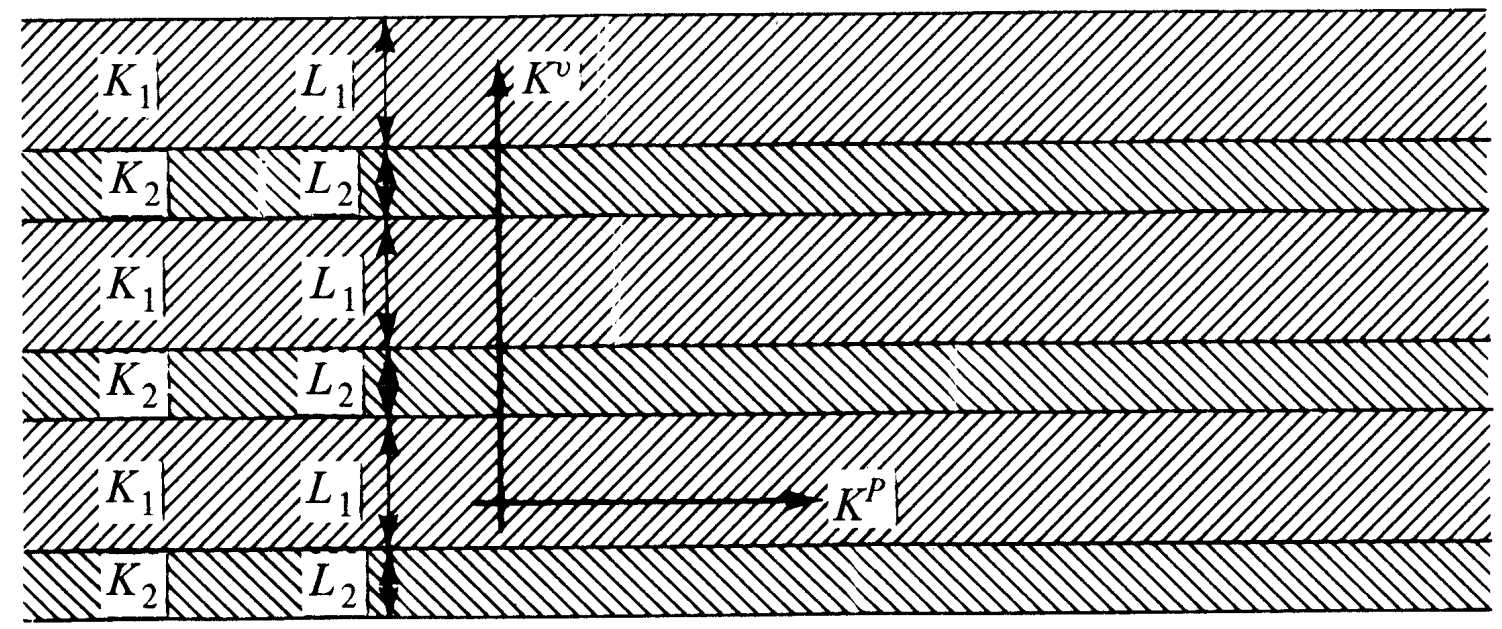

Aquifer composed of alternating layers exhibits anisotropy with $K^{v} \neq K^{p}$.

Figure 3.22. Illustration of an Equivalent, Anisotropic, Porous Medium Consisting of Alternating Layers of Differing Macroscopic Properties (Bear 1979).

considered in the process identification step since slower-acting processes not usually considered over shorter time frames may become important at these time scales. As was the case for the spatial domain, computational restrictions can affect the selection of temporal resolution. For example, the Courant condition requires the spatial resolution and temporal resolution to be related so that the contaminant fronts move exactly one grid block every time step in order to minimize numerical error. Violation of the Courant condition will lead to a more dispersive result or computational instability. Similar to the spatial domain discussion above, the temporal resolution selected for the modeling will introduce additional uncertainty related to upscaling and averaging of observations and parameters at one spatial and temporal scale for use in a model that involves a different scale. In the case of the Hanford Site, for example, 
issues that arise include 1) how should semiannual groundwater head measurements near the river, where groundwater levels vary daily in response to river flow fluctuations (e.g., Figure 3.15), be compared to model predictions made with a model using semiannual time steps, and 2) how should the daily fluctuations in river stage be averaged or upscaled to drive the boundary condition linking the river to the groundwater system.

\subsubsection{Process Identification, Simplification, and Representation}

An understanding of the various simple processes (e.g., Figure 3.23) at work in the porous media (e.g., Figures 3.14 and 3.24) is required to determine the appropriate representation of the processes (both physical and chemical) that control the state variables of interest at the spatial and temporal scales of interest. State variables of interest (head, temperature, fluid density, concentration, $\mathrm{pH}$ ) and the independent, interacting, and/or coupled processes that affect or control these state variables directly or indirectly (e.g., a process associated with a remediation activity or other natural process might slowly change system properties) must be identified while simultaneously considering the following:

- spatial domain and spatial resolution of interest including the external domain geometry and the internal domain geometry

- temporal domain and temporal resolution of interest

- media of interest (e.g., Figures 3.16, 3.17, and 3.24)

- spatial distribution of state variables and their temporal variations

- range in external and internal driving forces to be examined that includes past, present, and expected future conditions for the range of future scenarios of interest.

In addition to identifying the flow and transport processes of interest, the conceptual and mathematical representation and degree of simplification or aggregation and/or decoupling of these processes must be identified and investigated in order to understand the uncertainty implications. Simplification is required because of our inability to measure and represent all the actual details and because without simplifications, the resulting problem would be intractable. The medium of interest (e.g., Figures 3.16 and 3.17), scale and resolution of interest, and whether the medium and the processes will be represented at the microscopic (Figure 3.24), macroscopic (Figure 3.14), or megascopic scale (Figure 3.22) will affect the conceptual and thus the mathematical representation, the required parameterization, and even the way that measurements are carried out in the laboratory and field. Additional process simplification/resolution steps may be required (e.g., a three-dimensional system might need to be simplified to a two-dimensional system; a nonlinear model might need to be reduced to a simpler linear model for the media, scale, and parameter space of interest; additionally, when it can be justified, processes may need to be decoupled [Isukapalli 1999]). The appropriateness of process and representation simplifications and associated assumptions should be tested at the spatial, temporal, and parameter domains of interest through comparisons with more detailed models. This testing must also examine the uncertainty implications. For example, Sugita and Gillham (1995a,b) present a conceptual model that indicates a nonideal reactive 


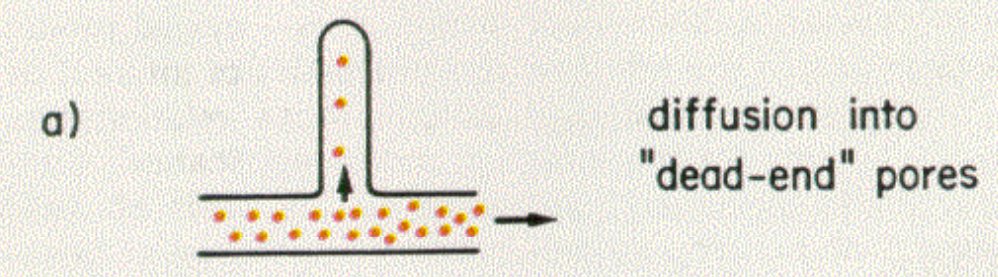

b)
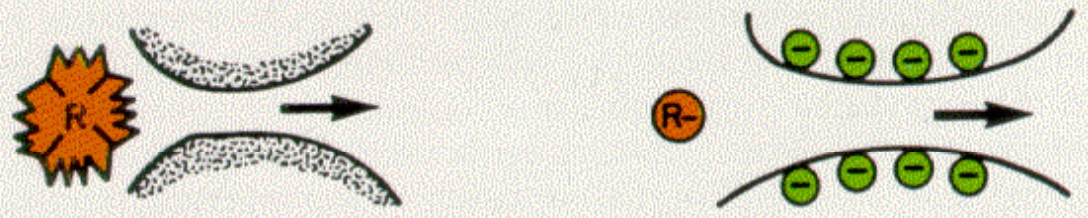

molecular

ion

filtration

exclusion

c)

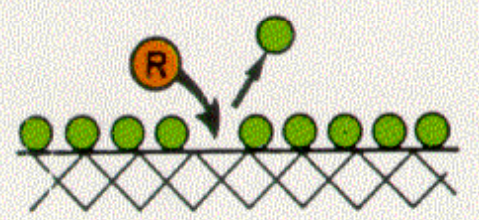

physical sorption

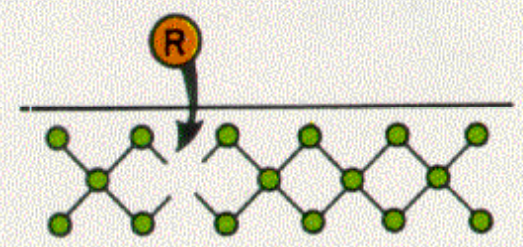

mineralisation

d)

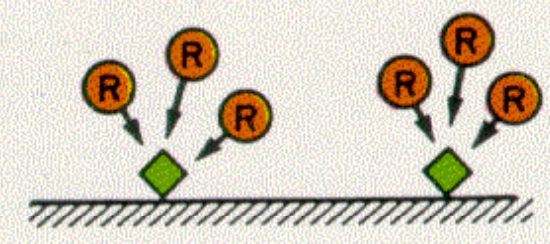

precipitation

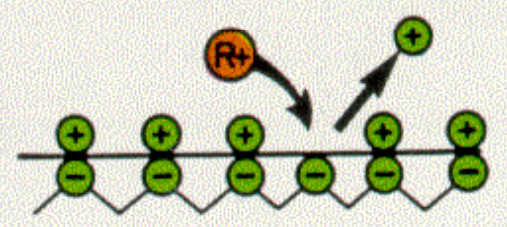

ion - exchange

Figure 3.23. Diagram of Various Simple Processes that Could Affect Solute Concentrations in Porous Media (from INTRAVAL 1990). 


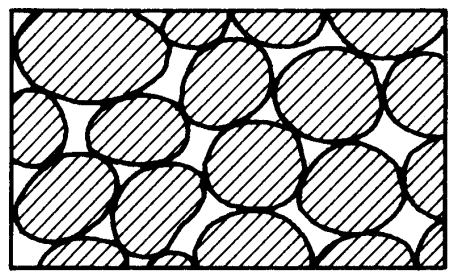

A

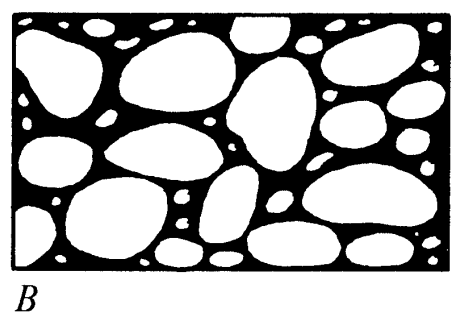

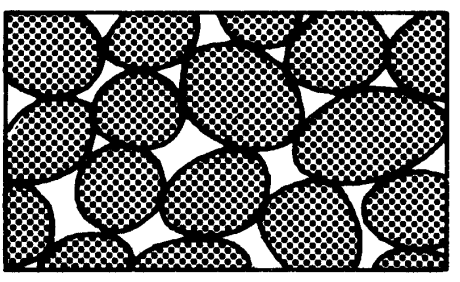

C

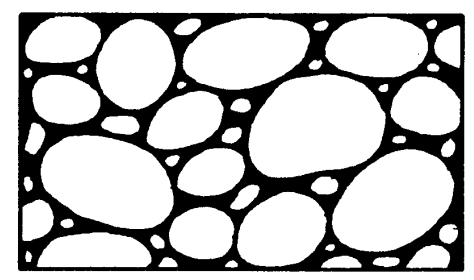

$D$

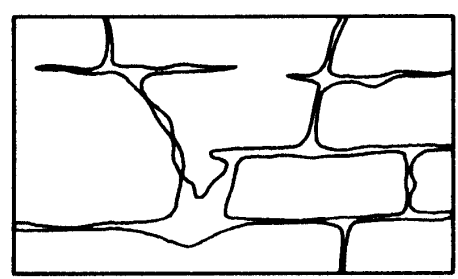

$E$

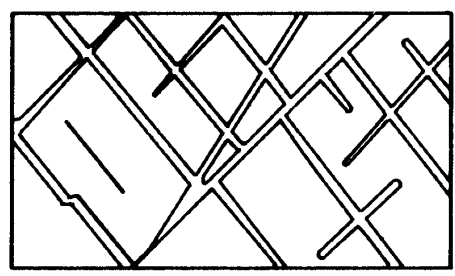

$F$

Figure 3.24. Diagram at the Microscopic Scale of Various Type of Media (from Bear 1979). Diagrams A through D illustrate sedimentary deposits of the following types: A) well sorted and high porosity, B) poorly sorted and low porosity, C) well sorted with porous clasts and thus very high porosity, and D) well sorted with low porosity due to mineral deposition in interstices. Diagrams E and F represent rock formations that have been rendered porous by: E) dissolution, and F) fracturing.

breakthrough (i.e., greater spreading and tailing) greater than predictable by the classical advectivedispersion equation with pore scale variations in retardation factor. Goode and Konikow (1990) illustrate that uncertainty in transient flow behavior gives rise to a greater apparent dispersivity. Tritscher et al. (2000) illustrate that neglecting unsaturated zone effects leads to a local specific yield strongly influenced by water table depth and mildly dependent on recharge rate. Lastly et al. (2000) illustrate that rate-limited mass transfer between mobile and small-scale immobile domains provides an alternative model that does not consider dispersion and can explain the evolution of the plumes at the Macrodispersion Experiment Site.

While most of the available information and observations will be related to current and recent past conditions, the effects of possible future conditions and the long time scale of interest for future predictions must be thoroughly considered in the process identification, process representation, and process simplification steps.

\subsubsection{Uncertainty in Model Parameters}

Section 3.2.1 introduced the subject of natural variability and made the point that subsurface hydrogeologic systems are inherently variable in space and time. Because of this variability and because we are able to sample the subsurface properties at only a limited number of locations, often along restricted linear paths (e.g., boreholes or seismic lines), the value of those properties remains highly uncertain for most locations in the groundwater system. Given the common groundwater model 
requirement for values of hydrogeologic properties at all grid locations within the bounds of the conceptual model, the properties must be interpolated between the sample locations. In addition, we need a quantitative model for the uncertainty associated with the interpolated hydrogeologic properties values. Webb and Davis (1998) and Koltermann and Gorelick (1996) provide excellent reviews of the problems involved with simulating the spatial heterogeneity of hydrogeologic properties and accounting for uncertainty.

A key issue in the representation of parameter variability is related to one's view of the best method to represent or characterize it. The characterization of the variability as discrete or continuous could be considered a key difference between geologists and hydrologists. Geologists tend to view a system as being composed of a number of discrete elements (e.g., formations, bed sets, beds, laminations, faults). These elements may grade into one another or have sharp interfaces, but they are separable features in either case. Hydrologists, however, particularly those using stochastic interpolation methods, traditionally base their models on physical principles and theories that treat model parameters (e.g., hydraulic conductivity, dispersivity, porosity) as continuously varying point wise in space. Therefore, hydrological models of spatial variability are often smoothly variable, without distinct, definable features. This polarization is discussed by Haldorsen and Damsleth (1990), who classify stochastic models of subsurface variability into two groups: discrete and continuous. Dubrule (1989) makes a similar distinction, dividing approaches to spatial variability into two groups: 1) object-based, corresponding to definition of discrete objects in space, and 2) sequence-based, corresponding to definition of continuous spatial relationships of point properties. An example of this difference is given in Figure 3.25, which displays two images. Each of two images represents the spatial distribution of hydraulic conductivity in a specific environment. The left image is taken from the numerical aquifer of Scheibe (1993) (see also Scheibe and Freyberg [1995]), and is based on a discrete geometric model of point bar sediments in the Wabash River. The right image is a continuous stochastic representation of the same conductivity field, which preserves the bivariate (point-to-point) spatial statistics of the left image. Both images are imperfect models of reality in that both represent some observable aspects of the real system and neglect others. However, they are strikingly different in appearance, and also differ in terms of predicted flow and transport behavior. An important question, then, is how to balance the discrete character of geological deposits (as observed by geologists) against the point-to-point variations in hydrological properties (as observed in hydrological data sets), when modeling such systems. Haldorsen and Damsleth (1990) advocate a hybrid model of spatial structure, which combines the discrete and continuous approaches. Figure 3.26 from Damsleth et al. (1992) illustrates this type of hybrid approach. In such a model, the large-scale heterogeneities are described using a discrete approach, such as the architectural elements of Miall (1985). The continuous portion of the model describes hydraulic conductivity variations within discrete elements and might be implemented using a geostatistical method. However, the appropriate approach may vary according to the nature of the geologic heterogeneity, the objective of the modeling effort, and the required/desired spatial and temporal resolution.

Recognizing that natural spatial structure is complex and exists on multiple scales, there is a need to understand which characteristics and scales of natural heterogeneity exert greatest control on subsurface flow and transport behavior at the scale needed to make our predictions. This understanding will allow those characteristics of spatial structure to be identified and represented in the groundwater flow and transport in order to provide meaningful predictions. In the face of the multiscale heterogeneity of natural 

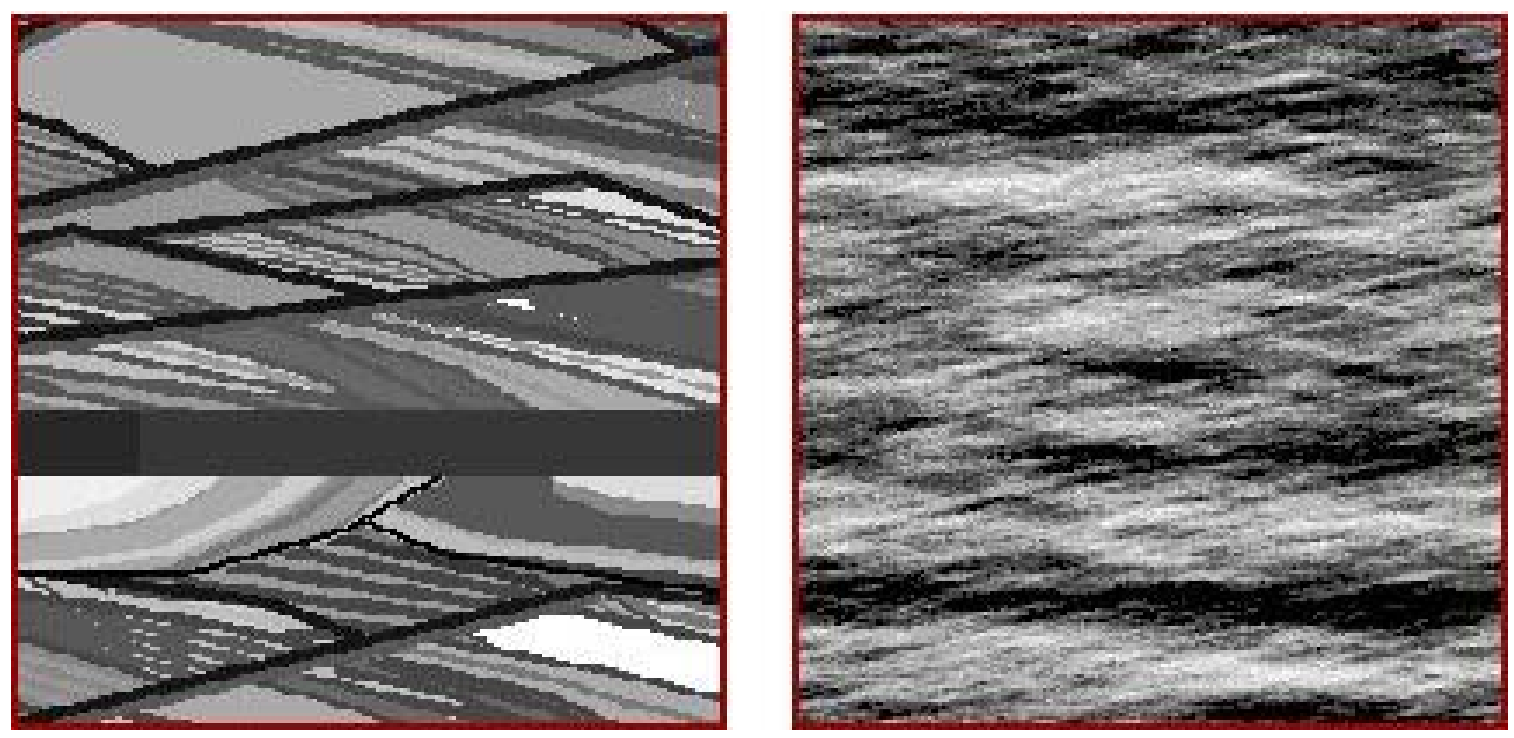

Figure 3.25. The Left Image is a Discrete Model Representation of Point Bar Sediments in the Wabash River (after Scheibe 1993). The right image is a continuous stochastic representation of the same parameter field, in which the first and second statistical moments are preserved using the spectral synthesis method of Gutjahr et al. (1987). In both images, permeability is represented by gray scale; high permeabilities have light tones and low permeabilities have dark tones.

systems, upscaling, which involves combining parameter estimates based on measurements at the local scale(s) to obtain model scale estimates (Section 3.2.1), is a major source of uncertainty and is one of the major issues that must be addressed. The conventional approach in hydrogeology assumes that Darcy's law for fluid flow and Fick's analogy for solute transport apply at each point in the subsurface. The corresponding constitutive parameters, most notably permeability and dispersivity, are viewed as local-continuum properties with values intrinsic to the porous material. Yet both theory (Cushman 1984, 1986; Baveye and Sposito 1984, 1985) and empirical evidence (Neuman 1992) suggest that parameters obtained from local measurements in heterogeneous media are generally nonunique, depending strongly on the scale (support volume) and mode (instruments and procedure) of measurement/interpretation. The upscaling problem is a central and ubiquitous problem: How to define effective parameters and the uncertainty in these effective parameters relevant to the scale of the problem being modeled and the applicable boundary conditions? This means that model parameters and their uncertainty in general must be specifically derived for each model in a way that factors in the specific constitutive model formulation (e.g., dual porosity illustrated in Figure 3.24C or fracture flow illustrated in Figure 3.24F), the aggregation scale (e.g., Figure 3.14), the actual spatial and temporal scale, dimensionality, and the desired resolution (e.g., Figures 3.19 and 3.20). While issues of measurement scale and aggregation of measurements apply to all models, the upscaling task is much more problematic for constitutive parameter models because parameters interpreted from widely different and many times uncertain spatial and temporal scales using different constitutive models and dimensionalities must be combined to derive estimates of model parameters and their uncertainty, for use at yet a different level of aggregation, spatial and 


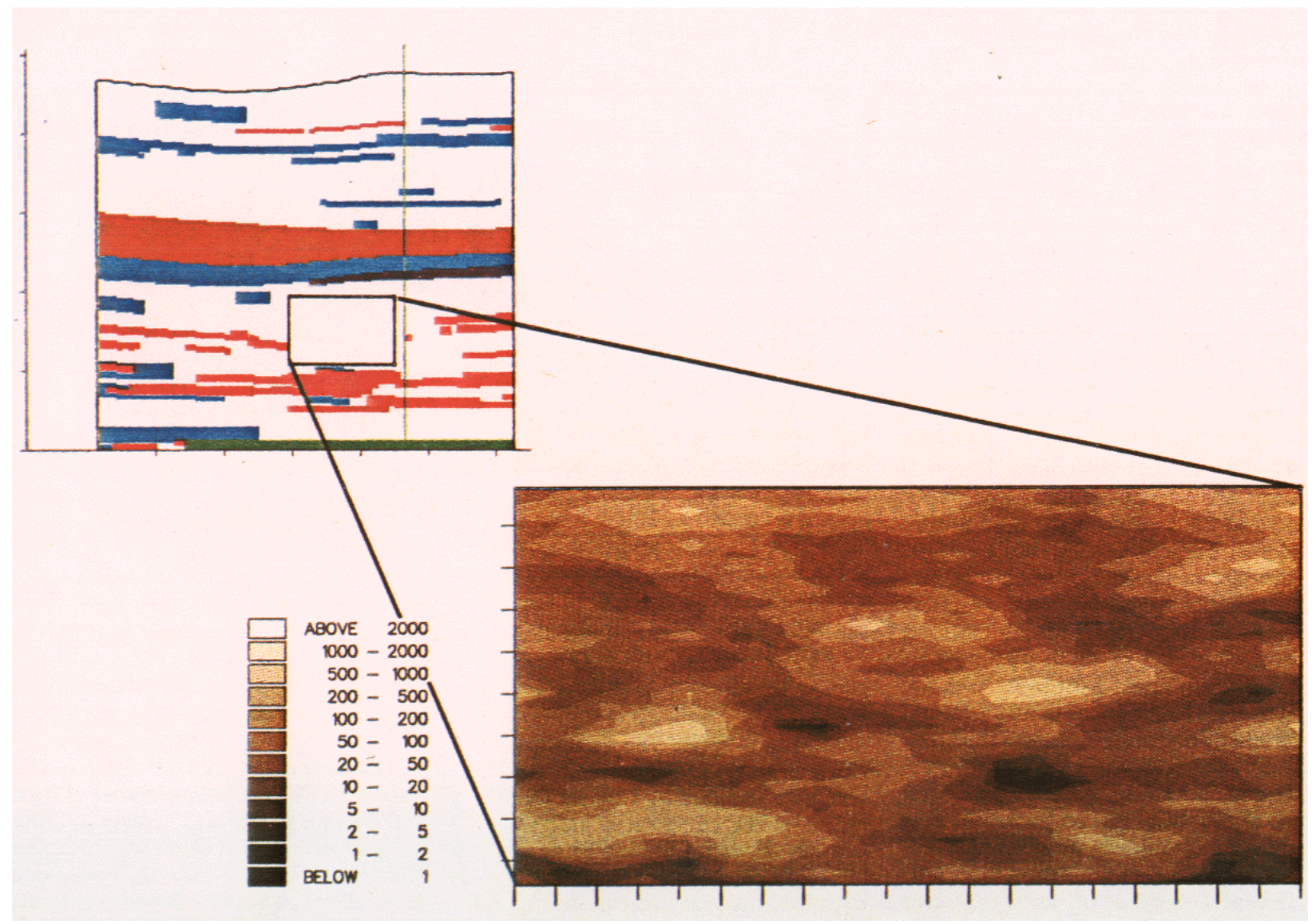

Figure 3.26. Illustration of Two-Scale Hybrid Model Approach (Figure 8 from Damsleth et al. 1992). Facies are represented as discrete units (small picture in upper left), while the variability in vertical hydraulic conductivity within the M1A facies of layer 7 of the discrete-layer model is represented by continuous stochastic realizations (large image in lower right).

temporal scale, dimensionality, or type of constitutive model. Even if it were possible to know the complete point wise variation of permeability and dispersivity of an aquifer at a scale considered generally well defined, it would not be feasible to model flow and transport in such a detailed system, because of computational limitations.

In the remaining parts of this section, two general approaches for dealing with estimating values for spatially variable parameters and their uncertainty are discussed: 1) geostatistical methods that deal directly with the data and its upscaling and interpolation, and 2) statistically based indirect inverse methods. These methods must also identify any correlation between the parameters identified because these correlations are an important part of the uncertainty propagation. For some sensitive model parameters, even though they may be spatially variable, the data may be too limited to apply either of 
these parameter identification methods. For sensitive data in this latter category, "expert elicitation" techniques can be used to select/develop (albeit in a subjective manner) a representative probability distribution and its coefficients based on the limited data available.

\subsubsection{Geostatistical Methods}

Freeze and others (Freeze et al. 1990, 1992; Massman et al. 1991; Sperling et al. 1992) broke down the uncertainty in the subsurface distribution of hydrogeologic properties into two components, which they label geologic uncertainty and parameter uncertainty. Geologic uncertainty refers to the uncertainty in the location of aquifer units and aquitards, as well as to uncertainty in the boundary conditions. Parameter uncertainty denotes the uncertainty in the values of hydrogeologic parameters through the spatial domain. They point out that by directly simulating the distribution of geologic units (e.g., sedimentary facies), we can directly reduce geologic uncertainty and indirectly reduce parameter uncertainty. The reduction in parameter uncertainty occurs because different sedimentary facies often contain different grain size distributions and there is a close association between the grain size distribution of sediments and their hydrogeologic properties. A review of this association and the various empirical equations that have been developed to exploit it (e.g., the Kozeny-Carman equation) can be found in Koltermann and Gorelick (1995). Note that diagenesis, especially cementation, can be a confounding factor in controlling the distribution of hydraulic conductivity within sediments, and it has been suggested as a cause of the lack of correlation sometimes observed between sedimentary facies, grain size, and permeability (Webb and Davis 1998).

The ability to simultaneously reduce the geologic and parameter uncertainty in hydrogeologic models by simulating the distribution of sedimentary units has led to a common strategy in aquifer and reservoir studies. First, simulate the distribution of sedimentary facies, then simulate the spatial distribution of hydrogeologic properties within each of the sedimentary facies (Deutsch and Journel 1998; Dominic et al. 1998; Murray 1995). Taking this two-staged approach helps decrease the problems caused by violation of stationarity assumptions that can arise when the distribution of hydrogeologic properties varies significantly from one type of sedimentary unit to another. Geostatistical methods can be used for generating these two-step simulations of geologic models, using different methods for the two stages of simulation.

Geostatistics is a field of statistics used to develop quantitative models of the spatial continuity of data in the earth sciences (Isaaks and Srivastava 1989; Goovaerts 1997). The most commonly used tool for describing the spatial continuity of geologic properties is the variogram (Isaaks and Srivastava 1989). Variables that result from geologic processes that vary spatially, e.g., sediment transport and deposition by rivers, often display spatial continuity that can be identified by variogram analysis. Variograms plot the average squared difference between data values separated by a given vector distance as a function of that distance. If a variable exhibits spatial continuity, then points that are close to one another will have smaller differences (and therefore lower variogram values) than pairs of points that are separated by greater distances. In variogram analysis, models that quantify the spatial continuity of the variable are fit to the experimental variograms. The weights assigned to data points in estimating or simulating the value of a variable at unsampled locations can be calculated using variogram models and the interpolation 
method known as kriging. Variogram analysis and kriging also form the basis of geostatistical stochastic simulation methods. See Isaaks and Srivastava (1989) or Goovaerts (1997) for an introduction to variogram analysis and kriging, and Goovaerts (1997) for an introduction to stochastic simulation.

One geostatistical technique that has been widely used for simulating the distribution of sedimentary units is indicator simulation because it can successfully deal with variables that are categories (e.g., an integer code for each sedimentary facies). The indicator simulation program provided by Deutsch and Journel (1998) includes the capability of simulating categorical variables. For curvilinear shapes, e.g., fluvial channels, other techniques may be more suitable, such as Boolean models or Marked Point process models (Koltermann and Gorelick 1996; Deutsch and Journel 1998). While geometric methods are better able to capture the geometric form of geologic deposits, they are difficult to condition to existing data (Deutsch and Journel 1998), e.g., well-bore data. Another alternative for simulation of geologic units is the Transition Probability/Markov approach developed by Carle et al. (1998). This approach does not necessarily capture the geometric shapes of units, but it does a good job of capturing the relationships between units more easily than other methods (e.g., if shale units are always found immediately above sandy units).

The distribution of hydraulic parameters within geological units can be handled in several ways. One possibility would be to use geostatistics to simulate their distribution using Gaussian or indicator methods. Indicator methods have the advantage of allowing one to honor the high connectivity of very high and low conductivity zones that often occur within sedimentary facies (Journel and Alabert 1989), which is not possible with a Gaussian approach. In Gaussian methods, the variable, or a transform of it such as the normal score transform or the logarithmic transform often suggested for hydraulic conductivity, is assumed to have a spatial multi-Gaussian distribution. Hydraulic conductivity distributions from natural environments often do not fit a lognormal distribution (Fogg 2000). Also, the rigorous assumptions associated with a multi-Gaussian distribution, which can be tested (Deutsch and Journel 1998), are rarely met in natural environments (Scheibe 1993). However, if a two-step approach is taken, with the simulation of sedimentary units first, then the high and low conductivity zones can be treated as separate facies, and Gaussian methods may be quite appropriate for simulating the spatial distribution of hydraulic conductivity within facies (Deutsch and Journel 1998).

Another approach for modeling the hydraulic parameters within hydrogeologic units might be to consider them as approximately homogeneous and treat the values of hydraulic properties within geologic units as a single parameter, an approach termed zoning by Peck et al. (1988). This simplification can be useful because the computational times for flow and transport models are highly sensitive to the number of parameters, and the computational costs for modeling the parameters at high resolution can be excessive. However, Peck et al. (1988) point out that one of the difficult aspects of zoning is determining the scaled-up, average value of a parameter to use over the zone. For tensor properties like hydraulic conductivity, which do not average linearly, the determination of the "average" value to use is particularly difficult. Even for cases where zonation is not used, it is normally necessary to upscale the hydraulic properties from the measurement scale to the scale of the model because of the computational difficulty of modeling flow and transport at the scale of the measurements, so the same question about the proper 
"average" for the model blocks will arise. Renard and de Marsily (1997) discuss several methods that have been proposed for upscaling hydraulic parameters. Zoning methods, as will be discussed, are common with many inverse methods.

The discussion in this section has emphasized the use of geostatistical simulation rather than kriging for the interpolation of hydrogeologic data between conditioning data. Kriging is a form of generalized linear regression often used for interpolating the spatial distribution of hydrogeological properties, and it forms the basis for almost all geostatistical stochastic simulation methods. However, the use of geostatistical simulation has several advantages over kriging for hydrogeological applications (Journel 1989). First, kriging provides a unique, best-fit interpolation, which is a smoothed interpolation of the data like all regression models. Stochastic simulation provides multiple, equally probable realizations (Goovaerts 1997), each of which reproduces the full spatial variability of the input variogram model. Also, the kriging variance, which was formerly used as a measure of the local uncertainty of the variable, is known to be a function of the spatial arrangement of the data values, but not of the data values themselves, and does not provide an accurate model of local uncertainty (Journel 1989). The suite of simulated values at each grid node taken from an ensemble of realizations does provide an estimate of the local CCDF and provides a suitable measure of local uncertainty.

By using a suite of stochastic simulations of the hydrogeologic parameters as input to flow and transport models, it is possible to generate an estimate of the uncertainty with respect to model outputs, e.g., the distribution of possible breakthrough curves at a compliance boundary. However, it may not be computationally feasible to run a flow and transport model for a large suite of stochastic simulations. In that case, it may be necessary to select a subset of simulations. Deutsch and Journel (1998) provide a discussion of the problems with selection of a subset of simulations and some selection methods that have proved useful for characterization of uncertainty.

\subsubsection{Indirect Inverse Methods}

Neuman (1973), Yeh (1986), and Peck et al. (1988) discuss both direct and indirect inverse (i.e., parameter estimation) methods that have been applied to groundwater modeling problems to estimate model parameters and their uncertainty. The following summarizes the two approaches and the historical experience at Hanford:

- Direct Methods. In direct methods, the dependent variables in the groundwater flow equation are assumed to be the unknown parameters. The values for these unknown parameters are determined through development of a formal inverse boundary value problem and then solving the resulting equation to minimize the mass balance error. From a historical perspective, it is interesting to note that the direct inverse methods developed by Nelson $(1960,1962,1968)$ for use at the Hanford Site that were implemented through graphical inverse techniques were among the first attempts at inversion (de Marsily et al. 2000). Nelson's technique used available field measurements of transmissivity, river stage, disposal rates to ground, and estimates of head and its spatial and temporal derivatives in an iterative approach to determine the Hanford Site unconfined aquifer transmissivity distribution (Cearlock et al. 1975). 
- Indirect Methods. In the indirect statistical inverse approach, (the subject of this section), the dependent variables are hydraulic heads, as is usual in a forward model, and model parameters are estimated by minimizing the head residuals (i.e., the difference between modeled and observed head) by iteratively updating an existing estimate of the parameters. As discussed in Galarza et al. (1996) and in Poeter and Hill (1999), indirect methods also allow for inclusion of terms in the objective function for "appropriately weighted" (e.g., to account for issues of upscaling and discretization) residuals related to prior information on parameters (i.e., the difference between "measured" and predicted parameter values) and for residuals related to aquifer stresses (e.g., the difference between model predicted and observed stream base flow). From a historical perspective, all the past applications of indirect statistical inverse methods at the Hanford Site were used to aid with deterministic model calibration of the existing two-dimensional conceptual model of the Hanford Site unconfined aquifer (Wurstner and Devary 1993) and not to address uncertainty in parameters. A steady state finite-element inverse calibration method developed by Neuman and Yakowitz (1979) and modified by Jacobson (1985) was the first application of an indirect statistical inverse method at Hanford. Available information on aquifer hydraulic properties (e.g., transmissivities), hydraulic heads, boundary conditions, and discharges to and withdrawals from the aquifer were included in this initial indirect statistical inverse calibration described in Evans et al. (1988) and Jacobson and Freshley (1990).

It is important to recognize that trial-and-error model calibration is also an indirect inverse parameter estimation method. However, it relies on subjective informal optimization methods that are not couched in a statistical framework and as a result provides no means for quantifying the uncertainty in the calibrated model parameters (Peck et al. 1988). In order to analyze the uncertainty in calibrated parameter estimates, the indirect inverse method must be statistically based. Peck et al. (1988) provides a summary discussion of the three most widely used statistical methodologies: Weighted least squares (Neuman 1980), Bayesian (Vicens et al. 1975), and Maximum Likelihood (Carrera and Neuman 1986a,b,c). Peck et al. (1988) indicate that it is the inherent nonlinear relationship between hydraulic head and the parameters to be estimated that presents the major parameter estimation difficulty.

Yeh (1986) provides an excellent summary and review on parameter estimation methods. Ginn and Cushman (1990) provide an updated summary that focuses on a discussion of the issues related to each of the statistical estimation methodologies: the distributional assumptions (e.g., Gaussian), appropriate use of prior information, and the related issues of effective parameters and upscaling. McLaughin and Townley (1996) provide a more recent general reassessment of the groundwater inverse problem that discusses the various indirect statistical approaches and issues. The McLaughin and Townley (1996) review also presents and describes a functional approach to the statistical inverse that is applicable to either approach to parameterization (i.e., discrete blocks of uniform properties, or the geostatistical view of stationary random fields smoothly varying over space). The most recent review by de Marsily et al. (2000) also focuses on the geostatistical inverse approaches to parameterization with an emphasis on the pilot point inverse method (de Marsily 1978).

The majority of the inverse literature focuses on the problem of estimating parameters and their uncertainty for the flow problem since the majority of the available data is related to the flow problem. However, as discussed in Peck et al. (1988), there have been limited ongoing efforts to formulate and 
apply indirect statistical inverse methods for the estimation of solute transport parameters and for simultaneous estimation of flow and transport parameters. These efforts have included one-dimensional solute transport in the unsaturated zone (e.g., Jury and Sposito 1985); formulation and demonstration of a statistical inverse approach for one-dimensional advective-dispersive transport (Wagner and Gorelick 1986); and simultaneous estimation of flow and transport parameters (hydraulic conductivity, dispersivity, and effective porosity) by Wagner and Gorelick (1987) for hypothetical two-dimensional systems. The Wagner and Gorelick (1987) effort is particularly relevant because the results were used for quantifying the uncertainty in model predictions through the use of a first-order uncertainty analysis (Dettinger and Wilson 1981). A few of the more recent efforts to formulate and apply inverse methods for coupled flow and transport parameter and source term estimation, complete with an estimate of the uncertainty, include Wagner (1992), Sidauruk et al. (1998), and Mayer and Huang (1998).

The natural, almost continuous variability (Figures 3.16 and 3.17) and scale dependence of parameters (Figure 3.14) are major sources of difficulty and ambiguity in applying the inverse methodology. They are the major contributor to the uncertainty in the resulting parameter estimates. McLaughin and Townley (1996) indicate that it is difficult to determine how all the inverse concepts and methods described in the literature are related, and they provide a method for characterizing the various methods based on the following four characteristics:

- Parameterization - the way the spatial variability is described (e.g., a discrete or zoned approach, a continuous geostatistical random field approach, or even the hybrid approach of Figure 3.26)

- Forward Equation - the forward equation used to relate parameters to measurements (e.g., the groundwater flow equations written in terms of upscaled or effective parameters)

- Performance Criterion - the performance criterion or objective function used to determine a "good" parameter estimate

- Solution Technique - the solution technique used to determine these parameter estimates.

McLaughin and Townley (1996) and Peck et al. (1988) classify the indirect inverse problem in two categories based on the approach to parameterization formulation:

- Blocked (or Zoned) - A more hydrogeologically based representation of variability where hydraulic parameters within various geologic units and facies might be grouped in order to consider them as approximately homogeneous with respect to hydrologic parameters

- Geostatistical - Parameters of interest are generally considered to be well represented by spatially random fields that vary relatively smoothly in space (Hoeksema and Kitanidis 1984; Dagan 1985).

Sun et al. (1995) and Galarza et al. (1996) present a comprehensive practical description of the various approaches to inverse parameterization. 
The study by Zimmerman et al. (1998) compares seven inverse methods for estimating transmissivities and the associated uncertainties for the purpose of modeling advective transport and its uncertainty for a two-dimensional representation of a synthetic groundwater system. The seven methods studied (described in Appendix B of Zimmerman et al. 1998), which included most of the current methods, were as follows:

- Fast Fourier Transform Method (Gutjahr and Wilson 1989)

- Linearized Semianalytical Method (Dagan 1985)

- Linearized Cokriging Method (Kitanidis and Vomvoris 1983)

- Fractal Simulation Method (Grindrod and Impey 1991)

- Pilot Point Method (RamaRao et al. 1995; Lavenue et al. 1995)

- Maximum Likelihood Method (Carrera et al. 1993)

- Sequential Self-Calibration (Gomez-Hernandez et al. 1997).

The nonlinear least squares approach of Cooley $(1977,1983)$, incorporated into Modular Flow Program (MODFLOWP) (Hill 1992) and which is also the methodology used in Universal Inverse Code (UCODE) (Poeter and Hill 1998), was not part of the comprehensive study comparing inverse methods discussed in Zimmerman et al. (1998).

UCODE (Poeter and Hill 1998) and a zoned approach to parameterization is being used in the initial inverse modeling at Hanford (Cole et al. 2001; Vermeul et al. 2001), because small-scale variability in parameters is not as important in large-scale regional groundwater modeling as identifying and accounting for the large-scale formations and trends in the geohydrology. Regional-scale modeling at the Hanford Site-wide scale creates additional difficulties related to the inability to reproduce responses to small-scale pumping tests and river-stage fluctuations because of space and time discretization limitations and because of the limitations in the processes that can be modeled at a site-wide scale (e.g., only saturated flow and not the unsaturated flow needed to deal with bank storage effects that control near river water level fluctuations in the unconfined aquifer). Parameter zones must be identified as part of the model structure identification process and each of these various model structures will then need to be evaluated for plausibility. In keeping with the principle of parsimony, and in order to have a tractable problem, we need to minimize the number of parameter zones and the number of parameters to be estimated to avoid the typical inverse problems of nonuniqueness expressed by instability and nonidentifiably. Sun and Yeh (1985) examined an inverse method that attempted to determine both the parameter structure and the parameter values. More recently Sun et al. (1995) proposed a geology-based geostatistical method for parameter identification that attempts to incorporate all available geologic and hydrologic data to identify the three-dimensional structure with an "appropriate level" of parameterization. This method allows one to avoid over parameterization, which generally provides a better fit of observations at the expense of an increase in the estimate of variance in the identified parameters. Model structure identification and 
mathematical approaches for development of parameter zones is still an area of research. Generally the determination of parameter zones is a subjective process considered as part of the conceptual $/$ model structure identification process discussed earlier (Section 3.2.2). For these cases the appropriateness, plausibility, or uncertainty associated with each of these model structures or conceptual models can be addressed only subjectively or through some statistically based measures of overall model fit as discussed in Hill (1998) or through the four statistically based measures of model fit initially described in Carrera and Neuman (1986a,b,c) and further discussed in a recent application by Hyun and Lee (1998).

Even when an inverse method is successful and a plausible model has been identified, additional analysis is required to determine the $95 \%$ confidence intervals for the inversed parameters and to determine whether they are meaningful for use in uncertainty assessment. Christensen and Cooley (1999) discuss this process for a study involving the application of MODFLOWP (Hill 1992) to a $450 \mathrm{~km}^{2}$ leaky aquifer in Quaternary deposits on the Danish island of Zeeland. The study indicates that since the residuals are normally distributed, nonlinear confidence intervals can be calculated, but that since the total nonlinearity of the regression model is significant, even with the addition of prior information on transmissivities, corrections to the size of the parameter confidence intervals are required. This is because nonlinear effects can cause nonlinear intervals to be offset from, and either smaller or larger than, the linear approximations. This study by Christensen and Cooley (1999) indicates the need for careful analysis of the usefulness and meaningfulness of uncertainty estimates.

\subsubsection{Numerical Error}

Assuming that all other aspects of the modeling and analysis have been properly carried out, numerical errors arise from a variety of sources. Numerical errors arise when the spatial and temporal discretizations are inadequate to resolve spatial and/or temporal details of interest or when a particular implementation results in violations of the numerical criteria such as grid Peclet and/or Courant restrictions as discussed in Campbell et al. (1981). Other numerical errors (James 1994) may be related to poor formulation of component process relations, the numerical mathematics used in the code, or just plain coding errors.

\subsubsection{Propagated Error}

Propagated error is the additional uncertainty that arises in an uncertainty analysis because of erroneously estimated values for input parameters and their uncertainty ranges. Propagated error arises from the error in the estimate of the uncertainty itself (e.g., the error in the uncertainty estimate that is propagated because a uniform distribution was assumed rather than the "real" distribution, which was normal). In the same way that uncertainty in input parameters propagates through a given model structure when quantifying the uncertainty in model predictions, errors in uncertainty estimates for the parameter inputs propagate to produce errors in these uncertainty estimates. Similarly, errors in the model resulting from conceptual, mathematical, numerical implementation, and discretization will result in both uncertainties and errors in uncertainties that will be erroneously propagated. 


\subsection{Approach for Addressing Groundwater Model Uncertainties}

NUREG/BR-0184 (USNRC 1997) presents a reasonable categorization or taxonomy of the various sources of uncertainty in PRAs and then provides guidance on how these various types of uncertainties should be addressed through combinations of sensitivity and uncertainty analysis. The seven categories of uncertainties encountered in PRAs identified by Vesely and Rasmuson (1984) and their recommended approach for addressing each category follow:

1. Data Uncertainty - (the most familiar and most often treated. It can be divided into four groups: population variation, imprecision in values, vagueness in values, and indefiniteness in applicability): Use uncertainty analysis for population variation and value imprecision, sensitivity analysis for value vagueness and indefiniteness in applicability.

2. Analyst Uncertainty - (refers to variations in modeling and quantification, which arise when different analysts perform different portions of the analysis and provides its own separate contribution to uncertainty.): Use sensitivity analysis.

3. Modeling Uncertainty - (arises from the indefiniteness in how comprehensive and how well characterized are the numerous models in the analysis. Do the models account for all significant variables? How well do the models represent the phenomena? Is the dependence between two phenomena accurately modeled?): Use sensitivity analysis.

4. Completeness Uncertainty - (is similar to modeling uncertainty, differing only in that it occurs at the initial, identification stage in the analysis. When the analytic "boundaries" are drawn at the start of the analysis, how can one be sure that all "important" items have been included [e.g., the Three-Mile Island core-damage scenario was not specifically identified in PRAs until it had occurred]? Even if the important items have been included, are their interrelationships adequately defined [if even known]?): Use sensitivity analysis.

5. Frequency Uncertainty - (accident frequency uncertainties arise from two sources: variations between accidents of the same type and limited knowledge of the data, models, and completeness.): Use uncertainty analysis for variation from one accident to another, sensitivity analysis for the limited knowledge of the data, models, and completeness.

6. Consequence Uncertainty - (accident consequence uncertainties parallel those in accident frequency, except that they involve consequence modeling rather than frequency estimation.): Use uncertainty analysis for variation from one accident to another, sensitivity analysis for the limited knowledge of the data, models, and completeness.

7. Interpretation Uncertainty - (deals with the interpretation of the analytic output and results that arises from the combination of all previous uncertainties plus the difficulty in conveying the information to the decision-maker. Even the most precise uncertainty analysis can be wasted if the meaning cannot be transferred to the decision-maker. Often, this results from difficulty in the way the results are 
presented. Ernst [1984] provides insight on reducing the uncertainty in interpretation of results.): Use sensitivity analysis.

While assessment of the uncertainty in groundwater flow and transport predictions does not involve all seven steps associated with a PRA above, the same general approach will be followed in addressing the uncertainty in groundwater system flow and transport predictions. The basic approach is to use sensitivity analysis for those aspects of the analysis related to vagueness and indefiniteness (e.g., alternative conceptual models (ACMs), model structure, and future scenarios) and uncertainty analysis for those situations where the uncertainty (e.g., for parameters) can and should be represented by a pdf (e.g., as interpreted from measurements using geostatistical methods, or determined from inverse modeling).

The general approach adopted for addressing groundwater flow and transport model uncertainties will consist of

1. identifying ACMs

2. development and evaluation of each ACM to determine plausibility or lack thereof

3. for plausible conceptual models, perform an uncertainty assessment as follows:

a. optimize model complexity

b. identify the important or relevant parameters (Figure 3.27) for the uncertainty analysis, based on the uncertainty in the parameter value and the sensitivity of the code to the parameter value (Meyer and Gee 1999), and develop pdfs for the important uncertain parameters

c. propagate the uncertainties (e.g., by a First Order Second Moment (FOSM) or a Monte Carlo approach) through the model to determine the uncertainty in predictions.

The combined uncertainty associated with the plausible models then represents the best estimate of uncertainty that can be developed based on current information and understanding. While the above list is a convenient way to describe the approach, there is considerable overlap in the first five items, because ACM identification and development requires examining and optimizing model complexity to be consistent with availability of information and the antithesis concept of model parsimony that requires the modeler to seek the simplest model parameterization consistent with the evidence (Kuczera and Mroczkowski 1998; Box and Jenkins 1976).

\subsubsection{Identify Alternative Conceptual Models}

The initial step in an uncertainty assessment involves identifying ACMs that can be supported by current understanding (both generic and site-specific) as well as site-specific observations and measurements. As discussed earlier, a conceptual model of a geohydrologic system, no matter how technically complex, will always be a simplified picture of the real system. Current computer technology and datagathering capabilities do not and will not allow a real geohydrologic system to be described in every 


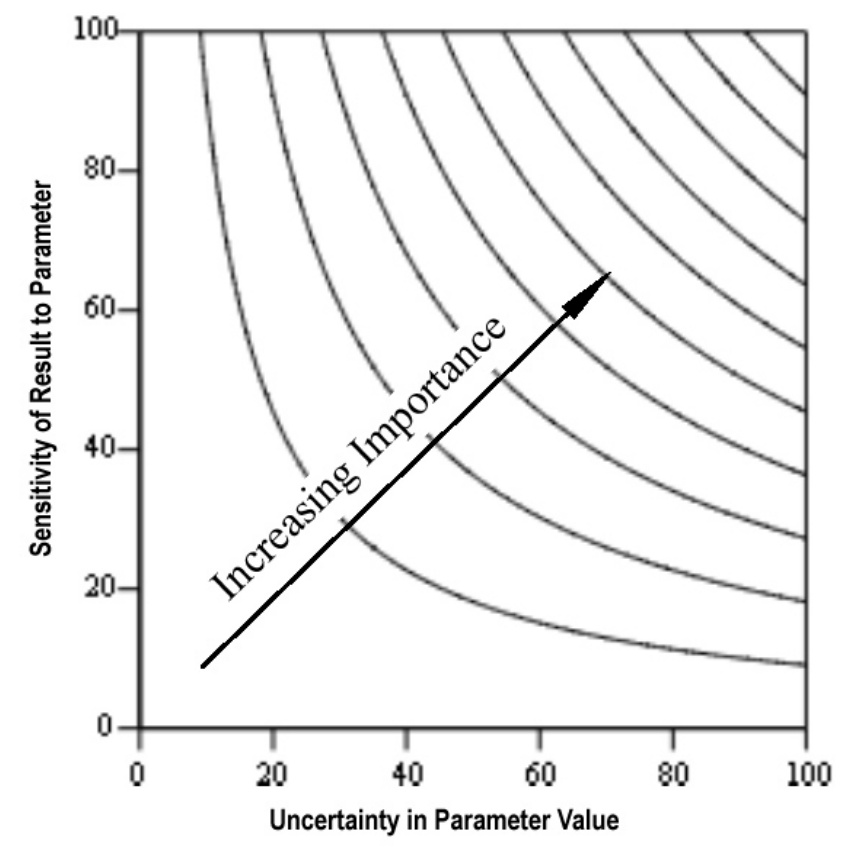

\begin{tabular}{|ccc|}
\hline $\begin{array}{c}\text { Importance of } \\
\text { Parameter to } \\
\text { Uncertainty } \\
\text { Assessment }\end{array} \quad \begin{array}{cc}\text { Uncertainty in } \\
\text { Parameter }\end{array}$ & $\begin{array}{c}\text { Result to } \\
\text { Value }\end{array}$ & $\begin{array}{c}\text { Parameter } \\
\text { Value }\end{array}$ \\
& & \\
\hline
\end{tabular}

Figure 3.27. Hypothetical Diagram (adapted from Meyer and Gee 1999) Illustrating the Importance Relationship that Links Parameter Uncertainty and Sensitivity to Parameter Importance in Uncertainty Assessment.

detail. In some respects, modeling a geohydrologic system is an art form. Conceptual model development involves forming a sufficiently accurate simplified picture of the aspects of the system important to making the desired predictions or performance assessment. In the process of forming this sufficiently accurate simplified picture, certain groundwater flow and transport modeling technical issues must be considered and addressed, and these decisions, supporting reasoning, and observations must be documented. As pointed out by James (1994) and Freeze et al. (1987), the effect of these assumptions and reasoning needs to be considered when evaluating the modeling results and estimates of uncertainty. The technical issues (Cole and Foley 1985) are simply questions as to what constitutes the correct way to describe the modeled system in terms of relevant processes, parameterization, and numerical models (e.g., saturated or unsaturated flow, buoyancy, importance of and method for describing dispersion, importance of and required dimensionality in properly representing the system and phenomena of interest). The issues stem from limitations on current physical and chemical theories, data gathering capabilities, and computer modeling capabilities; and it should be recognized that in some cases these technical issues might not be resolved absolutely (Cole and Foley 1985). 


\subsubsection{Develop and Evaluate Alternative Conceptual Models}

The second step involves developing and evaluating each of these alternative conceptual models to identify the plausible conceptual models that will be the subject of an appropriate uncertainty assessment. ACM evaluation (Section 3.2.2) will consist of the statistical measures of overall model fit discussed in Hill (1998) and examining as appropriate the four model structure identification measures suggested and discussed in Carrera and Neuman (1986). The issues and approach for identifying, developing, and evaluating ACMs are discussed more fully in Section 5.2.3 and will not be discussed further here except as they relate to optimizing model complexity.

\subsubsection{Perform Uncertainty Assessment for Plausible ACMs}

The three substeps (i.e., optimizing model complexity, identifying the relevant parameters and developing their pdfs, and propagating uncertainties through the model) associated with performing an uncertainty assessment are not totally independent from the first two steps needed to address groundwater flow and transport model uncertainties (i.e., ACM identification and ACM development and evaluation).

\subsubsection{Optimize Model Complexity}

Morgan and Henrion (1990) reproduce Albert Einstein's apt description of the complexity required in a good model:

"Everything must be made as simple as possible, but not simpler."

Optimization of model complexity is an issue and process that must be revisited at all steps of the uncertainty assessment. This is because the total number of parameters whose uncertainty could be characterized (i.e., with a pdf) and thus varied to produce uncertainty estimates, is large compared to the capacity to compute. As a result, only the most important sources of uncertainty can be included in an uncertainty analysis for a given conceptual model. This is consistent with the PRA guidance document (USNRC 1997), which indicates that an uncertainty assessment for a given model structure must include an initial scope definition step to determine what uncertainties should be included and with the concept of identifying relevant parameters discussed in Meyer and Gee (1999). This effort will involve identifying parameter ranges and conducting sensitivity studies to identify the most relevant sources of uncertainty affecting the model predictions of interest for the future scenarios of interest (i.e., the objective function). This effort, discussed in James (1994), is described as determining the optimal model complexity since he defines the number of uncertain parameters in a model as one measure of model complexity. However, James citing Seo (1991), indicates that while it is important to improve model accuracy and reliability by including as many relevant processes at spatial and temporal discretizations that are as fine as possible, there is no guarantee that model reliability will continue to increase with model complexity. This is because of the difficulty of getting good parameter estimates, and because of their combined effect on the computed response. As a result, James (1994) indicates that user controllable model complexity arising from process disaggregation (i.e., where an unnecessarily large number of processes, parameters, and variables are activated) and unnecessarily fine spatial and temporal discretization needs to be minimized, not only because of the model reliability issues already discussed, but also because of the costs associated 
with monitoring and analyzing data for a system with a large number of parameters and state variables. James suggests the use of an evaluation function for determining optimal order of model complexity based on cost (for complex models) and model reliability (i.e., by penalizing model inaccuracy for very simple, coarse, and inaccurate models). This thought is in line with the principle of parsimony discussed earlier (Hill 1998) and the discussion in Galarza et al. (1996) where they state

"For a model to have any chance of reproducing reality, it must include the relevant processes and represent them by an adequate model structure. Since one can neither consider jointly all possible processes affecting water flow and solute transport nor represent accurately their spatial variability, one makes simplifying assumptions about both."

Galarza et al. (1996) proceed to point out that it is this simplification process that makes the result very dependent on the modeler or analyst and identifies at least one source of the "analyst uncertainty" identified by Vesely and Rasmuson (1984).

\subsubsection{Define Probability Distributions}

Two general approaches for estimating values for spatially variable parameters along with their uncertainty and the correlations between these parameters were discussed in Sections 3.2.3.1 and 3.2.3.2. These approaches include geostatistical methods that deal directly with the data and their upscaling and interpolation, and the various statistically based indirect inverse methods. Additionally "expert elicitation" techniques may be required to develop uncertainty estimates for sensitive parameters, as might be determined through sensitivity analyses, for which available data may be too limited to apply either of these methods. These "expert elicitation" techniques can be used to select/develop (albeit in a subjective manner) a representative probability distribution and any of the necessary coefficients based on the limited data and understanding that is available. USNRC (2000) guidance in Appendix C, Section 8 on the assignment of distributions to relevant parameters based on the availability of data is paraphrased below:

- Ample data - Empirical distributions of a parameter can be generated directly

- Sufficient data - Standard distributional forms (e.g., normal, lognormal, and uniform) can be obtained by standard analysis means (e.g., plotting data as histograms or in probability coordinates)

- Some data - When the shape of an empirical distribution cannot be determined from available data, it may be supplemented by other soft information (e.g., a mechanistic basis exists for assigning a given distribution; the distribution was well known for the parameter regionally, so this same distribution could be used to estimate the shape of the distribution for this local data set)

- Insufficient information - When only incomplete information is known about the parameter (e.g., its mean or its range), and no correlations to other types of data are available, the parameter distribution selection should reflect this uncertainty. According to NRC (2000), Harr (1987) indicates that in this case the distribution should have the least-biased value, which is generally a wide distribution 
encompassing all the possible values and Harr indicates that the "maximum entropy formalism," based on Shannon's informational entropy is one procedure to ensure that the distribution has the least bias. Table 3.1 adapted from USNRC (2000) describes the maximum entropy solutions for several classes of available but insufficient information. This "maximum entropy formalism," provides a means to pick the distribution based on the kinds of information available for the parameter to ensure that the result is least-biased; for example, if only the range of the data is known, a uniform distribution between the range is least-biased as a uniform distribution provides the maximum uncertainty.

Table 3.1. Maximum Entropy Probability Distributions (adapted from USNRC 2000 with the original source being Harr 1987)

\begin{tabular}{|l|c|}
\hline \multicolumn{1}{|c|}{ Given Constraints on Data } & Assigned Probability Density \\
\hline Minimum and maximum only & Uniform \\
\hline Expected value only & Exponential \\
\hline Expected value and standard deviation & Normal \\
\hline $\begin{array}{l}\text { Expected value, standard deviation, } \\
\text { minimum and maximum }\end{array}$ & Beta \\
\hline $\begin{array}{l}\text { Mean occurrence rate between arrival of } \\
\text { independent events }\end{array}$ & Poisson \\
\hline
\end{tabular}

Discussions in USNRC (2000) also indicate that IAEA (1989) and NCRP (1996) provide guidance for choosing parameter distributions based on limited information. Additionally the appendix to the "Guiding Principles for Monte Carlo Analysis" document (EPA 1997) contains an excellent discussion on probability distribution selection issues. Peck et al. (1988) suggest that arbitrary selection of pdfs could result in "garbage in, garbage out."

\subsubsection{Propagation of Uncertainties}

The overview discussions on techniques for estimating uncertainties related to climate change by Katz (1999) are relevant to these discussions on prediction uncertainty propagation for groundwater flow and transport at the Hanford Site. Katz (1999) indicates that only a few formal probabilistic uncertainty analyses have been applied to global climate change models and that these existing analyses have focused on parameter/input uncertainty and ignored imperfections in model structure. Katz attributes this failure to apply full probabilistic uncertainty analyses to the complexity of the models and what he describes as the "curse of dimensionality" that arises in problems requiring optimization. As a result, Katz indicates that most global climate change analyses have generally dealt with simpler versions of global change models that have a high degree of aggregation.

The situation is generally the same for groundwater flow and transport uncertainty assessments. The majority of the full probabilistic uncertainty analyses generally use simpler highly aggregated models and are carried out with total system performance codes for probabilistic assessment (e.g., Repository Integration Program (RIP) by Golder Associates Inc. 1998; MEPAS Whelan et al. 1992, 1994). There are only a few of the more complete uncertainty assessments (e.g., Sandia National Laboratories 1992-1993; 
CRWMS M\&O 1998) that involve reasonably complex groundwater models and other components that deal with a full range of uncertainties (e.g., uncertainty regarding future conditions and the associated driving forces, related interactions triggered by these uncertainties, uncertainties in the effects of engineered solutions). These more complete uncertainty assessments (e.g., CRWMS M\&O 1998) identify scenarios for assessment that consist of expected features, events, and processes (see Section 2.2 of CRWMS M\&O 1999 for a discussion of the features, events, and processes approach) that attempt to represent the evolution of the natural system and any engineered components, including any interactions.

It is important to make a distinction between the steps and issues required in the propagation of a fuller range of uncertainty (e.g., those that address interacting features, events, and processes and the probability for each future state of system evolution such as a meandering river, catastrophic floods, climate change, and associated increased recharge) and the steps and issues involved with an uncertainty assessment focused on propagation of parameter uncertainty because additional information and steps are involved. While most of the efforts over the next few years will focus on parameter/input uncertainty for a series of plausible alternative conceptual models, as discussed in Section 5, it is important to understand that this discussion addresses only part of the uncertainty in future predictions. The approach to address scenario uncertainty, which also is required for a complete assessment of uncertainty, will be the subject of future efforts.

Katz (1999) uses a discussion of the simple rules associated with uncertainty propagation (i.e., the simple multiplication rule for probabilities and the simple addition rule for variances) to illustrate some of the issues and complexity associated with error propagation for uncertain future states and for parameter uncertainty for complex but plausible alternative conceptual models.

Future system states must be viewed as evolving as a sequential process with each stage occurring independently with a fixed probability in order for the simple "multiplication rule for probabilities" (i.e., the probability of a number of independent events jointly occurring equals the product of the probabilities of the individuals events) to be useful for estimating uncertainty in future system states and predictions (Katz 1999). As Katz points out, this simplistic view is not very applicable to the real world as many events are far from independent (e.g., a flood at Hanford that alters the river course could also alter groundwater pathways and cause a failure in an engineered barrier). The simple multiplication rule can be expanded to dependent events by replacing unconditional with conditional probabilities, but as Katz discusses, this requires that the conditional probabilities, which are much more difficult to determine, must now be developed for these possible future events. Finally, it must be recognized that the real evolution of the system is controlled by a combination of interactions between slowly acting processes (e.g., erosion, river course migration, climate changes) and what can be viewed as dependent and independent future events (e.g., floods and associated river course changes). The RIP code by Golder Associates Inc. (1998) attempts to address these types of interactions through use of a Monte Carlo process that considers interactions between slowly acting natural processes and the uncertainty in independent and dependent events including the uncertainty in their time of occurrence. These types of uncertainties associated with uncertain futures (or scenarios as they are often called) are beyond the scope of the uncertainty efforts currently being addressed by the Hanford SGM project. 
The heuristic for addition of variances (i.e., the variance of a sum of a number of uncorrelated random variables equals the sum of the variances of the individual random variables) is quite simple, but reality is more complicated (Katz 1999) because pairs or groups of random variables can be highly correlated, making this simple heuristic undependable unless a covariance term is incorporated into a modified addition rule. However, as was the case for conditional probabilities, information on the covariance structure can be difficult to obtain. On the other hand the more difficult issues, according to Katz (1999) and Meyer and Gee (1999), are related to how uncertainty depends on the level of aggregation and how it is affected by the upscaling process. The upscaling issue (i.e., How to define effective parameters and the uncertainty in these effective parameters relevant to the scale of the problem being modeled and the applicable boundary conditions?) as discussed in Section 3.2.3 is a central and ubiquitous problem in groundwater hydrology because of the heterogeneity (Figures 3.16 and 3.17), the use of constitutive models, the wide range of spatial and temporal scales over which measurements are made (Figures 3.22 and 3.25), the diversity of the constitutive models used to interpret parameters (Figures 3.22, 3.23, and 3.24), and the diversity in space and time scales for which predictions are needed (Figure 3.20). The use and behavior of the observation and parameter variances during the upscaling/ downscaling and aggregation process is the major source of the difficulty because the support volume for the parameters and observations varies with the scale and level of aggregation and with the actual laboratory and field methods used to interpret the field data. The effect of variable support volume on hydraulic conductivity parameter uncertainty related to natural variability is illustrated for a hypothetical example in Figure 3.28. Katz (1999) indicates that while for some models only simple adjustments may be needed to

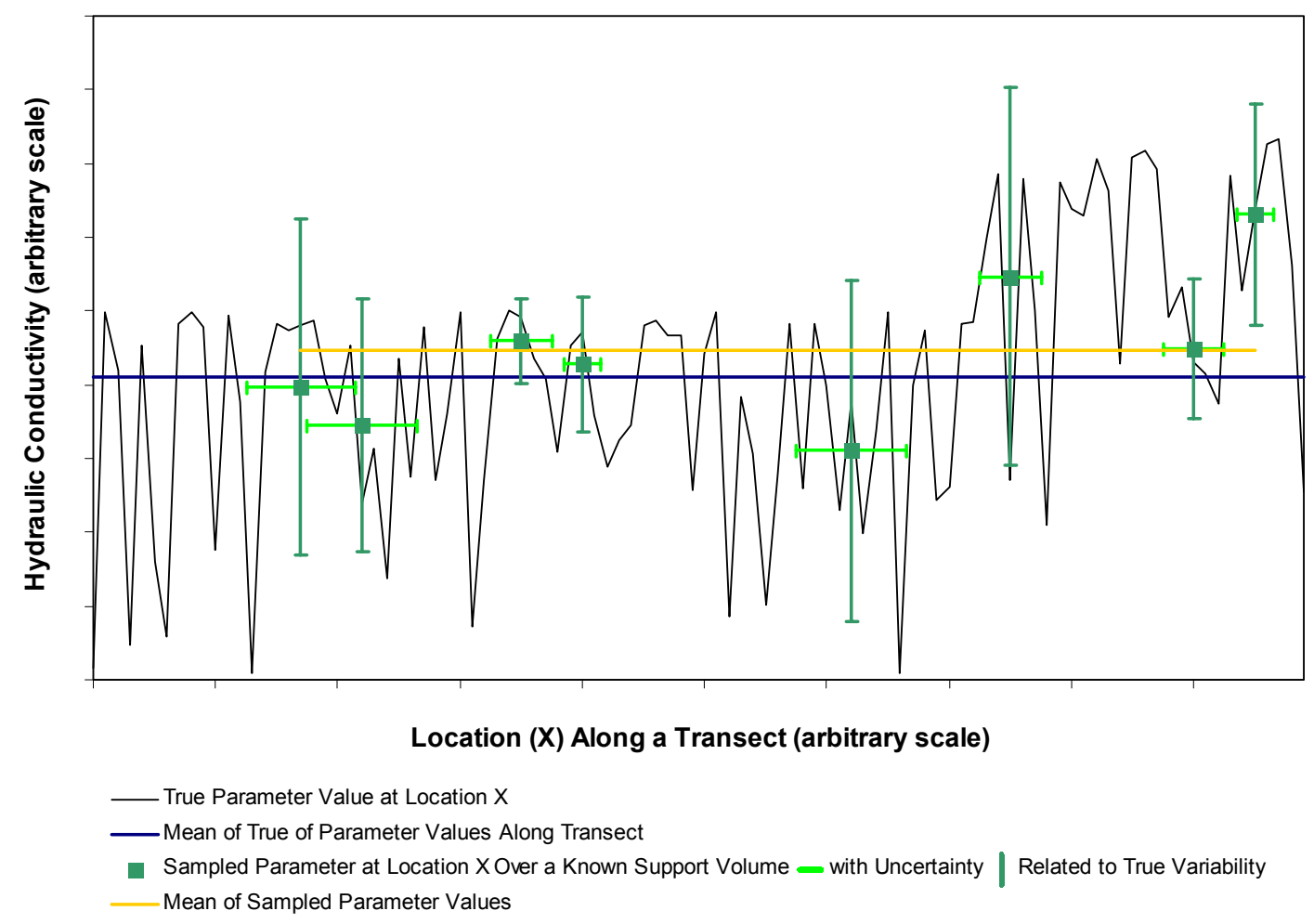

Figure 3.28. Hypothetical Illustration of the Effect of Support Volume on Hydraulic Conductivity Parameter Uncertainty Related to Natural Variability. 
use observations and variances made at one space-time scale, at some larger scale this is generally not possible because no simple invariance property holds and as a result a change in functional form is required. Katz goes on to suggest that for global climate change models:

"One could make the case that the issue of the level of aggregation is artificial and avoidable in principle. As such, uncertainties attributable to this source should not be regarded as inherent. But, in practice, the complexity of the problems (e.g., in integrated assessment) requires a substantial degree of aggregation to make implementation feasible (Rotmans and Dowlatabadi, 1998)"

As previously discussed (Sections 3.1.1.2, 3.2.3.1) the spatial correlation relationship for a given parameter (e.g., the interdependence of hydraulic conductivity at one point in an aquifer or aquitard with a nearby point in the same depositional environment) and the correlation that may exist between parameters (e.g., the correlation between hydraulic conductivity and porosity) must be appropriately accounted for in both the data gathering/interpretation phase as well as in the error propagation phase of the uncertainty/ variability analysis.

Prediction uncertainty propagation related to input uncertainty can proceed for each ACM once an ACM has been

1. developed

2. optimally implemented

3. evaluated and determined to be "plausible" (see Section 5.2.3)

4. estimates of the relevant sensitive parameters, their uncertainties, and any correlations have been identified.

Steps 1 through 3 of this process must be completed for all reasonable ACMs that have been identified, and step 4 as well as uncertainty propagation must be completed for each of the ACMs determined to be plausible.

An important part of an uncertainty analysis is the identification, enumeration, and documentation of all assumptions

- made during conceptual model development

- required by the mathematical model

- required by the numerical model

- made during the spatial and temporal discretization process (e.g., Figure 3.20) 
- needed to assign the statistical model and associated parameters that describe the uncertainty in the relevant input parameters

- required by the propagation method.

This list is important because the uncertainties related to these assumptions are not part of the uncertainty propagation and can be addressed only through sensitivity studies and alternative conceptual model evaluations.

To illustrate the importance of understanding the implications of the above assumptions made as part of an uncertainty analysis and the effect they have on both the modeling process and results, consider two different uncertainty approaches to represent the already simplified representation of the geohydrologic system shown in the upper part of Figure 3.20 (a). The simplified model representation assumes that the Bearpaw shale represents a no-flow boundary, which leaves the uncertainty related to interactions of the upper hydrologic system with the lower hydrologic system below the Bearpaw shale unaddressed. As already discussed, some simplification and parameter upscaling is always required because of the continuous variation in process and parameter scales. Assume for the purposes of this hypothetical uncertainty assessment that the simplified model representation, which involves a zonation approach as illustrated in Figure 3.20 (a, b, c), is appropriate. Implied in this assumption is the type of hydraulic parameterization required since it is a direct reflection of the level of aggregation. Under the above assumptions each zone is considered to be homogeneous and therefore each of the three zones (i.e., 1 - Glacial deposits and alluvium; 2 - Clay, silt members of Ravenscrag, Whitemud, and Eastend formations; and 3 - Sand members) can be fully represented in an uncertainty analysis by a single pdf if the hydraulic parameters for the three different zones can be shown to be uncorrelated. If they are correlated, then appropriate combinations of monovariate, bivariate, and trivariate pdfs or their equivalents are required to represent the uncertainty in these parameters. The required upscaled hydraulic parameters and the associated uncertainty estimates (e.g., pdfs) could be developed directly from the data sets as discussed in Section 3.2.3.1 or developed through the application of an inverse technique as discussed in Section 3.2.3.2. In the first approach, the uncertainties related to the spatial location and variation in thickness of each of the geohydrologic units would not affect the estimates of the hydraulic parameters except indirectly and only for those data points where the hydraulic properties were deduced from pump tests or improperly assigned to a wrong geohydrologic unit. However, the estimates developed from the hydraulic data alone would introduce additional uncertainty into both the upscaled parameter estimates as well as in the associated estimates of their uncertainty. The additional uncertainty introduced arises from the upscaling assumptions and methods used to develop the upscaled parameter estimates and because of the disparity in both the measurement scales and interpretation methods used to develop the small-scale parameter estimates that make up the available set of "field data" used to develop the upscaled parameter estimates for use in the model. Additionally the methods used in the first upscaled data approach, except for hybrid approaches involving cokriging, would not properly account for other observational data (e.g., head versus time). In a second approach, which involves inverse methods, uncertainty in the estimates of both the spatial location and thickness variation of the geohydrologic units and effects related to the actual model discretization, which are a deterministic component of the inverse model, will affect both the estimate of the expected value of each of the three parameters, the uncertainty in these estimates, and any correlation between the estimated parameters. This is because the inverse 
approach will seek out the best parameterization and the associated uncertainty estimate that is appropriate for the deterministic representation of the model structure posed. Because layering (e.g., aquifers and aquitards) in geohydrologic systems is generally represented deterministically, this aspect of uncertainty is not only not accounted for but gives rise to propagation errors even if the spatial variability in the upscaled hydraulic parameters (e.g., hydraulic conductivity and porosity) were perfectly known. This is because the transmissive properties of the aquifer are a function of the hydraulic parameters as well as the thickness, location, and areal extent of the various geohydrologic layers.

\subsection{Methods for Propagation of Uncertainty}

This section discusses some of the primary techniques employed in uncertainty analysis as they apply to parameter/input uncertainty. In these propagation methods the model itself is viewed, in effect, as being free of any uncertainties, only the inputs (e.g., parameter values) are assumed to be uncertain. The discussions focus on Monte Carlo and FOSM methods, which are the two approaches likely to be pursued in the assessment of parameter uncertainty for the Hanford Site-wide flow and transport modeling to determine the uncertainty in model results (i.e., predictions) related to input parameter uncertainty. These discussions will focus on parameter uncertainty, but it should be noted that when needed, the Monte Carlo approach, as discussed in CRWMS M\&O (1999) and Jow et al. (1997), can be expanded to include both stochastic and subjective uncertainty (Helton 1993). The stochastic uncertainty, or variability, is defined to be the uncertainty resulting from or attributable to geologic heterogeneity and natural variability and subjective uncertainty is defined to be the uncertainty arising from ignorance or imperfect knowledge about processes and/or parameters. The total uncertainty is then represented by two components: 1) due to stochastic uncertainty, represented by an individual CCDF in Figure 3.29 (source Jow et al. 1997), and 2) due to subjective uncertainty, which is represented by the family of CCDFs in Figure 3.29, which Jow et al. (1997) represent mathematically as the double sum given by Equation 3.11. In Equation 3.11

$$
\sum_{S U} \sum_{S T} F(x)
$$

the inner sum, denoted with the subscript $S T$, is a probabilistic characterization of the uncertainty associated with parameters that characterizes the stochastic uncertainty whose evaluation through the inner sum develops one of the individual CCDFs in Figure 3.29, while the outer sum, denoted with the subscript $S U$, characterizes the subjective uncertainty and gives rise to the family of CCDFs shown in Figure 3.29.

As discussed in Peck et al. (1988) partial differential equations for flow and transport are normally written for the deterministic case where both the dependent variables (e.g., head and concentration) are deterministic quantities with one unique value at each point in space and time. However, when some of the inputs are stochastic or uncertain, the results (i.e., the dependent variables) are also stochastic or uncertain and the deterministic partial differential equations must be rewritten in their stochastic form so that given the joint multivariate pdf describing all of the uncertain input parameters one could solve 


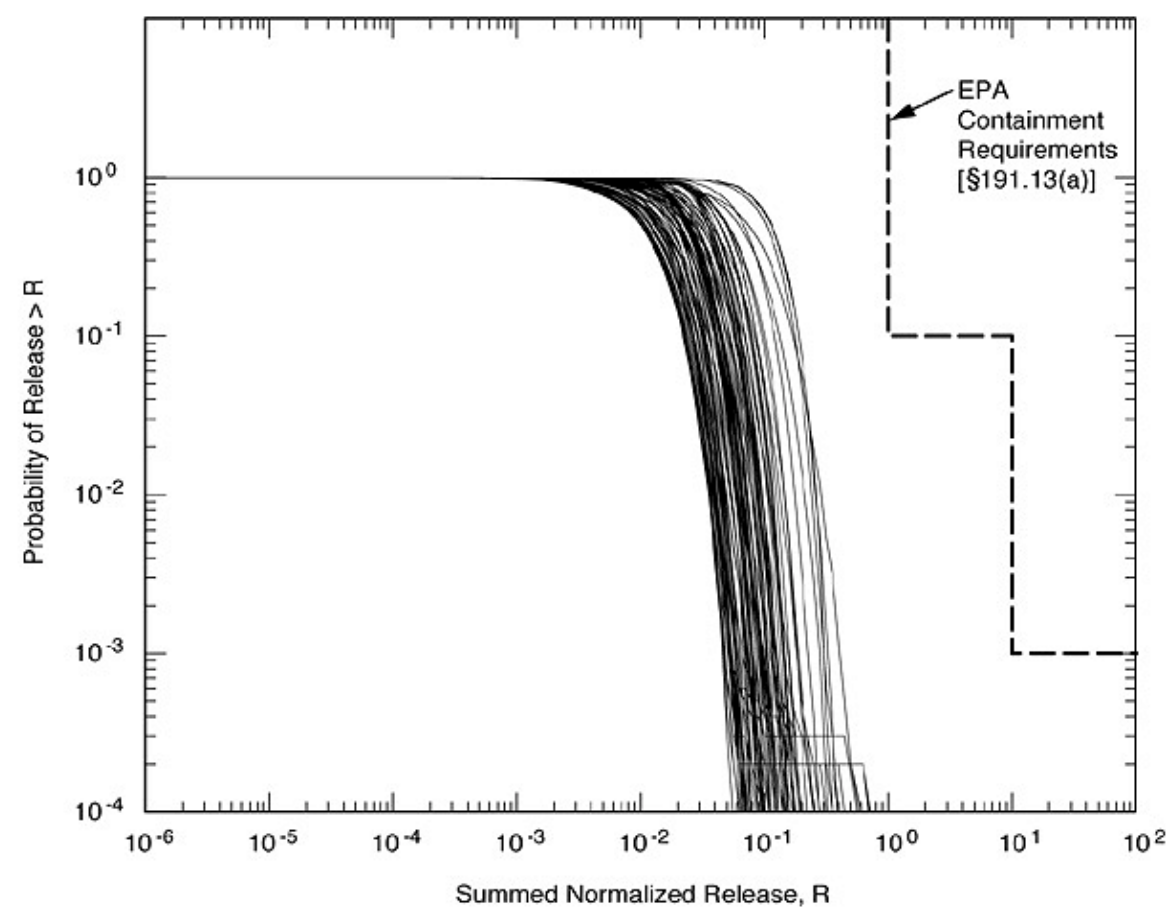

Figure 3.29. Example of CCDF that Attempts to Account for Both the Stochastic and Subjective Uncertainty Through a Family of CCDFs. The figure from Jow et al. (1997) shows a distribution of CCDFs for normalized radionuclide releases to the accessible environment from the Waste Isolation Pilot Plant, replicate 1.

directly for the joint multivariate pdf for the results (e.g., the dependent variables or any quantity that is a simple function of the dependent variables as discussed in Section 3.1.1.1.4). In practice this latter approach is impossible to achieve except for simple systems with relatively simple probability properties (Dettinger and Wilson 1981).

Dettinger and Wilson provide a useful taxonomy of the various techniques for propagation of parameter uncertainty. They divide the various approaches into two main groups as follows:

- First Order Second Moment Methods - These methods assume the first two moments of the random variable input parameters are sufficient to characterize the mean and variance/covariance of the results of interest. These methods include

1. The perturbation analysis approach in which the governing partial differential equation is perturbed slightly to yield a new equation for the variance of the dependent variable. The work by Tang and Pinder (1977) provides a good example of this approach.

2. The Taylor series expansion methods generally expand the analytical or numerical solution of the governing equation around the expected values of the parameters and independent variables to deduce various probabilistic moments (e.g., mean, variance/covariance) of the dependent variable 
(i.e., the results). According to Peck et al. (1988), Cornell (1972) was the first to apply these methods to water resource problems while Dettinger and Wilson (1981) extended the method to more complicated flow problems, and Wagner and Gorelick (1987) were the first to use the method in the assessment of uncertainty in combined flow and transport problems. These methods include the linear confidence and prediction interval codes developed by Hill (1994) based on the early work of Cooley (1977) for use with MODFLOWP (Hill 1992) and implemented in UCODE (Poeter and Hill 1998). They also include linear FOSM methods implemented in ITOUGH2 (Finsterle 1993) and discussed by James and Oldenburg (1997) as well as Peck et al. (1988).

To apply perturbation methods to nonlinear systems requires that the uncertainty in parameters be "small." For example, as discussed in Peck et al. (1988), the fluctuation around the mean as described by the Taylor series expansion still applies when the second and higher order terms are neglected if the uncertainty in parameters is small. The analysis and testing for "small" must be part of the application of these methods. Cornell (1972) suggested that for parameter coefficient of variations $\leq 0.2$ the method should be applicable to moderately nonlinear problems.

- Full Distribution Methods - These methods require a complete specification of all non-deterministic inputs and parameters and attempt to specify completely the probability distributions for the results of interest. The two most important of the full distribution methods include

1. The derived distribution approach, which is an analytical method with generally very limited applicability (Eagleson [1978] is an example of this approach).

2. The powerful Monte Carlo analysis (Monte Carlo simulation) method, which is a computer-based method of analysis developed in the 1940s (EPA 1997) with a wide range of applicability in the propagation of uncertainty that can be applied using either simple or complex models. The Monte Carlo method is both the most powerful and yet the simplest to understand (Peck et al. 1988). It involves numerous replications of the simulation model with the parameters and inputs for each simulation appropriately drawn at random from their respective pdfs (e.g., accounting for any correlations) so that the results from the random replicates can be compiled to form the probability distribution for the desired results (Dettinger and Wilson 1981). Limitations of the method are related to the number of runs required in order to fully sample the space of uncertainty, which may lead to long computational run times.

Each of these two main approaches can be solved numerically (at least theoretically) and under the right conditions, they can be solved analytically to produce a closed form solution that generally has limited applicability. The complexity of the site-wide flow and transport modeling effort at Hanford will require the use of the numerical based methods. With regard to the applicability of the these two main categories of approaches, Peck et al. (1988) and Hill (1994) discuss the importance of understanding the relationship between the "result" for which you wish to assess the uncertainty and the type of parameter uncertainty characterization that is available. This is because the type of parameter uncertainty characterization that is available limits the type of result uncertainty that can be computed. Therefore, the decision on uncertainty approach must factor in the type of result uncertainty that is needed, practical, and 
computable. For example, a first order second moment method, which is based strictly on means and variances may be sufficient if the data and physical arguments are generally insufficient to fully define the input pdfs, and the decisions to be made are generally not sensitive to moments higher than the mean and variance.

\subsection{First Order Second Moment Methods}

The First Order Second Moment method is quite simple. We generally follow the development in Dettinger and Wilson (1981), but use a slightly different nomenclature found in Jackson et al. (1995). Let $z$ be a quantity that is a function of a set of $n$ random variables $z_{i}$ :

$$
z=F\left(z_{1}, z_{2}, \ldots, z_{n}\right)
$$

The function expanded about the $n$ expected values, $E\left[z_{i}\right]$ (i.e., the means, $m_{i}$ ) of the $\mathrm{n}$ random variables is as follows:

$z=F\left(m_{1}, m_{2}, \ldots, m_{n}\right)+\sum_{i}\left(z_{i}-m_{i}\right) \frac{\partial F}{\partial z_{i}}\left(m_{i}, m_{2}, \ldots, m_{n}\right)+\frac{1}{2} \sum_{i, j}\left(z_{i}-m_{i}\right)\left(z_{j}-m_{j}\right) \frac{\partial^{2} F}{\partial z_{i} \partial z_{j}}\left(m_{1}, m_{2}, \ldots, m_{n}\right)+$ higher order terms.

Neglecting second and higher order terms and taking the expected value of the remaining terms leads to the following expression for the expected value of $z$ (i.e., $\left.E\left[z_{i}\right]\right)$ to the lowest order:

$$
E[z]=F\left(m_{1}, m_{2}, \ldots, m_{n}\right) .
$$

This follows because, as discussed in Dettinger and Wilson (1981), the expectation operator is linear and therefore $E[a+b]=E[a]+E[b]$ and $E[c b]=c E[b]$, where $c$ is a constant and $a$ and $b$ are random variables. As a result the second term involving the $\sum_{i}$ is zero because $E\left[z_{i}-m_{i}\right]$ is zero.

In a similar manner the variance, $\operatorname{var}(z)=E\left[(z-E[z])^{2}\right]$, is given to the lowest order by:

$$
\operatorname{var}(z)=\sum_{i, j} C\left(z_{i}, z_{j}\right) \frac{\partial F}{\partial z_{i}}\left(m_{1}, m_{2}, \ldots m_{n}\right) \frac{\partial F}{\partial z_{j}}\left(m_{1}, m_{2}, \ldots, m_{n}\right),
$$

where:

$$
C\left(z_{i}, z_{j}\right)=E\left[\left(z_{i}-m_{i}\right)\left(z_{j}-m_{j}\right)\right]
$$

is the covariance between $z_{i}$ and $z_{j}$. In numerical application $F()$ is the model and $z$ is the model result of interest and the $z_{i}$ are $n$ uncertain model input parameters and the required derivatives can be approximated numerically by differences. An approach involving forward differences could be carried out by 
using the model to calculate the quantity of interest, $z^{b}$, for a base-case set of parameters $z_{1}^{b}, z_{2}^{b}, \ldots, z_{n}^{b}$ and for a set of variants where each parameter is perturbed by an appropriate amount, $\Delta z_{i}$, to calculate values $F\left(z_{1}^{b}+\Delta z_{1}^{b}, z_{2}^{b}, \ldots, z_{n}^{b}\right), F\left(z_{2}^{b}, z_{2}^{b}+\Delta z_{2}^{b}, \ldots, z_{n}^{b}\right), \ldots, F\left(z_{2}^{b}, z_{2}^{b}, \ldots, z_{n}^{b}+\Delta z_{n}^{b}\right)$ so that the required derivatives could be estimated by forward differences as:

$$
\frac{\partial F}{\partial z_{i}}=\frac{F\left(z_{1}^{b}, \ldots, z_{i}^{b}+\Delta z_{i}, \ldots, z_{n}^{b}\right)-F\left(z_{1}^{b}, \ldots, z_{i}^{b}, \ldots, z_{n}^{b}\right)}{\Delta z_{i}} .
$$

The use of linear intervals to develop confidence and prediction intervals to indicate parameter and prediction uncertainty, respectively, is an application of the first order error analysis just described (Hill 1994). UCODE (Poeter and Hill 1998) calculates linear confidence and prediction intervals that approximate the likely uncertainty in predictions simulated using the application models and optimized parameter values. UCODE uses a slightly modified version of computer program YCINT developed by Hill (1994), which uses central-difference sensitivities in place of the forward difference sensitivities (e.g., Equation 3.17). Linear confidence and prediction intervals indicate the uncertainty with which the predictions are determined using the calibrated application model(s) and are defined by Poeter and Hill (1998) as follows:

Confidence intervals represent the uncertainty in the simulated values that is a propagation of the uncertainty in the estimated parameter values. For the purpose of calculating the confidence interval, the uncertainty in the estimated parameter values is expressed by the optimal parameter variance-covariance matrix. The validity of the confidence intervals depends on the calibrated application model(s) accurately representing the true system, the model being linear, and the weighted residuals being normally distributed.

Prediction intervals include the uncertainty in the estimated parameter values as described for confidence intervals, but also include the effects of the measurement error that is likely to be incurred if the predicted quantity were to be measured. Prediction intervals need to be used when a measured value is to be compared to the calculated interval.

As discussed in Hill (1994) the assumptions required to develop accurate confidence and prediction intervals are as follows:

1. uncertainty in parameters are normally distributed

2. model is correct

3. model is roughly linear. 
With regard to the first assumption, Hill (1994) indicates that a normal probability distribution has been found to be valid for many groundwater model calibrations performed using nonlinear regression. This may be related to the level of aggregation and averaging required by the large scale of most of these models and the effects of the central limit theorem discussed earlier. The second assumption, which Hill points out is clearly untrue in some ways, can be tested by various means provided by UCODE and discussed in Hill (1998). The third assumption can be tested directly using the modified Beale's measure (Cooley and Naff 1990), which is calculated directly by UCODE using the BEALP program developed by Hill (1994).

\subsection{Monte Carlo Method}

Nuclear weapons scientists at Los Alamos in the 1940s were the first to apply the name Monte Carlo (the famous gambling city in Monaco) to a class of mathematical methods for solving various problems through the use of random sampling (Kalos and Whitlock 1986). The obvious connection is the ability of the Monte Carlo random sampling methods to predict average or other statistical outcomes and not individual outcomes, just as in gambling at Monte Carlo, actual outcomes of rolling dice cannot be predicted but the frequencies of the various possible outcomes can be precisely determined. While the name Monte Carlo was not applied until the 1940s, Kalos and Whitlock state the earliest documented use of the random sampling approach to find the solution to an integral can be traced to Comte de Buffon (1777) and subsequent use of random sampling in the solution of mathematical problems and integrals by Laplace and Lord Kelvin. Also, according to Kalos and Whitlock, the numerical experiments on the newly discovered neutron by Enrico Fermi in the 1930s would now be classified as Monte Carlo calculations and the bringing together of Von Neumann, Fermi, Ulam, and Metropolis, as part of the Second World War effort, was a key step in the advancement of Monte Carlo methods to solve a variety of problems in the late 1940s and early 1950s (e.g., problems in statistical mechanics, radiation transport, and economic modeling).

As discussed in Smith (1991), Morgan and Henrion (1990), Kalos and Whitlock (1986), and Rice (1988), the Monte Carlo method can be used in the numerical solution of purely mathematical problems (e.g., determination of $\pi$ ), as well as in the simulation of both dynamic and equilibrium systems. Smith (1991) indicates that the Monte Carlo method has evolved into one of the most important tools in the numerical simulation of physical systems employed in nuclear science and technology with the main advantage that many problems that are virtually intractable by conventional deterministic simulation are relatively straightforward using Monte Carlo methods. Smith (1991) and Kalos and Whitlock (1986) discuss the distinction that is sometimes made between the uses of a Monte Carlo approach:

- in the simulation of stochastic processes (sometimes referred to as Monte Carlo simulation)

- for the solution of nonprobabilistic problems by probabilistic methods (sometimes referred to as just Monte Carlo methods).

Uncertainty propagation using the Monte Carlo simulation approach with a deterministic model/code (e.g., SGM) and Simple Random Sampling (SRS) is straightforward and, as discussed in Liebetrau and Doctor (1987), can be viewed as a simple sampling problem. Since the $n$-dimensional vector of inputs, 
$\boldsymbol{z}=\left(z_{1}, z_{2}, z_{3}, \cdots z_{n}\right)$, to the deterministic computer simulation model, $f(z)$, assumed to describe the phenomena and thus the result(s), $y=f(z)$, of interest are uncertain, then the result(s) is(are) also uncertain. The multiple realizations of the $n$-dimensional vector of deterministic model inputs that can be characterized by an appropriate combination of univariate and/or multivariate pdfs represent a population of possible model inputs, $\boldsymbol{Z}$, and each of these realizations, $k$, of that population of model inputs, $z^{k}=\left(z_{1}^{k}\right.$, $\left.z_{2}{ }^{k}, z_{3}{ }^{k}, \cdots z_{n}{ }^{k}\right)$, leads to a specific model result(s), $y^{k}=f\left(z^{k}\right)$, which will be an element of the population of model results, $\boldsymbol{Y}$, which, depending on the nature of the result(s) of interest, can be characterized by either a univariate or multivariate pdf. Monte Carlo simulation, as discussed in Liebetrau and Doctor (1987), is thus conducted using independent realizations of the n-dimensional vector of uncertain model inputs, $z^{k}$, constructed with standard statistical techniques using the pdfs assigned to this population of uncertain inputs, $\boldsymbol{Z}$. Each of these uncertain input samples, $\boldsymbol{z}^{k}$, is used as the input to the deterministic model to create a sample, $y^{k}$, of the population of uncertain model results of interest, $\boldsymbol{Y}$. With the ability to create as many samples, $y^{k}$, of the result population of interest as needed, then standard techniques can be used to estimate the various statistical properties (e.g., expected values, medians, ranges, variances, percentiles, and the cumulative distribution function) of the, $m$, samples of the result population. For example, if the model only produces one result of interest, then the expected value (i.e., the mean) of the uncertain result, $\bar{y}$, and the variance, $\sigma^{2}$, can be estimated by Equations 3.18 and 3.19 from $m$ samples of the result population as follows:

$$
\begin{gathered}
\bar{y}=1 / m \sum_{k=1}^{m} y_{k} \\
\sigma^{2}=[1 /(m-1)] \sum_{k=1}^{m}\left(y_{k}-\bar{y}\right)^{2}
\end{gathered}
$$

The big question with Monte Carlo simulation is how many samples are needed to obtain the desired precision in these estimates and how does the number of required samples change with model complexity as measured by the number of uncertain inputs, $n$. Morgan and Henrion (1990) discuss both of these issues and indicate the following:

- Precision - Monte Carlo with SRS has an advantage over other Monte Carlo methods in that the precision of the output distribution can be estimated directly from the sample, $m$, of the results directly or in an indirect manner through a bootstrap procedure. An example of the bootstrap approach discussed in Morgan and Henrion (1990) is discussed below that uses the standard error of the estimate, $\frac{\sigma}{\sqrt{m}}$, to determine the number of Monte Carlo runs needed to estimate the mean value to some desired level of precision and confidence. The same approach can also be used to select the sample size for estimating the median, other fractiles, and the precision of the CDF to a desired level of precision and confidence (Morgan and Henrion 1990).

- Complexity - As already discussed, Monte Carlo simulation is analogous to simple random sampling of a single output distribution, with the simulation model allowing as many samples, $m$, of the output 
distribution as might be needed to be created. Since a given output has a unique output distribution, the accuracy of the estimates for the parameters that describe this output distribution do not depend on the complexity of the model and its number, $n$, of uncertain inputs, but only on the number of samples, $m$, of this output distribution. As a result, Morgan and Henrion (1990) indicate there is no need to change the number of Monte Carlo runs as the model is made more complex, unless the additional complexity substantially increases the variance of the output distribution as discussed below.

The bootstrap procedure for estimating the number of samples of model output, $m$, required to determine that the estimate of the mean value, $\bar{y}$, of this output is within some interval, $w$, units wide to some desired level of confidence, $\alpha$, is based on the central limit theorem (Morgan and Herrion 1990). The theorem indicates that the distribution that describes the uncertainty in the estimated mean is a normal distribution with mean value $(\bar{y})$ and a standard deviation equal to the standard error of the estimate, $\frac{\sigma}{\sqrt{m}}$. Because a normal distribution represents the uncertainty in the estimate of the mean, the fact that as $m$ gets large, the uncertainty in the estimate of the mean $(\bar{y})$ approaches zero can be used to find an $m$ that will ensure that the estimate of the mean is within an interval of width, $w$. Let the desired level of confidence, $\alpha$, (e.g., 95\%) determine, $c$, the one-half width of the interval specified in terms of number of standard deviations required to achieve the desired confidence level (e.g., for $95 \%$ confidence $c=\sim 1.96$, also see Figure 3.8), then, $m$, should be selected such that the interval width, $w$, which specifies the desired precision is as follows:

$$
2 c \frac{\sigma}{\sqrt{m}}<w
$$

or

$$
m>(2 c \sigma / w)^{2}
$$

Figure 3.30 illustrates three CDFs constructed from various numbers $(10,396$, and 10,000) of random samples of a simulated output developed by taking random samples from a normal distribution with mean $(\bar{y}) 250$ and variance of 50. The figure also illustrates the parameters, formula, and results of bootstrap procedure for determining the number of samples of model output, $m=\sim 390$, required to resolve the estimate of the mean $(\bar{y})$ within an interval $w=10$ units wide with a 95\% level of confidence, $c=\sim 1.96$. In this procedure, 10 Monte Carlo simulations are used to estimate that $\sim 396$ samples were needed and then the sample of 396 was used to verify this estimate ( $\sim 390$ were indicated).

To further illustrate the use of the bootstrap approach, Figure 3.31 shows the number of Monte Carlo samples needed to achieve a desired level of precision in estimates of the mean for two different distributions:

1. The first problem (the one just discussed and illustrated in Figure 3.30) consists of a distribution with mean 250 and standard deviation of 50 (i.e., $20 \%$ of mean) 


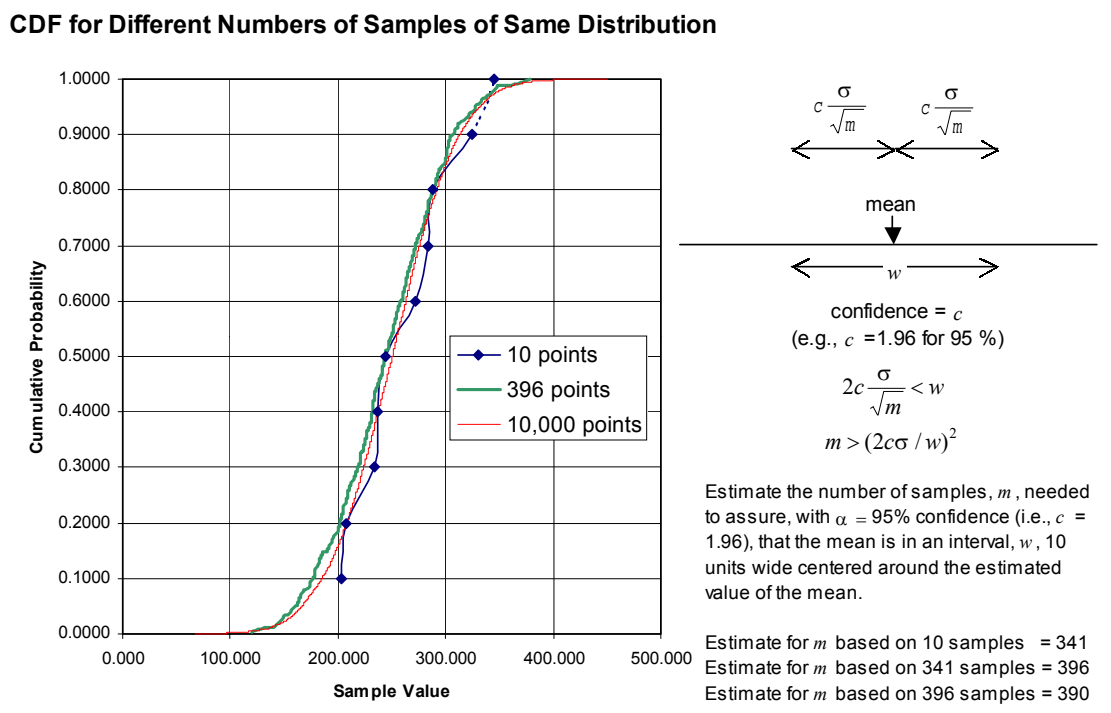

Figure 3.30. Three CDFs Constructed from Various Numbers (10, 396, and 10,000) of Random Samples of a Simulated Output Developed from a Normal Distribution to Illustrate the Bootstrap Procedure for Determining the Number of Monte Carlo Model Samples Needed to Achieve a Desired Level of Precision in the Estimate of the Mean $(\bar{y})$.

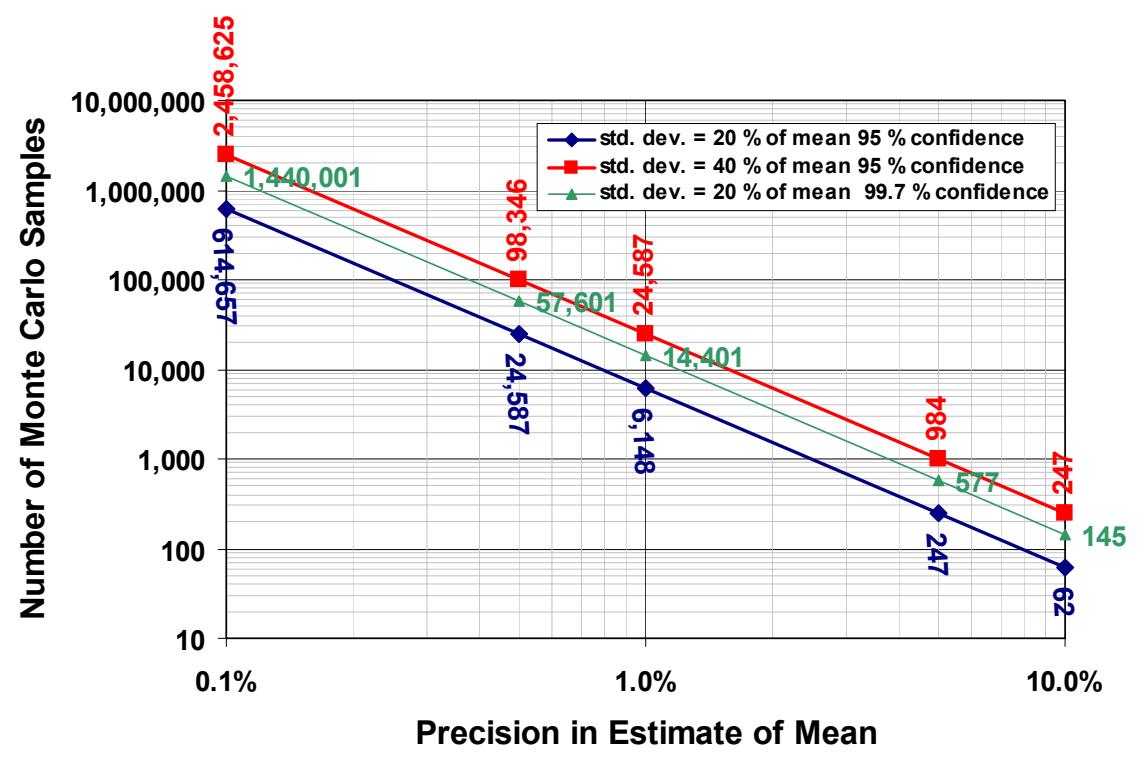

Figure 3.31. Number of Monte Carlo Samples to Achieve a Desired Level of Precision in Estimate of the Mean for Two Different Distributions. Problem 1 (Figure 3.30) - Distribution with Mean 250, Standard Deviation of 50 (i.e., 20\% of mean. Problem 2 - Distribution with Mean 250, Standard Deviation of 100 (i.e., 40\% of mean). Bottom line and middle line show number of samples to achieve desired precision for Problem 1 with 95 and 99.7\% confidence, respectively. Top line shows samples to achieve desired precision for Problem 2 with $95 \%$ confidence. 
2. The second problem consists of a distribution with the same mean of 250 , but with a standard deviation of 100 (i.e., $40 \%$ of mean and twice as large as the first problem).

Figure 3.31 illustrates the inverse straight line relationship on a log-log plot, $m^{-1 / 2}$, implied by Equation 3.21 between the number of Monte Carlo samples required and the level of precision desired in the estimate of the mean. Note that for Problem 1 only 62 samples are needed to determine with 95\% confidence that the mean lies in an interval that is within $10 \%$ of the mean [i.e., $(\bar{y}-12.5, \bar{y}+12.5)$ or $(237.5,262.5)]$ while more than double that number, 145 , are required to determine that the mean lies in this same interval with $99.7 \%$ confidence. Additionally, the figure indicates that 614,457 samples (more than 10,000 times the 62 required for $10 \%$ precision) are needed to determine with $95 \%$ confidence that the mean lies in an interval that is within $0.1 \%$ of the mean [i.e., $(\bar{y}-0.125, \bar{y}+0.125)$ or $(249.875$, 250.125)]. The graph and numbers for Problem 2 illustrate how the number of required samples is affected by the standard deviation of the output distribution. As indicated by Equation 3.21, doubling the standard deviation results in the need for 4 times as many samples to achieve the same precision with the same level of confidence [i.e., 247 samples are required to determine with $95 \%$ confidence that the mean lies in the $10 \%$ precision interval $(\bar{y}-12.5, \bar{y}+12.5)$ or $(237.5,262.5)]$.

As illustrated by the simple examples discussed above and illustrated in Figures 3.30 and 3.31, the number of runs required to obtain very precise estimates at high levels of confidence is staggering compared to the number of runs required for less precise estimates at lower levels of confidence. Compare

1. $>1.4$ million Monte Carlo runs, for the simple example presented above, if it is decided that the decision maker needs to be $99.7 \%$ confident that the estimate of the mean value is within $\pm 0.1 \%$ of the estimate

2. 62 Monte Carlo runs required if the decision maker needs to be only $95 \%$ confident that the estimate of the mean value is within $\pm 10 \%$ of the estimate.

Morgan and Henrion (1990) indicate that the need for a given level of precision and confidence in the uncertain outputs must be carefully examined relative to the planned use of the estimate, as well as the uncertainty in the input parameter distributions of the dominant uncertain parameters that give rise to this estimate.

Monte Carlo simulation implemented with the SRS, as discussed, requires each $n$-dimensional vector of uncertain inputs, $\boldsymbol{z}=\left(z_{1}, z_{2}, z_{3}, \cdots z_{n}\right)$, to the deterministic computer simulation model, $f(z)$, to be developed by randomly sampling from each of the probability distributions describing each of the $n$ uncertain inputs ( $n$-space). SRS, however, is susceptible to the creation of "clusters" and gaps as illustrated in Figure 3.32(a) for a two-dimensional uncertain input vector, $\boldsymbol{z}=\left(z_{1}, z_{2}\right)$ (after ANSYS 2001). As a result, only certain regions of the total multivariate space are well represented. To reduce this effect, sampling techniques that produce more systematic or stratified sampling of the multivariate space are often used because these techniques minimize the variance in the estimates of outputs of interest for the same number of samples (Morgan and Henrion 1990). The methods used to improve on SRS include 
(a)

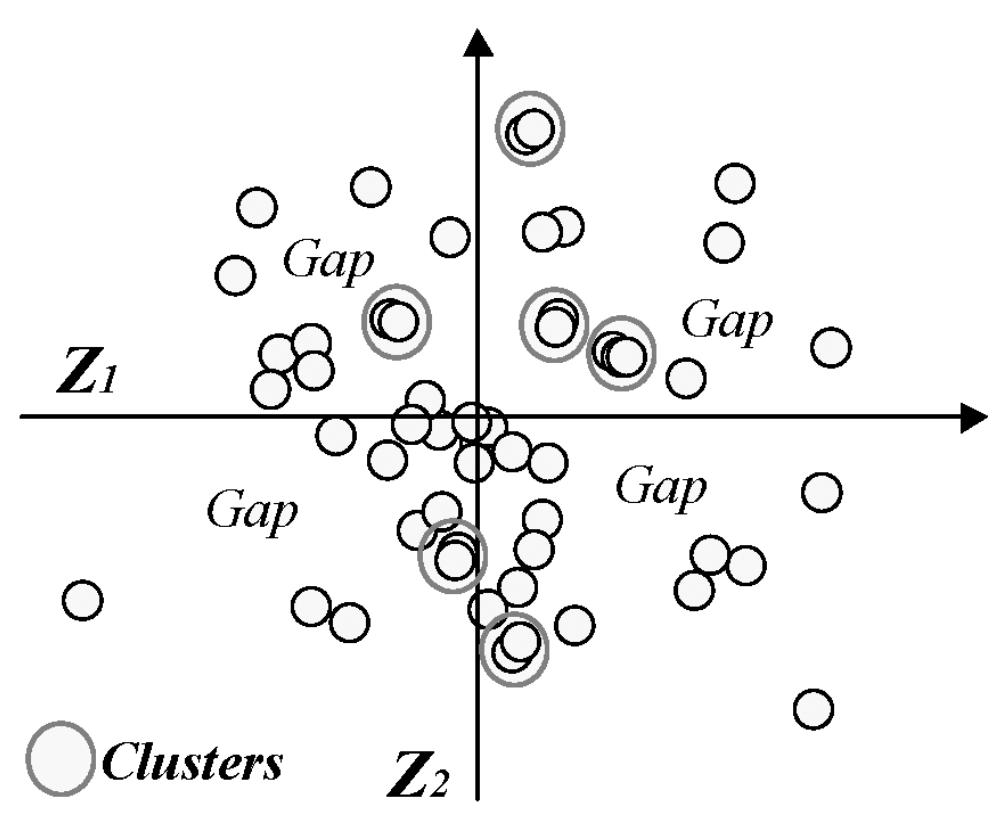

(b)
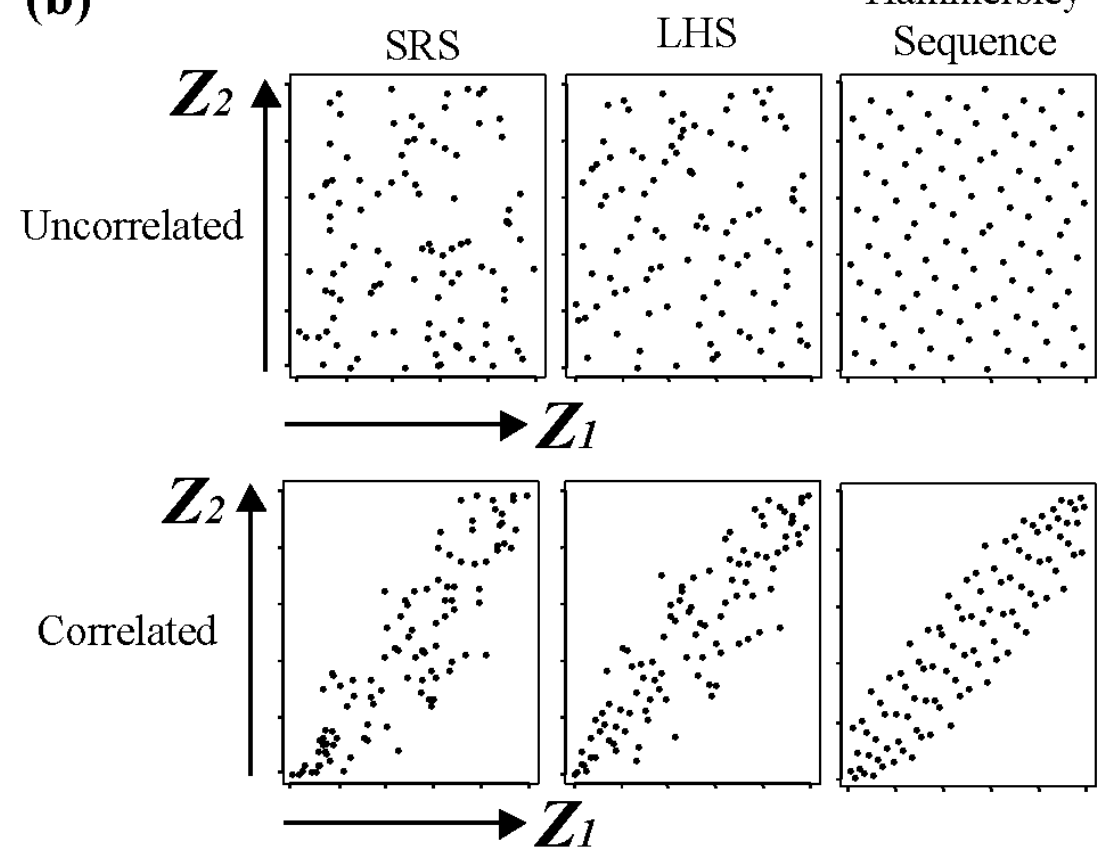

Figure 3.32. Sampling, (a) Gaps and Clusters Associated with SRS for a Two-Dimensional Uncertain Input Vector, $\boldsymbol{z}=\left(z_{1}, z_{2}\right)$ (after ANSYS 2001), (b) Comparison of Sampling Uniformity for a Uncorrelated and Highly Correlated Two-Dimensional Uncertain Input Vector, $\boldsymbol{z}=\left(z_{1}, z_{2}\right)$, for Three Different Sampling Approaches SRS, Latin Hypercube Sampling (LHS), and a Hammersley Sequence (Wang et al. in press). 
stratified sampling (e.g., Latin Hypercube Sampling, McKay et al. 1979), importance sampling, and the use of quasi-random low discrepancy sequences (e.g., Sobol et al. as discussed in Robinson and Atcitty 1999; Press et al. 1992). Importance sampling, through the use of prior knowledge of the character of the output, attempts to concentrate samples in the areas where they are more effective (Morgan and Henrion 1990), while stratified sampling tries to distribute samples more evenly by subdividing the domain into subregions such as grids (e.g., in the case of LHS, each component of the vector of $n$ uncertain inputs is divided over its range into $m$ equal strata with marginal probability of $1 / m$ and a sample is selected at random within each strata). The quasi-random sequences (e.g., Hammersley) used in the Quasi Monte Carlo (QMC) methods are designed to have low discrepancy where discrepancy is a measure of the uniformity of the distribution of finite point sets (Niederreiter 1992).

Figure 3.32(b) (after Wang et al. in press) illustrates the effect of sampling uniformity for both an uncorrelated and a highly correlated two-dimensional uncertain input vector, $\boldsymbol{z}=\left(z_{1}, z_{2}\right)$, for three different sampling approaches that include

\section{- SRS}

- Latin Hypercube Sampling, a stratified approach

- a Hammersley Sequence, a quasi-random low discrepancy sequence.

The motivation for the various methods (other than SRS) is to find point sets of $m$ points in the $n$-dimensional space $\left(z_{1}, z_{2}, z_{3}, \ldots z_{n}\right)$ that yield smaller estimation errors for the output results of interest than would be obtained with SRS. The goal is to reduce the estimation error faster than SRS, which decreases at a rate on the order of the inverse square root of $m$ \{i.e., $\boldsymbol{O}\left(\mathrm{m}^{-1 / 2}\right)$ \} and yet be able to slowly increase the number of samples until the desired estimation accuracy is achieved in the desired output estimates. Press et al. (1992) indicate that for Monte Carlo integration QMC methods (e.g., using Halton's sequence) provide this desired approach as points can be added iteratively and the estimation error decreases as $\boldsymbol{O}\left(\{\ln (m)\}^{k} m^{-1}\right)$. Recently Robinson and Atcitty (1999) compared the efficiency of QMC methods to LHS when applied to problems typically found in the reliability and uncertainty analysis field. They found that for their set of problems QMC methods generally provided estimates with lower average error and narrower error bounds than for identical sample sizes using LHS. In addition they concluded that certain QMC methods provide the additional advantage over LHS in that they can be applied in an iterative fashion. Although LHS is the method that we currently plan to employ in Monte Carlo simulation, the development of these QMC methods will need to be followed and assessed to determine their applicability to uncertainty estimation for the Hanford SGM ACMs.

\subsection{1 Latin Hypercube Sampling}

Latin Hypercube Sampling provides a sampling method that appears random but reproduces the input distribution with much greater efficiency than SRS through a technique known as stratified sampling without replacement. It breaks the probability distribution of each of the inputs into $n$ subintervals of 
equal probability, where $\mathrm{n}$ is the number of iterations to be performed with the model. One random sample is drawn from each subinterval thus providing for an equal-chance representation of all the portions of the distribution and thus a predictably uniform sampling of the distribution.

Latin Hypercube Sampling has been used extensively for uncertainty and probabilistic risk assessments based on Monte Carlo methods ever since the original LHS software packages were developed at Sandia National Laboratories by Ronald L. Iman, Michael J. Shortencarier, J. M. Davenport, and D. K. Ziegler during the late 1970s (documented in Iman et al. [1980]) and early 1980s (Iman and Shortencarier 1984) after the defining paper on the LHS approach by McKay et al. 1979. The users manual for the most recent update of the Sandia LHS software (Wyss and Jorgensen [1998]).

Press et al. (1992) describe LHS as a useful approach when you must sample a $n$-dimensional space very sparsely and therefore LHS is an important methodology for use in uncertainty assessments when the model computational times are large. LHS estimates have the desirable property of being unbiased estimators as discussed in McKay et al. (1979) but LHS like the other sampling approaches discussed above, except for SRS, provides no way to accurately determine the number of samples needed to achieve a specified level of precision in the desired estimate. However, Stein (1987) was able to show that LHS estimates of variance are asymptotically lower than with SRS. Charnes (2000) indicates that Avramidis and Wilson (1995) have demonstrated in their applications of LHS to stochastic activity networks that mean square errors are $40 \%$ less for LHS compared to SRS for the same number of samples. Morgan and Henrion (1990) indicate that even though LHS is sometimes much better, but never worse than SRS (Stein, 1987), there are still times when SRS may be needed because of the drawbacks discussed in the next paragraph.

Two basic drawbacks related to LHS, as well as most other methods other than SRS, are

1. it is not possible to derive accurate confidence limits as the SRS methods and theory (i.e., central limit theorem) for estimation of confidence limits discussed above do not apply to LHS and as a result underestimate the precision of LHS (Stein 1987)

2. more samples cannot be added incrementally to obtain the desired precision and as a result, if the original estimate of the number of samples required for LHS is too low and too few samples are drawn, then none of these samples (i.e., the expensive computer model runs) can be reused in development of a more precise estimate.

There are actually other drawbacks when using LHS for problems requiring very large numbers of samples (e.g., Monte Carlo integration). They are related to memory requirements, which become restrictive because all samples must be generated and stored, and related to efficiency, because the efficiency of the sample generation process is low compared to other methods. However, these drawbacks are not expected to be a problem for our uncertainty analyses for the Hanford SGM ACMs. 
Standard LHS generation (Wyss and Jorgensen 1998) in an uncertain parameter space of dimension $k$ is relatively straightforward and consists of the following three steps:

Step one - This is the stratification step. It involves using the probability distribution and range for each of the components, $z_{i}$, of the $n$-dimensional vector of uncertain inputs, $\boldsymbol{z}=\left(z_{1}, z_{2}, z_{3}, \cdots z_{n}\right)$ to create $m$ subintervals of equal probability $(1 / m)$, where $m$ is the number of model runs to be performed. Note that $m$ must be greater than $n$ in order to be able to easily deal with correlations between variables (Iman and Helton 1985).

Step two - This is a random sampling step in standard LHS. In this step, each of the $m$ subintervals of each uncertain input, $z_{i}$, is sampled according to the range of the subinterval and the probability distribution describing the uncertain input $z_{i}$. The $m$ samples representing all the strata for each uncertain input, $z_{i}$, are each placed in a separate bin for use in step three (i.e., $n$ bins, one for each component of $\boldsymbol{z}$ ).

Step three - This is a random sampling step without replacement. The $n$ bins containing the $m$ samples from each strata of each of the uncertain inputs, $z_{i}$, are lined up in order from bin $z_{1}$ to bin $z_{n}$. Each of the $m$ uncertain input vectors, $z_{j}$, is then constructed one at a time until all $m$ samples are constructed. Construction of each uncertain input vector, $\boldsymbol{z}_{j}$, consists of taking one random sample from each of the $n$ bins. The "sampling without replacement" refers to the fact that once the sample representing a given strata of a given component $z_{i}$ has been chosen, then that particular strata for that component will not be sampled again because it has been removed from the bin.

Morgan and Henrion (1990) indicate that a modification of the standard LHS they describe as midpoint LHS performs considerably better (e.g., sample mean and variance will often be exact) than standard LHS except in the rare circumstance when the system model exhibits periodicity with respect to an input induced by the stratification into $m$ subintervals of equal probability (a condition unlikely in our models). This modified or midpoint LHS involves replacing the random sampling of the $m$ subintervals of equal probability in step two above with a simple prescriptive step in which either the mean or median of the subinterval is used as the sample for the strata. Keramat and Kielbasa (1999) recently investigated the theoretical and practical aspects of this method and concluded that it provided for faster generation and it also provided a more precise estimate (smaller estimation variance) than standard LHS, which randomly samples each subinterval.

Another characteristic that LHS (or any other sampling method) must be able to deal with is both ensuring lack of correlation when the $n$ different inputs are independent and inducing the proper correlation relationship between correlated inputs. Morgan and Henrion (1990) suggest that the best approach for dealing with known correlations, when possible, is to build the known correlation or dependence directly into the structure of the model. Morgan and Henrion also indicate that while it is easy to generate correlated normally distributed random variables, the situation becomes "trickier" with other kinds of marginal distributions and that it is generally not possible to generate two random variables, each with an arbitrary marginal distribution and specified Spearman correlation. To ensure that the correlation (or lack of correlation) is obtained between the variables in LHS samples, the random pairing in step three of the 
simple description of LHS above must be replaced by the restricted pairing technique introduced by Iman and Conover (1982a) and implemented in the Sandia LHS program (Iman and Shortencarier 1984). The restricted pairing algorithm is needed even if uncorrelated samples are desired because as discussed and illustrated in Iman and Helton (1985), the random pairing step described above can produce undesired pairwise correlations. Dandekar et al. (2001) in their paper on the creation of multivariate synthetic micro data using LHS with the restricted pairing algorithm of Iman and Conover (1982a) discuss an additional step (developed by Dandekar) involving iterative refinement of the rank correlation matrix to reduce the gap between the desired rank correlations as computed for the actual data and the rank correlations achieved with the synthetic data. As discussed in Dandekar et al. (2001), Morgan and Henrion (1990), and Iman and Conover (1982a), the restricted pairing approach is able to generate samples with only a specified rank correlation structure. Dandekar et al. (2001) indicates that, in general, rank correlation provides a more useful summary of the relatedness of two non-normal variables that are monotonically but not linearly related and it is better suited for heavily skewed distributions, for which the Pearson correlation can be dominated by a small percentage of the data.

Iman and Helton (1985) provide general guidance on the number of samples, $m$, for sampling an $n$-dimensional space with LHS. The restricting pairing technique requires $m>n$ in order to avoid applying the technique in a piecewise fashion. Iman and Helton also indicate that while the choice of $m$ depends on a number of considerations, which includes the number of input variables, $n$, and the cost of making a single computer run (a dominant consideration), their experience shows that good results can be obtained with $m>(4 / 3) n$. 


\subsection{Conceptual Model of Aquifer System and Uncertainties}

The conceptual model (Section 3.12) is a working description of the characteristics and processes needed to describe the dynamics of the physical and chemical changes in the hydrogeologic system that is consistent with available data and understanding. It provides the means to consolidate our interpretations of the geologic, hydraulic, transport, chemical, and contaminant data to form a set of features, events, and processes that describe groundwater flow and contaminant transport. Assumptions are made in defining the conceptual model where information is lacking. However, assumptions must be consistent with the available data and understanding.

The term "conceptual model" is sometimes used to describe the way groundwater flow is implemented in a numerical model. For example, groundwater-river interactions may be implemented in a model using a constant-head boundary when only slowly acting, long-term interactions are being evaluated, but the actual expected groundwater-river interactions would be described in the "conceptual model" description. The simplification to constant head boundary would be part of the model specific implementation description. In this report, therefore, the term "implementation model" is used to describe a specific numerical model implementation, while conceptual model is reserved for the description of the actual groundwater flow and transport system as best we understand it based on available data and knowledge. Assumptions, parameters, and even processes in an implementation model may conflict with the available information regarding local details. This is part of the spatial and temporal aggregation process (Figure 3.11) associated with choosing the appropriate simplifications needed to model complex systems with uncertain model structure as was discussed in Sections 3.1.2 and 3.2.2. The simplifications are also consistent with the principle of parsimony discussed in Hill's 1998 methods and guidelines for effective model calibration which involves selecting the model with the greatest simplifications that adequately represents the processes affecting the phenomena of interest. These simplification decisions need to be evaluated to determine that the effect on model results is acceptable and thoroughly documented.

Uncertainties in the conceptual model arise from a lack of information concerning features and events, or a lack of understanding of the processes controlling groundwater flow and transport. The current understanding of the Hanford Site aquifer system is presented in this chapter and uncertainties in various components of the conceptual model are described. The SGM simulates groundwater flow and transport within the local groundwater flow system because it forms the uppermost aquifer system at the Site and is most affected by Site operations. However, the local groundwater flow system interacts with the underlying regional aquifers. Therefore, both the regional and local flow systems are described below.

\subsection{Regional Groundwater Flow System}

The Hanford Site lies within the Columbia Plateau, an approximately 70,000 square mile area including portions of Washington, Oregon, and Idaho (Figure 4.1). The Columbia Plateau is an intermountain basin bounded by the Cascade Mountains on the west, the Okanogan Highlands on the 


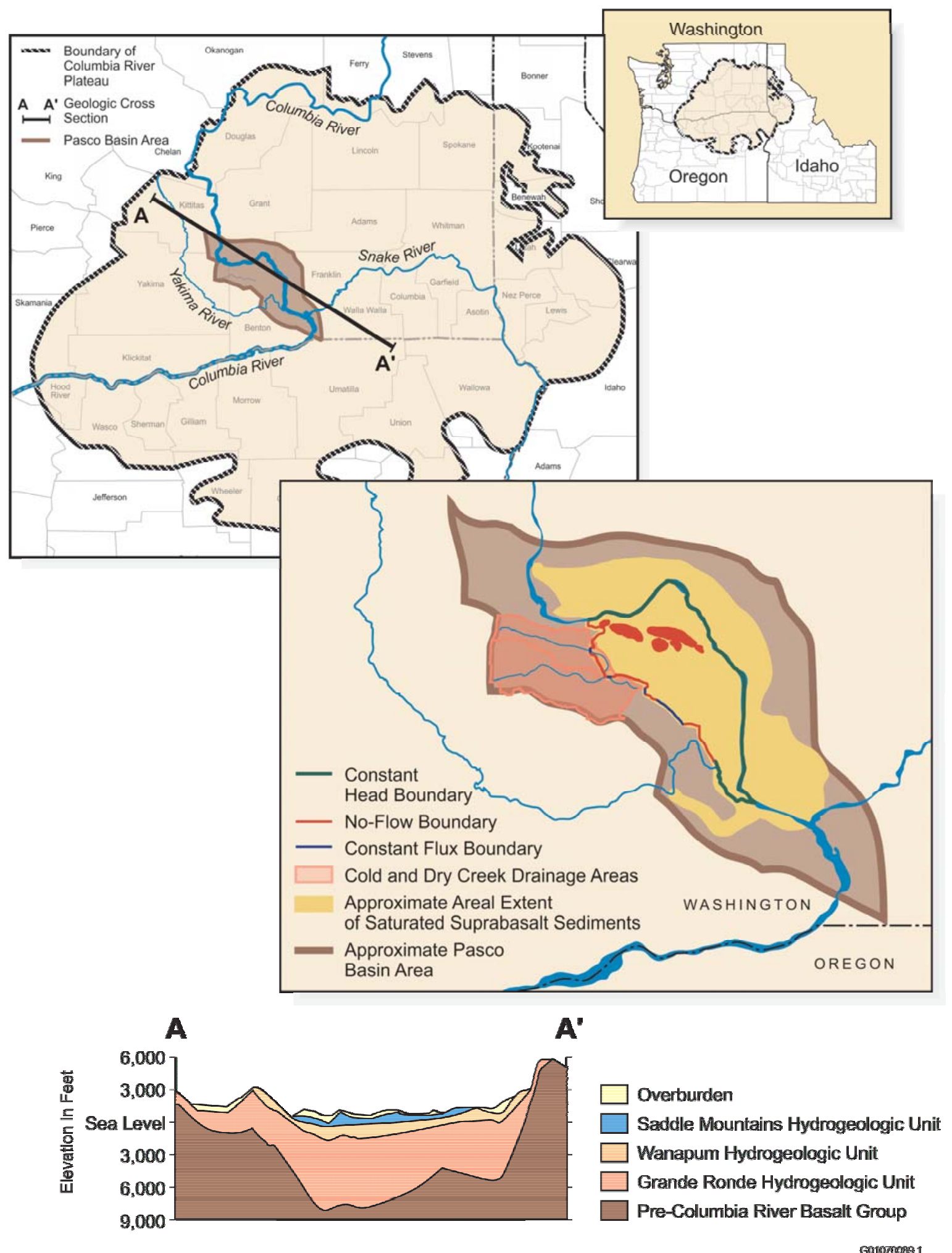

Figure 4.1. Extent and Cross Section of the Regional Columbia Plateau Aquifer System. 
north, the Rocky Mountains on the east, and the Blue Mountains on the south. Annual precipitation ranges from less than $20 \mathrm{~cm}$ in the central low-lying portion of the plateau to more than $60 \mathrm{~cm}$ on the elevated fringes. The Columbia River Basalt Group, a relatively thick sequence of basalt flows, underlies most of the Columbia Plateau region. The basalts have been exposed and eroded in some areas and are covered by thick sediments in other areas.

\subsubsection{Regional Geologic Setting}

The Columbia River Basalt Group was formed by a sequence of lava flows that periodically erupted from north-northwest trending fissures or linear vent systems in north central and northeastern Oregon, eastern Washington, and western Idaho (Swanson et al. 1979; Waters 1961). Isotopic age determinations indicate that flows of the Columbia River Basalt Group were erupted from approximately 17 to 6 million years ago, with more than $98 \%$ by volume being erupted between 17 and 14.5 million years ago. The thickness of the Columbia River Basalt Group reaches a maximum of about $3000 \mathrm{~m}$ in the vicinity of the Hanford Site and individual flows range from a few centimeters to about $100 \mathrm{~m}$ in thickness (DOE 1988).

The regional river system eroded the basalt and deposited sediments across the basalt surfaces between eruptions. Rubble zones between the basalt flows and sediments, which were deposited as interbeds between basalt eruptions, are frequently water-bearing zones that form the basalt-confined aquifer system. Additional information on the Columbia River Basalt Group and the basalt-confined aquifer system is available in DOE (1988).

Deformation of the basalts has resulted in the formation of structural basins, which have accumulated relatively thick sequences of fluvial and lacustrine sediments. Figure 4.1 shows the regional geologic structure, extent of the Columbia River Basalts, and locations of the sediment-filled basins. Anticlines within the basalt structure form ridges that often outcrop above the overlying sediments and form impediments to groundwater flow within the sedimentary aquifer systems.

\subsubsection{Regional Aquifers}

The regional aquifer system is composed of saturated transmissive units within the Columbia River Basalt Group. The brecciated tops of individual basalt flows and sedimentary interbeds between basalt flows form most of these transmissive units. However, interbeds of fine-grained material can also form aquitards. The basalts and associated interbeds form a confined aquifer system that extends from western Idaho through eastern Washington and northeastern Oregon (Figure 4.1). Important hydrostratigraphic units within the regional groundwater system include, from deepest to shallowest: the Grande Ronde unit, the Wanapum unit, and the Saddle Mountains unit. These units are named after the corresponding basalt formation and include associated sedimentary interbeds. Several aquifers are found within each of these units. Sedimentary and metamorphic rocks underlying the basalts generally have a much lower permeability and are considered to be the base of the regional aquifer system.

Recharge to the regional basalt groundwater system occurs primarily from infiltration of precipitation in elevated regions at the margins of the Columbia Plateau. Some recharge to the basalt aquifers also occurs from downward movement of irrigation water withdrawn from the Columbia River and its 
tributaries. Groundwater in the regional system eventually discharges to the Columbia River or its tributaries, which are the major regional drainage features for both surface water and groundwater. Significant amounts of water are withdrawn from water supply wells tapping the basalt aquifers in some areas.

Groundwater has accumulated within sediments deposited on top of the basalt bedrock, particularly within structural basins, to form local aquifer systems. These local aquifers are isolated from the underlying regional system to a varying degree depending on the nature of the uppermost confining basalt flows.

\subsection{Local Groundwater Flow System}

The Hanford Site lies within the Pasco Basin, a structural depression within the Columbia Plateau region that has accumulated a relatively thick sequence of fluvial, lacustrine, and glaciofluvial sediments above the basalt bedrock. The cross section in Figure 4.2 shows the basalt formations, structural features, and sediments accumulated within the Pasco Basin (Figure 4.1). The uppermost aquifer beneath the Hanford Site lies within these sediments. This aquifer provides a pathway for transport of contaminants released from past, present, and future Site activities. This uppermost saturated zone is termed the unconfined aquifer system, although locally confined conditions may exist in certain areas.

The SGM domain includes the unconfined aquifer system within the Pasco Basin that lies west and south of the Columbia River and east and north of the Yakima River. The unconfined aquifer also extends beneath these rivers and exists on both sides of the rivers. However, the SGM implementation (i.e., a no-flow boundary) assumes no flow communication under the rivers between the unconfined systems on opposite sides because the role of the river as a regional sink (i.e., a specified head boundary

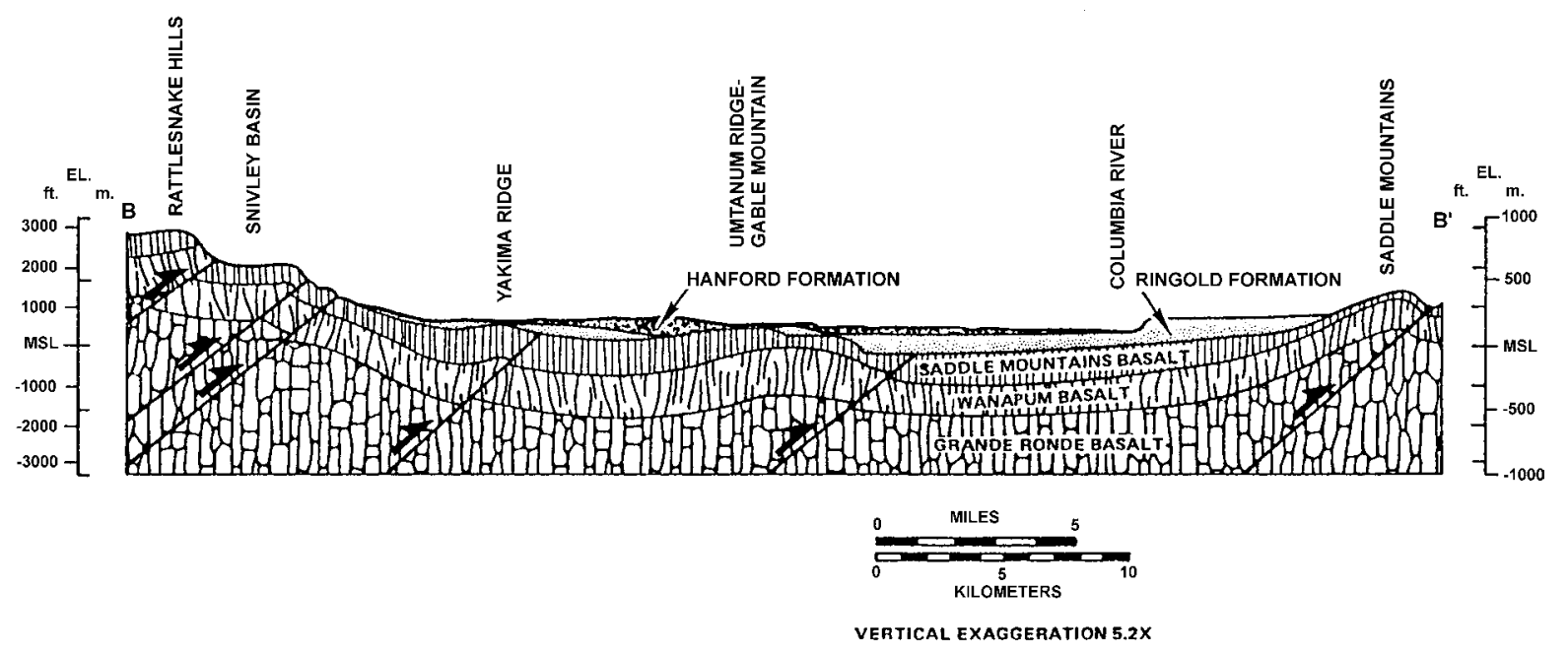

Figure 4.2. Schematic Cross Section of the Pasco Basin. 
implementation) is assumed to act to prevent all but minor flow communication between these parts of the same unconfined system. Additional information on the unconfined aquifer system is provided in DOE (1988) and Wurstner et al. (1995).

\subsubsection{Local Geologic Setting}

Principal sedimentary units that overlie the basalt at the Hanford Site include, in ascending order, the Ringold Formation and the Hanford formation (informal name). Less extensive sedimentary units include the Plio-Pleistocene, Early Palouse Soil, and pre-Missoula Gravel units, which lie stratigraphically between the Ringold and Hanford formations. A thin layer of recent aeolian and fluvial deposits is also present at the surface over much of the Site. The geologic units are described in the following sections.

\subsubsection{Ringold Formation}

After the last major eruption of basalt, the fluvial-lacustrine Ringold Formation was deposited in generally east-west trending valleys by the ancestral Columbia River and its tributaries. Following uplift of the basalts and overlying sediments, the Columbia River began to erode, rather than deposit, sediments in the Pasco Basin. The upper portion of the Ringold Formation was eroded from much of the Hanford Site and a caliche layer, part of the Plio-Pleistocene unit, developed in places on the eroded surface of the Ringold Formation. While exposures of the Ringold Formation are limited to the White Bluffs within the central Pasco Basin and to the Smyrna and Taunton Benches north of the Pasco Basin, extensive data on the Ringold Formation are available from boreholes. The White Bluffs outcrop on the east bank of the Columbia River shows a relatively thick section of the Ringold.

Fluvial deposits of the Ringold Formation have been historically grouped into three main facies associations based on proximity to the ancestral river channels. Gravel and associated sand and silt represent a migrating channel deposit of the major river systems and are generally confined to the central portion of the Pasco Basin. Overbank sand, silt, and clay deposits reflect occasional deposition and flooding beyond the influence of the main river channels, and are generally found along the margins of the Pasco Basin. Fanglomerates, composed of mostly angular basaltic debris derived from side-stream alluvium shed off bedrock ridges, occur locally around the extreme margins of the basin. Over time, the main river channels moved back and forth across the basin, causing a shift in location of the various facies. Periodically, the river channels were blocked, causing lakes to develop in which relatively thick layers of laminated mud with minor sand were deposited. In the literature prior to 1990, the Ringold Formation was usually divided into five informal lithofacies units. In ascending order, they are the gravels and sands of the basal Ringold unit, the clays and silts of the lower Ringold unit, the sandy gravel of the middle Ringold unit, the silts and sands of the upper Ringold unit, and the basaltic detritus of the fanglomerate unit (Newcomb et al. 1972; Tallman et al. 1979; Bjornstad 1985; DOE 1988).

Lindsey (1995) reevaluated the stratigraphy of the Ringold Formation across the Hanford Site and described it on the basis of five major sediment facies associations. This was a summary and extension of earlier, more localized geologic studies presented in Lindsey and Gaylord (1990), Lindsey et al. (1991, 
1992, 1994), and Lindsey and Jaeger (1993). Sediment facies types (based on Miall 1977, 1978, 1985), their characteristics, and depositional environments as defined by Lindsey (1995) for the Ringold Formation are summarized in Table 4.1.

Based on the distribution of dominant facies, three informal members of the Ringold Formation were defined by Lindsey (1995). Ringold sediments on the Hanford Site are dominated by the lowermost "member of Wooded Island," which is divided into five gravel-dominated units (A, B, C, D, and E). These units are separated by mud-dominated over bank and lacustrian deposits, including the extensive units referred to as the lower Ringold mud. This member encompasses the basal, lower, and middle Ringold units defined by Tallman et al. (1979). The "member of Taylor Flat" is dominated by fluvial sands and over bank fines. This member, referred to as the upper Ringold unit by Tallman et al. (1979), has been removed from most of the central and southern portions of the Hanford Site by post-Ringold erosion. The "member of Savage Island," is dominated by lacustrian deposits and has been almost completely removed from the Hanford Site by erosion. The facies definitions and unit groupings of Lindsey (1995) form the basis for the hydrogeologic structure of the Ringold sediments used to construct the SGM.

\subsubsection{Plio-Pleistocene Unit}

Deposition of the Ringold Formation was followed by a period of regional incision in the late Pliocene to early Pleistocene. Within the Pasco Basin, this is reflected by the abrupt termination and eroded nature of the top of the Ringold Formation (Bjornstad 1985; Newcomb et al. 1972). Following incision, a well-developed soil formed on top of the eroded surface. This unit was eroded from most of the Hanford Site by the catastrophic glacial floods that deposited the Hanford formation. Remnants of the fine-grained facies of the Plio-Pleistocene unit are found in the vicinity of the $200 \mathrm{West}$ Area. These sediments are above the water table and do not affect groundwater flow. However, they may have a significant impact on flow and contaminant migration through the vadose zone.

\subsubsection{Hanford Formation and Pre-Missoula Gravel}

Aggregation of sediments resumed during the Quaternary period, following the period of late Pliocene to early-Pleistocene incision. In the Pasco Basin, the Quaternary record is dominated by postglacial cataclysmic flood deposits with lesser amounts of fluvial and eolian deposits lying below, between, and above flood deposits (Lindsey 1995).

The informally named Hanford formation and the similar pre-Missoula gravel facies of the PlioPleistocene unit, which underlie the Hanford formation in the central part of the Hanford Site, are coarser and less consolidated than the Ringold. The pre-Missoula gravels are coarse non-indurated fluvial deposits. They are lithologically similar to the Ringold Formation main-channel gravel facies, consisting of dominantly nonbasaltic clasts. The pre-Missoula gravels occur in a relatively small area from the Old

Hanford Townsite to the central portion of the Site. Distribution of the pre-Missoula gravels and the finegrained facies of the Plio-Pleistocene unit is shown in Figure 4.3. The distribution of these sediments below the 1999 water table is also shown. 


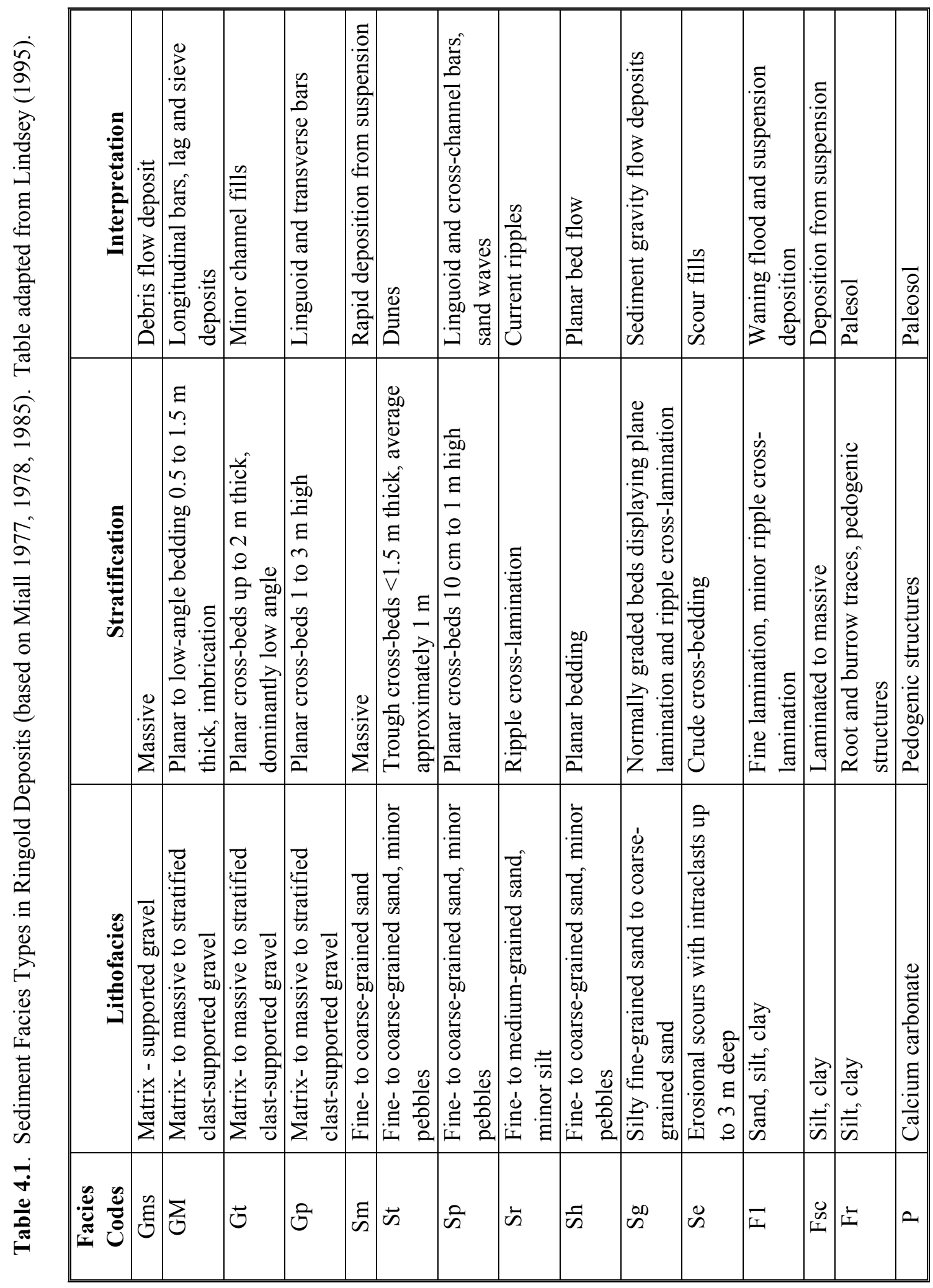




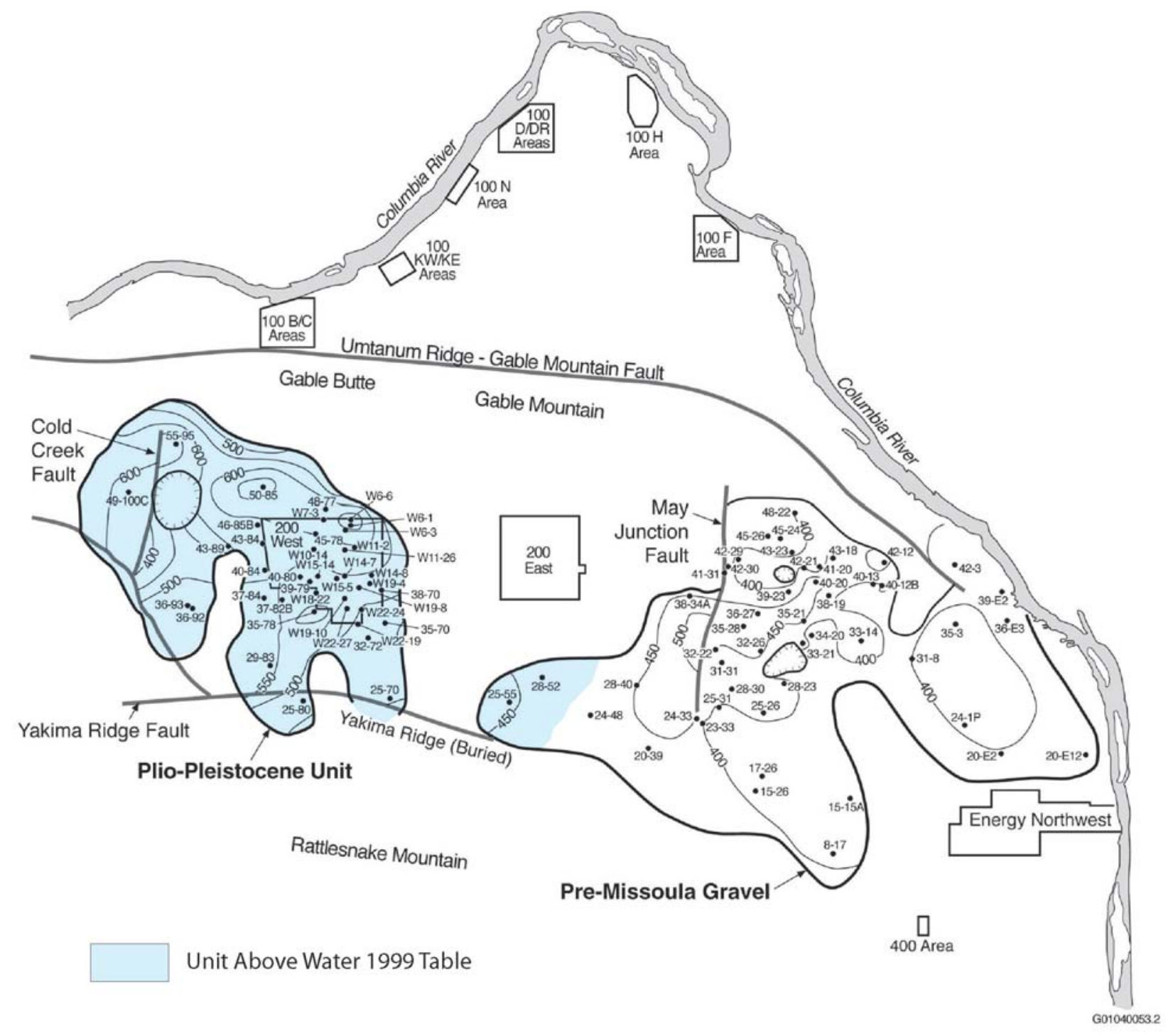

Figure 4.3. Distributions of the Pre-Missoula Gravel Facies and Fine-Grained Facies of the Plio-Pleistocene Unit on the Hanford Site (Lindsey 1995).

The Hanford formation was deposited by cataclysmic floods that inundated the Pasco Basin a number of times during the Pleistocene, beginning as early as 1 million years ago (Baker et al. 1991). The last major flood sequence is dated at about 13,000 years ago by the presence of Mount St. Helens "S" tephra (Mullineaux et al. 1978) interbedded with the flood deposits. The number and timing of cataclysmic floods continues to be debated. Baker et al. (1991) document as many as 10 flood events during the last ice age. The largest and most frequent floods came from glacial Lake Missoula in northwestern Montana. Cataclysmic floodwaters entering the Pasco Basin quickly became impounded behind Wallula Gap, which was too restrictive for the volume of water involved. Floodwaters formed temporary lakes with a shoreline up to $381.25 \mathrm{~m}$ (1250 ft) in elevation, which lasted only a few weeks or less (Baker 1991). The 
floods caused massive erosion of both earlier sediments and the basalt bedrock. These resulted in deposition of sediments in low-lying areas. Hanford formation sediments are found across nearly the entire Hanford Site, except where basalt outcrops occur.

The Hanford formation has been divided into three major facies: 1) gravel-dominated, 2) sanddominated, and 3) silt-dominated. These facies generally correspond to coarse gravels, laminated sands, and graded rhythmites, respectively (DOE 1988). Gravel-dominated strata consist of coarse-grained sand and granule-to-boulder sized, clast-supported gravel. As shown in Figure 4.4, the gravels can have an open matrix with large pore spaces and very high permeability. The sand-dominated facies consists of fine- to coarse-grained sand. Small pebbles and pebbly interbeds ( $<20 \mathrm{~cm}$ [8 in.] thick) may be encountered. The silt-dominated facies consists of silt and fine- to coarse-grained sand forming normally graded rhythmites. Plane lamination and ripple cross-lamination is common in outcrops in these facies. Hanford formation sediments tend to have a large proportion of basaltic fragments because of the upstream erosion of basalt bedrock in the channeled scablands.

Clastic dikes are commonly associated with, but not restricted to, cataclysmic flood deposits on the Columbia Plateau (Fecht et al. 1994). While there is general agreement that clastic dikes formed during cataclysmic flooding, a primary mechanism to satisfactorily explain the formation of all dikes has not

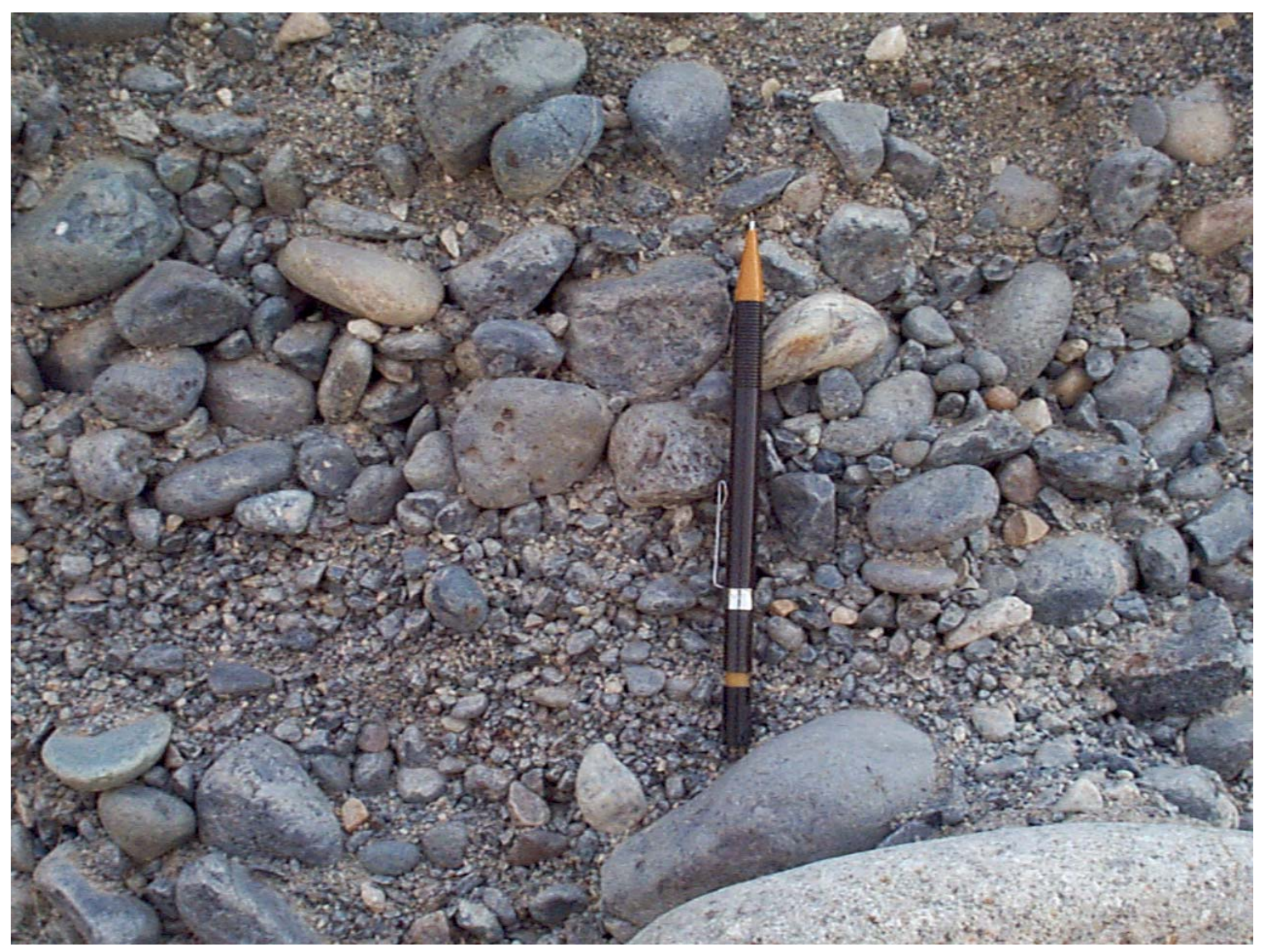

Figure 4.4. Photograph of Hanford Formation Gravel Showing Open Matrix Structure. 
been identified. Among the more probable explanations are fracturing initiated by hydrostatic loading and hydraulic injection associated with receding floodwaters. These dikes may provide vertical pathways for downward migration of water through the vadose zone. However, they are more likely to inhibit lateral flow because of the skin of fine-grained particles that form around the vertically oriented clastic dike (Lindsey 1995).

Recent alluvium is present, not only as a surficial deposit along major river and stream courses, but also in the subsurface, where it is sometimes found interbedded with Hanford formation flood deposits. Large areas of the Hanford Site are also covered by recent deposits of wind-blown loess and sand. However, these sediments are relatively thin and are nearly always above the water table. More detailed information on the geology of the Pasco Basin can be found in Lindsey (1995), Reidel et al. (1992), Connelly et al. (1992a,b), and DOE (1988).

\subsubsection{Local Structural Features}

The Hanford Site is located near the junction of the Yakima Fold Belt and the Palouse structural subprovinces (DOE 1988). The Yakima Fold Belt consists of a series of anticlines separated by broad synclines or basins that, in many cases, contain thick accumulations of sediments. The deformation of the Yakima Folds occurred under north-south compression. The fold belt was growing during the eruption of the Columbia River Basalt Group and continued to grow into the Pleistocene and probably into the present (Reidel 1984).

On the Hanford Site, the Wahluke syncline is found on the north side of the Umtanum Ridge-Gable Mountain anticline; the Cold Creek syncline is found on the south side of this structure (Figure 4.5). The Saddle Mountains uplift, located north of the Hanford Site, forms the northern boundary of the Pasco Basin. It is generally steepest on the north, with a gently dipping southern limb. A major thrust or highangle reverse fault occurs on the north side (Reidel 1984).

The Umtanum Ridge-Gable Mountain uplift is a segmented, asymmetrical anticlinal ridge extending $137 \mathrm{~km}$ (85 miles) in an east-west direction and passing north of the 200 areas (Figure 4.5). It separates the Cold Creek syncline from the Wahluke syncline. Three of this structure's segments are located on or adjacent to the Hanford Site. From the west, Umtanum Ridge plunges eastward toward the basin and merges with the Gable Mountain-Gable Butte segment. The latter segment then merges with the Southeast Anticline, which trends southeast before dying out near the Columbia River. There is a major thrust to high-angle reverse fault on the north side of the Umtanum Ridge-Gable Mountain structure (PSPL 1982). This fault dies out as it plunges eastward past the Gable Mountain-Gable Butte segment.

Rattlesnake Mountain is a steeply dipping and faulted asymmetrical anticline that forms the southern boundary of the Pasco Basin (Figure 4.5). It extends from the structurally complex Snively Basin area southeast to the Yakima River where the uplift continues as a series of doubly plunging anticlines (Fecht et al. 1984). At Snively Basin, the Rattlesnake Mountain structure intersects the Rattlesnake Hills anticline, which has an east-west trend. 


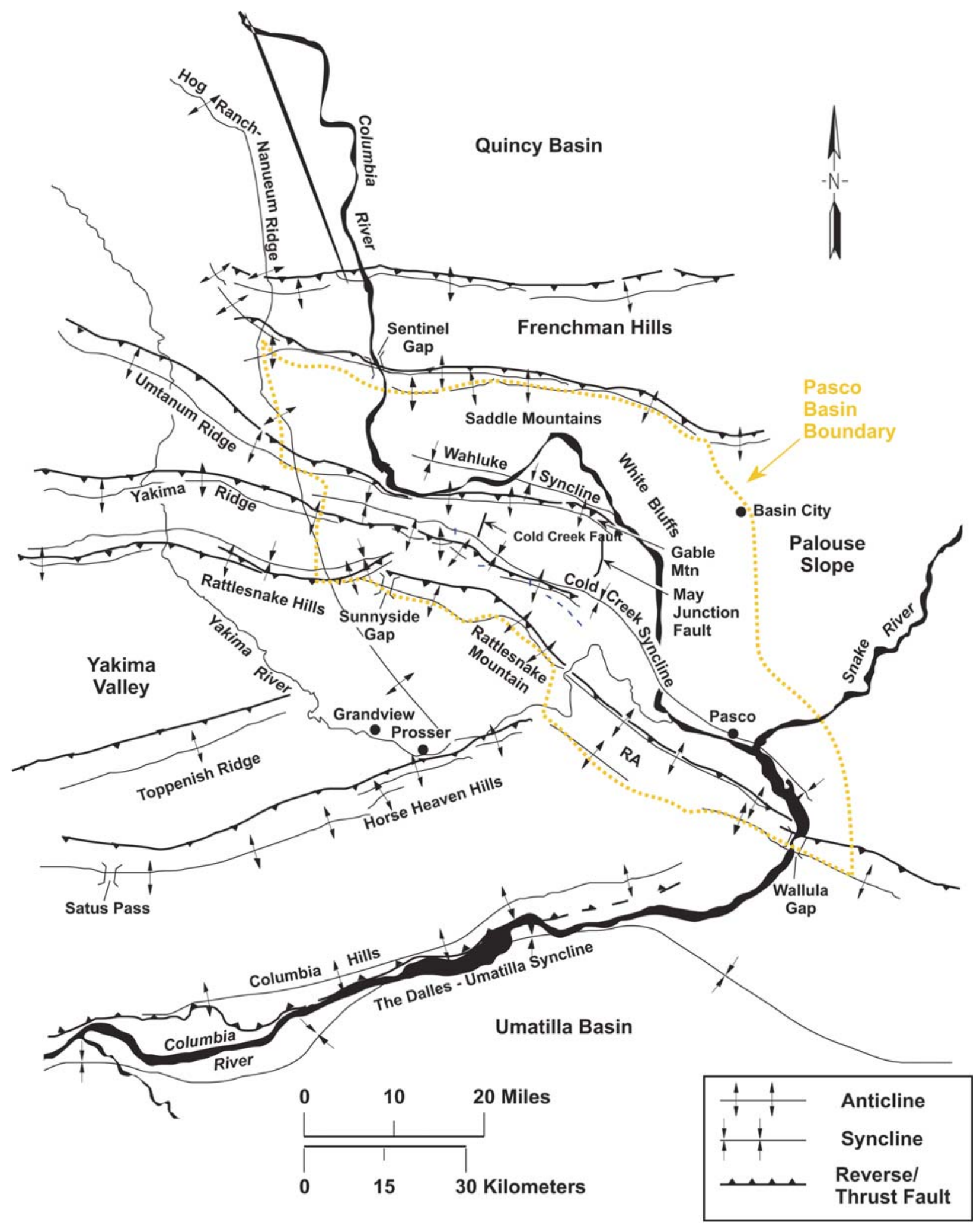

Figure 4.5. Structural Geologic Features of the Pasco Basin. 


\subsubsection{Local Unconfined Aquifer System}

Groundwater within the sediments described above forms an unconfined aquifer over most of the Hanford Site. However, the aquifer is locally confined in some areas where relatively impermeable mud units exist at the water table (Figure 4.6 and 4.7). The lower Ringold mud unit also locally confines deeper portions of the aquifer over large areas of the Site. This mud unit appears to be continuous in the area north of Gable Mountain.

Natural recharge to the local aquifer system occurs from infiltration of 1) runoff from elevated regions along the western boundary of the Hanford Site, 2) spring discharges originating from the basaltconfined aquifer system, and 3) precipitation falling across the Hanford Site. Some recharge to the unconfined aquifer also occurs along the Yakima River in the southern portion of the Hanford Site. Natural recharge from runoff and irrigation in the Cold Creek and Dry Creek valleys, upgradient of the Hanford Site, provides a source of groundwater inflow where these valleys enter the area of interest. The other source of recharge to the aquifer system is artificial recharge from wastewater disposal, which has significantly affected groundwater flow during the past 50 years. These recharge sources are discussed in detail in Sections 4.8 and 4.9 of this report.

Groundwater within the unconfined aquifer system flows from recharge areas toward the Columbia River, which is the major discharge area for the local aquifer. Water table elevations are routinely measured in wells and mapped (e.g., Hartman et al. 1999) to show groundwater flow direction and head gradients. Contaminant concentrations are also routinely measured and mapped.

\subsubsection{Water Table Elevation and Groundwater Flow Conditions}

Water levels have been measured on at least an annual basis using a site-wide well network since the 1940s. More than 600 wells are currently measured each year to determine the hydraulic head distribution for the unconfined aquifer on the Hanford Site and adjacent areas. Results of the 1999 measurements are presented in Hartman et al. (2000). Additional water-level data for the North Richland area are provided in Liikala (1994). The annual water-level measurements provide an extensive database that can be used to define initial head conditions for numerical modeling and for a comparison of modeling runs with historical data. Recent inverse modeling efforts (Cole et al. 2001a,b) used 76,000 historical water level measurements made through time since operations began in the 1940s. The interpreted water table for the March 1999 water level data is shown in Figure 4.7. This figure shows water table elevation contours for the unconfined aquifer system. Groundwater flow occurs at a right angle to these contour lines, moving from higher elevation to lower. Locations where the basalt is above the water table are also shown. The basalt is assumed to be relatively impermeable.

Prior to the mid-1980s, in response to wastewater disposal activities, hydraulic heads increased by more than $13 \mathrm{~m}$ over a period of 35 years in some areas of the Hanford Site. Before wastewater disposal operations began, the uppermost aquifer was almost entirely within the Ringold Formation and the water table extended into the Hanford formation at only a few locations near the Columbia River (Newcomb 


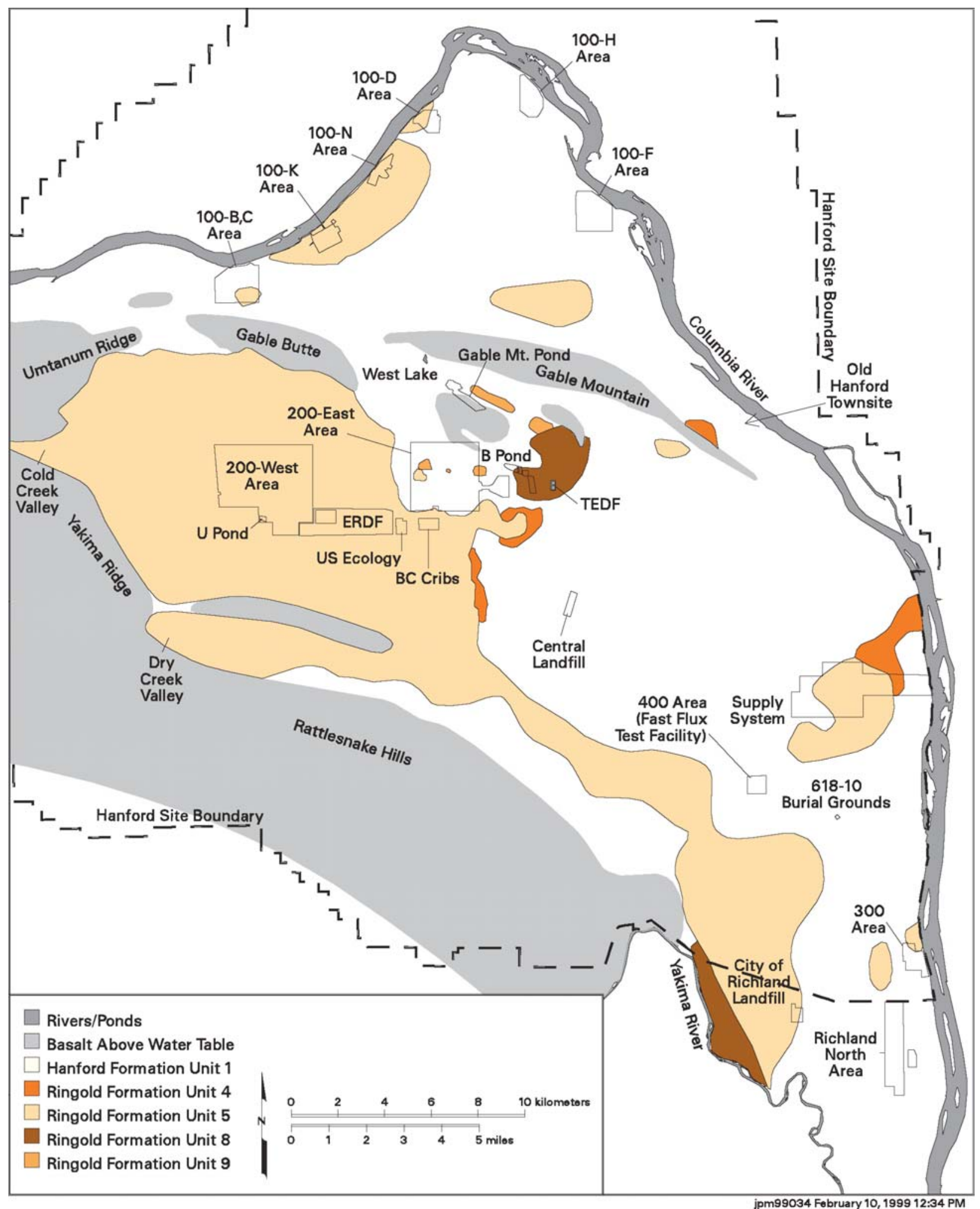

Figure 4.6. Map of SGM Hydrogeologic Units Containing the Water Table in March 1999. 


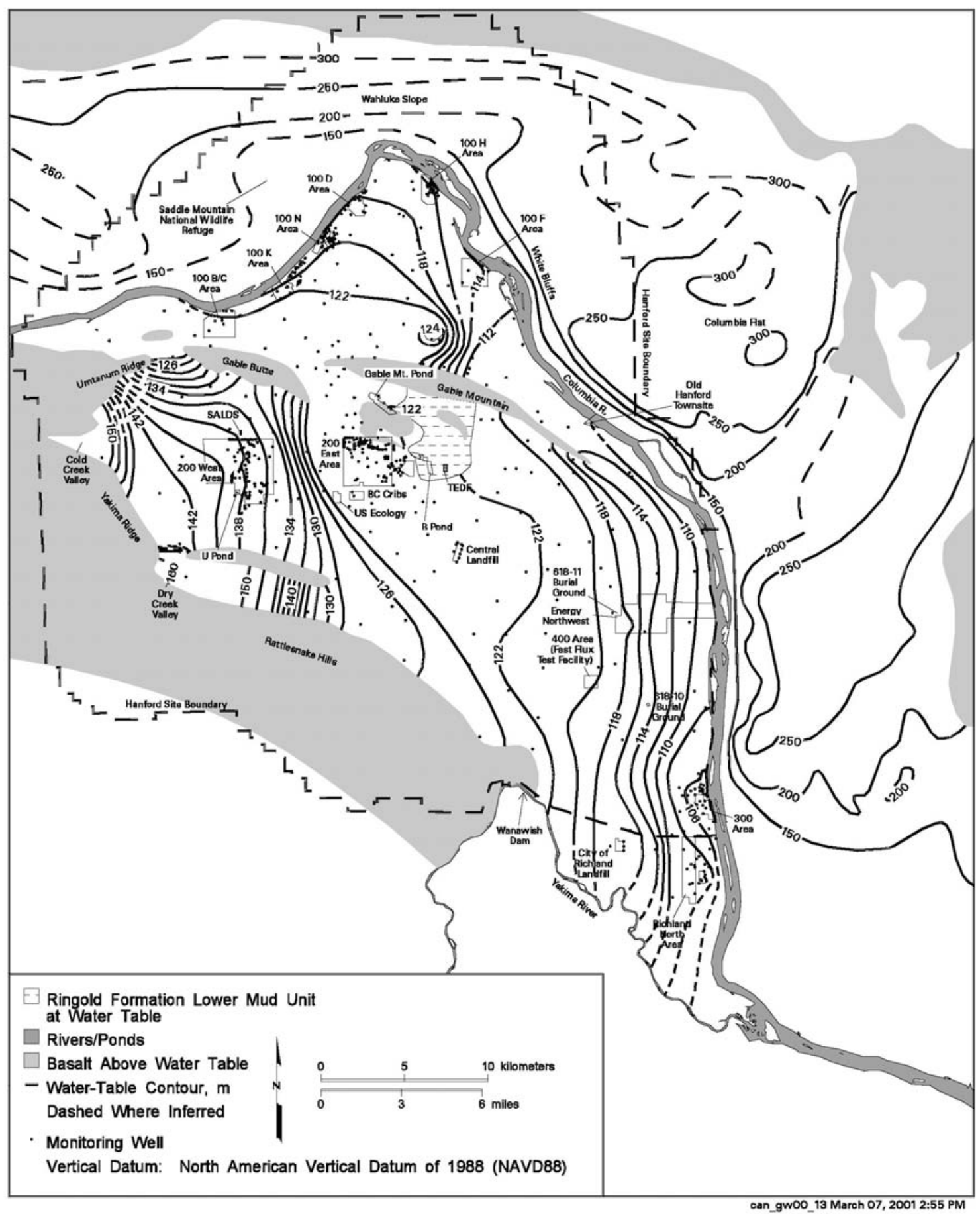

Figure 4.7. Interpreted Water Table for March 1999 Measurements. 
and Strand 1953). However, wastewater discharges have caused the water-table elevation to rise into the Hanford formation in the vicinity of the 200 East Area and in a wider area near the Columbia River. Water levels have begun to decrease over most of the Hanford Site during the last several years because of decreases in wastewater discharge (Dresel et al. 1995).

Most of the wells in the current unconfined aquifer-monitoring network are completed in the upper part of the aquifer, within $7 \mathrm{~m}$ of the water table. Most of the wells that were originally open to a greater depth interval were reconfigured in the early 1980s. The conceptual groundwater model, by its nature, is a three-dimensional problem and requires information on the vertical distribution of hydraulic head as well as the areal distribution. The locations and a listing of selected wells currently completed in the deeper part of the unconfined aquifer and wells with individual piezometers open to different depth intervals are presented in Wurstner et al. (1995).

Groundwater levels east and north of the Columbia River have been affected by irrigation recharge. A water table elevation map prepared by the U.S. Geological Survey (USGS) generally shows steep head gradients toward the river east of Hanford. The USGS study was conducted as part of the subsurface characterization around Franklin County, east of the Hanford Site. Water table elevations east of the river have increased during the past 50 years because of recharge from large-scale irrigation. The irrigationinduced rise of the water table is over $100 \mathrm{~m}$ in some areas (Drost et al. 1997). Part of the reason for these large increases is the relatively low permeability of upper Ringold sediments found in this area. Springs are seen in the bluffs on the eastern side of the river and landslides have occurred on these steep slopes because of the increased hydraulic pressure. A similar situation exists north of the Hanford Site, where large-scale irrigation with imported water has been developed on the Waluke Slope.

The water table currently lies within the Hanford formation/pre-Missoula over most of the eastern and northern parts of the Hanford Site (Figure 4.6). However, these deposits lie entirely above the water table in the western part of the Site and in some other localized areas (Figure 4.6). Where they exist below the water table, the Hanford/pre-Missoula gravel deposits usually dominate groundwater flow because they are generally 10 to 100 times more permeable than the Ringold gravels.

\subsubsection{Hydrogeologic Units}

Major hydrogeologic units within the sediments overlying the basalt bedrock can be correlated between boreholes over distances of several kilometers. This was recognized even in the earliest study of groundwater in the Hanford Site area conducted by Jenkins (1922). Since that time, the drilling of additional boreholes and further study of geological features has led to an improved understanding of the depositional history of the sediments and their resulting distribution and characteristics. The hydrogeologic structure of the conceptual model is designed to reflect differences in hydraulic properties of sediments such as effective porosity and hydraulic conductivity. These properties are related to sediment texture, which is a function of grain-size distribution, sorting, and consolidation/cementation. 
In developing the three-dimensional groundwater flow model, an effort was made to identify major textural units that influence groundwater flow directions and contaminant transport on a site-wide scale. Hydrogeologic units dominated by gravel and sand facies generally alternate with units dominated by mud facies.

The part of the Hanford formation/pre-Missoula gravel unit that lies below the water table is relatively thin and is usually composed of a vertically consistent textural facies. Therefore, a single model layer is used to represent the Hanford formation/pre-Missoula gravel unit, and lateral differences in facies type are represented by varying hydraulic properties assigned to this unit. The Hanford formation/pre-Missoula gravel is designated as unit 1 in the model. This unit also includes recent alluvium and eolian deposits that lie on top of the Hanford formation. However, these sediments are above the water table except in a few places next to the Columbia River. The difference in properties of these sediments is uncertain and has not been accounted for in the SGM.

The Ringold Formation is usually texturally distinct from the overlying Hanford/pre-Missoula unit and displays different hydraulic properties. Within the Ringold Formation, differences in texture are reflected by different facies associations defined by Lindsey (1995). Each facies association is dominated by a particular textural facies as shown in Table 4.2. Therefore, the facies associations and member/unit groupings presented in Lindsey (1995) are the basis for delineation of Ringold units within the threedimensional conceptual model. At some locations, however, sediments may be grouped slightly differently than by Lindsey (1995) to better reflect differences in hydraulic properties while ignoring other factors such as time of deposition or depositional environment. Because of these differences, the model hydrogeologic units have been numbered from top to bottom with units 4 through 9 representing the Ringold Formation and unit 1 representing the Hanford formation/pre-Missoula gravel unit. Figure 4.8 shows a comparison of Lindsey's stratigraphic column and the corresponding model units.

A major difference in the definition of model units is the grouping of Lindsey's (1995) "facies association II" (upper Ringold fine- to coarse-grained sands) with the gravel and sand dominated units E and $\mathrm{C}$ to form model unit 5. This left only the silt-dominated "facies association III" portion of the member of Taylor Flat assigned to model unit 4, also called the upper Ringold mud. The sands are grouped with the sandy gravels of units $\mathrm{E}$ and $\mathrm{C}$ because they are expected to have similar hydraulic properties. Ringold gravels are embedded in a poorly sorted sand matrix and have hydraulic properties that are dominated by the sand matrix. The hydraulic properties of these sediments are expected to be much different from the hydraulic properties of the silt-dominated "facies association III." Figures 4.9 and 4.10 show cross sections from Lindsey (1995) superimposed with cross sections along the same lines through the conceptual model units. Figures 4.11 and 4.12 show the extent of model units 4 and 5 , respectively.

Model unit 7 corresponds to Lindsey's (1995) units B and D, which occur at different locations on the Hanford Site. Extensive fine-grained over bank and paleosol deposits separate unit B from overlying unit $\mathrm{C}$ in the eastern part of the Hanford Site. These fine-grained sediments are expected to have much different hydraulic properties than the gravel units and were assigned to model unit 6 . Figures 4.13 and 4.14 show the extent of model units 6 and 7, respectively. Model unit 8 is equivalent to Lindsey's (1995) lower mud unit and forms an aquitard across much of the site. This unit is a combination of fine-grained 


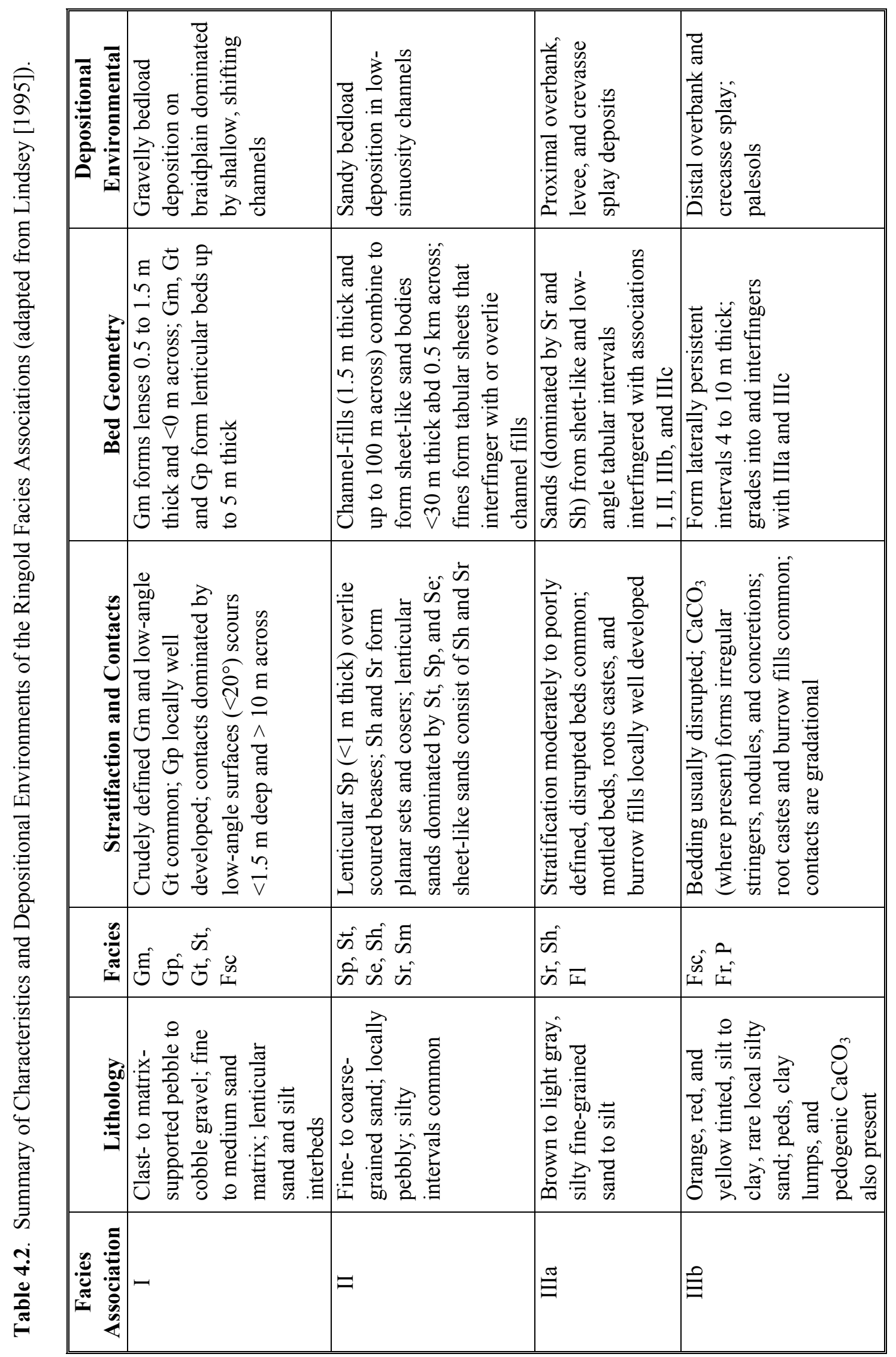




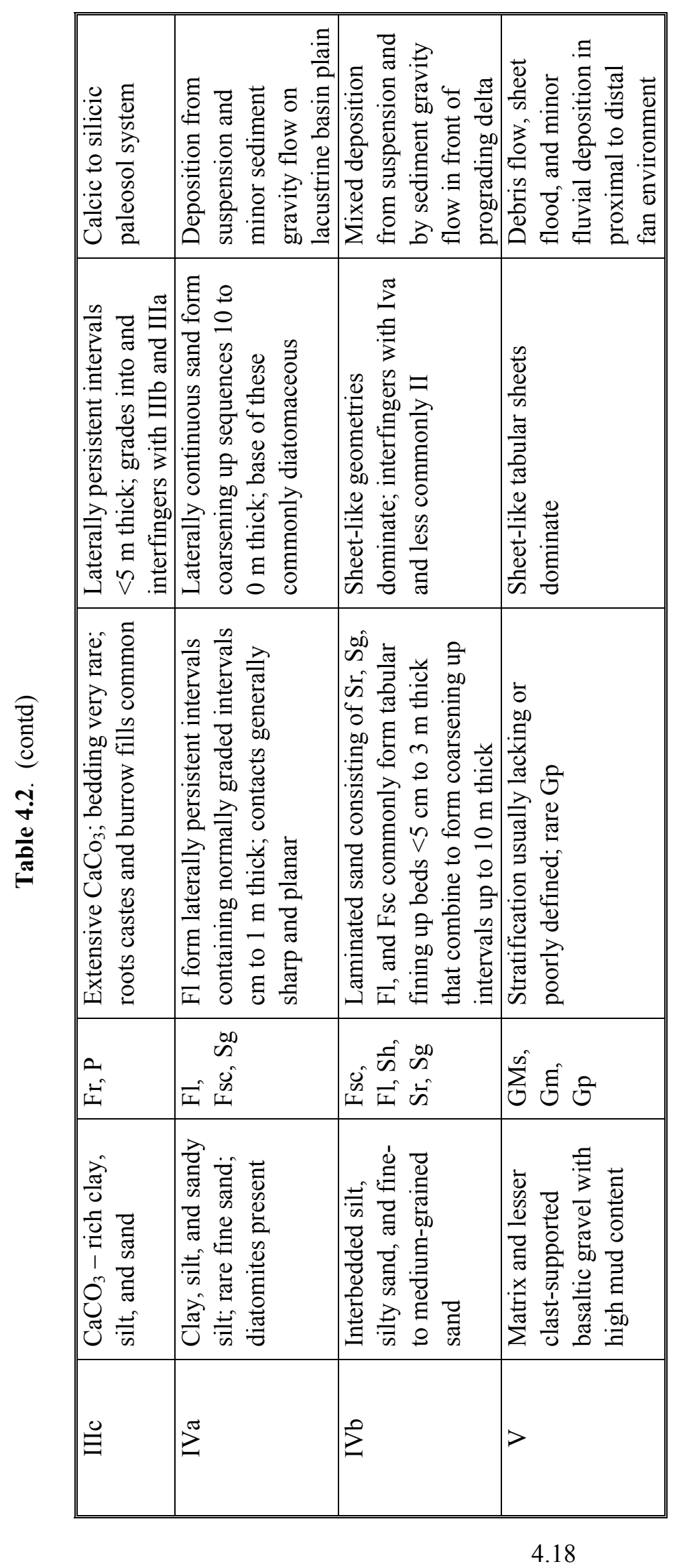




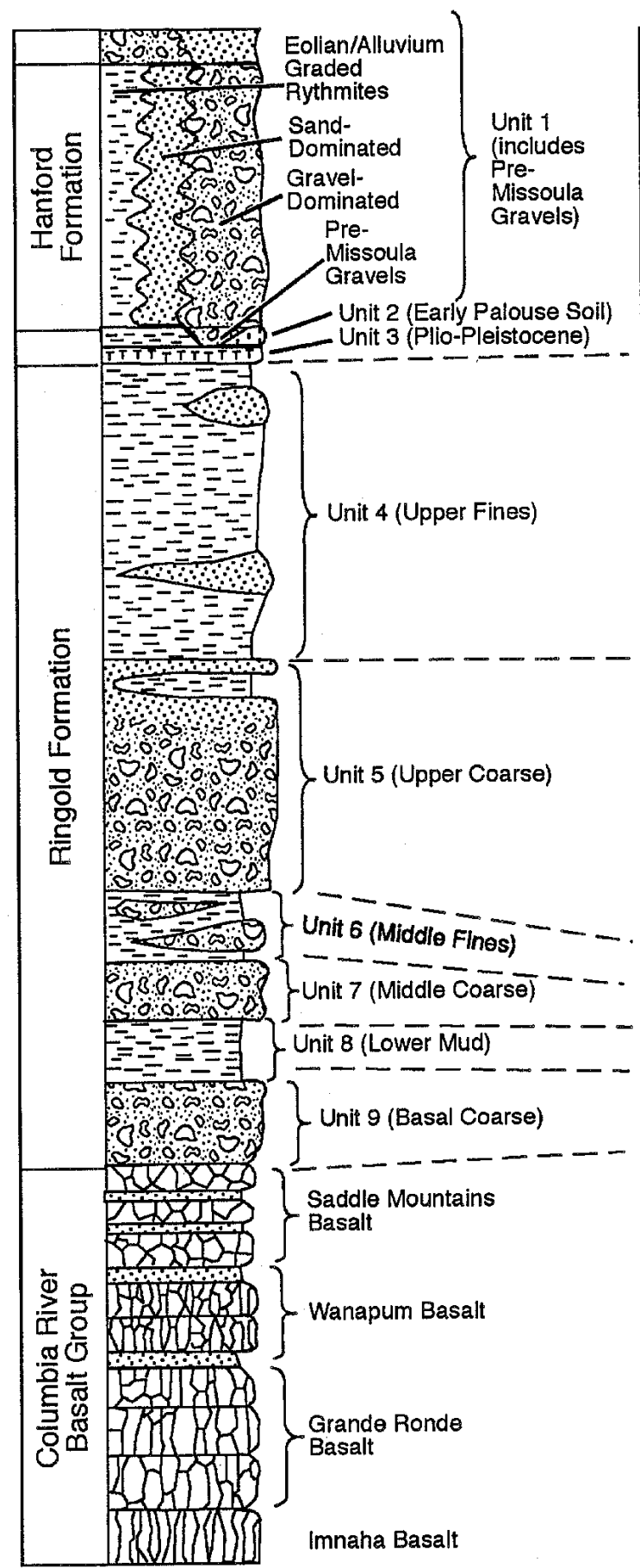

From PNL-8971
Not to Scale

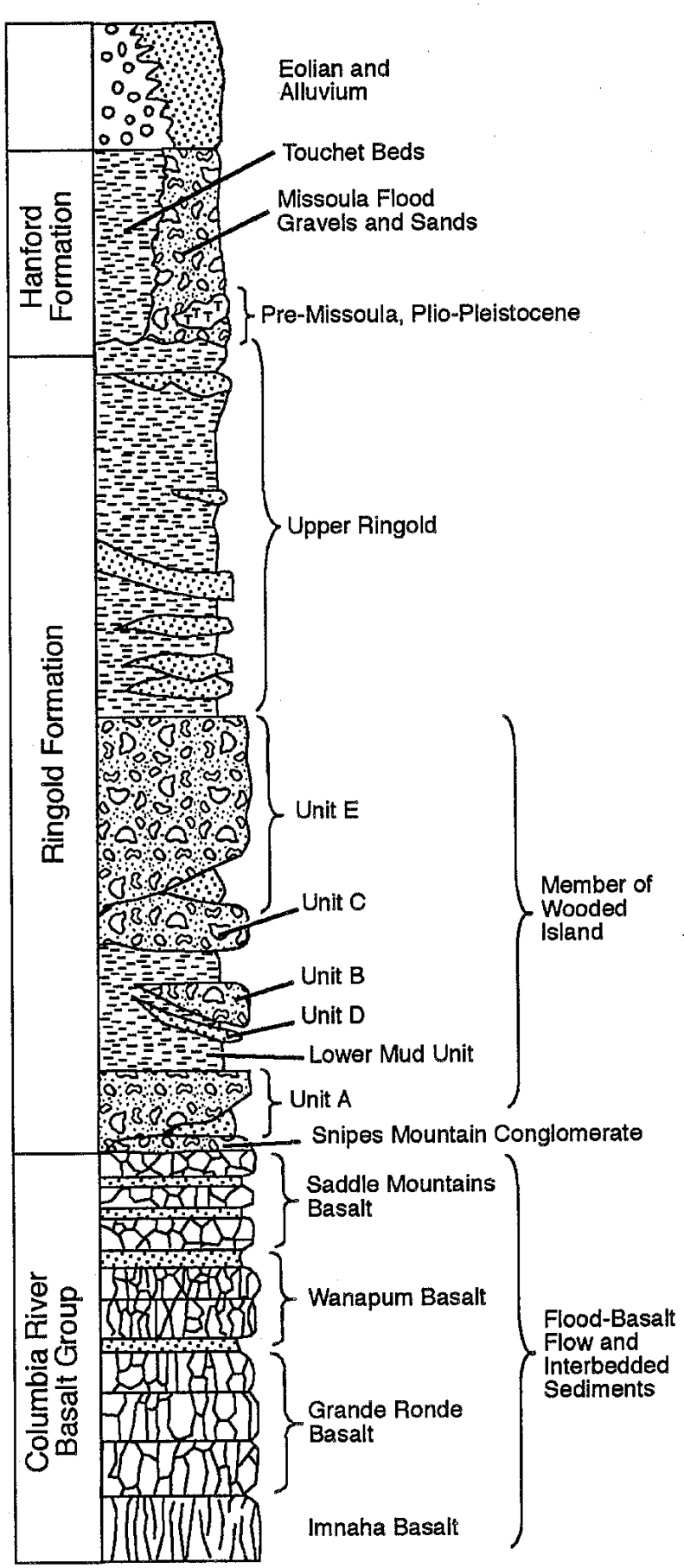

After BHI-00184

RG98120214.14

Figure 4.8. Comparison of Geologic Stratigraphy and Hydrogeologic Units Defined for the SGM. 


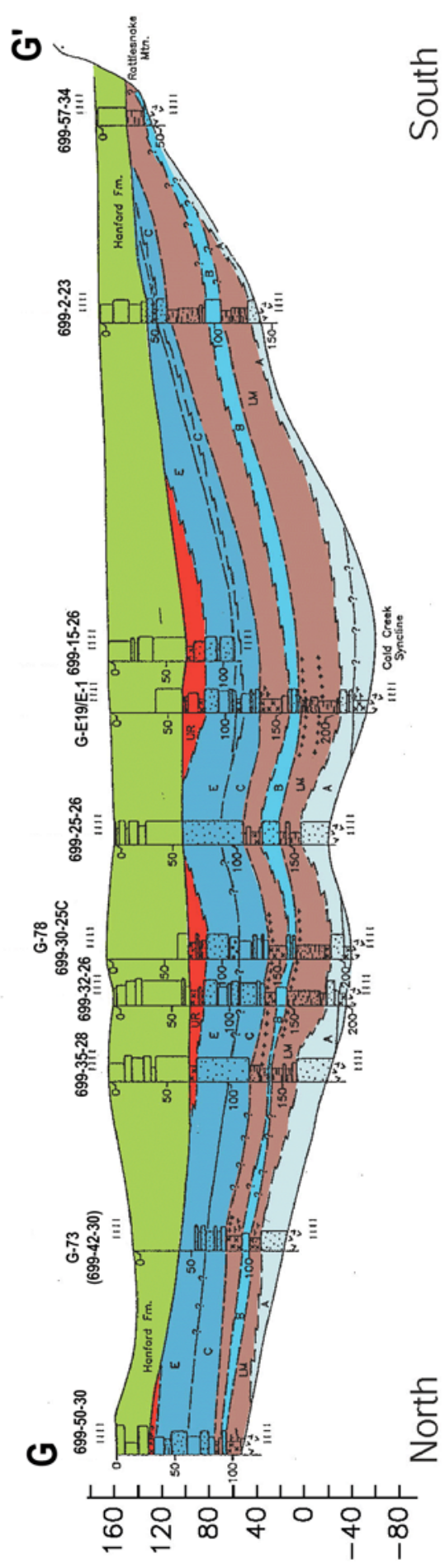

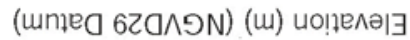

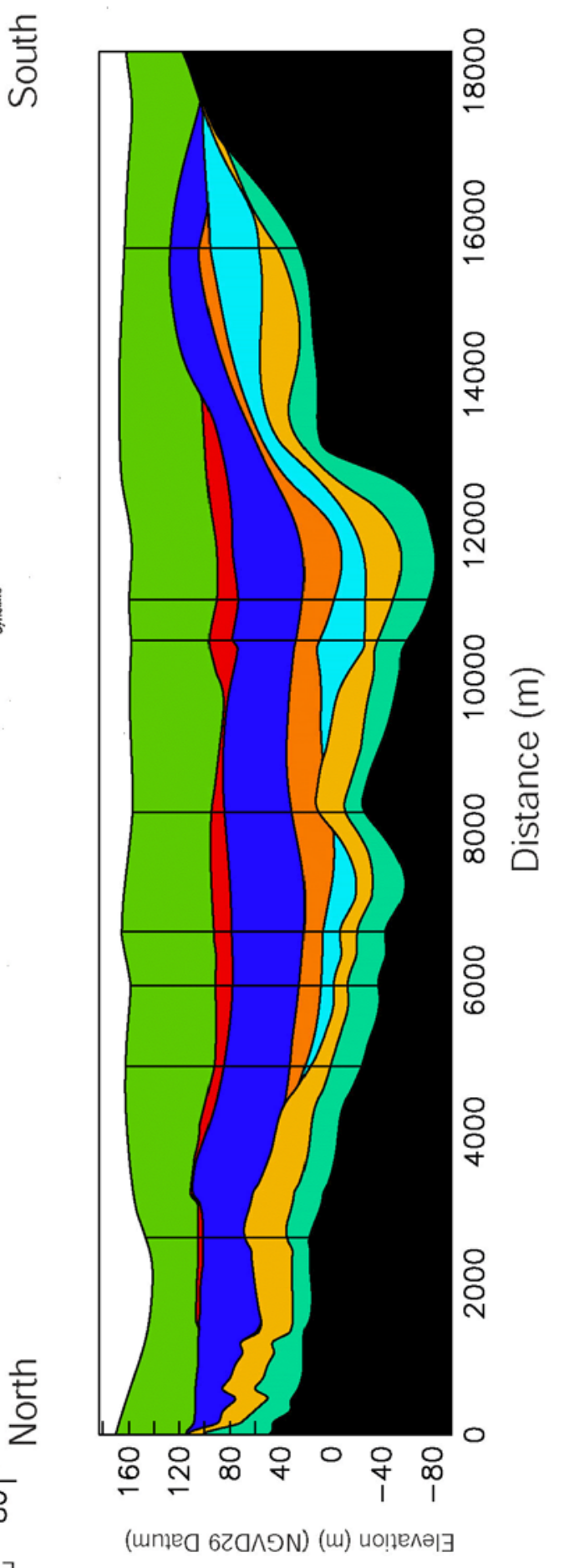




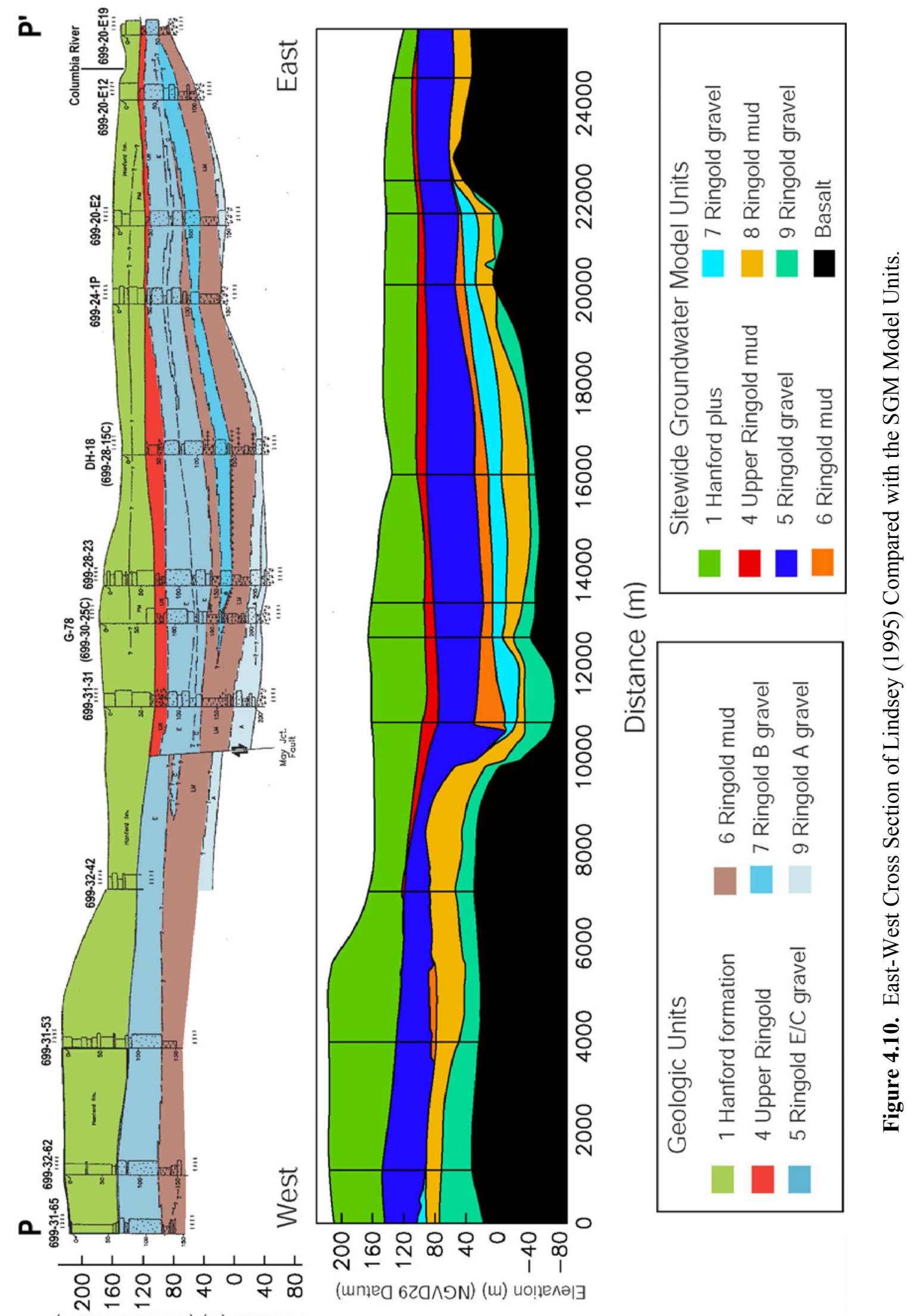

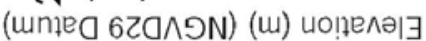




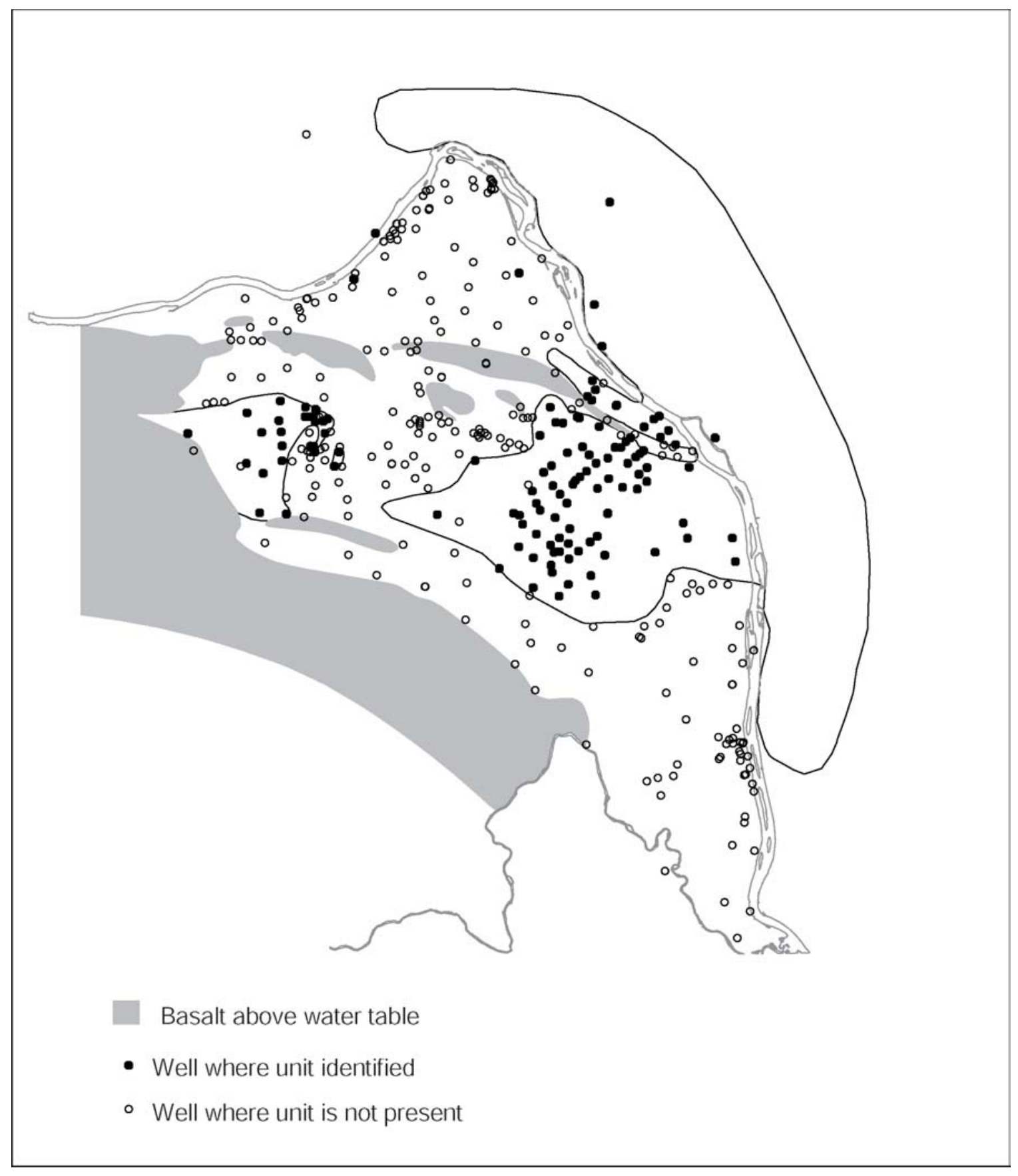

Figure 4.11. Extent of SGM Hydrogeologic Unit 4 (Upper Ringold Muds). 


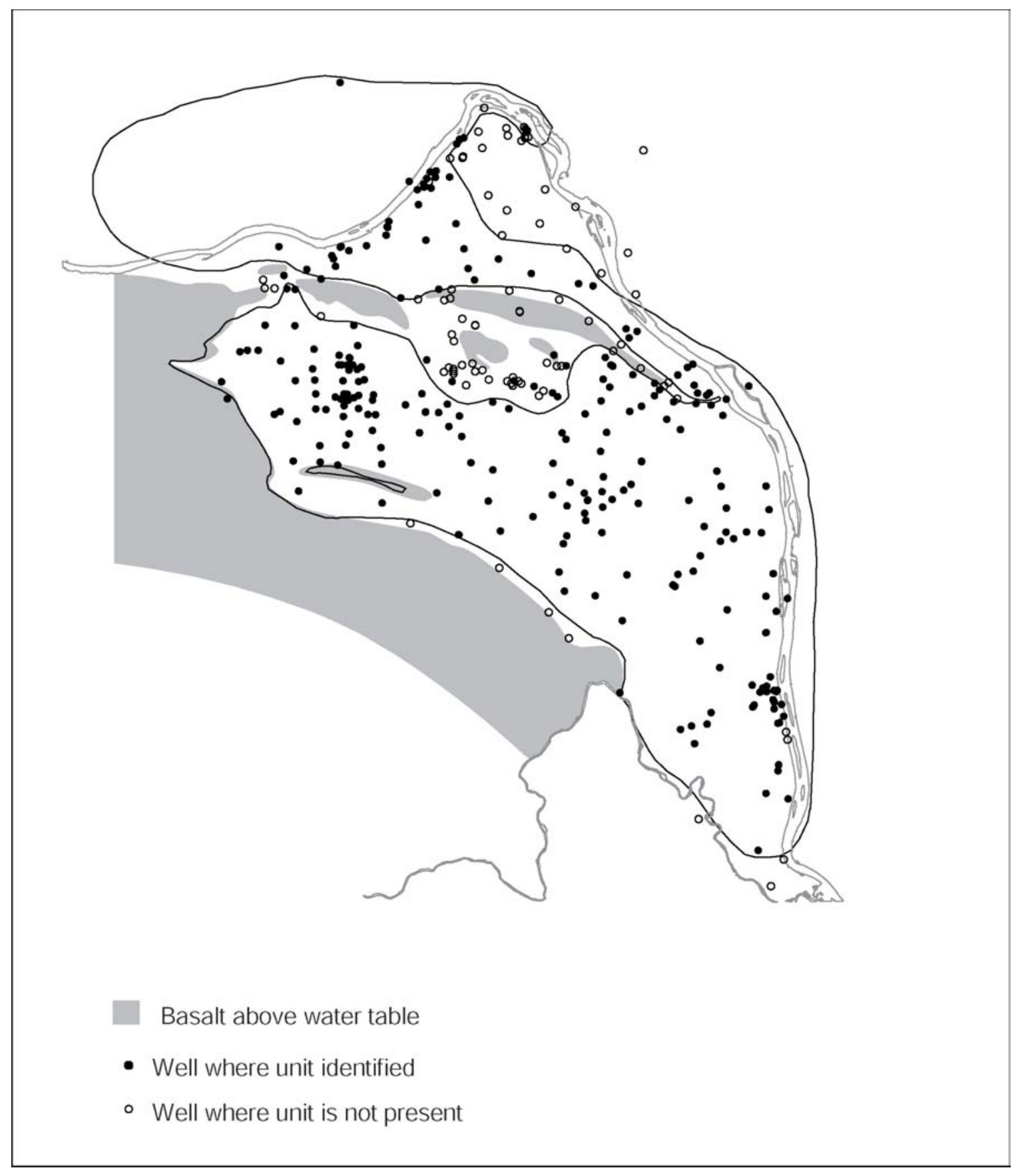

Figure 4.12. Extent of SGM Hydrogeologic Unit 5 (Ringold Gravel). 


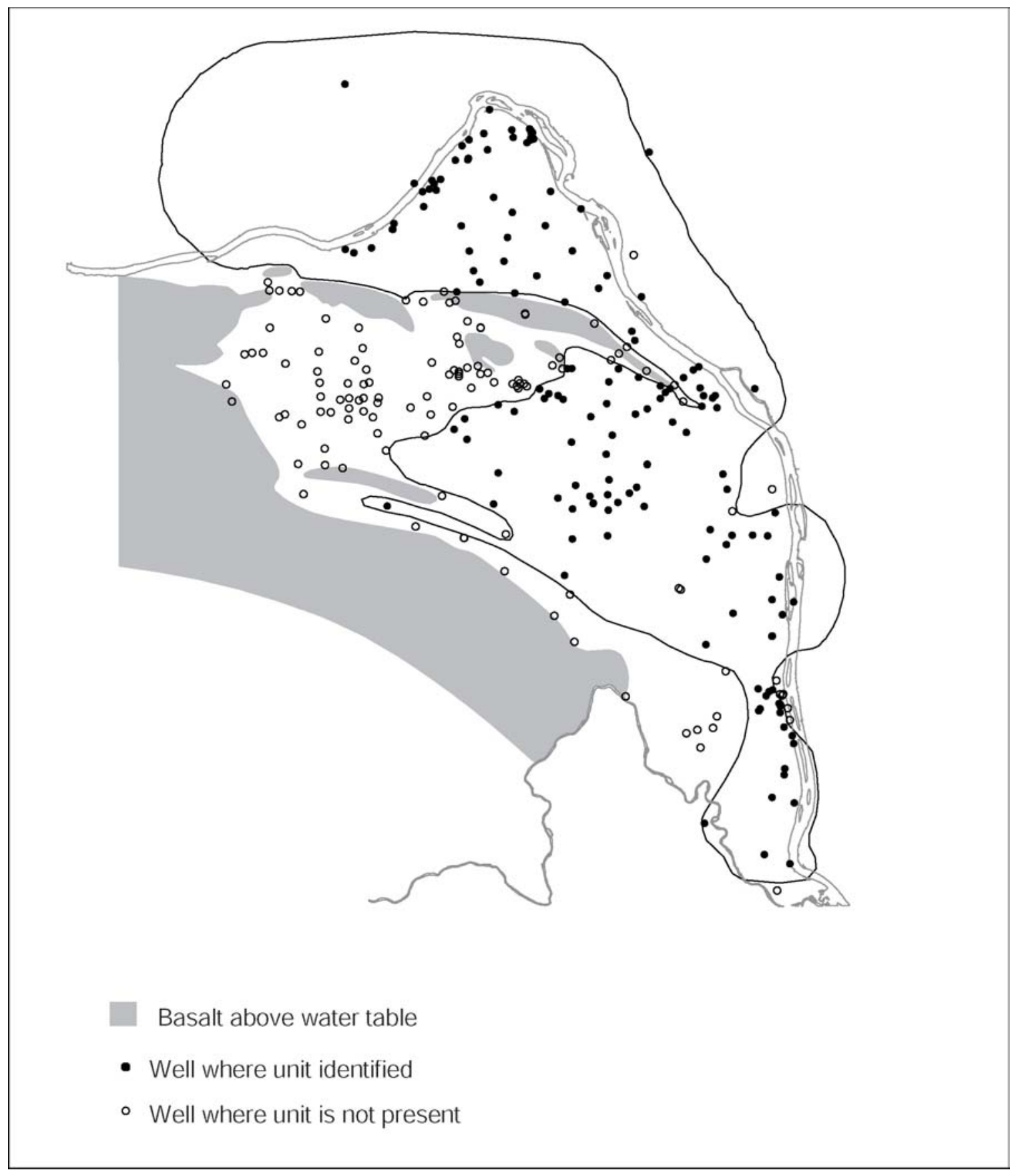

Figure 4.13. Extent of SGM Hydrogeologic Unit 6 (Ringold Over Bank Muds). 


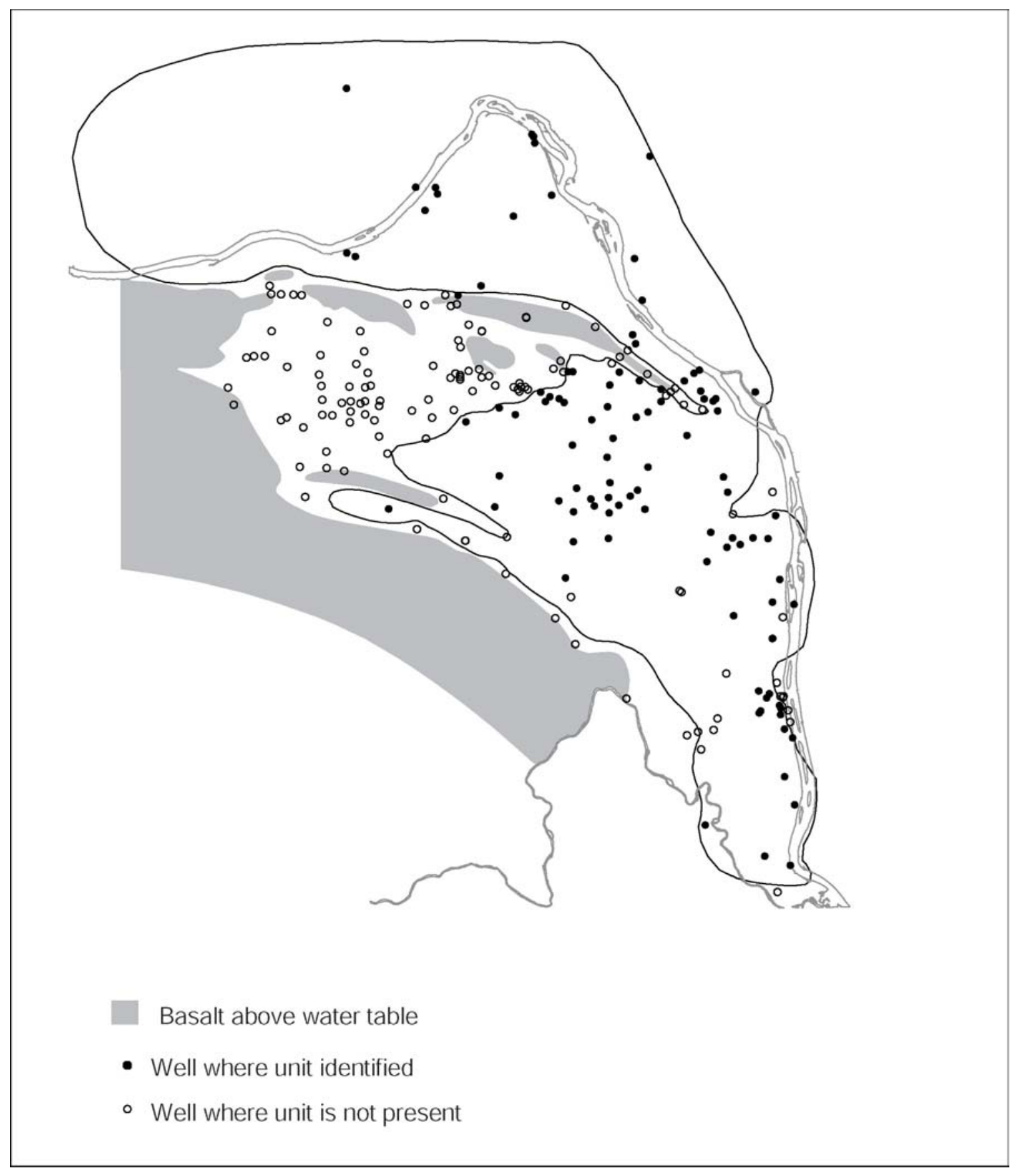

Figure 4.14. Extent of SGM Hydrogeologic Unit 7 (Ringold Gravel). 
paleosols and lacustrian deposits. Figure 4.15 shows the extent of model unit 8 . The mud in this unit is often described as blue or green, sticky clay, and frequently includes a white ash. Model unit 9 corresponds to Lindsey's (1995) unit A, a gravel and sand facies that is dominated by sand in the western part of the Pasco Basin. Figure 4.16 shows the extent of model unit 9.

\subsection{Criteria for Determining Hydrogeologic Unit Elevations and Extents}

Data from 426 boreholes across the Hanford Site were used to assign hydrogeologic unit elevations in the conceptual model. Top of basalt was identified in an additional 150 boreholes. The areal distribution of these boreholes is shown in Figure 4.17.

Information used in defining hydrogeologic units included geologic logs from boreholes, down hole geophysical logs, particle size analyses, calcium carbonate content, and geologic interpretations from other reports. However, not all these types of data were available for all boreholes. The primary source of information was geologic logs from boreholes. Quality of the geologic logs varies depending on the drilling method, the experience and knowledge of the individual describing the samples, and the purpose of the borehole. Archived samples are available for many boreholes and were examined to verify available geologic descriptions in some cases. Most boreholes were drilled using the cable-tool method, which breaks large clasts into smaller particles. Samples were collected from many boreholes using driven split-spoons, which also tend to break larger clasts and cause compaction of sediments. Cores are available from 28 boreholes (Figure 4.17). Detailed lithologic logs of these cores are presented in Lindsey (1995), who compared these and cuttings-based geologic logs to 29 measured geologic sections of the Ringold Formation exposed in the White Bluffs. The better quality data from cores and measured sections were used by Lindsey (1995) to identify the Ringold sedimentary facies associations described above and to establish analogs that are helpful in determining the facies type and other characteristics of cuttings and cuttings-based geologic descriptions. As stated in Lindsey (1995), "Use of analogs also allows identification of geologic properties fundamental to hydrologic interpretations that are otherwise incorrectly identified or not identified at all."

Characteristics used for correlating particular facies associations and model units to geologic descriptions are listed in Table 4.2. However, it should be understood that other information including consistency with neighboring borehole data and knowledge of the depositional environment were also considered in determining whether a facies change at a particular borehole reflected the presence of an extensive hydrogeologic unit or local heterogeneity within a unit.

Elevations of the tops and bottoms of each of the model hydrogeologic units were determined from borehole data as described above. The results are summarized in Appendix A. The texture of model unit 6 is more variable than the other units, therefore, available information on the percent mud for this unit is also listed in Appendix A. Model unit 6 corresponds to fine-grained over bank and paleosol deposits described by Lindsey (1995) that separate unit B from overlying unit C in the eastern part of the Hanford Site. After the distribution of each of the hydrogeologic units was understood, the areal extent of each unit on the Hanford Site was delineated, as shown in Figures 4.11 through 4.16. Note that the extents 


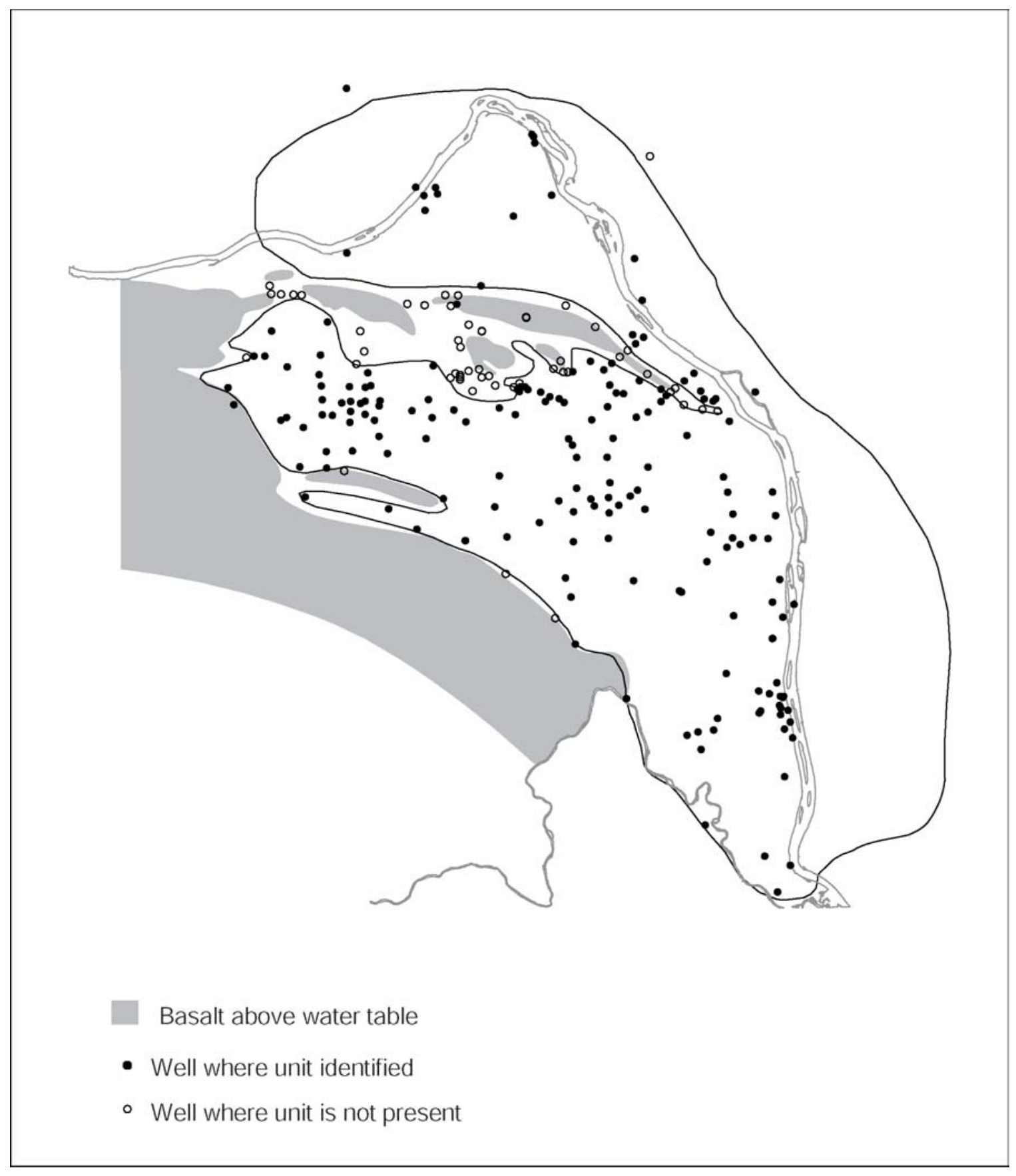

Figure 4.15. Extent of SGM Hydrogeologic Unit 8 (Lower Ringold Muds). 


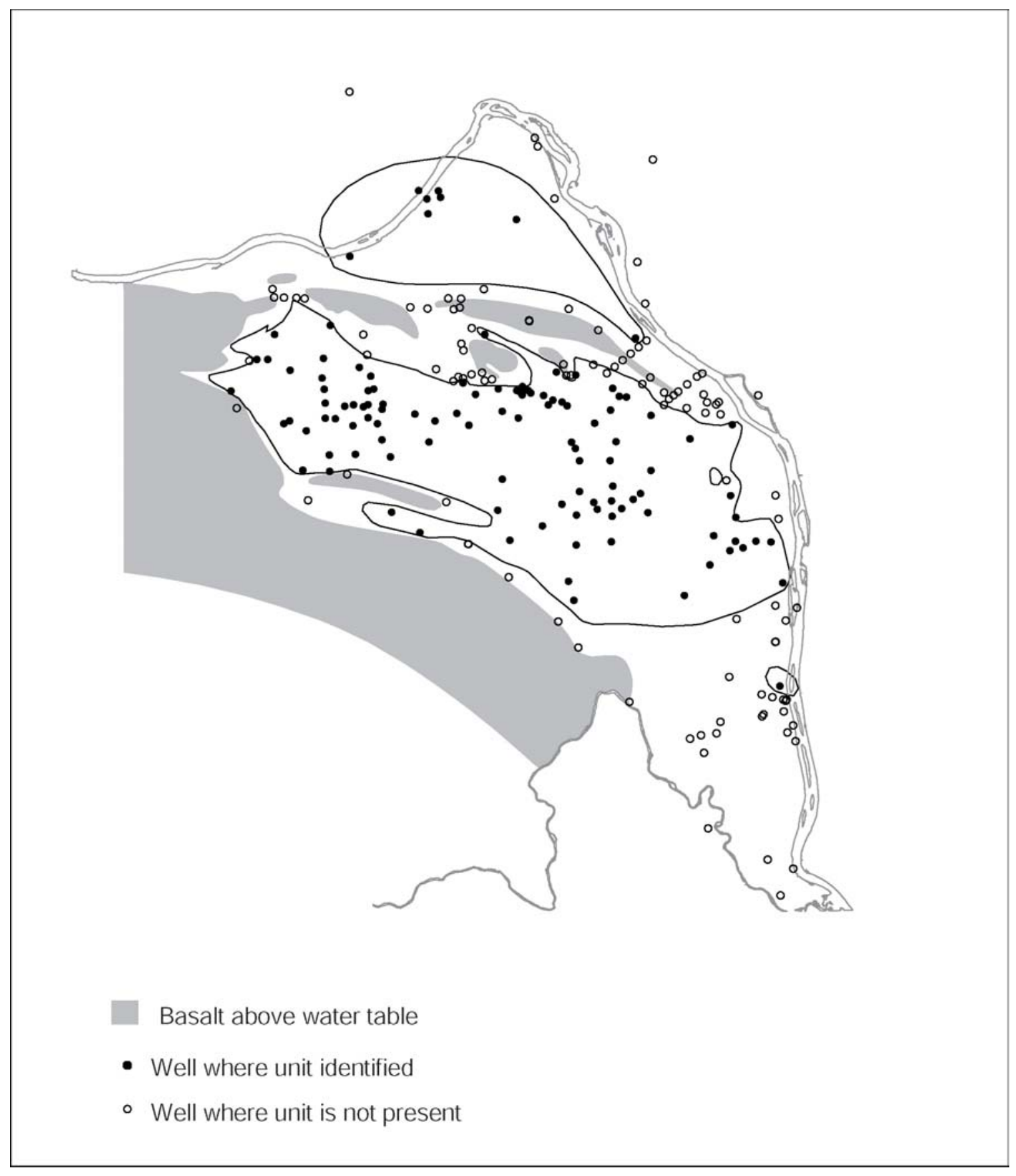

Figure 4.16. Extent of SGM Hydrogeologic Unit 9 (Basal Ringold Gravel). 


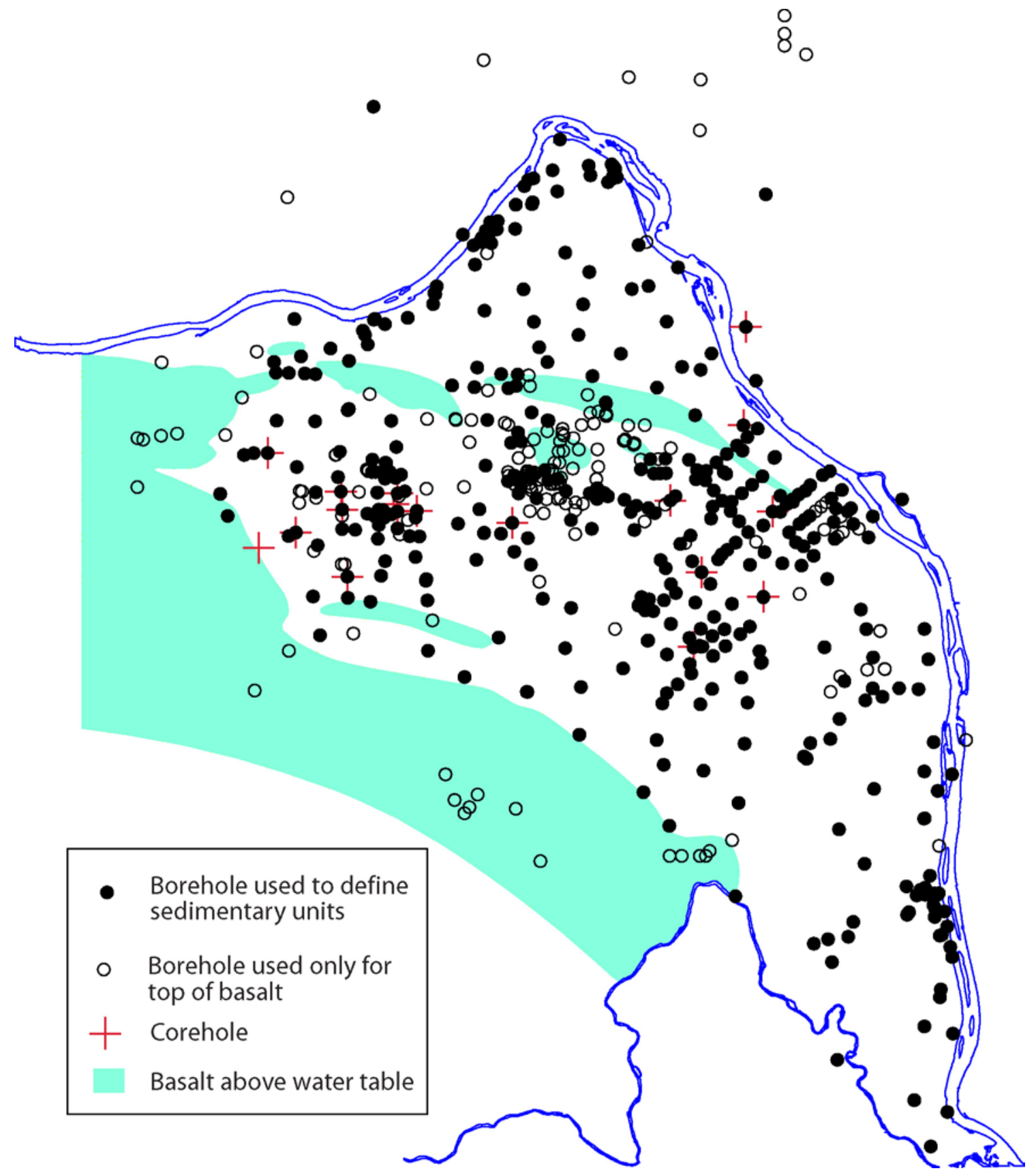

Figure 4.17. Distribution of Boreholes Used to Determine Hydrogeologic Structure for the SGM. 
shown have been truncated at the model boundary. A geologic modeling software package, Earth Vision $^{\mathrm{TM}}$ was used to assist in correlating unit elevations between boreholes and in visualizing the resulting unit surfaces in three dimensions.

\subsection{Uncertainty in the Distribution and Continuity of Hydrogeologic Units}

Uncertainty in the hydrogeologic structure of the model arises from both the possibility of misinterpretation and from actual spatial variability of the physical system. Uncertainty from misinterpretation can arise from the incorrect identification of a unit at a borehole or a unit being missed in the borehole interpretation. Samples are often logged only every 1.5 to $3 \mathrm{~m}$, which may not be enough to intercept a relatively thin unit. Incomplete descriptions or errors in descriptions and field interpretations can also lead to misinterpretation of units at a borehole location. However, even if the interpretation of units at boreholes were $100 \%$ accurate, unit continuity and elevation between boreholes would be uncertain because of the variability of the aquifer system between boreholes (i.e., the problem of interpolation and extrapolation under spatial variability). For example, erosional windows may exist in mud units but are not represented in the model. On the other hand, a unit may be present in an area where no boreholes exist and not be represented in the model.

\subsubsection{Effect of Faults Within the Sediments}

Two normal faults have been identified by apparent offset of both the basalts and units within the sediments overlying basalt on the Hanford Site. These are called the May Junction fault and the Cold Creek fault (Figure 4.18). Because of the offset of units within the aquifer, these faults may affect flow through the supra-basalt aquifer system and could potentially conduct some water from the basalts into the unconfined aquifer above it. Consequently, possible alternative conceptual models for flow behavior at these faults are also identified.

The May Junction fault (Reidel and Fecht 1994) is interpreted as a fault based on comparison to the aeromagnetic signatures of other faults in the area and the steep closure of contours on the top of the basalt. This fault is thought to be a normal or high-angle fault that has offset the basalts and the Ringold units.

The May Junction fault is approximately $3 \mathrm{~km}$ east of the 200 East Area (Figure 4.18). The fault is $6-\mathrm{km}$ long and trends generally in a north-south direction. It is completely buried along its length by sediments and dies out to the north before reaching Gable Mountain. The southern extent of the fault is difficult to estimate because borehole coverage is sparse in that region. Two boreholes that straddle the projection of the fault about $3 \mathrm{~km}$ south of the projected end of the fault indicate no offset.

The May Junction fault forms the western boundary of the Wye Barricade depression, a basin that lies between Gable Mountain and Rattlesnake Mountain east of the termination of Yakima Ridge. The Wye Barricade depression is a subfeature of the Cold Creek syncline within the Pasco Basin. These structures have been subsiding since the Miocene. The May Junction fault extends through the Columbia River basalt $(4 \mathrm{~km})$. The maximum displacement of the fault is about $60 \mathrm{~m}$ measured on the surface of the Columbia River Basalt Group, which is 10.5 million years old and overlying Ringold Formation 


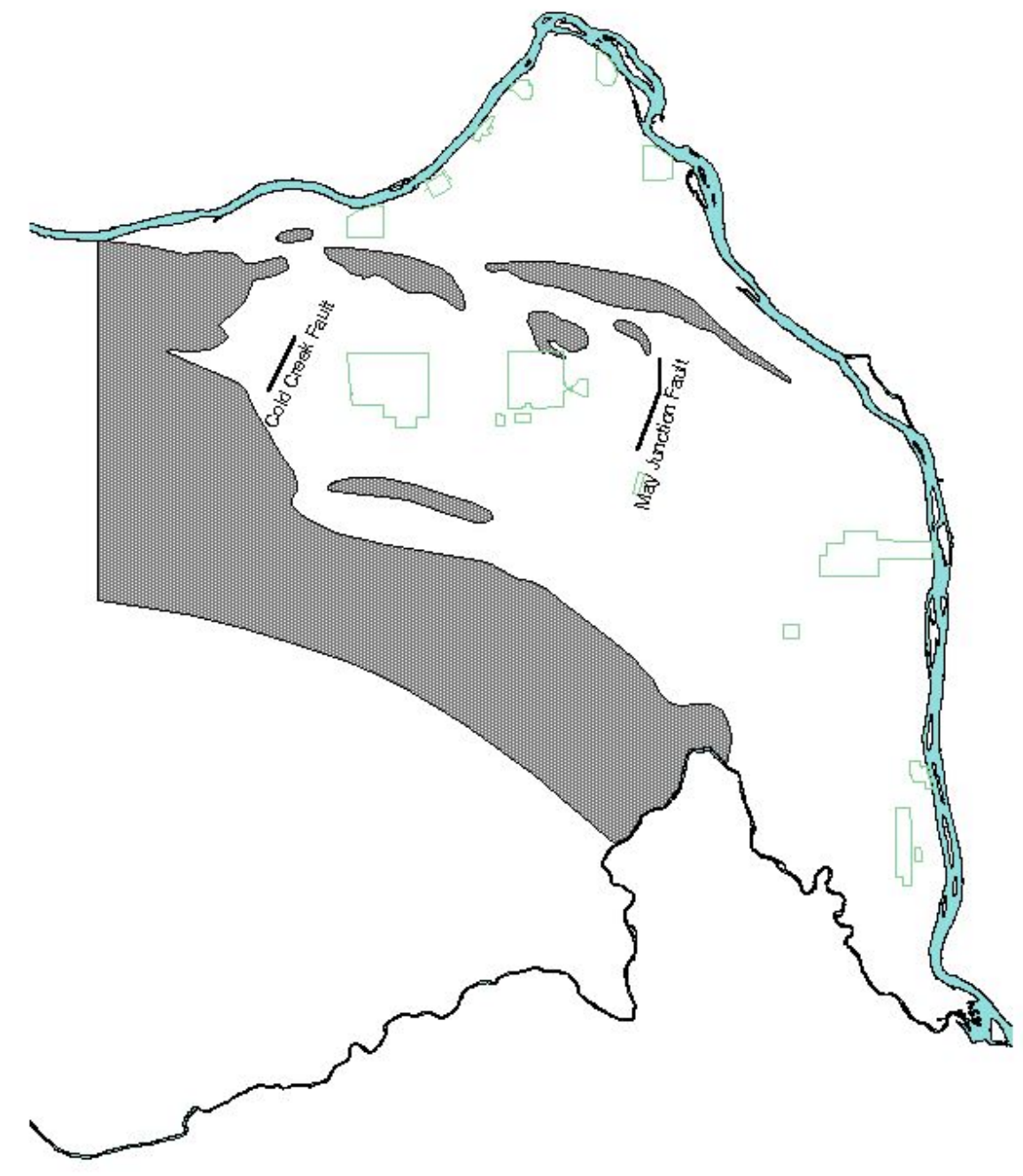

Figure 4.18. Location of the May Junction and Cold Creek Faults.

sediments, which are approximately 7 million years old. However, it is thought that the fault was active during the eruption of the Columbia River Basalts. Offset on the overlying Hanford formation, which is about 10 thousand to 1 million years old, cannot be estimated using available data. However, from comparing the elevations of subunits of the Hanford formation, any offset must be minor. The youngest movement of the fault is not known. The estimated long-term slip rate is $0.009 \mathrm{~mm} / \mathrm{yr}$.

Because of its location in the path of groundwater flow between the 200 Areas and the Columbia River, the May Junction fault may have a significant effect on transport of contaminants. There is little information on the hydrologic behavior of the fault. It was initially represented in the groundwater model as a steep-angled bend in the offset hydrogeologic units. The units are still connected, but the crosssectional area is greatly reduced. It is possible that the fault acts as a conduit for vertical flow between permeable units, and/or that it acts as a barrier to flow because of the "smearing" of fine-grained sediment 
along the fault. Again, these possible scenarios or large-scale uncertainties are included in our current SGM as alternative conceptual models to be investigated.

The Cold Creek fault (Figure 4.18) occurs on the west end of the Cold Creek syncline. This structure has previously been called the "Yakima Barricade geophysical anomaly" and the "Cold Creek flow impediment" in studies of the basalt-confined aquifer system (DOE 1988). The data suggest that this feature is a high-angle fault that has caused offset of the basalts and at least the older Ringold units (Johnson et al. 1993). This fault is upgradient of contamination sources at the Hanford Site, hence it is less significant in affecting results of transport modeling. However, behavior of flow in this area could affect the amount and location of recharge entering the Site from the Cold Creek Valley.

Like the May Junction fault, there is little information on the hydrologic behavior of the Cold Creek fault in the suprabasalt sediments. It may also act as either a conduit for vertical flow between permeable units, or as a barrier to flow. Within the basalt-confined aquifer system, the Cold Creek fault appears to act as a barrier to lateral flow because it coincides with a drastic west-to-east change in hydraulic gradient. The difference in head across the fault within the Wanapum Basalt Formation was more than $80 \mathrm{~m}$ (DOE 1988). It is thought that low-permeability fault gouge has created a barrier to lateral flow within the basalt-confined aquifers.

\subsubsection{Local Aquifer System Hydraulic Properties}

Hydraulic properties including both horizontal and vertical hydraulic conductivity $\left(\mathrm{K}_{\mathrm{h}}\right.$ and $\left.\mathrm{K}_{\mathrm{v}}\right)$, storativity $(\mathrm{S})$, and specific yield $\left(\mathrm{S}_{\mathrm{y}}\right)$ are key components of the groundwater conceptual model. Hydraulic conductivity controls the rate of water flow through a unit thickness of the aquifer at a given hydraulic gradient. Storativity and specific yield determine the change in water table elevation that will occur in response to a change in the volume of water stored in the aquifer.

Hydraulic property data for the Hanford Site unconfined aquifer have been derived mainly from aquifer pumping tests and, in a few cases, from laboratory permeameter tests. These results have been documented in dozens of published and unpublished reports over the past 50 years. A summary of available data for the unconfined aquifer was provided in DOE (1988) and an updated summary was provided in Thorne and Newcomer (1992) together with an evaluation of selected pumping test analyses. Additional tests have been conducted both to support the three-dimensional model and to support other Hanford Site projects (Spane et al. 2000). Some of the recent tests are documented in status reports on the development of the three-dimensional conceptual model (Thorne and Chamness 1992; Thorne et al. 1993, 1994; Wurstner et al. 1995).

Newcomb and Strand (1953) analyzed the growth of groundwater mounds beneath liquid disposal facilities in both the 200 West and 200 East areas between 1948 and 1953 to estimate hydraulic properties for these areas. Additional hydraulic property information has been determined from analyses of the decrease in groundwater mounds caused by recent decreases in disposal volumes. Details of the analysis of the mound dissipation are provided in Wurstner et al. (1995). Spane and Thorne (2000) analyzed water-level responses to a pump-and-treat operation in the 200 West Area to estimate hydraulic parameters. 


\subsection{Hydraulic Conductivity of Hydrogeologic Units}

Hydraulic conductivity values for sediments composing the unconfined aquifer system range from less than $10^{-4} \mathrm{~m} / \mathrm{d}$ for some mud units to about $10^{6} \mathrm{~m} / \mathrm{d}$ for coarse gravel flood deposits. The sand and gravel facies of the Ringold Formation are mostly between 1 and $50 \mathrm{~m} / \mathrm{d}$. The coarse sediments of the overlying Hanford formation range from 10 to $6,000 \mathrm{~m} / \mathrm{d}$ (DOE 1988). The Ringold Formation also contains relatively extensive layers of fine-grained, low permeability sediments such as silt or clay.

Most pumping test analyses result in estimates of aquifer transmissivity (T), which, for a vertically homogeneous aquifer, is the product of hydraulic conductivity in the horizontal plane $\left(\mathrm{K}_{\mathrm{h}}\right)$ and aquifer thickness (b). A listing of available hydraulic conductivity data obtained from pumping tests in the unconfined aquifer system is provided in Wurstner et al. (1995) and Figure 4.19, which show the distribution of the tested wells across the Hanford Site with the associated main geologic unit tested. Histograms of the data are also shown. The data provided in Wurstner et al. (1995) include 36 single-well pumping tests and 3 multiple-well pumping tests that pertain to the Hanford formation (unit 1). Thirtyseven single-well pumping tests and 12 multiple-well pumping tests pertain to Ringold Formation sand and gravel units (units 5, 7, and 9). An additional 32 single-well pumping tests, 7 multiple-well pumping tests, and 2 specific capacity tests for which the tested hydrogeologic unit has not been defined are included. The quality of these results is affected by both aquifer conditions and analysis procedures and varies widely (Thorne and Newcomer 1992). Slug tests have also been conducted at several Hanford Site wells. However, because many of the single-well slug test results are considered inaccurate, they have not been used to determine hydraulic properties for the proposed base conceptual model. Multiple-well slug tests have been conducted at a few wells in conjunction with multiple-well pumping tests. Because of vertical aquifer heterogeneity, and because most of the tested wells at Hanford partially penetrate the unconfined aquifer, it is sometimes difficult to determine the aquifer thickness that should be used in calculating hydraulic conductivity from the test results.

Aquifer tests (Thorne et al. 1993) indicate that the minimum $K_{h}$ is about $1 \mathrm{~m} / \mathrm{d}$ and the minimum $\mathrm{K}_{\mathrm{v}}$ is about $0.02 \mathrm{~m} / \mathrm{d}$ for unit 1 . The maximum measured value of $\mathrm{K}_{\mathrm{h}}$ for unit 1 on the Hanford Site is about $10,000 \mathrm{~m} / \mathrm{d}$ (Thorne and Newcomer 1992; DOE 1988). However, the maximum hydraulic conductivity that can be measured by an aquifer test is limited by the well efficiency and the flow rate that can be pumped with available equipment. As a result, the upper limit of $\mathrm{K}_{\mathrm{h}}$ for coarse gravel flood deposits of unit 1 is probably greater than the values interpreted from existing field tests. Maximum $\mathrm{K}_{\mathrm{v}}$ is unknown, but may approach the value for $\mathrm{K}_{\mathrm{h}}$ in relatively clean gravel zones where stratified layers of finer-grained material are not present.

Units 5, 7, and 9 are all within the Ringold Formation and consist of sand to muddy sandy gravel with varying degrees of consolidation and/or cementation. Unit 5 is the most widespread unit within the unconfined aquifer and is found below the water table across most of the model region. Hydraulic conductivities of units 5, 7, and 9 determined from aquifer tests vary within the range of about 0.1 to $200 \mathrm{~m} / \mathrm{d}$. Because these units are hydrologically similar, they were grouped together in areas where the intervening mud units do not exist. A few aquifer tests suggest vertical anisotropy is in the range of 0.01 to 0.1 . Therefore, the range of $\mathrm{K}_{\mathrm{v}}$ is estimated at about 0.001 to $20 \mathrm{~m} / \mathrm{d}$. 


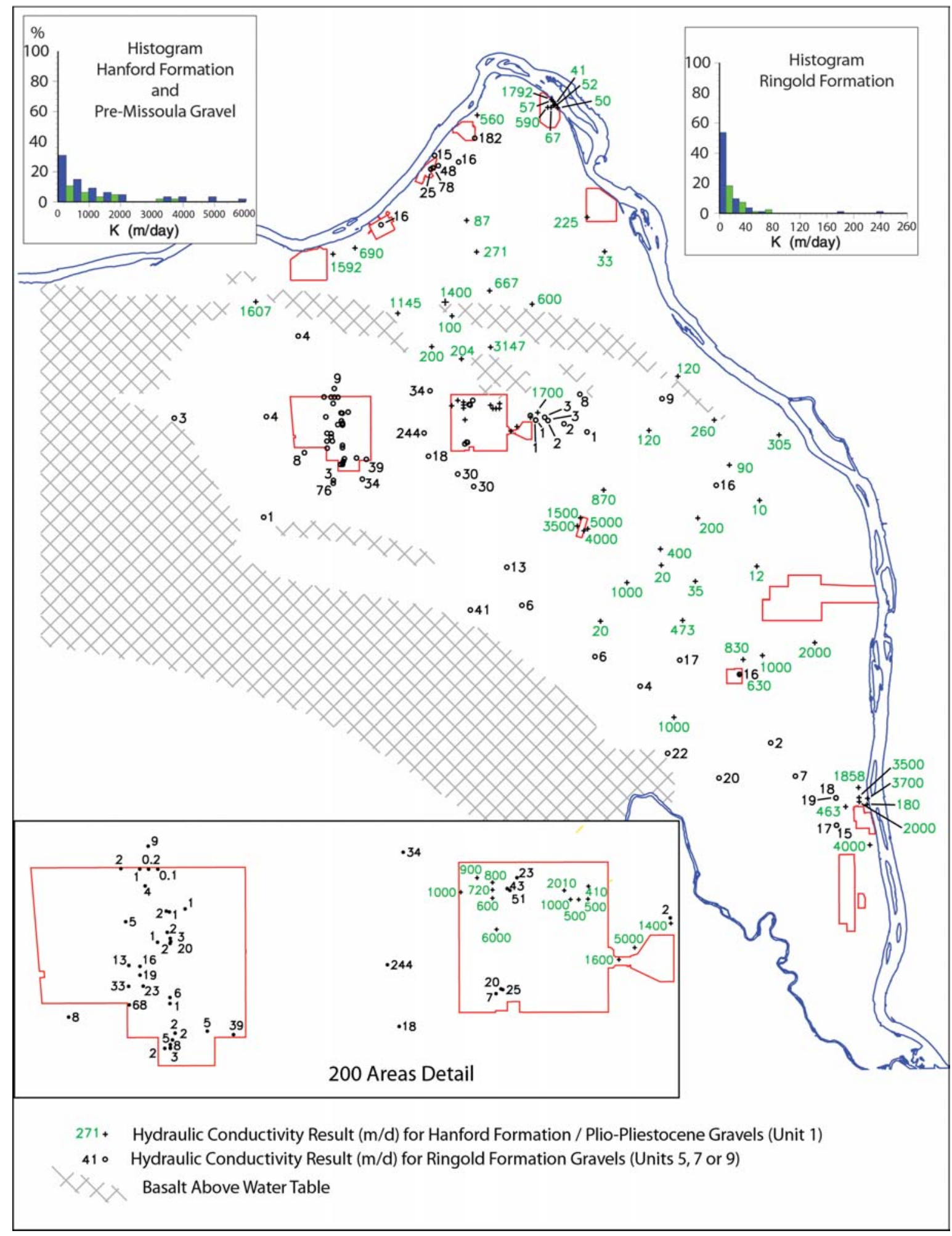

Figure 4.19. Distribution of Wells with Hydraulic Conductivity Determined from Aquifer Pumping Tests. 
Mud-dominated units within the unconfined aquifer system include unit 4, also known as the upper Ringold fines; unit 6, which is a composite of intercalated mud, sand, and gravel layers; and unit 8, which is an extensive lower Ringold mud unit. Hydraulic conductivity of these units is generally about 2 to 5 orders of magnitude less than that of the permeable sand and gravel units. Therefore, the mud units are essentially aquitards and are not expected to transmit significant quantities of water or contaminants in the horizontal direction. They are most significant in slowing the vertical migration of contaminants and influencing vertical head distributions. Therefore, the values of $\mathrm{K}_{\mathrm{v}}$ assigned to mud units are probably more important than the assigned values of $\mathrm{K}_{\mathrm{h}}$.

Hydraulic test results for mud-dominated units are listed in Table 4.3. These few tests yielded hydraulic conductivity (K) values of 0.0003 to $0.09 \mathrm{~m} / \mathrm{d}$. Some of the results are from well tests and some are from laboratory tests. Because of a tendency to complete wells only in zones that are likely to produce some water, these values may represent the higher range of $\mathrm{K}_{\mathrm{h}}$ for the mud units. Test results for unit 6 indicate that this unit has higher $\mathrm{K}_{\mathrm{h}}$ than unit 4 . This is expected because of the sand and gravel layers included in unit 6 . Unit 8 is expected to have hydraulic conductivity similar to unit 4 . Freeze and Cherry (1979) give a hydraulic conductivity range of 0.001 to $1 \mathrm{~m} / \mathrm{d}$ for silt and loess, and as low as $10^{-7} \mathrm{~m} / \mathrm{d}$ for clay. This range is partially based on a compilation of data by Davis (1969).

Table 4.3. Hydraulic Test Results for Mud-Dominated Units

\begin{tabular}{||l|c|l||}
\hline Hanford Well Number & Hydraulic Conductivity (K) (m/d) & Hydrogeologic Unit \\
\hline $299-W 7-9$ & 0.09 & Unit 4 (vadose) \\
\hline $699-20-39$ & $<0.06$ & Unit 6 \\
\hline $699-84-35$ A & 0.03 & Unit 6 \\
\hline $699-41-40$ & 0.0003 & Unit 4 \\
\hline
\end{tabular}

\subsection{Storativity and Specific Yield}

Storativity and specific yield can be calculated from multiple-well pumping and slug interference tests (Spane 1993, 1994). The average specific yield from these tests was 0.15. However, some of the test results are highly uncertain because of the effects of nonideal test conditions, such as partially penetrating wells and aquifer heterogeneity. Such conditions generally have a more significant effect on the determination of storage properties than on the determination of transmissivity. Moench (1994) demonstrated that these conditions can affect specific yield values calculated from type-curve analysis of aquifer pumping tests, and usually result in the calculated values being low.

Specific yield can also be calculated by measuring the change in saturated aquifer volume in response to the injection or withdrawal of a known volume of groundwater. This method was applied to the decreasing groundwater mound that occurred beneath the 200 West Area between 1985 and 1995 (Wurstner et al. 1995). The calculated specific yield was 0.17 , which is higher than the 0.11 value calculated by Newcomb and Strand (1953) when they analyzed the growth of groundwater mounds beneath liquid disposal facilities in both the 200 West and 200 East areas between 1948 and 1953. The accuracy of results from both these analyses is uncertain because the analyses assume that steady state 
conditions have been reached at the end of the analyzed period. Small head changes on the fringes of the mound are also difficult to measure and may have a significant impact because of the large area they cover.

Thorne et al. (1994) review of specific yield and storativity data for Hanford suprabasalt sediments is summarized here. Specific yield for unit 1 is estimated to range from about 0.1 to 0.3 . The specific yield is expected to be higher for coarse, well-sorted gravels than for poorly sorted mixtures of sand and gravel. Storativity is estimated to range from 0.0001 to 0.0005 . Specific yield is estimated to range from 0.05 to 0.2 for the generally poorly sorted sediments of units 5,7 , and 9 . Storativity is estimated to range from 0.0001 to 0.001 for these units.

A summary of the current estimates of hydraulic parameters and ranges interpreted from field and laboratory tests for the nine layers of the base case groundwater conceptual model is provided in Table 4.4.

\subsubsection{Local Aquifer Transport Parameters}

To accurately model contaminant transport using the advective-dispersion equation to describe spreading and the linear isotherm process to describe contaminant velocity, parameters including effective porosity, dispersivity, and retardation coefficients must be specified. Longitudinal and transverse dispersivity values are needed for a three-dimensional model. Retardation coefficients are specific to each contaminant species in association with the groundwater and host sediments. Thus, retardation coefficients may vary spatially and temporally depending on geochemical conditions within the aquifer. However, in the current SGM, retardation coefficients are spatially constant for each contaminant species. Information on retardation coefficients for Hanford unconfined aquifer sediments is available in Ames and Serne (1991) and Kaplan and Serne (1995).

\subsection{Effective Porosity}

Porosity is defined as the volume of void space divided by the total volume of the soil or rock matrix that contains it. Effective porosity does not include void space that is isolated from groundwater flow and, therefore, may be smaller than the total porosity. The average velocity of a conservative contaminant (nonsorbing and nondecaying) as it moves through an aquifer is equal to the average linear velocity of the groundwater, which is inversely proportional to the effective porosity of the aquifer matrix (Freeze and Cherry 1979). Porosity can be determined from laboratory measurements on samples of aquifer material or from field tracer tests. For unconfined aquifers, effective porosity can sometimes be approximated by the specific yield value determined from multiple-well hydraulic tests.

Laboratory measurements of porosity are available for samples from only a few of the available Hanford Site wells. Recently, 15 samples were collected from 6 wells at the $100 \mathrm{H}$ Area (Vermeul et al. 1995). Porosity ranged from 0.19 to 0.41 and averaged 0.33 for the Ringold Formation and 0.31 for the Hanford formation. Samples from five depth intervals within the Ringold Formation at the 200 West 
Table 4.4. Summary of Current Hydraulic Parameter Estimates Interpreted from Field and Laboratory Tests for the Conceptual Model Hydrogeologic Units.

\begin{tabular}{|c|c|c|c|c|}
\hline $\begin{array}{c}\text { Model } \\
\text { Unit } \\
\text { Number }\end{array}$ & $\begin{array}{l}\text { Horizontal } \\
\text { Hydraulic } \\
\text { Conductivity } \\
\mathbf{K}_{\mathrm{h}}(\mathbf{m} / \mathbf{d})\end{array}$ & $\begin{array}{c}\text { Storativity } \\
\text { (dimensionless) }\end{array}$ & $\begin{array}{l}\text { Specific Yield } \\
\text { (dimensionless) }\end{array}$ & Comments \\
\hline 1 & 10 to $>3.5 \mathrm{E}+3$ & 0.001 to 0.005 & 0.2 to 0.37 & $\begin{array}{l}\text { The water table surface is } \\
\text { present in this layer in most of } \\
\text { the eastern portion of the Site as } \\
\text { shown in Figure } 4.6 \text {. }\end{array}$ \\
\hline 2 & $\mathrm{~N} / \mathrm{A}$ & $\mathrm{N} / \mathrm{A}$ & $\mathrm{N} / \mathrm{A}$ & $\begin{array}{l}\text { Currently, where this unit } \\
\text { occurs on the Site, the water } \\
\text { table is below this unit. }\end{array}$ \\
\hline 3 & $\mathrm{~N} / \mathrm{A}$ & $\mathrm{N} / \mathrm{A}$ & $\mathrm{N} / \mathrm{A}$ & $\begin{array}{l}\text { Currently, where this unit } \\
\text { occurs on the Site, the water } \\
\text { table is below this unit. }\end{array}$ \\
\hline 4 & 0.0003 to 0.09 & no data & no data & $\begin{array}{l}\text { The hydraulic conductivity is } \\
\text { assumed to be a constant value } \\
\text { in this layer. }\end{array}$ \\
\hline 5 & 0.1 to 560 & 0.0001 to 0.06 & 0.05 to 0.37 & $\begin{array}{l}\text { This layer occurs at the water } \\
\text { table in the western portion of } \\
\text { the Site as shown in Figure 4.6. }\end{array}$ \\
\hline 6 & 0.002 to 0.03 & no data & no data & $\begin{array}{l}\text { Layer } 6 \text { is not present in } \\
\text { western portions of the Site. }\end{array}$ \\
\hline 7 & $\begin{array}{c}\text { no data except } \\
\text { composite zones, } \\
\text { assume similar to } \\
\text { unit } 5\end{array}$ & no data & no data & $\begin{array}{l}\text { The hydraulic conductivity is } \\
\text { assumed to be a constant value } \\
\text { in this layer. }\end{array}$ \\
\hline 8 & no data & no data & no data & $\begin{array}{l}\text { The hydraulic conductivity is } \\
\text { assumed to be a constant value } \\
\text { in this layer. }\end{array}$ \\
\hline 9 & 8 (only one test) & 0.002 & 0.15 & \\
\hline
\end{tabular}

Area were reported by Newcomer et al. (1995). The porosity ranged from 0.21 to 0.33 and averaged 0.27 . Laboratory porosity measurements are often considered unreliable because of the difficulty in obtaining undisturbed samples, especially for unconsolidated sediments.

A few tracer tests have been conducted within the unconfined aquifer. Bierschenk (1959) reported an effective porosity of 0.10 from a tracer test with fluoresce in dye under natural gradient conditions. Single borehole dilution tests, which do not provide information on porosity, were conducted by Graham et al. (1984). An effective porosity of 0.25 was assumed to calculate average groundwater velocity from 
the measurements. Borehole dilution tests and a two-well tracer test were conducted in the 200 West Area (Newcomer et al. 1995) under natural gradient conditions. However, porosity could not be determined from the two-well tracer test because the gradient was not well defined.

Porosity can also be estimated from measurements of aquifer specific yield. Specific yield is defined as the volume of water released from a unit area of an unconfined aquifer per unit decline in hydraulic head. Specific yield and effective porosity are equivalent if drainage of the aquifer matrix is complete. However, in reality, the specific yield may be lower than the effective porosity because of water held in pore spaces of the drained aquifer matrix by surface tension or adsorptive forces (Moench 1994).

Mud-dominated units generally have higher porosity than sand- and gravel-dominated units. Davis (1969) compiled porosity values that indicate ranges of 0.35 to 0.5 for silts and 0.4 to 0.7 for clays. However, because of the low permeability of such sediments, the porosity assigned to mud units in the model is not expected to have a major impact on model results.

\subsection{Dispersivity}

The following discussion on dispersivity summarized from Kincaid et al. (1995) illustrates the factors that go into the selection of dispersivity values. Dispersivity is determined by inverse modeling of tracer test breakthrough curves from tests performed at the transport scale of interest and in the geohydrologic system of interest (Farmer 1986). Dispersivity has been called "the most elusive of the solute transport parameters" (Freeze and Cherry 1979) because it cannot be directly measured in the field or laboratory. Freeze and Cherry (1979) indicate that field-scale values of longitudinal and transverse dispersivities are significantly larger than values obtained in laboratory experiments on homogeneous materials and materials with simple heterogeneity. No field tests have been conducted at the Hanford Site to develop an estimate for this parameter at the scale of transport appropriate for the Site Groundwater Conceptual Model.

General studies indicate that dispersivity is a function of both time and transport distance because of unaccounted for temporal changes and unaccounted for heterogeneities. The U.S. Environmental Protection Agency (EPA), in their guidance for water quality assessment screening for toxic and conventional pollutants in surface and groundwater (Mills et al. 1985), indicates "A rough estimate of longitudinal dispersivity in saturated porous media may be made by setting $\mathrm{D}_{1}(\mathrm{~cm})$ equal to $10 \%$ of the mean travel distance." This rule of thumb is based on analysis of tracer tests performed over a large range of laboratory and field scales and for a wide variety of aquifers.

The original work was performed by Lallemand-Barres and Peaudecerf (1978) and expanded by Gelhar and Axness (1981). Later in 1992, Gelhar, Welty, and Rehfeldt reexamined the data and indicated that because of the potential unreliability of the data, no definite conclusion regarding the rule could be reached beyond transport distances of $100 \mathrm{~m}$. However, this was later refuted by Neuman (1993).

Dispersivity is theoretically expected to have an asymptotic value that can be related to the scale of uncharacterized aquifer heterogeneity (Farmer 1986). In contaminant transport simulations, large values of dispersivity result in lower peak concentration estimates, but give rise to earlier first arrival times that 
can increase arrival concentrations of radionuclides with short half-lives. Freeze and Cherry (1979) observed that longitudinal dispersivities as large as $100 \mathrm{~m}$ and lateral dispersivities as large as $50 \mathrm{~m}$ have been used in migration studies of large contaminant plumes. As discussed in Wurstner et al. (1995), the 1/10 approach has generally been used in the past for determining dispersivity values for Hanford Site transport modeling. Law (1992) used values of $D_{1}=43 \mathrm{~m}$ and $D_{t}=12 \mathrm{~m}$ for a scale of $9500 \mathrm{~m}$ based on values compiled in Gelhar et al. (1985). An earlier model (Golder Associates 1990) used values of $15 \mathrm{~m}$ and $1.5 \mathrm{~m}$ for longitudinal and transverse dispersivity, which were also based on Gelhar et al. (1985).

It also should be recognized that the dispersivity values determined from field tests at 59 different sites, compiled by Gelhar et al. (1992), included results from two investigations at the Hanford Site. The first included two 1950s tracer tests that resulted in values of $D_{1}=6 \mathrm{~m}$ for the Hanford formation and $\mathrm{D}_{1}=460 \mathrm{~m}$ for the Ringold Formation, as reported by Bierschenk (1959) and analyzed by Cole (1979). The scales of these tests were $3500 \mathrm{~m}$ and $2600 \mathrm{~m}$, respectively. Also included are values of $\mathrm{D}_{1}=30.5 \mathrm{~m}$ and $D_{t}=18.3 \mathrm{~m}$ for a scale of $20,000 \mathrm{~m}$. These were calculated from two-dimensional transport modeling of the 200 East Area tritium plume as reported in Ahlstrom et al. (1977).

Dispersivity is likely to vary across the Hanford Site depending on the degree of heterogeneity and the temporal variability of flow gradients. Ahlstrom et al. (1977) noted that the ratio of $D_{1}$ to $D_{t}$ calculated from their model of the Hanford Site was much higher than the ratio expected. They attributed the high ratio to heterogeneity. However, horizontal dispersion may have been enhanced by temporal variations in flow gradients caused by disposal practices. The flow paths for the tritium transport from the 200 East Area have gradually shifted from due east to a south-easterly direction, in response to wastewater discharges to B Pond and the 200 East Area. This shift in the flow path has enhanced the apparent dispersion of the tritium plume emanating from the 200 East Area. More recent site-wide modeling studies (Law et al. 1996) used values of $D_{1}$ and $D_{t}$ of $30.5 \mathrm{~m}$ and $3 \mathrm{~m}$, respectively. They appear to be related to the transport grid spacing of $100 \mathrm{~m}$. In the recent Hanford Low-level Tank Waste Interim Performance Assessment (Mann et al. 1997) the horizontal dispersivity for aquifer transport was set at $10 \%$ of the travel length in the direction of flow and in the vertical direction at $1 \%$ of the travel length.

For the Composite Analysis (Kincaid et al. 1998), a longitudinal dispersivity, $\mathrm{D}_{1}$, of $95 \mathrm{~m}$ was selected. While the value of $\mathrm{D}_{1}=95 \mathrm{~m}$ is not based on any Hanford Site data, it satisfies all three of the following constraints on its value

1. The numerical constraint is related to the grid Peclet number, $P_{e}=$ (grid spacing)/ $D_{1}$. For finite element transport simulations $\mathrm{P}_{\mathrm{e}}<4$ are required for acceptable solutions (Campbell et al. 1981). The 95-m dispersivity estimate is approximately $1 / 4$ of the grid spacing in the finest part of the model grid in the 200 Area Plateau where the smallest grid spacing is on the order of about $375 \mathrm{~m}$ by $375 \mathrm{~m}$.

2. At the grid scale of $375 \mathrm{~m}$ used for the Composite Analysis modeling, the modeled system is homogeneous. Heterogeneities at scales less than $375 \mathrm{~m}$ are uncharacterized. The $95-\mathrm{m}$ dispersivity value selected satisfies this constraint. 
3. Finally, because it is more than $10 \mathrm{~km}$ from the closest source in the 200 East Area to the Columbia River, a nonasymptotic value of $1000 \mathrm{~m}$ for the longitudinal dispersivity could be appropriate if the $10 \%$ rule is applied and the heterogeneities at scales greater than the $375 \mathrm{~m}$ grid scale were not characterized. The 95-m dispersivity value selected for use in the Composite Analysis was the smallest value (i.e., the most conservative value from a plume spreading perspective) that could be used with the selected grid. Applying the rule of thumb, discussed above, estimates of concentration at a distance of $950 \mathrm{~m}$ from the source should be accurate, and for greater distances, they should be conservative if it is recognized that there is still a significant heterogeneities uncharacterized at scale greater than $375 \mathrm{~m}$.

With regard to transverse dispersivity the following is noted:

- EPA guidance (Mills et al. 1985) is $1 / 3$ for the ratio of $D_{1} / D_{t}$

- Freeze and Cherry (1979) indicate transverse dispersivity is lower by a range of 5 to 20 (i.e., 0.2 to 0.05 )

- Walton (1985) states that reported ratios of $\mathrm{D}_{\mathrm{l}} / \mathrm{D}_{\mathrm{t}}$ vary from 1 to 24 but that common values are $1 / 5$ and $1 / 10$.

As an example, the Composite Analysis in applying this guidance assumed the transverse dispersivity, $\mathrm{D}_{\mathrm{t}}$, was approximately $20 \%$ of the longitudinal dispersivity or about $20 \mathrm{~m}$.

In future ACMs, appropriate dispersivity values for movement of large-scale plumes may be determined as part of the flow and transport inverse modeling of the tritium disposals from 1943 to present.

\subsubsection{Uncertainty in Hydraulic and Transport Parameter Estimates}

Heterogeneity of a particular unit may not be adequately represented when assigning hydraulic properties based on relatively few measurements. Estimation of hydraulic properties through inverse modeling to match observed heads will lead to averaging of hydraulic properties over areas where head data is sparse. Geostatistical analyses coupled with inverse modeling could potentially provide best estimates of the hydraulic parameters and their spatial distribution and continuity. Further, geostatistics and inverse modeling can provide the basis for a complete stochastic model that quantifies the uncertainty in our estimated parameters, as well as the resulting uncertainty in predictions of flow and transport.

\subsection{Columbia River Boundary}

The Columbia River forms the northern and eastern boundary of the SGM domain. The Columbia River is believed to be the regional discharge zone for the entire unconfined aquifer system within the Pasco Basin. Interpreted water table elevation contours (Figure 4.7) support this assumption. All available data indicate that groundwater from the shallow flow system discharges to the Columbia River from both banks. 
The flow rate of the Columbia River in the Hanford Reach fluctuates significantly and is controlled primarily by releases from the upstream Priest Rapids Dam. Annual flows near Priest Rapids during the 68 years prior to 1985 averaged nearly $3360 \mathrm{~m}^{3} / \mathrm{s}$ (McGavock et al. 1987). Daily average flows during this period ranged from 1000 to $7000 \mathrm{~m}^{3} / \mathrm{s}$. During the last 10 years, the average daily flow was also about $3360 \mathrm{~m}^{3} / \mathrm{s}$. However, larger than normal snowpacks resulted in exceptionally high spring runoff during 1996 and 1997. The peak daily average flow rate during 1997 was nearly $11,750 \mathrm{~m}^{3} / \mathrm{s}$ (DART 1998). Average daily flows from 1991 through 2001 are plotted in Figure 4.20. Average flows during 2001 were lower than normal because of drought conditions.

There are both seasonal and daily fluctuations in flow, which also cause fluctuations in river stage. As shown in Figure 4.20, seasonal flows typically peak from April through June, during spring runoff from snowmelt, and are lowest from September through October. The seasonal change in average water level is up to about $2 \mathrm{~m}$. Daily fluctuations in discharge are caused by releases from Priest Rapids Dam based on demand for power production. Because of these changes in flow, the river stage varies significantly over a short time period. Vertical fluctuations of more than $1.5 \mathrm{~m}$ during a 24-hour period are common along the Hanford Reach (Dirkes and Hanf 1996).

The width of the river varies from approximately $300 \mathrm{~m}$ to $1000 \mathrm{~m}$ at the Hanford Site. Transects of the river bottom elevation have been measured about every $1 \mathrm{~km}$. Figure 4.21 shows the river cross section at three transect locations and the high and low river stage at each transect. The location of these transects is shown in Figure 4.22. The maximum depth of the Columbia River in the area adjacent to the Hanford Site is about $11 \mathrm{~m}$.

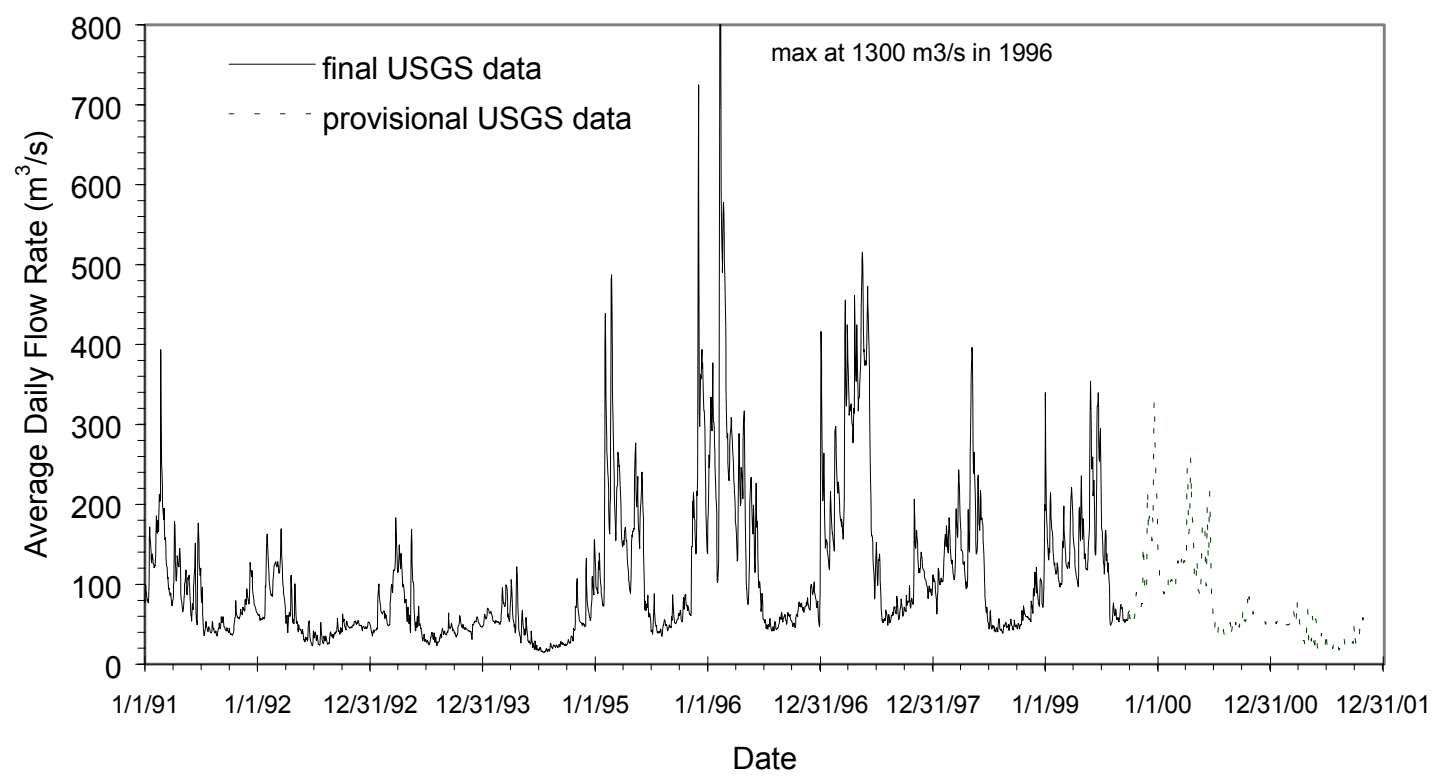

Figure 4.20. Average Daily Flows of the Columbia River from 1991 Through 2001. 

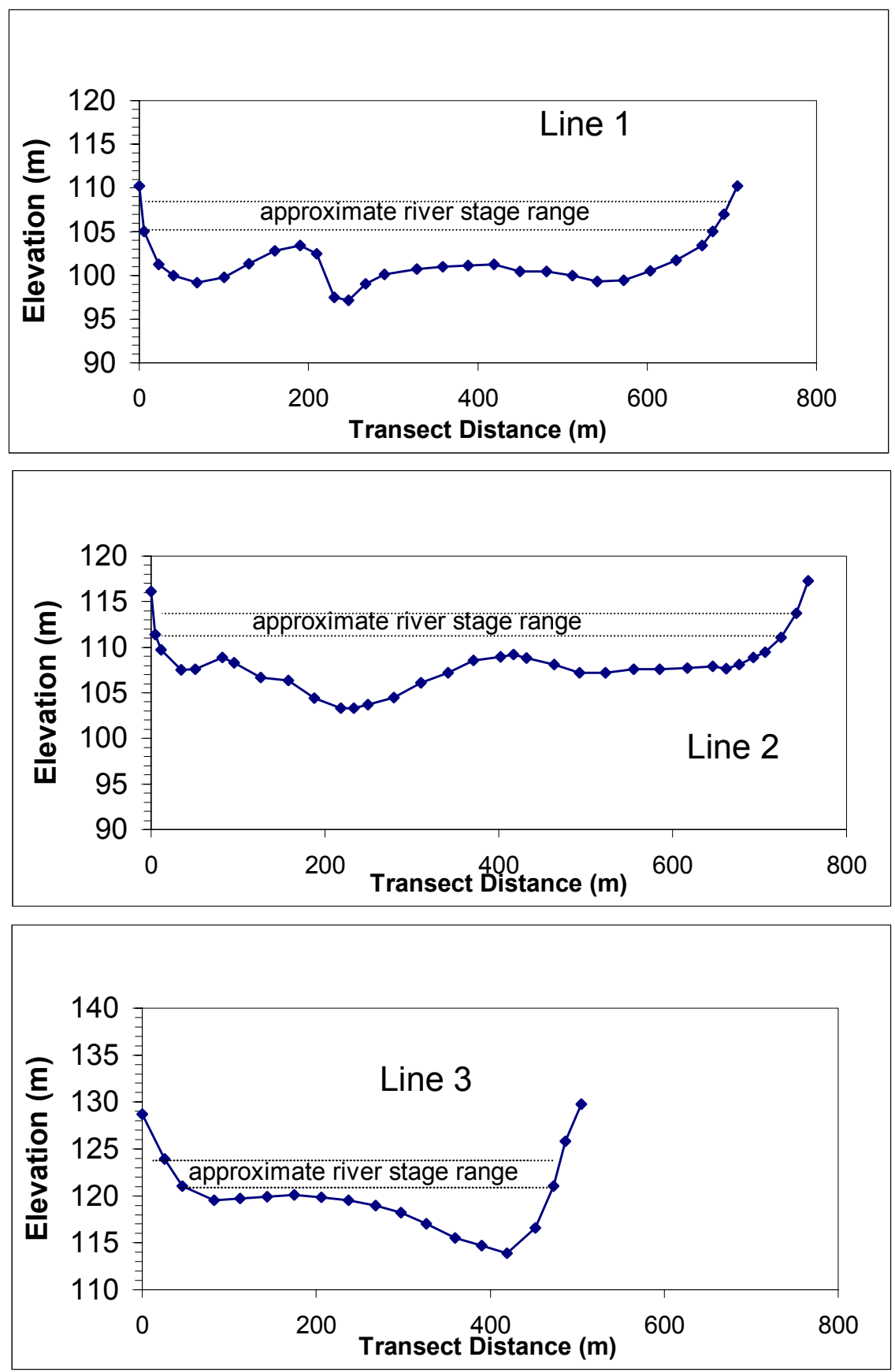

Figure 4.21. Cross Sections of the Columbia River at Three Locations Showing High and Low River Stages. 


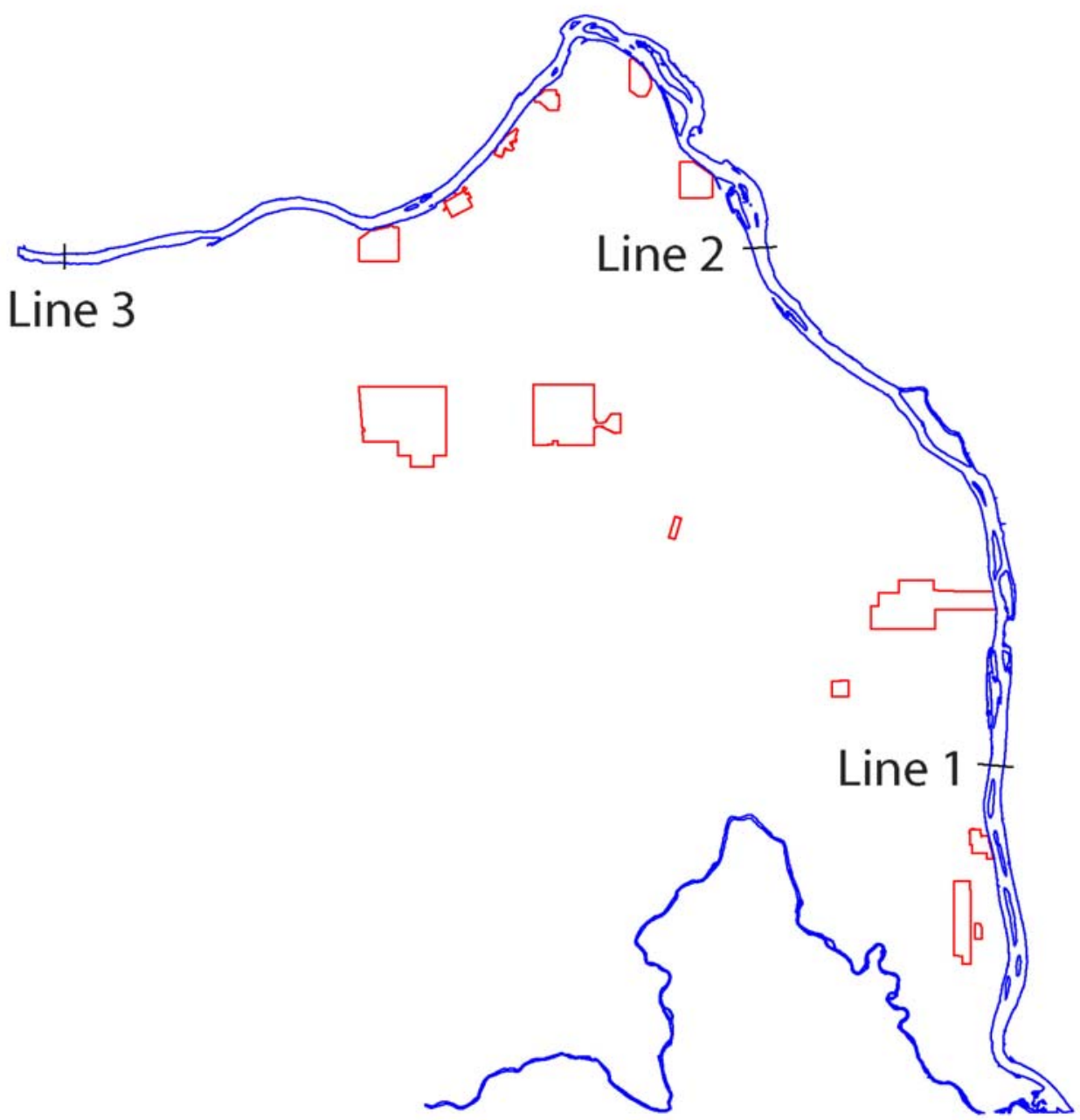

Figure 4.22. Location of Cross Sections Shown in Figure 4.21.

The Columbia River is generally in Hanford formation sediments at the Hanford Site. However, it may be in contact with Ringold sediments or recent alluvial deposits in some locations. Geologic cross sections, from Lindsey (1995), that cross the river are shown in Figures 4.23 through 4.25. The vertical scales of these cross sections and the transects shown in Figure 4.21 are exaggerated. The Columbia River is in fact relatively wide compared to the thickness of the aquifer. Figure 4.26 shows a cross section through the aquifer at $100 \mathrm{H}$ Area with no vertical exaggeration.

\subsubsection{Interaction Between Groundwater and the Columbia River}

Water levels in the aquifer adjacent to the Columbia River fluctuate in response to changes in river stage, following pressure "waves" transmitted through the unconfined aquifer. In addition, some water also moves temporarily between the aquifer and the river due to "bank storage" effects. Small-diameter 

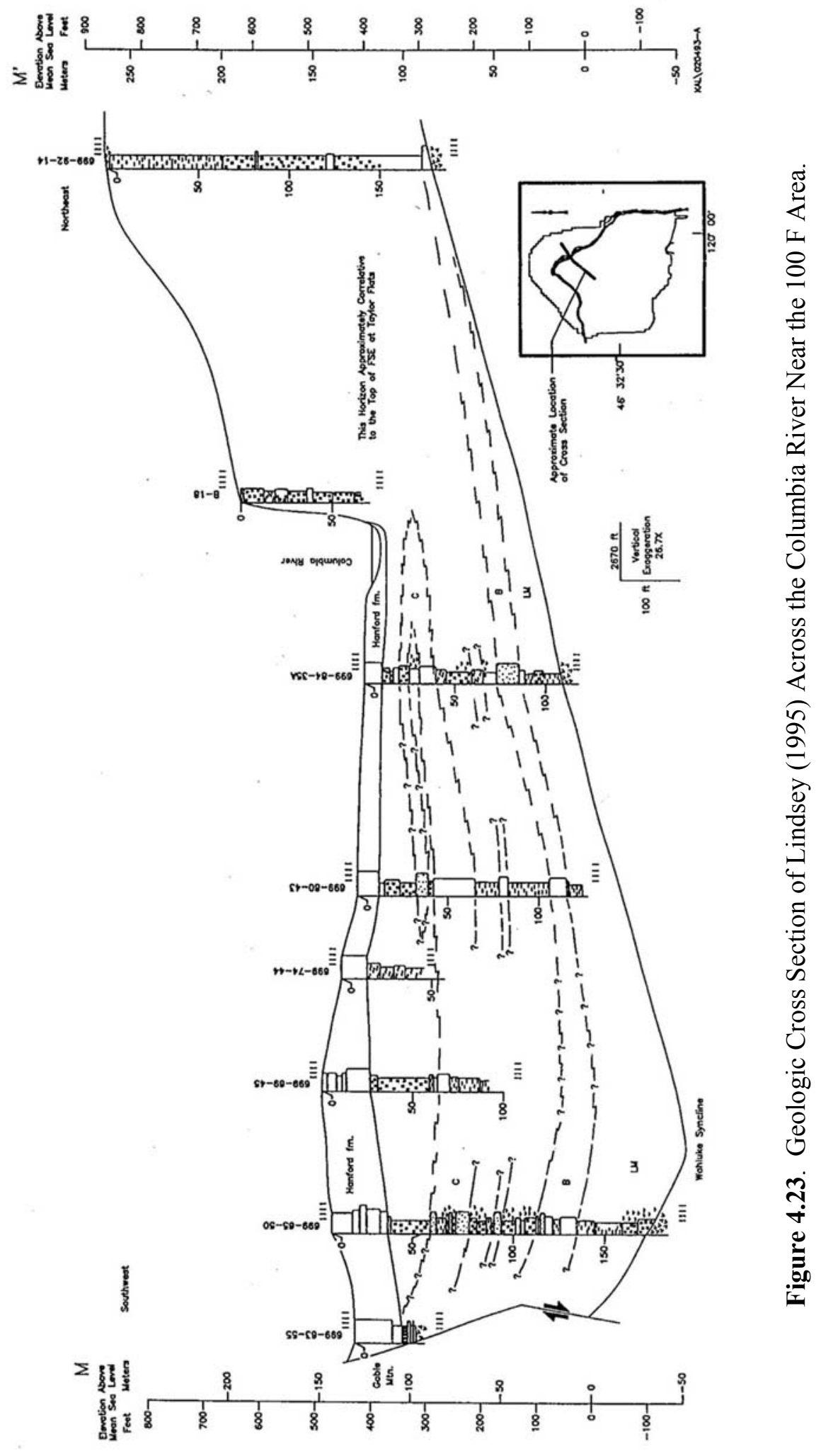


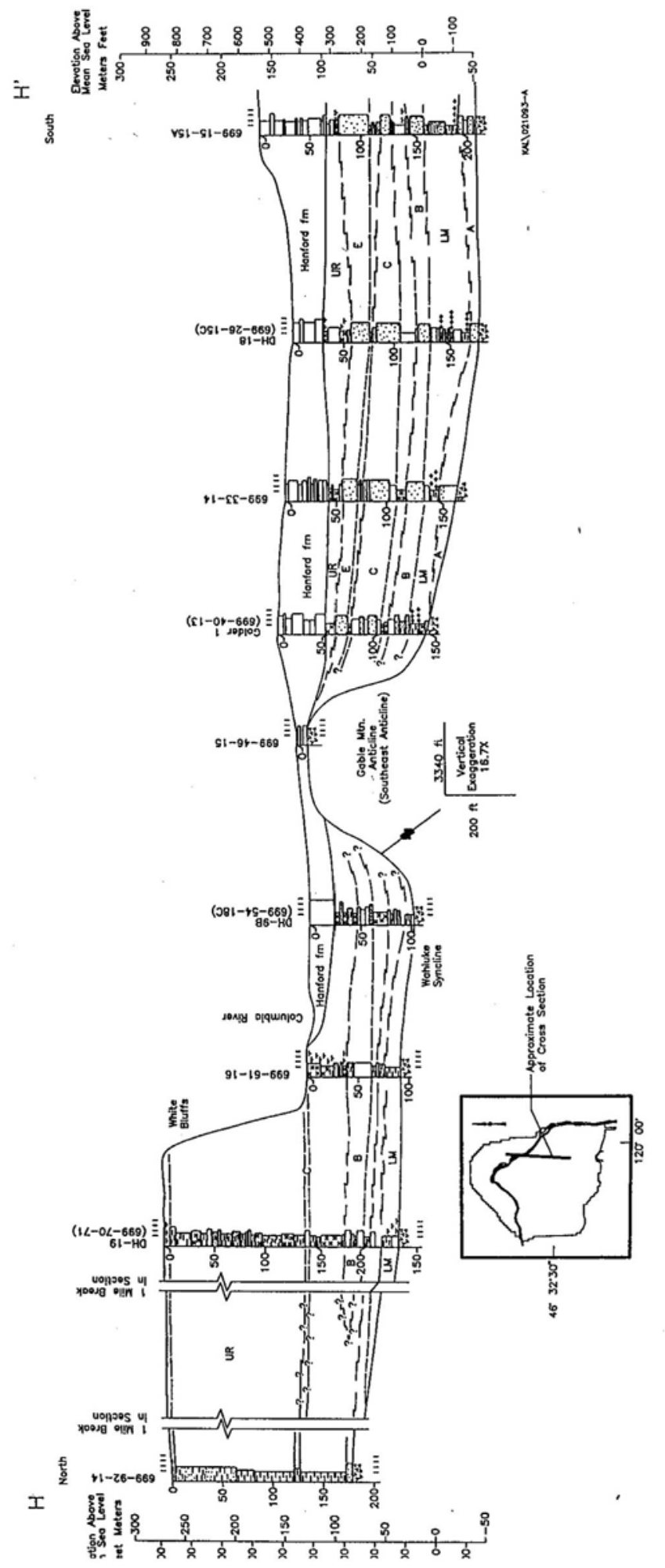

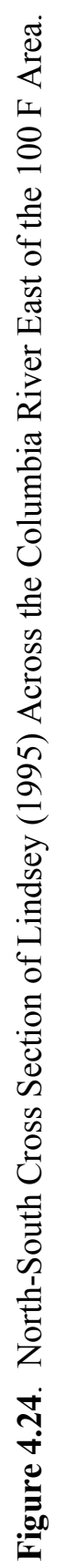




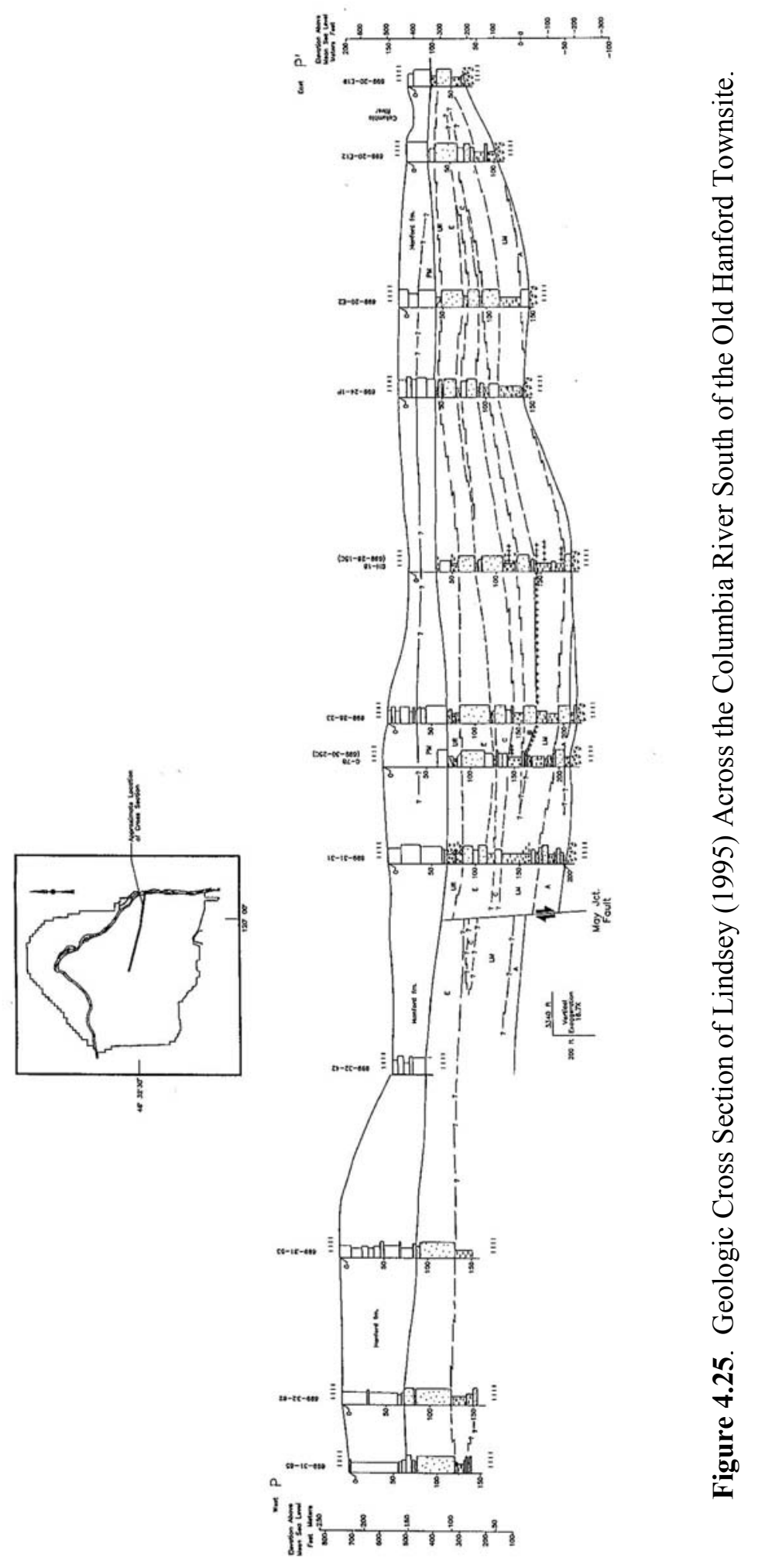



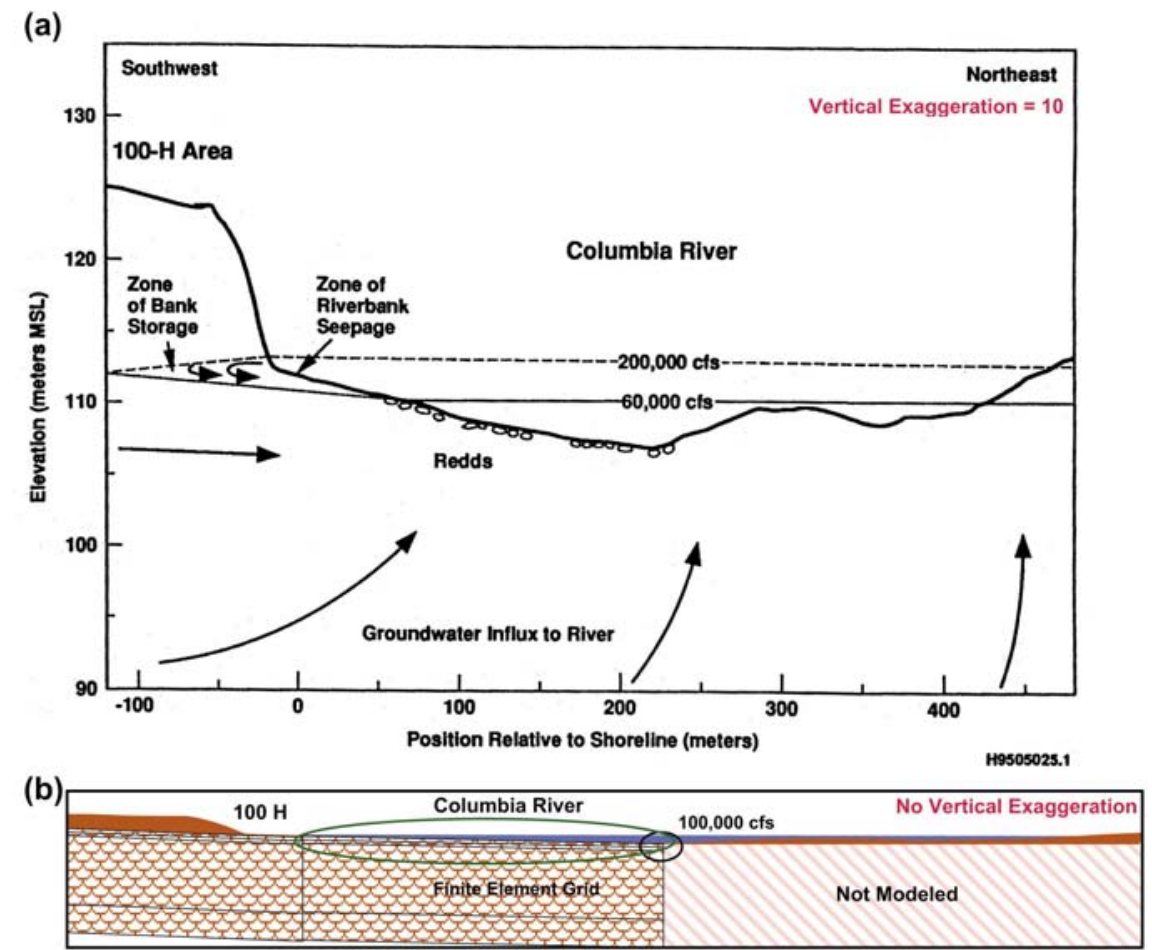

Figure 4.26. Generalized Cross Section of the Groundwater/Columbia River Interface at $100 \mathrm{H}$ (modified from Barnett et al. 1995). Illustrating: (a) The change in river width with stage, and (b) the relationship of river width to the thickness of the underlying suprabasalt sediments of the unconfined aquifer system and the finite element representation of a dirichlet river node.

plastic sampling tubes have recently been installed at multiple depths in the aquifer along the 100 Area shoreline to increase the understanding of the groundwater/river interface. These tubes monitor conditions within or very close to the interface between groundwater and river water.

Hydrographs showing the influence of the river stage on the unconfined aquifer at various locations along the Columbia River are presented by McMahon and Peterson (1992) and Campbell et al. (1993). Figure 4.27 shows water level responses at three wells compared to river stage fluctuations. The well locations are shown in Figure 4.28. Water-level responses to river stage have been observed up to $2 \mathrm{~km}$ from the river shore. Most of these wells show only a response to seasonal river stage changes. However, a few wells very near the river also respond to daily fluctuations as shown in Figure 4.27.

\subsubsection{Uncertainty in Model Representation of the Columbia River}

In the SGM, the Columbia River is presently represented as a prescribed head (Dirichlet) boundary over the entire thickness of the aquifer. In order to determine the appropriate head, the CHARIMA river-simulation model (Walters et al. 1994) was used to generate long-term average river-stage 


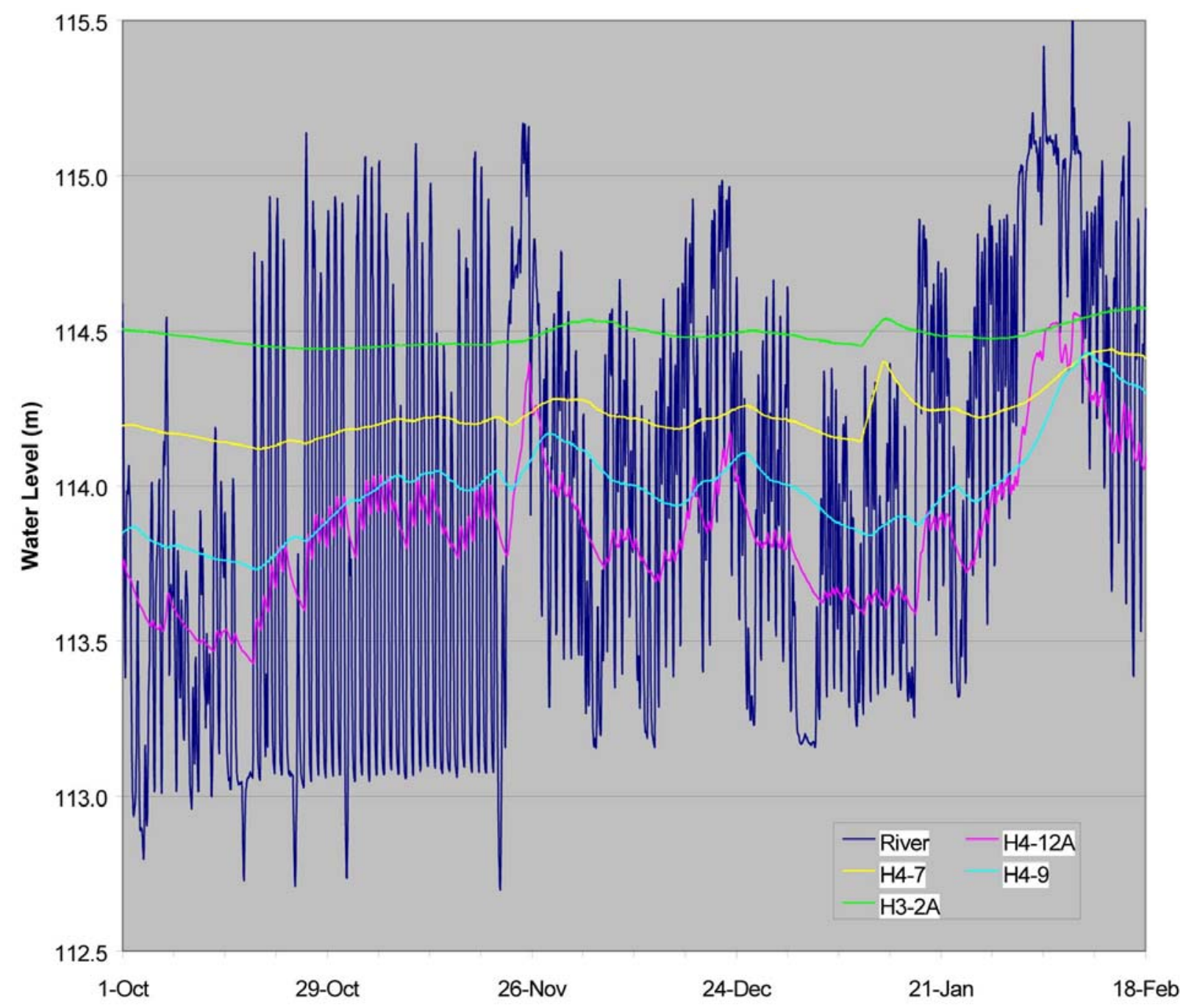

Figure 4.27. Water-Level Responses at Three Wells Compared to Columbia River Stage Fluctuations.

elevations for the Columbia River based on 1979 conditions. This boundary condition is placed at the middle of the river channel. The use of a held-head boundary at the Columbia River is based on several factors:

- the average river stage is lower than the water table elevation on both sides of the river

- groundwater appears to flow into the river from both sides and there is no evidence of flow across the river

- there is good hydraulic connection between the river and the aquifer based on the occurrence of water-level fluctuations in wells and the relatively high permeability of the sediments along the river

- the river is wide relative to the thickness of the aquifer. 


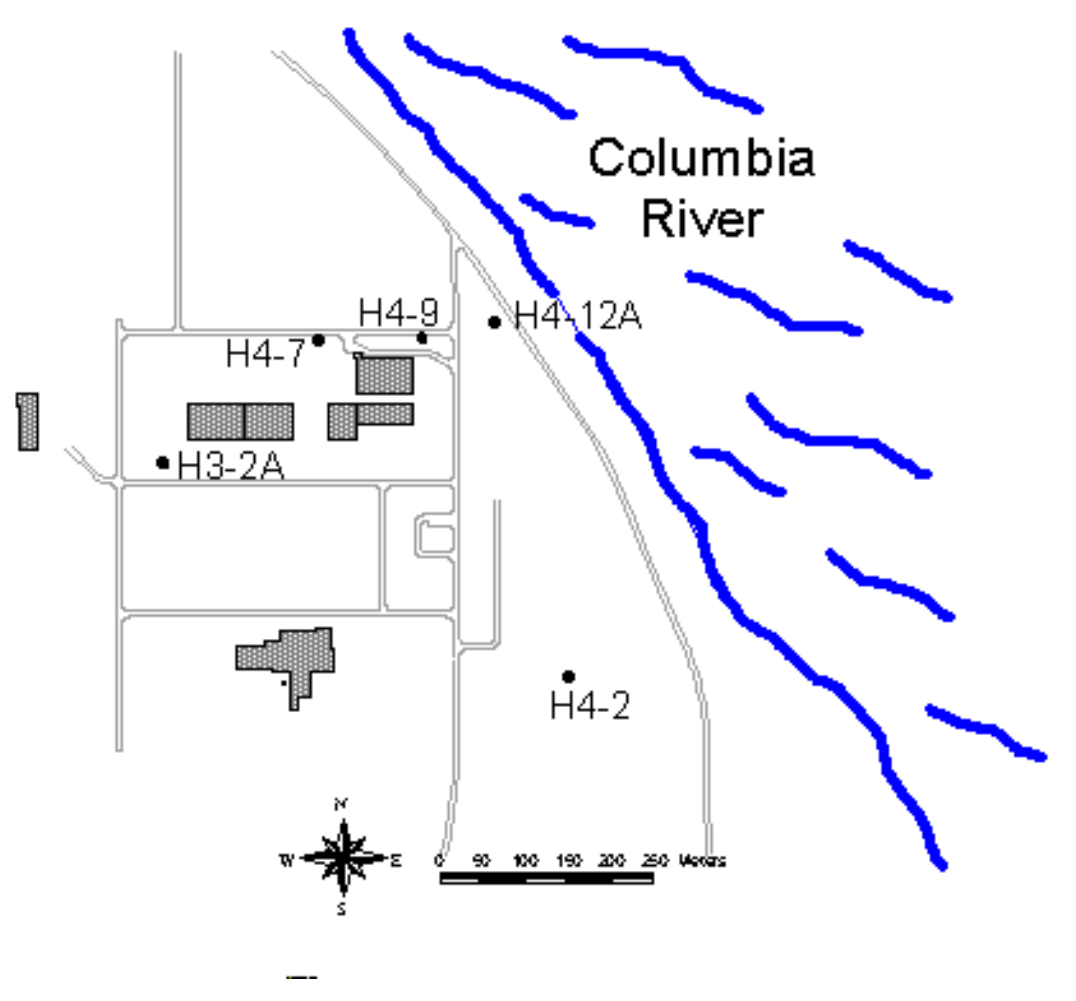

Figure 4.28. Locations of Wells in Figure 4.27.

The value of the prescribed head varies according to varying river stages over periods of time consistent with model time steps. The purpose and resolution required in the simulation determines the need for including the temporal variation in this boundary condition in a particular simulation. The Dirichlet condition is applied along the river bottom from the edge to the center of the river and a no-flow boundary is assumed to exist vertically under the middle of the river as illustrated in Figure 4.26. Other possible conceptual models include: 1) set boundary condition at the shoreline rather than center of the river, 2) seasonal river stage fluctuations, and 3) move boundary a certain distance beyond the river and implement it as a head-dependent flux boundary.

Due to the large spatial and time scales of simulations (tens of kilometers and up to thousands of years), the hydraulic heads along the river are assumed to be constant with time. This assumption ignores the seasonal and daily fluctuations in river stage. Near the river, these fluctuations may be important because of bank storage effects. Clean water moving into and out of the aquifer would dilute the concentration of contaminants in the groundwater and may cause contaminants to move some distance downstream parallel to the river before leaving the aquifer. Effects of river stage changes on groundwater near the river have been simulated by using a cross-sectional pathline model near the $100 \mathrm{~N}$ Area (Connelly 1998). This work showed a delay in contaminants reaching the river because each particle takes a circuitous route rather than moving directly to the riverbank. Movement of water in and out of the aquifer can also increase the release of sorbed contaminants in sediments near the river as clean water 
moves in and out of the contaminated material. However, these effects are only significant locally, close to the river, and are not expected to have an effect on large-scale and long-term transport predictions.

\subsection{Yakima River Boundary}

The Yakima River follows the southwestern boundary of the Hanford Site for about $2 \mathrm{~km}$, then flows southwest of Richland, partially defining the southern extent of the SGM. Approximately 1/3 of the Hanford Site is drained by the Yakima River system.

The Yakima River carries much less flow than the Columbia River. The average flow, based on nearly 60 years of records, is about $104 \mathrm{~m}^{3} / \mathrm{s}$, with an average monthly maximum of $490 \mathrm{~m}^{3} / \mathrm{s}$ and minimum of $4.6 \mathrm{~m}^{3} / \mathrm{s}$. Exceptionally high flows were observed during 1996 and 1997 . The peak average daily flow rate during 1997 was nearly $1300 \mathrm{~m}^{3} / \mathrm{s}$. Average daily flows for the Yakima River at Kiona from 1991 through 2001 are plotted in Figure 4.29. Flows during 2001 were lower than normal because of drought conditions.

There have been fewer than 20 major floods on the Yakima River since 1862 (DOE 1988). The most severe occurred in November 1906, December 1933, May 1948, and February 1996; discharge magnitudes at Kiona, Washington, were $1870,1900,1050$, and $1300 \mathrm{~m}^{3} / \mathrm{s}$, respectively. The recurrence intervals for the 1933 and 1948 floods are estimated at 170 and 33 years, respectively. The development of irrigation reservoirs within the Yakima River Basin has considerably reduced the flood potential of the river. The southern border of the Hanford Site could be susceptible to a 100-year flood on the Yakima River.

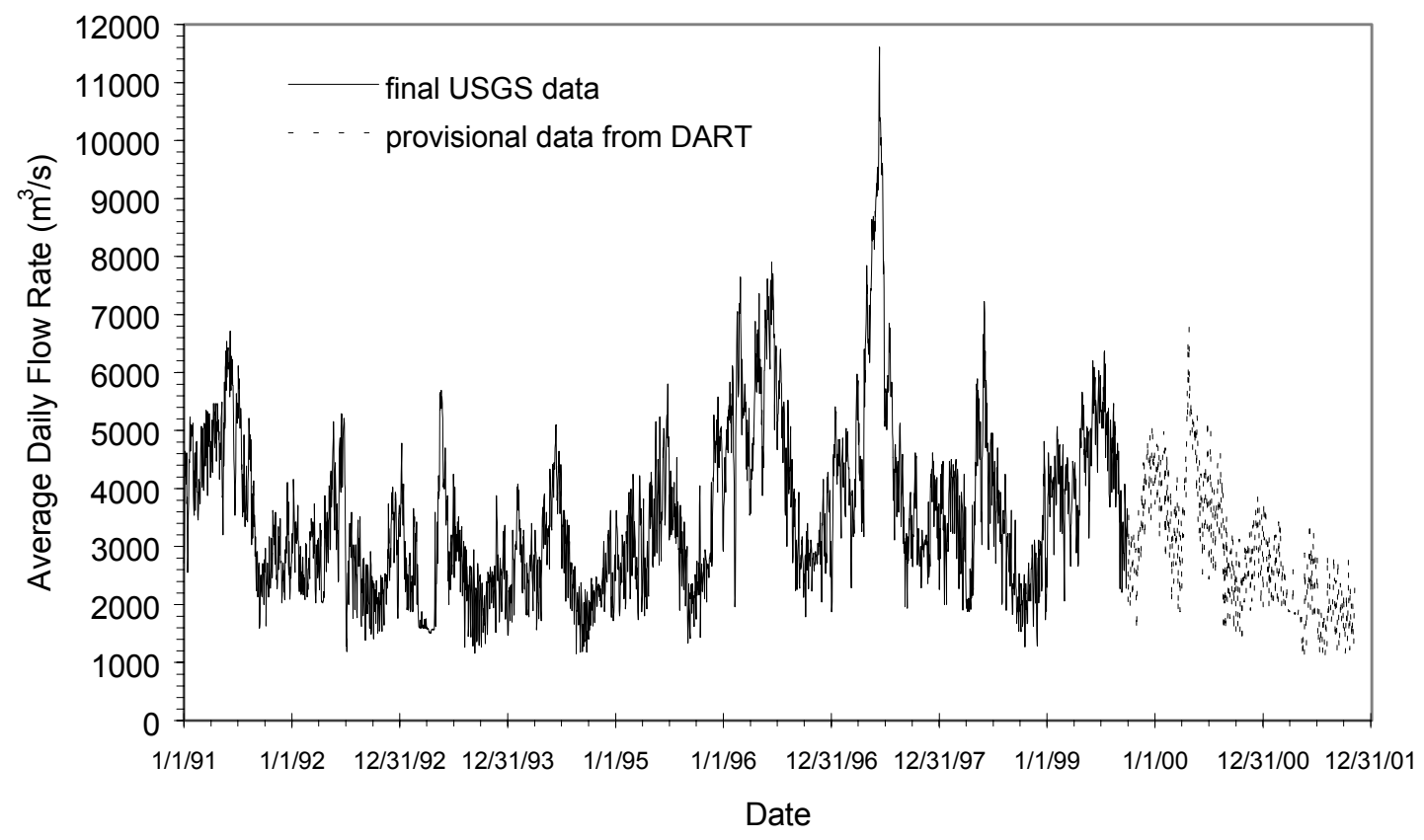

Figure 4.29. Average Daily Flows for the Yakima River at Kiona from 1991 Through 2001. 


\subsubsection{Groundwater-Yakima River Interaction}

The Yakima River is a potential source of recharge to the unconfined aquifer in the southern part of the Hanford Site because water levels in the river are higher than the heads within the adjacent aquifer. As part of a study of groundwater chemistry of the Pasco Basin (Ebbert et al. 1995), the U.S. Geological Survey found evidence that the Yakima River recharges into the unconfined aquifer adjacent to the Hanford Site. This conclusion was based on a comparison between the chemical composition of river water, groundwater from a well completed in the Saddle Mountains Basalt, and groundwater from an offsite well completed in the unconfined aquifer (Ringold Formation) near the river. The recharge rate from the river is controlled by the hydraulic conductivity of sediments adjacent to the river and the head difference between the river and aquifer. The rate of recharge at this boundary is uncertain because of a lack of wells and a corresponding lack of information concerning hydraulic properties and water-level elevations near the river.

There is a Yakima River gauging station upstream from the Hanford Site at Kiona, Washington. However, there are no gauging stations downstream. Determining recharge by measuring water loss in the river cannot be done with accepted accuracy because the volume of recharge to the aquifer along this section of the river is a very small fraction of the average flow through the river, which is $104 \mathrm{~m}^{3} / \mathrm{s}$ since the total estimated groundwater flow in the unconfined aquifer system is on the order of $1 \mathrm{~m}^{3} / \mathrm{s}$. The river also cuts through basalt bedrock between Kiona and the Hanford Site boundary and water may be recharged to the underlying basalt-confined aquifers as well as the sediments above the basalt.

To help define aquifer behavior near the Yakima River, river-stage elevation and water levels in an adjacent well were monitored at a location just below Horn Rapids Dam. As reported in Thorne et al. (1993), water levels in the unconfined aquifer at this well show very little response to changes in river stage. However, the water level of the unconfined aquifer does respond to the filling of a canal (the Horn Rapids Ditch) between the well and the river. The observed response indicates that at this location the Yakima River is isolated from the aquifer by relatively low-permeability sediments. The section of the Yakima River below Horn Rapids Dam flows through flood plain sediments that mainly consist of finegrained over bank and oxbow lake deposits. The adjacent canal is within the more permeable sediments lying above the water table.

\subsubsection{Uncertainty in Model Representation of the Yakima River}

In the area south of the Yakima Horn, the Yakima River boundary is represented as a no-flow boundary in the SGM because a mud-dominated unit is the only sediment below the water table adjacent to the river. Farther south, where units that are more permeable are present, the Yakima River is represented as a prescribed head (Dirichlet) boundary. The Yakima River has usually been represented by a prescribed-head boundary in earlier groundwater models. An alternative implementation model for the Yakima River could be a prescribed flux boundary with different levels of recharge. Earlier models (Jacobson and Freshley 1990; Chiramonte et al. 1996) have included only the short section of the Yakima River along the Hanford Site boundary because the southern extent of the model ended at this point. The current SGM domain includes the entire length of the Yakima River between the Hanford Site and its confluence with the Columbia River. 
The major uncertainty is the permeability of sediments along the Yakima River, which controls flow of water from the river to the aquifer. Few well test results are available in this area.

\subsection{Basalt Above the Water Table}

Where basalt rises above the water table in the local aquifer, an interface is formed that is usually considered an impermeable boundary in the SGM. Most of the exterior model boundary on the western side, and all the interior model boundaries are implemented as no-flow boundaries. There are spatial and temporal uncertainties associated with the location and fluxes along these boundaries. Spatial uncertainties associated with the location of this boundary arise from two (uncertain) sources of information: 1) extrapolation of water level data from well measurements, and 2) interpreted top of basalt surface from well logs and various remote sensing information about approximate interfaces between the sediments and the basalt.

Temporal uncertainties in locations arise from the rising and falling water table since the Hanford operations began in the $1940 \mathrm{~s}$, and that may take place in the future. These temporal uncertainties are small relative to the spatial resolution required for most SGM applications in those boundary areas where the top of basalt surface is steeply dipping over the range of water level changes since operations began. Along most of the western external boundary and along most of interior boundaries surrounding Gable Mountain and Gable Butte, particularly along the northern parts, this is the case. However, along the internal sub-crops south of Gable Mountain, the areas of sub-crop can and have changed significantly through time. These changes present a potentially significant uncertainty problem with regard to the routing of water and contaminants. Sub-crops can change volume and location of saturated sediments. Where such changes occur in a particularly critical area of high hydraulic conductivity, these sub-crop areas can block flow and could reroute water and contaminant movement. This uncertainty also creates a problem with respect to the distribution of recharge from the excluded model areas as will be discussed in a subsequent section on recharge. In a similar manner it also affects the influx of contaminants from waste disposal areas that may in the future be located over basalt sub-crop areas that have no underlying aquifer.

\subsection{Bottom Boundary of Local Aquifer System}

As discussed earlier, the underlying regional aquifer system that lies within the basalts is not considered within the current SGM domain. The base of the unconfined aquifer system in the SGM was initially assumed to be a no-flow boundary, and no interactions with the underlying basalt aquifer system were accounted for. However, it is known that some flow occurs between these aquifer systems and this creates a potential error in the model implementation. Including the entire regional aquifer within the model domain is not considered a reasonable option because flow through the local aquifer system is small compared to flow in the regional system and the uncertainties in regional recharge and discharge would be too large in comparison with the volume of interflow between these systems.

Several past investigations have provided important geologic, geophysical, hydrologic, and hydrochemical data relative to potential intercommunication between the unconfined aquifer and the uppermost confined aquifer. Contamination in the unconfined aquifer in the 200 areas was documented as early as 
1950 (HW-17088) and investigation of contaminants in the Mabton interbed was published in 1976 (ARH-SA-253) in which it was hypothesized that there may be an area of intercommunication in the vicinity of Gable Mountain Pond. The most focused and relevant information and data on potential intercommunication between the unconfined and uppermost confined aquifers are found in RHO-RE-ST-12P, DOE 1988, and PNL-6313. Appendix B of this report provides additional bibliographic material and summarizes the key sources of information and hydrogeologic data relative to the intercommunication between the unconfined aquifer and the uppermost confined aquifer at the Hanford Site.

\subsubsection{Upper Basalt-Confined Aquifer System}

Potential intercommunication between aquifer systems at the Hanford Site is between water-bearing units in the Saddle Mountains hydrostratigraphic unit and the overlying unconfined aquifer system. The Saddle Mountains unit at the Hanford Site consists of four basalt flows separated by interbedded sediments. The flows, in ascending order are the Umatilla, Esquatzel, Pomona, and Elephant Mountain. The major interbedded sediments are the Mabton, Cold Creek, Selah, and Rattlesnake Ridge.

The main water-bearing zones in the Saddle Mountains basalt are the sedimentary interbeds and the interflow zones between basalt flows associated with the interconnecting vesicles and fractures of basalt flow tops. Collectively, these interbedded sediments and the Saddle Mountains basalt interflow zones form an extensive confined aquifer system, which in this report is referred to as the uppermost basaltconfined aquifer.

\subsubsection{Interface Between Basalt-Confined and Unconfined Aquifer Systems}

The bottom of the unconfined aquifer throughout most of the Hanford Site is the top of the Elephant Mountain basalt flow interior where it is present and, in some areas, the basal Ringold where it occurs as a low permeability silt and clay. There are locations where the Elephant Mountain basalt has been fully eroded and is absent, specifically between Gable Butte and Gable Mountain in the vicinity of West Lake. Figure 4.30 is an isopach map of the Elephant Mountain basalt on the Hanford Site. Figure 4.31 is an isopach map of the Elephant Mountain basalt in the West Lake and B Pond area showing the area where the basalt is absent between Gable Butte and Gable Mountain and two other localized "pockets" to the southeast. The Rattlesnake Ridge interbed, which lies directly beneath the Elephant Mountain basalt, has also been removed by erosion in the gap area as illustrated in Figure 4.32 which is an isopach map of the Rattlesnake Ridge interbed in the vicinity of B Pond and West Lake. Likewise, a smaller area of Pomona basalt has been removed by erosion in the gap area as illustrated in the isopach map of the Pomona basalt (Figure 4.33).

\subsubsection{Potential Mechanisms for Aquifer Intercommunication}

Five principal intercommunication mechanisms have been identified as described in Appendix B:

1. Leakage through erosional windows where the confining layer is absent. 


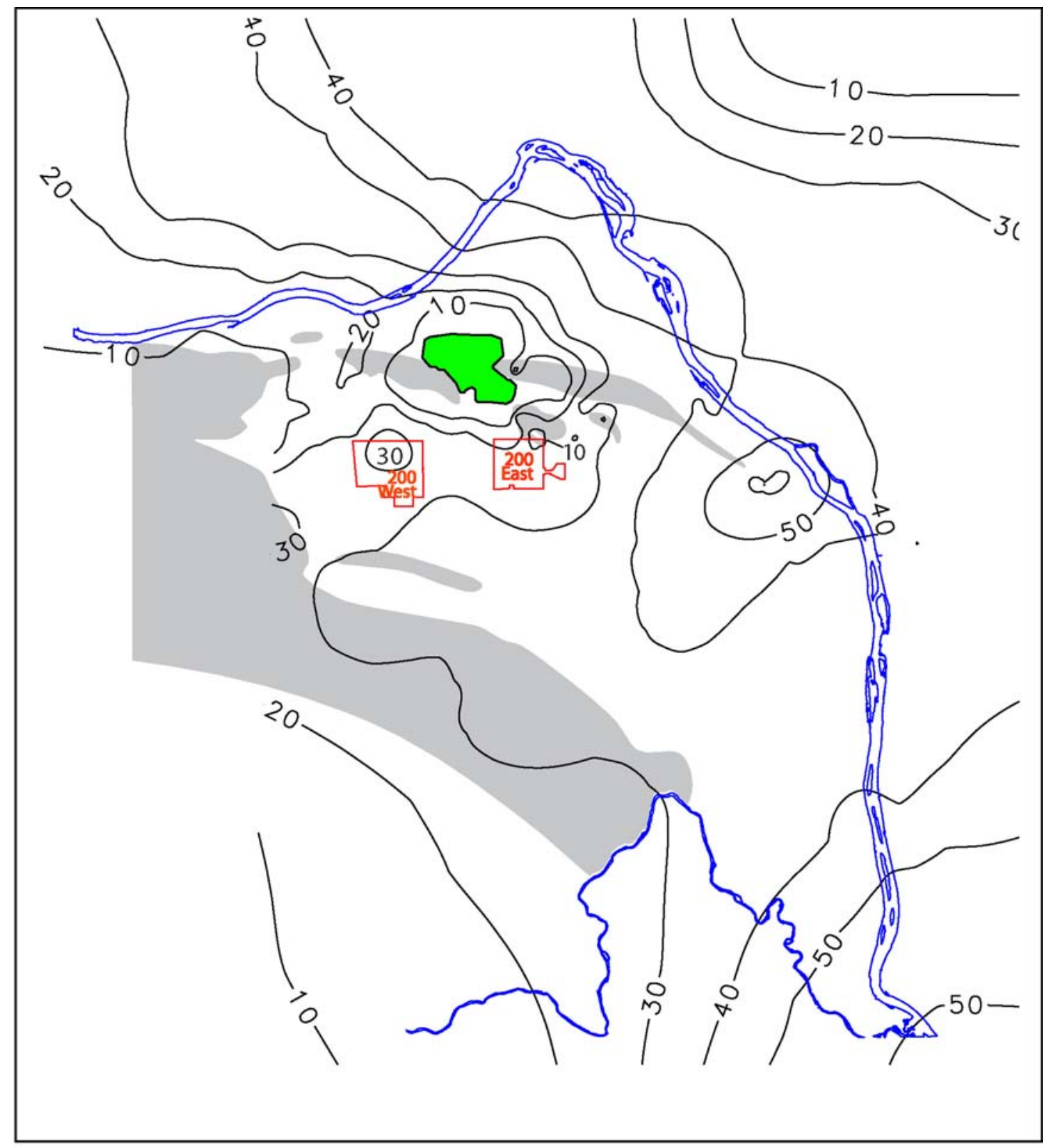

Basalt Above Unconfined Aquifer Water Table

Erosional Window in Contact with Unconfined Aquifer System

-10_ Estimated Elephant Mountain Thickness (m)

Figure 4.30. Isopach Map of the Elephant Mountain Basalt on the Hanford Site. 


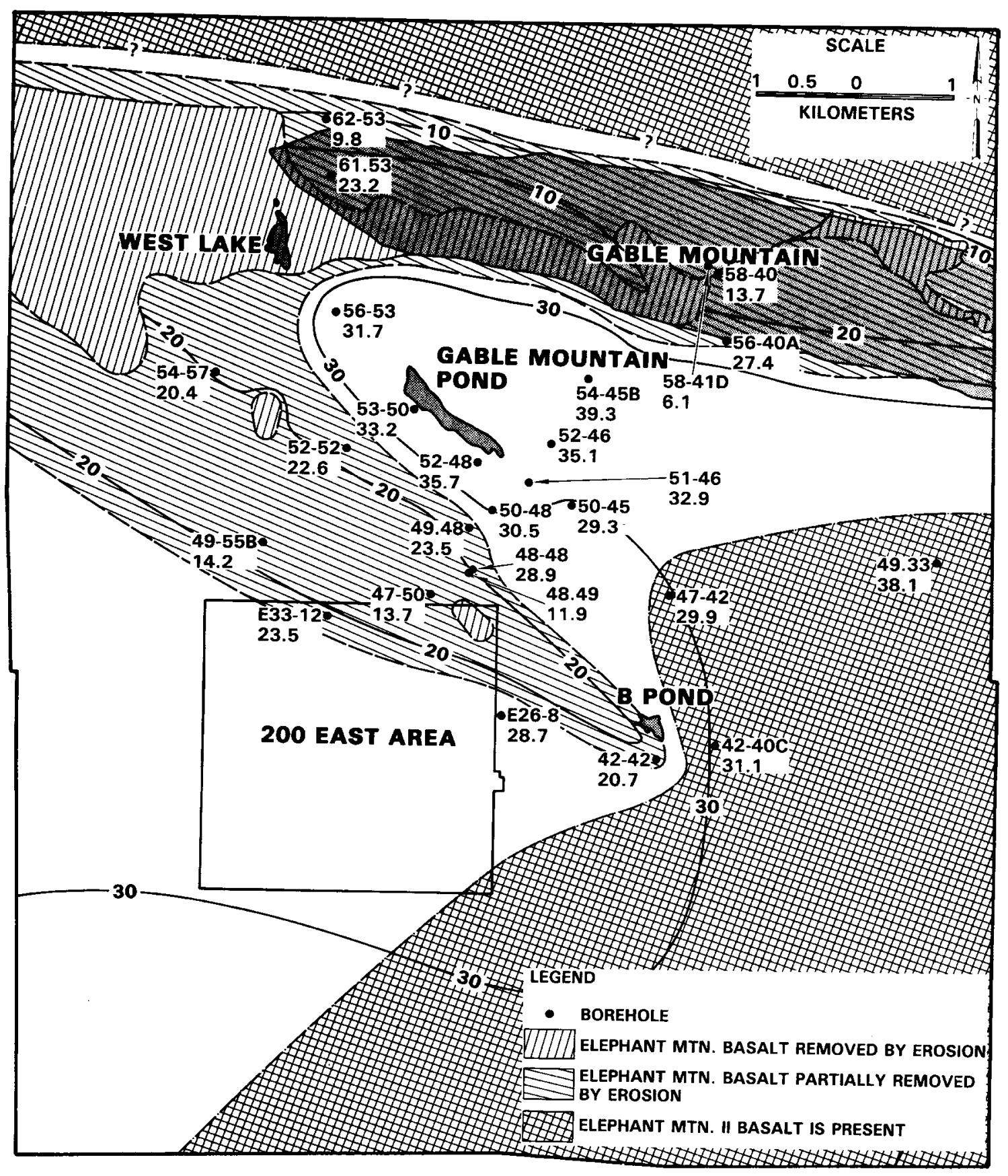

Figure 4.31. Isopach Map of the Elephant Mountain Basalt in the West Lake and B Pond Area. 


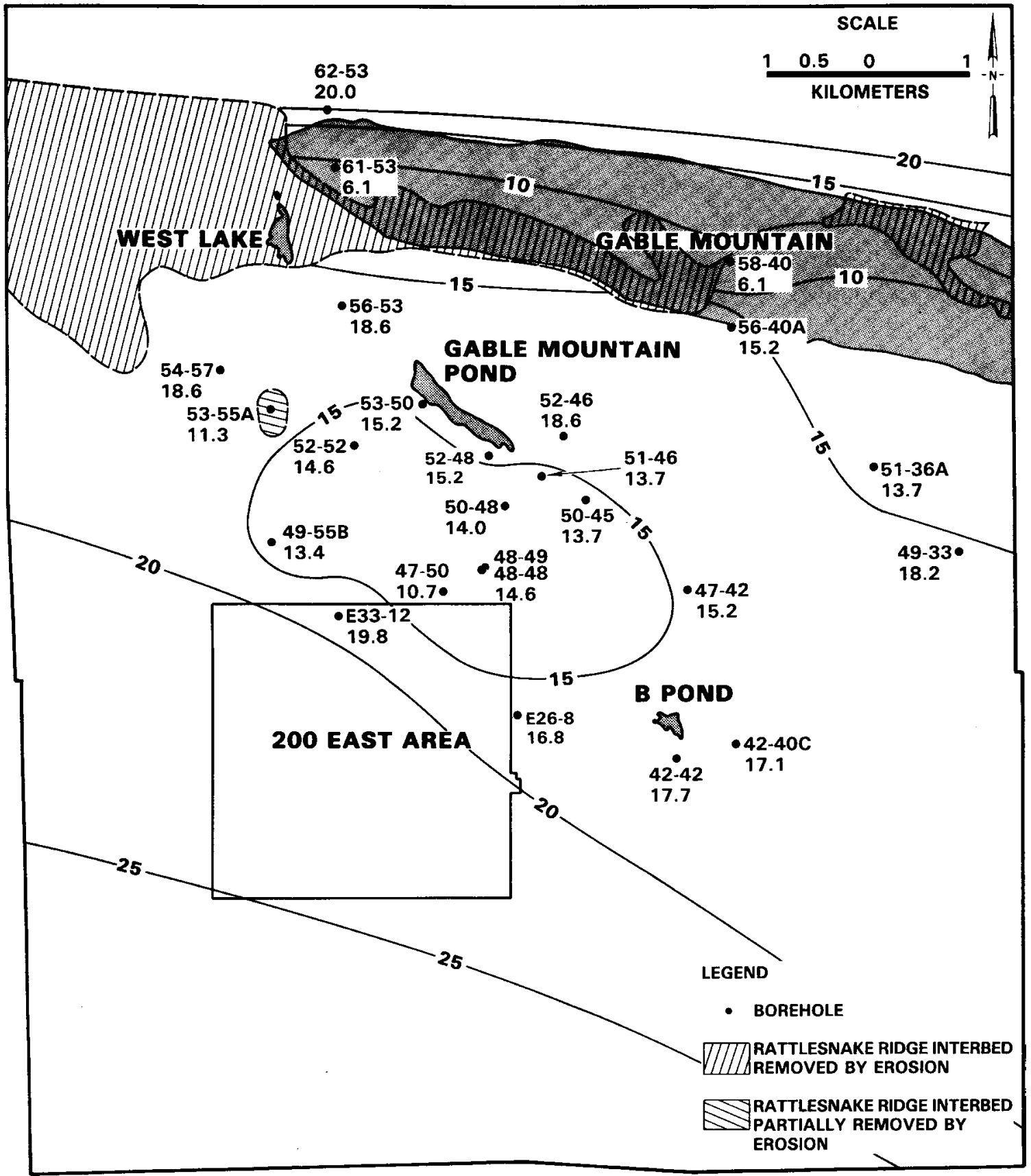

Figure 4.32. Isopach Map of the Rattlesnake Ridge Interbed in the West Lake and B Pond Area. 


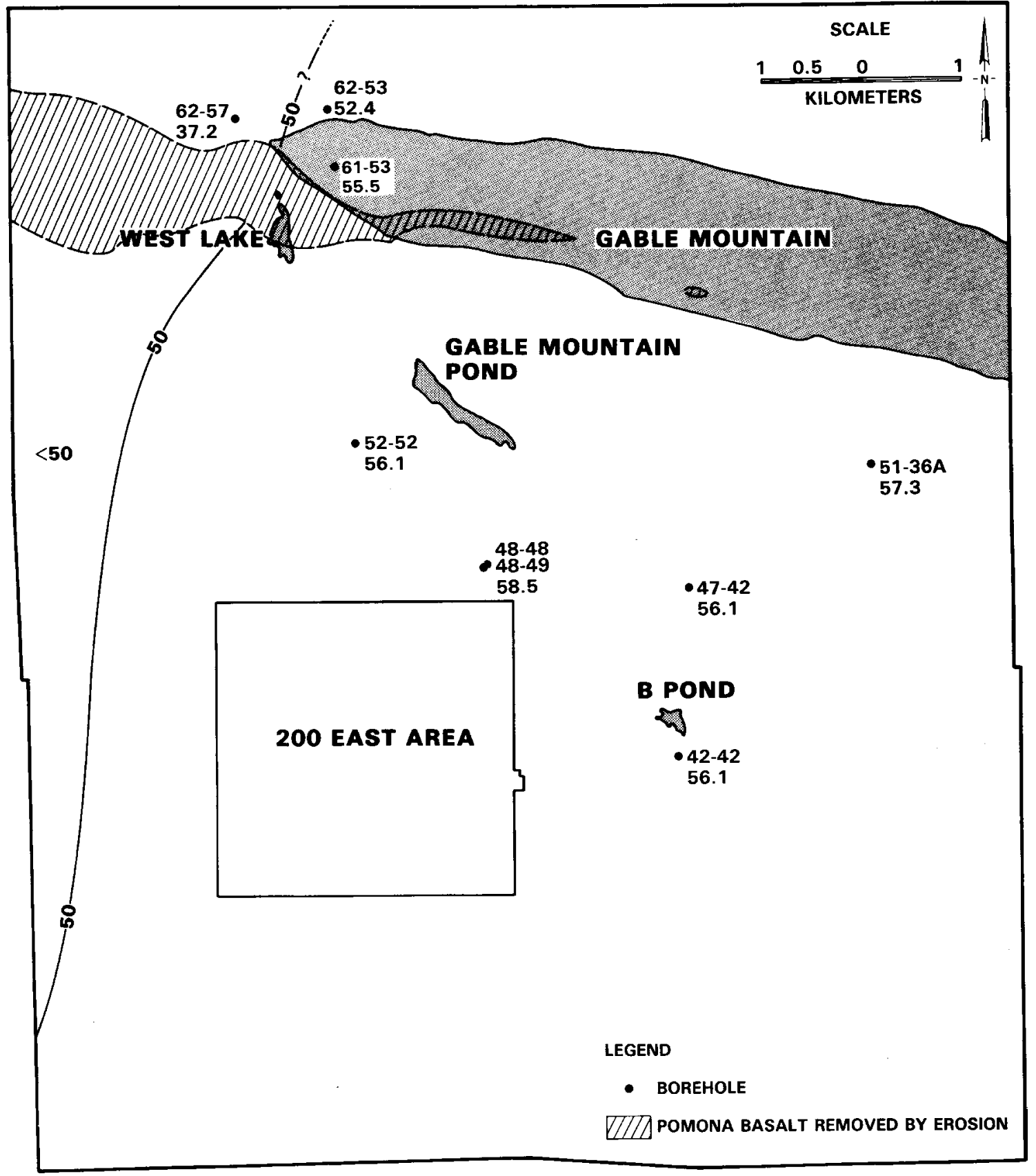

Figure 4.33. Isopach Map of the Pomona Basalt in the West Lake and B Pond Area. 
2. Flow along faults that connect the upper basalt-confined and unconfined aquifer systems.

3. Leakage in structurally deformed areas, such as anticlines, where dense interiors may be highly fractured.

4. Pervasive and relatively uniform leakage through the confining layer (basalt flow interior).

5. Human intrusion associated with improperly constructed wells and boreholes.

Evidence that hydraulic intercommunication, as described in Appendix B, is present in the Gable Butte-Gable Mountain gap area, where erosional windows have been identified, includes:

- chemical composition of groundwater indicating mixing

- presence of chemical species (i.e., nitrate ion) and radioisotopes (e.g., tritium and I-129) in the uppermost confined aquifer that are associated with near-surface wastewater disposal

- similarity of hydraulic heads in the unconfined and uppermost confined aquifers in the vicinity of the Gable Butte-Gable Mountain Gap where the Elephant Mountain basalt is absent

- geologic information from borehole logs and geophysical information indicating an area where the Elephant Mountain basalt (confining layer) is absent and within this area, locations where the underlying Rattlesnake Ridge interbed (water-bearing unit) and portions of the Pomona basalt (confining layer) are absent.

The area where the Elephant Mountain basalt is absent (see Figure 4.31) represents the area where aquifer intercommunication occurs, unimpeded by a confining layer. There are likely other localized areas to the southeast of the Gable Butte-Gable Mountain gap where the Elephant Mountain basalt has been fully or partially eroded, based on barometric efficiency information (RHO-ST-38, 1982).

Springs are present in the Rattlesnake Hills, along the western boundary of the SGM domain, that bring groundwater from the basalt-confined aquifer system to the surface. These springs are found where major thrust faults intersect the ground surface (DOE 1988). This provides evidence that the major thrust faults provide conduits for flow between aquifer systems. Anticlines may also be areas of increased communication because of fracturing. However, there is no direct evidence of intercommunication associated with anticlines other than in the area where erosional windows are also present.

Elsewhere on the Hanford Site, the Elephant Mountain basalt provides a significant impediment to vertical intercommunication between the aquifers owing to its thickness (Figure 4.30) and low vertical hydraulic conductivity that may range from $1 \mathrm{E}-8 \mathrm{~m} / \mathrm{d}(3.3 \mathrm{E}-8 \mathrm{ft} / \mathrm{d})(\mathrm{RHO}-\mathrm{RE}-\mathrm{ST}-12 \mathrm{P})$ to $2.6 \mathrm{E}-4 \mathrm{~m} / \mathrm{d}$ $(8.5 \mathrm{E}-4 \mathrm{ft} / \mathrm{d})$ (Nevulis et al. 1987). The effectiveness of the Elephant Mountain basalt as a confining layer and impediment to vertical communication between the unconfined and uppermost confined aquifers is evidenced by the hydraulic head difference between the two aquifers and difference in groundwater chemistry. However, the rate of pervasive flow through the confining unit may still be significant 
because it takes place over a large area. Gradient directions have generally been upward near the rivers and downward in the areas where discharges from Hanford Operations have built significant mounds in the unconfined systems.

\subsection{Top Aquifer Boundary}

The upper boundary of the SGM is defined by the water table. Natural recharge occurs at this boundary primarily from infiltration of precipitation that reaches the water table. However, during Hanford operations, the volume of artificial recharge from wastewater discharge was much greater than the natural recharge. In the current SGM, these artificial recharge sources are applied directly to the water table around surface facilities. No time delay is implemented for the vadose zone transit time, and no volume losses are implemented to account for evapotranspiration or other losses. We generally assume no source relocation due to preferential flow in the vadose zone, except for one known case of such relocation/diversion north of the 200 West Area (documented at the State Approved Land Disposal Site [SALDS]). Such possible relocation due to preferential flow (particularly, flow along dipping layers with contrasting permeability in the thick vadose zone) introduces an additional uncertainty.

Observations at the SALDS indicate that the source relocation can be on the order of $100 \mathrm{~m}$ or more and the delays on the order of 6 months to 1 year (Barnett et al. 1997). There are uncertainties in the identification of all the various disposals of liquids (contaminated or uncontaminated) to ground as well. There are also uncertainties in the spatial location (e.g., in a large crib or a long trench/ditch hundreds of meters or kilometers long) where the infiltration took place, and in the actual quantity versus time.

Additionally, the current SGM modeling does not account for any water importation losses (i.e., a water imbalance between imported water volumes and disposed water volumes) due to leaks. Spatial, temporal, and quantity uncertainties associated with the current SGM assumptions related to artificial sources of water are being investigated and implemented wherever known.

West Lake (Figure 4.34), whose location near the southwestern edge of Gable Mountain is shown in Figure 4.6, is the only natural water body internal to the SGM domain. Any losses of water from evaporation, although evident in the thick white residues around the lake, have not been considered in the previous SGM implementation.

\subsection{Natural Recharge from Precipitation and Springs}

Until the 1980s, natural recharge at Hanford was assumed to occur only from runoff from higher bordering elevations and infiltration from small ephemeral streams and springs. Natural recharge across the remainder of the Site was assumed to be nil. However, Gee et al. (1992) presented evidence from multiple experiments showing conclusively that measurable diffuse natural recharge occurs across the lower elevations of the Hanford Site, with rates ranging from near zero to more than $100 \mathrm{~mm} / \mathrm{yr}$. 


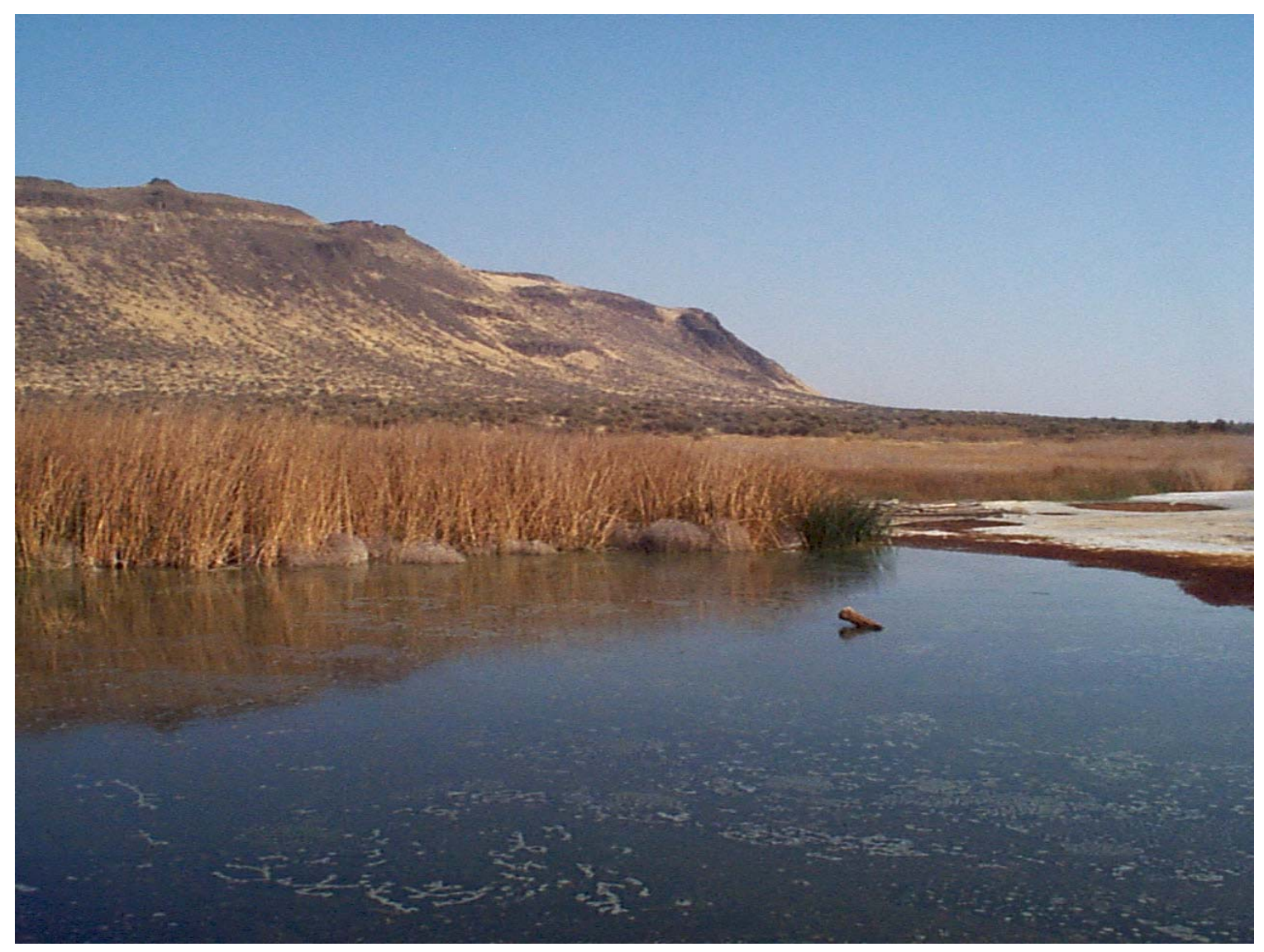

Figure 4.34. Photograph of West Lake Looking Northeast Toward Gable Mountain.

Bauer and Vaccaro (1990) simulated recharge at the Hanford Site using estimates of soil type and land use and a water balance model, which they called the Deep Percolation Model. The area of each of their simulation cells was roughly $1 \mathrm{~km}^{2}$. They estimated rates for most of the Site that ranged from 0 to $13 \mathrm{~mm} / \mathrm{yr}$. In the vicinity of the sand dunes in the middle of the Site, they estimated rates as high as $51 \mathrm{~mm} / \mathrm{yr}$. Bauer and Vaccaro (1990) did not account for the impacts of soil and vegetation disturbance caused by Hanford operations.

Fayer et al. (1996) used several types of field data and computer modeling results to estimate the areal distribution of mean recharge rates for the soil and vegetation conditions at the Hanford Site, including any disturbance by Hanford operations (Figure 4.35). Their estimates ranged from $2.6 \mathrm{~mm} / \mathrm{yr}$ for several soil and vegetation combinations to $127.1 \mathrm{~mm} / \mathrm{yr}$ for basalt outcrops with no vegetation at the crest of Rattlesnake Mountain. The annual volume of other groundwater inputs, the distribution of estimated recharge is highly skewed to the disturbed sandy soils (i.e., the 200 areas, where most contaminants originate). Such a recharge rate is by no means negligible, and consequently, uncertainty in recharge is a significant source of uncertainty in the groundwater conceptual model, especially under future conditions when groundwater flow magnitudes and directions will be controlled by natural recharge and groundwater fluxes between the unconfined system and the underlying basalts. 


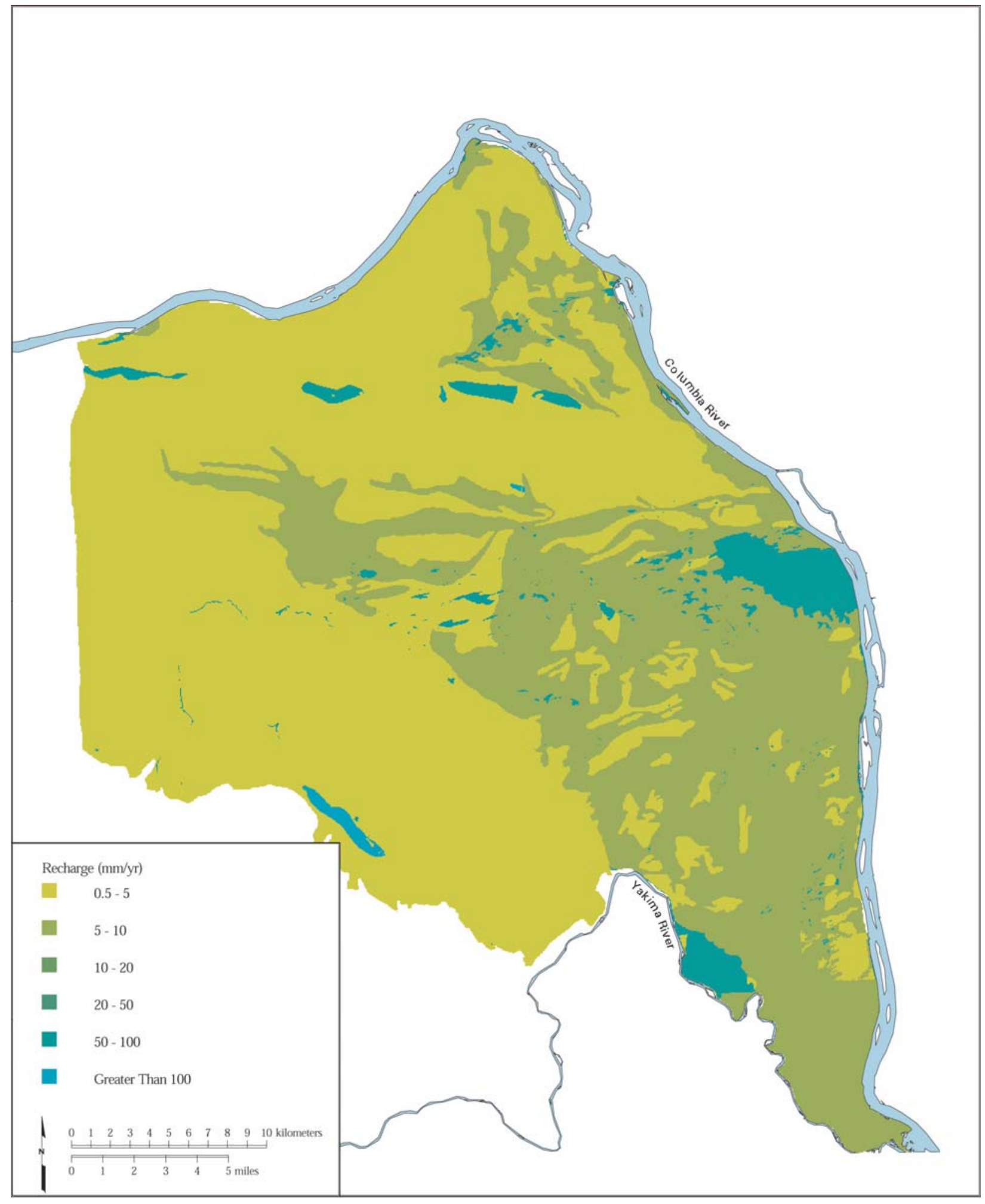

Figure 4.35. Estimates of Hanford Site Recharge for 1979 Conditions (Fayer and Walters 1995). 
The magnitude of recharge at a particular location is influenced by five main factors: climate, soils, vegetation, topography, and springs and streams. Other factors can significantly impact recharge by affecting one or more of the main factors. These other factors include soil development, animal activity, fire, water and wind erosion and deposition, plant community changes, disturbance, and human structures (e.g., roads, buildings). The five main factors are described below as they relate to recharge at the Hanford Site.

\subsubsection{Climate}

Precipitation is a prerequisite for natural recharge to occur. Precipitation that infiltrates the soil can return to the atmosphere via evaporation, a process that depends principally on the remaining variables. Annual precipitation varies between 76 to $291 \mathrm{~mm}$. Precipitation in the form of snow varies annually from $<1$ to about $140 \mathrm{~mm}$. A snow cover can have a significant impact on recharge by altering the receipt of solar radiation at the ground surface and by accumulating over many days and weeks and then melting rapidly within a day or two.

Air temperature plays a significant role in evapotranspiration, snowfall and melt, and the phenology and growth of vegetation, all of which impact recharge. The mean monthly temperature can be well below freezing for several winter months. The variation in average daily air temperatures given the desert environment is large.

Average monthly humidity ranges from 66 to $145 \%$ of the mean monthly value. Average monthly wind speeds at the $15.2-\mathrm{m}$ height range from 46 to $163 \%$ of the mean monthly value. Peak wind gusts have reached $35 \mathrm{~m} / \mathrm{s}$. Solar radiation changes in a fairly predictable way throughout the year yet still has daily variability. The record shows that daily values can range from 10 to $250 \%$ of the monthly mean value, with the greatest difference occurring in the winter months when the potential for recharge is highest.

The weather variations of a given climate are sufficient to influence recharge. When the time frame of interest is greater than decades, changes in weather statistics, known as a "change in climate," can also impact recharge. Thus, predictions of climate change are important to forecasts of recharge rates.

\subsubsection{Soils}

Soil type affects recharge by controlling the partitioning of precipitation into runoff and infiltration, the storage of water, the redistribution of water within the soil, evaporation, nutrient and water availability as it affects the status of vegetation, and percolation below the root zone (eventually to become recharge). The current map of soils at Hanford was published by Hajek (1966), who based the map on an earlier soil survey of Benton County by Kocher and Strahorn (1919). Hajek correlated the 1919 soil-mapping units to the mapping units used in the 1960s by the Soil Conservation Service, and the accuracy of the soil boundaries was spot-checked. The latest soil survey for Benton County (Rasmussen 1971) was derived from work conducted in the 1960s. It does not include the Hanford Site or the City of Richland. 


\subsubsection{Vegetation}

Vegetation plays a number of roles in the water budget of arid sites. Plants protect the soil from wind and water erosion, they are an integral part of the food chain, and they alter microclimates. Most important of all, plants remove water from the soil by the process of transpiration.

The vegetation at the Hanford Site is diverse; 590 species have been identified (Sackschewsky et al. 1992). This variety of vegetation species, each with different characteristics, is the reason that all plants do not affect the water budget in the same manner. Phenology, rooting depth, and fire resistance are some of the vegetation traits that impact the water budget.

The phenology of a plant refers to the time of year that it is active, (i.e., when the vegetation is growing and transpiring). Many annuals are predominantly active for a short period in spring, while water is available. However, some, such as tumbleweed, are more active in summer. Perennials such as bunchgrass and sagebrush are active for longer periods during the year, partly because of their established root systems. If conditions are right, sagebrush can remove water all year long. Rickard and Vaughan (1988) compared the phenology of 12 plant species in both a dry year and a wet year at the Hanford Site. The length of activity varied from 2 to 10 months, depending on the species.

The root depth of vegetation determines how much soil water can be accessed by the plant. The potential for recharge is reduced as more soil water becomes accessible to the plant. Perennials such as sagebrush and bunchgrass tend to have deep root systems, sometimes as deep as $3 \mathrm{~m}$, whereas annuals such as cheatgrass tend to have shallow root systems of less than $1 \mathrm{~m}$.

Fire is part of the natural cycle at the Hanford Site. After a fire, new annual plants appear the following year from seeds that survived the fire. The speed of propagation can influence the species composition of an area. Annuals tend to grow more quickly than perennials, but only if conditions are right. Some perennials, such as bunchgrass and rabbitbrush, can re-grow from their root system and others, such as sagebrush, cannot. Thus, fire can change a plant community composition quickly, and the effects can last for years (Rickard and Vaughan 1988; Link et al. 1990).

A vegetation map was prepared that shows the distribution of major vegetation/land use categories at the Hanford Site (Neitzel 1998). The map was developed from aerial photos and ground surveys. Industrial development and waste disposal activities can change the plant community distribution quickly. Other changes in the plant community composition (from diseases, insects, and alien infestations) are slower and may take several years to be recognized.

\subsubsection{Topography}

Variations in topography affect recharge in several ways. Large topographic features such as Rattlesnake Mountain alter weather by increasing precipitation and decreasing air temperatures. The crest of Rattlesnake Mountain is $1093 \mathrm{~m}$ (3586 ft). The Hanford Meteorological Station (HMS) is located at an elevation of $223 \mathrm{~m}$ (733 ft) between the 200 East and 200 West areas. Stone et al. (1983) report annual precipitation increases from $16 \mathrm{~cm}$ at the HMS to $28 \mathrm{~cm}$ at the top of Rattlesnake Mountain. Thorp and 
Hinds (1977) report that air temperatures decrease at higher elevations at the rate of $6^{\circ} \mathrm{C} / \mathrm{km}$ in winter and $10.4^{\circ} \mathrm{C} / \mathrm{km}$ in summer. These precipitation and temperature changes can increase diffuse recharge rates and lead to more runoff. Small variations in topography, while not influencing weather, can alter microclimates (e.g., causing drifting snow to accumulate).

The aspect of the soil surface affects the receipt of solar radiation. Hinds (1975) showed significant differences in water and plant behavior between north and south facing slopes. The degree of slope affects both the receipt of solar radiation and the runoff of surface water.

Variations of topography can focus surface runoff in such a way that infiltration is locally greater. Water runoff from the higher elevations occurs intermittently but can be extensive (e.g., Pearce et al. 1969). Cushing and Vaughan (1988) indicate surface runoff from higher elevations can occur, on average, about once every 3.8 years. Water runoff does not appear prevalent at the Hanford Site between Highway 240 and the Columbia River based on the absence of geomorphic features such as erosion rills and gullies. However, observations have revealed that local runoff does occur when there is a heavy rain, quick snowmelt, and the ground is frozen (e.g., Jones 1989).

\subsubsection{Springs and Streams}

Surface water at higher elevations can flow to lower elevations and infiltrate the soil and recharge the local aquifer. Cushing and Vaughan (1988) indicated that there may be more than 100 springs and spring locations in the higher elevations to the west of Hanford, but only two (Snively and Rattlesnake) appeared to be significant. Flow from both of these springs was said to originate from basalt aquifers. Studies of Rattlesnake Springs indicate that baseflow is $864 \mathrm{~m}^{3} / \mathrm{d}$ and can range up to $1340 \mathrm{~m}^{3} / \mathrm{d}$ in winter. Baseflow for Snively Springs was estimated to be $432 \mathrm{~m}^{3} / \mathrm{d}$.

\subsubsection{Uncertainties in Natural Recharge Estimates}

The estimates of recharge rates provided by Fayer et al. (1996) and applied in the SGM are affected by uncertainties that result from gaps in data and from not considering all of the processes involved in recharge.

The available information on recharge rates at the Hanford Site consists of measurements of water contents and drainage, tracer studies, and computer modeling of specific soil and vegetation combinations. All of these methods use near-surface measurements rather than measurements at the water table. Some techniques, like lysimetry, use data collected during a period of several years. In contrast, the chloride tracer technique estimates recharge for periods of 100 to 1000 years or more. Lysimetry measures drainage for a well-defined soil-vegetation combination, but this is an "interfering" method, both during construction and by the nature of its operation (particularly when the system is under high suction and lateral movement is limited). The influence of lysimeters and consequent errors have been studied by Selker and coworkers, and estimated to be $\sim 30 \%$. Besides, lysimeters provide "point" measurements that are likely to miss heterogeneity and preferential flow paths. Computer modeling of 
recharge relies on soil and vegetation parameters that are not well known. While the model has been tested successfully for conditions without plants, it has not been tested to demonstrate its ability to simulate deep drainage beneath a plant community. Additional sources of uncertainty are discussed below.

\subsubsection{Soil, Vegetation, and Climate Data}

The soils map was initially prepared in 1919 for agricultural purposes rather than for recharge estimation. Thus, soil features that might affect recharge (e.g., hydraulic conductivity, thin layers) were not recorded and no information is provided on the variability within soil types. Fayer et al. (1996) used the soils map to estimate recharge rates in the industrial zones, but they did not account for altered soils (e.g., gravel-covered tank farms; subsoil sand brought to the surface in many waste disposal areas). The land-use map used by Dinicola (1997) was made 20 years earlier. Much development has occurred since then and will continue to occur in the upper reaches of the Cold and Dry Creek basins. The vegetation and land-use map for the Hanford Site is continually updated to reflect changing conditions (Neitzel 1998). In addition to the uncertainty in soil and vegetation/land use coverage, there is a concern about the parameters used in any modeling study designed to estimate recharge. Many of these parameters are approximations or default values because a good set of measured values is lacking. The lack of measured parameters does not necessarily invalidate the recharge estimates, but it adds uncertainty.

Overall, the lack of good estimates of the means and variances of the supporting data (i.e., the soil map, the vegetation/land-use map, model parameters) translates into large uncertainties in the recharge estimates. For all three cases (soil, vegetation, climate), future conditions before and after Site closure cannot be expected to remain constant. Any attempt to predict future rates will have to specify the nature and degree of changes that are plausible.

\subsubsection{Spatial and Temporal Averaging}

Gee and Hillel (1988) observed that arid-site recharge can be generally low yet highly variable. The number of recharge measurements is usually limited and the sampling schemes are generally random. The results from these limited and random measurements yield an average recharge rate that might be practically negligible while most of the recharge is episodic and may be confined to small areas. Gee and Hillel referred to this result as the "fallacy of averaging" in arid regions. The propagation of storms and high precipitation events on recharge at Hanford has not been established. In fact, the amount of data and their temporal extent are very limited at Hanford. There are only 10 to 12 monitoring sites, some covering periods from 2 to 20 years, others averaging over 35 to thousands of years. Such limited data leads to averaging.

\subsubsection{Vadose Zone Hydraulic Properties}

Data on the distribution of hydraulic properties across the Site are also very limited and are concentrated in the 200 areas. This lack of information makes vadose zone/unsaturated flow modeling for other areas of the Site uncertain. Currently, only averaged soil and land-use classifications are available. Highly conductive features such as clastic dikes and permeable faults have not been characterized in 
terms of hydraulic properties and contribution to recharge. Creeks and basalt outcrops have not been treated as unique features in terms of both infiltration and accumulation of runoff waters as a function of season and time.

\subsubsection{Human Activities and Structures}

Human activities can disturb soil, vegetation, and topography, three of the factors that affect recharge rates. Disturbed areas can contribute the largest amount of site-specific recharge. The highest levels of disturbance occur in and around waste disposal areas, possibly increasing contaminant release and transport rates to the water table and affecting groundwater flow and direction. Unfortunately, little is known about the impact of disturbance on recharge in many areas of the Site.

Human structures can also affect recharge. Roads, power lines, firebreaks, and parking lots could increase recharge far in excess of expected recharge from similar undisturbed areas. The impact arises primarily from the concentration of precipitation in small infiltration areas or the removal and suppression of vegetation. Some facilities have storm-water discharge features that are controlled as miscellaneous streams (DOE 1998). Although controlled, these miscellaneous streams are not actually monitored; the control is primarily an upper limit on allowable discharge. Most buildings and all small structures such as sheds and Quonset huts do not have controlled discharge. Quantifying the contribution from these facilities is difficult given the lack of data. The same difficulty applies to paved roads and parking areas.

\subsubsection{Basalt Outcrops and Shallow Soils}

Shallow and stony silt loam soils, together with basalt outcrops, dominate the upper elevations of Rattlesnake Mountain, Gable Mountain, and Gable Butte. Fayer et al. (1996) estimated recharge for basalt outcrops by assuming the rates were similar to lysimeters containing gravel surface layers. This method ignores the presence of plants and the possibility of small amounts of soil that a soil surveyor might overlook, but that can have a large impact on recharge rates. For the shallow and stony soils (e.g., Lickskillet silt loam; Kiona silt loam), Fayer et al. (1996) assumed the rates were similar to the rates for deeper soils without stones.

\subsubsection{Recharge Above Basalt Zones}

Fayer et al. (1996) produced a map of estimated recharge rates for all areas of the Hanford Site regardless of whether an unconfined aquifer was present. As indicated by Cole et al. (1997), portions of the Hanford Site do not have an unconfined aquifer above the basalt. Furthermore, as the water table recedes with the cessation of liquid discharges, more of the basalt will be exposed above the water table.

\subsubsection{Uncertainty in Spring and Stream Estimates}

The available information on springs, streams, and runoff from higher elevations is limited. The available gauging data cover a period less than 3 years. In lieu of a long-term time series of gauge data, Dinicola (1997) used a model exercise to estimate stream flow and runoff. The model that was employed 
did not allow much detail in the landscape and used 20-year-old land-use maps. The study did not indicate how frequently overland flow reached the portion of the Hanford Site covered by the groundwater model domain, nor the volume of water and the areal extent of such events.

For the runoff modeling, Dinicola (1997) used data from a number of weather stations surrounding the modeled basins, and then corrected for altitude effects such as higher precipitation and lower air temperature.

\subsubsection{Time Delay Between Deep Drainage and Surface Infiltration Events}

The map of estimated recharge rates produced by Fayer et al. (1996) does not reflect historical recharge rates. Rather, the map reveals the distribution of recharge that was estimated using the current climate, the Hajek (1966) soil map, and the 1991 vegetation/land-use patterns. Exactly when these estimated rates apply to the water table depends on the propagation of the diffused pressure wave through the unsaturated zone, which varies as a function of precipitation intensity and duration, atmospheric conditions, initial water content, type of topsoil, geostratigraphy, and vadose zone thickness across the site. At this point, very little is known quantitatively about most of these characteristics and the complex relationships between them across the site.

\subsection{Artificial Recharge at the Hanford Site}

Artificial recharge is important information for groundwater modeling at Hanford because the volume of artificial recharge during most of the period of hydrogeologic records was significantly greater than the volume of recharge from natural sources. Reliable data on artificial recharge is important for inverse and forward modeling. Therefore, substantial efforts have been devoted to investigating and quantifying the various liquid sources.

\subsubsection{Brief History of Waste Disposal at Hanford}

Since 1943, Hanford operations have resulted in the production of liquid, solid, and gaseous wastes. Highly contaminated liquid waste was stored in large underground tanks. Less contaminated liquid waste streams were routed to underground structures of various types including cribs, French drains, injection wells, and trenches. Occasionally, trenches were filled with the liquid waste and then covered with soil after the waste soaked into the ground. Slightly contaminated liquid waste streams were usually routed to surface impoundments (ditches and ponds).

The large volume of wastewater discharged to disposal facilities on the Hanford Site over the past 50 years significantly affected the groundwater flow system. The volume of artificial recharge decreased significantly during the past 10 years and is currently still decreasing (Barnett et al. 1995; Dresel et al. 1995). Until it was taken out of service in 1984, Gable Mountain Pond received the largest volume of discharge on the Hanford Site. Major groundwater mounds occurred beneath B Pond, Gable Mountain Pond, and U Pond, and affected site-wide groundwater flow patterns (Bierschenk 1959; Dresel et al. 1995). Wastewater is no longer being discharged to U Pond and Gable Mountain Pond, which were decommissioned and are now dry. Other smaller-volume recharge sources existed until recently in the 
100, 200, and 300 areas and may affect groundwater flow on a local scale. B Pond was decommissioned in 1997. Currently, all tritiated water is disposed to the SALDS while the major artificial recharge source of clean water is the 200 Area Treated Effluent Disposal Facility. At the Richland City well field, there is a net recharge because of the input of Columbia River water to recharge basins.

\subsubsection{Uncertainty in Artificial Recharge}

The artificial recharge sources included in the current SGM are based on information reported in a series of reports on Effluent Discharges and Solid Waste Management issued by the Hanford Operations Contractor. At this point, only about $80 \%$ of the sources are known with some accuracy. A mass balance accounting of water withdrawn from the Columbia River, piped across the Site, and used by facilities has not been performed. Comparison of these volumes with the sum of discharge volumes at individual facilities could help quantify the uncertainty in artificial recharge. It is possible that a significant volume of artificial recharge occurred through leaks in the water distribution system, but has not been accounted for in the current SGM.

\subsubsection{Evaporation from Disposal Ponds and Ditches}

The current site-wide groundwater model does not account for evaporation from surface ponds and ditches. This can be estimated. For each ditch, the information needed to estimate evaporation includes the length of the ditch, the gradient of the ditch, a cross-sectional description with associated dimensions, the base material and its properties, the disposal history, the temperature of the water, and the estimate of the potential for overland flow and an accounting of it. For ponds, the information needed includes the surface area, the base material and its properties, the disposal history, the temperature of the water, locations and disposal history of all feeder pipes and ditches, and an estimate of the potential for overland flow and an accounting of it. Once these data are collected, estimates of the evaporation rates can be made and incorporated in the model.

\subsubsection{Additional Sources of Artificial Recharge}

Energy Northwest (formerly Washington Public Power Supply System), agricultural and lawn irrigation, and ground disposal of wastewater at a potato-processing plant are other sources of artificial recharge that may affect groundwater flow in the north Richland area and in the southern part of the Hanford Site (Liikala 1994). To estimate recharge from agricultural irrigation, the information needed includes the areal coverage of the irrigated land, the history of the farming practices, and the assumed or calculated deep drainage rate of the soil. To estimate recharge at Energy Northwest, the information needed includes the water use, water discharge practices, discharge locations and seasonality, discharge quality, and any additional impacts related to off-line conditions at the plant.

\subsubsection{Transit Time from Disposal to Aquifer}

The effluent discharge volumes in the current SGM are assumed to be discharged directly to ground. It is also assumed that there is no time lag between disposal and the time the discharge reaches groundwater. As in the case of natural recharge, there is a question of how much time it takes for water or 
contaminants to impact the aquifer below. We should keep in mind: 1) the diffusive propagation of liquid "wave" versus the different travel time of liquid particles through the vadose zone, 2) the almost saturated vadose zone conditions under most artificial recharge conditions, 3) different preferential flow mechanisms that are likely to affect transit times in different ways, and 4) the potentially significant volume of water that may be held up in the vadose zone upon initiation of artificial discharges that likely only reach the groundwater through slow drainage years later when the artificial discharge ceases. The uncertainty in soil structure and soil parameters beneath the sources, together with the uncertainty associated with unstable flow (that results in fingering) constitutes a significant uncertainty in travel times through the vadose zone. Because of its potential significance, the uncertainty in artificial recharge is currently under investigation for incorporation into the SGM. As with other uncertainties, we start with structural (or conceptual model) uncertainties, assuming different scenarios of source intensity and duration/timing, and different scenarios of "wave propagation" and solute travel times. We hope to recover much of the "wave propagation" and some of the travel times through our inverse modeling, particularly where aquifer and soil parameters are known with some certainty.

\subsubsection{Future Land Use}

Future land use on the Hanford Site is another source of uncertainty in predicted future artificial recharge. Previous analyses of post-Hanford Site unconfined aquifer conditions have considered land uses such as large-scale irrigation on the Hanford Site that could significantly alter the long-term behavior of the unconfined aquifer beneath the Hanford Site. The potential for large-scale agricultural irrigation on the Hanford Site in the future was examined for the Composite Analysis. Consultations with staff from the Agricultural Research Service at the Agricultural Experiment Station in Prosser, Washington, resulted in the conclusion that the prospect of large-scale irrigation occurring on the Hanford Site is unlikely because of limitations on regional water resources.

\subsection{Current Groundwater Use and Pumpage}

Wells used for water supply on the Hanford Site are shown in Figure 4.36. As shown, only a few of the more significant water supply wells are accounted for in the current SGM (e.g., Fast Flux Test Facility wells). Other water supply wells located on the Hanford Site affect local patterns of groundwater flow, but are not expected to have a significant effect on site-wide contaminant transport. Wells that have not been included in the current implementation of the SGM include irrigation and domestic wells located south of the Site boundary. Discharge from existing or future wells in this area has the potential to affect groundwater flow across the southern Site boundary. The Richland City well field and recharge basins located in north Richland are accounted for as a net recharge site because more water is pumped into the recharge basins than is removed.

\subsection{Anticipated Future Flow Conditions}

Future flow conditions in the unconfined aquifer will change as remaining wastewater discharges from Hanford Site operations are curtailed, and water table conditions are more strongly influenced by natural recharge. Past site-wide modeling of future water table conditions following elimination of 


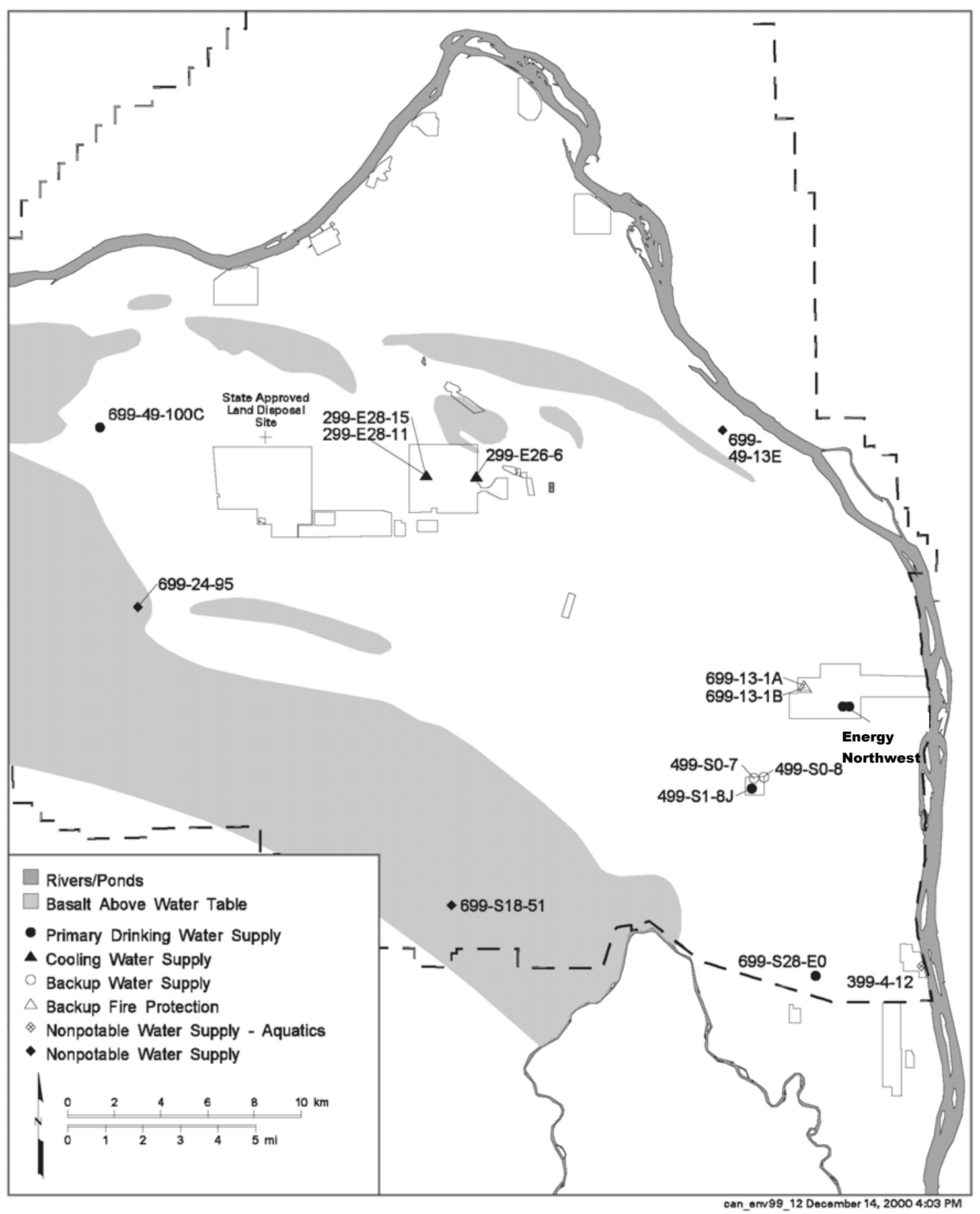

Figure 4.36. Water Supply Wells on the Hanford Site. 
wastewater discharges to the ground at the Hanford Site by Chiaramonte et al. (1997) and Cole et al. (1997) suggested that the water table will decline significantly over the next 200 to 300 years. These analyses also showed that the water table would return to near pre-Hanford Site conditions that were estimated to exist in 1944 (Kipp and Mudd 1974) over most of the Site. These simulations assumed no significant future recharge from irrigation or industrial wastewater on the Hanford Site.

In simulations documented in Section 4.3.2 of Cole et al. (1997), the areas where predicted future water conditions differ from those estimated for 1944 included: 1) the area west of the 200 West Area, where the water table is higher than pre-1944 conditions because it reflects the effect of higher irrigation in areas west of Hanford, and 2) the area north of Richland, where the model simulates the hydraulic effect of net recharge at the North Richland well field. The water table has been estimated to drop as much as $11 \mathrm{~m}$ beneath the 200 West Area near U Pond and $10 \mathrm{~m}$ beneath the 200 East Area near B Pond from 1996 to predicted post-Hanford steady state flow conditions (Cole et al. 1997). Steady state conditions were reached in many areas by the year 2100 and in all areas by 2350 .

Simulations from 1995 conditions made by Chiaramonte et al. (1997) (see Figures 3.2 through 3.6 in Chiaramonte et al. [1997]) showed the water table would decline for the first 100 years and stabilize within 200 years. A comparison of the water table after 200 years with the estimated water table map for 1944 showed a similar pattern of agreement as indicated in results by Cole et al. (1997) (see Figures 4.17 and 4.18 in Cole et al. [1997]). Good agreement with 1944 conditions was seen in areas north of the Gable Butte and Gable Mountain and in areas to the east of the 200 West Area. Future water table conditions that are higher than 1944 were predicted in and west of the 200 West Area. This was attributed by Chiaramonte et al. (1997) to a combination of uncertainties in natural recharge, hydraulic conductivity, and porosity estimates used in these areas of the model.

Past flow-modeling results also suggest that the water table in the central areas in the Site will decline from its current position in the Hanford formation into the uppermost units of the Ringold Formation. Consequently, future flow conditions and potential contaminant transport in areas east of the 200 Area Plateau will be more strongly influenced by the hydraulic characteristics of the subunits identified in the Ringold Formation. Of particular significance will be the influence of the low-permeability mud units identified in the upper part of the Ringold profile.

Future flow conditions simulated by Chiaramonte et al. (1997) (see Section 3.2 in Chiaramonte et al. [1997]) and Section 4.3.2 in Cole et al. [1997]) have suggested that the water table may decline to near the top of basalt in an area north of the 200 East Area. As the water table drops in the central portion of the Hanford Site, the saturated thickness of the unconfined aquifer will decrease and may eventually dry out over most of the area south of Gable Mountain along the southeast extension of the Gable Butte anticline. This could greatly reduce the aquifer cross section and the potential for flow to the north from the 200 areas in the future. However, a relatively deep but narrow channel apparently exists in the basalt surface north of the 200 East Area, so that some groundwater flow to the north will still be possible after the water table declines. 
More detailed investigations of local geologic and hydrologic conditions within the HGWP suggested that predictions of flow and potential contaminant transport through this region are uncertain and could be influenced by a number of factors.

- Interpretations of the top of basalt: In the region just east of Gable Butte, the top of basalt has been eroded and is difficult to delineate to the resolution needed to accurately model the position of the water table. Current interpretations of the top of basalt in this area are based on information from magnetic surveys.

- Interpretations of the areal extent and geometry of low-permeability mud units found in the Ringold Formation just east of 200 East plateau: Patterns of groundwater flow and contaminant transport will be influenced by the lower hydraulic characteristics of these units as the water table drops.

- Potential for upward leakage of water from the uppermost confined basalt aquifers: The region in the vicinity of Gable Butte and Gable Mountain is an area where the basalt is significantly deformed and fractured and an area of potential recharge to the unconfined aquifer system from the uppermost confined aquifers. As the unconfined aquifer becomes less influenced by the artificial recharge, upward leakage from the basalt-confined aquifer could influence the future position of the water table and future directions of groundwater flow.

- Uncertainty in the amount of recharge from the Cold Creek and Dry Creek Valleys: Increases or reductions in flow from these boundaries could have a significant influence on the future position of the water table in the aquifer system.

- Future off-site and on-site land uses: Future land uses, particularly the potential for large-scale irrigation, could have a significant influence on future water table conditions and resultant groundwater flow.

\subsection{Existing Radiological and Chemical Contamination}

Groundwater contamination at the Hanford Site is associated with a number of sources. However, most widespread contamination resulted from large volumes of liquid waste discharged to the ground since the 1940s. This wastewater percolated through the soil and reached the water table in many locations. Very little liquid waste is currently disposed to the soil, and cleanup of existing groundwater contamination, by pump-and-treat systems for example, is occurring at some locations. However, residual contamination in the vadose zone provides a continuing source of groundwater contamination in some areas. The potential for migration of these contaminants to the water table is increased by natural or artificial recharge. Other potential continuing sources include leaks from single-shell waste tanks and waste handling and disposal facilities. 


\subsubsection{Current Distribution of Radiological and Chemical Contaminants}

Monitoring of groundwater across the Hanford Site has detected a number of radioactive contaminant plumes (Figure 4.37) emanating from various operational areas (Hartman et al. 2000). The most widespread radionuclide contaminants in groundwater are tritium and iodine-129. Smaller plumes of strontium-90, technetium-99, cobalt-60, and uranium contain concentration levels exceeding EPA and state of Washington interim drinking water standards. Plutonium, americium, and cesium-137 contamination has also been found in groundwater at a few locations on the Site.

The extents of major chemical contaminant plumes in Hanford Site groundwater at levels above the regulatory concentration limits are shown in Figure 4.38. The most widespread contaminant is nitrate. Carbon tetrachloride is found over a relatively large area surrounding the 200 West Area. Other chemical contaminants include chloroform, chromium, cis-1, 2-dichloroethane, fluoride, and trichloroethylene (Hartman et al. 2000).

\subsubsection{Uncertainty in Distributions of Plumes}

The distributions of contaminant plumes at the Hanford Site are determined from analysis of groundwater samples taken from several hundred wells. The frequency of sampling varies from monthly to semiannually depending on the purpose of monitoring and the expected change in contaminant concentrations. Plume extents and concentrations are interpreted from these data and reported each year (e.g., Hartman et al. 2000). The monitoring data are also stored in the Hanford Environmental Information System database.

Uncertainty exists in both the measurements of contaminant concentrations and the plume interpretation process. Measurements are subject to detection limits and accuracy limits. Information on currently used analytical methods is provided in Hartman (2000b). Gross errors in analysis or in the reporting of contaminant data have also occurred and result in uncertainty. However, the most significant uncertainty is associated with the following plume interpretation factors.

- Limited well coverage - Plumes are based on a limited number of wells and must be interpolated between these measured concentration data. The distribution of sample locations has also changed over time, which has sometimes caused major changes in plume interpretation from one year to the next.

- Monitored depth interval - Depth of the well, length of the well screen, and the depth of the pump intake can affect contaminant concentrations in samples if concentrations vary with depth. Most wells are completed in the upper $10 \mathrm{~m}$ of the unconfined aquifer system. Higher concentration areas may exist below the well open interval. Also, groundwater samples may represent a mixture of contaminated water from a relatively thin contaminant plume and water from uncontaminated sections of the aquifer, resulting in a measured concentration that is lower than the actual plume concentration. The completion interval of many wells has also changed over time, which causes uncertainty when looking at historical data. 


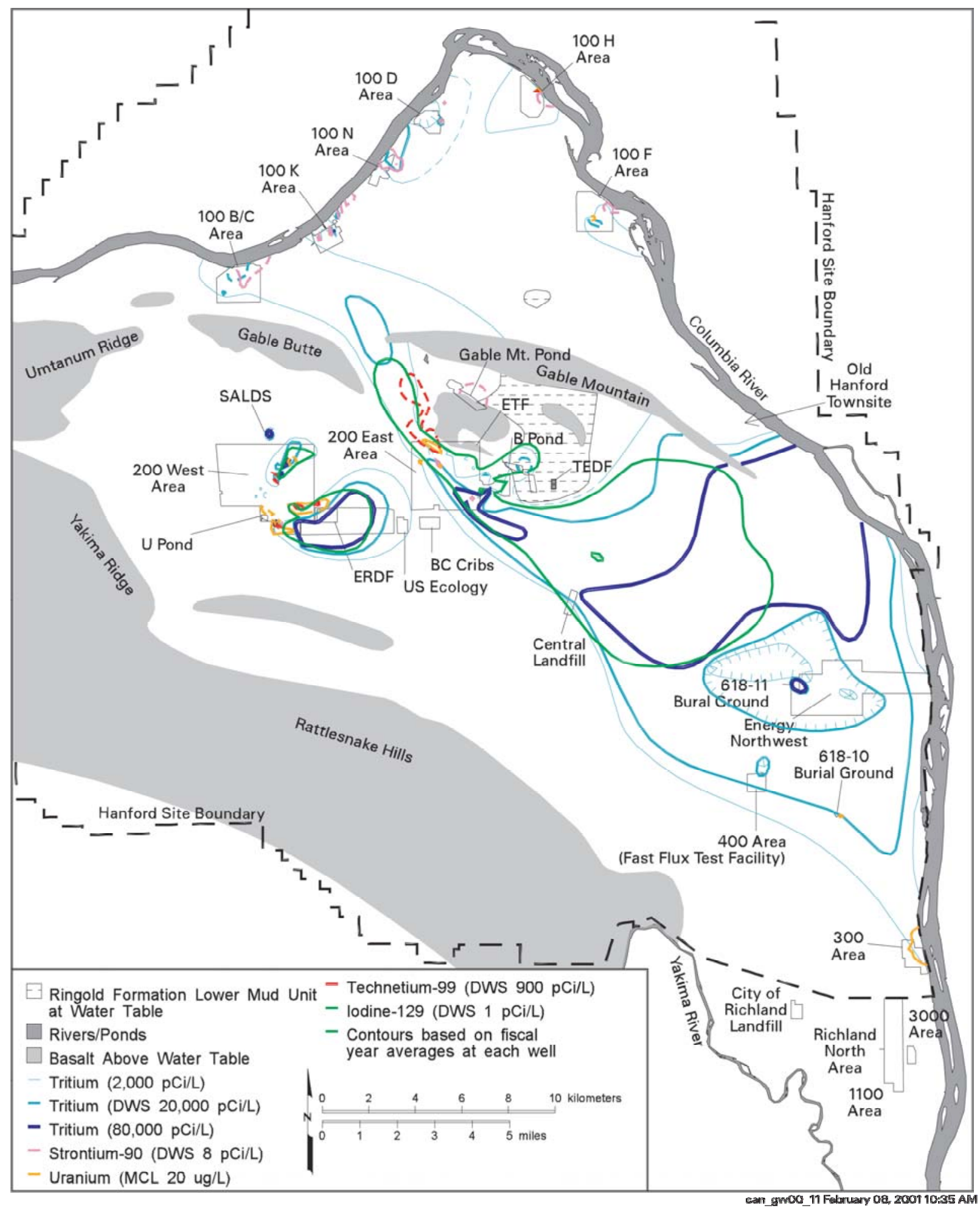

Figure 4.37. Extent of Radiological Contaminant Plumes at the Hanford Site. 


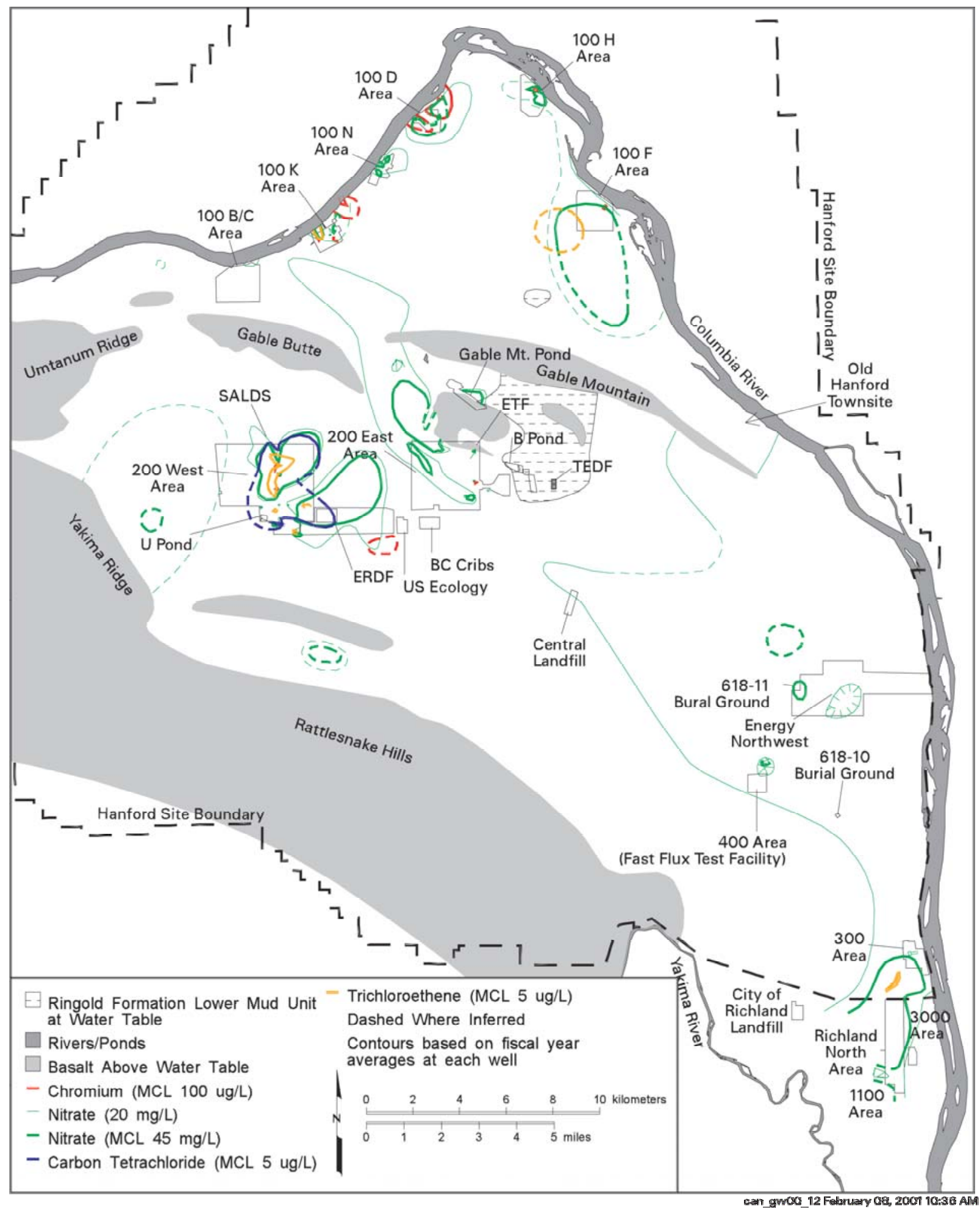

Figure 4.38. Extent of Hazardous Chemical Contaminant Plumes at the Hanford Site. 
- Vertical contaminant movement along well casing - For wells in contaminated areas, inadequate well seals have sometimes resulted in the movement of contaminants from the vadose zone or the surface to the water table. Therefore, data may represent this local contamination rather than a plume moving from some other source.

- Uncertainty in monitored interval - Inadequate casing integrity or well seals may result in contaminants entering the well from a different vertical section of the aquifer, or a different aquifer, than the well is thought to monitor. At the Hanford Site, this is most likely to affect older wells that monitor the deeper portion of the unconfined aquifer system or the basalt-confined aquifers.

- Heterogeneity - Groundwater tends to flow through sediments with higher hydraulic conductivity rather than less permeable units. Therefore, if a monitoring well happens to be completed where the permeability is low because of heterogeneity within the hydrogeologic unit, contaminants may move around the well through more permeable sediments.

- River stage fluctuations - Near the Columbia River, the distribution and concentration trends of contaminants may be affected by a changing direction of groundwater flow caused by bank storage. Movement of river water into the aquifer may dilute contaminants. Unusually high water levels in the river may also cause a water table increase near the river that remobilizes contaminants from the vadose zone.

- Groundwater remediation - Extraction and injection wells affect the direction of groundwater flow locally. This affects contaminant distributions. If treated water is injected back into the aquifer, it may contain contaminants that were not treated or may dilute local groundwater plumes.

\subsubsection{Potential Sources of Contaminants}

The unconfined aquifer will be affected by potential releases of radiological and chemical contaminants to the groundwater. These contaminants may result from a variety of waste sources, including:

- residual contamination left in the vadose zone from past waste-management operations: this includes liquid discharges to cribs, ditches, French drains, trenches, and ponds. Most of these sources are located in the 100, 200, and 300 areas

- past-practice (pre-1988) solid LLW burial grounds in the 200 areas and post-1988 solid LLW burial grounds in the 200 areas

- Environmental Restoration Disposal Facility located between 200 East and 200 West areas

- 149 single-shell tanks arrayed in 12 tank farms in the 200 areas 
- 28 double-shell tanks arrayed in 6 tank farms in the 200 areas

- ILAW disposed of in two locations in 200 East Area

- graphite cores from surplus reactors currently located in the 100 areas

- canyon buildings and related structures located in the 200 areas.

\subsubsection{Uncertainty Issues Related to Transport of Existing Plumes and Future Contaminant Sources}

In addition to uncertainty issues mentioned for the existing contaminant plumes in the previous section, additional uncertainty exists related to forward transport behavior of existing plumes and potential future sources of contamination. Some key areas of uncertainty include the following:

- Contamination mass in the vadose zone - A considerable amount of mass of both relatively mobile and immobile contaminants is suspected to be tied up in the vadose zone at residual levels from past and current releases. Additional contaminants will also be released eventually into the vadose zone from existing and future sources of contaminants. Contaminants in the vadose zone are expected to migrate slowly down to the underlying water before being transported laterally to discharge points at wells and/or the Columbia River.

- Release and mixing of contaminants upon arrival at the water table - Very little information exists on the behavior of contaminants being released to the water table from residual contamination in the vadose zone as it reaches the capillary fringe and enters into the upper part of the aquifer system.

- Regional dispersion of future sources of contamination - While much data and information are available about the regional transport of contamination released to the aquifer system from relatively high-volume liquid discharge sites, little field evidence is available about the regional transport behavior of future contaminant sources which will be released to the aquifer with very low fluid flux rates (i.e., natural and/or effective barrier infiltration rates).

- Diffusive mass transfer - Diffusive mass transfer, involving mass transfer between an immobile and a mobile domain, may be an important issue to consider in situations where the effective porosity is significantly smaller than the total porosity. The associated "tailing" (later mass arrival) of contaminant plumes may likely be significant at the Hanford Site. Since the current site-wide groundwater model does not consider this process, predictions made with this model will overestimate the rate at which these plumes migrate and dissipate after a source is removed.

- Adsorption processes and other potential chemical processes - The transport of most existing sitewide plumes and potentially important future plumes reflect relatively mobile constituents (tritium, iodine-129, technetium-99, and uranium) that are not significantly impacted by reactive processes other than adsorption. However, wastes sites do exist that have received wastes with complex 
chemistry, such as crib and trench sites that have received tank wastes or at sites near suspected tank leaks, that may have geochemical conditions that can influence the contaminant mobility of constituents that would normally be relatively immobile. These processes will likely be important in evaluating contaminant mobility of some of these constituents close to the waste sources, but whether the effect of these processes may have an influence on their mobility within the unconfined aquifer system is uncertain.

- Potential sources of uncharacterized sources of contamination at depth below the water table - The majority of groundwater monitoring being conducted across the Site has focused on monitoring the water table elevation and contaminant levels in the upper part of the aquifer system. Little is known about the levels of contamination found at depth in the aquifer system near wastes sites that received large volumes of wastewater or discharged large quantities of organic contaminants such as carbon tetrachloride in 200 West Area.

- Future land uses and potential impacts to on-site groundwater conditions - The focus of many predictions of contaminant fate and transport involve time scales well beyond the period of Site closure where land use and potential impacts to Site groundwater conditions are unknown.

- Effects of uncharacterized heterogeneity. 


\subsection{Technical Approach and Methods for Assessing Uncertainty in Hanford Site Groundwater Model Predictions}

This section of the report provides a discussion of the technical approach and methods that will be used to assess uncertainty in Hanford Site groundwater predictions.

\subsection{Overall Technical Approach for SGM Uncertainty Framework}

The overall technical approach being developed for use in assessing uncertainty in Hanford Site future predictions of groundwater and contaminant transport will closely follow the recommendations the external peer review panel made in 1999 (Gorelick et al. 1999). As the panel suggested, a new framework is being developed that acknowledges there is inherent uncertainty in conceptual representations and associated model inputs and thus in any predictions. This new framework acknowledges that prescribed processes, physical features, initial and boundary conditions, system stresses, field data, and model parameter values are not known and cannot be known with certainty, and as a result, predictions of heads and concentrations in three dimensions over time will be uncertain as well. The approach will specifically address those areas of special interest that were identified by the expert panel. These include uncertainty

- due to alternative model structures and constructs of processes (e.g., different zonation, different boundary conditions, large-scale features, stresses, chemical reactions)

- related to model parameters

- related to model scale and resolution issues.

This report represents one of the first steps in development of this new SGM uncertainty framework by

- identifying the types of assessments for which the SGM will likely be applied (Section 2)

- discussing the various sources of uncertainty and the issues associated with the assessment of uncertainty as they relate to the development of this new framework for assessment of uncertainty in the Hanford SGM predictions (Section 3)

- providing a detailed description of the current conceptual model of the Hanford Site unconfined aquifer system that includes an assessment of the uncertainties and issues (Section 4), the well picks used in the current interpretation of the hydrostratigraphic structure (Appendix A), and the results of a literature search that developed an initial bibliography and assessment regarding the various potential intercommunication mechanisms between the unconfined aquifer system and the uppermost confined aquifer system in the underlying basalts (Appendix B and attachments) 
- identifying the technical approach and initial plans and steps to be undertaken to develop the new framework (discussed in this section).

The uncertainty framework being developed is based on a classical approach as discussed in Section 3. The uncertainty assessment methods being adopted involve set and probability theory (Klir 1994) and will closely follow the approach discussed in Morgan and Henrion (1994). It will not address uncertainty using what Klir describes as novel uncertainty theories (e.g., fuzzy set theory). The approach used for uncertainty assessment, at least initially, will be probability-based like that developed and applied for probabilistic risk assessment of nuclear reactors (Vesely and Rasmuson 1984; USNRC 1997), and for the assessment of total system performance of potential geologic repositories at WIPP and Yucca Mountain (CRWMS M\&O 1999; Jow et al. 1997). As discussed in Section 3, the approach can be expanded to include both stochastic and subjective uncertainty (Helton 1993), where the stochastic uncertainty, or variability, is defined as the uncertainty resulting from or attributable to geologic heterogeneity and natural variability, and subjective uncertainty is defined as the uncertainty arising from ignorance or imperfect knowledge about processes (i.e., the conceptual model).

The general approach being adopted for addressing groundwater flow and transport model uncertainties in the Hanford SGM consists of addressing most of the blocks and issues identified in Beck's (1987) frame of reference diagram for the analysis of uncertainty (Figure 3.11). The emphasis will be on combining the knowledge and assumptions on the external and internal descriptions of the system in order to identify plausible alternative model structures capable of describing the uncertainty in the historical and current system state for use in the prediction phase (i.e., the uncertainty propagation phase). The only block in Beck's (1987) frame of reference diagram not being addressed in our initial efforts is the one that could be described as the scenario block in the lower left corner of the diagram (i.e., uncertainty in future observed inputs associated with the external description of the system or what might be thought of as the SGM system's environment). Future system states as discussed in Section 3 could be viewed as evolving as a sequential process with each stage occurring independently with a fixed probability; however, this simplistic view is not very applicable to the real world as many events are far from independent. At the Hanford Site, a flood that alters the river course could alter groundwater pathways and cause a failure in an engineered barrier, and fires that clear vegetation could also lead to increased recharge in a source area. Uncertainty related to future conditions and their uncertainty is generally not addressed by the methodology to be outlined here. Scenario uncertainty, because of its complexity, will need to be addressed by a separate effort. In our current assessments of uncertainty, we will treat uncertainty related to future system states essentially through sensitivity analysis since probabilities of occurrence will not be assigned. The uncertain predictions from one future state can be compared with those for another future state (e.g., no future development or climate change results could be compared to results for a scenario with full development and slowly changing climate). The effect of uncertainty related to scenarios is accounted for as part of the subjective uncertainty discussed in Section 3.3.3.3.1 and further discussed below. The exception to treatment of scenario uncertainty would be when the uncertainty of interest can be adequately treated by uncertainty in, for example, a boundary condition parameter or some other type of simple, characterizable parameter uncertainty and thus addressable through standard Monte Carlo methods. 
As discussed in Section 3.3, while assessment of the uncertainty in groundwater flow and transport predictions does not necessarily involve all seven categories of uncertainty (i.e., data, analyst, modeling, completeness, frequency, consequence, and interpretation uncertainty) associated with a PRA (Vesely and Rasmuson 1984), we will use the same general approach in addressing uncertainty in groundwater system flow and transport predictions. The basic approach is to use sensitivity analysis for those aspects of the analysis related to vagueness and indefiniteness (e.g., alternative conceptual models, model structure, and future scenarios) and uncertainty analysis for those situations where the uncertainty (e.g., for parameters) can and should be represented by a pdf (e.g., as interpreted from measurements using geostatistical methods, or determined from inverse modeling). The basic approach identified for addressing Hanford SGM uncertainty is as outlined in Section 3 and consists of the following basic steps:

1. Identify ACMs - This effort (see discussions in Sections 3.1.2, 3.2.2, and 3.3.1) consists of identifying and documenting the major features and assumptions associated with alternative conceptual models for the Hanford SGM as well as periodically reviewing this list of alternative conceptual models for the Hanford SGM based on any new data or understanding to see if any new ACMs should be added, developed, and evaluated against existing ones, or if existing ACMs should be eliminated. Totally new ACMs could be developed, but more likely the new ACMs will involve expanding and/or modifying existing ACMs (e.g., adding additional components, using different representations of processes, modifications or changes to the model structure based on new data and interpretations, altering/expanding the domain, and/or altering the model boundary conditions).

2. ACM Development - This step involves developing each new or altered ACM. In the approach identified this step will generally involve (if possible) an inverse modeling step (Section 3.2.3.2) in which historical site data on parameters, system responses (e.g., head and concentration), and external driving forces will be used in a parameter identification step to develop the best representation of this model and to help determine the associated uncertainty estimates in some of its parameters that is consistent with the available historical data available from the start of Hanford Site operations in 1943 (i.e., Beck's [1987] identification phase, Figure 3.11). Inverse modeling has already been completed for two ACMs and is documented in Cole et al. (2001) and Vermeul et al. (2001).

3. ACM Evaluation - This third step involves evaluating each ACM that has been developed to identify the plausible conceptual models that will be the subject of subsequent uncertainty assessments. Following the ACM development some ACMs may drop out without the need for further evaluation or comparison with existing "plausible" ACMs based on their inability to reasonably match historical site data on parameters, system responses. However, it is envisioned that there will be multiple plausible ACMs or ACM variations and some approach will be required to evaluate or rate them to determine those that are "plausible" enough to include in the outer summation, $\sum_{\text {SU }}$, of Equation 3.11

to capture the subjective uncertainty (Jow et al. 1997) associated with alternative plausible conceptual models. For example, each plausible ACM would be represented by an individual CCDF in Figure 3.29 or even several CCDFs if more than one future scenario is investigated for the ACM. Complete documentation of each ACM evaluation is an important part of the process. ACM evaluation as discussed in Section 3.2.2 will initially consist of examining statistical measures of overall model fit discussed in Hill (1998) as well as examining, as appropriate, the model structure 
identification measures suggested and discussed in Carrera and Neuman (1986) that they have found to be successful in discriminating between different parameterizations (e.g., transmissivity and recharge zones) of a test case of groundwater flow. These measures include the maximum likelihood objective function, Akaike's First Information Criterion (1974), Akaike's Second Information Criterion (1977), Hannan's Criterion (1980), and Kashyap's Criterion (1977).

4. ACM Uncertainty Assessment - Steps 1 through 3 of this process described above must be completed for all reasonable ACMs that have been identified, while this step (step 4) is only carried out for the ACMs determined to be plausible in step 3. The parameter uncertainty assessment process generally involves:

- Model Complexity Optimization - If as discussed in James (1994) the number of uncertain parameters in a model is considered to be one measure of model complexity, then this step involves sensitivity studies to identify the important or relevant parameters (Figure 3.27) for the uncertainty analysis. That identification will be based on the uncertainty in the parameter value and the sensitivity of model predictions of interest to variations in the parameter, as discussed in Meyer and Gee (1999). As mentioned in Section 3.3.3.1, optimization of model complexity is an issue and process that must be revisited at all steps of the uncertainty assessment because the total number of parameters whose uncertainty could be characterized with a pdf, and thus varied to produce uncertainty estimates, is large compared to the capacity to compute.

- Characterize Parameter Uncertainty - This step involves developing the pdfs for the important uncertain parameters as discussed in Section 3.3.3.2, including the identification of any correlations among parameters.

- Propagate Uncertainties - This step involves propagating the uncertainties (e.g., by first order second moment methods if applicable or by a Monte Carlo approach) through the model to determine the uncertainty in the model predictions of interest as discussed in Section 3.3.3.3.

5. Estimation of Combined ACM and Scenario Uncertainty - This step involves estimating the combined uncertainty as discussed in Section 3.3.3.3.1. The combined uncertainty is represented by the double sum presented in Equation 3.11. Each component of the inner sum represents the stochastic (or parameter) uncertainty associated with a particular scenario and plausible ACM and this uncertainty is represented by an individual CCDF (e.g., see Figure 3.29). The outer sum enumerates the various plausible ACMs and scenario combinations, which in the example from Jow et al. (1997), gives rise to the family of CCDFs in Figure 3.29 that represents the combined estimate of uncertainty.

The combined uncertainty associated with compositing uncertainties associated with the plausible ACMs for the various scenarios of interest, as outlined above, represents the best estimate of uncertainty that can be developed based on current information and understanding. While the above list is a convenient way to describe the approach, there is considerable overlap in some of the items. This is because ACM identification and development requires examining and optimizing model complexity (as measured by the number and complexity of processes and parameters included) to be consistent with availability of information while attempting to honor the antithetical concept of model parsimony that requires the 
modeler to seek the simplest model parameterization consistent with the evidence (Kuczera and Mroczkowski 1998; Box and Jenkins 1976). As discussed in Section 3.3.3.1, Seo (1991) indicated that while it is important to attempt to improve model accuracy and reliability by including as many relevant processes at the finest possible spatial and temporal discretizations, there is no guarantee that model reliability will continue to increase with model complexity.

Another important part of the process of performing an uncertainty analysis for each of the plausible ACMs of the Hanford SGM will be the identification, enumeration, and documentation of all the assumptions, which include those

- made during conceptual model development

- required by the mathematical model

- required by the numerical model

- made during the spatial and temporal descretization process (e.g., Figure 3.20)

- needed to assign the statistical model and associated parameters that describe the uncertainty in the relevant input parameters

- required by the propagation method.

Additionally any new issues, hypotheses, or ACM modifications identified during the development, testing, and uncertainty propagation step for a given ACM need to be documented.

Comprehensive documentation of the uncertainty analysis performed for each ACM is key to achieving transparency. Documentation of the information described above is important because the uncertainties related to these assumptions can be addressed only through additional sensitivity or uncertainty studies and/or alternative conceptual model evaluations. Additional sensitivity or uncertainty studies will be required to determine the impact of new findings (e.g., new data or new geologic models) that are not included in an uncertainty assessment. Table 5.1, which is adapted from the EPA (1997) document, Guiding Principles for Monte Carlo Analysis, provides a good guide for carrying out an uncertainty assessment. 
Table 5.1. Principles of Good Practice for Monte Carlo Simulation (adapted from EPA 1997)

\section{Selecting Input Data and Distributions for Use in Monte Carlo Analysis}

1. Conduct preliminary sensitivity analyses or numerical experiments to identify model structures, exposure pathways, and model input assumptions and parameters that make important contributions to the assessment endpoint and its overall variability and/or uncertainty.

2. Restrict the use of probabilistic assessment to significant pathways and parameters.

3. Use data to inform the choice of input distributions for model parameters.

4. Surrogate data can be used to develop distributions when they can be appropriately justified.

5. When obtaining empirical data to develop input distributions for exposure model parameters, the basic tenets of environmental sampling should be followed. Further, particular attention should be given to the quality of information at the tails of the distribution.

6. Depending on the objectives of the assessment, expert judgment can be included either within the computational analysis by developing distributions using various methods or by using judgments to select and separately analyze alternate, but plausible, scenarios. When expert judgment is employed, the analyst should be very explicit about its use.

\section{Evaluating Variability and Uncertainty}

7. The concepts of variability and uncertainty are distinct. They can be tracked and evaluated separately during an analysis, or they can be analyzed within the same computational framework. Separating variability and uncertainty is necessary to provide greater accountability and transparency. The decision about how to track them separately must be made on a case-by-case basis for each variable.

8. There are methodological differences regarding how variability and uncertainty are addressed in a Monte Carlo analysis. (Issues to be considered are enumerated in EPA 1997.)

9. Methods should investigate the numerical stability of the moments and the tails of the distributions.

10. There are limits to the assessor's ability to account for and characterize all sources of uncertainty. The analyst should identify areas of uncertainty and include them in the analysis, either quantitatively or qualitatively.

\section{Presenting the Results of a Monte Carlo Analysis}

11. Provide a complete and thorough description of the exposure model and its equations (including a discussion of the limitations of the methods and the results).

12. Provide detailed information on the input distributions selected. This information should identify whether the input represents largely variability, largely uncertainty, or some combination of both. Further, information on goodness-of-fit statistics should be discussed.

13. Provide detailed information and graphs for each output distribution.

14. Discuss the presence or absence of dependencies and correlations.

15. Calculate and present point estimates.

16. A tiered presentation style, in which briefing materials are assembled at various levels of detail, may be helpful. Presentations should be tailored to address the questions and information needs of the audience. 


\subsection{Uncertainty Framework Implementation}

The previous section outlined the basic approach identified for addressing Hanford SGM uncertainty. This section describes the implementation plans in more detail as well as the current status of ongoing efforts to develop the new uncertainty framework. As discussed above, the steps include

- identifying ACMs

- developing ACMs

- evaluating ACMs

- assessing ACM uncertainty

- optimizing model complexity

- characterizing parameter uncertainty

- propagating uncertainties

- estimating combined ACM and scenario uncertainty.

The final step involves identification, enumeration, and documentation of the all the assumptions as well as any issues or new hypotheses or ACM modifications identified during the development, testing, and uncertainty propagation step for each ACM.

\subsubsection{ACM Identification}

The model identification phase attempts to identify alternative interpretations of components of the site conceptual model that can be supported by direct measurements, data, observations, or indirect information such as interpretations based on technical judgment or scientific principles as well as the uncertainties associated with each entities. Section 4 and Appendix B of this report document the first attempt to examine various aspects of the Hanford SGM conceptual model components, which include model boundaries, structural features, and flow and transport parameters, process representation, and other features in order to identify key conceptual and data uncertainties. Many of the key components of the site conceptual model that will be considered initially have been developed based on technical issues identified by Hanford technical staff and through public and external review of the prior Hanford SGM. These public and external reviews have helped identify a number of components of the Hanford SGM conceptual model whose uncertainty warrants further investigation as discussed in Section 4. Continuing external review of the Hanford SGM and the new framework for uncertainty will likely lead to identification of other issues and additional improvements. Initial model and parameter identification efforts during the ACM development step will focus on the following list of ACMs to address issues already identified:

- Prior Hanford SGM - The prior conceptual model, described in Cole et al. (1997), was the first conceptual model to be evaluated. This assessment was simply to provide an assessment of the adequacy of the prior model and to provide a transient inverse calibration of this prior model for a baseline comparison with the multiple ACMs that will eventually be examined. This effort has been completed and the results are described in Cole et al. (2001). Figure 5.1 from Cole et al. (2001) 
(a) Prior Model

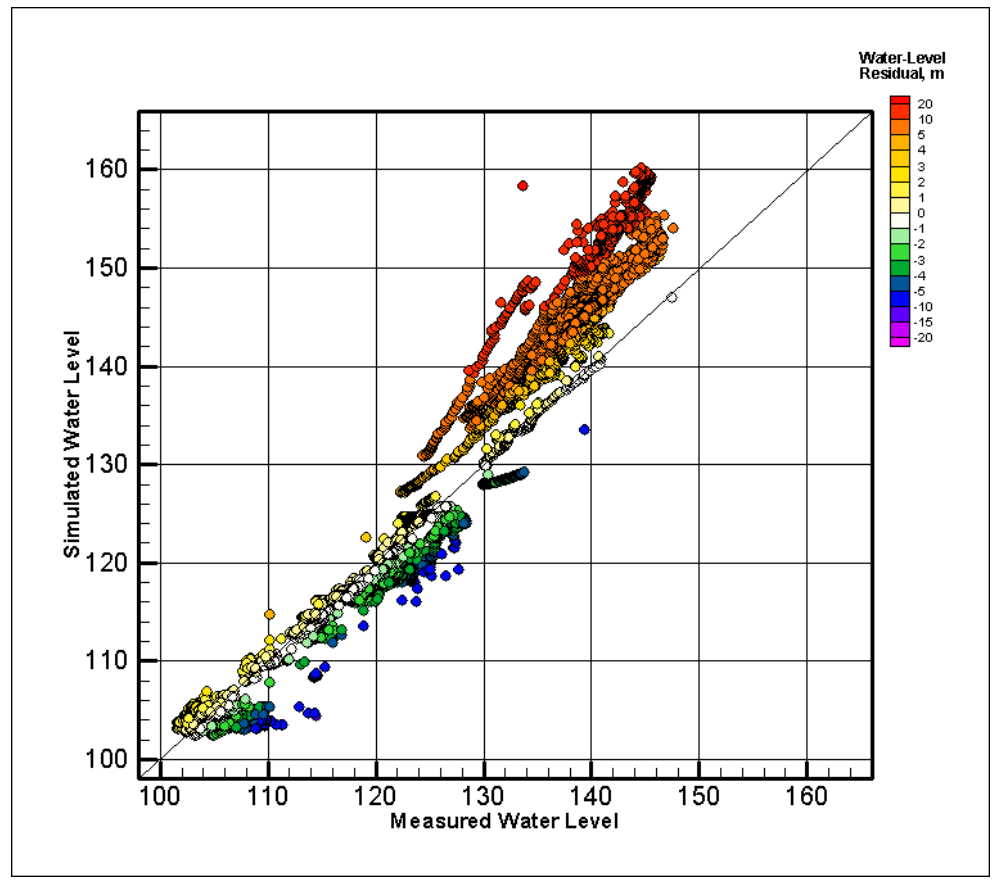

(b) Baseline Model

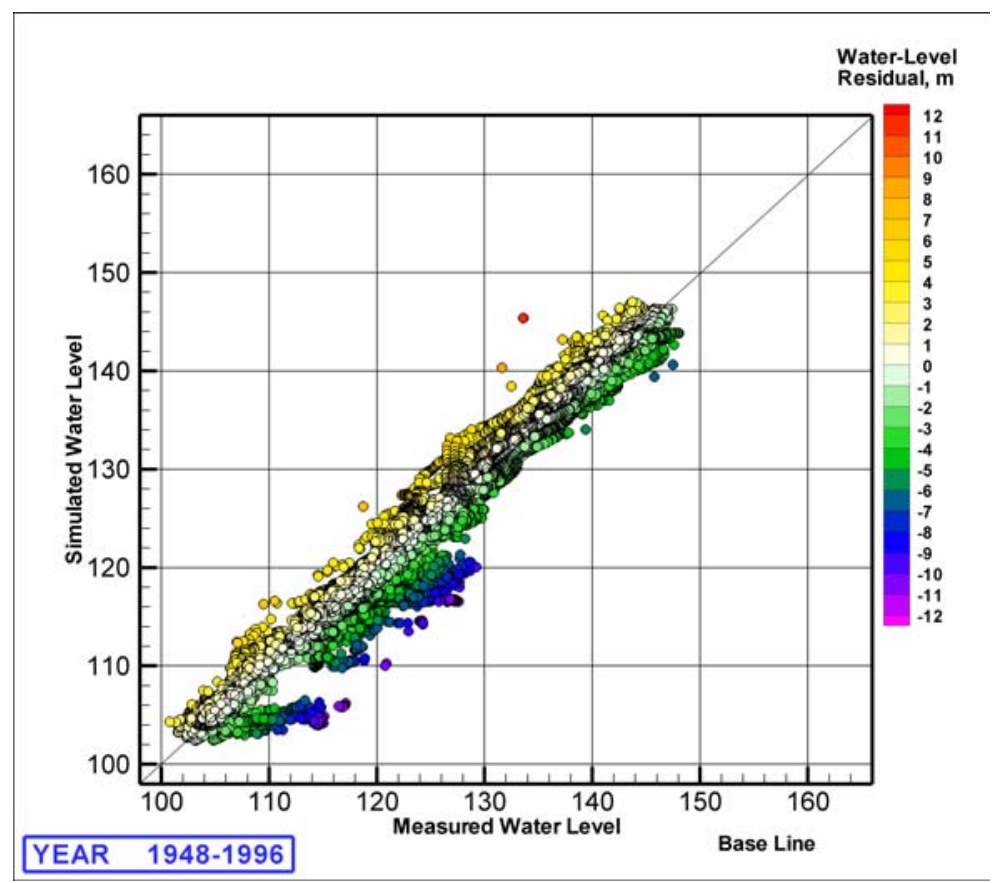

Figure 5.1. Simulated Versus Observed Heads for All Observations Through Time for (a) Prior Model and (b) Baseline Model. 
illustrates a comparison of the simulated versus observed heads for all observations through time for both the prior model and the transient inverse calibrated prior model, i.e., the baseline model.

- Basalt Interaction ACMs - These ACMs consider the interaction of the unconfined aquifer system with the uppermost confined aquifer associated with the underlying Columbia River basalt group. As discussed in Section 4.6.3, five intercommunication mechanisms were identified. The three most important mechanisms include the effects of 1) larger-scale regional flow and intercommunication of the unconfined aquifer with uppermost confined aquifers in the Columbia River basalt group, including the effect of regional upward discharge from the basalts into the Pasco Basin; 2) localized flow through two major structural features (Figures 4.3 and 4.5), which includes the thrust fault running along the north side of Gable Butte and Gable Mountain and the Yakima Ridge running along the southwestern boundary of the Hanford SGM; and 3) localized intercommunication between the unconfined aquifer and the uppermost confined unit through an erosional window in the vicinity of the 200 East Area between Gable Mountain and Gable Butte (Figure 4.30). These aspects of the Site conceptual model are important because of increased communication expected between the unconfined aquifer and the uppermost confined aquifer(s) as the effects of previous artificial discharges dissipate and the unconfined aquifer returns to more natural flow conditions. For postclosure at the Hanford Site, the influence of leakage from the basalt system could be even more important in influencing the regional position of the water table and in affecting overall direction of regional flow and transport of contaminants from waste sources in the 200 Area Plateau, either north through the gap between Gable Butte and Gable Mountain or east to the Old Hanford Townsite. The initial inverse modeling efforts that examined the various basalt interactions has been completed and is documented in Vermeul et al. (2001). Figure 5.2 illustrates histograms of predicted head residuals for all observations for both the baseline ACM (Cole et al. 2001) and the best-fit basalt interaction inverse model (Vermeul et al. 2001).

- ACMs with Alternative Structural Geometry and Alternative Zonation for Representation of Hydraulic and Transport Property Heterogeneity - Previous hydraulic parameter zonation within the conductive units of the Hanford SGM were based on parameter zonation patterns developed using two-dimensional steady state inverse models that employed both hard and soft geohydrologic evidence to estimate transmissivity distributions (Jacobson and Freshley 1990). As a result, existing zonation patterns generally reflect only the broad patterns of the more highly conductive depositional facies of the Hanford formation in areas where the water table is contained in the Hanford formation (Figure 4.6). Thus, the zonation is not reflective of the regional depositional facies needed to describe the heterogeneity of effective hydraulic properties of major permeable units within the Ringold Formation that underlie the Hanford formation in these areas. This effort involves evaluating ACMs that represent alternative zonation patterns, which are more reflective of site-wide scale variability in effective hydraulic and transport properties in the Ringold Formation based on interpretation of the geologic depositional history and resulting major geologic facies distributions and sediment characteristics. The multiple ACMs investigated in this effort will examine the effects of the uncertainty in this portion of the basic Hanford SGM structure. Some of these realizations will be interpretations made by Hanford Site hydrogeologists using standard interpretive methods (Thorne et al. 2001), while other interpretations will be developed through a joint effort between a scientific 
(a) Baseline Model

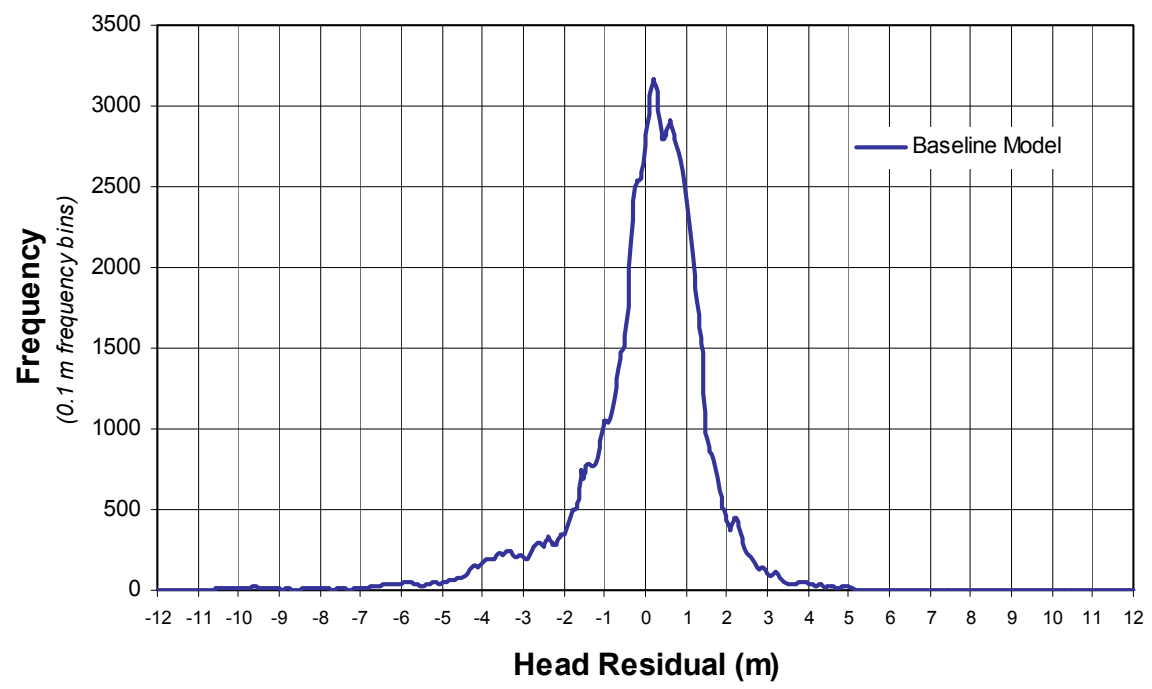

(b) Best-fit Basalt Model

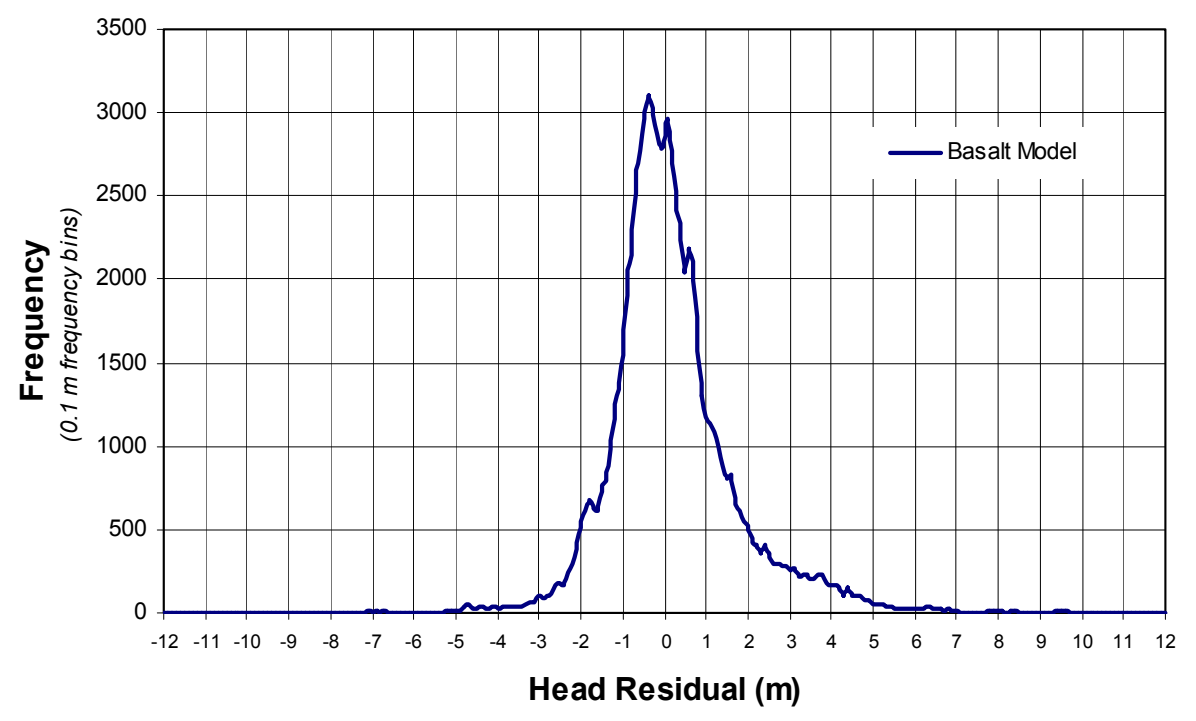

Figure 5.2. Histograms of Predicted Head Residuals for All Observations for (a) Baseline Model and (b) Best-fit Basalt Model. 
team of the Nuclear Safety Institute (IBRAE) of the Russian Academy of Sciences and U.S. Department of Energy scientists here at PNNL. The cooperative effort involves using the geologic and observational database for the unconfined aquifer system at the Hanford Site to develop and test methods for structure identification (i.e., areal extent and continuity of the mud units) and to identify plausible parameter zonation schemes for the coarse grained (i.e., aquifer) units. IBRAE scientists headed by Dr. Mikhail Kanevski are using various classification techniques and different kinds of stochastic simulations (e.g., Gaussian simulations, indicator simulations, simulated annealing, and other techniques) to define multiple realizations of the model structure associated with the areal extent/continuity of the fine-grained confining or semi-confining units (e.g., the Hanford Site unconfined system's mud units) as well as to define multiple plausible parameter zonation patterns for testing through inverse modeling (Kanevski et al. 2001). Dr. Kanevski and IBRAE scientists have provided preliminary results for identifying alternative geometric structures for the fine-grained units. Those results include geostatistical and neural network classification methods to identify the probability that Ringold Formation mud units 4, 6, and 8 are likely to be present throughout the Hanford Site. Figure 5.3 presents representative results for unit 4 that were provided by Dr. Kanevski. The geostatistical methods applied by IBRAE include sequential indicator simulation that will be used to produce multiple realizations of the presence/absence of the mud units. Those realizations will be used as an input layer in planned Monte Carlo uncertainty analysis of the impact of variability in the presence of the mud layers on flow and transport. In addition to the work performed by IBRAE, initial efforts by Hanford Site geologists, discussed in Vermeul et al. (2001),
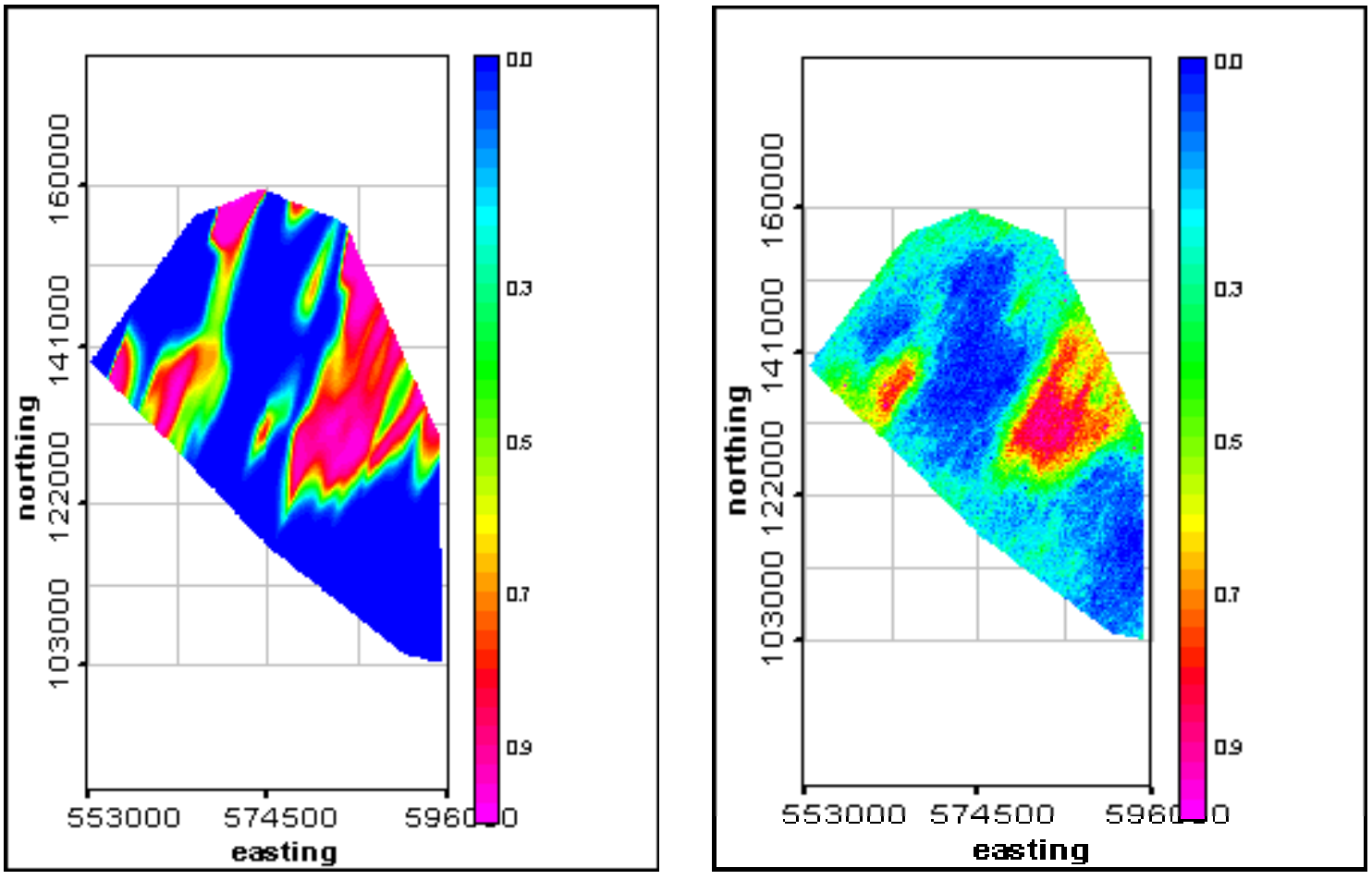

Figure 5.3. Probability of Unit 4 Presence Estimated by Probabilistic Neural Network Methods (left) and by Taking the Average of 50 Sequential Indicator Simulations (right). 
have used geologic and hydrologic information to make a preliminary map of zones (Figure 5.4) with relatively similar hydraulic properties within the saturated post-Ringold sediments (model Unit 1) and the upper Ringold Formation gravel and sand unit (model Unit 5) that subcrop at the water table.

Data used to develop this facies-based zonation included borehole records, aquifer test data, water table gradient, and the current understanding of the geologic depositional environment that existed when these sediments were deposited. Efforts at testing this new zonation realization have already begun as discussed in Vermeul et al. 2001. During FY 2002, IBRAE will perform additional work to identify alternative conceptual models for zonation of aquifer units within the Hanford and Ringold

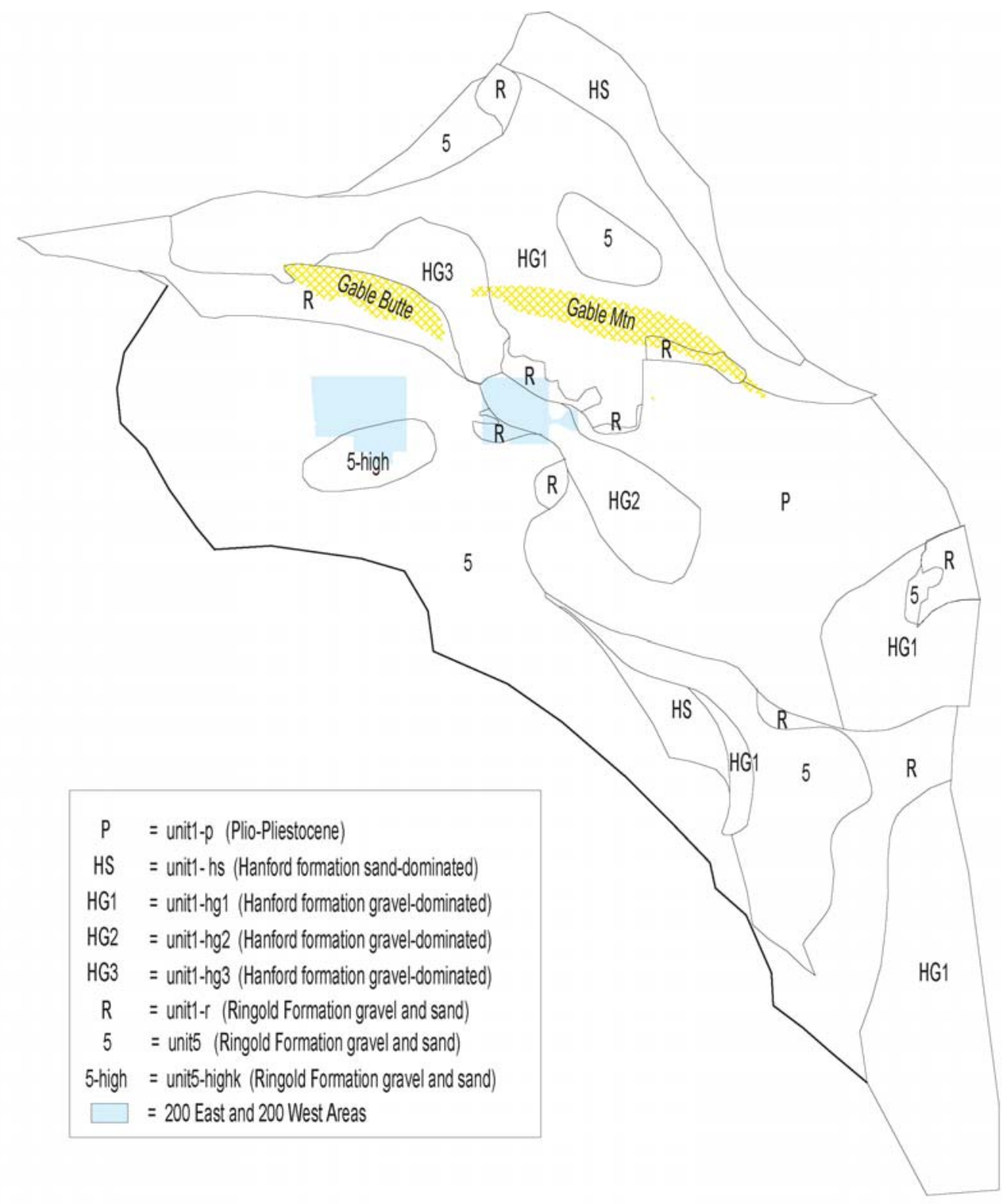

Figure 5.4. Facies-Based Zonation of Model Unit 1 and Unit 5 as Exposed at the Water Table. 
formations. A final aspect of model structure that will be investigated as a part of evaluating this set of ACMs will include examining the effect of alternative interpretations of flow and transport in the vicinity of a known geologic structure, the May Junction Fault (Figure 4.18) within the unconfined aquifer system. As discussed in Section 4.2.2.3, this fault is not currently represented in a specific way in the prior Hanford SGM. These aspects of the Site conceptual model will be examined in some detail because the potential significance of this fault in controlling and affecting regional flow and transport of contaminants from waste sources in the 200 Area Plateau as the water table returns to more natural conditions. Of specific interest is the multiple possible interpretations of the distribution of the low-permeability units in the vicinity of the May Junction Fault and their effect on transport.

- Further ACM Development Using Transport Observations - The best ACMs based on the initial flow model calibration above will be further developed using transient inverse methods that consider transport as well as flow by using the wealth of data on the Hanford Site tritium plume (Figure 4.37) discussed below in Section 5.2.2.1 and in also in Section 4.12. As part of this investigation, different model representations for transport such as diffusive mass transfer will be investigated as part of these efforts.

- ACMs to Address Other Conceptual Model Interpretations and Issues - Other alternative interpretations of conceptual components of the site-wide model can and will be investigated during the course of evaluating the larger-scale ACMs. The following list, which is not meant to be exhaustive, indicates the types of alternative representations that need to be investigated.

- The effect of the transient nature of the Columbia and Yakima Rivers on regional flow and contaminant transport

- The effect of higher water table conditions in heavily irrigated areas north and east of the Columbia River on Hanford Site-wide flow conditions and contaminant transport

- The effect of additional processes (direct evaporation, vadose zone transport, etc.) that could delay and/or reduce the amount of artificial discharge liquid and contamination sources from major wastewater discharge facilities.

\subsubsection{ACM Development}

This step involves developing each new or altered ACM through a transient inverse modeling approach in which historical site data on parameters, system responses (e.g., head and concentration), and external driving forces will be used in a parameter identification step to develop the ACM being studied and to help determine the associated uncertainty estimates in some of ACM parameters that are consistent with the available historical data and the assumptions of the ACM being studied.

\subsubsection{Data and Rationale for Transient Inverse Modeling}

Historical head $(\sim 76,000)$ and contaminant $(\sim 35,000$ tritium $)$ measurements and observations have been made in some 1200 wells over the historical period of Hanford Site operations (1943 to present). 
These measurements and observations provide realistic constraints that can help verify the reasonableness of the ACMs and also provide a common framework for intercomparison of the various ACMs. We believe, as suggested by the external review panel, that transient inverse calibration over the historical period of Hanford Site operations is a reasonable approach for model development, and that it is likely to be successful because of the following:

- Large regional-scale mound building and falling has occurred in response to waste and process water disposals over the period of Hanford Site operations. The large volumes of wastewater discharged to a variety of waste facilities over this historical period resulted in regional-scale rises in the water table and the creation of groundwater mounds under waste management facilities in the central part of the Hanford Site that by 1979 had risen $10 \mathrm{~m}$ and $22 \mathrm{~m}$ above pre-Hanford estimates of water levels in the 200 East and 200 West Areas, respectively. Similarly, since cessation of weapons production in 1988, declines in wastewater discharges have resulted in water table drops in these same areas (200 East and 200 West Areas) on the order of $2 \mathrm{~m}$ to $3 \mathrm{~m}$ and $8 \mathrm{~m}$, respectively.

- Disposal of tritium-contaminated waters has resulted in the formation of a large-scale tritium plume that is just now beginning to decrease in size (Hartman et al. 2001). Estimates for FY 1999 (Hartman et al. 2000) are that $\sim 254 \mathrm{~km}^{2}$ of Hanford Site unconfined aquifer system are above drinking water standards, and as can be seen in Figure 4.37, most of this area is associated with the large-scale tritium plumes.

Initial transient inverse evaluations of ACMs will focus on comparisons of predictions with data and information related to the hydraulic response of the aquifer system, rather than the transport of tritium, for logistical and technical reasons, which include

- logistical - from a logistical standpoint, the entire data set needed to carry out a transient inverse modeling effort involving both flow and the transport of tritium was not available, and the effort to construct early tritium disposal rates from existing data (e.g., gross beta) would be a time-consuming process and delay initiation of inverse work.

- technical - from a technical standpoint, two issues were considered: 1) the uncertainty introduced by the incomplete records on the large amounts of tritium disposed to ground prior to the time when good records were kept, and 2) performing a simultaneous flow and transport inverse would increase the model run times significantly because a finer horizontal and vertical grid is needed for contaminant transport.

These logistical and technical issues were discussed at a meeting held at PNNL that included experts in inverse modeling, Dr. Eileen Poeter (Colorado School of Mines) and Dr. Evan Anderman (Calibra Consulting Inc.), to plan the baseline inverse modeling effort that is now complete (Cole et al. 2001). Based on these discussions, it was concluded that the initial inverse efforts should concentrate on modeling the flow. 
While the observational data set consisting of $\sim 35,000$ tritium measurements has always been available, the disposal records for tritium prior to the late 1960s were not available and needed to be constructed from other records. This task was recently undertaken as part of the development of information and data supporting the initial development of the System Assessment Capability (SAC), Rev. 0 (Kincaid et al. 2000). SAC Rev. 0 and its associated assessments are an ongoing effort that is part of the Groundwater/Vadose Zone Integration Project (Integration Project) at the Hanford Site (DOE 1998). Under the SAC project, an inventory and disposal history for tritium and other contaminants (including estimates of uncertainty) have been prepared based on available historical records and process knowledge (Kincaid et al. 2001). We have completed (FY 2001) initial deterministic simulations using this disposal history as part of the effort to prepare for a transient contaminant transport inverse effort that is planned for FY 2003.

\subsubsection{Methodology/Tools}

The first implementation of the methodology and tools developed for transient inverse modeling are described in Cole et al. (2001). The following is a brief summary extracted from that document. The codes selected for implementation of three-dimensional transient inverse calibration (involving either flow, transport, or both) consists of

- UCODE, a universal inverse modeling code developed jointly by the U.S. Geological Survey and the International Groundwater Modeling Center of the Colorado School of Mines (Poeter and Hill 1998)

- CFEST, the Coupled Fluid Energy and Solute Transport code (Gupta et al. 1987; Cole et al. 1988; Gupta 1997), which is the forward model whose parameters are estimated by UCODE.

The long simulation run times for fully three-dimensional transient groundwater modeling over the historical period of interest and the large number of simulations required for transient inverse modeling indicated that a serial computational approach for the coupled flow and transport inverse was unacceptable (estimates indicated a year or more of computational effort). As a result, an innovative parallel computational approach that uses an isolated network of 23 computers was developed. The approach uses a recently developed parallel version of UCODE developed by Eileen Poeter (Colorado School of Mines) as part of this effort that communicates with a parallel task manager, MasterTasker, developed at PNNL to propagate the multiple simulation tasks (i.e., the forward model runs) for simultaneous computation on the isolated network of dedicated computers. In addition, customized modules CFUCODE and LP3UCODE, modules of the forward model code CFEST, were developed to work directly with the enhanced parallel version of UCODE. The methodology is fully described in the first inverse modeling effort that used the prior conceptual model (Cole et al. 2001), and it has since been applied to the basalt ACM (Vermeul et al. 2001).

\subsubsection{ACM Evaluation}

Once each ACM is calibrated to the same historical observations, the reasonableness and intercomparison of alternative conceptual models and their associated model parameter estimates and 
uncertainty can be evaluated and compared with information primarily provided by the inverse method. These include the following measures:

- residual of simulated versus observed values

- normality of residuals both spatially and temporarily

- composite-scaled sensitivity coefficients of model parameters

- confidence intervals around best-fit model parameter estimates (ability of model to estimate selected model parameters)

- consistency of best-model parameter estimates with prior information, basic scientific principles, or other knowledge.

Use of the model structure criteria suggested by Carrera and Neuman (1986a) and discussed in Section 3.2.2 will be investigated for use in the evaluation and comparison of the various ACMs. These four model structure identification criteria, which include: 1) Akaike's First Information Criterion (1974), 2) Akaike's Second Information Criterion (1977), 3) Hannan's Criterion (1980), and 4) Kashyap's Criterion (1977), were developed to address the criticism that the calculated error variance and standard error do not sufficiently represent the drawbacks associated with increasing the number of estimated parameters. As such, they start with the log-likelihood criterion as a basic measure and add penalty terms that reflect the fact that adding too many parameters produces unreliable parameter estimates (i.e., 2 NP, $\mathrm{NP} \ln [\mathrm{ND}+\mathrm{NPR}], \mathrm{c} \mathrm{NP} \ln [\ln (\mathrm{ND}+\mathrm{NPR})]$, and $\mathrm{NP} \ln [(\mathrm{ND}+\mathrm{NPR}) / 2 \pi]+\ln \left(\left|\mathrm{F}_{\mathrm{M}}\right|\right)$, respectively, for the four criteria referenced above where NP is the number of unknown parameters, ND is the number of observations, NPR is the number of observations of prior information, $\mathrm{c}$ is an arbitrary constant, and $\left|\mathrm{F}_{\mathrm{M}}\right|$ is the determinant of the Fisher information matrix, Carrera [1984]). As discussed in Section 3.2.2, Carrera and Neuman (1986b) were able to successfully use these four criteria to discriminate between different parameterizations of a test case of groundwater flow even though the four criteria were originally developed for time-series problems.

\subsubsection{ACM Uncertainty Assessment}

This step of the new uncertainty framework involves translating model parameter uncertainty for each of the ACMs identified, developed, and evaluated as plausible into model prediction uncertainty regarding predictions of groundwater flow and contaminant transport important to Hanford Site decision making for the various scenarios of interest. Most of the initial efforts will involve acquiring and testing the codes and approaches, with simple problems to work out the mechanics of implementation. Then the methodology will be demonstrated through development of Hanford Site-related simple demonstrations (e.g., unit source releases from critical waste disposal areas) prior to full application for Hanford Sitespecific problems. 


\subsubsection{Model Complexity Optimization}

This step involves two parts: 1) identifying the future scenarios and flow and transport predictions of interest, and 2) conducting sensitivity studies to identify the most "relevant sources of uncertainty" affecting the model predictions of interest for the future scenarios of interest over the identified ranges for the various model parameters (see Section 3.3 and Figure 3.27 for a discussion of "relevant sources of uncertainty" from Meyer and Gee [1999]). The rationale for this step, which is equivalent to the first two steps in the EPA (1997) Principles of Good Practice for Monte Carlo Simulation presented in Table 5.1 above, is discussed in Section 3.3.3.1. Model complexity optimization, as discussed in Section 3.3.3.1, must be revisited at all steps of an uncertainty assessment because only the most important sources of uncertainty should be included in an uncertainty analysis for a given conceptual model.

Uncertainty analysis will be performed in FY 2002 using the tools and methodologies embodied in UCODE. This analysis will likely focus on simulations of unit releases of a nonreactive, long-lived contaminant from selected waste site areas based on post-Hanford Site water table conditions to examine the uncertainty in the contaminant concentrations and other appropriate metrics at selected locations between the waste sites and the Columbia River. The metrics of interest for this transport problem will be used as the basis for a sensitivity analysis to identify the sensitive parameters important to transport predictions in order to identify which parameters should be treated as uncertain when carrying out a Monte Carlo uncertainty assessment demonstration for these same unit release problems.

\subsubsection{Characterize Parameter Uncertainty}

Some estimates for model parameters and their uncertainty will be derived from the inversing process. Uncertainty for other parameters will have to be derived from available data and understanding as is discussed in Section 3.3.3.2, which states that NRC (2000), EPA (1997), NCRP (1996), and IAEA (1989) provide guidance for choosing parameter distributions based on limited information. We will observe the warning in Peck et al. (1988) that arbitrary selection of pdfs could result in "garbage in, garbage out."

Of particular importance in this step is 1) properly identifying and accounting for correlation between parameters (see Figures 3.6 and 3.7 and discussions in Sections 3.1.1.1.3, 3.1.1.4, 3.2.3, 3.3.3.2, and 3.3.3.3.1.2.1) and 2) the approach used to upscale parameter measurements and estimate the uncertainty in the upscaled or model parameters that were not determined from the inverse process. The upscaling approach is important because, as discussed in Section 3.2.1 on natural variability model, coefficients are derived to correspond to a specific model, the upscaling process provides a new source of uncertainty, and the upscaling process also affects the uncertainty estimates for these upscaled parameters.

With regard to the upscaling issue, Figure 3.18 from Meyer and Gee (1999) illustrates the effect of the averaging process, related to upscaling both spatially variable and temporally varying parameter estimates, on the appropriate parameter variability of these averaged or upscaled parameters.

Section 3.3.3.3 provides additional discussion on this difficult upscaling issue, which Katz (1999) indicates is related to how uncertainty depends on the level of aggregation and is affected by the upscaling process. As discussed in Section 3.3.3.3, the issue is particularly relevant to groundwater 
systems because of the wide range of spatial and temporal scales over which measurements are made, the diversity of the constitutive models used to interpret parameters, and the diversity in space and time scales for which predictions are needed. The use and behavior of the observation and parameter variances during the upscaling/downscaling and aggregation process is the major source of the difficulty.

Figure 3.28 presents a hypothetical example indicating how the support volume for the parameters and observations could vary with the scale and level of aggregation and actual laboratory and field methods used to interpret the field data.

\subsubsection{Propagate Uncertainties}

Propagation of uncertainties is more fully covered in Section 3.3.3.3, which discusses the robust Monte Carlo method and more limited FOSM method for uncertainty assessment. These are the two approaches that will be initially implemented in development of the new uncertainty framework. Since FSOM methods are more limited, only the FSOM methods provided in UCODE will be implemented. Use of FSOM methods, therefore will be appropriate only when the model is determined to be roughly linear (e.g., as determined by Beal's measure provided by UCODE phase 33) and when the parameters for which uncertainty are to be assessed are parameters that were inversed by UCODE. The most appropriate of these two methods will be used for the assessment of uncertainty in flow and transport modeling results (i.e., predictions) related to input parameter uncertainty. The most appropriate method will be applied for each of the future scenarios of interest and for each of the plausible Hanford SGM ACMs for use in assessment of the total uncertainty. The method for assessment of the total uncertainty is discussed in Section 5.2.5.

\subsection{First Order Second Moment Methods}

The FOSM methods (Dettinger and Wilson 1981) are more fully discussed in Sections 3.3.3.3.1 and 3.3.3.3.1.1. These methods assume the first two moments of the random variable input parameters are sufficient to characterize the mean and variance/covariance of the results of interest. To apply to nonlinear systems, these methods require that the uncertainty in parameters be "small" so that the fluctuation around the mean still applies when the second and higher order terms are neglected, for example in a Taylor series expansion. As a result, the analysis and testing for "small" must be part of the application of these methods. Of the two possible approaches discussed in Section 3.3.3.3.1 (perturbation analysis discussed in Tang and Pinder [1977] and Taylor series expansion methods), the Taylor series expansion methods are more generally applicable to the Hanford SGM uncertainty assessment problem. These methods expand the analytical or numerical solution of the governing equation around the expected values of the parameters and independent variables to deduce various probabilistic moments (e.g., mean, variance/covariance) of the dependent variable (i.e., the results) as is presented in Section 3.3.3.3.1.1. They have been routinely applied to groundwater flow and transport problems (e.g., Dettinger and Wilson [1981]; Wagner and Gorelick [1987]; James and Oldenburg [1997]). More importantly, the FSOM methods include the linear confidence and prediction interval codes developed by Hill (1994) based on the early work of Cooley (1977) for use with MODFLOWP (Hill 1992) and implemented in UCODE (Poeter and Hill 1998). This allows us to use these uncertainty analysis methods directly since UCODE will be used for the transient inverse analysis (Section 5.2.2.2). 
Currently, the only planned implementation of FOSM is that provided by UCODE through its phase 33, phase 44 and phase 45 options (Poeter and Hill 1998). As discussed in Section 3.3.3.3.1.1, UCODE, through its various outputs and operational phases, allows for the required evaluations of the three assumptions discussed in Hill (1994) that are needed to develop accurate confidence and prediction intervals. These assumptions include

- uncertainty in parameters are normally distributed

- model is correct

- model is roughly linear.

As discussed in Section 3.3.3.3.1.1, Hill indicates the following:

- The first assumption of normal probability distributions has generally been found to be valid for many groundwater model calibrations performed using nonlinear regression.

- The second assumption, while clearly untrue in some ways, can be tested by various means provided by UCODE phase 3 output.

- The third assumption can be tested directly using the results from UCODE phase 33 output. Phase 33 of UCODE calculates a modified Beale's measure.

Efforts to deploy FOSM for uncertainty assessment of Hanford SGM results were begun in FY 2001. The initial effort ${ }^{(a)}$ consisted of implementing the UCODE methodology for analyzing uncertainty with the Hanford SGM modeling tools and demonstrating its use for a simple problem. The demonstration problem illustrates how the UCODE uncertainty methodology could be used to estimate uncertainty in transport predictions of the type that might be useful in Hanford SGM applications and provided the means to gain an understanding of the strengths and limitations of UCODE approach. In FY 2002, the tools and methodologies embodied in UCODE will be applied using one or more of the ACMs (e.g., Cole et al. 2001; Vermeul et al. 2001) to demonstrate the UCODE capabilities for quantifying uncertainty in predictions for Hanford Site-specific problems. These analyses will likely focus on simulations of unit releases of a nonreactive, long-lived contaminant from selected waste site areas. The simulations will be based on post-Hanford Site water table conditions and will be used to examine the uncertainty in contaminant concentrations and other appropriate metrics at selected locations between the waste sites and the Columbia River.

(a) Informal project report, Status Report on Uncertainty Analysis Task FY 2001, V.L. Freedman, C.R. Cole, and M.P. Bergeron, September 2001, Prepared for the U.S. Department of Energy under Contract DE-AC06-76RL01830, Pacific Northwest National Laboratory, Richland, Washington 99352. 


\subsection{Monte Carlo Method}

The powerful Monte Carlo analysis method, which is a full distribution method (Dettinger and Wilson 1981), requires a complete specification of all nondeterministic inputs and parameters. The Monte Carlo method attempts to specify completely the probability distributions for the results of interest and has been selected as the primary method for propagation of uncertainty in Hanford SGM predictions. The Monte Carlo approach was selected because it is a computer-based, full-distribution method that is often used in the propagation of uncertainty. The method can be applied to either simple or complex models (see Section 3.3.3.3.1.2). As discussed in Peck et al. (1988), it is both the most powerful method and yet the simplest to understand because it simply involves numerous replications of the simulation model with the parameters and inputs for each simulation appropriately drawn at random from their respective pdfs. The results from the random replicates can be compiled to form the probability distribution for the desired results (Dettinger and Wilson 1981). The major limitation of the method is the number of runs that may be required.

Implementation of the Monte Carlo methodology is relatively simple, requiring only the ability to run the Hanford SGM model multiple times for different realizations of the important Hanford SGM parameters by randomly sampling the parameter distributions. A method to sample the output of the model for each random sample of model inputs is also needed to determine the values of the model result(s) that will be used in the decision-making process (e.g., maximum concentration at a compliance boundary, time of maximum concentration, maximum area of aquifer above a specified concentration). The suite (or distribution) of model results are then used to calculate the desired statistical description of the uncertainty in the decision-making results (e.g., mean, variance, $\mathrm{CDF}$ ).

Based on the sensitivity study described in Section 5.2.4.1, the Monte Carlo analysis methodology will be implemented during FY 2002 and demonstrated during FY 2003 for the same unit release problems for which the UCODE uncertainty estimate demonstrations were performed. The only new capability required for a Monte Carlo analysis is a sampling method and code to create the multiple realizations. A Latin Hypercube Sampling (LHS) approach that uses the Sandia National Laboratories (SNL) LHS methodology (Wyss and Jorgensen 1998) embedded in the SNL Advanced Risk and Reliability Assessment Model Integrated S/W (ARRAMIS) code will be used to develop the random samples for the Monte Carlo assessment.

\subsubsection{Estimation of Combined ACM and Scenario Uncertainty}

This step simply involves estimating the combined uncertainty by the double sum presented in Equation 3.1, where each component of the inner sum (a single CCDF in Figure 3.29) represents the parameter uncertainty associated with a particular scenario and plausible ACM. The enumeration of all the various plausible ACMs and scenario combinations gives rise to the family of CCDFs in Figure 3.29 that represents the combined estimate of uncertainty. 


\subsubsection{ACM Assumption and Issue Tracking}

The purpose of this step, as discussed in Section 5.1, is to achieve transparency by ensuring that there is comprehensive documentation of each uncertainty analysis performed for each ACM. This entails complete documentation of the all the assumptions including those 1) made during conceptual model development, 2) required by the mathematical model, 3) required by the numerical model, 4) made during the spatial and temporal descretization process, 5) made regarding the statistical nature of the model input parameters, 6) needed to assign the statistical parameters that describe the uncertainty in the relevant input parameters, 7) required by the propagation method, and 8) identification and documentation of any issues or new hypotheses or ACM modifications identified during the development, testing, and uncertainty propagation step for a given ACM. 


\subsection{References}

Abbaspour KC, R Schulin, MT van Genuchten, and E Schlappi. 1998. "Procedures for uncertainty analysis applied to a landfill leachate plume." Groundwater 36(6):874-883.

Adams EE, and LW Gelhar. 1992. "Field study of dispersion in a heterogeneous aquifer, 2. Spatial moments analysis." Water Resour. Res. 28(12):3293-3307.

Ahlstrom SW, HP Foote, RC Arnett, CR Cole, and RJ Serne. 1977. Multicomponent mass transport model: Theory and numerical implementation (Discrete-Parcel-Random-Walk Version). BNWL-2127, Pacific Northwest Laboratory, Richland, Washington.

Akaike H. 1974. “A new look at statistical model identification.” IEEE Trans. Automat. Contr. AC-19, pp. 716-722.

Akaike H. 1977. On entropy maximization principle, in applications of statistics. PR Krishnaiah ed., pp. 27-41, North-Holland, Amsterdam.

Ames LL, and RJ Serne. 1991. Compilation of data to estimate groundwater migration potential for constituents in active liquid discharges at the Hanford Site. PNL-7660, Pacific Northwest Laboratory, Richland, Washington.

Anderson MP, and WW Woessner. 1992. Applied groundwater modeling simulation of flow and advective transport. Academic Press Inc., Harcourt Brace Jovanovich, Publishers, San Diego, California.

ANSYS, Inc. 2001. Theory reference. ANSYS Release 6.0 (October 2001), ANSYS, Inc., Canonsburg, Pennsylvania.

Avramidis AN, and JR Wilson. 1995. Correlation induction techniques for estimating quantiles in simulation experiments. Technical Report 95-5, Department of Industrial Engineering, North Carolina State University, Raleigh, North Carolina.

Baker V, BN Bjornstad, AJ Busacca, KR Fecht, EP Kiver, UL Moody, JG Rigby, DF Stradling, and AM Tallman. 1991. "Quaternary geology of the Columbia Plateau." In quaternary nonglacial geology; Conterminous US, ed. RB Morrison. The Geological Society of America. K-2:215-250.

Barnett DB, MD Freshley, MP Bergeron, SK Wurstner, and CR Cole. 1997. Tritium monitoring in groundwater and evaluation of model predictions for the Hanford Site 200 Area Effluent Treatment Facility. PNNL-1 1665, Pacific Northwest National Laboratory, Richland, Washington. 
Barnett DB, JS Schmid, SS Lowe, WL Allen, NA Ballantyne, CH Dohrer, MJ Hartman, FN Hodges, DG Horton, VG Johnson, KJ Lueck, DJ Ortiz, AJ Knepp, BH Ford, SJ Hope, DK Tyler, RD Hildebrand, DE Olson, RE Peterson, GL Kasza, DA Myers, SP Luttrell, PD Thorne, and RK Moser. 1995. Hanford Site Ground Water Protection Management Plan. DOE/RL-89-12, Rev. 2, US Department of Energy, Richland, Washington.

Bauer HH, and JJ Vaccaro. 1990. Estimates of ground-water recharge to the Columbia Plateau Regional Aquifer System, Washington, Oregon, and Idaho, for predevelopment and current land-use conditions. WRIR 88-4108, US Geological Survey, Tacoma, Washington.

Baveye P, and G Sposito. 1984. "The operational significance of the continuum hypothesis in the theory of water movement through soils and aquifers." Water Resour. Res. 20(5):521-530.

Baveye P, and G Sposito. 1985. "Macroscopic balance equations in soils and aquifers: The case of space- and time-dependent instrument response.” Water Resour. Res. 21(8):1116-1120.

Bear J. 1972. Dynamics of fluids in porous media. American Elsevier, New York.

Bear J. 1979. Hydraulics of groundwater. McGraw-Hill, London.

Bear J. 1985. "Conceptual and mathematical modeling of groundwater flow and pollution: An overview." Presented at the American Society of Civil Engineers Hydraulics Specialty Conference, August 12-17, 1985, Lake Buena Vista, Florida.

Bear J, and Y Bachmat. 1990. Introduction to modeling of transport phenomena in porous media. Kluwer Academic Publishers, Dordrecht/Boston/London.

Beck JV, and KJ Arnold. 1977. Parameter estimation in engineering and science. John Wiley \& Sons, New York.

Beck MB. 1987. "Water quality modeling: A review of the analysis of uncertainty." Water Resour. Res. 23(8):1393-1442.

Bentley RD, JL Anderson, NP Campbell, and DA Swanson. 1980. Stratigraphy and structure of the Yakima Indian Reservation, with emphasis on the Columbia River Basalt Group. US Geological Survey Open-File Report 80-200, p. 85, 1 plate.

Bergeron MP, and SK Wurstner. 2000. Groundwater transport calculations supporting the immobilized low-activity disposal facility performance assessment. PNNL-13400, Pacific Northwest National Laboratory, Richland, Washington.

Bierschenk WH. 1959. Aquifer characteristics and ground-water movement at Hanford. HW-60601, General Electric Company, Hanford Atomic Products Operation, Richland, Washington.

Bjornstad BN. 1984. Suprabasalt stratigraphy within and adjacent to the reference repository location. SD-BWI-DP-039, Rockwell Hanford Operations, Richland, Washington. 
Bjornstad BN. 1985. "Late cenozoic stratigraphy and tectonic evolution within a subsiding basin, South-Central Washington.” Geological Society of America, 17:524 (Abstracts with Programs).

Brown RE, and HG Ruppert. 1950. The underground disposal of liquid waste at the Hanford Works, Washington. HW-17088, General Electric Hanford Company, Richland, Washington.

Box GEP, and GM Jenkins. 1976. Time series analysis: Forecasting and control. Holden-Day, San Francisco.

Campbell JE, DE Longsine, and M Reeves. 1981. "Distributed velocity method of solving the convective-dispersion equation: 1 . Introduction, mathematical theory, and numerical implementation." Advances in Water Resources 4:102.

Campbell MD, WJ McMahon, and KR Simpson. 1993. Water level measurements for modeling hydraulic properties in the 300-FF-5 and 100 Aggregate Area operable units. PNL-8580, Pacific Northwest Laboratory, Richland, Washington.

Carle SF, EM Labolle, GS Weissmann, and D van Brocklin. 1998. "Conditional simulation of hydrofacies architecture: A Transition Probability/Markov Approach.” In uses of sedimentologic and stratigraphic information in predicting reservoir heterogeneity: Special publications, eds. GS Fraser and JM Davis, pp. 147-170. SEPM (Society for Sedimentary Geology), Tulsa, Oklahoma,.

Carrera J. 1993. "Groundwater inverse problem: Discussion on geostatistical formulations and validation." Hydrogeologie 4:313-324.

Carrera J. 1984. Estimation of aquifer parameter under transient and steady state conditions. Ph.D. dissertation, Department of Hydrology and Water Resources, University of Arizona, Tucson, Arizona.

Carrera J, and SP Neuman. 1986a. "Estimation of aquifer parameter under transient and steady state conditions: 1. Maximum likelihood method incorporating prior information." Water Resour. Res. 22(2):199-210.

Carrera J, and SP Neuman. 1986b. "Estimation of aquifer parameter under transient and steady state conditions: 2. Uniqueness, stability, and solution algorithms.” Water Resour. Res. 22(2):211-227.

Carrera J, and SP Neuman. 1986c. "Estimation of aquifer parameter under transient and steady state conditions: 3. Application to synthetic and field data." Water Resour. Res. 22(2):228-242.

Cearlock DB, KL Kipp, and DR Friedrichs. 1975. The transmissivity iterative calculation routine theory and numerical implementation. BNWL-1706, Pacific Northwest Laboratory, Richland, Washington.

Charnes JM. 2000. "Using simulation for option pricing." In Proceedings of the 2000 Winter Simulation Conference, eds. JA Joines, RR Barton, K Kang, and PA Fishwick, , pp 151-157. Published by IEEE, ISBN: 0780365798. 
Cherry JA, RW Gilliam, and JF Pickens. 1979. "Contaminant hydrogeology: Part 1, Physical processes." Geoscience Canada 2(2):76-84.

Chiaramonte GR, CW Denslow, AJ Knepp, RD Landon, and S Panday. 1997. Hanford Site-wide groundwater remediation strategy - groundwater contaminant predictions. BHI-00469, Bechtel Hanford, Inc., Richland, Washington.

Chilakapati A, M Williams, S Yabusaki, C Cole, and J Szecsody. 2000. Optimal design of an in situ Fe(II) barrier: Transport limited reoxidation. Environmental Science and Technology 34(24):5215-5221.

Christensen S, and RL Cooley. 1999. "Evaluation of confidence intervals for a steady-state leaky aquifer model." Advances in Water Resources 22(8):807-817.

Chow VT. 1970. "An introduction to systems analysis of hydrologic problems." In Proceedings of the second international seminar for hydrology professors, Logan, Utah.

Cole CR, and MG Foley. 1985. "The link between laboratory/field observations and models." In Proceedings of the Nuclear Energy Agency Workshop, pp. 127-140. October 22, 1985, Paris, France.

Cole CR, SK Wurstner, MP Bergeron, MD Williams, and PD Thorne. 1997. Three-dimensional analysis of future groundwater flow conditions and contaminant plume transport in the Hanford Site Unconfined Aquifer System: FY 1996 and 1997 Status Report. PNNL-11801, Pacific Northwest National Laboratory, Richland, Washington.

Cole CR, SB Yabusaki, and CT Kincaid. 1988. CFEST-SC: Coupled fluid, energy, and solute transport code, super computer version, documentation, and user's manual. Pacific Northwest Laboratory, Richland, Washington.

Cole JA, ed. 1972. "Some interpretations of dispersion measurements in aquifers." Groundwater pollution in Europe, pp. 86-95. Water Research Association, Reading, England.

Comte de Buffon G. 1777. Essai d'arithmetique morale. Suppletment a l'Historie Naturelle, Vol. 4.

Connelly MP. 1998. "Modeling to support permeable barrier technology testing." Hanford Site groundwater monitoring for Fiscal Year 1997, eds. MJ Hartman and PE Drese, pp. 6.6-6.10, PNNL-11793, Pacific Northwest National Laboratory, Richland, Washington.

Connelly MP, CR Cole, and MD Williams. 1997. Bank storage modeling of the 100-N Area. CH2MHill Letter Report to Pacific Northwest National Laboratory, CH2M-Hill Hanford, Inc., Richland, Washington.

Connelly MP, JD Davis, and PD Rittman. 1991. Numerical simulation of strontium-90 transport from the 100-N Liquid Waste Disposal Facilities. WHC-SD-ER-TA-001, Rev. 0, Westinghouse Hanford Company, Richland, Washington. 
Connelly MP, BH Ford, and JV Borghese. 1992b. Hydrogeologic model for the 200 West Groundwater Aggregate Area. WHC-SD-EN-TI-014, Westinghouse Hanford Company, Richland, Washington.

Connelly MP, BH Ford, JW Lindberg, SJ Trent, CD Delaney, and JV Borghese. 1992a. Hydrogeologic model for the 200 East Groundwater Aggregate Area. WHC-SD-EN-TI-019, Rev. 0, Westinghouse Hanford Company, Richland, Washington.

Cooley RL. 1977. "A method of estimating parameters and assessing reliability for models of steady state groundwater flow 1. Theory and numerical properties." Water Resour. Res. 13(2):318-324.

Cooley RL. 1982. "Incorporation of prior information on parameters into nonlinear regression groundwater flow models, I. Theory.” Water Resour. Res. 18(4):965-976.

Cooley RL, and RL Naff. 1990. Regression modeling of ground-water flow, Book 3, in Chapter B4 in US Geological Survey Techniques in Water-Resources Investigations, p. 232.

Cornell CA. 1972. "First order analysis of model parameter uncertainty." International symposium on uncertainties in hydrologic and water resource systems, University of Arizona, Tucson, Arizona.

CRWMS M\&O. 1998. Total System Performance Assessment-Viability Assessment (TSPA-VA) Analyses Technical Basis Document. Las Vegas, Nevada.

CRWMS M\&O. 1999. Total system performance assessment-site recommendation methods and assumptions. TDR-MGR-MD-000001 Rev. 00 ICN 01, Las Vegas, Nevada.

Cushing CE, and BE Vaughan. 1988. "Springs and streams in shrub-steppe balance and change in a semi-arid terrestrial ecosystem." Developments in Agricultural and Managed-Forest Ecology 20, eds. WH Rickard et al. Elsevier Science Publishers, New York.

Cushman JH. 1984. "On unifying concepts of scale, instrumentation, and stochastics in the development of multiphase transport theory." Water Resour. Res. 20(11):1668-1676.

Cushman JH. 1986. “On measurement, scale, and scaling.” Water Resour. Res. 22(2):129-134.

Dagan G. 1985. "Stochastic modeling of groundwater flow by unconditional and conditional probabilities: The inverse problem." Water Resour. Res. 21(1):65-72.

Damsleth E, CB Tjolsen, A Omre, and HH Haldorsen. 1992. "A two-stage stochastic model applied to a North Sea Reservoir.” J. Petrol. Tech., Trans. AIME 291:402-408.

Dandekar DA, M Cohen, and N Kirkendall. 2001. "Applicability of Latin Hypercube Sampling to create multi variate synthetic micro data." Pre-proceedings of the conference, ETK-NTTS2001, pp 839-847.

June 18-22, 2001, Hersonissos (Crete). 
DART (Data Aquisition in Real Time). 1998. Data published on the internet at http://www.cqs.washington.edu/index.html. April 1998. Columbia Basin Research Project, Department of Fisheries, University of Washington, Seattle, Washington.

Davis JC. 1986. Statistics and data analysis in geology. John Wiley \& Sons, New York, p. 646.

Davis SN. 1969. "Porosity and permeability of natural materials." In flow through porous media, ed. RJM DeWiest, pp. 54-89. Academic Press, New York.

de Marsily G. 1978. De I'identification des systemes en hydrogeologiques (tome 1), Ph.D. thesis, pp. 58-130, L’Univ. Pierre et Marie Curie-Paris VI, Paris.

de Marsily G. 1986. Quantative hydrology. Academic Press, Orlando, Florida.

de Marsily G, JP Delhomme, A Coudrain-Ribstein, and AM Lavenue. 2000. "Four decades of inverse problems in hydrogeology." In theory, modeling, and field investigation in hydrogeology: A special volume in honor of Shlomo P. Neuman's 60th birthday, eds. D Zhang and CL Winter, pp. 1-17. Geological Society of America Special Paper 348, Boulder, Colorado.

Desbarats AJ. 1998. "Scaling of constitutive relationships in unsaturated heterogeneous media: A numerical investigation." Water Resour. Res. 34(6):1427-1435.

Dettinger MD and JL Wilson. 1981. "First order analysis of uncertainty in numerical models of groundwater flow. Part 1: Mathematical development.” Water Resour. Res. 17(1):149-161.

Deutsch CV, and AG Journel. 1998. GSLIB: Geostatistical software library and user's guide. Oxford University Press, New York.

Devary JL. 1987. The CFEST-INV Stochastic Hydrology Code: Mathematical formulation, application, and user's manual. ICF Northwest, Richland, Washington.

Dinicola RS. 1997. Estimates of recharge from runoff at the Hanford Site, Washington. WaterResources Investigations Report 97-4038, US Geological Survey, Tacoma, Washington.

Dirkes RL, and RW Hanf. 1996. Hanford Site Environmental Report for Calendar Year 1995. PNNL-11139, Pacific Northwest National Laboratory, Richland, Washington.

Dominic DF, RW Ritzi, Jr., EC Reboulet, and AC Zimmer. 1998. “Geostatistical analysis of facies distributions: Elements of a quantitative facies model." In uses of sedimentologic and stratigraphic information in predicting reservoir heterogeneity, special publications, eds. GS Fraser and JM Davis, pp. 137-146. SEPM (Society for Sedimentary Geology), Tulsa, Oklahoma.

Dove FH, CR Cole, MG Foley, FW Bond, RE Brown, WJ Deutsch, MD Freshley, SK Gupta, PJ Gutknecht, WL Kuhn, JW Lindberg, WA Rice, R Schalla, JF Washburn, and JT Zellmer. 1982. AEGIS technology demonstration for a nuclear waste repository in basalt. PNL-3632, Pacific Northwest Laboratory, Richland, Washington. 
Dresel PE, PD Thorne, SP Luttrell, BM Gillespie, WD Webber, JK Merz, JT Rieger, MA Chamness, SK Wurstner, and BE Opitz. 1995. Hanford Site ground-water monitoring for 1994. PNL-10698, Pacific Northwest Laboratory, Richland, Washington.

Droppo JG, Jr. 1991. Multimedia Environmental Pollutant Assessment System (MEPAS) application guidance, Volume 1 - User's Guide and Volume 2 - Guidelines for Evaluating MEPAS Input Parameters. PNL-7216, Pacific Northwest Laboratory, Richland, Washington.

Drost BW, SE Cox, and KM Schurr. 1997. Changes in ground-water levels and ground-water budgets, from predevelopment to 1986, in parts of the Pasco Basin, Washington. Water-Resources Investigations Report 96-4086, US Geological Survey in cooperation with the Washington State Department of Ecology, Tacoma, Washington.

Drost BW, KJ Whiteman, and JB Gonthier. 1990. Geologic framework of the Columbia Plateau Aquifer System, Washington, Oregon, and Idaho. Water-Resources Investigations Report 87-4238, US Geological Survey, Portland, Oregon.

Dubois D, and H Prade. 1988. Possibility Theory - An approach to computerized processing of uncertainty. Plenum Press, New York and London.

Dubrule O. 1989. "A review of stochastic models for petroleum reservoirs." Geostatistics, Vol.2, ed. M Armstrong, pp. 493-506. Kluwer Academic Pub., Dordrecht.

Eagleson PS. 1970. Dynamic hydrology. McGraw-Hill, New York.

Eagleson PS. 1978. "Climate, soil, and vegetation--5. A derived distribution of storm surface runoff." Water Resour. Res. 14(5):741-748.

Ebbert JC, SE Cox, BW Drost, and KM Shurr. 1995. Distribution and sources of nitrate and presence of fluoride and pesticides in parts of the Pasco Basin, Washington, 1986-1988. Water-Resources Investigations Report 93-4197, US Geological Survey, Washington, D.C.

Eggleston JR, SA Rojstaczer, and JJ Peirce. 1996. "Identification of hydraulic conductivity structure in sand and gravel aquifers: Cape code data set.” Water Resour. Res. 32(5):1209-1222.

Eisenberg NA, LD Rickertsen, and C Voss. 1987. "Performance assessment, site characterization, and sensitivity and uncertainty methods: Their necessary association for licensing." In Proceedings of the conference on geostatistical, sensitivity, and uncertainty methods for ground-water flow and radionuclide transport modeling, pp 9-38. Battelle Press, Columbus, Ohio.

Evans JC, DI Dennison, RW Bryce, PJ Mitchell, DR Sherwood, KM Krupka, NW Hinman, EA Jacobson, and MD Freshley. 1988. Hanford Site ground-water monitoring for July through December 1987. PNL-6315-2, Pacific Northwest Laboratory, Richland, Washington. 
Farmer CL. 1986. The dispersal of contaminants in heterogeneous aquifers: A review of methods of estimating scale dependent parameters. AEEW R-2085, United Kingdom Atomic Energy Authority, Atomic Energy Establishment, Winfrith, Dorchester, Dorset, England.

Fayer MJ, GW Gee, ML Rockhold, MD Freshley, and TB Walters. 1996. "Estimating recharge rates for a groundwater model using a GIS.” J. Environ. Qual. 25:510-518.

Fayer MJ, and TL Jones. 1990. UNSAT-H Version 2.0: Unsaturated soil water and heat flow model. PNL-6779, Pacific Northwest Laboratory, Richland, Washington.

Fayer MJ, and TB Walters. 1995. Estimated recharge rates at the Hanford Site. PNL-10285, Pacific Northwest Laboratory, Richland, Washington.

Fecht KR, DB Barnett, AG Law, KA Lindsey, and VJ Rohay. 1994. "Pleistocene clastic dikes and their influence on moisture flow in the vadose zone of South-Central Washington State." Geological Society of America 26(7):A204 (Abstracts with Programs).

Finsterle S. 1993. ITOUGH2 User's Guide Version 2.2. Rep. LBL-34581 UC-600, Lawerence Berkeley Laboratory, University of California, Berkeley.

Fogg G. 2000. "Facies connectivity and scale dependence of flow and transport properties: Perspective on Gaussian and non-Gaussian heterogeneity." In SEPM/IAS research conference in environmental sedimentology: Hydrogeology of sedimentary aquifers, Santa Fe, New Mexico.

Freeze RA. 1969. Regional groundwater flow - Old Wives Lake Drainage Basin, Saskatchewan. Inland Waters Branch, Department of Energy, Mines and Resources, Canada Scientific Series, No. 5.

Freeze RA, and JA Cherry. 1979. Ground water. Prentice-Hall, Englewood Cliffs, New Jersey.

Freeze RA, B James, J Massmann, T Sperling, and L Smith. 1992. "Hydrogeological decision analysis, Part 4 - The concept of data worth and its use in the development of site investigation strategies." Groundwater 30:574-588.

Freeze RA, J Massmann, L Smith, T Sperling, and B James. 1990. "Hydrogeological decision analysis, Part 1 - A framework." Groundwater 28:738-766.

Freeze RA, L Smith, G de Marsily, and J Massmann. 1987. "Some uncertainties about uncertainty." In Proceedings of the conference on geostatistical, sensitivity, and uncertainty methods for ground-water flow and radionuclide transport modeling, pp. 231-260. Battelle Press, Columbus, Ohio.

Frey HC. 1998. Briefing paper Part 1: Introduction to uncertainty analysis, Department of Civil Engineering, North Carolina State University, Raleigh, North Carolina.

Freyberg DL. 1986. "A natural gradient experiment on solute transport in a sand aquifer: 2. Spatial moments and the advection and dispersion of nonreactive tracers." Water Resour. Res.

22(13):2031-2046. 
Galarza G, A Medina, and J Carrera. 1996. TRANSIN-III: FORTRAN code for solving the coupled nonlinear flow and transport inverse problem: User's guide. E.T.S.I. Caminos, Canales y Puertos C/ Gran Capitan s/n 08034 Barcelona, Spain.

Gee GW, MJ Fayer, ML Rockhold, and MD Campbell. 1992. "Variations in recharge at the Hanford Site." Northwest Sci. 66:237-250.

Gee GW, and D Hillel. 1988. "Groundwater recharge in arid regions: Review and critique of estimation methods." Hydrologic Processes 2:255-266.

Gelhar LW, and CL Axness. 1981. Stochastic analysis of macro-dispersion in three-dimensionally heterogeneous aquifers. Report No. H-8, Hydraulic Research Program, New Mexico Institute of Mining and Technology, Soccorro, New Mexico.

Gelhar LW, A Mantoglou, C Welty, and KR Rehfeldt. 1985. A review of field-scale physical solute transport processes in saturated and unsaturated porous media. EPRI EA-4190, Project 2485-5, Electric Power Research Institute, Palo Alto, California.

Gelhar LW, C Welty, and KR Rehfeldt. 1992. "A critical review of data on field-scale dispersion in aquifers.” Water Resour. Res. 28:1955-1974.

Ginn TR, and JH Cushman. 1990. "Review - Inverse methods for subsurface flow: A critical review of stochastic techniques." Stochastic Hydrol. Hydraul. 4(1990):1-26.

Golder Associates Inc. 1990. TIC: 238560. Golder Associates.

Golder Associates Inc. 1998. RIP integrated probabilistic simulator for environmental systems, theory manual and user's guide. Redmond, Washington.

Gomez-Hernandez JJ, A Sahuquillo, JE Capilla. 1997. "Stochastic simulation of transmissivity fields conditional to both transmissivity and piezometric data. 1. Theory." Journal of Hydrology 203:162-174.

Goode DJ, and LF Konikow. 1990. "Apparent dispersion in transient groundwater flow." Water Resour. Res. 26(10):2339-2351.

Goovaerts P. 1997. Geostatistics for natural resources evaluation, applied geostatistics series. Oxford University Press, New York.

Gorelick S, C Andrews, and J Mercer. 1999. Report of the peer review panel on the proposed Hanford Site-wide groundwater model. Stanford, California.

Graham MJ, GV Last, and KR Fecht. 1984. An assessment of aquifer intercommunication in the B Pond, Gable Mountain Pond Area. RHO-RE-ST-12P, Rockwell Hanford Operations, Richland, Washington.

Grindrod P, and MD Impey. 1991. "Fractal filed simulations of tracer test migration within the WIPP Culebra Dolomite." Intera Inf. Technol., Henley-upon-Thames, United Kingdom. 
Gupta SK, CR Cole, CT Kincaid, and AM Monti. 1987. Coupled Fluid, Energy, and Solute Transport (CFEST) Model: Formulation and Users Manual. BMI/ONWI-660; Prepared for the US Department of Energy by Battelle Project Management Division, Office of Nuclear Waste Isolation, Columbus, Ohio, and Pacific Northwest Laboratory, Richland, Washington.

Gutjahr A, D McKay, and JL Wilson. 1987. "Fast fourier transform methods for random field generation.” EOS 68(44):1265.

Gutjahr A, and JL Wilson. 1989. "Co-kriging for stochastic flow models.” Transp. Pourous Media. 4(6):585-598.

Haan CT. 1977. Statistical methods in hydrology. The Iowa State University Press, Ames, Iowa.

Hagood MC. 1985. Structure and evolution of the Horse Heaven Hills in South-Central Washington. RHO-BWI-SA-344 P, Rockwell Hanford Operations, Richland, Washington.

Hajek BF. 1966. Soil survey Hanford Project in Benton County, Washington. BNWL-243, Pacific Northwest Laboratory, Richland, Washington.

Haldorsen HH, and E Damsleth. 1990. “Stochastic modeling.” SPE JPT, pp. 404-412.

Hannan ES. 1980. "The estimation of the order of an Arma Process.” Ann. Stat. 8:1071-1081.

Harr ME, 1987. Reliability based design in civil engineering. McGraw-Hill, New York.

Hartman MJ, and PE Dresel, eds. 1998. Hanford Site groundwater monitoring for Fiscal Year 1997. PNNL-11793, Pacific Northwest National Laboratory, Richland, Washington.

Hartman MJ, ed. 1999. Hanford Site groundwater monitoring for Fiscal Year 1998. PNNL-12086, Pacific Northwest National Laboratory, Richland, Washington.

Hartman MJ, LF Morasch, and WD Webber, eds. 2000. Hanford Site groundwater monitoring for Fiscal Year 1999. PNNL-11470, Pacific Northwest National Laboratory, Richland, Washington.

Hartman MJ, ed. 2000. Hanford Site groundwater monitoring, setting, sources, and methods.

PNNL-13404, Pacific Northwest National Laboratory, Richland, Washington.

Harvey C, and SM Gorelick. 2000. "Rate-limited mass transfer or macrodispersion: which dominates plume evolution at the MacroDispersion Experiment (MADE) site?" Water Resour. Res. 36(3):637-650.

Helton JC. 1993. "Uncertainty and sensitivity analysis techniques for use in performance assessment for radioactive waste disposal.” Reliability Engineering \& System Safety. Barking, Essex, England 42(2-3):327-367. Elsevier Applied Science Publishers, New York. 
Hill MC. 1992. A computer program (MODFLOWP) for estimating parameters of a transient, threedimensional, ground-water flow model using nonlinear regression. US Geological Survey Open-File Report 91-484.

Hill MC. 1994. Five computer programs for testing weighted residuals and calculating linear confidence and prediction intervals on results from the Groundwater Computer Program MODFLOWP. US Geological Survey Open-File Report 93-481.

Hill MC. 1998. Methods and guidelines for effective model calibration. US Geological Survey WaterResources Investigations Report 98-4005, Denver, Coloradro.

Hills RG, and PJ Wierenga. 1994. INTRAVAL Phase 2 model testing at the Las Cruces Trench Site. NUREG/CR-6063, US Nuclear Regulatory Commisson, Washington, D.C.

Hinds WT. 1975. "Energy and carbon balances in cheatgrass: An essay in autecology." Ecological Monographs 45:367-388.

Hoeksema RJ, and PK Kitanidis. 1984. "An application of the geostatistical approach to the inverse problem in two-dimensional groundwater modeling." Water Resour. Res. 20(7):1003-1020.

Hoitink DJ and KW Burk. 1994. Climatological Data Summary 1993 with historical data. PNL-9809, Pacific Northwest Laboratory, Richland, Washington.

Huyakorn PS, JB Kool, and YS Wu. 1991. VAM2D - Variably saturated analysis in two dimensions, Version 5.2 with hysteresis and chained decay transport, documentation and user's guide. NUREG/CR5352, Rev. 1, US Nuclear Regulatory Commission, Washington, D.C.

Huyakorn PS, and S Panday. 1994. VAM3DCG: Variable saturated analysis model in three dimensions with preconditioned conjugate gradient matrix solver, documentation and user's guide, Version 3.1. HydroGeoLogic, Inc., Herndon, Virginia.

HYDROCOIN. 1992. The International HYDROCOIN Project groundwater hydrology modeling strategies for performance assessment of nuclear waste disposal, Level 3: Uncertainty and sensitivity analysis. The coordinating group of the HYDROCOIN Project, Swedish Nuclear Power Inspectorate (SKI), Nuclear Energy Agency Organisation for Economic Co-Operation and Development, Paris, France.

Hyun Y, and K Lee 1998. "Model identification criteria for inverse estimation of hydraulic parameters." Groundwater 36(2):230-239.

Iman RL, and WJ Conover. 1982a. "A distribution-free approach to inducing rank correlation among input variables." Communications in Statistics B11(3):311-334. 
Iman RL, and WJ Conover. 1982b. Sensitivity analysis techniques: Self teaching cirriculum. NUREG/CR-2350 (SAND81-1978), Sandia National Laboratories, Albuquerque, New Mexico. Division of Waste Management, Office of Nuclear Material Safety and Safeguards, US Nuclear Regulatory Commission, Washington, D.C.

Iman RL, JM Davenport, and DK Ziegler. 1980. Latin Hypercube Sampling (Program User's Guide). Technical Report SAND79-1473, Sandia National Laboratories, Albuquerque, New Mexico.

Iman RL, and JC Helton. 1985. A comparison of uncertainty and sensitivity analysis techniques for computer models. NUREG/CR-33904, Technical Report SAND84-1461, Sandia National Laboratories, Albuquerque, New Mexico.

Iman RL, and MJ Shortencarier. 1984. A Fortran 77 Program and User's Guide for the generation of Latin Hypercube and random samples for use with computer models. NUREG/CR-3624, Technical Report SAND83-2365, Sandia National Laboratories, Albuquerque, New Mexico.

International Atomic Energy Agency. 1989. Evaluating the reliability of predictions made using environmental transfer models. IAEA Safety Series 100, International Atomic Energy Agency.

INTRAVAL. 1990. The International INTRAVAL Project: Background and results. The coordinating group of the INTRAVAL Project, Swedish Nuclear Power Inspectorate (SKI), Nuclear Energy Agency Organisation for Economic Co-Operation and Development, Paris, France.

Isaaks EH, and RM Srivastava. 1989. An introduction to applied geostatistics. Oxford University Press, New York.

Isukapalli SS. 1999. Uncertainty analysis of transport-transformation models. Ph.D. Dissertation, New Jersey State University, New Brunswick, New Jersey.

Jackson CP, AR Hoch, M Milicky, D Brear, K Ghabaee, and MJ Poole. 1995. Nirex 95: A preliminary analysis of the groundwater pathway for a deep repository at Sellafield. Volume 2 - Derivation of effective hydrogeological parameters for regional modeling. Nirex. Report S/95/012, Volume 2, Nirex Limited, United Kingdom.

Jacobson EA. 1985. A statistical parameter estimation method using singular value decomposition with application to Avra Valley Aquifer in Southern Arizona. Dissertation, Department of Hydrology and Water Resources, University of Arizona, Tucson.

Jacobson EA, and MD Freshley. 1990. An initial inverse calibration of the ground-water flow model for the Hanford unconfined aquifer. PNL-7144, Pacific Northwest Laboratory, Richland, Washington.

James AL, and CM Oldenburg. 1997. "Linear and Monte Carlo uncertainty analysis for subsurface contaminant transport simulation." Water Resour. Res. 33(11):2595-2598.

James W. 1994. Rules for responsible modeling. Computational Hydraulics International (CHI), Guelph, Canada. 
Jenkins OP. 1922. Undergroundwater supply of the region about White Bluffs and Hanford. Bulletin No. 26, State of Washington Department of Conservation and Development, Division of Geology, Olympia, Washington.

Jensen EJ. 1987. An evaluation of aquifer intercommunication between the unconfined and Rattlesnake Ridge aquifers on the Hanford Site. PNL-6313, Pacific Northwest Laboratory, Richland, Washington.

Johnson VG, DL Graham, and SP Reidel. 1993. Methane in Columbia River basalt aquifers: Isotopic and geohydrologic evidence for a deep coal-bed gas source in the Columbia Basin, Washington.

American Association of Petroleum Geologists Bulletin 77:1192-1207.

Jones TL. 1989. Simulating the water balance of an arid site. PNL-SA-17633, Pacific Northwest Laboratory, Richland, Washington.

Journel AG. 1989. Fundamentals of geostatistics in five lessons, short courses in geology: American Geophysical Union, Washington, D.C.

Journel AG, and F Alabert. 1989. "Non-Gaussian data expansion in the earth sciences." Terra Nova $1: 123-134$.

Jow H-N, DR Anderson, M Marietta, J Helton, and G Basabilvazo. 1997. “An overview of performance assessment for the Waste Isolation Pilot Plant." In Proceedings of the Waste Management 97

Conference, WM Symposia, Inc. Tucson, Arizona.

Jury WA, and G Sposito. 1985. "Field calibration of and validation of solute transport models for the unsaturated zone." Soil Sci. AM. J. 49:1331-1341.

Kalos MH, and PA Whitlock. 1986. Monte Carlo Methods Volume I: Basics. John Wiley \& Sons, New York.

Kanasewich ER. 1981. Time sequence analysis in geophyics. University of Alberta Press, Edmonton, Alberta, Canada.

Kanevski M, L Bolshov, E Savelieva, A Pozdnukhov, V Timonin, and S Chernov. 2001. "Characterization of hydrogeologic systems with machine learning algorithms and geostatistical models." Presented at the 2001 International Containment and Remediation Technology Conference, June 12, Orlando, Florida.

Kaplan DI, and RJ Serne. 1995. Distribution coefficient values describing iodine, neptunium, selenium, technetium, and uranium sorption to Hanford sediments. PNL-10379, Sup. 1, Pacific Northwest

Laboratory, Richland, Washington.

Keramat M, and R Kielbasa. 1999. "Modified Latin Hypercube Sampling Monte Carlo (MLHSMC) estimation for average quality index." Analog Integrated Circuits and Signal Processing, Vol. 19, No. 1.

Kashyap RL. 1977. "Bayesian comparison of dynamic models." IEEE Trans. Automat. Contr. AC-22, pp. $715-802$ 
Katz RW. 1999. "Techniques for estimating uncertainty in climate change scenarios and impact studies." In Proceedings of first ECLAT-2 Workshop on representing uncertainty in climate change scenarios and impact studies. Finnish Environment Institute, Helsinki, Finland.

Kincaid CT, MP Bergeron, CR Cole, MD Freshley, NL Hassig, VG Johnson, DI Kaplan, RJ Serne, GP Streile, DL Strenge, PD Thorne, LW Vail, GA Whyatt, and SK Wurstner. 1998. Composite analysis for low-level waste disposal in the 200-Area Plateau of the Hanford Site. PNNL-11800, Pacific Northwest National Laboratory, Richland, Washington.

Kincaid CT, FM Coony, DL Strenge, RL Aaberg, and PW Eslinger. 2001. “Appendix A: Inventory data for initial assessment performed with the system assessment capability (Rev. 0)." In addendum to composite analysis for low-level waste disposal in the 200 Area Plateau of the Hanford Site.

PNNL 11800-Addendum 1, Pacific Northwest National Laboratory, Richland, Washington.

Kincaid CT, PW Eslinger, WE Nichols, AL Bunn, RW Bryce, TB Miley, MC Richmond, SF Snyder, and RL Aaberg. 2000. System Assessment Capability (Revision 0): Assessment Description, Requirements, Software Design, and Test Plan. BHI-01365, Draft A. Bechtel Hanford, Inc., Richland, Washington.

Kincaid CT, JW Shade, GA Whyatt, MG Piepho, K Rhoads, JA Voogd, JH Westsik, Jr., MD Freshley, KA Blanchard, and BG Lauzon. 1995. Volumes 1 and 2: Performance assessment of grouted doubleshell tank waste disposal at Hanford. WHC-SD-WM-EE-004, Rev. 1, Westinghouse Hanford Company, Richland, Washington.

Kitanidis PK, and EG Vomvoris. 1983. "A geostatistical approach to the inverse problem in groundwater modeling, steady state, and one-dimensional simulations." Water Resour. Res. 19(13):677-690.

Kocher AE, and AT Strahorn. 1919. "Soil survey of Benton County, Washington." US Government Printing Office, Washington, D.C.

Koltermann CE, and SM Gorelick. 1995. "Fractional packing model for hydraulic conductivity derived from sedimentary mixtures." Water Resour. Res. 31(12)3283-3297.

Koltermann CE, and SM Gorelick. 1996. "Heterogeneity in sedimentary deposits - A review of structure-imitating, process-imitating, and descriptive approaches.” Water Resour. Res. 32:2617-2658.

Konikow LF, and RC Ewing. 1999. Editorial: Is a probabilistic performance assessment enough? Groundwater 37(4):481-482.

Kuczera G, and M Mroczkowski. 1998. "Assessment of hydrologic parameter uncertainty and the worth of multiresponse data." Water Resour. Res. 34(6):1481-1489.

Lallemand-Barres A, and P Peaudecerf. 1978. "Recherche des Relations Entre La Valeur de la Dispersivite Macroscopique D'un Milieu Aquifere, Ses Autres Caracteristiques et les Conditions de Mesure.” Bulletin de Recherches Geologiques Minieres, 2e Serie, Section III, Number 4, Orleans, France. 
Lavenue AM, BS RamaRao, G de Marsily, and MG Marietta. 1995. "Pilot point methodology for automated calibration of an ensemble of conditionally simulated transmissivity fields: Part 2 application.” Water Resour. Res. 31(3):495-516.

Law AG. 1992. "Groundwater modeling and plume migration analyses for candidate soil column disposal sites Hanford, Washington.” WHC-SD-EN-ES-022, Westinghouse Hanford Company, Richland, Washington.

Law A, S Panday, C Denslow, K Fecht, and A Knepp. 1996. Hanford Site-wide groundwater flow and transport model calibration report. BHI-00608, Bechtel Hanford, Inc., Richland, Washington.

LeBlanc DR, SP Garabedian, KM Hess, LW Gelhar, RD Quadri, KG Stollenwerk, and WW Wood. 1991. "Large-scale natural gradient tracer test in sand and gravel, Cape Cod, Massachusetts: 1. Experimental design and observed tracer movement." Water Resour. Res. 27(5):895-910.

Ledgerwood RK, and RA Deju. 1976. Hydrogeology of the uppermost confined aquifers underlying the Hanford Reservation. Atlantic Richfield Hanford Company, Richland, Washington.

Lence BJ, and A Ruszczynski. 1996. Managing water quality under uncertainty: Application of a new stochastic branch and bound method. WP-96-066, International Institute for Applied Systems Analysis A-2361, Laxenburg, Austria.

Liebetrau AM, and PG Doctor. 1987. "The generation of dependent input variables to a performance assessment simulation code." In Proceedings of the Nuclear Energy Agency Workshop, pp. 85-115, February 24-26, 1987, Paris, France.

Liikala TL. 1994. Hydrogeology along the southern boundary of the Hanford Site between the Yakima and Columbia Rivers, Washington. PNL-10094, Pacific Northwest Laboratory, Richland, Washington.

Lindsey KA. 1995. Miocene- to pliocene-aged suprabasalt sediments of the Hanford Site, South-Central Washington. BHI-00184 Rev. 00, Bechtel Hanford Inc., Richland, Washington.

Lindsey KA, BN Bjornstad, and MP Connelly. 1991. Geologic setting of the 200 West Area: An update. WHC-SD-EN-TI-008, Westinghouse Hanford Company, Richland, Washington.

Lindsey KA, BN Bjornstad, JW Lindberg, and KM Hoffman. 1992. Geologic setting of the 200 East Area: An update. WHC-SD-EN-TI-012, Westinghouse Hanford Company, Richland, Washington.

Lindsey KA, and DR Gaylord. 1990. "Sedimentology and stratigraphy of the miocene-pliocene Ringold Formation, Hanford Site, South-Central Washington.” Northwest Science 64:165-180.

Lindsey KA, and GK Jaeger. 1993. Geologic setting of the 100-HR-3 Operable Unit, Hanford Site, South-Central Washington. WHC-SD-EN-TI-132, Westinghouse Hanford Company, Richland, Washington. 
Lindsey KA, JL Slate, GK Jaeger, KJ Swett, and RB Mercer. 1994. Geologic setting of the low-level burial grounds. WHC-SD-EN-TI-290, Rev. 0, Westinghouse Hanford Company, Richland, Washington.

Link SO, GW Gee, ME Thiede, and PA Beedlow. 1990. "Response of a shrub-steppe ecosystem to fire: Soil, water and vegetational change." Arid Soil Research and Rehabilitation 4:163-172.

Lu AH. 1990. Simulation of strontium-90 transport from the 100-N Area to the Columbia River using VAM2DH. WHC-EP-0369, Westinghouse Hanford Company, Richland, Washington.

Mann FM, CR Eiholzer, Y Chen, NW Kline, AH Lu, BP McGrail, PD Rittmann, GF Williamson, JA Voogd, NR Brown, and PE LaMont. 1997. Hanford low-level tank waste interim performance assessment. HNF-EP-0884, Rev. 1, Lockheed Martin Hanford Corporation, Richland, Washington.

Mayer AS, and C Huang. 1999. "Development and application of a coupled-process parameter inversion model based on the maximum-likelihood estimation method." Advances in Water Resources 22(8):841-853.

Massman J, RA Freeze, L Smith, T Sperling, and B James. 1991. "Hydrogeologic decision analysis, Part 2 - Applications to ground-water contamination." Groundwater 29:536-548.

McGavock EH, WD Wiggens, RL Blazs, PR Boucher, LL Reed, and MC Smith. 1987. Water resources data Washington Water Year 1985. US Geological Survey, Tacoma, Washington.

McGraw-Hill. 1983. McGraw-Hill Dictionary of Scientific and Technical Terms (Third Edition). Sybil P. Parker Editor in Chief, McGraw-Hill Book Company, New York.

McKay MD, WJ Conover, and RJ Beckman. 1979. "A comparison of three methods for selecting values of input variables in the analysis of output from a computer code." Technometrics 221, 239-245.

McLaughlin D, and LR Townley. 1996. "A reassessment of the groundwater inverse problem." Water Resour. Res. 32(5):1131-1161.

McLaughlin D, and EF Wood. 1988. "A distributed parameter approach for evaluating the accuracy of groundwater model predictions, 1. Theory.” Water Resour. Res. 24(7):1037-1047.

McMahon WJ, and RE Peterson. 1992. Estimating aquifer hydraulic properties using the Ferris Method, Hanford Site, Washington. DOE/RL-92-64, US Department of Energy, Richland, Washington.

Meyer PD, and GW Gee. 1999. Information on hydrologic conceptual models, parameters, uncertainty analysis, and data sources for dose assessments at decommissioning sites, NUREG/CR-6656, US Nuclear Regulatory Commission, Washington, D.C.

Miall AD. 1985. "Architectural-Element Analysis: A new method of facies analysis applied to fluvial deposits." Earth-Science Reviews 22:261-308. 
Mills DB, DB Porcella, MJ Ungs, SA Gherini, KV Summers, Lingfung Mok, GL Rupp, GL Bowie, and DA Haith. 1985. Water quality assessment: A screening procedure for toxic and conventional pollutants (Revised 1985) Part II. EPA/600/6-85/002b, Environmental Research Laboratory Office of Research and Development, US Environmental Protection Agency, Athens, Georgia.

Moench AF. 1994. "Specific yield as determined by type-curve analysis of aquifer-test data." Groundwater 32(6):949-957.

Moltyaner GL. 1987. "Mixing cup and through-the-wall measurements in field-scale tracer tests and their related scales of averaging." J. Hydrol. 89:281-302.

Morgan MG, and M Henrion. 1990. Uncertainty: A guide to dealing with uncertainty in quantitative risk and policy analysis. Cambridge University Press, Cambridge, United Kingdom.

Mullineaux DR, RE Wilcox, WF Ebaugh, R Fryxell, and M Rubin. 1978. “Age of the last major scabland flood of the Columbia Plateau in Eastern Washington.” Quaternary Research 10:171-180.

Murray CJ. 1994. "Identification and 3-D modeling of petrophysical rock types." In stochastic modeling and geostatistics, eds. JM Yarus and RL Chambers, pp. 323-336. American Association of Petroleum Geologists, Tulsa, Oklahoma.

NAS. 1996. Fractures and fluid flow: Contemporary understanding and applications, committee on fracture characterization and fluid flow. National Academy of Sciences, Washington, D.C.

National Council on Radiation Protection and Measurements (NCRP). 1996. A guide for uncertainty analysis in dose and risk assessments related to environmental contamination. NCRP Commentary No. 14, Bethesda, Maryland.

National Research Council. 1990. Ground water models, scientific and regulatory applications. National Academy Press, Washington, D.C.

National Research Council. 1994. Science and judgment in risk assessment. National Academy Press, Washington, D.C.

National Research Council. 1999. Environmental cleanup at Navy facilities: Risk based methods. National Academy Press, Washington, D.C.

Neitzel DA (ed.). 1998. Hanford Site National Environmental Policy Act (NEPA) Characterization. PNL-6415, Rev. 10, Pacific Northwest Laboratory, Richland, Washington.

Nelson RW. 1960. "In place measurement of permeability in heterogeneous media, 1. Theory of a proposed method." Journal of Geophysical Research 65(6):1753-1760.

Nelson RW. 1961. "In place measurement of permeability in heterogeneous media, 2. Experimental and computational considerations." Journal of Geophysical Research 66(5):2469-2478. 
Nelson RW. 1968. "In-place determination of permeability distribution of heterogeneous porous media through analysis of energy dissipation." Society of Petroleum Engineers Journal, 3:33-42.

Neuman SP. 1973. "Calibration of distributed parameter groundwater flow models viewed as a multiple objective decision process under uncertainty." Water Resour. Res. 9(4):1006-1021.

Neuman SP. 1980. "A statistical approach to the inverse problem of aquifer hydrology, 3. Improved solution method and added perspective." Water Resour. Res. 16(2):331-346.

Neuman SP. 1992. "Validation of safety assessment models as a process of scientific and public confidence building." In Proceedings International High Level Radioactive Waste Management Conference, pp. 1404-1420. US Department of Energy, Las Vegas, Nevada.

Neuman SP. 1993. "Comment on 'A Critical Review of Data on Field-Scale Dispersion in Aquifers' by LW Gelhar, C Welty, and KR Rehfeldt.” Water Resour. Res. 29(6):1863-1865.

Neuman SP. 1999. "Methodology to identify and evaluate conceptual models and uncertainty related to groundwater transport at nuclear facilities and sites." Draft report for US Nuclear Regulatory Commission presented at Research Symposium: Hydrologic Conceptual Model and Parameter Uncertainty, July 25-26, 2000, at NRC Headquarters in Rockville, Maryland.

Neuman SP, and S Yakowitz. 1979. "A statistical approach to the inverse problem of aquifer hydrology, 1. Theory." Water Resour. Res. 15(4):845-860.

Nevulis R, S Sorooshian, and DR Davis. 1987. Effects of surface waste disposal activity on groundwater levels in the Saddle Mountain Basalt. Department of Hydrology and Water Resources, University of Arizona, Tucson, Arizona.

Newcomb RC, and JR Strand. 1953. Geology and ground-water characteristics of the Hanford Reservation of the US Atomic Energy Commission, Washington. US Geological Survey Administrative Report WP-8, US Geological Survey, Washington, D.C.

Newcomb RC, JR Strand, and FJ Frank. 1972. Geology and ground-water characteristics of the Hanford Reservation of the US Atomic Energy Commission, Washington. Professional Paper 717, US Geological Survey, Washington, D.C.

Newcomer DR, LA Doremus, SH Hall, MJ Truex, VR Vermeul, and RE Engelman. 1995. Geology, hydrology, chemistry, and microbiology of the in situ bioremediation demonstration site. PNNL-10422, Pacific Northwest National Laboratory, Richland, Washington.

Nichols WE, NJ Aimo, M Oostrom, and MD White. 1997. STOMP subsurface transport over multiple phases: Application guide. PNNL-11216, Pacific Northwest National Laboratory, Richland, Washington.

Niederreiter H. 1992. "Random number generation and Quasi-Monte Carlo Methods." CBMS-NSF, 63, SIAM, Philadelphia, Pennsylvania. 
Pearce DW, RE Brown, and TP O'Farrell. 1969. The Arid Lands Ecology Reserve at Pacific Northwest Laboratory, Richland, Washington. BNWL-SA-2574, Pacific Northwest Laboratory, Richland, Washington.

Peck A, S Gorelick, G de Marsily, S Foster, and V Kovalevsky. 1988. Consequences of spatial variability in aquifer properties and data limitations for groundwater modelling practice. IAHS Publication No. 175, International Association of Hydrological Sciences, IAHS Press. Institute of Hydrology, Wallingford, Oxfordshire, United Kingdom.

Poeter EP, and MC Hill. 1998. Documentation of UCODE, a computer code for universal inverse modeling. Report 98-4080, US Geological Survey Water Resources Investigations.

Press WH, SA Teukolsky, WT Vetterling, and BP Flannery. 1992. Numerical recipes in C: The art of scientific computing. Cambridge University Press.

Puget Sound Power and Light Company (PSPL). 1982. Skagit/Hanford nuclear project, preliminary safety analysis report. Appendix 20, Amendment 23, Puget Sound Power and Light Company, Bellevue, Washington.

RamaRao BS, AM Lavenue, G de Marsily, MG Marietta. 1995. "Pilot point methodology for automated calibration of an ensemble of conditionally simulated transmissivity fields: Part 1 - Theory and computational experiments." Water Resour. Res. 31(3):475-493.

Randall JD, EJ Bonano, FA Kulacki, FA Costanzi, and P Davis (eds.). 1990. Validation of mathematical models for Waste Repository Performance Assessment - Confidence building through synthesis of experiments and calculations. NUREG Report, US Nuclear Regulatory Commission, Washington D.C.

Rasmussen JJ. 1971. “Soil survey of Benton County area, Washington.” US Government Printing Office, Washington, D.C.

Reidel SP. 1984. "The Saddle Mountains: The evolution of an anticline in the Yakima Fold Belt." American Journal of Science 284:942-978.

Reidel SP, and K. R. Fecht. 1994. Geologic map of the Priest Rapids 1:100,000 Quadrangle, Washington. Open File Report 94-13, Washington Division of Geology and Earth Resources, Washington State Department of Natural Resources, Olympia, Washington.

Reidel SP, KR Fecht, MC Hagood, and TL Tolan. 1989. "Wanapum and Saddle Mountains Basalt of the Cold Creek Syncline Area." In subsurface geology of the Cold Creek syncline, eds. CW Myers and SM Price. RHO-BWI-ST-14, Rockwell Hanford Operations, Richland, Washington.

Reidel SP, KA Lindsey, and KR Fecht. 1992. Field trip guide to the Hanford Site. WHC-MR-0391, Westinghouse Hanford Company, Richland, Washington.

Renard P, and G de Marsily. 1997. "Calculating equivalent permeability: A review." Advances in Water Resources 20:253-278. 
Rice JA. 1987. Mathematical statistics and data analysis. Wadsworth \& Brooks/Cole Advanced Books \& Software, Pacific Grove, California.

Rickard WH, and BE Vaughan. 1988. "Plant community characteristics and responses in shrub-steppe balance and change in a semi-arid terrestrial ecosystem." Developments in Agricultural and ManagedForest Ecology 20, eds. WH Rickard et al. Elsevier Science Publishers, New York.

Robinson D, and C Atcitty. 1999. "Comparison of Quasi- and Pseudo-Monte Carlo Sampling for reliability and uncertainty analysis." Collection of technical papers - AIAA/ASME/ASCE/AHS/ASC Structures, Structural Dynamics and Materials Conference, AIAA 4:2942-2949.

Rockhold ML, CS Simmons, and MJ Fayer. 1997. "An analytical solution technique for onedimensional steady water flow in layered soils." Water Resour. Res. 33:897-902.

Rotmans J, and H. Dowlatabadi. 1998. "Integrated assessment modeling." In human choice and climate change. Volume 3: The tools for policy analysis, eds. S Rayner and EL Malone, pp. 91-377. Battelle Press, Columbus, Ohio.

Sackschewsky MR, DS Landeen, GI Baird, WH Rickard, and JL Downs. 1992. Vascular plants of the Hanford Site. WHC-EP-0554, Westinghouse Hanford Company, Richland, Washington.

Sandia National Laboratories. 1992-1993. Preliminary performance assessment for the Waste Isolation Pilot Plant, December 1992. SAND92-0700, Vols. 1 - 5. Sandia National Laboratories, WIPP Performance Assessment Division, Albuquerque, New Mexico.

Scheibe TD. 1993. Characterization of the spatial structuring of natural porous media and its impacts on subsurface flow and transport. Ph.D. Dissertation, Stanford University, Stanford, California.

Scheibe TD, and DL Freyberg. 1995. "Use of sedimentological information for geometric simulation of natural porous media structure." Water Resour. Res. 31(12):3259-3270.

Schultz K, and B Huwe. 1999. "Uncertainty and sensitivity analysis of water transport modeling in layered soil profile using fuzzy set theory." Journal of Hydroinformatics 01.2:127-138.

Schulze-Makuch D, DA Carlson, DS Cherkauer, and P Malik. 1999. "Scale dependency of hydraulic conductivity in heterogeneous media." Groundwater 37(6):904-919.

Seo Dong-il. 1991. Optimal complexity analysis of total phosphorus models. Ph.D. thesis, University of Michigan Ann Arbor, Michigan.

Sidarauruk P, AH-D Cheng, and D Ouazar. 1998. "Ground water contaminant source and transport parameter identification by correlation coefficient optimization.” Groundwater 36(2):208-214.

Simmons CS, and CR Cole. 1985. Guidelines approach. Volume 1 of Guidelines for selecting codes for ground-water transport modeling of low-level waste burial sites. PNL-4980, Pacific Northwest Laboratory, Richland, Washington. 
Skaggs RL, and WH Walters. 1981. Flood risk analysis of Cold Creek near the Hanford Site. RHO-BWI-C-120 (PNL-4219), Rockwell Hanford Operations, Richland, Washington.

Smith AE, PB Ryan, and JS Evans. 1992 "The effect of neglecting correlations when propagating uncertainty and estimating the population distribution of risk." Risk Analysis 12(4):467-474.

Smith DL 1991. Probability, statistics, and data uncertainties in nuclear science and technology. American Nuclear Society, LaGrange Park, Illinois.

Spane FA, Jr. 1993. Selected hydraulic test analysis techniques for constant-rate discharge tests. PNL-8539, Pacific Northwest Laboratory, Richland, Washington.

Spane FA, Jr. 1994. Applicability of slug interference tests for hydraulic characterization of unconfined aquifers: (1) Analytical assessment. PNL-SA-24283, Pacific Northwest Laboratory, Richland, Washington.

Spane FA, Jr., and PD Thorne. 2000. Analysis of the hydrologic response associated with shutdown and restart of the 200-ZP-1 pump-and-treat system. PNNL-13342, Pacific Northwest National Laboratory, Richland, Washington.

Spane FA, Jr., PD Thorne, and DR Newcomer. 2001. Results of detailed hydrologic characterization tests - Fiscal Year 1999. PNNL-13378, Pacific Northwest National Laboratory, Richland, Washington.

Sperling T, RA Freeze, J Massmann, L Smith, and B James. 1992. "Hydrogeologic decision analysis, Part 3 - Application to design of a ground-water control system at an open pit mine." Groundwater 30:376-389.

Stein M. 1987. "Large sample properties of simulations using Latin Hypercube Sampling." Technometrics 29:143-151.

Stone WA, JM Thorp, OP Gifford, and DJ Hoitink. 1983. Climatological summary for the Hanford Area. PNL-4622, Pacific Northwest Laboratory, Richland, Washington.

Sugita F, and RW Gillham. 1995a. "Pore scale variation in retardation factor as a cause of nonideal reactive breakthrough curves 1 . Conceptual model and its evaluation." Water Resour. Res.

31(1):103-112.

Sugita F, and RW Gillham. 1995b. "Pore scale variation in retardation factor as a cause of nonideal reactive breakthrough curves 2. Pore network analysis.” Water Resour. Res. 31(1):113-119.

Sun N-Z, M-C Jeng, and W-G Yeh. 1995. "A propose geological method for parameter identification in three-dimensional groundwater modeling." Water Resour. Res. 31(1):89-102.

Sun N-Z, and W-G Yeh. 1985. "Identification of parameter structure in groundwater inverse problem." Water Resour. Res. 21(6):869-883. 
Swanson DA, JL Anderson, RD Bentley, VE Camp, JN Gardner, and TL Wright. 1979. Reconnaissance geologic map of the Columbia River basalt group in Washington and adjacent Idaho. Open-File Report 79-1363, US Geological Survey, Washington, D.C.

Tallman AM, KR Fecht, MC Marratt, and GV Last. 1979. Geology of the separations areas, Hanford Site, South-Central Washington. RHO-ST-23, Rockwell Hanford Operations, Richland, Washington.

Tang DH, and GF Pinder. 1977. "Simulation of groundwater flow and mass transport under uncertainty." Advances in Water Resources 1(1):25-30.

Theis CV. 1935. "The relation between the lowering of the piezometric surface and the rate and duration of discharge of a well using groundwater storage." Americal Union Transactions 16:519-524.

Thorne PD, and MA Chamness. 1992. Status report on the development of a three-dimensional conceptual model for the Hanford Site unconfined aquifer system. PNL-8332, Pacific Northwest Laboratory, Richland, Washington.

Thorne PD, MA Chamness, FA Spane, Jr., VR Vermeul, and WD Webber. 1993. Three-dimensional conceptual model for the Hanford Site unconfined aquifer system, FY 93 status report. PNL-8971, Pacific Northwest Laboratory, Richland, Washington.

Thorne PD, MA Chamness, VR Vermeul, QC MacDonald, and SE Schubert. 1994. Three-dimensional conceptual model for the Hanford Site unconfined aquifer system, FY 1994 status report. PNL-10195, Pacific Northwest Laboratory, Richland, Washington.

Thorne P, CJ Murray, CR Cole, MP Bergeron, and SK Wurstner. 2001. "Capturing hydrogeologic uncertainty in transport predictions at the Hanford Site, Washington." Presented at the 2001 International Containment and Remediation Technology Conference, June 12, Orlando, Florida.

Thorne PD, and DR Newcomer. 1992. Summary and evaluation of available hydraulic property data for the Hanford Site unconfined aquifer system. PNL-8337, Pacific Northwest Laboratory, Richland, Washington.

Thorp JM, and WT Hinds. 1977. Microclimates of the arid lands ecology reserve. BNWL-SA-6231, Pacific Northwest Laboratory, Richland, Washington.

Toth J. 1963. "A theoretical analysis of groundwater flow in small drainage basins." J. Geophys. Res. 68:4795-4812.

Tritscher P, WW Read, and P Broadbridge. 2000. "Specific yield for a two-dimensional flow." Water Resour. Res. 36(6):1393-1402.

US Department of Energy (DOE). 1987. Disposal of Hanford defense high-level, transuranic and tank wastes. DOE/EIS-0113, Richland, Washington. 
US Department of Energy (DOE). 1988. Consultation draft, Site characterization plan, reference repository location, Hanford Site, Washington. DOE/RW-0164, Vols. 1 and 2, Richland, Washington.

US Department of Energy (DOE). 1996. Draft Hanford Remedial Action Environmental Impact Statement and Comprehensive Land Use Plan. DOE/EIS-0222D, Washington, D.C.

US Department of Energy - Richland Operations Office (DOE-RL). 1995a. Hanford Site risk assessment methodology. DOE/RL-91-45, Richland, Washington.

US Department of Energy - Richland Operations Office (DOE-RL). 1995b. Modeling evaluation of the $N$-Springs barrier and pump-and-treat system. DOE/RL-94-132, Rev. 0, Richland, Washington.

US Department of Energy - Richland Operations Office (DOE-RL). 1996a. N-Springs expedited response action performance evaluation report. DOE/RL-95-110, Rev. 0, Richland, Washington.

US Department of Energy - Richland Operations Office (DOE-RL). 1996b. Remedial design and remedial action work plan for the 100 HR-3 and 100-KR-4 Groundwater Operable Units Interim Action. DOE/RL-96-84, Rev. 0, Richland, Washington.

US Department of Energy - Richland Operations Office (DOE-RL). 1998a. Inventory of miscellaneous streams. DOE/RL-95-82, Rev. 3, Richland, Washington.

US Department of Energy - Richland Operations Office (DOE-RL). 1998b. Groundwater/Vadose Zone Integration Project Specification. DOE/RL-98-48, Draft C, Richland, Washington.

US Department of Energy - Richland Operations Office (DOE-RL). 1999a. Fiscal Year 1998 Annual Summary Report - January - December 1998 for the 200-ZP-1, 200-ZP-1, and 100-NR-2 pump-and-treat operations and operable units. 1999-02s, Richland Operations Office, Richland, Washington.

US Department of Energy - Richland Operations Office (DOE-RL). 1999b. Hanford Comprehensive Land Use Plan Environmnetal Impact Statement. DOE/EIS-0222-F, Richland, Washington.

US Department of Energy - Richland Operations Office (DOE-RL). 1999c. Selection and review of a site-wide groundwater model at the Hanford Site. DOE/RL-2000-11, Richland, Washington.

US Department of Energy - Richland Operations Office (DOE-RL). 1999d. Annual Summary ReportJanuary - December 1998 for the 100-H-3 and 100-K pump-and-treat operations and operable units. DOE/RL-1999-13, Richland, Washington.

US Department of Energy - Richland Operations Office (DOE-RL). 2000. Fiscal Year 1999 Annual Summary Report - January - December 1998 for the 200-ZP-1, 200-ZP-1, and 100-NR-2 pump-and-treat operations and operable units. DOE/RL-1999-79, Richland, Washington.

US Department of Energy (DOE) and State of Washington Department of Ecology (Ecology). 1996. Tank Waste Remediation System, Hanford Site, Richland, Washington, Final Environmental Impact Statement. DOE/EIS-0189, Washington, D.C. 
US Environmental Protection Agency (EPA). 1997. Guiding principles for Monte-Carlo Analysis. EPA/630/R-97/001. TIC: 244010, Washington, D.C.

US Nuclear Regulatory Commission (USNRC). 2000. NMSS Decommissioning Standard Review Plan. NUREG-1727, Division of Waste Management, Office of Nuclear Material Safety and Safegards, Washington, D.C.

US Nuclear Regulatory Commission (USNRC). 1997. Regulatory Analysis Technical Evaluation Handbook. NUREG/BR-0184, Division of Regulatory Applications, Office of Nuclear Regulatory Research, Washington, D.C.

Vermeul VR, CR Cole, MP Bergeron, PD Thorne, and SK Wurstner. 2001. Transient inverse calibration of site-wide groundwater model to Hanford Operational Impacts from 1943 to 1996alternative conceptual model considering interaction with uppermost basalt confined aquifer. PNNL-13623, Pacific Northwest National Laboratory, Richland, Washington.

Vesely W, and D Rasmuson. 1984. "PRA uncertainties and the roles of sensitivity and uncertainty analyses." Proceedings of the 9th annual statistics symposium on national energy issues, NUREG/CP-0053. Los Alamos National Laboratory, Los Alamos, New Mexico.

Vincens GJ, I Rodrigues-Iturbe, and JC Schaake. 1975. "A Bayesian framework for the use of regional information in hydrology." Water Resour. Res. 11(3):405-414.

Wagner BJ. 1992. "Simultaneous parameter estimation and contaminant source characterization for coupled groundwater flow and contaminant transport modeling." Journal of Hydrology 135:275-303.

Wagner BJ, and SM Gorelick. 1986. "A statistical methodology for estimating transport parameters: theory and applications to one-dimensional systems." Water Resour. Res. 22(8):303-315.

Wagner BJ, and SM Gorelick. 1987. "Optimal groundwater quality management under uncertainty." Water Resour. Res. 23(7):1162-1174.

Walton WC. 1985. Practical aspects of groundwater modeling. Second edition, National Water Well Association, Worthington, Ohio.

Wang R, U Diwekar, and CE Padro. "Latin Hypercube Hammesley Squence Sampling Technique for risk and uncertainty analysis." Risk Analysis (in press).

Waters AC. 1961. "Stratigraphic and lithologic variations in the Columbia River basalt." American Journal of Science 259:583-611.

Walters WH, MC Richmond, and BG Gilmore. 1994. Reconstruction of radionuclide concentrations in the Columbia River from Hanford, Washington to Portland, Oregon January 1950-January 1971. PNWD-2225 HEDR, Pacific Northwest Laboratory, Richland, Washington. 
Washington State Department of Ecology (Ecology). 1986. State Waste Discharge Program. WAC 173-126, Olympia, Washington.

Webb EK, and JM Davis. 1998. "Simulation of the spatial heterogeneity of geologic properties: An overview." In uses of sedimentologic and stratigraphic information in predicting reservoir heterogeneity: Special publications, eds. GS Fraser and JM Davis, pp. 1-24. SEPM (Society for Sedimentary Geology), Tulsa, Oklahoma.

Weinberg AM. 1985. "Science and its limits: The regulator's dilemma." In Issues in Science and Technology, 2(1):59-72. National Academy of Sciences, Washington, D.C.

Whelan G, JW Buck, and A Nazarali. 1994. "Modular risk analysis for assessing multiple waste sites." PNL-SA-24239. In Proceedings of the US DOE Integrated Planning Workshop, June 1-2, Denver, Colorado, Pacific Northwest Laboratory, Richland, Washington.

Whelan G, JW Buck, DL Strenge, JG Droppo, Jr., and BL Hoopes. 1992. "Overview of the Multimedia Environmental Pollutant Assessment System (MEPAS)." Hazardous Waste \& Hazardous Materials 9(2):191-208.

White MD, and M Oostrom. 1996. STOMP subsurface transport over multiple phases: User's guide. PNNL-11217, Pacific Northwest National Laboratory, Richland, Washington.

White MD, and M Oostrom. 1997. STOMP subsurface transport over multiple phases: User's guide. PNNL-11218, Pacific Northwest National Laboratory, Richland, Washington.

Wigmosta MS, LW Vail, and DP Lettenmaier. 1994. "A distributed hydrology-vegetation model for complex terrain.” Water Resour. Res. 30(6):1665-1679.

Williams BA, BN Bjornstad, R Schalla, and WD Webber. 2000. Revised hydrogeology for the suprabasalt upper aquifer system, 200 East Area, Hanford Site, Washington. PNNL-12261, Pacific Northwest National Laboratory, Richland Washington.

Williams MD, and M Oostrom. 2000. "Oxygenation of anoxic water in a fluctuating water table system." Journal of Hydrology 230:70-85.

Williams MD, VR Vermeul, M Oostrom, JC Evans, JS Fruchter, JD Istok, MD Humphrey, DC Lanigan, JE Szecsody, MD White, TW Wietsma, and CR Cole. 1999. Anoxic plume attenuation in a fluctuating water table system: Impact of 100-D Area in situ redox manipulation on downgradient dissolved oxygen concentrations. PNNL-12192, Pacific Northwest National Laboratory, Richland, Washington.

Williams MD, SB Yabusaki, CR Cole, and VR Vermeul. 1994. In situ redox manipulation field experiment: Design analysis." In situ remediation: Scientific basis for current and future technologies, eds. GW Gee and NR Wing, pp. 1131-1152. Battelle Press, Columbus, Ohio. 
Wilson R, and A Shlyakhter. 1995. Uncertainty and variability in risk assessment. Department of Physics, Harvard Center for Risk Analysis, and Northeast Regional Center for Global Environmental Change, Harvard University, Cambridge, Massachusetts.

Wood MI, R Khaleel, PD Rittmann, SH Finfrock, TH DeLorenzo, and DY Garbrick. 1996. Performance assessment for the disposal of low-level waste in the 200-East Area burial grounds. WHC-SD-WM-TI730, Rev. 0, Westinghouse Hanford Company, Richland, Washington.

Wood MI, R Khaleel, PD Rittmann, AH Lu, SH Finfrock, RJ Serne, KJ Cantrell, and TH DeLorenzo. 1995. Performance assessment for the disposal of low-level waste in the 200 West Area burial grounds. WHC-ED-0645, Westinghouse Hanford Company, Richland, Washington.

Wurstner SK, and JL Devary. 1993. Hanford Site ground-water model: Geographic information system linkages and model enhancements, FY 1993. PNL-8991, Pacific Northwest Laboratory, Richland, Washington.

Wurstner SK, PD Thorne, MA Chamness, MD Freshley, and MD Williams. 1995. Development of a three-dimensional groundwater model of the Hanford Site unconfined aquifer system: FY 1995 Status Report. PNL-10886, Pacific Northwest Laboratory, Richland, Washington.

Wyss GD, and KH Jorgensen. 1998. A user's guide to LHS: Sandia's Latin Hypercube Sampling software. Technical Report SAND98-0210, Sandia National Laboratories, Albuquerque, New Mexico.

Yeh AW-G. 1986. "Review of parameter identification proceedures in groundwater hydrology: The inverse problem.” Water Resour. Res. 22(2):95-108.

Zimmerman DA, RT Hanson, and PA Davis. 1991. A comparison of parameter estimation and sensitivity techniques on the uncertainty in groundwater flow predictions. NUREG/CR-5522 (SAND900128, Sandia National Laboratories, Albuquerque, New Mexico), Division of High Level Waste Management, Office of Nuclear Material Safety and Safeguards, US Nuclear Regulatory Commission, Washington, D.C.

Zimmerman DA, G de Marsily, CA Gotway, MG Marietta, CL Axness, R Beauheim, R Bras, J Carrera., G Dagan, PB Davies, D Gallegos, A Galli, J Gomez-Hernandez, P Grindrod, AL Gutjahr, P Kitanidis, A M Lavenue, D McLaughlin, SP Neuman, BS RamaRao, C Ravenne, and Y Rubin. 1998. "A comparison of seven geostatistically-based inverse approaches to estimate transmissivities for modeling advective transport by groundwater flow." Water Resour. Res. 34(6):1373-1413. 


\section{Appendix A}

\section{Interpreted Top and Bottom Elevations of Major Hydrogeologic Units}




\section{Appendix A}

\section{Interpreted Top and Bottom Elevations of Major Hydrogeologic Units}

\section{A.1 Introduction}

This appendix provides a summary of top and bottom elevations of each hydrogeologic unit used in the Hanford Site-wide groundwater flow and transport model as interpreted from well data. These interpretations of well-log data provide the basis for the interpreted distributions and thicknesses of major hydrogeologic units summarized in Section 4 of this report and in reports by Thorne and Chamness (1992), Thorne et al. (1993, 1994), and Wurstner et al. (1995).

Information used to pick these elevations at each well includes the geologist's or driller's geologic description of core samples or drill cuttings, geophysical logs, and sieve analyses of samples. However, not all of these types of data were available for all of the wells. The quality of the geologic descriptions varies widely and, in some cases, it was not possible to determine if certain units were present. For many wells, it was only possible to determine the top of basalt elevation. Unit 6 , which represents generally finer-grained over-bank deposits within the Ringold gravels, varies more in texture across the Hanford Site than the other units identified in the model. Therefore, where available, the percent mud determined from sieve analysis for this unit is listed in the last column of the table. 
Table A.1. Interpreted Top and Bottom Elevation of Major Hydrogeologic Units

$\mathrm{NP}=$ unit not present

$\mathrm{NC}=$ not certain whether unit is present or not

$\mathrm{ND}=$ well not deep enough to determine

$\mathrm{NI}=$ not interpreted or unknown

Elevations in $\mathrm{m}$ above mean sea level based on the National Geodetic Vertical Datum of 1929 (NGVD29 datum)

\begin{tabular}{|c|c|c|c|c|c|c|c|c|c|c|c|c|c|c|c|c|c|c|c|c|}
\hline Well number & $\begin{array}{l}\text { Casing } \\
\text { Elev. }\end{array}$ & Bot 1 & Top 2 & Bot 2 & Top 3 & Bot 3 & Top 4 & Bot 4 & Top 5 & Bot 5 & Top 6 & Bot 6 & Top 7 & Bot 7 & Top 8 & Bot 8 & Top 9 & Bot 9 & $\begin{array}{l}\text { Top of } \\
\text { Basalt }\end{array}$ & $\begin{array}{l}\text { Layer } 6 \\
\% \text { mud }\end{array}$ \\
\hline 699-LANDFILL & 146.9 & 120.7 & NP & NP & NP & NP & NP & NP & 120.7 & 85.3 & NP & NP & NP & NP & 85.3 & 71.3 & NP & NP & 71.3 & NP \\
\hline 699-ORV-1 & 143.9 & 121.0 & NP & NP & NP & NP & NP & NP & 121.0 & 95.7 & NP & NP & NP & NP & 95.7 & 82.9 & NP & NP & 82.9 & NP \\
\hline 699-ORV-2 & 138.7 & 120.4 & NP & NP & NP & NP & NP & NP & 120.4 & 89.9 & NP & NP & NP & NP & 89.9 & 77.7 & NP & NP & 77.7 & NP \\
\hline 699-S51-2 & 117.7 & 100.0 & NP & NP & NP & NP & NP & NP & NP & NP & 100.0 & 90.2 & 90.2 & 86.3 & 86.3 & 64.9 & NP & NP & 64.9 & 23.8 \\
\hline $699-S 31-1$ & 139.6 & 100.6 & $\mathrm{NP}$ & NP & NP & NP & NP & NP & 100.6 & 83.2 & $\mathrm{NP}$ & $\mathrm{NP}$ & $\mathrm{NP}$ & $\mathrm{NP}$ & 83.2 & 72.2 & NP & NP & 72.2 & NP \\
\hline 699-S30-E15A & 122.0 & 101.2 & NP & NP & NP & NP & NP & NP & 101.2 & ND & ND & ND & ND & ND & ND & ND & ND & ND & ND & ND \\
\hline 699-S30-E14 & 122.3 & 102.5 & NP & NP & NP & NP & NP & NP & 102.4 & 84.7 & 84.7 & 79.6 & 79.6 & 72.2 & 72.2 & 61.3 & NP & NP & 61.4 & 30.5 \\
\hline 699-S29-E16C & 114.9 & 102.4 & NP & NP & NP & NP & NP & NP & 102.4 & 84.4 & NP & NP & NP & NP & 84.4 & 61.6 & NP & NP & 61.6 & NP \\
\hline 699-S28-E0 & 136.7 & 103.2 & NP & NP & NP & NP & NP & NP & 103.0 & 79.6 & NP & NP & NP & NP & 79.6 & 68.6 & NP & NP & 68.7 & NP \\
\hline 699-S27-E14 & 121.8 & 102.6 & $\mathrm{NP}$ & NP & NP & NP & NP & NP & 102.7 & 96.9 & 96.9 & 82.3 & 82.3 & 71.6 & 71.6 & ND & ND & ND & ND & 15.2 \\
\hline 699-S27-E9C & 118.3 & 109.4 & $\mathrm{NP}$ & NP & NP & NP & NP & NP & 109.4 & 95.7 & 95.7 & 78.3 & 78.3 & 65.8 & 65.8 & 59.1 & NP & NP & 59.1 & 21.9 \\
\hline 699-S24-19 & 130.1 & 120.7 & NP & NP & NP & NP & NP & NP & 120.7 & 116.7 & NP & NP & NP & NP & 116.7 & 116.4 & NP & NP & 116.4 & NP \\
\hline 699-S22-E9C & 113.1 & 105.5 & NP & NP & NP & NP & NP & NP & 105.5 & 99.4 & 99.4 & 78.0 & 78.0 & 67.7 & 67.7 & 61.3 & NP & NP & 61.3 & 11.9 \\
\hline 699-S18-E2A & 132.6 & 109.7 & NP & NP & NP & NP & NP & NP & 109.7 & 70.1 & NP & NP & NP & NP & 70.1 & 57.0 & NP & NP & 57.0 & NP \\
\hline 699-S18-51 & $\mathrm{NI}$ & $\mathrm{NI}$ & $\mathrm{NI}$ & $\mathrm{NI}$ & $\mathrm{NI}$ & $\mathrm{NI}$ & $\mathrm{NI}$ & $\mathrm{NI}$ & $\mathrm{NI}$ & $\mathrm{NI}$ & $\mathrm{NI}$ & $\mathrm{NI}$ & $\mathrm{NI}$ & $\mathrm{NI}$ & $\mathrm{NI}$ & $\mathrm{NI}$ & $\mathrm{NI}$ & $\mathrm{NI}$ & 356.3 & $\mathrm{NI}$ \\
\hline 699-S17-24 & $\mathrm{NI}$ & $\mathrm{NI}$ & $\mathrm{NI}$ & $\mathrm{NI}$ & $\mathrm{NI}$ & $\mathrm{NI}$ & $\mathrm{NI}$ & $\mathrm{NI}$ & $\mathrm{NI}$ & $\mathrm{NI}$ & $\mathrm{NI}$ & $\mathrm{NI}$ & $\mathrm{NI}$ & $\mathrm{NI}$ & $\mathrm{NI}$ & $\mathrm{NI}$ & $\mathrm{NI}$ & $\mathrm{NI}$ & 152.4 & $\mathrm{NI}$ \\
\hline 699-S17-25 & $\mathrm{NI}$ & $\mathrm{NI}$ & $\mathrm{NI}$ & $\mathrm{NI}$ & $\mathrm{NI}$ & $\mathrm{NI}$ & $\mathrm{NI}$ & $\mathrm{NI}$ & $\mathrm{NI}$ & $\mathrm{NI}$ & $\mathrm{NI}$ & $\mathrm{NI}$ & $\mathrm{NI}$ & $\mathrm{NI}$ & $\mathrm{NI}$ & $\mathrm{NI}$ & $\mathrm{NI}$ & $\mathrm{NI}$ & 137.2 & $\mathrm{NI}$ \\
\hline 699-S17-28 & $\mathrm{NI}$ & $\mathrm{NI}$ & $\mathrm{NI}$ & $\mathrm{NI}$ & $\mathrm{NI}$ & $\mathrm{NI}$ & $\mathrm{NI}$ & $\mathrm{NI}$ & $\mathrm{NI}$ & $\mathrm{NI}$ & $\mathrm{NI}$ & $\mathrm{NI}$ & $\mathrm{NI}$ & $\mathrm{NI}$ & $\mathrm{NI}$ & $\mathrm{NI}$ & $\mathrm{NI}$ & $\mathrm{NI}$ & 134.1 & $\mathrm{NI}$ \\
\hline 699-S17-30A & $\mathrm{NI}$ & $\mathrm{NI}$ & $\mathrm{NI}$ & $\mathrm{NI}$ & $\mathrm{NI}$ & $\mathrm{NI}$ & $\mathrm{NI}$ & $\mathrm{NI}$ & $\mathrm{NI}$ & $\mathrm{NI}$ & $\mathrm{NI}$ & $\mathrm{NI}$ & $\mathrm{NI}$ & $\mathrm{NI}$ & $\mathrm{NI}$ & $\mathrm{NI}$ & $\mathrm{NI}$ & $\mathrm{NI}$ & 132.6 & $\mathrm{NI}$ \\
\hline 699-S17-30B & $\mathrm{NI}$ & $\mathrm{NI}$ & $\mathrm{NI}$ & $\mathrm{NI}$ & $\mathrm{NI}$ & $\mathrm{NI}$ & $\mathrm{NI}$ & $\mathrm{NI}$ & $\mathrm{NI}$ & $\mathrm{NI}$ & $\mathrm{NI}$ & $\mathrm{NI}$ & $\mathrm{NI}$ & $\mathrm{NI}$ & $\mathrm{NI}$ & $\mathrm{NI}$ & $\mathrm{NI}$ & $\mathrm{NI}$ & 129.5 & $\mathrm{NI}$ \\
\hline 699-S17-30C & $\mathrm{NI}$ & $\mathrm{NI}$ & $\mathrm{NI}$ & $\mathrm{NI}$ & $\mathrm{NI}$ & $\mathrm{NI}$ & $\mathrm{NI}$ & $\mathrm{NI}$ & $\mathrm{NI}$ & $\mathrm{NI}$ & $\mathrm{NI}$ & $\mathrm{NI}$ & $\mathrm{NI}$ & $\mathrm{NI}$ & $\mathrm{NI}$ & $\mathrm{NI}$ & $\mathrm{NI}$ & $\mathrm{NI}$ & 129.5 & $\mathrm{NI}$ \\
\hline 699-S16-E14 & 122.5 & $\mathrm{NI}$ & $\mathrm{NI}$ & $\mathrm{NI}$ & $\mathrm{NI}$ & $\mathrm{NI}$ & $\mathrm{NI}$ & $\mathrm{NI}$ & $\mathrm{NI}$ & $\mathrm{NI}$ & $\mathrm{NI}$ & $\mathrm{NI}$ & $\mathrm{NI}$ & $\mathrm{NI}$ & $\mathrm{NI}$ & $\mathrm{NI}$ & $\mathrm{NI}$ & $\mathrm{NI}$ & 62.2 & $\mathrm{NI}$ \\
\hline 699-S16-24 & $\mathrm{NI}$ & $\mathrm{NI}$ & $\mathrm{NI}$ & $\mathrm{NI}$ & $\mathrm{NI}$ & $\mathrm{NI}$ & $\mathrm{NI}$ & $\mathrm{NI}$ & $\mathrm{NI}$ & $\mathrm{NI}$ & $\mathrm{NI}$ & $\mathrm{NI}$ & $\mathrm{NI}$ & $\mathrm{NI}$ & $\mathrm{NI}$ & $\mathrm{NI}$ & $\mathrm{NI}$ & $\mathrm{NI}$ & 162.2 & $\mathrm{NI}$ \\
\hline 699-S14-20A & $\mathrm{NI}$ & $\mathrm{NI}$ & $\mathrm{NI}$ & $\mathrm{NI}$ & $\mathrm{NI}$ & $\mathrm{NI}$ & $\mathrm{NI}$ & $\mathrm{NI}$ & $\mathrm{NI}$ & $\mathrm{NI}$ & $\mathrm{NI}$ & $\mathrm{NI}$ & $\mathrm{NI}$ & $\mathrm{NI}$ & $\mathrm{NI}$ & $\mathrm{NI}$ & $\mathrm{NI}$ & $\mathrm{NI}$ & 101.5 & $\mathrm{NI}$ \\
\hline 699-S12-3 & 132.7 & 119.3 & $\mathrm{NP}$ & NP & NP & NP & NP & NP & 119.5 & 99.7 & 99.7 & ND & ND & ND & ND & ND & ND & ND & ND & ND \\
\hline 699-S12-29 & 148.4 & 110.6 & $\mathrm{NP}$ & NP & NP & NP & NP & NP & NP & $\mathrm{NP}$ & $\mathrm{NP}$ & NP & NP & $\mathrm{NP}$ & 110.6 & 93.9 & NP & NP & 93.9 & NP \\
\hline 699-S11-E12A & 111.3 & 93.0 & $\mathrm{NP}$ & NP & NP & NP & NP & NP & 93.0 & 76.2 & 76.2 & 67.1 & 67.1 & 57.9 & 57.9 & 47.2 & NP & NP & 47.2 & 30.5 \\
\hline
\end{tabular}


Table A.1. Interpreted Top and Bottom Elevation of Major Hydrogeologic Units (continued)

$\mathrm{NP}=$ unit not present

$\mathrm{NC}=$ not certain whether unit is present or not

$\mathrm{ND}=$ well not deep enough to determine

$\mathrm{NI}=$ not interpreted or unknown

Elevations in $\mathrm{m}$ above mean sea level based on the National Geodetic Vertical Datum of 1929 (NGVD29 datum)

\begin{tabular}{|c|c|c|c|c|c|c|c|c|c|c|c|c|c|c|c|c|c|c|c|c|}
\hline Well number & $\begin{array}{l}\text { Casing } \\
\text { Elev. }\end{array}$ & Bot 1 & Top 2 & Bot 2 & Top 3 & Bot 3 & Top 4 & Bot 4 & Top 5 & Bot 5 & Top 6 & Bot 6 & Top 7 & Bot 7 & Top 8 & Bot 8 & Top 9 & Bot 9 & $\begin{array}{l}\text { Top of } \\
\text { Basalt }\end{array}$ & $\begin{array}{l}\text { Layer } 6 \\
\% \text { mud }\end{array}$ \\
\hline 699-S11-E12B & 111.3 & 94.5 & NP & NP & NP & NP & NP & NP & 94.5 & 77.7 & 77.7 & 67.1 & 67.1 & 57.9 & 57.9 & 45.7 & NP & NP & 45.7 & 7.9 \\
\hline 699-S9-56 & $\mathrm{NI}$ & $\mathrm{NI}$ & $\mathrm{NI}$ & $\mathrm{NI}$ & $\mathrm{NI}$ & $\mathrm{NI}$ & $\mathrm{NI}$ & $\mathrm{NI}$ & $\mathrm{NI}$ & $\mathrm{NI}$ & $\mathrm{NI}$ & $\mathrm{NI}$ & $\mathrm{NI}$ & $\mathrm{NI}$ & $\mathrm{NI}$ & $\mathrm{NI}$ & $\mathrm{NI}$ & $\mathrm{NI}$ & 338.9 & $\mathrm{NI}$ \\
\hline 699-S9-63B & 437.7 & $\mathrm{NI}$ & $\mathrm{NI}$ & $\mathrm{NI}$ & $\mathrm{NI}$ & $\mathrm{NI}$ & $\mathrm{NI}$ & $\mathrm{NI}$ & $\mathrm{NI}$ & $\mathrm{NI}$ & $\mathrm{NI}$ & $\mathrm{NI}$ & $\mathrm{NI}$ & $\mathrm{NI}$ & $\mathrm{NI}$ & $\mathrm{NI}$ & $\mathrm{NI}$ & $\mathrm{NI}$ & 425.5 & $\mathrm{NI}$ \\
\hline 699-S8-19 & 153.6 & 119.5 & NP & NP & NP & NP & NP & NP & 119.5 & ND & ND & ND & ND & ND & ND & ND & ND & ND & ND & ND \\
\hline 699-S7-34 & 160.6 & 116.7 & $\mathrm{NP}$ & NP & NP & $\mathrm{NP}$ & $\mathrm{NP}$ & NP & $\mathrm{NP}$ & $\mathrm{NP}$ & NP & NP & NP & NP & NP & NP & NP & NP & 116.7 & NP \\
\hline 699-S7-62A & 381.0 & $\mathrm{NI}$ & $\mathrm{NI}$ & $\mathrm{NI}$ & $\mathrm{NI}$ & $\mathrm{NI}$ & $\mathrm{NI}$ & $\mathrm{NI}$ & $\mathrm{NI}$ & $\mathrm{NI}$ & $\mathrm{NI}$ & $\mathrm{NI}$ & $\mathrm{NI}$ & $\mathrm{NI}$ & $\mathrm{NI}$ & $\mathrm{NI}$ & $\mathrm{NI}$ & $\mathrm{NI}$ & 381.0 & $\mathrm{NI}$ \\
\hline 699-S7-62B & $\mathrm{NI}$ & $\mathrm{NI}$ & $\mathrm{NI}$ & $\mathrm{NI}$ & $\mathrm{NI}$ & $\mathrm{NI}$ & $\mathrm{NI}$ & $\mathrm{NI}$ & $\mathrm{NI}$ & $\mathrm{NI}$ & $\mathrm{NI}$ & $\mathrm{NI}$ & $\mathrm{NI}$ & $\mathrm{NI}$ & $\mathrm{NI}$ & $\mathrm{NI}$ & $\mathrm{NI}$ & $\mathrm{NI}$ & 339.2 & $\mathrm{NI}$ \\
\hline 699-S7-62C & $\mathrm{NI}$ & $\mathrm{NI}$ & $\mathrm{NI}$ & $\mathrm{NI}$ & $\mathrm{NI}$ & $\mathrm{NI}$ & $\mathrm{NI}$ & $\mathrm{NI}$ & $\mathrm{NI}$ & $\mathrm{NI}$ & $\mathrm{NI}$ & $\mathrm{NI}$ & $\mathrm{NI}$ & $\mathrm{NI}$ & $\mathrm{NI}$ & $\mathrm{NI}$ & $\mathrm{NI}$ & $\mathrm{NI}$ & 310.9 & $\mathrm{NI}$ \\
\hline 699-S6-E14A & 114.6 & 103.3 & NP & NP & NP & NP & NP & NP & 103.3 & 61.0 & 61.0 & 57.3 & NP & NP & 57.3 & 55.2 & NP & NP & 55.2 & 30.5 \\
\hline 699-S6-E14B & $\mathrm{NI}$ & $\mathrm{NI}$ & $\mathrm{NI}$ & $\mathrm{NI}$ & $\mathrm{NI}$ & $\mathrm{NI}$ & $\mathrm{NI}$ & $\mathrm{NI}$ & $\mathrm{NI}$ & $\mathrm{NI}$ & $\mathrm{NI}$ & $\mathrm{NI}$ & $\mathrm{NI}$ & $\mathrm{NI}$ & $\mathrm{NI}$ & $\mathrm{NI}$ & $\mathrm{NI}$ & $\mathrm{NI}$ & 56.4 & $\mathrm{NI}$ \\
\hline 699-S6-E4C & 132.0 & 107.6 & NP & NP & NP & NP & $\mathrm{NP}$ & $\mathrm{NP}$ & 107.6 & 69.5 & 69.5 & 48.2 & 48.2 & 42.1 & 42.1 & 31.7 & NP & $\mathrm{NP}$ & 31.7 & 28.3 \\
\hline 699-S4-E16 & 105.2 & 97.5 & $\mathrm{NP}$ & NP & NP & NP & NP & NP & 97.5 & 83.2 & 83.2 & 74.4 & 74.4 & 57.3 & 57.3 & 49.4 & NP & NP & 49.4 & 19.8 \\
\hline 699-S3-E12 & 121.0 & 97.8 & $\mathrm{NP}$ & NP & NP & NP & NP & NP & 97.8 & 76.8 & 76.8 & 70.4 & 70.4 & 58.5 & 58.5 & 44.8 & NP & NP & 45.1 & 21.3 \\
\hline 699-S3-25 & 159.7 & 122.5 & $\mathrm{NP}$ & NP & NP & NP & NP & NP & 122.5 & ND & ND & ND & ND & ND & ND & ND & ND & ND & ND & ND \\
\hline 699-S3-67 & $\mathrm{NI}$ & $\mathrm{NI}$ & $\mathrm{NI}$ & $\mathrm{NI}$ & $\mathrm{NI}$ & $\mathrm{NI}$ & $\mathrm{NI}$ & $\mathrm{NI}$ & $\mathrm{NI}$ & $\mathrm{NI}$ & $\mathrm{NI}$ & $\mathrm{NI}$ & $\mathrm{NI}$ & $\mathrm{NI}$ & $\mathrm{NI}$ & $\mathrm{NI}$ & $\mathrm{NI}$ & $\mathrm{NI}$ & 353.6 & $\mathrm{NI}$ \\
\hline 699-S2-34B & 164.6 & 126.5 & NP & NP & NP & NP & NP & NP & 126.5 & 83.8 & NP & NP & NP & NP & 83.8 & 65.5 & 65.5 & 59.4 & 59.4 & NP \\
\hline 699-1-18 & 164.0 & 124.4 & $\mathrm{NP}$ & NP & NP & NP & NP & NP & 124.4 & 64.9 & $\mathrm{NC}$ & $\mathrm{NC}$ & $\mathrm{NC}$ & $\mathrm{NC}$ & 64.9 & ND & ND & ND & ND & $\mathrm{NI}$ \\
\hline 699-2-E19 & $\mathrm{NI}$ & $\mathrm{NI}$ & $\mathrm{NI}$ & $\mathrm{NI}$ & $\mathrm{NI}$ & $\mathrm{NI}$ & $\mathrm{NI}$ & $\mathrm{NI}$ & $\mathrm{NI}$ & $\mathrm{NI}$ & $\mathrm{NI}$ & $\mathrm{NI}$ & $\mathrm{NI}$ & $\mathrm{NI}$ & $\mathrm{NI}$ & $\mathrm{NI}$ & $\mathrm{NI}$ & $\mathrm{NI}$ & 31.1 & $\mathrm{NI}$ \\
\hline 699-2-E14 & 118.5 & 98.8 & NP & NP & NP & NP & NP & NP & 98.8 & 75.9 & 75.9 & 65.2 & 65.2 & 48.5 & 48.5 & 34.7 & 34.7 & 21.0 & 21.0 & 12.8 \\
\hline $699-2-3$ & 145.4 & 116.4 & $\mathrm{NP}$ & NP & NP & NP & NP & $\mathrm{NP}$ & 116.4 & ND & ND & ND & ND & ND & ND & ND & ND & ND & ND & ND \\
\hline $699-2-7$ & 156.1 & 118.0 & NP & NP & NP & NP & NP & $\mathrm{NP}$ & 118.0 & ND & ND & ND & ND & ND & ND & ND & ND & ND & ND & ND \\
\hline $699-2-33 A$ & 163.4 & 126.8 & $\mathrm{NP}$ & NP & NP & NP & NP & NP & 126.8 & 104.2 & 104.2 & 95.7 & 95.7 & 57.0 & 57.0 & 42.4 & 42.4 & 25.6 & 25.6 & 30.5 \\
\hline $699-2-33 B$ & $\mathrm{NI}$ & $\mathrm{NI}$ & $\mathrm{NI}$ & $\mathrm{NI}$ & $\mathrm{NI}$ & $\mathrm{NI}$ & $\mathrm{NI}$ & $\mathrm{NI}$ & $\mathrm{NI}$ & $\mathrm{NI}$ & $\mathrm{NI}$ & $\mathrm{NI}$ & $\mathrm{NI}$ & $\mathrm{NI}$ & $\mathrm{NI}$ & $\mathrm{NI}$ & $\mathrm{NI}$ & $\mathrm{NI}$ & 26.2 & $\mathrm{NI}$ \\
\hline $699-3-45$ & 153.6 & 112.8 & $\mathrm{NP}$ & NP & NP & NP & NP & NP & NP & $\mathrm{NP}$ & NP & NP & NP & NP & NP & NP & NP & NP & 112.8 & NP \\
\hline $699-6-2 A$ & 137.5 & 118.3 & NP & NP & NP & NP & NP & NP & 118.3 & 56.4 & 56.4 & 35.7 & 35.7 & 20.4 & 20.4 & -10.7 & -10.7 & -23.8 & -23.8 & 28.3 \\
\hline $699-8-17$ & 159.2 & 101.2 & NP & NP & NP & NP & 101.2 & ND & ND & ND & ND & ND & ND & ND & ND & ND & ND & ND & ND & ND \\
\hline $699-8-25$ & 155.1 & 104.9 & NP & NP & NP & NP & 104.9 & ND & ND & ND & ND & ND & ND & ND & ND & ND & ND & ND & ND & ND \\
\hline
\end{tabular}


Table A.1. Interpreted Top and Bottom Elevation of Major Hydrogeologic Units (continued)

$\mathrm{NP}=$ unit not present

$\mathrm{NC}=$ not certain whether unit is present or not

$\mathrm{ND}=$ well not deep enough to determine

$\mathrm{NI}=$ not interpreted or unknown

Elevations in $\mathrm{m}$ above mean sea level based on the National Geodetic Vertical Datum of 1929 (NGVD29 datum)

\begin{tabular}{|c|c|c|c|c|c|c|c|c|c|c|c|c|c|c|c|c|c|c|c|c|}
\hline Well number & $\begin{array}{l}\text { Casing } \\
\text { Elev. }\end{array}$ & Bot 1 & Top 2 & Bot 2 & Top 3 & Bot 3 & Top 4 & Bot 4 & Top 5 & Bot 5 & Top 6 & Bot 6 & Top 7 & Bot 7 & Top 8 & Bot 8 & Top 9 & Bot 9 & $\begin{array}{l}\text { Top of } \\
\text { Basalt }\end{array}$ & $\begin{array}{l}\text { Layer } 6 \\
\% \text { mud }\end{array}$ \\
\hline 699-8-32 & 168.9 & 116.4 & NP & NP & $\mathrm{NP}$ & NP & $\mathrm{NP}$ & $\mathrm{NP}$ & 116.4 & ND & ND & ND & ND & ND & ND & ND & ND & ND & ND & ND \\
\hline 699-9-E5A & 138.4 & 118.3 & NP & NP & NP & NP & NP & NP & 118.3 & 76.2 & $\mathrm{NC}$ & $\mathrm{NC}$ & 76.2 & 13.4 & 13.4 & 2.7 & 2.7 & -6.7 & -6.7 & $\mathrm{NC}$ \\
\hline 699-9-E2 & 127.4 & 112.2 & NP & NP & $\mathrm{NP}$ & NP & NP & NP & 112.2 & 69.5 & 69.5 & 43.6 & 43.6 & 31.4 & 31.4 & 2.4 & 2.4 & -1.2 & -1.2 & 12.5 \\
\hline 699-10-E12 & 131.3 & 105.5 & NP & NP & $\mathrm{NP}$ & NP & NP & $\mathrm{NP}$ & 105.5 & 89.6 & 89.6 & 61.9 & 61.9 & 41.5 & 41.5 & 27.7 & 27.7 & 23.2 & 23.2 & 16.2 \\
\hline $699-10-3 A$ & $\mathrm{NI}$ & $\mathrm{NI}$ & $\mathrm{NI}$ & $\mathrm{NI}$ & $\mathrm{NI}$ & $\mathrm{NI}$ & $\mathrm{NI}$ & $\mathrm{NI}$ & $\mathrm{NI}$ & $\mathrm{NI}$ & $\mathrm{NI}$ & $\mathrm{NI}$ & $\mathrm{NI}$ & $\mathrm{NI}$ & $\mathrm{NI}$ & $\mathrm{NI}$ & $\mathrm{NI}$ & $\mathrm{NI}$ & -25.6 & $\mathrm{NI}$ \\
\hline $699-10-30 \mathrm{~A}$ & 168.4 & 100.0 & NP & NP & NP & NP & 100.0 & 89.3 & 89.3 & 61.9 & 61.9 & 60.0 & 60.0 & 49.7 & 49.7 & 45.1 & 45.1 & 13.1 & 13.1 & 30.5 \\
\hline 699-10-54B & 157.4 & 128.3 & NP & NP & NP & NP & $\mathrm{NP}$ & $\mathrm{NP}$ & 128.3 & 105.5 & $\mathrm{NP}$ & $\mathrm{NP}$ & NP & $\mathrm{NP}$ & 105.5 & 93.3 & NP & NP & 93.3 & NP \\
\hline 699-10-99 & $\mathrm{NI}$ & $\mathrm{NI}$ & $\mathrm{NI}$ & $\mathrm{NI}$ & $\mathrm{NI}$ & $\mathrm{NI}$ & $\mathrm{NI}$ & $\mathrm{NI}$ & $\mathrm{NI}$ & $\mathrm{NI}$ & $\mathrm{NI}$ & $\mathrm{NI}$ & $\mathrm{NI}$ & $\mathrm{NI}$ & $\mathrm{NI}$ & $\mathrm{NI}$ & $\mathrm{NI}$ & $\mathrm{NI}$ & 320.0 & $\mathrm{NI}$ \\
\hline 699-11-E8B & 136.9 & 118.6 & NP & NP & $\mathrm{NP}$ & $\mathrm{NP}$ & $\mathrm{NP}$ & $\mathrm{NP}$ & 118.6 & 78.9 & 78.9 & 75.9 & 75.9 & 39.3 & 39.3 & 27.1 & 27.1 & 19.5 & 19.5 & 30.5 \\
\hline 699-11-E4E & 134.4 & 115.8 & NP & $\mathrm{NP}$ & $\mathrm{NP}$ & NP & $\mathrm{NP}$ & $\mathrm{NP}$ & 115.8 & 62.5 & 62.5 & 44.2 & 44.2 & 35.1 & 35.1 & 7.6 & 7.6 & -7.0 & -7.0 & 30.5 \\
\hline $699-11-23 A$ & 166.1 & 93.0 & NP & NP & NP & NP & 93.0 & 79.2 & 79.2 & 24.4 & 24.4 & 7.6 & 7.6 & -21.3 & -21.3 & -41.1 & -41.1 & ND & ND & 25.0 \\
\hline $699-11-29$ & 166.1 & 108.2 & NP & NP & NP & NP & $\mathrm{NI}$ & $\mathrm{NI}$ & $\mathrm{NI}$ & $\mathrm{NI}$ & $\mathrm{NI}$ & $\mathrm{NI}$ & $\mathrm{NI}$ & $\mathrm{NI}$ & $\mathrm{NI}$ & $\mathrm{NI}$ & $\mathrm{NI}$ & $\mathrm{NI}$ & -50.0 & $\mathrm{NI}$ \\
\hline $699-11-45 A$ & 176.2 & 125.0 & NP & NP & NP & NP & $\mathrm{NP}$ & $\mathrm{NP}$ & 125.0 & 107.6 & $\mathrm{NP}$ & $\mathrm{NP}$ & $\mathrm{NP}$ & $\mathrm{NP}$ & 107.6 & 95.7 & 95.7 & 63.4 & 63.4 & $\mathrm{NP}$ \\
\hline $699-12-1 \mathrm{~A}$ & 134.7 & 115.5 & NP & NP & $\mathrm{NP}$ & NP & $\mathrm{NP}$ & $\mathrm{NP}$ & 115.5 & 58.2 & 58.2 & 42.7 & 42.7 & 8.8 & 8.8 & -6.7 & -6.7 & -18.6 & -18.6 & 23.5 \\
\hline $699-12-18$ & 167.2 & 83.5 & NP & $\mathrm{NP}$ & $\mathrm{NP}$ & $\mathrm{NP}$ & 83.5 & 80.5 & $\mathrm{NI}$ & $\mathrm{NI}$ & $\mathrm{NI}$ & $\mathrm{NI}$ & $\mathrm{NI}$ & $\mathrm{NI}$ & $\mathrm{NI}$ & $\mathrm{NI}$ & $\mathrm{NI}$ & $\mathrm{NI}$ & -57.3 & $\mathrm{NI}$ \\
\hline $699-13-2 B$ & $\mathrm{NI}$ & $\mathrm{NI}$ & $\mathrm{NI}$ & $\mathrm{NI}$ & $\mathrm{NI}$ & $\mathrm{NI}$ & $\mathrm{NI}$ & $\mathrm{NI}$ & $\mathrm{NI}$ & $\mathrm{NI}$ & $\mathrm{NI}$ & $\mathrm{NI}$ & $\mathrm{NI}$ & $\mathrm{NI}$ & $\mathrm{NI}$ & $\mathrm{NI}$ & $\mathrm{NI}$ & $\mathrm{NI}$ & -19.5 & $\mathrm{NI}$ \\
\hline 699-13-26 & 156.2 & 93.3 & NP & NP & NP & NP & 93.3 & 87.2 & 87.2 & $\mathrm{NI}$ & $\mathrm{NI}$ & $\mathrm{NI}$ & $\mathrm{NI}$ & $\mathrm{NI}$ & $\mathrm{NI}$ & $\mathrm{NI}$ & $\mathrm{NI}$ & $\mathrm{NI}$ & -71.6 & $\mathrm{NI}$ \\
\hline 699-13-64 & 168.2 & 161.8 & NP & NP & 161.8 & 156.1 & $\mathrm{NP}$ & $\mathrm{NP}$ & $\mathrm{NP}$ & NP & NP & $\mathrm{NP}$ & NP & NP & 156.1 & 136.6 & 136.6 & 119.2 & 119.2 & NP \\
\hline 699-14-E6P & $\mathrm{NI}$ & $\mathrm{NI}$ & $\mathrm{NI}$ & $\mathrm{NI}$ & $\mathrm{NI}$ & $\mathrm{NI}$ & $\mathrm{NI}$ & $\mathrm{NI}$ & $\mathrm{NI}$ & $\mathrm{NI}$ & $\mathrm{NI}$ & $\mathrm{NI}$ & $\mathrm{NI}$ & $\mathrm{NI}$ & $\mathrm{NI}$ & $\mathrm{NI}$ & $\mathrm{NI}$ & $\mathrm{NI}$ & 17.1 & $\mathrm{NI}$ \\
\hline 699-14-E6Q & $\mathrm{NI}$ & $\mathrm{NI}$ & $\mathrm{NI}$ & $\mathrm{NI}$ & $\mathrm{NI}$ & $\mathrm{NI}$ & $\mathrm{NI}$ & $\mathrm{NI}$ & $\mathrm{NI}$ & $\mathrm{NI}$ & $\mathrm{NI}$ & $\mathrm{NI}$ & $\mathrm{NI}$ & $\mathrm{NI}$ & $\mathrm{NI}$ & $\mathrm{NI}$ & $\mathrm{NI}$ & $\mathrm{NI}$ & 17.1 & $\mathrm{NI}$ \\
\hline 699-14-E3В & $\mathrm{NI}$ & $\mathrm{NI}$ & $\mathrm{NI}$ & $\mathrm{NI}$ & $\mathrm{NI}$ & $\mathrm{NI}$ & $\mathrm{NI}$ & $\mathrm{NI}$ & $\mathrm{NI}$ & $\mathrm{NI}$ & $\mathrm{NI}$ & $\mathrm{NI}$ & $\mathrm{NI}$ & $\mathrm{NI}$ & $\mathrm{NI}$ & $\mathrm{NI}$ & $\mathrm{NI}$ & $\mathrm{NI}$ & 2.1 & $\mathrm{NI}$ \\
\hline $699-14-38$ & 157.0 & 102.1 & NP & $\mathrm{NP}$ & $\mathrm{NP}$ & NP & 102.1 & 87.2 & 87.2 & 55.8 & $\mathrm{NC}$ & $\mathrm{NC}$ & $\mathrm{NC}$ & $\mathrm{NC}$ & 55.8 & 40.5 & 40.5 & 28.0 & 28.0 & $\mathrm{NC}$ \\
\hline 699-15-E13 & 125.6 & 105.8 & NP & NP & $\mathrm{NP}$ & NP & 105.8 & 104.2 & 104.2 & 90.5 & 90.5 & 70.7 & 70.7 & 58.5 & 58.5 & 32.6 & $\mathrm{NP}$ & $\mathrm{NP}$ & 32.6 & 9.4 \\
\hline $699-15-15 A$ & 166.7 & 99.7 & $\mathrm{NI}$ & $\mathrm{NI}$ & $\mathrm{NI}$ & $\mathrm{NI}$ & $\mathrm{NI}$ & $\mathrm{NI}$ & $\mathrm{NI}$ & $\mathrm{NI}$ & $\mathrm{NI}$ & $\mathrm{NI}$ & $\mathrm{NI}$ & $\mathrm{NI}$ & $\mathrm{NI}$ & $\mathrm{NI}$ & $\mathrm{NI}$ & $\mathrm{NI}$ & -49.7 & $\mathrm{NI}$ \\
\hline 699-15-15B & 167.0 & ND & ND & ND & ND & ND & ND & ND & ND & ND & ND & ND & ND & ND & ND & ND & ND & ND & ND & ND \\
\hline $699-15-15 C$ & $\mathrm{NI}$ & $\mathrm{NI}$ & $\mathrm{NI}$ & $\mathrm{NI}$ & $\mathrm{NI}$ & $\mathrm{NI}$ & $\mathrm{NI}$ & $\mathrm{NI}$ & $\mathrm{NI}$ & $\mathrm{NI}$ & $\mathrm{NI}$ & $\mathrm{NI}$ & $\mathrm{NI}$ & $\mathrm{NI}$ & $\mathrm{NI}$ & $\mathrm{NI}$ & $\mathrm{NI}$ & $\mathrm{NI}$ & -41.1 & $\mathrm{NI}$ \\
\hline
\end{tabular}


Table A.1. Interpreted Top and Bottom Elevation of Major Hydrogeologic Units (continued)

$\mathrm{NP}=$ unit not present

$\mathrm{NC}=$ not certain whether unit is present or not

$\mathrm{ND}=$ well not deep enough to determine

$\mathrm{NI}=$ not interpreted or unknown

Elevations in $\mathrm{m}$ above mean sea level based on the National Geodetic Vertical Datum of 1929 (NGVD29 datum)

\begin{tabular}{|c|c|c|c|c|c|c|c|c|c|c|c|c|c|c|c|c|c|c|c|c|}
\hline Well number & $\begin{array}{l}\text { Casing } \\
\text { Elev. }\end{array}$ & Bot 1 & Top 2 & Bot 2 & Top 3 & Bot 3 & Top 4 & Bot 4 & Top 5 & Bot 5 & Top 6 & Bot 6 & Top 7 & Bot 7 & Top 8 & Bot 8 & Top 9 & Bot 9 & $\begin{array}{l}\text { Top of } \\
\text { Basalt }\end{array}$ & $\begin{array}{l}\text { Layer } \\
\% \text { mu }\end{array}$ \\
\hline $699-15-15 F$ & $\mathrm{NI}$ & $\mathrm{NI}$ & $\mathrm{NI}$ & $\mathrm{NI}$ & $\mathrm{NI}$ & $\mathrm{NI}$ & $\mathrm{NI}$ & $\mathrm{NI}$ & $\mathrm{NI}$ & $\mathrm{NI}$ & $\mathrm{NI}$ & $\mathrm{NI}$ & $\mathrm{NI}$ & $\mathrm{NI}$ & $\mathrm{NI}$ & $\mathrm{NI}$ & $\mathrm{NI}$ & $\mathrm{NI}$ & -53.0 & $\mathrm{NI}$ \\
\hline $699-15-15 G$ & $\mathrm{NI}$ & $\mathrm{NI}$ & $\mathrm{NI}$ & $\mathrm{NI}$ & $\mathrm{NI}$ & $\mathrm{NI}$ & $\mathrm{NI}$ & $\mathrm{NI}$ & $\mathrm{NI}$ & $\mathrm{NI}$ & $\mathrm{NI}$ & $\mathrm{NI}$ & $\mathrm{NI}$ & $\mathrm{NI}$ & $\mathrm{NI}$ & $\mathrm{NI}$ & $\mathrm{NI}$ & $\mathrm{NI}$ & -47.9 & $\mathrm{NI}$ \\
\hline 699-15-26 & 159.7 & 90.2 & NP & NP & NP & NP & 90.2 & 73.5 & 73.5 & ND & ND & ND & ND & ND & ND & ND & ND & ND & ND & ND \\
\hline 699-16-E4A & 139.9 & 112.8 & NP & NP & NP & NP & NC & NC & 112.8 & 74.7 & NP & NP & NP & NP & 74.7 & 53.3 & 53.3 & 47.2 & 47.2 & NP \\
\hline 699-16-23 & 169.0 & 95.7 & NP & NP & NP & NP & 95.7 & 78.9 & 78.9 & 31.7 & 31.7 & 11.9 & 11.9 & -4.6 & -4.6 & -33.5 & -33.5 & -51.2 & -51.2 & 18.9 \\
\hline 699-16-30A & 164.6 & 73.5 & NP & NP & NP & NP & 73.5 & 55.2 & 55.2 & 3.0 & 3.0 & -27.4 & -27.4 & -39.6 & -39.6 & -65.5 & -65.5 & -93.3 & -93.3 & 27.4 \\
\hline $699-17-5$ & 132.0 & 111.3 & NP & NP & NP & NP & 111.3 & 102.4 & 102.4 & ND & ND & ND & ND & ND & ND & ND & ND & ND & ND & ND \\
\hline 699-17-15 & 163.1 & 93.3 & NP & NP & NP & NP & 93.3 & 87.2 & 87.2 & 36.9 & 36.9 & 33.8 & 33.8 & -19.5 & -19.5 & -25.6 & -25.6 & -41.8 & -41.8 & 30.5 \\
\hline 699-17-26F & 158.3 & 95.7 & NP & NP & NP & NP & 95.7 & 77.4 & 77.4 & 27.1 & 27.1 & ND & ND & ND & ND & ND & ND & ND & ND & ND \\
\hline $699-17-26 G$ & 158.3 & 80.8 & NP & NP & NP & NP & 80.8 & 74.7 & 74.7 & 28.0 & 28.0 & 10.4 & 10.4 & -26.8 & -26.8 & -36.0 & -36.0 & -58.2 & -58.2 & 30.5 \\
\hline $699-17-47$ & 176.1 & 124.4 & NP & NP & NP & NP & NP & NP & 124.4 & 109.1 & 109.1 & 95.4 & 95.4 & 82.0 & 82.0 & 79.6 & 79.6 & 72.5 & 72.5 & 30.5 \\
\hline $699-17-70$ & 171.6 & 160.0 & NP & NP & 160.0 & 156.4 & NP & NP & 156.4 & 135.0 & 135.0 & 128.3 & 128.3 & 106.1 & 106.1 & 103.0 & 103.0 & 89.3 & 89.3 & 30.5 \\
\hline $699-17-93$ & $\mathrm{NI}$ & $\mathrm{NI}$ & $\mathrm{NI}$ & $\mathrm{NI}$ & $\mathrm{NI}$ & $\mathrm{NI}$ & $\mathrm{NI}$ & $\mathrm{NI}$ & $\mathrm{NI}$ & $\mathrm{NI}$ & $\mathrm{NI}$ & $\mathrm{NI}$ & $\mathrm{NI}$ & $\mathrm{NI}$ & $\mathrm{NI}$ & $\mathrm{NI}$ & $\mathrm{NI}$ & $\mathrm{NI}$ & 224.3 & $\mathrm{NI}$ \\
\hline $699-18-21$ & 163.8 & 92.0 & NP & NP & NP & NP & 92.0 & 87.5 & 87.5 & 35.7 & 35.7 & 18.9 & 18.9 & -7.0 & -7.0 & -31.4 & -31.4 & -49.7 & -49.7 & 25.0 \\
\hline 699-18-25A & $\mathrm{NI}$ & $\mathrm{NI}$ & $\mathrm{NI}$ & $\mathrm{NI}$ & $\mathrm{NI}$ & $\mathrm{NI}$ & $\mathrm{NI}$ & $\mathrm{NI}$ & $\mathrm{NI}$ & $\mathrm{NI}$ & $\mathrm{NI}$ & $\mathrm{NI}$ & $\mathrm{NI}$ & $\mathrm{NI}$ & $\mathrm{NI}$ & $\mathrm{NI}$ & $\mathrm{NI}$ & $\mathrm{NI}$ & -61.0 & $\mathrm{NI}$ \\
\hline $699-18-25 C$ & $\mathrm{NI}$ & $\mathrm{NI}$ & $\mathrm{NI}$ & $\mathrm{NI}$ & $\mathrm{NI}$ & $\mathrm{NI}$ & $\mathrm{NI}$ & $\mathrm{NI}$ & $\mathrm{NI}$ & $\mathrm{NI}$ & $\mathrm{NI}$ & $\mathrm{NI}$ & $\mathrm{NI}$ & $\mathrm{NI}$ & $\mathrm{NI}$ & $\mathrm{NI}$ & $\mathrm{NI}$ & $\mathrm{NI}$ & -57.6 & $\mathrm{NI}$ \\
\hline 699-18-25E & 158.9 & 95.1 & NP & NP & NP & NP & 95.1 & 93.6 & $\mathrm{NI}$ & $\mathrm{NI}$ & $\mathrm{NI}$ & $\mathrm{NI}$ & $\mathrm{NI}$ & $\mathrm{NI}$ & $\mathrm{NI}$ & $\mathrm{NI}$ & $\mathrm{NI}$ & $\mathrm{NI}$ & -63.4 & $\mathrm{NI}$ \\
\hline 699-19-23 & 163.3 & 92.0 & NP & NP & NP & NP & 92.0 & 84.1 & 84.1 & 35.4 & 35.4 & 15.5 & 15.5 & -5.8 & -5.8 & -28.7 & -28.7 & -43.0 & -43.0 & 23.5 \\
\hline $699-19-27$ & 160.4 & 81.4 & NP & NP & NP & NP & 81.4 & 78.3 & 78.3 & 29.6 & 29.6 & 2.1 & 2.1 & -8.5 & -8.5 & -40.8 & -40.8 & -56.1 & -56.1 & 30.5 \\
\hline 699-19-34A & 163.3 & 95.1 & NP & NP & NP & NP & 95.1 & 89.0 & 89.0 & 49.4 & 49.4 & 18.9 & 18.9 & -21.9 & -21.9 & -29.9 & -29.9 & -52.4 & -52.4 & 10.7 \\
\hline 699-19-34B & $\mathrm{NI}$ & $\mathrm{NI}$ & $\mathrm{NI}$ & $\mathrm{NI}$ & $\mathrm{NI}$ & $\mathrm{NI}$ & $\mathrm{NI}$ & $\mathrm{NI}$ & $\mathrm{NI}$ & $\mathrm{NI}$ & $\mathrm{NI}$ & $\mathrm{NI}$ & $\mathrm{NI}$ & $\mathrm{NI}$ & & $\mathrm{NI}$ & $\mathrm{NI}$ & $\mathrm{NI}$ & -52.4 & $\mathrm{NI}$ \\
\hline 699-19-58 & 174.7 & 144.2 & NP & NP & NP & NP & NP & NP & 144.2 & 116.7 & NP & NP & NP & NP & 116.7 & 112.2 & NP & NP & 112.2 & NP \\
\hline 699-19-88 & 196.3 & 178.0 & NP & NP & 178.0 & 173.4 & NP & NP & 173.4 & 94.2 & NP & NP & NP & NP & 94.2 & 89.3 & NP & NP & 89.3 & NP \\
\hline 699-20-E12 & 133.3 & 108.8 & NP & NP & NP & NP & 108.8 & 102.7 & 102.7 & 55.5 & NP & NP & NP & NP & 55.5 & 32.6 & NP & NP & 32.6 & NP \\
\hline 699-20-E5P & $\mathrm{NI}$ & $\mathrm{NI}$ & $\mathrm{NI}$ & $\mathrm{NI}$ & $\mathrm{NI}$ & $\mathrm{NI}$ & $\mathrm{NI}$ & $\mathrm{NI}$ & $\mathrm{NI}$ & $\mathrm{NI}$ & $\mathrm{NI}$ & $\mathrm{NI}$ & $\mathrm{NI}$ & $\mathrm{NI}$ & $\mathrm{NI}$ & $\mathrm{NI}$ & $\mathrm{NI}$ & $\mathrm{NI}$ & 44.8 & $\mathrm{NI}$ \\
\hline 699-20-E5Q & $\mathrm{NI}$ & $\mathrm{NI}$ & $\mathrm{NI}$ & $\mathrm{NI}$ & $\mathrm{NI}$ & $\mathrm{NI}$ & $\mathrm{NI}$ & $\mathrm{NI}$ & $\mathrm{NI}$ & $\mathrm{NI}$ & $\mathrm{NI}$ & $\mathrm{NI}$ & $\mathrm{NI}$ & $\mathrm{NI}$ & $\mathrm{NI}$ & $\mathrm{NI}$ & $\mathrm{NI}$ & $\mathrm{NI}$ & 44.8 & $\mathrm{NI}$ \\
\hline 699-20-E5R & $\mathrm{NI}$ & $\mathrm{NI}$ & $\mathrm{NI}$ & $\mathrm{NI}$ & $\mathrm{NI}$ & $\mathrm{NI}$ & $\mathrm{NI}$ & $\mathrm{NI}$ & $\mathrm{NI}$ & $\mathrm{NI}$ & $\mathrm{NI}$ & $\mathrm{NI}$ & $\mathrm{NI}$ & $\mathrm{NI}$ & $\mathrm{NI}$ & $\mathrm{NI}$ & $\mathrm{NI}$ & $\mathrm{NI}$ & 44.8 & $\mathrm{NI}$ \\
\hline
\end{tabular}


Table A.1. Interpreted Top and Bottom Elevation of Major Hydrogeologic Units (continued)

$\mathrm{NP}=$ unit not present

$\mathrm{NC}=$ not certain whether unit is present or not

$\mathrm{ND}=$ well not deep enough to determine

$\mathrm{NI}=$ not interpreted or unknown

Elevations in $\mathrm{m}$ above mean sea level based on the National Geodetic Vertical Datum of 1929 (NGVD29 datum)

\begin{tabular}{|c|c|c|c|c|c|c|c|c|c|c|c|c|c|c|c|c|c|c|c|c|}
\hline Well number & $\begin{array}{l}\text { Casing } \\
\text { Elev. }\end{array}$ & Bot 1 & Top 2 & Bot 2 & Top 3 & Bot 3 & Top 4 & Bot 4 & Top 5 & Bot 5 & Top 6 & Bot 6 & Top 7 & Bot 7 & Top 8 & Bot 8 & Top 9 & Bot 9 & $\begin{array}{l}\text { Top of } \\
\text { Basalt }\end{array}$ & $\begin{array}{l}\text { Layer } 6 \\
\% \text { mud }\end{array}$ \\
\hline 699-20-E2 & 142.0 & 101.8 & NP & NP & NP & NP & 101.8 & 95.1 & 95.1 & 49.7 & 49.7 & 46.6 & 46.6 & 27.7 & 27.7 & 3.7 & 3.7 & -5.2 & -5.2 & 30.5 \\
\hline $699-20-18 A$ & 162.2 & 96.6 & NP & NP & NP & NP & 96.6 & 93.6 & 93.6 & 49.4 & 49.4 & 40.2 & 40.2 & 9.8 & 9.8 & -9.8 & -9.8 & -22.9 & -22.9 & 30.5 \\
\hline $699-20-20$ & 153.9 & ND & ND & ND & ND & ND & ND & ND & ND & ND & ND & ND & ND & ND & ND & ND & ND & ND & ND & ND \\
\hline $699-20-25$ & 158.7 & 87.2 & NP & NP & NP & NP & 87.2 & 78.0 & $\mathrm{NI}$ & $\mathrm{NI}$ & $\mathrm{NI}$ & $\mathrm{NI}$ & $\mathrm{NI}$ & $\mathrm{NI}$ & $\mathrm{NI}$ & $\mathrm{NI}$ & $\mathrm{NI}$ & $\mathrm{NI}$ & -44.2 & $\mathrm{NI}$ \\
\hline $699-20-39$ & $\mathrm{NI}$ & $\mathrm{NI}$ & $\mathrm{NI}$ & $\mathrm{NI}$ & $\mathrm{NI}$ & $\mathrm{NI}$ & $\mathrm{NI}$ & $\mathrm{NI}$ & $\mathrm{NI}$ & $\mathrm{NI}$ & $\mathrm{NI}$ & $\mathrm{NI}$ & $\mathrm{NI}$ & $\mathrm{NI}$ & $\mathrm{NI}$ & $\mathrm{NI}$ & $\mathrm{NI}$ & $\mathrm{NI}$ & -21.3 & $\mathrm{NI}$ \\
\hline $699-20-82$ & $\mathrm{NI}$ & $\mathrm{NI}$ & $\mathrm{NI}$ & $\mathrm{NI}$ & $\mathrm{NI}$ & $\mathrm{NI}$ & $\mathrm{NI}$ & $\mathrm{NI}$ & $\mathrm{NI}$ & $\mathrm{NI}$ & $\mathrm{NI}$ & $\mathrm{NI}$ & $\mathrm{NI}$ & $\mathrm{NI}$ & $\mathrm{NI}$ & $\mathrm{NI}$ & $\mathrm{NI}$ & $\mathrm{NI}$ & 82.0 & $\mathrm{NI}$ \\
\hline $699-21-17$ & 160.6 & 98.5 & NP & NP & NP & NP & 98.5 & 92.4 & 92.4 & 41.8 & 41.8 & 32.6 & 32.6 & 10.1 & 10.1 & -2.1 & -2.1 & -28.0 & -28.0 & 15.2 \\
\hline $699-21-30 \mathrm{~A}$ & $\mathrm{NI}$ & $\mathrm{NI}$ & $\mathrm{NI}$ & $\mathrm{NI}$ & $\mathrm{NI}$ & $\mathrm{NI}$ & $\mathrm{NI}$ & $\mathrm{NI}$ & $\mathrm{NI}$ & $\mathrm{NI}$ & $\mathrm{NI}$ & $\mathrm{NI}$ & $\mathrm{NI}$ & $\mathrm{NI}$ & $\mathrm{NI}$ & $\mathrm{NI}$ & $\mathrm{NI}$ & $\mathrm{NI}$ & -51.2 & $\mathrm{NI}$ \\
\hline 699-21-30B & 164.2 & 95.7 & NP & NP & NP & NP & 95.7 & 91.1 & 91.1 & 25.6 & 25.6 & 10.4 & 10.4 & -11.0 & -11.0 & -26.2 & -26.2 & -53.9 & -53.9 & 30.5 \\
\hline $699-22-23$ & 156.4 & 90.8 & NP & NP & NP & NP & 90.8 & 84.7 & 84.7 & 40.5 & 40.5 & 14.6 & 14.6 & 0.9 & 0.9 & -29.6 & -29.6 & -40.2 & -40.2 & 30.5 \\
\hline $699-22-70$ & $\mathrm{NI}$ & $\mathrm{NI}$ & $\mathrm{NI}$ & $\mathrm{NI}$ & $\mathrm{NI}$ & $\mathrm{NI}$ & $\mathrm{NI}$ & $\mathrm{NI}$ & $\mathrm{NI}$ & $\mathrm{NI}$ & $\mathrm{NI}$ & $\mathrm{NI}$ & $\mathrm{NI}$ & $\mathrm{NI}$ & $\mathrm{NI}$ & $\mathrm{NI}$ & $\mathrm{NI}$ & $\mathrm{NI}$ & 150.3 & $\mathrm{NI}$ \\
\hline $699-23-33$ & 168.3 & 95.1 & NP & NP & NP & NP & 95.1 & 92.0 & $\mathrm{NI}$ & $\mathrm{NI}$ & $\mathrm{NI}$ & $\mathrm{NI}$ & $\mathrm{NI}$ & $\mathrm{NI}$ & $\mathrm{NI}$ & $\mathrm{NI}$ & $\mathrm{NI}$ & $\mathrm{NI}$ & -21.0 & $\mathrm{NI}$ \\
\hline 699-24-1P & 144.7 & 102.1 & NP & NP & NP & NP & 102.1 & 93.0 & 93.0 & 54.3 & 54.3 & 41.5 & 41.5 & 27.4 & 27.4 & 4.6 & NP & NP & 4.6 & 30.5 \\
\hline $699-24-33$ & 159.7 & ND & ND & ND & ND & ND & ND & ND & ND & ND & ND & ND & ND & ND & ND & ND & ND & ND & ND & ND \\
\hline $699-24-34 \mathrm{~A}$ & 162.8 & ND & ND & ND & ND & ND & ND & ND & ND & ND & ND & ND & ND & ND & ND & ND & ND & ND & ND & ND \\
\hline $699-24-35$ & 164.3 & ND & ND & ND & ND & ND & ND & ND & ND & ND & ND & ND & ND & ND & ND & ND & ND & ND & ND & ND \\
\hline $699-24-46$ & 180.1 & 119.2 & NP & NP & NP & NP & NP & NP & 119.2 & 76.5 & 76.5 & 71.9 & 71.9 & 59.7 & 59.7 & -4.3 & -4.3 & -24.7 & -24.7 & 30.5 \\
\hline $699-25-20$ & 159.1 & 102.7 & NP & NP & NP & NP & $\mathrm{NI}$ & $\mathrm{NI}$ & $\mathrm{NI}$ & $\mathrm{NI}$ & $\mathrm{NI}$ & $\mathrm{NI}$ & $\mathrm{NI}$ & $\mathrm{NI}$ & $\mathrm{NI}$ & $\mathrm{NI}$ & $\mathrm{NI}$ & $\mathrm{NI}$ & -58.2 & $\mathrm{NI}$ \\
\hline $699-25-26$ & 157.1 & 94.8 & NP & NP & NP & NP & 94.8 & 93.3 & $\mathrm{NI}$ & $\mathrm{NI}$ & $\mathrm{NI}$ & $\mathrm{NI}$ & $\mathrm{NI}$ & $\mathrm{NI}$ & $\mathrm{NI}$ & $\mathrm{NI}$ & $\mathrm{NI}$ & $\mathrm{NI}$ & -27.7 & $\mathrm{NI}$ \\
\hline $699-25-31$ & 155.9 & 120.7 & NP & NP & NP & NP & $\mathrm{NI}$ & $\mathrm{NI}$ & $\mathrm{NI}$ & $\mathrm{NI}$ & $\mathrm{NI}$ & $\mathrm{NI}$ & $\mathrm{NI}$ & $\mathrm{NI}$ & $\mathrm{NI}$ & $\mathrm{NI}$ & $\mathrm{NI}$ & $\mathrm{NI}$ & -14.9 & $\mathrm{NI}$ \\
\hline $699-25-33 A$ & 161.1 & 104.9 & NP & NP & NP & NP & 104.9 & 97.2 & 97.2 & ND & ND & ND & ND & ND & ND & ND & ND & ND & ND & ND \\
\hline $699-25-70$ & 191.7 & 152.1 & 152.1 & 149.0 & 149.0 & 135.3 & NP & NP & 135.3 & ND & ND & ND & ND & ND & ND & ND & ND & ND & ND & ND \\
\hline $699-25-80$ & 188.1 & 179.5 & NP & NP & 179.5 & 175.6 & NP & NP & 175.6 & 154.2 & NP & NP & NP & NP & NP & NP & NP & NP & 154.2 & NP \\
\hline $699-26-15 A$ & 134.9 & 103.0 & NP & NP & NP & NP & 103.0 & 90.8 & 90.8 & 29.3 & 29.3 & ND & ND & ND & ND & ND & ND & ND & ND & ND \\
\hline $699-26-15 C$ & 135.4 & 103.3 & NP & NP & $\mathrm{NP}$ & NP & 103.3 & 78.3 & 78.3 & 29.0 & 29.0 & 11.3 & 11.3 & -7.9 & -7.9 & -42.7 & -42.7 & -49.1 & -49.1 & 30.5 \\
\hline $699-26-29 A$ & 157.6 & 96.6 & NP & NP & NP & NP & 96.6 & 83.2 & $\mathrm{NI}$ & $\mathrm{NI}$ & $\mathrm{NI}$ & $\mathrm{NI}$ & $\mathrm{NI}$ & $\mathrm{NI}$ & $\mathrm{NI}$ & $\mathrm{NI}$ & $\mathrm{NI}$ & $\mathrm{NI}$ & -58.8 & $\mathrm{NI}$ \\
\hline $699-26-35 C$ & 162.2 & 100.3 & NP & NP & NP & NP & 100.3 & ND & ND & ND & ND & ND & ND & ND & ND & ND & ND & ND & ND & ND \\
\hline
\end{tabular}


Table A.1. Interpreted Top and Bottom Elevation of Major Hydrogeologic Units (continued)

$\mathrm{NP}=$ unit not present

$\mathrm{NC}=$ not certain whether unit is present or not

$\mathrm{ND}=$ well not deep enough to determine

$\mathrm{NI}=$ not interpreted or unknown

Elevations in $\mathrm{m}$ above mean sea level based on the National Geodetic Vertical Datum of 1929 (NGVD29 datum)

\begin{tabular}{|c|c|c|c|c|c|c|c|c|c|c|c|c|c|c|c|c|c|c|c|c|}
\hline Well number & $\begin{array}{l}\text { Casing } \\
\text { Elev. }\end{array}$ & Bot 1 & Top 2 & Bot 2 & Top 3 & Bot 3 & Top 4 & Bot 4 & Top 5 & Bot 5 & Top 6 & Bot 6 & Top 7 & Bot 7 & Top 8 & Bot 8 & Top 9 & Bot 9 & $\begin{array}{l}\text { Top of } \\
\text { Basalt }\end{array}$ & $\begin{array}{l}\text { Layer } 6 \\
\% \text { mud }\end{array}$ \\
\hline 699-26-51 & 205.7 & 132.6 & NP & NP & NP & NP & 132.6 & 126.5 & 126.5 & ND & ND & ND & ND & ND & ND & ND & ND & ND & ND & ND \\
\hline 699-26-83A & 194.2 & 173.7 & NP & NP & NC & NC & 173.7 & 170.7 & 170.7 & 131.7 & NP & NP & NP & NP & 131.7 & 112.8 & 112.8 & 91.1 & 91.1 & NP \\
\hline 699-26-89 & 199.0 & 189.9 & NP & NP & 189.9 & 165.8 & 165.8 & 157.3 & 157.3 & 107.0 & NP & NP & NP & NP & 107.0 & 84.1 & 84.1 & 51.8 & 51.8 & NP \\
\hline 699-27-8 & $\mathrm{NI}$ & $\mathrm{NI}$ & $\mathrm{NI}$ & $\mathrm{NI}$ & $\mathrm{NI}$ & $\mathrm{NI}$ & $\mathrm{NI}$ & $\mathrm{NI}$ & $\mathrm{NI}$ & $\mathrm{NI}$ & $\mathrm{NI}$ & $\mathrm{NI}$ & $\mathrm{NI}$ & $\mathrm{NI}$ & $\mathrm{NI}$ & $\mathrm{NI}$ & $\mathrm{NI}$ & $\mathrm{NI}$ & -30.8 & $\mathrm{NI}$ \\
\hline $699-28-23$ & 161.1 & 93.9 & NP & NP & NP & NP & 93.9 & 81.7 & 81.7 & 18.0 & 18.0 & 5.8 & 5.8 & -11.0 & -11.0 & -32.3 & -32.3 & -48.8 & -48.8 & 26.8 \\
\hline 699-28-30 & 162.1 & 81.4 & NP & NP & NP & NP & 81.4 & 79.9 & $\mathrm{NI}$ & $\mathrm{NI}$ & $\mathrm{NI}$ & $\mathrm{NI}$ & $\mathrm{NI}$ & $\mathrm{NI}$ & -32.6 & -38.7 & -38.7 & -53.6 & -53.6 & $\mathrm{NI}$ \\
\hline 699-28-52B & $\mathrm{NI}$ & $\mathrm{NI}$ & $\mathrm{NI}$ & $\mathrm{NI}$ & $\mathrm{NI}$ & $\mathrm{NI}$ & $\mathrm{NI}$ & $\mathrm{NI}$ & $\mathrm{NI}$ & $\mathrm{NI}$ & $\mathrm{NI}$ & $\mathrm{NI}$ & $\mathrm{NI}$ & $\mathrm{NI}$ & $\mathrm{NI}$ & $\mathrm{NI}$ & $\mathrm{NI}$ & $\mathrm{NI}$ & -15.2 & $\mathrm{NI}$ \\
\hline 699-29-4 & 148.7 & ND & ND & ND & ND & ND & ND & ND & ND & ND & ND & ND & ND & ND & ND & ND & ND & ND & ND & ND \\
\hline 699-29-70A & $\mathrm{NI}$ & $\mathrm{NI}$ & $\mathrm{NI}$ & $\mathrm{NI}$ & $\mathrm{NI}$ & $\mathrm{NI}$ & $\mathrm{NI}$ & $\mathrm{NI}$ & $\mathrm{NI}$ & $\mathrm{NI}$ & $\mathrm{NI}$ & $\mathrm{NI}$ & $\mathrm{NI}$ & $\mathrm{NI}$ & $\mathrm{NI}$ & $\mathrm{NI}$ & $\mathrm{NI}$ & $\mathrm{NI}$ & 26.5 & $\mathrm{NI}$ \\
\hline $699-29-70 \mathrm{C}$ & 192.9 & 152.4 & NP & NP & 152.4 & 147.2 & $\mathrm{NP}$ & NP & 147.2 & 80.8 & NP & NP & NP & NP & 80.8 & 64.9 & 64.9 & 27.1 & 27.1 & NP \\
\hline $699-29-78$ & 197.2 & 159.7 & NP & NP & 159.7 & 152.4 & $\mathrm{NP}$ & NP & 152.4 & 82.9 & $\mathrm{NC}$ & NC & NC & NC & 63.1 & 39.9 & 39.9 & 18.0 & 18.0 & 30.5 \\
\hline 699-29-83 & 189.9 & 169.2 & $\mathrm{NP}$ & NP & 169.2 & 155.8 & $\mathrm{NP}$ & NP & 155.8 & 75.6 & NP & NP & NP & NP & 75.6 & 52.7 & 52.7 & 20.4 & 20.4 & NP \\
\hline $699-30-25 C$ & 165.4 & 93.0 & NP & NP & NP & NP & 93.0 & 82.9 & $\mathrm{NI}$ & $\mathrm{NI}$ & $\mathrm{NI}$ & $\mathrm{NI}$ & $\mathrm{NI}$ & $\mathrm{NI}$ & $\mathrm{NI}$ & $\mathrm{NI}$ & $\mathrm{NI}$ & $\mathrm{NI}$ & -43.9 & $\mathrm{NI}$ \\
\hline 699-31-8 & 145.1 & 97.8 & NP & NP & NP & NP & 97.8 & 84.1 & $\mathrm{NI}$ & $\mathrm{NI}$ & $\mathrm{NI}$ & $\mathrm{NI}$ & $\mathrm{NI}$ & $\mathrm{NI}$ & $\mathrm{NI}$ & $\mathrm{NI}$ & $\mathrm{NI}$ & $\mathrm{NI}$ & -19.5 & $\mathrm{NI}$ \\
\hline 699-31-11 & 146.8 & 98.5 & NP & NP & NP & NP & 98.5 & 84.4 & $\mathrm{NI}$ & $\mathrm{NI}$ & $\mathrm{NI}$ & $\mathrm{NI}$ & $\mathrm{NI}$ & $\mathrm{NI}$ & $\mathrm{NI}$ & $\mathrm{NI}$ & $\mathrm{NI}$ & $\mathrm{NI}$ & -23.2 & $\mathrm{NI}$ \\
\hline $699-31-17$ & 133.8 & 92.7 & NP & NP & NP & NP & 92.7 & 82.0 & $\mathrm{NI}$ & $\mathrm{NI}$ & $\mathrm{NI}$ & $\mathrm{NI}$ & $\mathrm{NI}$ & $\mathrm{NI}$ & $\mathrm{NI}$ & $\mathrm{NI}$ & $\mathrm{NI}$ & $\mathrm{NI}$ & -35.1 & $\mathrm{NI}$ \\
\hline 699-31-31 & 161.3 & 96.0 & NP & NP & NP & NP & 96.0 & 75.9 & 75.9 & 30.2 & 30.2 & -10.1 & -10.1 & -21.3 & -21.3 & -31.7 & -31.7 & ND & ND & 18.3 \\
\hline 699-31-53B & 215.7 & 129.8 & NP & NP & NP & NP & NP & NP & 129.8 & 86.0 & 86.0 & ND & ND & ND & ND & ND & ND & ND & ND & ND \\
\hline 699-31-84A & 190.5 & $\mathrm{NI}$ & $\mathrm{NI}$ & $\mathrm{NI}$ & $\mathrm{NI}$ & $\mathrm{NI}$ & $\mathrm{NI}$ & $\mathrm{NI}$ & $\mathrm{NI}$ & $\mathrm{NI}$ & $\mathrm{NI}$ & $\mathrm{NI}$ & $\mathrm{NI}$ & $\mathrm{NI}$ & $\mathrm{NI}$ & $\mathrm{NI}$ & $\mathrm{NI}$ & $\mathrm{NI}$ & 6.4 & $\mathrm{NI}$ \\
\hline 699-31-84B & $\mathrm{NI}$ & $\mathrm{NI}$ & $\mathrm{NI}$ & $\mathrm{NI}$ & $\mathrm{NI}$ & $\mathrm{NI}$ & $\mathrm{NI}$ & $\mathrm{NI}$ & $\mathrm{NI}$ & $\mathrm{NI}$ & $\mathrm{NI}$ & $\mathrm{NI}$ & $\mathrm{NI}$ & $\mathrm{NI}$ & $\mathrm{NI}$ & $\mathrm{NI}$ & $\mathrm{NI}$ & $\mathrm{NI}$ & 3.0 & $\mathrm{NI}$ \\
\hline 699-31-84C & $\mathrm{NI}$ & $\mathrm{NI}$ & $\mathrm{NI}$ & $\mathrm{NI}$ & $\mathrm{NI}$ & $\mathrm{NI}$ & $\mathrm{NI}$ & $\mathrm{NI}$ & $\mathrm{NI}$ & $\mathrm{NI}$ & $\mathrm{NI}$ & $\mathrm{NI}$ & $\mathrm{NI}$ & $\mathrm{NI}$ & $\mathrm{NI}$ & $\mathrm{NI}$ & $\mathrm{NI}$ & $\mathrm{NI}$ & 3.0 & $\mathrm{NI}$ \\
\hline 699-32-22B & 156.8 & 88.4 & NP & NP & NP & NP & 88.4 & 82.3 & 82.3 & 29.0 & 29.0 & 18.3 & 18.3 & 3.0 & 3.0 & -25.9 & -25.9 & -35.1 & -35.1 & 30.5 \\
\hline 699-32-26 & 158.5 & 91.4 & NP & NP & NP & NP & 91.4 & 83.8 & $\mathrm{NI}$ & $\mathrm{NI}$ & $\mathrm{NI}$ & $\mathrm{NI}$ & $\mathrm{NI}$ & $\mathrm{NI}$ & $\mathrm{NI}$ & $\mathrm{NI}$ & $\mathrm{NI}$ & $\mathrm{NI}$ & -37.2 & $\mathrm{NI}$ \\
\hline 699-32-32 & 158.7 & 87.5 & NP & NP & NP & NP & NP & NP & 87.5 & 4.6 & $\mathrm{NC}$ & $\mathrm{NC}$ & $\mathrm{NC}$ & $\mathrm{NC}$ & 4.6 & -17.7 & -17.7 & -56.7 & -56.7 & $\mathrm{NC}$ \\
\hline 699-32-62 & 215.5 & 147.5 & NP & NP & NP & NP & NP & NP & 147.5 & 92.7 & NP & NP & NP & NP & 92.7 & 72.2 & 72.2 & ND & ND & NP \\
\hline $699-32-72 A$ & 203.6 & 148.4 & NP & NP & 148.4 & 144.2 & $\mathrm{NP}$ & NP & 144.2 & 77.1 & NP & NP & NP & NP & 77.1 & 66.1 & 66.1 & 29.3 & 29.3 & NP \\
\hline 699-32-77 & 199.3 & 167.3 & 167.3 & 159.7 & 159.7 & 156.7 & $\mathrm{NP}$ & NP & 156.7 & ND & ND & ND & ND & ND & ND & ND & ND & ND & ND & ND \\
\hline
\end{tabular}


Table A.1. Interpreted Top and Bottom Elevation of Major Hydrogeologic Units (continued)

$\mathrm{NP}=$ unit not present

$\mathrm{NC}=$ not certain whether unit is present or not

$\mathrm{ND}=$ well not deep enough to determine

$\mathrm{NI}=$ not interpreted or unknown

Elevations in $\mathrm{m}$ above mean sea level based on the National Geodetic Vertical Datum of 1929 (NGVD29 datum)

\begin{tabular}{|c|c|c|c|c|c|c|c|c|c|c|c|c|c|c|c|c|c|c|c|c|}
\hline Well number & $\begin{array}{l}\text { Casing } \\
\text { Elev. }\end{array}$ & Bot 1 & Top 2 & Bot 2 & Top 3 & Bot 3 & Top 4 & Bot 4 & Top 5 & Bot 5 & Top 6 & Bot 6 & Top 7 & Bot 7 & Top 8 & Bot 8 & Top 9 & Bot 9 & $\begin{array}{l}\text { Top of } \\
\text { Basalt }\end{array}$ & $\begin{array}{l}\text { Layer } 6 \\
\% \text { mud }\end{array}$ \\
\hline 699-33-6 & 153.3 & 100.0 & NP & NP & NP & NP & 100.0 & 92.4 & 92.4 & 72.2 & 72.2 & 57.3 & 57.3 & 10.1 & 10.1 & -11.3 & -11.3 & -12.5 & -12.5 & 30.5 \\
\hline 699-33-14 & 143.3 & 100.6 & NP & NP & NP & NP & 100.6 & 89.9 & $\mathrm{NI}$ & $\mathrm{NI}$ & $\mathrm{NI}$ & $\mathrm{NI}$ & $\mathrm{NI}$ & $\mathrm{NI}$ & $\mathrm{NI}$ & $\mathrm{NI}$ & $\mathrm{NI}$ & $\mathrm{NI}$ & -24.1 & $\mathrm{NI}$ \\
\hline 699-33-21A & 152.2 & 103.3 & NP & NP & NP & NP & 103.3 & 86.6 & $\mathrm{NI}$ & $\mathrm{NI}$ & $\mathrm{NI}$ & $\mathrm{NI}$ & $\mathrm{NI}$ & $\mathrm{NI}$ & $\mathrm{NI}$ & $\mathrm{NI}$ & $\mathrm{NI}$ & $\mathrm{NI}$ & -35.4 & $\mathrm{NI}$ \\
\hline 699-33-56 & 218.6 & 130.1 & NP & NP & NP & NP & NP & NP & 130.1 & 96.6 & 96.6 & ND & ND & ND & ND & ND & ND & ND & ND & ND \\
\hline $699-34-8$ & 148.1 & 99.7 & NP & NP & NP & NP & 99.7 & 90.5 & $\mathrm{NI}$ & $\mathrm{NI}$ & $\mathrm{NI}$ & $\mathrm{NI}$ & $\mathrm{NI}$ & $\mathrm{NI}$ & $\mathrm{NI}$ & $\mathrm{NI}$ & $\mathrm{NI}$ & $\mathrm{NI}$ & -9.4 & $\mathrm{NI}$ \\
\hline $699-34-20$ & 152.5 & 94.5 & NP & NP & NP & NP & 94.5 & 84.1 & $\mathrm{NI}$ & $\mathrm{NI}$ & $\mathrm{NI}$ & $\mathrm{NI}$ & $\mathrm{NI}$ & $\mathrm{NI}$ & $\mathrm{NI}$ & $\mathrm{NI}$ & $\mathrm{NI}$ & $\mathrm{NI}$ & -31.4 & $\mathrm{NI}$ \\
\hline 699-34-88 & 192.6 & 172.8 & NP & NP & 172.8 & 157.6 & 157.6 & 156.1 & 156.1 & 63.1 & NP & NP & NP & NP & 63.1 & 32.6 & 32.6 & -7.0 & -7.0 & NP \\
\hline 699-34-89B & $\mathrm{NI}$ & $\mathrm{NI}$ & $\mathrm{NI}$ & $\mathrm{NI}$ & $\mathrm{NI}$ & $\mathrm{NI}$ & $\mathrm{NI}$ & $\mathrm{NI}$ & $\mathrm{NI}$ & $\mathrm{NI}$ & $\mathrm{NI}$ & $\mathrm{NI}$ & $\mathrm{NI}$ & $\mathrm{NI}$ & $\mathrm{NI}$ & $\mathrm{NI}$ & $\mathrm{NI}$ & $\mathrm{NI}$ & -2.4 & $\mathrm{NI}$ \\
\hline 699-35-3B & 146.2 & 100.6 & NP & NP & NP & NP & $\mathrm{NI}$ & $\mathrm{NI}$ & $\mathrm{NI}$ & $\mathrm{NI}$ & $\mathrm{NI}$ & $\mathrm{NI}$ & $\mathrm{NI}$ & $\mathrm{NI}$ & $\mathrm{NI}$ & $\mathrm{NI}$ & $\mathrm{NI}$ & $\mathrm{NI}$ & 30.5 & $\mathrm{NI}$ \\
\hline 699-35-6 & 153.2 & 101.5 & NP & NP & NP & NP & 101.5 & 98.5 & $\mathrm{NI}$ & $\mathrm{NI}$ & $\mathrm{NI}$ & $\mathrm{NI}$ & $\mathrm{NI}$ & $\mathrm{NI}$ & $\mathrm{NI}$ & $\mathrm{NI}$ & $\mathrm{NI}$ & $\mathrm{NI}$ & 1.5 & $\mathrm{NI}$ \\
\hline 699-35-9 & 152.3 & 117.0 & NP & NP & NP & NP & NC & NC & 117.0 & 98.8 & 98.8 & ND & ND & ND & ND & ND & ND & ND & ND & ND \\
\hline 699-35-16 & $\mathrm{NI}$ & $\mathrm{NI}$ & $\mathrm{NI}$ & $\mathrm{NI}$ & $\mathrm{NI}$ & $\mathrm{NI}$ & $\mathrm{NI}$ & $\mathrm{NI}$ & $\mathrm{NI}$ & $\mathrm{NI}$ & $\mathrm{NI}$ & $\mathrm{NI}$ & $\mathrm{NI}$ & $\mathrm{NI}$ & $\mathrm{NI}$ & $\mathrm{NI}$ & $\mathrm{NI}$ & $\mathrm{NI}$ & -20.1 & $\mathrm{NI}$ \\
\hline $699-35-19 A$ & 144.4 & 100.3 & NP & NP & NP & NP & 100.3 & 86.6 & $\mathrm{NI}$ & $\mathrm{NI}$ & $\mathrm{NI}$ & $\mathrm{NI}$ & $\mathrm{NI}$ & $\mathrm{NI}$ & $\mathrm{NI}$ & $\mathrm{NI}$ & $\mathrm{NI}$ & $\mathrm{NI}$ & -23.5 & $\mathrm{NI}$ \\
\hline 699-35-27 & $\mathrm{NI}$ & $\mathrm{NI}$ & $\mathrm{NI}$ & $\mathrm{NI}$ & $\mathrm{NI}$ & $\mathrm{NI}$ & $\mathrm{NI}$ & $\mathrm{NI}$ & $\mathrm{NI}$ & $\mathrm{NI}$ & $\mathrm{NI}$ & $\mathrm{NI}$ & $\mathrm{NI}$ & $\mathrm{NI}$ & $\mathrm{NI}$ & $\mathrm{NI}$ & $\mathrm{NI}$ & $\mathrm{NI}$ & -26.5 & $\mathrm{NI}$ \\
\hline $699-35-28$ & 162.3 & 92.4 & NP & NP & NP & NP & 92.4 & 86.3 & $\mathrm{NI}$ & $\mathrm{NI}$ & $\mathrm{NI}$ & $\mathrm{NI}$ & $\mathrm{NI}$ & $\mathrm{NI}$ & $\mathrm{NI}$ & $\mathrm{NI}$ & $\mathrm{NI}$ & $\mathrm{NI}$ & -25.9 & $\mathrm{NI}$ \\
\hline 699-35-78B & 201.2 & 168.2 & 168.2 & 158.5 & 158.5 & 155.4 & NP & NP & 155.4 & 73.5 & NP & NP & NP & NP & 73.5 & 51.5 & 51.5 & 24.1 & 24.1 & NP \\
\hline 699-36-E3 & 141.8 & 100.9 & $\mathrm{NP}$ & $\mathrm{NP}$ & NP & $\mathrm{NP}$ & 100.9 & 93.3 & 93.3 & 61.3 & $\mathrm{NC}$ & NC & $\mathrm{NC}$ & NC & 61.3 & 44.2 & 44.2 & 42.7 & 42.7 & NC \\
\hline 699-36-1 & 148.0 & $\mathrm{NI}$ & $\mathrm{NI}$ & $\mathrm{NI}$ & $\mathrm{NI}$ & $\mathrm{NI}$ & $\mathrm{NI}$ & $\mathrm{NI}$ & $\mathrm{NI}$ & $\mathrm{NI}$ & $\mathrm{NI}$ & $\mathrm{NI}$ & $\mathrm{NI}$ & $\mathrm{NI}$ & $\mathrm{NI}$ & $\mathrm{NI}$ & $\mathrm{NI}$ & $\mathrm{NI}$ & 66.4 & $\mathrm{NI}$ \\
\hline 699-36-2 & 146.8 & $\mathrm{NI}$ & $\mathrm{NI}$ & $\mathrm{NI}$ & $\mathrm{NI}$ & $\mathrm{NI}$ & $\mathrm{NI}$ & $\mathrm{NI}$ & $\mathrm{NI}$ & $\mathrm{NI}$ & $\mathrm{NI}$ & $\mathrm{NI}$ & $\mathrm{NI}$ & $\mathrm{NI}$ & $\mathrm{NI}$ & $\mathrm{NI}$ & $\mathrm{NI}$ & $\mathrm{NI}$ & 55.8 & $\mathrm{NI}$ \\
\hline 699-36-10 & 160.3 & 100.9 & NP & NP & NP & NP & 100.9 & 93.3 & $\mathrm{NI}$ & $\mathrm{NI}$ & $\mathrm{NI}$ & $\mathrm{NI}$ & $\mathrm{NI}$ & $\mathrm{NI}$ & $\mathrm{NI}$ & $\mathrm{NI}$ & $\mathrm{NI}$ & $\mathrm{NI}$ & -6.7 & $\mathrm{NI}$ \\
\hline 699-36-17 & 134.1 & 99.1 & NP & NP & NP & NP & 99.1 & 89.9 & 89.9 & 39.6 & 39.6 & 24.4 & 24.4 & 7.6 & 7.6 & -12.2 & NC & NC & -12.2 & 30.5 \\
\hline 699-36-27 & 162.1 & 98.1 & NP & NP & NP & NP & 98.1 & 90.5 & 90.5 & 35.7 & 35.7 & 23.5 & 23.5 & -4.0 & -4.0 & -13.1 & -13.1 & -19.8 & -19.8 & 30.5 \\
\hline 699-36-46P & $\mathrm{NI}$ & $\mathrm{NI}$ & $\mathrm{NI}$ & $\mathrm{NI}$ & $\mathrm{NI}$ & $\mathrm{NI}$ & $\mathrm{NI}$ & $\mathrm{NI}$ & $\mathrm{NI}$ & $\mathrm{NI}$ & $\mathrm{NI}$ & $\mathrm{NI}$ & $\mathrm{NI}$ & $\mathrm{NI}$ & $\mathrm{NI}$ & $\mathrm{NI}$ & $\mathrm{NI}$ & $\mathrm{NI}$ & 56.7 & $\mathrm{NI}$ \\
\hline $699-36-58 A$ & 224.3 & 129.8 & NP & NP & NP & NP & NP & NP & 129.8 & ND & ND & ND & ND & ND & ND & ND & ND & ND & ND & ND \\
\hline 699-36-61A & 228.0 & 129.2 & NP & NP & NP & NP & NP & NP & 129.2 & ND & ND & ND & ND & ND & ND & ND & ND & ND & ND & ND \\
\hline 699-36-61B & 228.4 & 133.8 & NP & NP & NP & NP & NP & NP & 133.8 & 104.9 & NP & NP & NP & NP & 104.9 & 85.0 & 85.0 & 61.0 & 61.0 & NP \\
\hline 699-36-93 & 196.3 & 184.1 & NP & NP & 184.1 & 159.7 & NP & NP & 159.7 & 80.5 & NP & NP & NP & NP & 80.5 & 13.4 & 13.4 & -13.4 & -13.4 & NP \\
\hline
\end{tabular}


Table A.1. Interpreted Top and Bottom Elevation of Major Hydrogeologic Units (continued)

$\mathrm{NP}=$ unit not present

$\mathrm{NC}=$ not certain whether unit is present or not

$\mathrm{ND}=$ well not deep enough to determine

$\mathrm{NI}=$ not interpreted or unknown

Elevations in $\mathrm{m}$ above mean sea level based on the National Geodetic Vertical Datum of 1929 (NGVD29 datum)

\begin{tabular}{|c|c|c|c|c|c|c|c|c|c|c|c|c|c|c|c|c|c|c|c|c|}
\hline Well number & $\begin{array}{l}\text { Casing } \\
\text { Elev. }\end{array}$ & Bot 1 & Top 2 & Bot 2 & Top 3 & Bot 3 & Top 4 & Bot 4 & Top 5 & Bot 5 & Top 6 & Bot 6 & Top 7 & Bot 7 & Top 8 & Bot 8 & Top 9 & Bot 9 & $\begin{array}{l}\text { Top of } \\
\text { Basalt }\end{array}$ & $\begin{array}{l}\text { Layer } 6 \\
\% \text { mud }\end{array}$ \\
\hline 699-37-E4 & 118.0 & 101.2 & NP & NP & NP & NP & NP & NP & 101.2 & ND & ND & ND & ND & ND & ND & ND & ND & ND & ND & ND \\
\hline 699-37-E1 & 140.1 & $\mathrm{NI}$ & $\mathrm{NI}$ & $\mathrm{NI}$ & $\mathrm{NI}$ & $\mathrm{NI}$ & $\mathrm{NI}$ & $\mathrm{NI}$ & $\mathrm{NI}$ & $\mathrm{NI}$ & $\mathrm{NI}$ & $\mathrm{NI}$ & $\mathrm{NI}$ & $\mathrm{NI}$ & $\mathrm{NI}$ & $\mathrm{NI}$ & $\mathrm{NI}$ & $\mathrm{NI}$ & 74.4 & $\mathrm{NI}$ \\
\hline 699-37-4 & 148.6 & $\mathrm{NI}$ & $\mathrm{NI}$ & $\mathrm{NI}$ & $\mathrm{NI}$ & $\mathrm{NI}$ & $\mathrm{NI}$ & $\mathrm{NI}$ & $\mathrm{NI}$ & $\mathrm{NI}$ & $\mathrm{NI}$ & $\mathrm{NI}$ & $\mathrm{NI}$ & $\mathrm{NI}$ & $\mathrm{NI}$ & $\mathrm{NI}$ & $\mathrm{NI}$ & $\mathrm{NI}$ & 78.0 & $\mathrm{NI}$ \\
\hline 699-37-36 & 165.5 & 118.3 & NP & NP & NP & NP & $\mathrm{NI}$ & $\mathrm{NI}$ & $\mathrm{NI}$ & $\mathrm{NI}$ & $\mathrm{NI}$ & $\mathrm{NI}$ & $\mathrm{NI}$ & $\mathrm{NI}$ & $\mathrm{NI}$ & $\mathrm{NI}$ & $\mathrm{NI}$ & $\mathrm{NI}$ & 37.5 & $\mathrm{NI}$ \\
\hline $699-37-43$ & 210.4 & 125.0 & $\mathrm{NP}$ & NP & NP & NP & 125.0 & 120.4 & 120.4 & 105.2 & 105.2 & 88.4 & 88.4 & 80.8 & 80.8 & 67.1 & 67.1 & 54.9 & 54.9 & 30.5 \\
\hline 699-37-82B & 193.9 & 171.0 & 171.0 & 164.9 & 164.9 & 160.3 & NP & NP & 160.3 & 71.0 & NP & NP & NP & NP & 71.0 & 43.9 & 43.9 & 10.4 & 10.4 & NP \\
\hline 699-37-84 & 193.2 & 173.1 & NP & NP & NP & NP & 173.1 & 169.8 & 169.8 & 63.7 & NP & NP & NP & NP & 63.7 & 36.6 & 36.6 & 4.9 & 4.9 & NP \\
\hline $699-37-92$ & 196.6 & 181.4 & NP & NP & 181.4 & 160.0 & 160.0 & 149.7 & 149.7 & 61.6 & NP & NP & NP & NP & 61.6 & 34.1 & 34.1 & -9.1 & -9.1 & NP \\
\hline 699-38-E0 & 143.1 & 99.1 & NP & NP & NP & NP & NP & NP & 99.1 & 88.4 & 88.4 & 83.8 & 83.8 & 77.7 & NP & NP & NP & NP & 77.7 & 30.5 \\
\hline $699-38-3$ & 151.7 & 99.1 & $\mathrm{NP}$ & NP & NP & NP & NP & NP & 99.1 & 97.5 & 97.5 & 87.8 & NP & $\mathrm{NP}$ & NP & NP & NP & NP & 87.8 & NP \\
\hline 699-38-8A & 146.3 & 99.1 & NP & NP & NP & NP & 99.1 & 79.2 & 79.2 & $\mathrm{NI}$ & $\mathrm{NI}$ & $\mathrm{NI}$ & $\mathrm{NI}$ & $\mathrm{NI}$ & $\mathrm{NI}$ & $\mathrm{NI}$ & $\mathrm{NI}$ & $\mathrm{NI}$ & 22.9 & $\mathrm{NI}$ \\
\hline 699-38-9 & 152.7 & 107.0 & $\mathrm{NP}$ & NP & NP & NP & 107.0 & 97.8 & $\mathrm{NI}$ & $\mathrm{NI}$ & $\mathrm{NI}$ & $\mathrm{NI}$ & $\mathrm{NI}$ & $\mathrm{NI}$ & $\mathrm{NI}$ & $\mathrm{NI}$ & $\mathrm{NI}$ & $\mathrm{NI}$ & 6.7 & $\mathrm{NI}$ \\
\hline 699-38-15 & 137.7 & 101.2 & $\mathrm{NP}$ & NP & NP & NP & 101.2 & 98.1 & 98.1 & 38.7 & 38.7 & 35.7 & 35.7 & 12.2 & 12.2 & 2.1 & 2.1 & -6.4 & -6.4 & 30.5 \\
\hline 699-38-19 & 140.6 & 100.6 & $\mathrm{NP}$ & NP & NP & NP & 100.6 & 93.0 & $\mathrm{NI}$ & $\mathrm{NI}$ & $\mathrm{NI}$ & $\mathrm{NI}$ & $\mathrm{NI}$ & $\mathrm{NI}$ & $\mathrm{NI}$ & $\mathrm{NI}$ & $\mathrm{NI}$ & $\mathrm{NI}$ & -8.8 & $\mathrm{NI}$ \\
\hline 699-38-34A & $\mathrm{NI}$ & $\mathrm{NI}$ & $\mathrm{NI}$ & $\mathrm{NI}$ & $\mathrm{NI}$ & $\mathrm{NI}$ & $\mathrm{NI}$ & $\mathrm{NI}$ & $\mathrm{NI}$ & $\mathrm{NI}$ & $\mathrm{NI}$ & $\mathrm{NI}$ & $\mathrm{NI}$ & $\mathrm{NI}$ & $\mathrm{NI}$ & $\mathrm{NI}$ & $\mathrm{NI}$ & $\mathrm{NI}$ & 53.6 & $\mathrm{NI}$ \\
\hline 699-38-65 & 229.6 & 144.2 & $\mathrm{NP}$ & NP & NP & NP & $\mathrm{NP}$ & NP & 144.2 & 110.6 & $\mathrm{NP}$ & $\mathrm{NP}$ & NP & $\mathrm{NP}$ & 110.6 & 93.9 & 93.9 & 69.2 & 69.2 & NP \\
\hline 699-39-E2 & 123.4 & 85.3 & $\mathrm{NP}$ & NP & NP & NP & NP & NP & NP & NP & 85.3 & 76.2 & 76.2 & 73.2 & 73.2 & 67.1 & NP & NP & 67.1 & 30.5 \\
\hline 699-39-1 & 144.5 & $\mathrm{NI}$ & $\mathrm{NI}$ & $\mathrm{NI}$ & $\mathrm{NI}$ & $\mathrm{NI}$ & $\mathrm{NI}$ & $\mathrm{NI}$ & $\mathrm{NI}$ & $\mathrm{NI}$ & $\mathrm{NI}$ & $\mathrm{NI}$ & $\mathrm{NI}$ & $\mathrm{NI}$ & $\mathrm{NI}$ & $\mathrm{NI}$ & $\mathrm{NI}$ & $\mathrm{NI}$ & 68.3 & $\mathrm{NI}$ \\
\hline $699-39-2 A$ & 142.2 & $\mathrm{NI}$ & $\mathrm{NI}$ & $\mathrm{NI}$ & $\mathrm{NI}$ & $\mathrm{NI}$ & $\mathrm{NI}$ & $\mathrm{NI}$ & $\mathrm{NI}$ & $\mathrm{NI}$ & $\mathrm{NI}$ & $\mathrm{NI}$ & $\mathrm{NI}$ & $\mathrm{NI}$ & $\mathrm{NI}$ & $\mathrm{NI}$ & $\mathrm{NI}$ & $\mathrm{NI}$ & 64.0 & $\mathrm{NI}$ \\
\hline 699-39-7A & 149.3 & $\mathrm{NI}$ & $\mathrm{NI}$ & $\mathrm{NI}$ & $\mathrm{NI}$ & $\mathrm{NI}$ & $\mathrm{NI}$ & $\mathrm{NI}$ & $\mathrm{NI}$ & $\mathrm{NI}$ & $\mathrm{NI}$ & $\mathrm{NI}$ & $\mathrm{NI}$ & $\mathrm{NI}$ & $\mathrm{NI}$ & $\mathrm{NI}$ & $\mathrm{NI}$ & $\mathrm{NI}$ & 103.0 & $\mathrm{NI}$ \\
\hline 699-39-7B & 149.2 & 106.7 & $\mathrm{NP}$ & NP & NP & NP & 106.7 & 104.9 & NP & NP & $\mathrm{NP}$ & $\mathrm{NP}$ & NP & NP & NP & NP & NP & NP & 104.9 & NP \\
\hline 699-39-23 & 145.0 & 103.9 & NP & $\mathrm{NP}$ & NP & $\mathrm{NP}$ & 103.9 & 96.3 & 96.3 & 50.6 & 50.6 & 46.0 & 46.0 & 17.1 & 17.1 & 1.8 & 1.8 & -4.6 & -4.6 & 30.5 \\
\hline 699-39-79 & 205.1 & 179.2 & 179.2 & 162.5 & 162.5 & 157.9 & NP & $\mathrm{NP}$ & 157.9 & $\mathrm{NI}$ & $\mathrm{NI}$ & $\mathrm{NI}$ & $\mathrm{NI}$ & $\mathrm{NI}$ & $\mathrm{NI}$ & $\mathrm{NI}$ & $\mathrm{NI}$ & $\mathrm{NI}$ & $\mathrm{NI}$ & $\mathrm{NI}$ \\
\hline 699-39-84B & $\mathrm{NI}$ & $\mathrm{NI}$ & $\mathrm{NI}$ & $\mathrm{NI}$ & $\mathrm{NI}$ & $\mathrm{NI}$ & $\mathrm{NI}$ & $\mathrm{NI}$ & $\mathrm{NI}$ & $\mathrm{NI}$ & $\mathrm{NI}$ & $\mathrm{NI}$ & $\mathrm{NI}$ & $\mathrm{NI}$ & $\mathrm{NI}$ & $\mathrm{NI}$ & $\mathrm{NI}$ & $\mathrm{NI}$ & 8.8 & $\mathrm{NI}$ \\
\hline $699-39-103$ & 271.3 & 266.7 & $\mathrm{NP}$ & NP & 266.7 & 265.2 & $\mathrm{NP}$ & $\mathrm{NP}$ & 266.7 & 237.7 & NP & NP & NP & NP & 237.7 & 229.2 & $\mathrm{NP}$ & NP & 229.2 & NP \\
\hline $699-40-0$ & 128.2 & 106.7 & $\mathrm{NP}$ & NP & NP & NP & 106.7 & 93.0 & 93.0 & 86.9 & 86.9 & 67.1 & 67.1 & 35.1 & 35.1 & 32.0 & NP & NP & 32.0 & 25.0 \\
\hline $699-40-1$ & 133.7 & 100.3 & $\mathrm{NP}$ & NP & NP & NP & NP & NP & 100.3 & 91.1 & 91.1 & 81.7 & 81.7 & 46.9 & 46.9 & 37.5 & NP & NP & 37.5 & 30.5 \\
\hline
\end{tabular}


Table A.1. Interpreted Top and Bottom Elevation of Major Hydrogeologic Units (continued)

$\mathrm{NP}=$ unit not present

$\mathrm{NC}=$ not certain whether unit is present or not

$\mathrm{ND}=$ well not deep enough to determine

$\mathrm{NI}=$ not interpreted or unknown

Elevations in $\mathrm{m}$ above mean sea level based on the National Geodetic Vertical Datum of 1929 (NGVD29 datum)

\begin{tabular}{|c|c|c|c|c|c|c|c|c|c|c|c|c|c|c|c|c|c|c|c|c|}
\hline Well number & $\begin{array}{l}\text { Casing } \\
\text { Elev. }\end{array}$ & Bot 1 & Top 2 & Bot 2 & Top 3 & Bot 3 & Top 4 & Bot 4 & Top 5 & Bot 5 & Top 6 & Bot 6 & Top 7 & Bot 7 & Top 8 & Bot 8 & Top 9 & Bot 9 & $\begin{array}{l}\text { Top of } \\
\text { Basalt }\end{array}$ & $\begin{array}{l}\text { Layer } 6 \\
\% \text { mud }\end{array}$ \\
\hline $699-40-2$ & 140.6 & 108.2 & NP & NP & NP & NP & NP & NP & 108.2 & 93.0 & 93.0 & 88.4 & 88.4 & 48.8 & 48.8 & 32.3 & NP & NP & 32.3 & 30.5 \\
\hline $699-40-6$ & 147.9 & $\mathrm{NI}$ & $\mathrm{NI}$ & $\mathrm{NI}$ & $\mathrm{NI}$ & $\mathrm{NI}$ & $\mathrm{NI}$ & $\mathrm{NI}$ & $\mathrm{NI}$ & $\mathrm{NI}$ & $\mathrm{NI}$ & $\mathrm{NI}$ & $\mathrm{NI}$ & $\mathrm{NI}$ & $\mathrm{NI}$ & $\mathrm{NI}$ & $\mathrm{NI}$ & $\mathrm{NI}$ & 78.0 & $\mathrm{NI}$ \\
\hline $699-40-12 B$ & 157.1 & 106.7 & NP & NP & NP & NP & 106.7 & 100.6 & 100.6 & 88.4 & 88.4 & 39.6 & 39.6 & 29.0 & 29.0 & 15.2 & NP & NP & 15.2 & 8.5 \\
\hline $699-40-13$ & 154.7 & 106.1 & NP & NP & NP & NP & 106.1 & 100.9 & $\mathrm{NI}$ & $\mathrm{NI}$ & $\mathrm{NI}$ & $\mathrm{NI}$ & $\mathrm{NI}$ & $\mathrm{NI}$ & $\mathrm{NI}$ & $\mathrm{NI}$ & $\mathrm{NI}$ & $\mathrm{NI}$ & 6.1 & $\mathrm{NI}$ \\
\hline $699-40-20$ & 145.2 & 102.7 & $\mathrm{NP}$ & NP & NP & NP & 102.7 & 101.2 & $\mathrm{NI}$ & $\mathrm{NI}$ & $\mathrm{NI}$ & $\mathrm{NI}$ & $\mathrm{NI}$ & $\mathrm{NI}$ & $\mathrm{NI}$ & $\mathrm{NI}$ & $\mathrm{NI}$ & $\mathrm{NI}$ & -1.5 & $\mathrm{NI}$ \\
\hline $699-40-32$ & 159.2 & 119.5 & $\mathrm{NP}$ & NP & NP & NP & NP & NP & 119.5 & 118.3 & 118.3 & 104.2 & 104.2 & 92.0 & 92.0 & 82.9 & 82.9 & 61.0 & 61.0 & 30.5 \\
\hline $699-40-33 A$ & 157.9 & 125.6 & $\mathrm{NP}$ & NP & NP & NP & NP & NP & 125.6 & 122.8 & 122.8 & 94.5 & 94.5 & 85.3 & 85.3 & 81.1 & 81.1 & 73.2 & 73.2 & 13.7 \\
\hline $699-40-33 C$ & $\mathrm{NI}$ & $\mathrm{NI}$ & $\mathrm{NI}$ & $\mathrm{NI}$ & $\mathrm{NI}$ & $\mathrm{NI}$ & $\mathrm{NI}$ & $\mathrm{NI}$ & $\mathrm{NI}$ & $\mathrm{NI}$ & $\mathrm{NI}$ & $\mathrm{NI}$ & $\mathrm{NI}$ & $\mathrm{NI}$ & $\mathrm{NI}$ & $\mathrm{NI}$ & $\mathrm{NI}$ & $\mathrm{NI}$ & 71.6 & $\mathrm{NI}$ \\
\hline 699-40-36 & 160.9 & 126.5 & $\mathrm{NP}$ & NP & NP & NP & NP & NP & NP & NP & 126.5 & 100.9 & 100.9 & 91.7 & 91.7 & 84.7 & 84.7 & 78.0 & 78.0 & 30.5 \\
\hline $699-40-62$ & 228.0 & 132.0 & $\mathrm{NP}$ & NP & NP & NP & NP & NP & 132.0 & 113.7 & NP & NP & NP & NP & 113.7 & ND & ND & ND & ND & NP \\
\hline $699-40-80$ & 199.9 & 172.2 & 172.2 & 164.6 & 164.6 & 153.6 & $\mathrm{NP}$ & NP & 153.6 & 65.5 & NP & NP & NP & NP & 65.5 & 50.0 & 50.0 & 32.9 & 32.9 & NP \\
\hline $699-40-84$ & 194.5 & 173.1 & 173.1 & 168.6 & 168.6 & 166.1 & 166.1 & 160.6 & 160.6 & 71.9 & NP & NP & NP & NP & 71.9 & 49.7 & 49.7 & 12.2 & 12.2 & NP \\
\hline 699-41-4 & $\mathrm{NI}$ & $\mathrm{NI}$ & $\mathrm{NI}$ & $\mathrm{NI}$ & $\mathrm{NI}$ & $\mathrm{NI}$ & $\mathrm{NI}$ & $\mathrm{NI}$ & $\mathrm{NI}$ & $\mathrm{NI}$ & $\mathrm{NI}$ & $\mathrm{NI}$ & $\mathrm{NI}$ & $\mathrm{NI}$ & $\mathrm{NI}$ & $\mathrm{NI}$ & $\mathrm{NI}$ & $\mathrm{NI}$ & 43.0 & $\mathrm{NI}$ \\
\hline $699-41-5$ & 146.6 & $\mathrm{NI}$ & $\mathrm{NI}$ & $\mathrm{NI}$ & $\mathrm{NI}$ & $\mathrm{NI}$ & $\mathrm{NI}$ & $\mathrm{NI}$ & $\mathrm{NI}$ & $\mathrm{NI}$ & $\mathrm{NI}$ & $\mathrm{NI}$ & $\mathrm{NI}$ & $\mathrm{NI}$ & $\mathrm{NI}$ & $\mathrm{NI}$ & $\mathrm{NI}$ & $\mathrm{NI}$ & 79.6 & $\mathrm{NI}$ \\
\hline $699-41-10$ & 152.0 & 92.4 & NP & NP & NP & NP & $\mathrm{NI}$ & $\mathrm{NI}$ & $\mathrm{NI}$ & $\mathrm{NI}$ & $\mathrm{NI}$ & $\mathrm{NI}$ & $\mathrm{NI}$ & $\mathrm{NI}$ & $\mathrm{NI}$ & $\mathrm{NI}$ & $\mathrm{NI}$ & $\mathrm{NI}$ & 68.6 & $\mathrm{NI}$ \\
\hline 699-41-11 & 155.5 & 102.1 & $\mathrm{NP}$ & NP & NP & NP & 102.1 & 99.1 & 99.1 & 70.1 & 70.1 & 57.9 & NP & NP & 57.9 & 39.6 & NP & NP & 39.6 & 26.8 \\
\hline $699-41-20$ & 147.7 & 93.0 & $\mathrm{NP}$ & NP & NP & NP & $\mathrm{NI}$ & $\mathrm{NI}$ & $\mathrm{NI}$ & $\mathrm{NI}$ & $\mathrm{NI}$ & $\mathrm{NI}$ & $\mathrm{NI}$ & $\mathrm{NI}$ & 30.5 & 6.1 & 6.1 & 5.2 & 5.2 & $\mathrm{NI}$ \\
\hline $699-41-23$ & 142.0 & ND & ND & ND & ND & ND & ND & ND & ND & ND & ND & ND & ND & ND & ND & ND & ND & ND & ND & ND \\
\hline $699-41-31$ & $\mathrm{NI}$ & $\mathrm{NI}$ & $\mathrm{NI}$ & $\mathrm{NI}$ & $\mathrm{NI}$ & $\mathrm{NI}$ & $\mathrm{NI}$ & $\mathrm{NI}$ & $\mathrm{NI}$ & $\mathrm{NI}$ & $\mathrm{NI}$ & $\mathrm{NI}$ & $\mathrm{NI}$ & $\mathrm{NI}$ & $\mathrm{NI}$ & $\mathrm{NI}$ & $\mathrm{NI}$ & $\mathrm{NI}$ & 59.4 & $\mathrm{NI}$ \\
\hline 699-41-35 & 158.5 & 127.7 & $\mathrm{NP}$ & NP & NP & NP & NP & NP & NP & NP & 127.7 & 116.4 & 116.4 & 96.6 & 96.6 & 90.5 & 90.5 & 83.2 & 83.2 & 30.5 \\
\hline 699-41-91 & $\mathrm{NI}$ & $\mathrm{NI}$ & $\mathrm{NI}$ & $\mathrm{NI}$ & $\mathrm{NI}$ & $\mathrm{NI}$ & $\mathrm{NI}$ & $\mathrm{NI}$ & $\mathrm{NI}$ & $\mathrm{NI}$ & $\mathrm{NI}$ & $\mathrm{NI}$ & $\mathrm{NI}$ & $\mathrm{NI}$ & $\mathrm{NI}$ & $\mathrm{NI}$ & $\mathrm{NI}$ & $\mathrm{NI}$ & -8.8 & $\mathrm{NI}$ \\
\hline 699-42-E9B & 116.9 & 89.6 & $\mathrm{NP}$ & NP & NP & NP & 89.6 & 80.5 & 80.5 & 72.8 & 72.8 & 69.8 & 69.8 & 60.7 & 60.7 & 48.5 & NP & NP & 48.5 & 30.5 \\
\hline $699-42-3$ & 135.3 & 100.3 & $\mathrm{NP}$ & NP & NP & NP & 100.3 & 85.0 & 85.0 & 77.4 & 77.4 & 64.3 & 64.3 & 18.0 & 18.0 & 16.2 & NP & NP & 16.2 & 30.5 \\
\hline 699-42-10 & 150.4 & 100.0 & $\mathrm{NP}$ & NP & NP & NP & 100.0 & 98.5 & NP & $\mathrm{NP}$ & 98.5 & 88.7 & NP & NP & $\mathrm{NP}$ & NP & NP & NP & 88.7 & 30.5 \\
\hline $699-42-12 A$ & 156.7 & 115.5 & $\mathrm{NP}$ & NP & NP & NP & $\mathrm{NP}$ & NP & 115.5 & 92.7 & 92.7 & 85.0 & 85.0 & 71.3 & 71.3 & 56.7 & NP & NP & 56.7 & 30.5 \\
\hline $699-42-21$ & 140.6 & 104.2 & $\mathrm{NP}$ & NP & NP & NP & NC & NC & 104.2 & 26.2 & NC & NC & NC & NC & 26.2 & 21.6 & 21.6 & 0.3 & 0.3 & $\mathrm{NC}$ \\
\hline $699-42-29$ & 138.3 & 104.5 & $\mathrm{NP}$ & NP & NP & NP & 104.5 & 101.5 & $\mathrm{NI}$ & $\mathrm{NI}$ & $\mathrm{NI}$ & $\mathrm{NI}$ & $\mathrm{NI}$ & $\mathrm{NI}$ & $\mathrm{NI}$ & $\mathrm{NI}$ & $\mathrm{NI}$ & $\mathrm{NI}$ & 6.7 & $\mathrm{NI}$ \\
\hline
\end{tabular}


Table A.1. Interpreted Top and Bottom Elevation of Major Hydrogeologic Units (continued)

$\mathrm{NP}=$ unit not present

$\mathrm{NC}=$ not certain whether unit is present or not

$\mathrm{ND}=$ well not deep enough to determine

$\mathrm{NI}=$ not interpreted or unknown

Elevations in $\mathrm{m}$ above mean sea level based on the National Geodetic Vertical Datum of 1929 (NGVD29 datum)

\begin{tabular}{|c|c|c|c|c|c|c|c|c|c|c|c|c|c|c|c|c|c|c|c|c|}
\hline Well number & $\begin{array}{l}\text { Casing } \\
\text { Elev. }\end{array}$ & Bot 1 & Top 2 & Bot 2 & Top 3 & Bot 3 & Top 4 & Bot 4 & Top 5 & Bot 5 & Top 6 & Bot 6 & Top 7 & Bot 7 & Top 8 & Bot 8 & Top 9 & Bot 9 & $\begin{array}{l}\text { Top of } \\
\text { Basalt }\end{array}$ & $\begin{array}{l}\text { Layer } 6 \\
\% \text { mud }\end{array}$ \\
\hline $699-42-30$ & 146.5 & 103.9 & NP & NP & NP & NP & $\mathrm{NI}$ & $\mathrm{NI}$ & $\mathrm{NI}$ & $\mathrm{NI}$ & $\mathrm{NI}$ & $\mathrm{NI}$ & $\mathrm{NI}$ & $\mathrm{NI}$ & $\mathrm{NI}$ & $\mathrm{NI}$ & $\mathrm{NI}$ & $\mathrm{NI}$ & 17.1 & $\mathrm{NI}$ \\
\hline 699-42-37 & 158.2 & 136.9 & $\mathrm{NP}$ & NP & NP & NP & NP & NP & 136.9 & 130.1 & 130.1 & 120.1 & 120.1 & 104.5 & 104.5 & 100.0 & 100.0 & 82.6 & 82.6 & 30.5 \\
\hline $699-42-40 \mathrm{~A}$ & 166.4 & 126.8 & NP & NP & NP & NP & NP & NP & NP & NP & NP & NP & NP & NP & 126.8 & 122.2 & 122.2 & ND & ND & NP \\
\hline $699-42-40 \mathrm{C}$ & 166.4 & 125.3 & NP & NP & NP & NP & NP & NP & NP & NP & NP & NP & NP & NP & 125.3 & 122.8 & 122.8 & 99.4 & 99.4 & NP \\
\hline $699-42-42 A$ & 189.0 & 124.7 & NP & NP & NP & NP & NP & NP & NP & NP & NP & NP & NP & NP & 124.7 & 120.4 & 120.4 & 90.8 & 90.8 & NP \\
\hline $699-42-42 B$ & 177.7 & 121.3 & NP & NP & NP & NP & NP & NP & NP & NP & NP & NP & NP & NP & NP & NP & 121.3 & ND & ND & NP \\
\hline 699-43-2 & 123.2 & 104.9 & $\mathrm{NP}$ & NP & NP & NP & 104.9 & 83.5 & $\mathrm{NI}$ & $\mathrm{NI}$ & $\mathrm{NI}$ & $\mathrm{NI}$ & $\mathrm{NI}$ & $\mathrm{NI}$ & $\mathrm{NI}$ & $\mathrm{NI}$ & $\mathrm{NI}$ & $\mathrm{NI}$ & 21.3 & $\mathrm{NI}$ \\
\hline $699-43-8$ & 144.0 & 98.5 & $\mathrm{NP}$ & NP & NP & NP & $\mathrm{NI}$ & $\mathrm{NI}$ & $\mathrm{NI}$ & $\mathrm{NI}$ & $\mathrm{NI}$ & $\mathrm{NI}$ & $\mathrm{NI}$ & $\mathrm{NI}$ & $\mathrm{NI}$ & $\mathrm{NI}$ & $\mathrm{NI}$ & $\mathrm{NI}$ & 65.5 & $\mathrm{NI}$ \\
\hline $699-43-9$ & 149.1 & 99.7 & NP & NP & NP & NP & NP & NP & NP & NP & NP & NP & NP & NP & NP & NP & NP & NP & 99.7 & NP \\
\hline 699-43-18 & 157.1 & 102.4 & NP & NP & NP & NP & $\mathrm{NI}$ & $\mathrm{NI}$ & $\mathrm{NI}$ & $\mathrm{NI}$ & $\mathrm{NI}$ & $\mathrm{NI}$ & $\mathrm{NI}$ & $\mathrm{NI}$ & $\mathrm{NI}$ & $\mathrm{NI}$ & $\mathrm{NI}$ & $\mathrm{NI}$ & 19.8 & $\mathrm{NI}$ \\
\hline 699-43-23 & 158.1 & 103.3 & $\mathrm{NP}$ & NP & NP & NP & NC & NC & 103.3 & 59.1 & 59.1 & 56.1 & 56.1 & 46.9 & 46.9 & 44.2 & 44.2 & 14.9 & 14.9 & 30.5 \\
\hline 699-43-41G & 167.9 & 130.8 & NP & NP & NP & NP & NP & NP & NP & NP & NP & NP & NP & NP & 130.8 & 124.7 & 124.7 & ND & ND & NP \\
\hline 699-43-42 & 173.4 & 126.5 & NP & NP & NP & NP & NP & NP & 126.5 & 117.0 & NP & NP & NP & NP & 117.0 & 113.1 & 113.1 & 108.8 & 108.8 & NP \\
\hline $699-43-42 \mathrm{~K}$ & 177.1 & 128.6 & NP & NP & NP & NP & NP & NP & $\mathrm{NP}$ & NP & NP & NP & NP & NP & 128.6 & 121.6 & 121.6 & 103.0 & 103.0 & NP \\
\hline $699-43-43$ & 176.5 & 123.7 & NP & NP & NP & NP & NP & NP & NP & NP & NP & NP & NP & NP & NP & NP & 123.7 & ND & ND & NP \\
\hline $699-43-81$ & $\mathrm{NI}$ & $\mathrm{NI}$ & $\mathrm{NI}$ & $\mathrm{NI}$ & $\mathrm{NI}$ & $\mathrm{NI}$ & $\mathrm{NI}$ & $\mathrm{NI}$ & $\mathrm{NI}$ & $\mathrm{NI}$ & $\mathrm{NI}$ & $\mathrm{NI}$ & $\mathrm{NI}$ & $\mathrm{NI}$ & $\mathrm{NI}$ & $\mathrm{NI}$ & $\mathrm{NI}$ & $\mathrm{NI}$ & 30.2 & $\mathrm{NI}$ \\
\hline $699-43-84$ & 193.2 & 173.7 & 173.7 & 169.8 & 169.8 & 163.7 & 163.7 & 159.7 & 159.7 & 60.0 & NP & NP & NP & NP & 60.0 & 46.0 & 46.0 & 21.3 & 21.3 & NP \\
\hline 699-43-89 & 196.3 & 171.0 & 171.0 & 168.9 & 168.9 & 165.8 & 165.8 & 153.6 & 153.6 & ND & ND & ND & ND & ND & ND & ND & ND & ND & ND & ND \\
\hline 699-43-91A & $\mathrm{NI}$ & $\mathrm{NI}$ & $\mathrm{NI}$ & $\mathrm{NI}$ & $\mathrm{NI}$ & $\mathrm{NI}$ & $\mathrm{NI}$ & $\mathrm{NI}$ & $\mathrm{NI}$ & $\mathrm{NI}$ & $\mathrm{NI}$ & $\mathrm{NI}$ & $\mathrm{NI}$ & $\mathrm{NI}$ & $\mathrm{NI}$ & $\mathrm{NI}$ & $\mathrm{NI}$ & $\mathrm{NI}$ & 2.4 & $\mathrm{NI}$ \\
\hline 699-43-91D & $\mathrm{NI}$ & $\mathrm{NI}$ & $\mathrm{NI}$ & $\mathrm{NI}$ & $\mathrm{NI}$ & $\mathrm{NI}$ & $\mathrm{NI}$ & $\mathrm{NI}$ & $\mathrm{NI}$ & $\mathrm{NI}$ & $\mathrm{NI}$ & $\mathrm{NI}$ & $\mathrm{NI}$ & $\mathrm{NI}$ & $\mathrm{NI}$ & $\mathrm{NI}$ & $\mathrm{NI}$ & $\mathrm{NI}$ & 3.7 & $\mathrm{NI}$ \\
\hline $699-43-104$ & 233.5 & 196.9 & NP & NP & NP & NP & 196.9 & 187.8 & 187.8 & 140.8 & NP & NP & NP & NP & 140.8 & 123.7 & 123.7 & 117.0 & 117.0 & NP \\
\hline $699-44-7$ & 132.4 & 104.9 & $\mathrm{NP}$ & NP & NP & NP & 104.9 & 75.9 & 75.9 & 72.8 & 72.8 & 46.9 & 46.9 & 30.2 & 30.2 & 4.0 & NP & NP & 4.0 & 25.0 \\
\hline $699-44-16$ & 135.7 & 94.5 & NP & NP & NP & NP & 94.5 & 93.0 & 93.0 & 76.2 & 76.2 & 73.2 & 73.2 & 33.5 & 33.5 & 29.9 & NP & NP & 29.9 & 30.5 \\
\hline 699-44-42 & 176.5 & 126.8 & $\mathrm{NP}$ & NP & NP & NP & NP & NP & NP & NP & NP & NP & NP & NP & NP & NP & 126.8 & ND & ND & NP \\
\hline $699-44-64$ & $\mathrm{NI}$ & $\mathrm{NI}$ & $\mathrm{NI}$ & $\mathrm{NI}$ & $\mathrm{NI}$ & $\mathrm{NI}$ & $\mathrm{NI}$ & $\mathrm{NI}$ & $\mathrm{NI}$ & $\mathrm{NI}$ & $\mathrm{NI}$ & $\mathrm{NI}$ & $\mathrm{NI}$ & $\mathrm{NI}$ & $\mathrm{NI}$ & $\mathrm{NI}$ & $\mathrm{NI}$ & $\mathrm{NI}$ & 86.6 & $\mathrm{NI}$ \\
\hline $699-44-70$ & $\mathrm{NI}$ & $\mathrm{NI}$ & $\mathrm{NI}$ & $\mathrm{NI}$ & $\mathrm{NI}$ & $\mathrm{NI}$ & $\mathrm{NI}$ & $\mathrm{NI}$ & $\mathrm{NI}$ & $\mathrm{NI}$ & $\mathrm{NI}$ & $\mathrm{NI}$ & $\mathrm{NI}$ & $\mathrm{NI}$ & $\mathrm{NI}$ & $\mathrm{NI}$ & $\mathrm{NI}$ & $\mathrm{NI}$ & 64.6 & $\mathrm{NI}$ \\
\hline
\end{tabular}


Table A.1. Interpreted Top and Bottom Elevation of Major Hydrogeologic Units (continued)

$\mathrm{NP}=$ unit not present

$\mathrm{NC}=$ not certain whether unit is present or not

$\mathrm{ND}=$ well not deep enough to determine

$\mathrm{NI}=$ not interpreted or unknown

Elevations in $\mathrm{m}$ above mean sea level based on the National Geodetic Vertical Datum of 1929 (NGVD29 datum)

\begin{tabular}{|c|c|c|c|c|c|c|c|c|c|c|c|c|c|c|c|c|c|c|c|c|}
\hline Well number & $\begin{array}{l}\text { Casing } \\
\text { Elev. }\end{array}$ & Bot 1 & Top 2 & Bot 2 & Top 3 & Bot 3 & Top 4 & Bot 4 & Top 5 & Bot 5 & Top 6 & Bot 6 & Top 7 & Bot 7 & Top 8 & Bot 8 & Top 9 & Bot 9 & $\begin{array}{l}\text { Top of } \\
\text { Basalt }\end{array}$ & $\begin{array}{l}\text { Layer } 6 \\
\% \text { mud }\end{array}$ \\
\hline $699-44-118$ & $\mathrm{NI}$ & $\mathrm{NI}$ & $\mathrm{NI}$ & $\mathrm{NI}$ & $\mathrm{NI}$ & $\mathrm{NI}$ & $\mathrm{NI}$ & $\mathrm{NI}$ & $\mathrm{NI}$ & $\mathrm{NI}$ & $\mathrm{NI}$ & $\mathrm{NI}$ & $\mathrm{NI}$ & $\mathrm{NI}$ & $\mathrm{NI}$ & $\mathrm{NI}$ & $\mathrm{NI}$ & $\mathrm{NI}$ & 274.3 & $\mathrm{NI}$ \\
\hline $699-45-24$ & 155.5 & 106.7 & NP & NP & NP & NP & 106.7 & 105.2 & $\mathrm{NI}$ & $\mathrm{NI}$ & $\mathrm{NI}$ & $\mathrm{NI}$ & $\mathrm{NI}$ & $\mathrm{NI}$ & $\mathrm{NI}$ & $\mathrm{NI}$ & $\mathrm{NI}$ & $\mathrm{NI}$ & 26.5 & $\mathrm{NI}$ \\
\hline $699-45-26$ & 158.0 & 106.1 & NP & NP & NP & NP & 106.1 & 101.5 & $\mathrm{NI}$ & $\mathrm{NI}$ & $\mathrm{NI}$ & $\mathrm{NI}$ & $\mathrm{NI}$ & $\mathrm{NI}$ & $\mathrm{NI}$ & $\mathrm{NI}$ & $\mathrm{NI}$ & $\mathrm{NI}$ & 21.3 & $\mathrm{NI}$ \\
\hline $699-45-42$ & $\mathrm{NI}$ & $\mathrm{NI}$ & $\mathrm{NI}$ & $\mathrm{NI}$ & $\mathrm{NI}$ & $\mathrm{NI}$ & $\mathrm{NI}$ & $\mathrm{NI}$ & $\mathrm{NI}$ & $\mathrm{NI}$ & $\mathrm{NI}$ & $\mathrm{NI}$ & $\mathrm{NI}$ & $\mathrm{NI}$ & $\mathrm{NI}$ & $\mathrm{NI}$ & $\mathrm{NI}$ & $\mathrm{NI}$ & 118.0 & $\mathrm{NI}$ \\
\hline $699-46-3$ & 115.7 & 102.1 & NP & NP & NP & NP & 102.1 & 89.9 & 89.9 & 74.7 & 74.7 & 71.6 & 71.6 & 23.8 & $\mathrm{NC}$ & $\mathrm{NC}$ & NP & NP & 23.8 & 30.5 \\
\hline $699-46-5$ & 116.3 & 104.2 & $\mathrm{NP}$ & NP & NP & NP & 104.2 & 82.9 & 82.9 & 72.5 & 72.5 & 60.4 & 60.4 & 31.1 & 31.1 & 17.1 & NP & NP & 17.1 & 30.5 \\
\hline $699-46-15$ & 135.0 & 125.9 & $\mathrm{NP}$ & NP & NP & NP & NP & NP & NP & $\mathrm{NP}$ & $\mathrm{NP}$ & NP & NP & NP & $\mathrm{NP}$ & NP & NP & NP & 125.9 & $\mathrm{NP}$ \\
\hline 699-46-21B & 159.1 & 135.0 & $\mathrm{NP}$ & NP & NP & NP & 135.0 & 121.3 & 121.3 & 78.3 & 78.3 & 61.6 & 61.6 & ND & ND & ND & ND & ND & ND & 30.5 \\
\hline $699-46-21 C$ & 158.5 & 135.9 & $\mathrm{NP}$ & NP & NP & NP & 135.9 & 122.5 & 122.5 & ND & ND & ND & ND & ND & ND & ND & ND & ND & ND & ND \\
\hline 699-46-31 & 145.2 & 106.7 & $\mathrm{NP}$ & NP & NP & NP & $\mathrm{NP}$ & NP & 106.7 & 73.2 & 73.2 & 70.1 & 70.1 & 65.5 & 65.5 & 45.7 & 45.7 & 29.0 & 29.0 & 30.5 \\
\hline 699-46-32 & 143.3 & 117.3 & $\mathrm{NP}$ & NP & NP & NP & NP & NP & NP & NP & 117.3 & 88.4 & 88.4 & 80.8 & $\mathrm{NP}$ & NP & NP & NP & 80.8 & 25.9 \\
\hline 699-46-33 & 142.8 & 125.9 & $\mathrm{NP}$ & NP & NP & NP & NP & NP & NP & $\mathrm{NP}$ & NP & $\mathrm{NP}$ & NP & NP & $\mathrm{NP}$ & NP & NP & NP & 125.9 & $\mathrm{NP}$ \\
\hline 699-46-85B & 241.4 & 176.8 & $\mathrm{NP}$ & NP & 176.8 & 173.7 & 173.7 & 157.6 & 157.6 & 68.3 & NP & NP & NP & NP & 68.3 & 56.1 & 56.1 & 25.9 & 25.9 & NP \\
\hline $699-47-24$ & 157.7 & 107.6 & $\mathrm{NP}$ & NP & $\mathrm{NP}$ & NP & $\mathrm{NI}$ & $\mathrm{NI}$ & $\mathrm{NI}$ & $\mathrm{NI}$ & $\mathrm{NI}$ & $\mathrm{NI}$ & $\mathrm{NI}$ & $\mathrm{NI}$ & 46.3 & 37.5 & NP & NP & 37.5 & $\mathrm{NI}$ \\
\hline $699-47-25$ & 163.0 & 101.8 & $\mathrm{NP}$ & NP & NP & NP & $\mathrm{NI}$ & $\mathrm{NI}$ & $\mathrm{NI}$ & $\mathrm{NI}$ & $\mathrm{NI}$ & $\mathrm{NI}$ & $\mathrm{NI}$ & $\mathrm{NI}$ & $\mathrm{NI}$ & $\mathrm{NI}$ & $\mathrm{NI}$ & $\mathrm{NI}$ & 32.0 & $\mathrm{NI}$ \\
\hline $699-47-35 A$ & 145.2 & 120.1 & $\mathrm{NP}$ & NP & NP & $\mathrm{NP}$ & NP & NP & NP & NP & NP & NP & NP & NP & NP & NP & 120.1 & 114.0 & 114.0 & NP \\
\hline $699-47-35 C$ & $\mathrm{NI}$ & $\mathrm{NI}$ & $\mathrm{NI}$ & $\mathrm{NI}$ & $\mathrm{NI}$ & $\mathrm{NI}$ & $\mathrm{NI}$ & $\mathrm{NI}$ & $\mathrm{NI}$ & $\mathrm{NI}$ & $\mathrm{NI}$ & $\mathrm{NI}$ & $\mathrm{NI}$ & $\mathrm{NI}$ & $\mathrm{NI}$ & $\mathrm{NI}$ & $\mathrm{NI}$ & $\mathrm{NI}$ & 113.4 & $\mathrm{NI}$ \\
\hline 699-47-42 & $\mathrm{NI}$ & $\mathrm{NI}$ & $\mathrm{NI}$ & $\mathrm{NI}$ & $\mathrm{NI}$ & $\mathrm{NI}$ & $\mathrm{NI}$ & $\mathrm{NI}$ & $\mathrm{NI}$ & $\mathrm{NI}$ & $\mathrm{NI}$ & $\mathrm{NI}$ & $\mathrm{NI}$ & $\mathrm{NI}$ & $\mathrm{NI}$ & $\mathrm{NI}$ & $\mathrm{NI}$ & $\mathrm{NI}$ & 122.2 & $\mathrm{NI}$ \\
\hline 699-47-46A & $\mathrm{NI}$ & $\mathrm{NI}$ & $\mathrm{NI}$ & $\mathrm{NI}$ & $\mathrm{NI}$ & $\mathrm{NI}$ & $\mathrm{NI}$ & $\mathrm{NI}$ & $\mathrm{NI}$ & $\mathrm{NI}$ & $\mathrm{NI}$ & $\mathrm{NI}$ & $\mathrm{NI}$ & $\mathrm{NI}$ & $\mathrm{NI}$ & $\mathrm{NI}$ & $\mathrm{NI}$ & $\mathrm{NI}$ & 123.7 & $\mathrm{NI}$ \\
\hline 699-47-60 & 198.1 & 128.9 & $\mathrm{NP}$ & NP & NP & NP & NP & NP & 128.9 & 113.4 & $\mathrm{NP}$ & NP & NP & NP & 113.4 & 112.8 & $\mathrm{NP}$ & NP & 112.8 & $\mathrm{NP}$ \\
\hline $699-47-80 A$ & $\mathrm{NI}$ & $\mathrm{NI}$ & $\mathrm{NI}$ & $\mathrm{NI}$ & $\mathrm{NI}$ & $\mathrm{NI}$ & $\mathrm{NI}$ & $\mathrm{NI}$ & $\mathrm{NI}$ & $\mathrm{NI}$ & $\mathrm{NI}$ & $\mathrm{NI}$ & $\mathrm{NI}$ & $\mathrm{NI}$ & $\mathrm{NI}$ & $\mathrm{NI}$ & $\mathrm{NI}$ & $\mathrm{NI}$ & 54.9 & $\mathrm{NI}$ \\
\hline 699-47-80B & $\mathrm{NI}$ & $\mathrm{NI}$ & $\mathrm{NI}$ & $\mathrm{NI}$ & $\mathrm{NI}$ & $\mathrm{NI}$ & $\mathrm{NI}$ & $\mathrm{NI}$ & $\mathrm{NI}$ & $\mathrm{NI}$ & $\mathrm{NI}$ & $\mathrm{NI}$ & $\mathrm{NI}$ & $\mathrm{NI}$ & $\mathrm{NI}$ & $\mathrm{NI}$ & $\mathrm{NI}$ & $\mathrm{NI}$ & 54.3 & $\mathrm{NI}$ \\
\hline $699-47-80 \mathrm{C}$ & $\mathrm{NI}$ & $\mathrm{NI}$ & $\mathrm{NI}$ & $\mathrm{NI}$ & $\mathrm{NI}$ & $\mathrm{NI}$ & $\mathrm{NI}$ & $\mathrm{NI}$ & $\mathrm{NI}$ & $\mathrm{NI}$ & $\mathrm{NI}$ & $\mathrm{NI}$ & $\mathrm{NI}$ & $\mathrm{NI}$ & $\mathrm{NI}$ & $\mathrm{NI}$ & $\mathrm{NI}$ & $\mathrm{NI}$ & 54.9 & $\mathrm{NI}$ \\
\hline 699-47-80D & $\mathrm{NI}$ & $\mathrm{NI}$ & $\mathrm{NI}$ & $\mathrm{NI}$ & $\mathrm{NI}$ & $\mathrm{NI}$ & $\mathrm{NI}$ & $\mathrm{NI}$ & $\mathrm{NI}$ & $\mathrm{NI}$ & $\mathrm{NI}$ & $\mathrm{NI}$ & $\mathrm{NI}$ & $\mathrm{NI}$ & $\mathrm{NI}$ & $\mathrm{NI}$ & $\mathrm{NI}$ & $\mathrm{NI}$ & 54.6 & $\mathrm{NI}$ \\
\hline 699-47-92 & 246.0 & 192.6 & NP & NP & 192.6 & 191.1 & 191.1 & 167.3 & 167.3 & 77.4 & NP & NP & NP & NP & 77.4 & 50.3 & 50.3 & 20.4 & 20.4 & NP \\
\hline $699-47-118$ & $\mathrm{NI}$ & $\mathrm{NI}$ & $\mathrm{NI}$ & $\mathrm{NI}$ & $\mathrm{NI}$ & $\mathrm{NI}$ & $\mathrm{NI}$ & $\mathrm{NI}$ & $\mathrm{NI}$ & $\mathrm{NI}$ & $\mathrm{NI}$ & $\mathrm{NI}$ & $\mathrm{NI}$ & $\mathrm{NI}$ & $\mathrm{NI}$ & $\mathrm{NI}$ & $\mathrm{NI}$ & $\mathrm{NI}$ & 155.4 & $\mathrm{NI}$ \\
\hline $699-48-18$ & 129.2 & 106.1 & NP & NP & NP & NP & 106.1 & ND & ND & ND & ND & ND & ND & ND & ND & ND & ND & ND & ND & ND \\
\hline
\end{tabular}


Table A.1. Interpreted Top and Bottom Elevation of Major Hydrogeologic Units (continued)

$\mathrm{NP}=$ unit not present

$\mathrm{NC}=$ not certain whether unit is present or not

$\mathrm{ND}=$ well not deep enough to determine

$\mathrm{NI}=$ not interpreted or unknown

Elevations in m above mean sea level based on the National Geodetic Vertical Datum of 1929 (NGVD29 datum)

\begin{tabular}{|c|c|c|c|c|c|c|c|c|c|c|c|c|c|c|c|c|c|c|c|c|}
\hline Well number & $\begin{array}{l}\text { Casing } \\
\text { Elev. }\end{array}$ & Bot 1 & Top 2 & Bot 2 & Top 3 & Bot 3 & Top 4 & Bot 4 & Top 5 & Bot 5 & Top 6 & Bot 6 & Top 7 & Bot 7 & Top 8 & Bot 8 & Top 9 & Bot 9 & $\begin{array}{l}\text { Top of } \\
\text { Basalt }\end{array}$ & $\begin{array}{l}\text { Layer } 6 \\
\% \text { mud }\end{array}$ \\
\hline $699-48-22$ & 157.3 & 108.5 & NP & NP & NP & NP & NP & NP & 108.5 & 64.0 & NP & NP & NP & NP & 64.0 & 43.3 & NP & NP & 43.3 & NP \\
\hline $699-48-27$ & 160.1 & 106.7 & $\mathrm{NP}$ & NP & NP & NP & 106.7 & 105.2 & $\mathrm{NI}$ & $\mathrm{NI}$ & $\mathrm{NI}$ & $\mathrm{NI}$ & $\mathrm{NI}$ & $\mathrm{NI}$ & 56.7 & 39.0 & NP & NP & 39.0 & $\mathrm{NI}$ \\
\hline $699-48-35$ & 148.1 & $\mathrm{NI}$ & $\mathrm{NI}$ & $\mathrm{NI}$ & $\mathrm{NI}$ & $\mathrm{NI}$ & $\mathrm{NI}$ & $\mathrm{NI}$ & $\mathrm{NI}$ & $\mathrm{NI}$ & $\mathrm{NI}$ & $\mathrm{NI}$ & $\mathrm{NI}$ & $\mathrm{NI}$ & $\mathrm{NI}$ & $\mathrm{NI}$ & $\mathrm{NI}$ & $\mathrm{NI}$ & 97.8 & $\mathrm{NI}$ \\
\hline $699-48-48 A$ & $\mathrm{NI}$ & $\mathrm{NI}$ & $\mathrm{NI}$ & $\mathrm{NI}$ & $\mathrm{NI}$ & $\mathrm{NI}$ & $\mathrm{NI}$ & $\mathrm{NI}$ & $\mathrm{NI}$ & $\mathrm{NI}$ & $\mathrm{NI}$ & $\mathrm{NI}$ & $\mathrm{NI}$ & $\mathrm{NI}$ & $\mathrm{NI}$ & $\mathrm{NI}$ & $\mathrm{NI}$ & $\mathrm{NI}$ & $\mathrm{NI}$ & $\mathrm{NI}$ \\
\hline $699-48-49$ & $\mathrm{NI}$ & $\mathrm{NI}$ & $\mathrm{NI}$ & $\mathrm{NI}$ & $\mathrm{NI}$ & $\mathrm{NI}$ & $\mathrm{NI}$ & $\mathrm{NI}$ & $\mathrm{NI}$ & $\mathrm{NI}$ & $\mathrm{NI}$ & $\mathrm{NI}$ & $\mathrm{NI}$ & $\mathrm{NI}$ & $\mathrm{NI}$ & $\mathrm{NI}$ & $\mathrm{NI}$ & $\mathrm{NI}$ & $\mathrm{NI}$ & $\mathrm{NI}$ \\
\hline 699-48-77C & 205.4 & 197.8 & $\mathrm{NP}$ & NP & 197.8 & 185.6 & 185.6 & 180.1 & 180.1 & 117.0 & NP & NP & NP & NP & NP & NP & 117.0 & 68.0 & 68.0 & NP \\
\hline 699-49-13A & 125.0 & 98.1 & $\mathrm{NP}$ & NP & $\mathrm{NP}$ & NP & 98.1 & ND & ND & ND & ND & ND & ND & ND & ND & ND & ND & ND & ND & ND \\
\hline 699-49-13B & 125.3 & 102.1 & $\mathrm{NP}$ & NP & NP & NP & 102.1 & ND & ND & ND & ND & ND & ND & ND & ND & ND & ND & ND & ND & ND \\
\hline $699-49-13 C$ & 125.9 & 106.7 & $\mathrm{NP}$ & NP & NP & NP & 106.7 & ND & ND & ND & ND & ND & ND & ND & ND & ND & ND & ND & ND & ND \\
\hline 699-49-13E & 126.8 & 103.6 & NP & NP & NP & NP & 103.6 & ND & ND & ND & ND & ND & ND & ND & ND & ND & ND & ND & ND & ND \\
\hline $699-49-21$ & 150.4 & 117.7 & $\mathrm{NP}$ & NP & NP & NP & $\mathrm{NP}$ & NP & NP & NP & NP & NP & NP & NP & NP & NP & NP & NP & 117.7 & NP \\
\hline 699-49-31 & 159.6 & 98.8 & $\mathrm{NP}$ & NP & NP & NP & $\mathrm{NI}$ & $\mathrm{NI}$ & $\mathrm{NI}$ & $\mathrm{NI}$ & $\mathrm{NI}$ & $\mathrm{NI}$ & $\mathrm{NI}$ & $\mathrm{NI}$ & $\mathrm{NI}$ & $\mathrm{NI}$ & $\mathrm{NI}$ & $\mathrm{NI}$ & 39.9 & $\mathrm{NI}$ \\
\hline 699-49-32B & 156.4 & $\mathrm{NI}$ & $\mathrm{NI}$ & $\mathrm{NI}$ & $\mathrm{NI}$ & $\mathrm{NI}$ & $\mathrm{NI}$ & $\mathrm{NI}$ & $\mathrm{NI}$ & $\mathrm{NI}$ & $\mathrm{NI}$ & $\mathrm{NI}$ & $\mathrm{NI}$ & $\mathrm{NI}$ & $\mathrm{NI}$ & $\mathrm{NI}$ & $\mathrm{NI}$ & $\mathrm{NI}$ & 109.7 & $\mathrm{NI}$ \\
\hline $699-49-33$ & 152.9 & 134.7 & $\mathrm{NP}$ & NP & NP & NP & NP & NP & 134.7 & 130.1 & NP & NP & NP & NP & NP & NP & NP & NP & 130.1 & NP \\
\hline 699-49-55A & $\mathrm{NI}$ & $\mathrm{NI}$ & $\mathrm{NI}$ & $\mathrm{NI}$ & $\mathrm{NI}$ & $\mathrm{NI}$ & $\mathrm{NI}$ & $\mathrm{NI}$ & $\mathrm{NI}$ & $\mathrm{NI}$ & $\mathrm{NI}$ & $\mathrm{NI}$ & $\mathrm{NI}$ & $\mathrm{NI}$ & $\mathrm{NI}$ & $\mathrm{NI}$ & $\mathrm{NI}$ & $\mathrm{NI}$ & 120.1 & $\mathrm{NI}$ \\
\hline 699-49-55B & $\mathrm{NI}$ & $\mathrm{NI}$ & $\mathrm{NI}$ & $\mathrm{NI}$ & $\mathrm{NI}$ & $\mathrm{NI}$ & $\mathrm{NI}$ & $\mathrm{NI}$ & $\mathrm{NI}$ & $\mathrm{NI}$ & $\mathrm{NI}$ & $\mathrm{NI}$ & $\mathrm{NI}$ & $\mathrm{NI}$ & $\mathrm{NI}$ & $\mathrm{NI}$ & $\mathrm{NI}$ & $\mathrm{NI}$ & 120.1 & $\mathrm{NI}$ \\
\hline $699-49-57$ & $\mathrm{NI}$ & $\mathrm{NI}$ & $\mathrm{NI}$ & $\mathrm{NI}$ & $\mathrm{NI}$ & $\mathrm{NI}$ & $\mathrm{NI}$ & $\mathrm{NI}$ & $\mathrm{NI}$ & $\mathrm{NI}$ & $\mathrm{NI}$ & $\mathrm{NI}$ & $\mathrm{NI}$ & $\mathrm{NI}$ & $\mathrm{NI}$ & $\mathrm{NI}$ & $\mathrm{NI}$ & $\mathrm{NI}$ & 119.2 & $\mathrm{NI}$ \\
\hline 699-49-79 & 210.0 & 187.1 & NP & NP & 187.1 & 182.6 & 182.6 & 179.5 & 179.5 & ND & ND & ND & ND & ND & ND & ND & ND & ND & ND & ND \\
\hline $699-49-85 A$ & $\mathrm{NI}$ & $\mathrm{NI}$ & $\mathrm{NI}$ & $\mathrm{NI}$ & $\mathrm{NI}$ & $\mathrm{NI}$ & $\mathrm{NI}$ & $\mathrm{NI}$ & $\mathrm{NI}$ & $\mathrm{NI}$ & $\mathrm{NI}$ & $\mathrm{NI}$ & $\mathrm{NI}$ & $\mathrm{NI}$ & $\mathrm{NI}$ & $\mathrm{NI}$ & $\mathrm{NI}$ & $\mathrm{NI}$ & 41.1 & $\mathrm{NI}$ \\
\hline 699-49-85B & $\mathrm{NI}$ & $\mathrm{NI}$ & $\mathrm{NI}$ & $\mathrm{NI}$ & $\mathrm{NI}$ & $\mathrm{NI}$ & $\mathrm{NI}$ & $\mathrm{NI}$ & $\mathrm{NI}$ & $\mathrm{NI}$ & $\mathrm{NI}$ & $\mathrm{NI}$ & $\mathrm{NI}$ & $\mathrm{NI}$ & $\mathrm{NI}$ & $\mathrm{NI}$ & $\mathrm{NI}$ & $\mathrm{NI}$ & 37.2 & $\mathrm{NI}$ \\
\hline $699-49-100 A$ & $\mathrm{NI}$ & $\mathrm{NI}$ & $\mathrm{NI}$ & $\mathrm{NI}$ & $\mathrm{NI}$ & $\mathrm{NI}$ & $\mathrm{NI}$ & $\mathrm{NI}$ & $\mathrm{NI}$ & $\mathrm{NI}$ & $\mathrm{NI}$ & $\mathrm{NI}$ & $\mathrm{NI}$ & $\mathrm{NI}$ & $\mathrm{NI}$ & $\mathrm{NI}$ & $\mathrm{NI}$ & $\mathrm{NI}$ & 116.7 & $\mathrm{NI}$ \\
\hline $699-49-100 C$ & 241.7 & 174.7 & NP & NP & NP & NP & NP & NP & 174.7 & 116.7 & NP & NP & NP & NP & NP & NP & NP & NP & 116.7 & NP \\
\hline $699-49-111$ & $\mathrm{NI}$ & $\mathrm{NI}$ & $\mathrm{NI}$ & $\mathrm{NI}$ & $\mathrm{NI}$ & $\mathrm{NI}$ & $\mathrm{NI}$ & $\mathrm{NI}$ & $\mathrm{NI}$ & $\mathrm{NI}$ & $\mathrm{NI}$ & $\mathrm{NI}$ & $\mathrm{NI}$ & $\mathrm{NI}$ & $\mathrm{NI}$ & $\mathrm{NI}$ & $\mathrm{NI}$ & $\mathrm{NI}$ & 231.6 & $\mathrm{NI}$ \\
\hline $699-50-30$ & $\mathrm{NI}$ & $\mathrm{NI}$ & $\mathrm{NI}$ & $\mathrm{NI}$ & $\mathrm{NI}$ & $\mathrm{NI}$ & $\mathrm{NI}$ & $\mathrm{NI}$ & $\mathrm{NI}$ & $\mathrm{NI}$ & $\mathrm{NI}$ & $\mathrm{NI}$ & $\mathrm{NI}$ & $\mathrm{NI}$ & $\mathrm{NI}$ & $\mathrm{NI}$ & $\mathrm{NI}$ & $\mathrm{NI}$ & 47.5 & $\mathrm{NI}$ \\
\hline $699-50-42$ & $\mathrm{NI}$ & $\mathrm{NI}$ & $\mathrm{NI}$ & $\mathrm{NI}$ & $\mathrm{NI}$ & $\mathrm{NI}$ & $\mathrm{NI}$ & $\mathrm{NI}$ & $\mathrm{NI}$ & $\mathrm{NI}$ & $\mathrm{NI}$ & $\mathrm{NI}$ & $\mathrm{NI}$ & $\mathrm{NI}$ & $\mathrm{NI}$ & $\mathrm{NI}$ & $\mathrm{NI}$ & $\mathrm{NI}$ & 122.5 & $\mathrm{NI}$ \\
\hline 699-50-45 & $\mathrm{NI}$ & $\mathrm{NI}$ & $\mathrm{NI}$ & $\mathrm{NI}$ & $\mathrm{NI}$ & $\mathrm{NI}$ & $\mathrm{NI}$ & $\mathrm{NI}$ & $\mathrm{NI}$ & $\mathrm{NI}$ & $\mathrm{NI}$ & $\mathrm{NI}$ & $\mathrm{NI}$ & $\mathrm{NI}$ & $\mathrm{NI}$ & $\mathrm{NI}$ & $\mathrm{NI}$ & $\mathrm{NI}$ & 126.2 & $\mathrm{NI}$ \\
\hline
\end{tabular}


Table A.1. Interpreted Top and Bottom Elevation of Major Hydrogeologic Units (continued)

$\mathrm{NP}=$ unit not present

$\mathrm{NC}=$ not certain whether unit is present or not

$\mathrm{ND}=$ well not deep enough to determine

$\mathrm{NI}=$ not interpreted or unknown

Elevations in $\mathrm{m}$ above mean sea level based on the National Geodetic Vertical Datum of 1929 (NGVD29 datum)

\begin{tabular}{|c|c|c|c|c|c|c|c|c|c|c|c|c|c|c|c|c|c|c|c|c|}
\hline Well number & $\begin{array}{l}\text { Casing } \\
\text { Elev. }\end{array}$ & Bot 1 & Top 2 & Bot 2 & Top 3 & Bot 3 & Top 4 & Bot 4 & Top 5 & Bot 5 & Top 6 & Bot 6 & Top 7 & Bot 7 & Top 8 & Bot 8 & Top 9 & Bot 9 & $\begin{array}{l}\text { Top of } \\
\text { Basalt }\end{array}$ & $\begin{array}{l}\text { Layer } 6 \\
\% \text { mud }\end{array}$ \\
\hline $699-50-48 A$ & $\mathrm{NI}$ & $\mathrm{NI}$ & $\mathrm{NI}$ & $\mathrm{NI}$ & $\mathrm{NI}$ & $\mathrm{NI}$ & $\mathrm{NI}$ & $\mathrm{NI}$ & $\mathrm{NI}$ & $\mathrm{NI}$ & $\mathrm{NI}$ & $\mathrm{NI}$ & $\mathrm{NI}$ & $\mathrm{NI}$ & $\mathrm{NI}$ & $\mathrm{NI}$ & $\mathrm{NI}$ & $\mathrm{NI}$ & 124.4 & $\mathrm{NI}$ \\
\hline 699-50-48B & $\mathrm{NI}$ & $\mathrm{NI}$ & $\mathrm{NI}$ & $\mathrm{NI}$ & $\mathrm{NI}$ & $\mathrm{NI}$ & $\mathrm{NI}$ & $\mathrm{NI}$ & $\mathrm{NI}$ & $\mathrm{NI}$ & $\mathrm{NI}$ & $\mathrm{NI}$ & $\mathrm{NI}$ & $\mathrm{NI}$ & $\mathrm{NI}$ & $\mathrm{NI}$ & $\mathrm{NI}$ & $\mathrm{NI}$ & 124.7 & $\mathrm{NI}$ \\
\hline 699-50-53B & $\mathrm{NI}$ & $\mathrm{NI}$ & $\mathrm{NI}$ & $\mathrm{NI}$ & $\mathrm{NI}$ & $\mathrm{NI}$ & $\mathrm{NI}$ & $\mathrm{NI}$ & $\mathrm{NI}$ & $\mathrm{NI}$ & $\mathrm{NI}$ & $\mathrm{NI}$ & $\mathrm{NI}$ & $\mathrm{NI}$ & $\mathrm{NI}$ & $\mathrm{NI}$ & $\mathrm{NI}$ & $\mathrm{NI}$ & 121.6 & $\mathrm{NI}$ \\
\hline 699-50-85 & 225.2 & 197.8 & NP & NP & 197.8 & 193.2 & 193.2 & 160.6 & 160.6 & 81.4 & NP & NP & NP & NP & 81.4 & 63.7 & 63.7 & 45.4 & 45.4 & NP \\
\hline $699-50-96$ & 243.8 & 171.9 & NP & NP & $\mathrm{NP}$ & NP & NP & NP & 171.9 & 72.5 & NP & NP & NP & NP & 72.5 & 52.1 & 52.1 & 25.0 & 25.0 & NP \\
\hline $699-50-98$ & $\mathrm{NI}$ & $\mathrm{NI}$ & $\mathrm{NI}$ & $\mathrm{NI}$ & $\mathrm{NI}$ & $\mathrm{NI}$ & $\mathrm{NI}$ & $\mathrm{NI}$ & $\mathrm{NI}$ & $\mathrm{NI}$ & $\mathrm{NI}$ & $\mathrm{NI}$ & $\mathrm{NI}$ & $\mathrm{NI}$ & $\mathrm{NI}$ & $\mathrm{NI}$ & $\mathrm{NI}$ & $\mathrm{NI}$ & 24.4 & $\mathrm{NI}$ \\
\hline 699-51-19 & 129.2 & 109.4 & NP & NP & NP & NP & 109.4 & 104.9 & $\mathrm{NP}$ & NP & NP & NP & NP & NP & NP & $\mathrm{NP}$ & NP & NP & 104.5 & NP \\
\hline 699-51-36A & $\mathrm{NI}$ & $\mathrm{NI}$ & $\mathrm{NI}$ & $\mathrm{NI}$ & $\mathrm{NI}$ & $\mathrm{NI}$ & $\mathrm{NI}$ & $\mathrm{NI}$ & $\mathrm{NI}$ & $\mathrm{NI}$ & $\mathrm{NI}$ & $\mathrm{NI}$ & $\mathrm{NI}$ & $\mathrm{NI}$ & $\mathrm{NI}$ & $\mathrm{NI}$ & $\mathrm{NI}$ & $\mathrm{NI}$ & 148.4 & $\mathrm{NI}$ \\
\hline 699-51-36B & $\mathrm{NI}$ & $\mathrm{NI}$ & $\mathrm{NI}$ & $\mathrm{NI}$ & $\mathrm{NI}$ & $\mathrm{NI}$ & $\mathrm{NI}$ & $\mathrm{NI}$ & $\mathrm{NI}$ & $\mathrm{NI}$ & $\mathrm{NI}$ & $\mathrm{NI}$ & $\mathrm{NI}$ & $\mathrm{NI}$ & $\mathrm{NI}$ & $\mathrm{NI}$ & $\mathrm{NI}$ & $\mathrm{NI}$ & 146.6 & $\mathrm{NI}$ \\
\hline $699-51-36 \mathrm{C}$ & $\mathrm{NI}$ & $\mathrm{NI}$ & $\mathrm{NI}$ & $\mathrm{NI}$ & $\mathrm{NI}$ & $\mathrm{NI}$ & $\mathrm{NI}$ & $\mathrm{NI}$ & $\mathrm{NI}$ & $\mathrm{NI}$ & $\mathrm{NI}$ & $\mathrm{NI}$ & $\mathrm{NI}$ & $\mathrm{NI}$ & $\mathrm{NI}$ & $\mathrm{NI}$ & $\mathrm{NI}$ & $\mathrm{NI}$ & 143.6 & $\mathrm{NI}$ \\
\hline 699-51-36D & $\mathrm{NI}$ & $\mathrm{NI}$ & $\mathrm{NI}$ & $\mathrm{NI}$ & $\mathrm{NI}$ & $\mathrm{NI}$ & $\mathrm{NI}$ & $\mathrm{NI}$ & $\mathrm{NI}$ & $\mathrm{NI}$ & $\mathrm{NI}$ & $\mathrm{NI}$ & $\mathrm{NI}$ & $\mathrm{NI}$ & $\mathrm{NI}$ & $\mathrm{NI}$ & $\mathrm{NI}$ & $\mathrm{NI}$ & 148.4 & $\mathrm{NI}$ \\
\hline 699-51-63 & $\mathrm{NI}$ & $\mathrm{NI}$ & $\mathrm{NI}$ & $\mathrm{NI}$ & $\mathrm{NI}$ & $\mathrm{NI}$ & $\mathrm{NI}$ & $\mathrm{NI}$ & $\mathrm{NI}$ & $\mathrm{NI}$ & $\mathrm{NI}$ & $\mathrm{NI}$ & $\mathrm{NI}$ & $\mathrm{NI}$ & $\mathrm{NI}$ & $\mathrm{NI}$ & $\mathrm{NI}$ & $\mathrm{NI}$ & 118.6 & $\mathrm{NI}$ \\
\hline 699-51-75 & 195.5 & 166.7 & NP & NP & NP & NP & NP & NP & 166.7 & 81.4 & NP & NP & NP & NP & NP & NP & NP & NP & 81.4 & NP \\
\hline $699-52-17$ & 121.5 & 92.7 & NP & NP & NP & NP & 92.7 & 86.6 & 86.6 & 77.4 & 77.4 & 71.3 & 71.3 & 28.7 & 28.7 & 14.9 & NP & NP & 14.9 & 30.5 \\
\hline 699-52-37A & $\mathrm{NI}$ & $\mathrm{NI}$ & $\mathrm{NI}$ & $\mathrm{NI}$ & $\mathrm{NI}$ & $\mathrm{NI}$ & $\mathrm{NI}$ & $\mathrm{NI}$ & $\mathrm{NI}$ & $\mathrm{NI}$ & $\mathrm{NI}$ & $\mathrm{NI}$ & $\mathrm{NI}$ & $\mathrm{NI}$ & $\mathrm{NI}$ & $\mathrm{NI}$ & $\mathrm{NI}$ & $\mathrm{NI}$ & 148.4 & $\mathrm{NI}$ \\
\hline 699-52-37B & $\mathrm{NI}$ & $\mathrm{NI}$ & $\mathrm{NI}$ & $\mathrm{NI}$ & $\mathrm{NI}$ & $\mathrm{NI}$ & $\mathrm{NI}$ & $\mathrm{NI}$ & $\mathrm{NI}$ & $\mathrm{NI}$ & $\mathrm{NI}$ & $\mathrm{NI}$ & $\mathrm{NI}$ & $\mathrm{NI}$ & $\mathrm{NI}$ & $\mathrm{NI}$ & $\mathrm{NI}$ & $\mathrm{NI}$ & 152.1 & $\mathrm{NI}$ \\
\hline 699-52-38A & $\mathrm{NI}$ & $\mathrm{NI}$ & $\mathrm{NI}$ & $\mathrm{NI}$ & $\mathrm{NI}$ & $\mathrm{NI}$ & $\mathrm{NI}$ & $\mathrm{NI}$ & $\mathrm{NI}$ & $\mathrm{NI}$ & $\mathrm{NI}$ & $\mathrm{NI}$ & $\mathrm{NI}$ & $\mathrm{NI}$ & $\mathrm{NI}$ & $\mathrm{NI}$ & $\mathrm{NI}$ & $\mathrm{NI}$ & 152.7 & $\mathrm{NI}$ \\
\hline 699-52-46A & 138.7 & $\mathrm{NI}$ & $\mathrm{NI}$ & $\mathrm{NI}$ & $\mathrm{NI}$ & $\mathrm{NI}$ & $\mathrm{NI}$ & $\mathrm{NI}$ & $\mathrm{NI}$ & $\mathrm{NI}$ & $\mathrm{NI}$ & $\mathrm{NI}$ & $\mathrm{NI}$ & $\mathrm{NI}$ & $\mathrm{NI}$ & $\mathrm{NI}$ & $\mathrm{NI}$ & $\mathrm{NI}$ & 123.4 & $\mathrm{NI}$ \\
\hline 699-52-48 & $\mathrm{NI}$ & $\mathrm{NI}$ & $\mathrm{NI}$ & $\mathrm{NI}$ & $\mathrm{NI}$ & $\mathrm{NI}$ & $\mathrm{NI}$ & $\mathrm{NI}$ & $\mathrm{NI}$ & $\mathrm{NI}$ & $\mathrm{NI}$ & $\mathrm{NI}$ & $\mathrm{NI}$ & $\mathrm{NI}$ & $\mathrm{NI}$ & $\mathrm{NI}$ & $\mathrm{NI}$ & $\mathrm{NI}$ & 133.5 & $\mathrm{NI}$ \\
\hline $699-52-52$ & $\mathrm{NI}$ & $\mathrm{NI}$ & $\mathrm{NI}$ & $\mathrm{NI}$ & $\mathrm{NI}$ & $\mathrm{NI}$ & $\mathrm{NI}$ & $\mathrm{NI}$ & $\mathrm{NI}$ & $\mathrm{NI}$ & $\mathrm{NI}$ & $\mathrm{NI}$ & $\mathrm{NI}$ & $\mathrm{NI}$ & $\mathrm{NI}$ & $\mathrm{NI}$ & $\mathrm{NI}$ & $\mathrm{NI}$ & 125.9 & $\mathrm{NI}$ \\
\hline $699-52-54$ & 173.1 & 122.2 & NP & NP & NP & NP & NP & NP & NP & NP & NP & NP & NP & NP & NP & NP & NP & NP & 122.2 & NP \\
\hline $699-52-57$ & 171.3 & ND & ND & ND & ND & ND & ND & ND & ND & ND & ND & ND & ND & ND & ND & ND & ND & ND & ND & ND \\
\hline $699-52-117$ & $\mathrm{NI}$ & $\mathrm{NI}$ & $\mathrm{NI}$ & $\mathrm{NI}$ & $\mathrm{NI}$ & $\mathrm{NI}$ & $\mathrm{NI}$ & $\mathrm{NI}$ & $\mathrm{NI}$ & $\mathrm{NI}$ & $\mathrm{NI}$ & $\mathrm{NI}$ & $\mathrm{NI}$ & $\mathrm{NI}$ & $\mathrm{NI}$ & $\mathrm{NI}$ & $\mathrm{NI}$ & $\mathrm{NI}$ & 284.4 & $\mathrm{NI}$ \\
\hline 699-52-118 & $\mathrm{NI}$ & $\mathrm{NI}$ & $\mathrm{NI}$ & $\mathrm{NI}$ & $\mathrm{NI}$ & $\mathrm{NI}$ & $\mathrm{NI}$ & $\mathrm{NI}$ & $\mathrm{NI}$ & $\mathrm{NI}$ & $\mathrm{NI}$ & $\mathrm{NI}$ & $\mathrm{NI}$ & $\mathrm{NI}$ & $\mathrm{NI}$ & $\mathrm{NI}$ & $\mathrm{NI}$ & $\mathrm{NI}$ & 338.3 & $\mathrm{NI}$ \\
\hline 699-53-47A & $\mathrm{NI}$ & $\mathrm{NI}$ & $\mathrm{NI}$ & $\mathrm{NI}$ & $\mathrm{NI}$ & $\mathrm{NI}$ & $\mathrm{NI}$ & $\mathrm{NI}$ & $\mathrm{NI}$ & $\mathrm{NI}$ & $\mathrm{NI}$ & $\mathrm{NI}$ & $\mathrm{NI}$ & $\mathrm{NI}$ & $\mathrm{NI}$ & $\mathrm{NI}$ & $\mathrm{NI}$ & $\mathrm{NI}$ & 124.4 & $\mathrm{NI}$ \\
\hline 699-53-47B & $\mathrm{NI}$ & $\mathrm{NI}$ & $\mathrm{NI}$ & $\mathrm{NI}$ & $\mathrm{NI}$ & $\mathrm{NI}$ & $\mathrm{NI}$ & $\mathrm{NI}$ & $\mathrm{NI}$ & $\mathrm{NI}$ & $\mathrm{NI}$ & $\mathrm{NI}$ & $\mathrm{NI}$ & $\mathrm{NI}$ & $\mathrm{NI}$ & $\mathrm{NI}$ & $\mathrm{NI}$ & $\mathrm{NI}$ & 124.7 & $\mathrm{NI}$ \\
\hline
\end{tabular}


Table A.1. Interpreted Top and Bottom Elevation of Major Hydrogeologic Units (continued)

$\mathrm{NP}=$ unit not present

$\mathrm{NC}=$ not certain whether unit is present or not

$\mathrm{ND}=$ well not deep enough to determine

$\mathrm{NI}=$ not interpreted or unknown

Elevations in $\mathrm{m}$ above mean sea level based on the National Geodetic Vertical Datum of 1929 (NGVD29 datum)

\begin{tabular}{|c|c|c|c|c|c|c|c|c|c|c|c|c|c|c|c|c|c|c|c|c|}
\hline Well number & $\begin{array}{l}\text { Casing } \\
\text { Elev. }\end{array}$ & Bot 1 & Top 2 & Bot 2 & Top 3 & Bot 3 & Top 4 & Bot 4 & Top 5 & Bot 5 & Top 6 & Bot 6 & Top 7 & Bot 7 & Top 8 & Bot 8 & Top 9 & Bot 9 & $\begin{array}{l}\text { Top of } \\
\text { Basalt }\end{array}$ & $\begin{array}{l}\text { Layer } 6 \\
\% \text { mud }\end{array}$ \\
\hline $699-53-48 A$ & $\mathrm{NI}$ & $\mathrm{NI}$ & $\mathrm{NI}$ & $\mathrm{NI}$ & $\mathrm{NI}$ & $\mathrm{NI}$ & $\mathrm{NI}$ & $\mathrm{NI}$ & $\mathrm{NI}$ & $\mathrm{NI}$ & $\mathrm{NI}$ & $\mathrm{NI}$ & $\mathrm{NI}$ & $\mathrm{NI}$ & $\mathrm{NI}$ & $\mathrm{NI}$ & $\mathrm{NI}$ & $\mathrm{NI}$ & 121.9 & $\mathrm{NI}$ \\
\hline 699-53-50 & $\mathrm{NI}$ & $\mathrm{NI}$ & $\mathrm{NI}$ & $\mathrm{NI}$ & $\mathrm{NI}$ & $\mathrm{NI}$ & $\mathrm{NI}$ & $\mathrm{NI}$ & $\mathrm{NI}$ & $\mathrm{NI}$ & $\mathrm{NI}$ & $\mathrm{NI}$ & $\mathrm{NI}$ & $\mathrm{NI}$ & $\mathrm{NI}$ & $\mathrm{NI}$ & $\mathrm{NI}$ & $\mathrm{NI}$ & 124.7 & $\mathrm{NI}$ \\
\hline $699-53-55 A$ & 175.9 & 85.3 & NP & NP & NP & NP & NP & NP & NP & NP & NP & NP & NP & NP & NP & NP & NP & NP & 85.3 & NP \\
\hline $699-53-103$ & 255.4 & $\mathrm{NI}$ & $\mathrm{NI}$ & $\mathrm{NI}$ & $\mathrm{NI}$ & $\mathrm{NI}$ & $\mathrm{NI}$ & $\mathrm{NI}$ & $\mathrm{NI}$ & $\mathrm{NI}$ & $\mathrm{NI}$ & $\mathrm{NI}$ & $\mathrm{NI}$ & $\mathrm{NI}$ & $\mathrm{NI}$ & $\mathrm{NI}$ & $\mathrm{NI}$ & $\mathrm{NI}$ & 78.9 & $\mathrm{NI}$ \\
\hline 699-53-111 & $\mathrm{NI}$ & $\mathrm{NI}$ & $\mathrm{NI}$ & $\mathrm{NI}$ & $\mathrm{NI}$ & $\mathrm{NI}$ & $\mathrm{NI}$ & $\mathrm{NI}$ & $\mathrm{NI}$ & $\mathrm{NI}$ & $\mathrm{NI}$ & $\mathrm{NI}$ & $\mathrm{NI}$ & $\mathrm{NI}$ & $\mathrm{NI}$ & $\mathrm{NI}$ & $\mathrm{NI}$ & $\mathrm{NI}$ & 229.2 & $\mathrm{NI}$ \\
\hline $699-53-114$ & $\mathrm{NI}$ & $\mathrm{NI}$ & $\mathrm{NI}$ & $\mathrm{NI}$ & $\mathrm{NI}$ & $\mathrm{NI}$ & $\mathrm{NI}$ & $\mathrm{NI}$ & $\mathrm{NI}$ & $\mathrm{NI}$ & $\mathrm{NI}$ & $\mathrm{NI}$ & $\mathrm{NI}$ & $\mathrm{NI}$ & $\mathrm{NI}$ & $\mathrm{NI}$ & $\mathrm{NI}$ & $\mathrm{NI}$ & 257.6 & $\mathrm{NI}$ \\
\hline $699-54-18 C$ & 122.6 & 89.6 & NP & NP & NP & NP & 89.6 & 82.0 & 82.0 & 61.6 & 61.6 & 40.8 & 40.8 & 31.1 & 31.1 & 18.0 & 18.0 & 15.8 & 15.8 & 26.5 \\
\hline 699-54-34 & $\mathrm{NI}$ & $\mathrm{NI}$ & $\mathrm{NI}$ & $\mathrm{NI}$ & $\mathrm{NI}$ & $\mathrm{NI}$ & $\mathrm{NI}$ & $\mathrm{NI}$ & $\mathrm{NI}$ & $\mathrm{NI}$ & $\mathrm{NI}$ & $\mathrm{NI}$ & $\mathrm{NI}$ & $\mathrm{NI}$ & $\mathrm{NI}$ & $\mathrm{NI}$ & $\mathrm{NI}$ & $\mathrm{NI}$ & 121.9 & $\mathrm{NI}$ \\
\hline 699-54-37B & $\mathrm{NI}$ & $\mathrm{NI}$ & $\mathrm{NI}$ & $\mathrm{NI}$ & $\mathrm{NI}$ & $\mathrm{NI}$ & $\mathrm{NI}$ & $\mathrm{NI}$ & $\mathrm{NI}$ & $\mathrm{NI}$ & $\mathrm{NI}$ & $\mathrm{NI}$ & $\mathrm{NI}$ & $\mathrm{NI}$ & $\mathrm{NI}$ & $\mathrm{NI}$ & $\mathrm{NI}$ & $\mathrm{NI}$ & 121.0 & $\mathrm{NI}$ \\
\hline $699-54-42$ & $\mathrm{NI}$ & $\mathrm{NI}$ & $\mathrm{NI}$ & $\mathrm{NI}$ & $\mathrm{NI}$ & $\mathrm{NI}$ & $\mathrm{NI}$ & $\mathrm{NI}$ & $\mathrm{NI}$ & $\mathrm{NI}$ & $\mathrm{NI}$ & $\mathrm{NI}$ & $\mathrm{NI}$ & $\mathrm{NI}$ & $\mathrm{NI}$ & $\mathrm{NI}$ & $\mathrm{NI}$ & $\mathrm{NI}$ & 95.7 & $\mathrm{NI}$ \\
\hline 699-54-45B & $\mathrm{NI}$ & $\mathrm{NI}$ & $\mathrm{NI}$ & $\mathrm{NI}$ & $\mathrm{NI}$ & $\mathrm{NI}$ & $\mathrm{NI}$ & $\mathrm{NI}$ & $\mathrm{NI}$ & $\mathrm{NI}$ & $\mathrm{NI}$ & $\mathrm{NI}$ & $\mathrm{NI}$ & $\mathrm{NI}$ & $\mathrm{NI}$ & $\mathrm{NI}$ & $\mathrm{NI}$ & $\mathrm{NI}$ & 96.9 & $\mathrm{NI}$ \\
\hline $699-54-48$ & $\mathrm{NI}$ & $\mathrm{NI}$ & $\mathrm{NI}$ & $\mathrm{NI}$ & $\mathrm{NI}$ & $\mathrm{NI}$ & $\mathrm{NI}$ & $\mathrm{NI}$ & $\mathrm{NI}$ & $\mathrm{NI}$ & $\mathrm{NI}$ & $\mathrm{NI}$ & $\mathrm{NI}$ & $\mathrm{NI}$ & $\mathrm{NI}$ & $\mathrm{NI}$ & $\mathrm{NI}$ & $\mathrm{NI}$ & 110.6 & $\mathrm{NI}$ \\
\hline $699-54-57$ & $\mathrm{NI}$ & $\mathrm{NI}$ & $\mathrm{NI}$ & $\mathrm{NI}$ & $\mathrm{NI}$ & $\mathrm{NI}$ & $\mathrm{NI}$ & $\mathrm{NI}$ & $\mathrm{NI}$ & $\mathrm{NI}$ & $\mathrm{NI}$ & $\mathrm{NI}$ & $\mathrm{NI}$ & $\mathrm{NI}$ & $\mathrm{NI}$ & $\mathrm{NI}$ & $\mathrm{NI}$ & $\mathrm{NI}$ & 121.0 & $\mathrm{NI}$ \\
\hline $699-55-50 \mathrm{~A}$ & 135.0 & 110.6 & NP & NP & $\mathrm{NP}$ & $\mathrm{NP}$ & $\mathrm{NP}$ & $\mathrm{NP}$ & $\mathrm{NP}$ & NP & $\mathrm{NP}$ & $\mathrm{NP}$ & $\mathrm{NP}$ & $\mathrm{NP}$ & NP & NP & 110.6 & 104.5 & 104.5 & NP \\
\hline 699-55-50B & 135.0 & 112.2 & NP & NP & NP & NP & NP & NP & NP & NP & NP & NP & NP & NP & NP & NP & 112.2 & 106.7 & 106.7 & NP \\
\hline 699-55-50D & $\mathrm{NI}$ & $\mathrm{NI}$ & $\mathrm{NI}$ & $\mathrm{NI}$ & $\mathrm{NI}$ & $\mathrm{NI}$ & $\mathrm{NI}$ & $\mathrm{NI}$ & $\mathrm{NI}$ & $\mathrm{NI}$ & $\mathrm{NI}$ & $\mathrm{NI}$ & $\mathrm{NI}$ & $\mathrm{NI}$ & $\mathrm{NI}$ & $\mathrm{NI}$ & $\mathrm{NI}$ & $\mathrm{NI}$ & 106.4 & $\mathrm{NI}$ \\
\hline $699-55-57$ & $\mathrm{NI}$ & $\mathrm{NI}$ & $\mathrm{NI}$ & $\mathrm{NI}$ & $\mathrm{NI}$ & $\mathrm{NI}$ & $\mathrm{NI}$ & $\mathrm{NI}$ & $\mathrm{NI}$ & $\mathrm{NI}$ & $\mathrm{NI}$ & $\mathrm{NI}$ & $\mathrm{NI}$ & $\mathrm{NI}$ & $\mathrm{NI}$ & $\mathrm{NI}$ & $\mathrm{NI}$ & $\mathrm{NI}$ & 117.3 & $\mathrm{NI}$ \\
\hline $699-55-60 A$ & 174.7 & ND & ND & ND & ND & ND & ND & ND & ND & ND & ND & ND & ND & ND & ND & ND & ND & ND & ND & ND \\
\hline $699-55-63$ & $\mathrm{NI}$ & $\mathrm{NI}$ & $\mathrm{NI}$ & $\mathrm{NI}$ & $\mathrm{NI}$ & $\mathrm{NI}$ & $\mathrm{NI}$ & $\mathrm{NI}$ & $\mathrm{NI}$ & $\mathrm{NI}$ & $\mathrm{NI}$ & $\mathrm{NI}$ & $\mathrm{NI}$ & $\mathrm{NI}$ & $\mathrm{NI}$ & $\mathrm{NI}$ & $\mathrm{NI}$ & $\mathrm{NI}$ & 119.8 & $\mathrm{NI}$ \\
\hline 699-55-65B & $\mathrm{NI}$ & $\mathrm{NI}$ & $\mathrm{NI}$ & $\mathrm{NI}$ & $\mathrm{NI}$ & $\mathrm{NI}$ & $\mathrm{NI}$ & $\mathrm{NI}$ & $\mathrm{NI}$ & $\mathrm{NI}$ & $\mathrm{NI}$ & $\mathrm{NI}$ & $\mathrm{NI}$ & $\mathrm{NI}$ & $\mathrm{NI}$ & $\mathrm{NI}$ & $\mathrm{NI}$ & $\mathrm{NI}$ & 134.1 & $\mathrm{NI}$ \\
\hline $699-55-65 C$ & $\mathrm{NI}$ & $\mathrm{NI}$ & $\mathrm{NI}$ & $\mathrm{NI}$ & $\mathrm{NI}$ & $\mathrm{NI}$ & $\mathrm{NI}$ & $\mathrm{NI}$ & $\mathrm{NI}$ & $\mathrm{NI}$ & $\mathrm{NI}$ & $\mathrm{NI}$ & $\mathrm{NI}$ & $\mathrm{NI}$ & $\mathrm{NI}$ & $\mathrm{NI}$ & $\mathrm{NI}$ & $\mathrm{NI}$ & 134.1 & $\mathrm{NI}$ \\
\hline $699-55-70$ & $\mathrm{NI}$ & $\mathrm{NI}$ & $\mathrm{NI}$ & $\mathrm{NI}$ & $\mathrm{NI}$ & $\mathrm{NI}$ & $\mathrm{NI}$ & $\mathrm{NI}$ & $\mathrm{NI}$ & $\mathrm{NI}$ & $\mathrm{NI}$ & $\mathrm{NI}$ & $\mathrm{NI}$ & $\mathrm{NI}$ & $\mathrm{NI}$ & $\mathrm{NI}$ & $\mathrm{NI}$ & $\mathrm{NI}$ & 112.5 & $\mathrm{NI}$ \\
\hline $699-55-76$ & 177.7 & 132.9 & NP & $\mathrm{NP}$ & 132.9 & 131.4 & $\mathrm{NP}$ & $\mathrm{NP}$ & 131.4 & 109.7 & NP & $\mathrm{NP}$ & $\mathrm{NP}$ & NP & NP & NP & $\mathrm{NP}$ & $\mathrm{NP}$ & 109.7 & $\mathrm{NP}$ \\
\hline $699-55-89$ & 188.1 & 151.5 & NP & $\mathrm{NP}$ & 151.5 & 149.4 & $\mathrm{NP}$ & $\mathrm{NP}$ & 149.4 & ND & ND & ND & ND & ND & ND & ND & ND & ND & ND & ND \\
\hline $699-55-95$ & 236.8 & 206.3 & NP & NP & NP & NP & NP & NP & 206.3 & 116.4 & NP & NP & NP & NP & 116.4 & 99.7 & 99.7 & 80.8 & 80.8 & NP \\
\hline 699-56-26A & 124.7 & 123.7 & NP & NP & NP & NP & $\mathrm{NP}$ & NP & NP & NP & NP & NP & NP & NP & NP & $\mathrm{NP}$ & $\mathrm{NP}$ & $\mathrm{NP}$ & 123.7 & $\mathrm{NP}$ \\
\hline
\end{tabular}


Table A.1. Interpreted Top and Bottom Elevation of Major Hydrogeologic Units (continued)

$\mathrm{NP}=$ unit not present

$\mathrm{NC}=$ not certain whether unit is present or not

$\mathrm{ND}=$ well not deep enough to determine

$\mathrm{NI}=$ not interpreted or unknown

Elevations in $\mathrm{m}$ above mean sea level based on the National Geodetic Vertical Datum of 1929 (NGVD29 datum)

\begin{tabular}{|c|c|c|c|c|c|c|c|c|c|c|c|c|c|c|c|c|c|c|c|c|}
\hline Well number & $\begin{array}{l}\text { Casing } \\
\text { Elev. }\end{array}$ & Bot 1 & Top 2 & Bot 2 & Top 3 & Bot 3 & Top 4 & Bot 4 & Top 5 & Bot 5 & Top 6 & Bot 6 & Top 7 & Bot 7 & Top 8 & Bot 8 & Top 9 & Bot 9 & $\begin{array}{l}\text { Top of } \\
\text { Basalt }\end{array}$ & $\begin{array}{l}\text { Layer } 6 \\
\% \text { mud }\end{array}$ \\
\hline $699-56-40 A$ & $\mathrm{NI}$ & $\mathrm{NI}$ & $\mathrm{NI}$ & $\mathrm{NI}$ & $\mathrm{NI}$ & $\mathrm{NI}$ & $\mathrm{NI}$ & $\mathrm{NI}$ & $\mathrm{NI}$ & $\mathrm{NI}$ & $\mathrm{NI}$ & $\mathrm{NI}$ & $\mathrm{NI}$ & $\mathrm{NI}$ & $\mathrm{NI}$ & $\mathrm{NI}$ & $\mathrm{NI}$ & $\mathrm{NI}$ & 154.8 & $\mathrm{NI}$ \\
\hline 699-56-40B & $\mathrm{NI}$ & $\mathrm{NI}$ & $\mathrm{NI}$ & $\mathrm{NI}$ & $\mathrm{NI}$ & $\mathrm{NI}$ & $\mathrm{NI}$ & $\mathrm{NI}$ & $\mathrm{NI}$ & $\mathrm{NI}$ & $\mathrm{NI}$ & $\mathrm{NI}$ & $\mathrm{NI}$ & $\mathrm{NI}$ & $\mathrm{NI}$ & $\mathrm{NI}$ & $\mathrm{NI}$ & $\mathrm{NI}$ & 176.5 & $\mathrm{NI}$ \\
\hline $699-56-40 \mathrm{C}$ & $\mathrm{NI}$ & $\mathrm{NI}$ & $\mathrm{NI}$ & $\mathrm{NI}$ & $\mathrm{NI}$ & $\mathrm{NI}$ & $\mathrm{NI}$ & $\mathrm{NI}$ & $\mathrm{NI}$ & $\mathrm{NI}$ & $\mathrm{NI}$ & $\mathrm{NI}$ & $\mathrm{NI}$ & $\mathrm{NI}$ & $\mathrm{NI}$ & $\mathrm{NI}$ & $\mathrm{NI}$ & $\mathrm{NI}$ & 168.6 & $\mathrm{NI}$ \\
\hline $699-56-41$ & $\mathrm{NI}$ & $\mathrm{NI}$ & $\mathrm{NI}$ & $\mathrm{NI}$ & $\mathrm{NI}$ & $\mathrm{NI}$ & $\mathrm{NI}$ & $\mathrm{NI}$ & $\mathrm{NI}$ & $\mathrm{NI}$ & $\mathrm{NI}$ & $\mathrm{NI}$ & $\mathrm{NI}$ & $\mathrm{NI}$ & $\mathrm{NI}$ & $\mathrm{NI}$ & $\mathrm{NI}$ & $\mathrm{NI}$ & 166.7 & $\mathrm{NI}$ \\
\hline $699-56-43$ & $\mathrm{NI}$ & $\mathrm{NI}$ & $\mathrm{NI}$ & $\mathrm{NI}$ & $\mathrm{NI}$ & $\mathrm{NI}$ & $\mathrm{NI}$ & $\mathrm{NI}$ & $\mathrm{NI}$ & $\mathrm{NI}$ & $\mathrm{NI}$ & $\mathrm{NI}$ & $\mathrm{NI}$ & $\mathrm{NI}$ & $\mathrm{NI}$ & $\mathrm{NI}$ & $\mathrm{NI}$ & $\mathrm{NI}$ & 148.4 & $\mathrm{NI}$ \\
\hline $699-56-53$ & 132.3 & 101.8 & NP & NP & NP & NP & NP & NP & NP & NP & NP & NP & NP & NP & NP & NP & NP & NP & 101.8 & NP \\
\hline $699-57-83 A$ & 176.2 & 114.3 & NP & NP & NP & NP & NP & NP & $\mathrm{NP}$ & NP & NP & NP & $\mathrm{NP}$ & $\mathrm{NP}$ & 114.3 & 93.9 & 93.9 & 71.6 & 71.6 & NP \\
\hline $699-57-83 C$ & $\mathrm{NI}$ & $\mathrm{NI}$ & $\mathrm{NI}$ & $\mathrm{NI}$ & $\mathrm{NI}$ & $\mathrm{NI}$ & $\mathrm{NI}$ & $\mathrm{NI}$ & $\mathrm{NI}$ & $\mathrm{NI}$ & $\mathrm{NI}$ & $\mathrm{NI}$ & $\mathrm{NI}$ & $\mathrm{NI}$ & $\mathrm{NI}$ & $\mathrm{NI}$ & $\mathrm{NI}$ & $\mathrm{NI}$ & 63.7 & $\mathrm{NI}$ \\
\hline $699-58-40$ & 226.2 & $\mathrm{NP}$ & NP & NP & $\mathrm{NP}$ & $\mathrm{NP}$ & $\mathrm{NP}$ & $\mathrm{NP}$ & $\mathrm{NP}$ & NP & NP & $\mathrm{NP}$ & $\mathrm{NP}$ & $\mathrm{NP}$ & NP & NP & $\mathrm{NP}$ & $\mathrm{NP}$ & 226.2 & NP \\
\hline $699-58-41 \mathrm{~A}$ & 215.2 & $\mathrm{NP}$ & NP & NP & $\mathrm{NP}$ & NP & NP & $\mathrm{NP}$ & NP & $\mathrm{NP}$ & NP & NP & NP & NP & NP & NP & $\mathrm{NP}$ & NP & 215.2 & NP \\
\hline $699-59-44$ & $\mathrm{NI}$ & $\mathrm{NI}$ & $\mathrm{NI}$ & $\mathrm{NI}$ & $\mathrm{NI}$ & $\mathrm{NI}$ & $\mathrm{NI}$ & $\mathrm{NI}$ & $\mathrm{NI}$ & $\mathrm{NI}$ & $\mathrm{NI}$ & $\mathrm{NI}$ & $\mathrm{NI}$ & $\mathrm{NI}$ & $\mathrm{NI}$ & $\mathrm{NI}$ & $\mathrm{NI}$ & $\mathrm{NI}$ & 226.5 & $\mathrm{NI}$ \\
\hline 699-59-80B & $\mathrm{NI}$ & $\mathrm{NI}$ & $\mathrm{NI}$ & $\mathrm{NI}$ & $\mathrm{NI}$ & $\mathrm{NI}$ & $\mathrm{NI}$ & $\mathrm{NI}$ & $\mathrm{NI}$ & $\mathrm{NI}$ & $\mathrm{NI}$ & $\mathrm{NI}$ & $\mathrm{NI}$ & $\mathrm{NI}$ & $\mathrm{NI}$ & $\mathrm{NI}$ & $\mathrm{NI}$ & $\mathrm{NI}$ & 120.7 & $\mathrm{NI}$ \\
\hline $699-59-101$ & $\mathrm{NI}$ & $\mathrm{NI}$ & $\mathrm{NI}$ & $\mathrm{NI}$ & $\mathrm{NI}$ & $\mathrm{NI}$ & $\mathrm{NI}$ & $\mathrm{NI}$ & $\mathrm{NI}$ & $\mathrm{NI}$ & $\mathrm{NI}$ & $\mathrm{NI}$ & $\mathrm{NI}$ & $\mathrm{NI}$ & $\mathrm{NI}$ & $\mathrm{NI}$ & $\mathrm{NI}$ & $\mathrm{NI}$ & 141.1 & $\mathrm{NI}$ \\
\hline $699-60-32$ & 129.5 & 109.7 & NP & NP & $\mathrm{NP}$ & NP & $\mathrm{NP}$ & $\mathrm{NP}$ & $\mathrm{NP}$ & NP & 109.7 & 105.5 & $\mathrm{NP}$ & $\mathrm{NP}$ & NP & NP & $\mathrm{NP}$ & $\mathrm{NP}$ & 105.5 & 30.5 \\
\hline $699-60-57$ & 143.1 & 99.1 & NP & $\mathrm{NP}$ & NP & NP & NP & $\mathrm{NP}$ & $\mathrm{NP}$ & $\mathrm{NP}$ & NP & NP & NP & NP & NP & NP & NP & NP & 99.1 & NP \\
\hline $699-60-60$ & $\mathrm{NI}$ & $\mathrm{NI}$ & $\mathrm{NI}$ & $\mathrm{NI}$ & $\mathrm{NI}$ & $\mathrm{NI}$ & $\mathrm{NI}$ & $\mathrm{NI}$ & $\mathrm{NI}$ & $\mathrm{NI}$ & $\mathrm{NI}$ & $\mathrm{NI}$ & $\mathrm{NI}$ & $\mathrm{NI}$ & $\mathrm{NI}$ & $\mathrm{NI}$ & $\mathrm{NI}$ & $\mathrm{NI}$ & 116.1 & $\mathrm{NI}$ \\
\hline $699-61-16 A$ & 125.6 & NP & NP & NP & NP & NP & 125.6 & 108.2 & NP & NP & 108.2 & 63.1 & 63.1 & 54.6 & 54.6 & 33.2 & NP & NP & 33.2 & 30.5 \\
\hline $699-61-53$ & $\mathrm{NI}$ & $\mathrm{NI}$ & $\mathrm{NI}$ & $\mathrm{NI}$ & $\mathrm{NI}$ & $\mathrm{NI}$ & $\mathrm{NI}$ & $\mathrm{NI}$ & $\mathrm{NI}$ & $\mathrm{NI}$ & $\mathrm{NI}$ & $\mathrm{NI}$ & $\mathrm{NI}$ & \#VALUE! & $\mathrm{NI}$ & $\mathrm{NI}$ & $\mathrm{NI}$ & $\mathrm{NI}$ & 232.9 & $\mathrm{NI}$ \\
\hline 699-61-62 & 151.5 & 97.5 & NP & $\mathrm{NP}$ & $\mathrm{NP}$ & NP & $\mathrm{NP}$ & $\mathrm{NP}$ & $\mathrm{NP}$ & NP & NP & NP & $\mathrm{NP}$ & NP & $\mathrm{NP}$ & NP & $\mathrm{NP}$ & $\mathrm{NP}$ & 97.5 & $\mathrm{NP}$ \\
\hline $699-61-55 A$ & 140.7 & 138.1 & NP & NP & NP & NP & NP & NP & NP & NP & NP & NP & NP & NP & 138.1 & 137.2 & NP & NP & 137.2 & NP \\
\hline 699-61-66 & 159.1 & 110.3 & $\mathrm{NP}$ & $\mathrm{NP}$ & $\mathrm{NP}$ & NP & NP & $\mathrm{NP}$ & 110.3 & 93.3 & NP & $\mathrm{NP}$ & NP & $\mathrm{NP}$ & NP & NP & $\mathrm{NP}$ & $\mathrm{NP}$ & 93.3 & NP \\
\hline $699-62-43 C$ & 130.8 & 110.3 & $\mathrm{NP}$ & $\mathrm{NP}$ & $\mathrm{NP}$ & NP & NP & NP & NP & $\mathrm{NP}$ & 110.3 & ND & ND & ND & ND & ND & ND & ND & ND & $\mathrm{NI}$ \\
\hline $699-62-43 F$ & 129.0 & 107.6 & NP & NP & NP & NP & NP & NP & NP & NP & 107.6 & ND & ND & ND & ND & ND & ND & ND & ND & $\mathrm{NI}$ \\
\hline $699-62-53$ & $\mathrm{NI}$ & $\mathrm{NI}$ & $\mathrm{NI}$ & $\mathrm{NI}$ & $\mathrm{NI}$ & $\mathrm{NI}$ & $\mathrm{NI}$ & $\mathrm{NI}$ & $\mathrm{NI}$ & $\mathrm{NI}$ & $\mathrm{NI}$ & $\mathrm{NI}$ & $\mathrm{NI}$ & $\mathrm{NI}$ & $\mathrm{NI}$ & $\mathrm{NI}$ & $\mathrm{NI}$ & $\mathrm{NI}$ & 135.3 & $\mathrm{NI}$ \\
\hline $699-63-25 A$ & 120.4 & 101.5 & NP & NP & NP & NP & NP & NP & 101.5 & 96.3 & 96.3 & ND & ND & ND & ND & ND & ND & ND & ND & $\mathrm{NI}$ \\
\hline 699-63-55 & 130.1 & 105.2 & $\mathrm{NP}$ & NP & NP & NP & $\mathrm{NP}$ & NP & NP & $N P$ & 105.2 & 103.3 & 103.3 & 96.0 & NP & NP & $\mathrm{NP}$ & $\mathrm{NP}$ & 96.0 & 30.5 \\
\hline
\end{tabular}


Table A.1. Interpreted Top and Bottom Elevation of Major Hydrogeologic Units (continued)

$\mathrm{NP}=$ unit not present

$\mathrm{NC}=$ not certain whether unit is present or not

$\mathrm{ND}=$ well not deep enough to determine

$\mathrm{NI}=$ not interpreted or unknown

Elevations in $\mathrm{m}$ above mean sea level based on the National Geodetic Vertical Datum of 1929 (NGVD29 datum)

\begin{tabular}{|c|c|c|c|c|c|c|c|c|c|c|c|c|c|c|c|c|c|c|c|c|}
\hline Well number & $\begin{array}{l}\text { Casing } \\
\text { Elev. }\end{array}$ & Bot 1 & Top 2 & Bot 2 & Top 3 & Bot 3 & Top 4 & Bot 4 & Top 5 & Bot 5 & Top 6 & Bot 6 & Top 7 & Bot 7 & Top 8 & Bot 8 & Top 9 & Bot 9 & $\begin{array}{l}\text { Top of } \\
\text { Basalt }\end{array}$ & $\begin{array}{l}\text { Layer } 6 \\
\% \text { mud }\end{array}$ \\
\hline $699-63-58$ & 149.9 & 116.4 & NP & NP & NP & NP & NP & NP & 116.4 & 113.4 & NP & NP & NP & NP & NP & NP & NP & NP & 113.4 & ND \\
\hline 699-63-89 & 156.2 & 122.5 & NP & NP & NP & NP & NP & NP & 122.5 & 101.8 & NP & NP & NP & NP & NP & NP & NP & NP & 101.8 & NP \\
\hline 699-63-90 & 155.4 & 117.3 & $\mathrm{NP}$ & NP & NP & NP & NP & NP & 117.3 & 82.9 & NP & NP & NP & NP & NP & NP & NP & NP & 82.9 & NP \\
\hline 699-63-92 & 151.5 & 104.9 & $\mathrm{NP}$ & NP & NP & NP & NP & NP & NP & $\mathrm{NP}$ & NP & NP & NP & NP & $\mathrm{NP}$ & NP & NP & NP & 104.9 & NP \\
\hline $699-63-95$ & 147.5 & 124.7 & NP & NP & NP & NP & NP & NP & NP & NP & NP & NP & NP & NP & $\mathrm{NP}$ & NP & NP & NP & 124.7 & NP \\
\hline 699-64-27 & 126.2 & 103.3 & $\mathrm{NP}$ & NP & NP & NP & NP & NP & 103.3 & ND & ND & ND & ND & ND & ND & ND & ND & ND & ND & ND \\
\hline 699-64-62 & 152.4 & ND & ND & ND & ND & ND & ND & ND & ND & ND & ND & ND & ND & ND & ND & ND & ND & ND & ND & ND \\
\hline 699-65-83 & 147.8 & 121.3 & $\mathrm{NP}$ & NP & NP & NP & NP & NP & 121.3 & $\mathrm{NI}$ & $\mathrm{NI}$ & $\mathrm{NI}$ & $\mathrm{NI}$ & $\mathrm{NI}$ & $\mathrm{NI}$ & $\mathrm{NI}$ & $\mathrm{NI}$ & $\mathrm{NI}$ & $\mathrm{NI}$ & $\mathrm{NI}$ \\
\hline 699-65-95 & 137.8 & 120.7 & NP & NP & NP & NP & NP & NP & NP & NP & NP & NP & NP & NP & NP & NP & NP & NP & 120.7 & NP \\
\hline $699-65-114 A$ & $\mathrm{NI}$ & $\mathrm{NI}$ & $\mathrm{NI}$ & $\mathrm{NI}$ & $\mathrm{NI}$ & $\mathrm{NI}$ & $\mathrm{NI}$ & $\mathrm{NI}$ & $\mathrm{NI}$ & $\mathrm{NI}$ & $\mathrm{NI}$ & $\mathrm{NI}$ & $\mathrm{NI}$ & $\mathrm{NI}$ & $\mathrm{NI}$ & $\mathrm{NI}$ & $\mathrm{NI}$ & $\mathrm{NI}$ & 92.4 & $\mathrm{NI}$ \\
\hline $699-65-50$ & 142.3 & 112.8 & $\mathrm{NP}$ & NP & NP & NP & NP & NP & 112.8 & 110.9 & 110.9 & 40.2 & 40.2 & -1.8 & -1.8 & -33.8 & NP & NP & -33.8 & 17.1 \\
\hline 699-66-23 & 118.6 & 106.4 & $\mathrm{NP}$ & NP & NP & NP & NP & NP & NP & NP & 106.4 & ND & ND & ND & ND & ND & ND & ND & ND & $\mathrm{NI}$ \\
\hline 699-66-38 & 132.9 & 123.1 & NP & NP & NP & NP & NP & NP & 123.1 & 114.3 & 114.3 & ND & ND & ND & ND & ND & ND & ND & ND & $\mathrm{NI}$ \\
\hline 699-66-91 & 142.3 & 124.7 & $\mathrm{NP}$ & NP & NP & NP & NP & NP & 124.7 & $\mathrm{NI}$ & $\mathrm{NI}$ & $\mathrm{NI}$ & $\mathrm{NI}$ & $\mathrm{NI}$ & $\mathrm{NI}$ & $\mathrm{NI}$ & $\mathrm{NI}$ & $\mathrm{NI}$ & 112.8 & $\mathrm{NI}$ \\
\hline $699-67-51$ & 159.7 & 115.5 & $\mathrm{NP}$ & NP & NP & NP & NP & NP & 115.5 & 114.0 & 114.0 & ND & ND & ND & ND & ND & ND & ND & ND & $\mathrm{NI}$ \\
\hline $699-67-86$ & 143.9 & 107.3 & $\mathrm{NP}$ & NP & NP & $\mathrm{NP}$ & NP & $\mathrm{NP}$ & 107.3 & $\mathrm{NC}$ & $\mathrm{NC}$ & $\mathrm{NC}$ & $\mathrm{NC}$ & NC & $\mathrm{NC}$ & NC & NC & $\mathrm{NC}$ & $\mathrm{NC}$ & $\mathrm{NC}$ \\
\hline 699-67-98 & $\mathrm{NI}$ & $\mathrm{NI}$ & $\mathrm{NI}$ & $\mathrm{NI}$ & $\mathrm{NI}$ & $\mathrm{NI}$ & $\mathrm{NI}$ & $\mathrm{NI}$ & $\mathrm{NI}$ & $\mathrm{NI}$ & $\mathrm{NI}$ & $\mathrm{NI}$ & $\mathrm{NI}$ & $\mathrm{NI}$ & $\mathrm{NI}$ & $\mathrm{NI}$ & $\mathrm{NI}$ & $\mathrm{NI}$ & 86.9 & $\mathrm{NI}$ \\
\hline 699-69-45 & 148.1 & 120.7 & $\mathrm{NP}$ & NP & NP & NP & NP & $\mathrm{NP}$ & 120.7 & 107.6 & 107.6 & ND & ND & ND & ND & ND & ND & ND & ND & 30.5 \\
\hline $699-70-17$ & 269.4 & NP & $\mathrm{NP}$ & NP & NP & NP & 269.4 & 91.4 & NP & NP & $\mathrm{NP}$ & $\mathrm{NP}$ & 91.4 & 56.4 & 56.4 & 36.0 & NP & NP & 36.0 & NP \\
\hline $699-71-30$ & 121.9 & 110.6 & $\mathrm{NP}$ & NP & NP & NP & $\mathrm{NP}$ & $\mathrm{NP}$ & NP & NP & 110.6 & ND & ND & ND & ND & ND & ND & ND & ND & $\mathrm{NI}$ \\
\hline $699-71-52$ & 159.4 & 120.1 & NP & NP & NP & NP & NP & NP & 120.1 & 112.8 & 112.8 & ND & ND & ND & ND & ND & ND & ND & ND & $\mathrm{NI}$ \\
\hline 699-71-77 & 143.9 & 118.0 & $\mathrm{NP}$ & NP & NP & NP & NP & NP & 118.0 & 89.9 & 89.9 & 55.2 & 55.2 & ND & ND & ND & ND & ND & ND & 12.2 \\
\hline $699-72-73$ & 146.9 & 121.0 & $\mathrm{NP}$ & NP & NP & NP & NP & NP & 121.0 & 96.0 & 96.0 & ND & ND & ND & ND & ND & ND & ND & ND & $\mathrm{NI}$ \\
\hline $699-72-92$ & 137.8 & 92.0 & $\mathrm{NP}$ & NP & NP & NP & NP & NP & 92.0 & ND & ND & ND & ND & ND & ND & ND & ND & ND & ND & ND \\
\hline $699-73-61$ & 161.8 & 117.7 & $\mathrm{NP}$ & NP & NP & NP & NP & NP & 117.7 & ND & ND & ND & ND & ND & ND & ND & ND & ND & ND & ND \\
\hline $699-74-44$ & 135.6 & 112.8 & $\mathrm{NP}$ & NP & NP & NP & NP & NP & NP & NP & 112.8 & ND & ND & ND & ND & ND & ND & ND & ND & $\mathrm{NI}$ \\
\hline 699-77-36 & 125.6 & 112.2 & $\mathrm{NP}$ & NP & NP & NP & NP & NP & NP & NP & 112.2 & ND & ND & ND & ND & ND & ND & ND & ND & $\mathrm{NI}$ \\
\hline
\end{tabular}


Table A.1. Interpreted Top and Bottom Elevation of Major Hydrogeologic Units (continued)

$\mathrm{NP}=$ unit not present

$\mathrm{NC}=$ not certain whether unit is present or not

$\mathrm{ND}=$ well not deep enough to determine

$\mathrm{NI}=$ not interpreted or unknown

Elevations in $\mathrm{m}$ above mean sea level based on the National Geodetic Vertical Datum of 1929 (NGVD29 datum)

\begin{tabular}{|c|c|c|c|c|c|c|c|c|c|c|c|c|c|c|c|c|c|c|c|c|}
\hline Well number & $\begin{array}{l}\text { Casing } \\
\text { Elev. }\end{array}$ & Bot 1 & Top 2 & Bot 2 & Top 3 & Bot 3 & Top 4 & Bot 4 & Top 5 & Bot 5 & Top 6 & Bot 6 & Top 7 & Bot 7 & Top 8 & Bot 8 & Top 9 & Bot 9 & $\begin{array}{l}\text { Top of } \\
\text { Basalt }\end{array}$ & $\begin{array}{l}\text { Layer } 6 \\
\% \text { mud }\end{array}$ \\
\hline $699-77-54$ & 146.5 & 117.3 & NP & NP & NP & NP & NP & NP & 117.3 & 100.0 & 100.0 & ND & ND & ND & ND & ND & ND & ND & ND & $\mathrm{NI}$ \\
\hline 699-80-43P & 126.2 & 112.2 & NP & NP & NP & NP & NP & NP & NP & NP & 112.2 & 81.7 & 81.7 & 39.0 & 39.0 & 17.7 & 17.7 & -7.9 & -7.9 & 30.5 \\
\hline 699-81-62 & 134.4 & 123.4 & NP & NP & NP & NP & NP & NP & 123.4 & 103.0 & 103.0 & 26.5 & 26.5 & 12.2 & 12.2 & -22.3 & -22.3 & -26.5 & -26.5 & 27.4 \\
\hline $699-83-47$ & 132.6 & 107.3 & NP & NP & NP & NP & NP & NP & NP & NP & 107.3 & ND & ND & ND & ND & ND & ND & ND & ND & $\mathrm{NI}$ \\
\hline $699-83-60$ & $\mathrm{NI}$ & $\mathrm{NI}$ & $\mathrm{NI}$ & $\mathrm{NI}$ & $\mathrm{NI}$ & $\mathrm{NI}$ & $\mathrm{NI}$ & $\mathrm{NI}$ & $\mathrm{NI}$ & $\mathrm{NI}$ & $\mathrm{NI}$ & $\mathrm{NI}$ & $\mathrm{NI}$ & $\mathrm{NI}$ & $\mathrm{NI}$ & $\mathrm{NI}$ & $\mathrm{NI}$ & $\mathrm{NI}$ & -20.1 & $\mathrm{NI}$ \\
\hline 699-84-34B & $\mathrm{NI}$ & $\mathrm{NI}$ & $\mathrm{NI}$ & $\mathrm{NI}$ & $\mathrm{NI}$ & $\mathrm{NI}$ & $\mathrm{NI}$ & $\mathrm{NI}$ & $\mathrm{NI}$ & $\mathrm{NI}$ & $\mathrm{NI}$ & $\mathrm{NI}$ & $\mathrm{NI}$ & $\mathrm{NI}$ & $\mathrm{NI}$ & $\mathrm{NI}$ & $\mathrm{NI}$ & $\mathrm{NI}$ & 10.7 & $\mathrm{NI}$ \\
\hline 699-84-35A & 121.9 & 109.7 & $\mathrm{NP}$ & NP & NP & NP & NP & NP & NP & NP & 109.7 & 91.4 & 91.4 & 33.5 & 33.5 & 11.9 & NP & NP & 11.9 & 15.2 \\
\hline 699-84-59 & 140.2 & 122.5 & $\mathrm{NP}$ & NP & NP & NP & NP & NP & 122.5 & 107.3 & 107.3 & 37.2 & 37.2 & 17.7 & 17.7 & -16.8 & -16.8 & -19.5 & -19.5 & 25.9 \\
\hline 699-84-62A & 137.5 & 122.2 & $\mathrm{NP}$ & NP & NP & NP & NP & NP & 122.2 & 104.5 & 104.5 & 3.4 & $\mathrm{NC}$ & $\mathrm{NC}$ & 3.4 & -18.9 & -18.9 & -20.4 & -20.4 & 18.6 \\
\hline 699-86-60 & 138.1 & 108.8 & $\mathrm{NP}$ & NP & NP & NP & NP & NP & NP & NP & 108.8 & 50.9 & 50.9 & 40.5 & 40.5 & -9.8 & -9.8 & -20.4 & -20.4 & 19.2 \\
\hline $699-86-64$ & 123.1 & 105.5 & $\mathrm{NP}$ & NP & NP & NP & 105.5 & 92.7 & 92.7 & 59.1 & 59.1 & 40.8 & 40.8 & 32.9 & 32.9 & -14.6 & -14.6 & -16.2 & -16.2 & 18.3 \\
\hline 699-87-55 & 139.8 & 127.4 & $\mathrm{NP}$ & NP & NP & NP & NP & NP & 127.4 & ND & ND & ND & ND & ND & ND & ND & ND & ND & ND & ND \\
\hline 699-92-14 & 262.7 & 261.8 & $\mathrm{NP}$ & NP & NP & NP & 261.8 & 200.3 & NP & NP & 200.3 & 88.1 & 88.1 & 83.2 & NP & NP & NP & NP & 83.2 & NP \\
\hline 699-93-48A & 133.2 & 111.9 & $\mathrm{NP}$ & NP & NP & NP & NP & NP & NP & NP & 111.9 & ND & ND & ND & ND & ND & ND & ND & ND & ND \\
\hline 699-93-93 & $\mathrm{NI}$ & $\mathrm{NI}$ & $\mathrm{NI}$ & $\mathrm{NI}$ & $\mathrm{NI}$ & $\mathrm{NI}$ & $\mathrm{NI}$ & $\mathrm{NI}$ & $\mathrm{NI}$ & $\mathrm{NI}$ & $\mathrm{NI}$ & $\mathrm{NI}$ & $\mathrm{NI}$ & $\mathrm{NI}$ & $\mathrm{NI}$ & $\mathrm{NI}$ & $\mathrm{NI}$ & $\mathrm{NI}$ & 36.6 & $\mathrm{NI}$ \\
\hline $699-96-43$ & 128.3 & 114.0 & $\mathrm{NP}$ & NP & NP & $\mathrm{NP}$ & NP & NP & NP & $\mathrm{NP}$ & 114.0 & ND & ND & ND & ND & ND & ND & ND & ND & $\mathrm{NI}$ \\
\hline 699-96-49 & 127.7 & 109.4 & $\mathrm{NP}$ & NP & NP & NP & NP & NP & NP & NP & 109.4 & ND & ND & ND & ND & ND & ND & ND & ND & $\mathrm{NI}$ \\
\hline $699-97-43$ & 128.3 & 114.3 & $\mathrm{NP}$ & NP & NP & NP & NP & NP & NP & NP & 114.3 & ND & ND & ND & ND & ND & ND & ND & ND & $\mathrm{NI}$ \\
\hline $699-101-48 C$ & 118.3 & 103.6 & NP & NP & NP & NP & NP & NP & NP & NP & 103.6 & ND & ND & ND & ND & ND & ND & ND & ND & $\mathrm{NI}$ \\
\hline 699-103-25 & $\mathrm{NI}$ & $\mathrm{NI}$ & $\mathrm{NI}$ & $\mathrm{NI}$ & $\mathrm{NI}$ & $\mathrm{NI}$ & $\mathrm{NI}$ & $\mathrm{NI}$ & $\mathrm{NI}$ & $\mathrm{NI}$ & $\mathrm{NI}$ & $\mathrm{NI}$ & $\mathrm{NI}$ & $\mathrm{NI}$ & $\mathrm{NI}$ & $\mathrm{NI}$ & $\mathrm{NI}$ & $\mathrm{NI}$ & 95.1 & $\mathrm{NI}$ \\
\hline 699-107-79 & 200.9 & 197.2 & $\mathrm{NP}$ & NP & NP & NP & NP & NP & 197.2 & 182.0 & 182.0 & 166.7 & 166.7 & 111.6 & 111.6 & 93.0 & NP & NP & 93.0 & 30.5 \\
\hline 699-107-83 & $\mathrm{NI}$ & $\mathrm{NI}$ & $\mathrm{NI}$ & $\mathrm{NI}$ & $\mathrm{NI}$ & $\mathrm{NI}$ & $\mathrm{NI}$ & $\mathrm{NI}$ & $\mathrm{NI}$ & $\mathrm{NI}$ & $\mathrm{NI}$ & $\mathrm{NI}$ & $\mathrm{NI}$ & $\mathrm{NI}$ & $\mathrm{NI}$ & $\mathrm{NI}$ & $\mathrm{NI}$ & $\mathrm{NI}$ & 103.6 & $\mathrm{NI}$ \\
\hline $699-109-80$ & $\mathrm{NI}$ & $\mathrm{NI}$ & $\mathrm{NI}$ & $\mathrm{NI}$ & $\mathrm{NI}$ & $\mathrm{NI}$ & $\mathrm{NI}$ & $\mathrm{NI}$ & $\mathrm{NI}$ & $\mathrm{NI}$ & $\mathrm{NI}$ & $\mathrm{NI}$ & $\mathrm{NI}$ & $\mathrm{NI}$ & $\mathrm{NI}$ & $\mathrm{NI}$ & $\mathrm{NI}$ & $\mathrm{NI}$ & 131.1 & $\mathrm{NI}$ \\
\hline $699-111-24$ & $\mathrm{NI}$ & $\mathrm{NI}$ & $\mathrm{NI}$ & $\mathrm{NI}$ & $\mathrm{NI}$ & $\mathrm{NI}$ & $\mathrm{NI}$ & $\mathrm{NI}$ & $\mathrm{NI}$ & $\mathrm{NI}$ & $\mathrm{NI}$ & $\mathrm{NI}$ & $\mathrm{NI}$ & $\mathrm{NI}$ & $\mathrm{NI}$ & $\mathrm{NI}$ & $\mathrm{NI}$ & $\mathrm{NI}$ & 149.4 & $\mathrm{NI}$ \\
\hline $699-112-37$ & 225.9 & NP & NP & NP & NP & NP & 225.9 & 201.8 & 201.8 & 193.2 & 193.2 & 181.7 & 181.7 & 171.9 & 171.9 & 142.0 & 142.0 & 141.1 & 141.1 & 30.5 \\
\hline $699-115-7$ & $\mathrm{NI}$ & $\mathrm{NI}$ & $\mathrm{NI}$ & $\mathrm{NI}$ & $\mathrm{NI}$ & $\mathrm{NI}$ & $\mathrm{NI}$ & $\mathrm{NI}$ & $\mathrm{NI}$ & $\mathrm{NI}$ & $\mathrm{NI}$ & $\mathrm{NI}$ & $\mathrm{NI}$ & $\mathrm{NI}$ & $\mathrm{NI}$ & $\mathrm{NI}$ & $\mathrm{NI}$ & $\mathrm{NI}$ & 268.2 & $\mathrm{NI}$ \\
\hline $699-115-61$ & 240.8 & 233.8 & $\mathrm{NP}$ & NP & NP & NP & 233.8 & 164.6 & 164.6 & 156.7 & NP & NP & NP & NP & NP & NP & NP & NP & 156.7 & NP \\
\hline
\end{tabular}


Table A.1. Interpreted Top and Bottom Elevation of Major Hydrogeologic Units (continued)

$\mathrm{NP}=$ unit not present

$\mathrm{NC}=$ not certain whether unit is present or not

$\mathrm{ND}=$ well not deep enough to determine

$\mathrm{NI}=$ not interpreted or unknown

Elevations in $\mathrm{m}$ above mean sea level based on the National Geodetic Vertical Datum of 1929 (NGVD29 datum)

\begin{tabular}{|c|c|c|c|c|c|c|c|c|c|c|c|c|c|c|c|c|c|c|c|c|}
\hline Well number & $\begin{array}{l}\text { Casing } \\
\text { Elev. }\end{array}$ & Bot 1 & Top 2 & Bot 2 & Top 3 & Bot 3 & Top 4 & Bot 4 & Top 5 & Bot 5 & Top 6 & Bot 6 & Top 7 & Bot 7 & Top 8 & Bot 8 & Top 9 & Bot 9 & $\begin{array}{l}\text { Top of } \\
\text { Basalt }\end{array}$ & $\begin{array}{l}\text { Layer } 6 \\
\% \text { mud }\end{array}$ \\
\hline $699-115-77$ & $\mathrm{NI}$ & $\mathrm{NI}$ & $\mathrm{NI}$ & $\mathrm{NI}$ & $\mathrm{NI}$ & $\mathrm{NI}$ & $\mathrm{NI}$ & $\mathrm{NI}$ & $\mathrm{NI}$ & $\mathrm{NI}$ & $\mathrm{NI}$ & $\mathrm{NI}$ & $\mathrm{NI}$ & $\mathrm{NI}$ & $\mathrm{NI}$ & $\mathrm{NI}$ & $\mathrm{NI}$ & $\mathrm{NI}$ & 179.8 & $\mathrm{NI}$ \\
\hline 699-117-11 & $\mathrm{NI}$ & $\mathrm{NI}$ & $\mathrm{NI}$ & $\mathrm{NI}$ & $\mathrm{NI}$ & $\mathrm{NI}$ & $\mathrm{NI}$ & $\mathrm{NI}$ & $\mathrm{NI}$ & $\mathrm{NI}$ & $\mathrm{NI}$ & $\mathrm{NI}$ & $\mathrm{NI}$ & $\mathrm{NI}$ & $\mathrm{NI}$ & $\mathrm{NI}$ & $\mathrm{NI}$ & $\mathrm{NI}$ & 271.9 & $\mathrm{NI}$ \\
\hline $699-119-11$ & $\mathrm{NI}$ & $\mathrm{NI}$ & $\mathrm{NI}$ & $\mathrm{NI}$ & $\mathrm{NI}$ & $\mathrm{NI}$ & $\mathrm{NI}$ & $\mathrm{NI}$ & $\mathrm{NI}$ & $\mathrm{NI}$ & $\mathrm{NI}$ & $\mathrm{NI}$ & $\mathrm{NI}$ & $\mathrm{NI}$ & $\mathrm{NI}$ & $\mathrm{NI}$ & $\mathrm{NI}$ & $\mathrm{NI}$ & 299.0 & $\mathrm{NI}$ \\
\hline $699-122-11$ & $\mathrm{NI}$ & $\mathrm{NI}$ & $\mathrm{NI}$ & $\mathrm{NI}$ & $\mathrm{NI}$ & $\mathrm{NI}$ & $\mathrm{NI}$ & $\mathrm{NI}$ & $\mathrm{NI}$ & $\mathrm{NI}$ & $\mathrm{NI}$ & $\mathrm{NI}$ & $\mathrm{NI}$ & $\mathrm{NI}$ & $\mathrm{NI}$ & $\mathrm{NI}$ & $\mathrm{NI}$ & $\mathrm{NI}$ & 363.0 & $\mathrm{NI}$ \\
\hline 299-E13-20 & 226.4 & 130.5 & $\mathrm{NP}$ & NP & $\mathrm{NP}$ & $\mathrm{NP}$ & $\mathrm{NP}$ & $\mathrm{NP}$ & 130.5 & 103.0 & 103.0 & 84.7 & 84.7 & 79.9 & 79.9 & 70.4 & 70.4 & 48.2 & 48.2 & 30.5 \\
\hline 299-E16-1 & 212.3 & 128.3 & $\mathrm{NP}$ & NP & NP & NP & NP & $\mathrm{NP}$ & 128.3 & 110.0 & 110.0 & 101.5 & 101.5 & 81.7 & 81.7 & 77.1 & 77.1 & 68.0 & 68.0 & 30.5 \\
\hline 299-E23-2 & 219.5 & $\mathrm{NI}$ & $\mathrm{NI}$ & $\mathrm{NI}$ & $\mathrm{NI}$ & $\mathrm{NI}$ & $\mathrm{NI}$ & $\mathrm{NI}$ & $\mathrm{NI}$ & $\mathrm{NI}$ & $\mathrm{NI}$ & $\mathrm{NI}$ & $\mathrm{NI}$ & $\mathrm{NI}$ & $\mathrm{NI}$ & $\mathrm{NI}$ & $\mathrm{NI}$ & $\mathrm{NI}$ & 85.6 & $\mathrm{NI}$ \\
\hline 299-E24-7 & 218.2 & $\mathrm{NI}$ & $\mathrm{NI}$ & $\mathrm{NI}$ & $\mathrm{NI}$ & $\mathrm{NI}$ & $\mathrm{NI}$ & $\mathrm{NI}$ & $\mathrm{NI}$ & $\mathrm{NI}$ & $\mathrm{NI}$ & $\mathrm{NI}$ & $\mathrm{NI}$ & $\mathrm{NI}$ & $\mathrm{NI}$ & $\mathrm{NI}$ & $\mathrm{NI}$ & $\mathrm{NI}$ & 82.3 & $\mathrm{NI}$ \\
\hline 299-E24-8 & 209.7 & $\mathrm{NI}$ & $\mathrm{NI}$ & $\mathrm{NI}$ & $\mathrm{NI}$ & $\mathrm{NI}$ & $\mathrm{NI}$ & $\mathrm{NI}$ & $\mathrm{NI}$ & $\mathrm{NI}$ & $\mathrm{NI}$ & $\mathrm{NI}$ & $\mathrm{NI}$ & $\mathrm{NI}$ & $\mathrm{NI}$ & $\mathrm{NI}$ & $\mathrm{NI}$ & $\mathrm{NI}$ & 96.0 & $\mathrm{NI}$ \\
\hline 299-E25-2 & 205.7 & $\mathrm{NI}$ & $\mathrm{NI}$ & $\mathrm{NI}$ & $\mathrm{NI}$ & $\mathrm{NI}$ & $\mathrm{NI}$ & $\mathrm{NI}$ & $\mathrm{NI}$ & $\mathrm{NI}$ & $\mathrm{NI}$ & $\mathrm{NI}$ & $\mathrm{NI}$ & $\mathrm{NI}$ & $\mathrm{NI}$ & $\mathrm{NI}$ & $\mathrm{NI}$ & $\mathrm{NI}$ & 93.0 & $\mathrm{NI}$ \\
\hline 299-E25-28 & 201.8 & $\mathrm{NI}$ & $\mathrm{NI}$ & $\mathrm{NI}$ & $\mathrm{NI}$ & $\mathrm{NI}$ & $\mathrm{NI}$ & $\mathrm{NI}$ & $\mathrm{NI}$ & $\mathrm{NI}$ & $\mathrm{NI}$ & $\mathrm{NI}$ & $\mathrm{NI}$ & $\mathrm{NI}$ & $\mathrm{NI}$ & $\mathrm{NI}$ & $\mathrm{NI}$ & $\mathrm{NI}$ & 97.8 & $\mathrm{NI}$ \\
\hline 299-E25-32P & 204.0 & $\mathrm{NI}$ & $\mathrm{NI}$ & $\mathrm{NI}$ & $\mathrm{NI}$ & $\mathrm{NI}$ & $\mathrm{NI}$ & $\mathrm{NI}$ & $\mathrm{NI}$ & $\mathrm{NI}$ & $\mathrm{NI}$ & $\mathrm{NI}$ & $\mathrm{NI}$ & $\mathrm{NI}$ & $\mathrm{NI}$ & $\mathrm{NI}$ & $\mathrm{NI}$ & $\mathrm{NI}$ & 97.5 & $\mathrm{NI}$ \\
\hline 299-E25-33 & 198.1 & $\mathrm{NI}$ & $\mathrm{NI}$ & $\mathrm{NI}$ & $\mathrm{NI}$ & $\mathrm{NI}$ & $\mathrm{NI}$ & $\mathrm{NI}$ & $\mathrm{NI}$ & $\mathrm{NI}$ & $\mathrm{NI}$ & $\mathrm{NI}$ & $\mathrm{NI}$ & $\mathrm{NI}$ & $\mathrm{NI}$ & $\mathrm{NI}$ & $\mathrm{NI}$ & $\mathrm{NI}$ & 80.8 & $\mathrm{NI}$ \\
\hline 299-E26-1 & 188.1 & $\mathrm{NI}$ & $\mathrm{NI}$ & $\mathrm{NI}$ & $\mathrm{NI}$ & $\mathrm{NI}$ & $\mathrm{NI}$ & $\mathrm{NI}$ & $\mathrm{NI}$ & $\mathrm{NI}$ & $\mathrm{NI}$ & $\mathrm{NI}$ & $\mathrm{NI}$ & $\mathrm{NI}$ & $\mathrm{NI}$ & $\mathrm{NI}$ & $\mathrm{NI}$ & $\mathrm{NI}$ & $\mathrm{NI}$ & $\mathrm{NI}$ \\
\hline 299-E26-8 & 188.8 & 127.7 & NP & NP & NP & NP & NP & NP & NP & NP & NP & NP & NP & NP & NP & NP & 127.7 & 114.0 & 114.0 & NP \\
\hline 299-E27-3 & 208.2 & $\mathrm{NI}$ & $\mathrm{NI}$ & $\mathrm{NI}$ & $\mathrm{NI}$ & $\mathrm{NI}$ & $\mathrm{NI}$ & $\mathrm{NI}$ & $\mathrm{NI}$ & $\mathrm{NI}$ & $\mathrm{NI}$ & $\mathrm{NI}$ & $\mathrm{NI}$ & $\mathrm{NI}$ & $\mathrm{NI}$ & $\mathrm{NI}$ & $\mathrm{NI}$ & $\mathrm{NI}$ & 101.8 & $\mathrm{NI}$ \\
\hline 299-E27-6 & 204.5 & $\mathrm{NI}$ & $\mathrm{NI}$ & $\mathrm{NI}$ & $\mathrm{NI}$ & $\mathrm{NI}$ & $\mathrm{NI}$ & $\mathrm{NI}$ & $\mathrm{NI}$ & $\mathrm{NI}$ & $\mathrm{NI}$ & $\mathrm{NI}$ & $\mathrm{NI}$ & $\mathrm{NI}$ & $\mathrm{NI}$ & $\mathrm{NI}$ & $\mathrm{NI}$ & $\mathrm{NI}$ & 100.9 & $\mathrm{NI}$ \\
\hline 299-E27-8 & 194.4 & $\mathrm{NI}$ & $\mathrm{NI}$ & $\mathrm{NI}$ & $\mathrm{NI}$ & $\mathrm{NI}$ & $\mathrm{NI}$ & $\mathrm{NI}$ & $\mathrm{NI}$ & $\mathrm{NI}$ & $\mathrm{NI}$ & $\mathrm{NI}$ & $\mathrm{NI}$ & $\mathrm{NI}$ & $\mathrm{NI}$ & $\mathrm{NI}$ & $\mathrm{NI}$ & $\mathrm{NI}$ & $\mathrm{NI}$ & $\mathrm{NI}$ \\
\hline 299-E27-9 & 191.7 & $\mathrm{NI}$ & $\mathrm{NI}$ & $\mathrm{NI}$ & $\mathrm{NI}$ & $\mathrm{NI}$ & $\mathrm{NI}$ & $\mathrm{NI}$ & $\mathrm{NI}$ & $\mathrm{NI}$ & $\mathrm{NI}$ & $\mathrm{NI}$ & $\mathrm{NI}$ & $\mathrm{NI}$ & $\mathrm{NI}$ & $\mathrm{NI}$ & $\mathrm{NI}$ & $\mathrm{NI}$ & $\mathrm{NI}$ & $\mathrm{NI}$ \\
\hline 299-E27-10 & 190.2 & $\mathrm{NI}$ & $\mathrm{NI}$ & $\mathrm{NI}$ & $\mathrm{NI}$ & $\mathrm{NI}$ & $\mathrm{NI}$ & $\mathrm{NI}$ & $\mathrm{NI}$ & $\mathrm{NI}$ & $\mathrm{NI}$ & $\mathrm{NI}$ & $\mathrm{NI}$ & $\mathrm{NI}$ & $\mathrm{NI}$ & $\mathrm{NI}$ & $\mathrm{NI}$ & $\mathrm{NI}$ & $\mathrm{NI}$ & $\mathrm{NI}$ \\
\hline 299-E28-5 & 204.8 & $\mathrm{NI}$ & $\mathrm{NI}$ & $\mathrm{NI}$ & $\mathrm{NI}$ & $\mathrm{NI}$ & $\mathrm{NI}$ & $\mathrm{NI}$ & $\mathrm{NI}$ & $\mathrm{NI}$ & $\mathrm{NI}$ & $\mathrm{NI}$ & $\mathrm{NI}$ & $\mathrm{NI}$ & $\mathrm{NI}$ & $\mathrm{NI}$ & $\mathrm{NI}$ & $\mathrm{NI}$ & $\mathrm{NI}$ & $\mathrm{NI}$ \\
\hline 299-E28-7 & 209.1 & $\mathrm{NI}$ & $\mathrm{NI}$ & $\mathrm{NI}$ & $\mathrm{NI}$ & $\mathrm{NI}$ & $\mathrm{NI}$ & $\mathrm{NI}$ & $\mathrm{NI}$ & $\mathrm{NI}$ & $\mathrm{NI}$ & $\mathrm{NI}$ & $\mathrm{NI}$ & $\mathrm{NI}$ & $\mathrm{NI}$ & $\mathrm{NI}$ & $\mathrm{NI}$ & $\mathrm{NI}$ & $\mathrm{NI}$ & $\mathrm{NI}$ \\
\hline 299-E28-8 & 203.6 & $\mathrm{NI}$ & $\mathrm{NI}$ & $\mathrm{NI}$ & $\mathrm{NI}$ & $\mathrm{NI}$ & $\mathrm{NI}$ & $\mathrm{NI}$ & $\mathrm{NI}$ & $\mathrm{NI}$ & $\mathrm{NI}$ & $\mathrm{NI}$ & $\mathrm{NI}$ & $\mathrm{NI}$ & $\mathrm{NI}$ & $\mathrm{NI}$ & $\mathrm{NI}$ & $\mathrm{NI}$ & $\mathrm{NI}$ & $\mathrm{NI}$ \\
\hline 299-E28-10 & 206.3 & $\mathrm{NI}$ & $\mathrm{NI}$ & $\mathrm{NI}$ & $\mathrm{NI}$ & $\mathrm{NI}$ & $\mathrm{NI}$ & $\mathrm{NI}$ & $\mathrm{NI}$ & $\mathrm{NI}$ & $\mathrm{NI}$ & $\mathrm{NI}$ & $\mathrm{NI}$ & $\mathrm{NI}$ & $\mathrm{NI}$ & $\mathrm{NI}$ & $\mathrm{NI}$ & $\mathrm{NI}$ & $\mathrm{NI}$ & $\mathrm{NI}$ \\
\hline 299-E28-16 & 214.3 & 127.4 & NP & NP & NP & NP & NP & NP & 127.4 & ND & ND & ND & ND & ND & ND & ND & ND & ND & ND & ND \\
\hline 299-E28-22 & 213.5 & 114.3 & NP & NP & NP & NP & $\mathrm{NP}$ & NP & NP & NP & $\mathrm{NP}$ & $\mathrm{NP}$ & NP & NP & NP & $\mathrm{NP}$ & 114.3 & 97.2 & 97.2 & NP \\
\hline
\end{tabular}


Table A.1. Interpreted Top and Bottom Elevation of Major Hydrogeologic Units (continued)

$\mathrm{NP}=$ unit not present

$\mathrm{NC}=$ not certain whether unit is present or not

$\mathrm{ND}=$ well not deep enough to determine

$\mathrm{NI}=$ not interpreted or unknown

Elevations in $\mathrm{m}$ above mean sea level based on the National Geodetic Vertical Datum of 1929 (NGVD29 datum)

\begin{tabular}{|c|c|c|c|c|c|c|c|c|c|c|c|c|c|c|c|c|c|c|c|c|}
\hline Well number & $\begin{array}{l}\text { Casing } \\
\text { Elev. }\end{array}$ & Bot 1 & Top 2 & Bot 2 & Top 3 & Bot 3 & Top 4 & Bot 4 & Top 5 & Bot 5 & Top 6 & Bot 6 & Top 7 & Bot 7 & Top 8 & Bot 8 & Top 9 & Bot 9 & $\begin{array}{l}\text { Top of } \\
\text { Basalt }\end{array}$ & $\begin{array}{l}\text { Layer } 6 \\
\% \text { mud }\end{array}$ \\
\hline 299-E28-26 & 209.4 & $\mathrm{NI}$ & $\mathrm{NI}$ & $\mathrm{NI}$ & $\mathrm{NI}$ & $\mathrm{NI}$ & $\mathrm{NI}$ & $\mathrm{NI}$ & $\mathrm{NI}$ & $\mathrm{NI}$ & $\mathrm{NI}$ & $\mathrm{NI}$ & $\mathrm{NI}$ & $\mathrm{NI}$ & $\mathrm{NI}$ & $\mathrm{NI}$ & $\mathrm{NI}$ & $\mathrm{NI}$ & $\mathrm{NI}$ & $\mathrm{NI}$ \\
\hline 299-E28-27 & 207.4 & 121.9 & NP & NP & NP & NP & NP & NP & NP & NP & NP & NP & NP & NP & NP & NP & 121.9 & ND & ND & NP \\
\hline 299-E32-1 & 199.9 & $\mathrm{NI}$ & $\mathrm{NI}$ & $\mathrm{NI}$ & $\mathrm{NI}$ & $\mathrm{NI}$ & $\mathrm{NI}$ & $\mathrm{NI}$ & $\mathrm{NI}$ & $\mathrm{NI}$ & $\mathrm{NI}$ & $\mathrm{NI}$ & $\mathrm{NI}$ & $\mathrm{NI}$ & $\mathrm{NI}$ & $\mathrm{NI}$ & $\mathrm{NI}$ & $\mathrm{NI}$ & $\mathrm{NI}$ & $\mathrm{NI}$ \\
\hline 299-E32-2 & 204.2 & $\mathrm{NI}$ & $\mathrm{NI}$ & $\mathrm{NI}$ & $\mathrm{NI}$ & $\mathrm{NI}$ & $\mathrm{NI}$ & $\mathrm{NI}$ & $\mathrm{NI}$ & $\mathrm{NI}$ & $\mathrm{NI}$ & $\mathrm{NI}$ & $\mathrm{NI}$ & $\mathrm{NI}$ & $\mathrm{NI}$ & $\mathrm{NI}$ & $\mathrm{NI}$ & $\mathrm{NI}$ & $\mathrm{NI}$ & $\mathrm{NI}$ \\
\hline 299-E32-4 & 209.0 & 118.9 & NP & NP & NP & NP & NP & NP & NP & NP & NP & NP & NP & NP & NP & NP & NP & NP & ND & NP \\
\hline 299-E33-2 & 191.7 & $\mathrm{NI}$ & $\mathrm{NI}$ & $\mathrm{NI}$ & $\mathrm{NI}$ & $\mathrm{NI}$ & $\mathrm{NI}$ & $\mathrm{NI}$ & $\mathrm{NI}$ & $\mathrm{NI}$ & $\mathrm{NI}$ & $\mathrm{NI}$ & $\mathrm{NI}$ & $\mathrm{NI}$ & $\mathrm{NI}$ & $\mathrm{NI}$ & $\mathrm{NI}$ & $\mathrm{NI}$ & $\mathrm{NI}$ & $\mathrm{NI}$ \\
\hline 299-E33-5 & 192.3 & $\mathrm{NI}$ & $\mathrm{NI}$ & $\mathrm{NI}$ & $\mathrm{NI}$ & $\mathrm{NI}$ & $\mathrm{NI}$ & $\mathrm{NI}$ & $\mathrm{NI}$ & $\mathrm{NI}$ & $\mathrm{NI}$ & $\mathrm{NI}$ & $\mathrm{NI}$ & $\mathrm{NI}$ & $\mathrm{NI}$ & $\mathrm{NI}$ & $\mathrm{NI}$ & $\mathrm{NI}$ & $\mathrm{NI}$ & $\mathrm{NI}$ \\
\hline 299-E33-7 & 190.8 & $\mathrm{NI}$ & $\mathrm{NI}$ & $\mathrm{NI}$ & $\mathrm{NI}$ & $\mathrm{NI}$ & $\mathrm{NI}$ & $\mathrm{NI}$ & $\mathrm{NI}$ & $\mathrm{NI}$ & $\mathrm{NI}$ & $\mathrm{NI}$ & $\mathrm{NI}$ & $\mathrm{NI}$ & $\mathrm{NI}$ & $\mathrm{NI}$ & $\mathrm{NI}$ & $\mathrm{NI}$ & $\mathrm{NI}$ & $\mathrm{NI}$ \\
\hline 299-E33-8 & 198.1 & $\mathrm{NI}$ & $\mathrm{NI}$ & $\mathrm{NI}$ & $\mathrm{NI}$ & $\mathrm{NI}$ & $\mathrm{NI}$ & $\mathrm{NI}$ & $\mathrm{NI}$ & $\mathrm{NI}$ & $\mathrm{NI}$ & $\mathrm{NI}$ & $\mathrm{NI}$ & $\mathrm{NI}$ & $\mathrm{NI}$ & $\mathrm{NI}$ & $\mathrm{NI}$ & $\mathrm{NI}$ & $\mathrm{NI}$ & $\mathrm{NI}$ \\
\hline 299-E33-10 & 204.5 & $\mathrm{NI}$ & $\mathrm{NI}$ & $\mathrm{NI}$ & $\mathrm{NI}$ & $\mathrm{NI}$ & $\mathrm{NI}$ & $\mathrm{NI}$ & $\mathrm{NI}$ & $\mathrm{NI}$ & $\mathrm{NI}$ & $\mathrm{NI}$ & $\mathrm{NI}$ & $\mathrm{NI}$ & $\mathrm{NI}$ & $\mathrm{NI}$ & $\mathrm{NI}$ & $\mathrm{NI}$ & $\mathrm{NI}$ & $\mathrm{NI}$ \\
\hline 299-E33-11 & 189.0 & $\mathrm{NI}$ & $\mathrm{NI}$ & $\mathrm{NI}$ & $\mathrm{NI}$ & $\mathrm{NI}$ & $\mathrm{NI}$ & $\mathrm{NI}$ & $\mathrm{NI}$ & $\mathrm{NI}$ & $\mathrm{NI}$ & $\mathrm{NI}$ & $\mathrm{NI}$ & $\mathrm{NI}$ & $\mathrm{NI}$ & $\mathrm{NI}$ & $\mathrm{NI}$ & $\mathrm{NI}$ & $\mathrm{NI}$ & $\mathrm{NI}$ \\
\hline 299-E33-12 & 190.0 & 119.5 & NP & NP & NP & NP & NP & NP & NP & NP & NP & NP & NP & NP & NP & NP & NP & NP & 119.5 & NP \\
\hline 299-E33-14 & 189.6 & $\mathrm{NI}$ & $\mathrm{NI}$ & $\mathrm{NI}$ & $\mathrm{NI}$ & $\mathrm{NI}$ & $\mathrm{NI}$ & $\mathrm{NI}$ & $\mathrm{NI}$ & $\mathrm{NI}$ & $\mathrm{NI}$ & $\mathrm{NI}$ & $\mathrm{NI}$ & $\mathrm{NI}$ & $\mathrm{NI}$ & $\mathrm{NI}$ & $\mathrm{NI}$ & $\mathrm{NI}$ & $\mathrm{NI}$ & $\mathrm{NI}$ \\
\hline 299-E33-15 & 191.1 & $\mathrm{NI}$ & $\mathrm{NI}$ & $\mathrm{NI}$ & $\mathrm{NI}$ & $\mathrm{NI}$ & $\mathrm{NI}$ & $\mathrm{NI}$ & $\mathrm{NI}$ & $\mathrm{NI}$ & $\mathrm{NI}$ & $\mathrm{NI}$ & $\mathrm{NI}$ & $\mathrm{NI}$ & $\mathrm{NI}$ & $\mathrm{NI}$ & $\mathrm{NI}$ & $\mathrm{NI}$ & $\mathrm{NI}$ & $\mathrm{NI}$ \\
\hline 299-E33-18 & 198.4 & $\mathrm{NI}$ & $\mathrm{NI}$ & $\mathrm{NI}$ & $\mathrm{NI}$ & $\mathrm{NI}$ & $\mathrm{NI}$ & $\mathrm{NI}$ & $\mathrm{NI}$ & $\mathrm{NI}$ & $\mathrm{NI}$ & $\mathrm{NI}$ & $\mathrm{NI}$ & $\mathrm{NI}$ & $\mathrm{NI}$ & $\mathrm{NI}$ & $\mathrm{NI}$ & $\mathrm{NI}$ & $\mathrm{NI}$ & $\mathrm{NI}$ \\
\hline 299-E33-19 & 194.5 & $\mathrm{NI}$ & $\mathrm{NI}$ & $\mathrm{NI}$ & $\mathrm{NI}$ & $\mathrm{NI}$ & $\mathrm{NI}$ & $\mathrm{NI}$ & $\mathrm{NI}$ & $\mathrm{NI}$ & $\mathrm{NI}$ & $\mathrm{NI}$ & $\mathrm{NI}$ & $\mathrm{NI}$ & $\mathrm{NI}$ & $\mathrm{NI}$ & $\mathrm{NI}$ & $\mathrm{NI}$ & $\mathrm{NI}$ & $\mathrm{NI}$ \\
\hline 299-E33-20 & 195.1 & $\mathrm{NI}$ & $\mathrm{NI}$ & $\mathrm{NI}$ & $\mathrm{NI}$ & $\mathrm{NI}$ & $\mathrm{NI}$ & $\mathrm{NI}$ & $\mathrm{NI}$ & $\mathrm{NI}$ & $\mathrm{NI}$ & $\mathrm{NI}$ & $\mathrm{NI}$ & $\mathrm{NI}$ & $\mathrm{NI}$ & $\mathrm{NI}$ & $\mathrm{NI}$ & $\mathrm{NI}$ & $\mathrm{NI}$ & $\mathrm{NI}$ \\
\hline 299-E33-28 & 202.5 & 117.3 & NP & NP & NP & NP & NP & NP & NP & NP & NP & NP & NP & NP & NP & NP & NP & NP & 117.3 & NP \\
\hline 299-E33-29 & 205.4 & 132.3 & NP & NP & NP & NP & NP & NP & NP & NP & NP & NP & NP & NP & NP & NP & $\mathrm{NC}$ & $\mathrm{NC}$ & 117.0 & NP \\
\hline 299-E33-30 & 202.3 & 118.0 & NP & NP & NP & NP & NP & NP & NP & NP & NP & NP & NP & NP & NP & NP & NP & NP & 118.0 & NP \\
\hline 299-E33-41 & 199.6 & $\mathrm{NI}$ & $\mathrm{NI}$ & $\mathrm{NI}$ & $\mathrm{NI}$ & $\mathrm{NI}$ & $\mathrm{NI}$ & $\mathrm{NI}$ & $\mathrm{NI}$ & $\mathrm{NI}$ & $\mathrm{NI}$ & $\mathrm{NI}$ & $\mathrm{NI}$ & $\mathrm{NI}$ & $\mathrm{NI}$ & $\mathrm{NI}$ & $\mathrm{NI}$ & $\mathrm{NI}$ & $\mathrm{NI}$ & $\mathrm{NI}$ \\
\hline 299-E34-1 & 191.7 & $\mathrm{NI}$ & $\mathrm{NI}$ & $\mathrm{NI}$ & $\mathrm{NI}$ & $\mathrm{NI}$ & $\mathrm{NI}$ & $\mathrm{NI}$ & $\mathrm{NI}$ & $\mathrm{NI}$ & $\mathrm{NI}$ & $\mathrm{NI}$ & $\mathrm{NI}$ & $\mathrm{NI}$ & $\mathrm{NI}$ & $\mathrm{NI}$ & $\mathrm{NI}$ & $\mathrm{NI}$ & $\mathrm{NI}$ & $\mathrm{NI}$ \\
\hline 299-E34-2 & 192.3 & 118.9 & NP & NP & NP & NP & NP & NP & NP & NP & NP & NP & NP & NP & NP & NP & NP & NP & 118.9 & NP \\
\hline 299-E34-3 & 186.4 & 121.3 & NP & NP & NP & NP & NP & NP & NP & NP & NP & NP & NP & NP & NP & NP & NP & NP & 121.3 & NP \\
\hline 299-E34-4 & 178.9 & $\mathrm{NI}$ & $\mathrm{NI}$ & $\mathrm{NI}$ & $\mathrm{NI}$ & $\mathrm{NI}$ & $\mathrm{NI}$ & $\mathrm{NI}$ & $\mathrm{NI}$ & $\mathrm{NI}$ & $\mathrm{NI}$ & $\mathrm{NI}$ & $\mathrm{NI}$ & $\mathrm{NI}$ & $\mathrm{NI}$ & $\mathrm{NI}$ & $\mathrm{NI}$ & $\mathrm{NI}$ & $\mathrm{NI}$ & $\mathrm{NI}$ \\
\hline 299-E34-5 & 179.8 & $\mathrm{NI}$ & $\mathrm{NI}$ & $\mathrm{NI}$ & $\mathrm{NI}$ & $\mathrm{NI}$ & $\mathrm{NI}$ & $\mathrm{NI}$ & $\mathrm{NI}$ & $\mathrm{NI}$ & $\mathrm{NI}$ & $\mathrm{NI}$ & $\mathrm{NI}$ & $\mathrm{NI}$ & $\mathrm{NI}$ & $\mathrm{NI}$ & $\mathrm{NI}$ & $\mathrm{NI}$ & $\mathrm{NI}$ & $\mathrm{NI}$ \\
\hline
\end{tabular}


Table A.1. Interpreted Top and Bottom Elevation of Major Hydrogeologic Units (continued)

$\mathrm{NP}=$ unit not present

$\mathrm{NC}=$ not certain whether unit is present or not

$\mathrm{ND}=$ well not deep enough to determine

$\mathrm{NI}=$ not interpreted or unknown

Elevations in m above mean sea level based on the National Geodetic Vertical Datum of 1929 (NGVD29 datum)

\begin{tabular}{|c|c|c|c|c|c|c|c|c|c|c|c|c|c|c|c|c|c|c|c|c|}
\hline Well number & $\begin{array}{l}\text { Casing } \\
\text { Elev. }\end{array}$ & Bot 1 & Top 2 & Bot 2 & Top 3 & Bot 3 & Top 4 & Bot 4 & Top 5 & Bot 5 & Top & Bot 6 & Top 7 & Bot 7 & Top 8 & Bot 8 & Top 9 & Bot 9 & $\begin{array}{l}\text { Top of } \\
\text { Basalt }\end{array}$ & $\begin{array}{l}\text { Layer } 6 \\
\% \text { mud }\end{array}$ \\
\hline 299-E34-6 & 182.2 & 114.0 & NP & NP & NP & NP & NP & NP & NP & NP & NP & NP & NP & NP & NP & NP & NP & NP & 114.0 & NP \\
\hline 299-E34-7 & 184.1 & $\mathrm{NI}$ & $\mathrm{NI}$ & $\mathrm{NI}$ & $\mathrm{NI}$ & $\mathrm{NI}$ & $\mathrm{NI}$ & $\mathrm{NI}$ & $\mathrm{NI}$ & $\mathrm{NI}$ & $\mathrm{NI}$ & $\mathrm{NI}$ & $\mathrm{NI}$ & $\mathrm{NI}$ & $\mathrm{NI}$ & $\mathrm{NI}$ & $\mathrm{NI}$ & $\mathrm{NI}$ & $\mathrm{NI}$ & $\mathrm{NI}$ \\
\hline 299-E34-8 & 195.1 & $\mathrm{NI}$ & $\mathrm{NI}$ & $\mathrm{NI}$ & $\mathrm{NI}$ & $\mathrm{NI}$ & $\mathrm{NI}$ & $\mathrm{NI}$ & $\mathrm{NI}$ & $\mathrm{NI}$ & $\mathrm{NI}$ & $\mathrm{NI}$ & $\mathrm{NI}$ & $\mathrm{NI}$ & $\mathrm{NI}$ & $\mathrm{NI}$ & $\mathrm{NI}$ & $\mathrm{NI}$ & $\mathrm{NI}$ & $\mathrm{NI}$ \\
\hline 299-W6-1 & 214.0 & 199.6 & NP & NP & 199.6 & 195.7 & 195.7 & 183.5 & 183.5 & 87.5 & NP & NP & NP & NP & 87.5 & 83.5 & 83.5 & 74.7 & 74.7 & NP \\
\hline 299-W6-2 & 211.1 & 194.2 & NP & NP & 194.2 & 185.0 & 185.0 & 183.5 & 183.5 & ND & ND & ND & ND & ND & ND & ND & ND & ND & ND & ND \\
\hline 299-W7-1 & 210.5 & 192.3 & 192.3 & 189.3 & 189.3 & 177.1 & 177.1 & 167.9 & 167.9 & ND & ND & ND & ND & ND & ND & ND & ND & ND & ND & ND \\
\hline 299-W7-2 & 205.9 & 195.4 & 195.4 & 192.3 & 192.3 & 180.1 & 180.1 & 178.6 & 178.6 & ND & ND & ND & ND & ND & ND & ND & ND & ND & ND & ND \\
\hline 299-W7-3 & 206.0 & $\mathrm{NI}$ & $\mathrm{NI}$ & $\mathrm{NI}$ & $\mathrm{NI}$ & $\mathrm{NI}$ & $\mathrm{NI}$ & $\mathrm{NI}$ & $\mathrm{NI}$ & $\mathrm{NI}$ & $\mathrm{NI}$ & $\mathrm{NI}$ & $\mathrm{NI}$ & $\mathrm{NI}$ & $\mathrm{NI}$ & $\mathrm{NI}$ & $\mathrm{NI}$ & $\mathrm{NI}$ & 61.3 & $\mathrm{NI}$ \\
\hline 299-W7-4 & 204.7 & 189.6 & NP & NP & 189.6 & 182.0 & 182.0 & 174.3 & 174.3 & ND & ND & ND & ND & ND & ND & ND & ND & ND & ND & ND \\
\hline 299-W7-5 & 205.1 & 194.5 & NP & NP & 194.5 & 182.3 & NP & NP & 182.3 & ND & ND & ND & ND & ND & ND & ND & ND & ND & ND & ND \\
\hline 299-W7-6 & 206.8 & 200.9 & NP & NP & 200.9 & 184.1 & NP & NP & 184.1 & ND & ND & ND & ND & ND & ND & ND & ND & ND & ND & ND \\
\hline 299-W8-1 & 213.8 & 185.9 & NP & NP & 185.9 & 177.1 & 177.1 & 167.9 & 167.9 & ND & ND & ND & ND & ND & ND & ND & ND & ND & ND & ND \\
\hline 299-W10-13 & 213.1 & 179.5 & 179.5 & 178.0 & 178.0 & 170.4 & NP & NP & 170.4 & ND & ND & ND & ND & ND & ND & ND & ND & ND & ND & ND \\
\hline 299-W10-14 & 213.2 & 176.5 & 176.5 & 173.4 & 173.4 & 168.9 & NP & NP & 168.9 & 75.9 & NP & NP & NP & NP & 75.9 & ND & ND & ND & ND & NP \\
\hline 299-W11-2 & 217.0 & 183.2 & 183.2 & 178.9 & 178.9 & 171.9 & NP & NP & 171.9 & 88.1 & NP & NP & NP & NP & 88.1 & 82.9 & 82.9 & 61.9 & 61.9 & NP \\
\hline 299-W11-26 & 211.5 & 185.6 & 185.6 & 179.8 & 179.8 & 171.3 & 171.3 & 168.9 & 168.9 & 86.0 & NP & NP & NP & NP & 86.0 & 80.8 & 80.8 & 57.9 & 57.9 & NP \\
\hline 299-W11-13 & 210.9 & $\mathrm{NI}$ & $\mathrm{NI}$ & $\mathrm{NI}$ & $\mathrm{NI}$ & $\mathrm{NI}$ & $\mathrm{NI}$ & $\mathrm{NI}$ & $\mathrm{NI}$ & $\mathrm{NI}$ & $\mathrm{NI}$ & $\mathrm{NI}$ & $\mathrm{NI}$ & $\mathrm{NI}$ & $\mathrm{NI}$ & $\mathrm{NI}$ & $\mathrm{NI}$ & $\mathrm{NI}$ & 62.5 & $\mathrm{NI}$ \\
\hline 299-W14-7 & 206.7 & 167.6 & NP & NP & 167.6 & 166.4 & NP & NP & 166.4 & 77.4 & NP & NP & NP & NP & 77.4 & 61.9 & 61.9 & 48.5 & 48.5 & NP \\
\hline 299-W14-8A & 221.0 & 173.7 & 173.7 & 169.2 & 169.2 & 164.3 & NP & NP & 164.3 & 86.9 & NP & NP & NP & NP & 86.9 & 84.7 & 84.7 & 57.6 & 57.6 & NP \\
\hline 299-W15-5 & 204.2 & 170.7 & 170.7 & 167.3 & 167.3 & 161.5 & NP & NP & 161.5 & 74.7 & NP & NP & NP & NP & 74.7 & 68.6 & 68.6 & 44.2 & 44.2 & NP \\
\hline 299-W15-14 & 212.8 & 163.1 & NP & NP & 163.1 & 159.1 & 159.1 & 155.8 & 155.8 & 73.2 & NP & NP & NP & NP & 73.2 & 58.2 & 58.2 & 40.2 & 40.2 & NP \\
\hline 299-W15-15 & 212.7 & 170.1 & 170.1 & 167.0 & 167.0 & 159.4 & NP & NP & 159.4 & ND & ND & ND & ND & ND & ND & ND & ND & ND & ND & ND \\
\hline 299-W15-16 & 208.8 & 167.6 & 167.6 & 166.1 & 166.1 & 160.0 & 160.0 & 157.0 & 157.0 & ND & ND & ND & ND & ND & ND & ND & ND & ND & ND & ND \\
\hline 299-W15-18 & 209.0 & 167.9 & NP & NP & 167.9 & 163.4 & 163.4 & 157.3 & 157.3 & ND & ND & ND & ND & ND & ND & ND & ND & ND & ND & ND \\
\hline 299-W18-21 & 203.8 & 167.3 & 167.3 & 162.8 & 162.8 & 159.7 & NP & NP & 159.7 & ND & ND & ND & ND & ND & ND & ND & ND & ND & ND & ND \\
\hline 299-W18-22 & 203.8 & 167.3 & 167.3 & 161.2 & 161.2 & 158.2 & NP & NP & 158.2 & 67.7 & NP & NP & NP & NP & 67.7 & ND & ND & ND & ND & NP \\
\hline 299-W18-23 & 212.4 & 163.4 & 163.4 & 160.6 & 160.6 & 157.3 & NP & NP & 157.3 & ND & ND & ND & ND & ND & ND & ND & ND & ND & ND & ND \\
\hline
\end{tabular}


Table A.1. Interpreted Top and Bottom Elevation of Major Hydrogeologic Units (continued)

$\mathrm{NP}=$ unit not present

$\mathrm{NC}=$ not certain whether unit is present or not

$\mathrm{ND}=$ well not deep enough to determine

$\mathrm{NI}=$ not interpreted or unknown

Elevations in $\mathrm{m}$ above mean sea level based on the National Geodetic Vertical Datum of 1929 (NGVD29 datum)

\begin{tabular}{|c|c|c|c|c|c|c|c|c|c|c|c|c|c|c|c|c|c|c|c|c|}
\hline Well number & $\begin{array}{l}\text { Casing } \\
\text { Elev. }\end{array}$ & Bot 1 & Top 2 & Bot 2 & Top 3 & Bot 3 & Top 4 & Bot 4 & Top 5 & Bot 5 & Top 6 & Bot 6 & Top 7 & Bot 7 & Top 8 & Bot 8 & Top 9 & Bot 9 & $\begin{array}{l}\text { Top of } \\
\text { Basalt }\end{array}$ & $\begin{array}{l}\text { Layer } 6 \\
\% \text { mud }\end{array}$ \\
\hline 299-W18-24 & 208.6 & 167.3 & 167.3 & 165.8 & 165.8 & 159.7 & 159.7 & 155.1 & 155.1 & ND & ND & ND & ND & ND & ND & ND & ND & ND & ND & ND \\
\hline 299-W19-4 & 217.3 & 166.1 & 166.1 & 157.3 & 157.3 & 143.3 & 143.3 & 138.1 & 138.1 & 83.2 & NP & NP & NP & NP & 83.2 & 80.2 & 80.2 & 53.0 & 53.0 & NP \\
\hline 299-W19-8 & 213.4 & $\mathrm{NI}$ & $\mathrm{NI}$ & $\mathrm{NI}$ & $\mathrm{NI}$ & $\mathrm{NI}$ & $\mathrm{NI}$ & $\mathrm{NI}$ & $\mathrm{NI}$ & $\mathrm{NI}$ & $\mathrm{NI}$ & $\mathrm{NI}$ & $\mathrm{NI}$ & $\mathrm{NI}$ & $\mathrm{NI}$ & $\mathrm{NI}$ & $\mathrm{NI}$ & $\mathrm{NI}$ & 44.2 & $\mathrm{NI}$ \\
\hline 299-W19-10 & 207.9 & 165.2 & 165.2 & 159.1 & 159.1 & 155.8 & NP & NP & 155.8 & 68.0 & NP & NP & NP & NP & 68.0 & 60.0 & 60.0 & 38.7 & 38.7 & NP \\
\hline 299-W21-1 & 213.1 & 160.0 & 160.0 & 152.1 & 152.1 & 143.6 & NP & NP & 143.6 & ND & ND & ND & ND & ND & ND & ND & ND & ND & ND & ND \\
\hline 299-W22-24 & 210.3 & 167.9 & 167.9 & 154.8 & 154.8 & 142.6 & 142.6 & 139.6 & 139.6 & 90.8 & NP & NP & NP & NP & 90.8 & 77.1 & 77.1 & 38.7 & 38.7 & NP \\
\hline 299-W22-27 & 207.3 & $\mathrm{NI}$ & $\mathrm{NI}$ & $\mathrm{NI}$ & $\mathrm{NI}$ & $\mathrm{NI}$ & $\mathrm{NI}$ & $\mathrm{NI}$ & $\mathrm{NI}$ & $\mathrm{NI}$ & $\mathrm{NI}$ & $\mathrm{NI}$ & $\mathrm{NI}$ & $\mathrm{NI}$ & $\mathrm{NI}$ & $\mathrm{NI}$ & $\mathrm{NI}$ & $\mathrm{NI}$ & 38.4 & $\mathrm{NI}$ \\
\hline 199-B3-2 & 135.0 & 118.3 & NP & NP & NP & NP & NP & NP & 118.3 & 88.4 & 88.4 & 54.9 & 54.9 & 19.8 & 19.8 & -42.7 & -42.7 & -64.9 & -64.9 & 27.1 \\
\hline 199-B3-46 & 134.4 & 118.0 & NP & NP & NP & NP & NP & NP & 118.0 & $\mathrm{NI}$ & $\mathrm{NI}$ & $\mathrm{NI}$ & $\mathrm{NI}$ & $\mathrm{NI}$ & $\mathrm{NI}$ & $\mathrm{NI}$ & $\mathrm{NI}$ & $\mathrm{NI}$ & $\mathrm{NI}$ & $\mathrm{NI}$ \\
\hline 199-B4-3 & 140.5 & 119.2 & NP & NP & NP & NP & NP & NP & 119.2 & $\mathrm{NI}$ & $\mathrm{NI}$ & $\mathrm{NI}$ & $\mathrm{NI}$ & $\mathrm{NI}$ & $\mathrm{NI}$ & $\mathrm{NI}$ & $\mathrm{NI}$ & $\mathrm{NI}$ & $\mathrm{NI}$ & $\mathrm{NI}$ \\
\hline 199-B4-9 & $\mathrm{NI}$ & 116.1 & NP & NP & NP & NP & NP & NP & 116.1 & $\mathrm{NI}$ & $\mathrm{NI}$ & $\mathrm{NI}$ & $\mathrm{NI}$ & $\mathrm{NI}$ & $\mathrm{NI}$ & $\mathrm{NI}$ & $\mathrm{NI}$ & $\mathrm{NI}$ & $\mathrm{NI}$ & $\mathrm{NI}$ \\
\hline 199-B9-2 & 151.5 & 123.1 & NP & NP & NP & NP & NP & NP & 123.1 & $\mathrm{NI}$ & $\mathrm{NI}$ & $\mathrm{NI}$ & $\mathrm{NI}$ & $\mathrm{NI}$ & $\mathrm{NI}$ & $\mathrm{NI}$ & $\mathrm{NI}$ & $\mathrm{NI}$ & $\mathrm{NI}$ & $\mathrm{NI}$ \\
\hline 199-D2-5 & 140.2 & 114.0 & NP & NP & NP & NP & NP & NP & NP & NP & 114.0 & ND & ND & ND & ND & ND & ND & ND & ND & $\mathrm{NI}$ \\
\hline 199-D2-6 & 142.3 & 110.9 & NP & NP & NP & NP & NP & NP & NP & NP & 110.9 & ND & ND & ND & ND & ND & ND & ND & ND & $\mathrm{NI}$ \\
\hline 199-D5-19 & 140.8 & 112.2 & NP & NP & NP & NP & NP & NP & NP & NP & 112.2 & ND & ND & ND & ND & ND & ND & ND & ND & $\mathrm{NI}$ \\
\hline 199-D8-6 & 145.1 & 127.1 & NP & NP & NP & NP & NP & NP & 127.1 & 114.6 & 114.6 & ND & ND & ND & ND & ND & ND & ND & ND & $\mathrm{NI}$ \\
\hline 199-D8-53 & 132.9 & 118.6 & NP & NP & NP & NP & NP & NP & 118.6 & 112.2 & 112.2 & ND & ND & ND & ND & ND & ND & ND & ND & $\mathrm{NI}$ \\
\hline 199-D8-55 & 133.8 & 111.9 & NP & NP & NP & NP & NP & NP & 111.9 & $\mathrm{NI}$ & $\mathrm{NI}$ & $\mathrm{NI}$ & $\mathrm{NI}$ & $\mathrm{NI}$ & $\mathrm{NI}$ & $\mathrm{NI}$ & $\mathrm{NI}$ & $\mathrm{NI}$ & $\mathrm{NI}$ & $\mathrm{NI}$ \\
\hline 199-F5-43B & 119.5 & 106.4 & NP & NP & NP & NP & NP & NP & NP & NP & 106.4 & ND & ND & ND & ND & ND & ND & ND & ND & $\mathrm{NI}$ \\
\hline 199-F7-1 & 118.8 & 111.6 & NP & NP & NP & NP & 111.6 & $\mathrm{NI}$ & $\mathrm{NI}$ & $\mathrm{NI}$ & $\mathrm{NI}$ & $\mathrm{NI}$ & $\mathrm{NI}$ & $\mathrm{NI}$ & $\mathrm{NI}$ & $\mathrm{NI}$ & $\mathrm{NI}$ & $\mathrm{NI}$ & $\mathrm{NI}$ & $\mathrm{NI}$ \\
\hline 199-H4-2 & 128.4 & 108.8 & NP & NP & NP & NP & NP & NP & NP & NP & 108.8 & 70.1 & 70.1 & 50.6 & 50.6 & 26.2 & NP & NP & 26.2 & 30.5 \\
\hline 199-H4-3 & 128.1 & 112.8 & NP & NP & NP & NP & NP & NP & 112.8 & 111.3 & 111.3 & ND & ND & ND & ND & ND & ND & ND & ND & $\mathrm{NI}$ \\
\hline 199-H4-10 & 123.1 & 110.9 & NP & NP & NP & NP & NP & NP & NP & NP & 110.9 & ND & ND & ND & ND & ND & ND & ND & ND & $\mathrm{NI}$ \\
\hline 199-H4-12C & 125.9 & 107.6 & NP & NP & NP & NP & NP & NP & NP & NP & 107.6 & 71.0 & 71.0 & 57.3 & 57.3 & ND & ND & ND & ND & 30.5 \\
\hline 199-H4-15C & 123.9 & 110.0 & NP & NP & NP & NP & NP & NP & 110.0 & 99.7 & 99.7 & 63.1 & 63.1 & 57.0 & 57.0 & 28.3 & NP & NP & 28.3 & 30.5 \\
\hline 199-H4-46 & 129.2 & 109.7 & NP & NP & NP & NP & NP & NP & NP & NP & 109.7 & ND & ND & ND & ND & ND & ND & ND & ND & $\mathrm{NI}$ \\
\hline 199-H4-47 & 129.2 & 112.8 & NP & NP & NP & NP & NP & NP & NP & NP & 112.8 & ND & ND & ND & ND & ND & ND & ND & ND & $\mathrm{NI}$ \\
\hline
\end{tabular}


Table A.1. Interpreted Top and Bottom Elevation of Major Hydrogeologic Units (continued)

$\mathrm{NP}=$ unit not present

$\mathrm{NC}=$ not certain whether unit is present or not

$\mathrm{ND}=$ well not deep enough to determine

$\mathrm{NI}=$ not interpreted or unknown

Elevations in $\mathrm{m}$ above mean sea level based on the National Geodetic Vertical Datum of 1929 (NGVD29 datum)

\begin{tabular}{|c|c|c|c|c|c|c|c|c|c|c|c|c|c|c|c|c|c|c|c|c|}
\hline Well number & $\begin{array}{l}\text { Casing } \\
\text { Elev. }\end{array}$ & Bot 1 & Top 2 & Bot 2 & Top 3 & Bot 3 & Top 4 & Bot 4 & Top 5 & Bot 5 & Top 6 & Bot 6 & Top 7 & Bot 7 & Top 8 & Bot 8 & Top 9 & Bot 9 & $\begin{array}{l}\text { Top of } \\
\text { Basalt }\end{array}$ & $\begin{array}{l}\text { Layer } 6 \\
\% \text { mud }\end{array}$ \\
\hline 199-H5-1A & 128.0 & 111.3 & NP & NP & NP & NP & NP & NP & NP & NP & 111.3 & ND & ND & ND & ND & ND & ND & ND & ND & $\mathrm{NI}$ \\
\hline 199-K-10 & 142.2 & 133.2 & NP & NP & NP & NP & 133.2 & 128.3 & 128.3 & 91.7 & 91.7 & ND & ND & ND & ND & ND & ND & ND & ND & $\mathrm{NI}$ \\
\hline 199-K-11 & 142.5 & 134.1 & NP & NP & NP & NP & 134.1 & 129.8 & 129.8 & 90.8 & 90.8 & ND & ND & ND & ND & ND & ND & ND & ND & $\mathrm{NI}$ \\
\hline 199-K-12 & 142.2 & 131.1 & NP & NP & NP & NP & NP & NP & 131.1 & ND & ND & ND & ND & ND & ND & ND & ND & ND & ND & ND \\
\hline 199-K-14 & 143.0 & 131.7 & NP & NP & NP & NP & NP & NP & 131.7 & ND & ND & ND & ND & ND & ND & ND & ND & ND & ND & ND \\
\hline 199-K-32B & 135.7 & 124.7 & NP & NP & NP & NP & NP & NP & 124.7 & 94.2 & 94.2 & ND & ND & ND & ND & ND & ND & ND & ND & $\mathrm{NI}$ \\
\hline 199-K-35 & 150.9 & 127.4 & NP & NP & NP & NP & NP & NP & 127.4 & ND & ND & ND & ND & ND & ND & ND & ND & ND & ND & ND \\
\hline $199-N-14$ & 138.1 & 120.4 & NP & NP & NP & NP & NP & NP & 120.4 & ND & ND & ND & ND & ND & ND & ND & ND & ND & ND & ND \\
\hline $199-\mathrm{N}-16$ & 139.0 & 122.2 & NP & NP & NP & NP & NP & NP & 122.2 & ND & ND & ND & ND & ND & ND & ND & ND & ND & ND & ND \\
\hline $199-N-50$ & 138.1 & 119.2 & NP & NP & NP & NP & NP & NP & 119.2 & ND & ND & ND & ND & ND & ND & ND & ND & ND & ND & ND \\
\hline $199-N-69$ & 139.6 & 119.5 & NP & NP & NP & NP & NP & NP & 119.5 & 108.8 & 108.8 & ND & ND & ND & ND & ND & ND & ND & ND & $\mathrm{NI}$ \\
\hline $199-\mathrm{N}-70$ & 137.8 & 124.1 & NP & NP & NP & NP & NP & NP & 124.1 & 106.4 & 106.4 & ND & ND & ND & ND & ND & ND & ND & ND & $\mathrm{NI}$ \\
\hline $199-N-77$ & 139.9 & 120.1 & NP & NP & NP & NP & NP & NP & 120.1 & 109.4 & 109.4 & ND & ND & ND & ND & ND & ND & ND & ND & $\mathrm{NI}$ \\
\hline 399-1-9 & 117.3 & 100.8 & NP & NP & NP & NP & NP & NP & 100.9 & 81.4 & NP & NP & NP & NP & 81.4 & 64.3 & NP & NP & 64.3 & NP \\
\hline $399-1-16 C$ & 116.5 & 102.8 & NP & NP & NP & NP & NP & NP & 102.7 & 80.8 & NP & NP & NP & NP & 80.8 & 64.0 & NP & NP & 64.1 & NP \\
\hline $399-1-17 C$ & 115.3 & 104.3 & NP & NP & NP & NP & NP & NP & 115.2 & 80.5 & NP & NP & NP & NP & 80.5 & 64.6 & NP & NP & 64.7 & NP \\
\hline $399-1-18 C$ & 118.3 & 102.7 & NP & NP & NP & NP & NP & NP & 102.7 & 82.3 & NP & NP & NP & NP & 82.3 & 80.2 & 80.2 & 74.4 & 74.4 & NP \\
\hline $399-1-20$ & 117.5 & $\mathrm{NI}$ & $\mathrm{NI}$ & $\mathrm{NI}$ & $\mathrm{NI}$ & $\mathrm{NI}$ & $\mathrm{NI}$ & $\mathrm{NI}$ & $\mathrm{NI}$ & $\mathrm{NI}$ & $\mathrm{NI}$ & $\mathrm{NI}$ & $\mathrm{NI}$ & $\mathrm{NI}$ & $\mathrm{NI}$ & $\mathrm{NI}$ & $\mathrm{NI}$ & $\mathrm{NI}$ & 63.1 & $\mathrm{NI}$ \\
\hline 399-3-3 & 121.2 & 103.6 & NP & NP & NP & NP & NP & NP & 103.6 & 89.6 & 89.6 & 77.7 & 77.7 & 73.5 & 73.5 & ND & ND & ND & ND & 20.1 \\
\hline $399-4-5$ & 122.5 & 103.6 & NP & NP & NP & NP & NP & NP & 103.6 & 96.9 & 96.9 & 89.9 & 89.9 & 78.6 & 78.6 & 63.1 & NP & NP & 63.1 & 30.5 \\
\hline $399-4-6$ & 118.9 & $\mathrm{NI}$ & $\mathrm{NI}$ & $\mathrm{NI}$ & $\mathrm{NI}$ & $\mathrm{NI}$ & $\mathrm{NI}$ & $\mathrm{NI}$ & $\mathrm{NI}$ & $\mathrm{NI}$ & $\mathrm{NI}$ & $\mathrm{NI}$ & $\mathrm{NI}$ & $\mathrm{NI}$ & $\mathrm{NI}$ & $\mathrm{NI}$ & $\mathrm{NI}$ & $\mathrm{NI}$ & 63.1 & $\mathrm{NI}$ \\
\hline 399-4-7 & 114.9 & 102.7 & NP & NP & NP & NP & NP & NP & 102.7 & 78.9 & NP & NP & NP & NP & 78.9 & ND & ND & ND & ND & NP \\
\hline $399-5-2$ & 119.1 & 104.5 & NP & NP & NP & NP & NP & NP & 104.5 & 98.1 & 98.1 & 92.7 & 92.7 & 69.8 & 69.2 & 61.3 & NP & NP & 61.3 & 30.5 \\
\hline $399-8-1$ & 120.8 & 100.0 & NP & NP & NP & NP & NP & NP & 100.0 & ND & ND & ND & ND & ND & ND & ND & ND & ND & ND & ND \\
\hline 399-8-2 & 120.7 & 104.9 & NP & NP & NP & NP & NP & NP & 104.9 & 99.4 & 99.4 & 87.2 & 87.2 & ND & ND & ND & ND & ND & ND & 4.0 \\
\hline $399-8-3$ & 119.8 & 101.8 & NP & NP & NP & NP & NP & NP & 101.8 & 93.0 & 93.0 & ND & ND & ND & ND & ND & ND & ND & ND & $\mathrm{NI}$ \\
\hline $399-8-5 C$ & 121.2 & 103.6 & NP & NP & NP & NP & NP & NP & 103.6 & 98.8 & 98.8 & 93.0 & 93.0 & 71.0 & 71.0 & 61.6 & NP & NP & 61.6 & 30.5 \\
\hline
\end{tabular}


Table A.1. Interpreted Top and Bottom Elevation of Major Hydrogeologic Units (continued)

$\mathrm{NP}=$ unit not present

$\mathrm{NC}=$ not certain whether unit is present or not

$\mathrm{ND}=$ well not deep enough to determine

$\mathrm{NI}=$ not interpreted or unknown

Elevations in m above mean sea level based on the National Geodetic Vertical Datum of 1929 (NGVD29 datum)

\begin{tabular}{|c|c|c|c|c|c|c|c|c|c|c|c|c|c|c|c|c|c|c|c|c|}
\hline Well number & $\begin{array}{l}\text { Casing } \\
\text { Elev. }\end{array}$ & Bot 1 & Top 2 & Bot 2 & Top 3 & Bot 3 & Top 4 & Bot 4 & Top 5 & Bot 5 & Top 6 & Bot 6 & Top 7 & Bot 7 & Top 8 & Bot 8 & Top 9 & Bot 9 & $\begin{array}{l}\text { Top of } \\
\text { Basalt }\end{array}$ & $\begin{array}{l}\text { Layer } 6 \\
\% \text { mud }\end{array}$ \\
\hline 499-S1-7B & 168.9 & 111.6 & NP & NP & NP & NP & NP & NP & 111.6 & 65.8 & NP & NP & NP & NP & 65.8 & 25.6 & 25.6 & -12.5 & -12.5 & 15.2 \\
\hline 499-S1-7A & 168.9 & 111.6 & NP & NP & NP & NP & NP & NP & 111.6 & ND & ND & ND & ND & ND & ND & ND & ND & ND & ND & ND \\
\hline 499-S1-8J & 166.4 & 111.6 & NP & NP & NP & NP & NP & NP & 111.6 & 67.1 & NP & NP & NP & NP & 67.1 & ND & ND & ND & ND & 15.2 \\
\hline 499-S1-8H & 166.4 & $\mathrm{NI}$ & $\mathrm{NI}$ & $\mathrm{NI}$ & $\mathrm{NI}$ & $\mathrm{NI}$ & $\mathrm{NI}$ & $\mathrm{NI}$ & $\mathrm{NI}$ & $\mathrm{NI}$ & $\mathrm{NI}$ & $\mathrm{NI}$ & $\mathrm{NI}$ & $\mathrm{NI}$ & $\mathrm{NI}$ & $\mathrm{NI}$ & $\mathrm{NI}$ & $\mathrm{NI}$ & -19.8 & $\mathrm{NI}$ \\
\hline 1199-14-14 & 108.2 & 98.1 & NP & NP & NP & NP & NP & NP & NP & NP & NP & NP & NP & NP & 98.1 & 77.4 & NP & NP & 77.4 & NP \\
\hline 1199-20-17 & 108.2 & 99.7 & NP & NP & NP & NP & NP & NP & NP & NP & 99.7 & 86.9 & 86.9 & 81.4 & 81.4 & 66.1 & NP & NP & 66.1 & 30.5 \\
\hline 1199-22-11A & 123.1 & 101.8 & NP & NP & NP & NP & NP & NP & 101.8 & 97.5 & 97.5 & 94.5 & 94.5 & 89.3 & 89.3 & 80.5 & NP & NP & 80.5 & 30.5 \\
\hline 1199-33-18D & 111.3 & 100.0 & NP & NP & NP & NP & NP & NP & 100.0 & 97.8 & 97.8 & 85.3 & 85.3 & ND & ND & ND & ND & ND & ND & 27.4 \\
\hline 1199-34-13 & 120.1 & 103.3 & NP & NP & NP & NP & NP & NP & 103.3 & 97.2 & 97.2 & ND & ND & ND & ND & ND & ND & ND & ND & $\mathrm{NI}$ \\
\hline 1199-39-16B & 113.1 & 100.3 & NP & NP & NP & NP & NP & NP & 100.3 & 93.9 & 93.9 & 88.7 & 88.7 & 85.6 & 85.6 & ND & ND & ND & ND & 30.5 \\
\hline $1199-40-16 \mathrm{~A}$ & 118.9 & 102.1 & NP & NP & NP & NP & NP & NP & 102.1 & 100.6 & 100.6 & ND & ND & ND & ND & ND & ND & ND & ND & $\mathrm{NI}$ \\
\hline $3099-45-18 A$ & 110.0 & 98.8 & NP & NP & NP & NP & NP & NP & NP & NP & 98.8 & ND & ND & ND & ND & ND & ND & ND & ND & $\mathrm{NI}$ \\
\hline $3099-47-18 A$ & 114.0 & 100.9 & NP & NP & NP & NP & NP & NP & NP & NP & 100.9 & 77.4 & 77.4 & 69.5 & 69.5 & 60.4 & NP & NP & 60.4 & 19.8 \\
\hline TW-16 & 114.9 & 109.4 & NP & NP & NP & NP & NP & NP & 109.4 & 101.8 & 101.8 & ND & ND & ND & ND & ND & ND & ND & ND & $\mathrm{NI}$ \\
\hline W-5 & 110.0 & 99.7 & NP & NP & NP & NP & NP & NP & NP & NP & 99.7 & 78.0 & 78.0 & ND & ND & ND & ND & ND & ND & 23.5 \\
\hline
\end{tabular}




\section{A.2 References}

Thorne PD, and MA Chamness. 1992. Status report on the development of a three-dimensional conceptual model for the Hanford Site unconfined aquifer system. PNL-8332, Pacific Northwest Laboratory, Richland, Washington.

Thorne PD, MA Chamness, FA Spane Jr, VR Vermeul, and WD Webber. 1993. Three-dimensional conceptual model for the Hanford Site unconfined aquifer system, FY93 status report. PNL-8971, Pacific Northwest Laboratory, Richland, Washington.

Thorne PD, MA Chamness, VR Vermeul, QC MacDonald, and SE Schubert. 1994. Three-dimensional conceptual model for the Hanford Site unconfined aquifer system, FY 1994 status report. PNL-10195, Pacific Northwest Laboratory, Richland, Washington.

Wurstne SK, PD Thorne, MA Chamness, MD Freshley, and MD Williams. 1995. Development of a three-dimensional ground-water model of the Hanford Site unconfined aquifer system: FY 1995 status report. PNL-10886, Pacific Northwest Laboratory, Richland, Washington. 


\title{
Appendix B
}

Bibliography and Preliminary Information on Intercommunication Between the Unconfined Aquifer and the Uppermost Confined Aquifer at the Hanford Site

\author{
by \\ Philip Rogers \\ Jacobs Engineering Group \\ Richland, Washington
}

February 15, 2000 


\section{Contents}

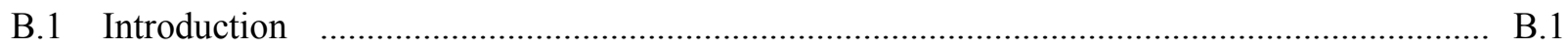

B.2 Hydrogeologic Setting And Alternative Conceptual Models............................................ B.1

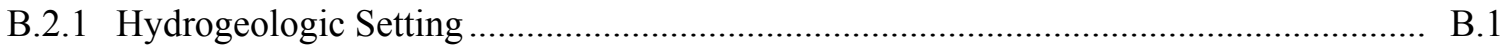

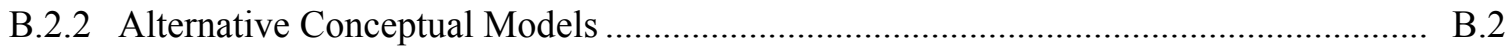

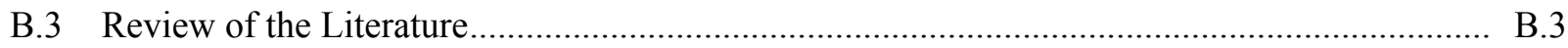

B.3.1 Geohydrology of the Rattlesnake Ridge Interbed in the Gable Mountain Pond Area.... B.4

B.3.2 An Assessment of Aquifer Intercommunication in the B Pond-Gable Mountain

Pond Area of the Hanford Site............................................................................ B.6

B.3.3 Consultation Draft, Site Characterization Plan, Reference Repository Location, Hanford, Site, Washington ...................................................................... B. 8

B.3.4 An Evaluation of Aquifer Intercommunication Between the Unconfined and

Rattlesnake Ridge Aquifers on the Hanford Site ................................................... B. 10

B.3.5 Hydrologic Model for the 200 East Groundwater Aggregate Area .............................. B.11

B.3.6 Hydrochemistry and Hydrogeologic Conditions Within the Hanford Site Upper Basalt Confined Aquifer System ............................................................. B. B. 11

B.3.7 Conclusions and Data from Other Documents ..................................................... B. 13

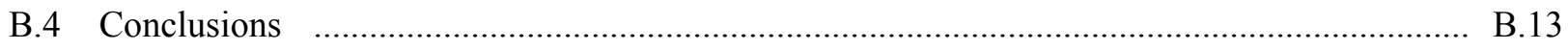

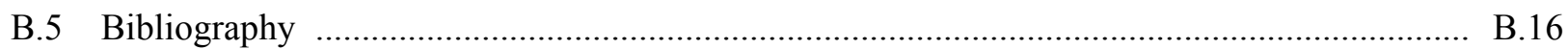

Exhibit 1 - Task 4.2: Effects of Surface Waste Disposal Activity on Ground-Water Levels in the Saddle Mountains Basalt.......................................................................................... B. 51

\section{Figures}

B.1 Geologic Structures of the Pasco Basin and the Hanford Site. ........................................... B.20

B.2 Stratigraphic Location of Principal Aquifers at the Hanford Site....................................... B. 21

B.3 Hypothetical Composite Cross Section of Possible Geologic Features in the

Layered Basalt Sequence. ........................................................................................ B. 22

B.4 Isopach Map of Rattlesnake Ridge Interbed on the Hanford Site...................................... B.23

B.5 Isopach Map of the Elephant Mountain Member in Vicinity of 200 East Area.................... B. B.24

B.6 Isopach Map of the Rattlesnake Ridge Interbed in Vicinity of 200 East Area. ................... B. B.25

B.7 Isopach Map of the Pomona Member in Vicinity of 200 East Area. ................................. B. B.26

B.8 Areas of Hydraulic Potential for Aquifer Communication Under Hydraulic Head
Conditions as they Existed in the Early 1980's................................................................ B. 27

B.9 Location of 14 Confined Aquifer Test Wells Used in the RHO-RE-ST-12P Study............. B.28

B.10 Stiff Diagrams from Samples in the Unconfined Aquifer................................................. B.29

B.11 Stiff Diagrams from Samples in the Rattlesnake Ridge Interbed ...................................... B. B.

B.13 Barometric Efficiencies for the Uppermost Confined Aquifer as Estimated in
RHO-RE-ST-12P Study ........................................................................................ B. 
B.13 Location of Wells and Boreholes Where Groundwater Levels were Measured for the Basalt Waste Isolation Project.

B.14 Vertical Head Distributions Within the Columbia River Basalt from Boreholes DC-14 and DC-15.

B.16 Vertical Head Distributions Within the Columbia River Basalt from

Boreholes DC-1419, DC-20, and DC-22.

B.16 Comparison of Hydraulic Heads Between the Unconfined Aquifer and the Rattlesnake Ridge Interbed for Conditions in the Early 1980's.

B.17 Hydrographs for Selected Boreholes in the Vicinity of Gable Mountain and Gable Butte. .

B.18 Correlation of Geochemical Depth Profiles for Saddle Mountains and Wanapum Basalt Groundwaters in Borehole DC-14.

B.19 Structural Features on or Near the Hanford Site that Could be Associated with Vertical Groundwater Flow.

B.20 Isopach Map for the Elephant Mountain Member.

B.21 Isopach Map of the Saddle Mountains Basalt.

B.22 Isopach Map of the Pomona Member.

B.23 Locations of Aquifer Intercommunication as Estimated in WHC-SD-EN-TI-019, Rev. 0. ..

B.24 Tri-Linear Diagrams Showing Hydro-chemical Evolution of Groundwater Within the Upper Basalt Confined Aquifer System.

B.25 Comparison of Observed Hydraulic Heads for the Upper Basalt and Overlying Unconfined Aquifer Systems.

B.26 Isopach Map of the Elephant Mountain Member from 200 BP-1 Operable Unit.

B.27 200-BP-1 Operable Unit Cross Section Locations

B.28 Cross Section A-A' 200-BP-1 Operable Unit.

B.29 Cross Section B-B' 200-BP-1 Operable Unit.

B.30 Tritium, Nitrate, Technetium-99, and Cobalt-60 Detected in Confined Aquifer Wells, 1988.

B.31 Stratigraphy of the Columbia River Basalt.

B.32 Estimated Water Table Elevation, 1944

B. 55

B.33 Water Table Elevation Map in December 1984.

B. 55

B.34 Contour Map of the Piezometric Levels in the Rosalia Flow Top

B.35 Contour Map of the Piezometric Levels in the Mabton Interbed.

B.36 Contour Map of the Piezometric Levels in the Rattlesnake Ridge Interbed.

B.37 Estimation of the Change in the Maximum Height of the Water Table with Time:

A) Height Decreasing in 1985, and B) Height Increasing Through 1985.

B.38 Load Response at Depth Due to a Growth in the Water Table Mound

B.39 Piezometric Mound and the Finite Difference Grid Used to Model the Dissipation.

B.40 Vertical Hydraulic Gradient through the Saddle Mountain Basalt at DC-19, DC-20, and DC-22.

B.41 Conceptualization of the Flow through a Volume of the Saddle Mountain Basalt.

B. 66

\section{Table}

B.1 Assessment of Alternative Conceptual Models

B. 15

B.iv 


\section{Bibliography and Preliminary Information on Intercommunication Between the Unconfined Aquifer and the Uppermost Confined Aquifer at the Hanford Site}

\section{B.1 Introduction}

The purpose of this appendix is to provide an initial bibliography and summation of hydrogeologic data relative to the intercommunication between the unconfined aquifer and the uppermost confined aquifer at the Hanford Site. This information is needed to support the continued refinement of the Hanford site-wide Groundwater Model (SGM). The present conceptualization assumes that there is no intercommunication between the unconfined aquifer that is being modeled and the uppermost confined aquifer. This assumption needs to be tested and alternatives considered. Testing the intercommunication assumption and potential revisions to the conceptual model and its numerical implementation requires knowledge of 1) the spatially varying hydrogeologic characteristics of the confining layer(s) that separate the unconfined and the uppermost confined aquifers and 2) the spatially and temporally varying hydraulic heads within the two aquifers. With these data needs in mind, the literature has been reviewed for data, assumptions, and approaches that previous investigators have used in addressing aquifer intercommunication at the Hanford Site and surrounding region.

This report documents the sources of information identified in the preliminary literature review and assessment. A qualitative assessment of the key sources of information and data is also provided for consideration by PNNL in incorporating the basalt system in future model applications at the Hanford Site.

\section{B.2 Hydrogeologic Setting And Alternative Conceptual Models}

This section provides the hydrogeologic framework in which aquifer intercommunication occurs. Alternative conceptualizations of aquifer intercommunication are offered to help focus the information from the literature.

\section{B.2.1 Hydrogeologic Setting}

The Hanford Site is located within the Pasco Basin, a structural and topographic basin in south central Washington. The basin is bounded by anticlinal structures of the Saddle Mountains to the north; Umtanum Ridge, Yakima Ridge, and Rattlesnake Hills to the west; and the Rattlesnake Hills and a series of doubly plunging anticlines to the south (Figure B.1). The major geologic units, in ascending order beneath the Hanford Site are basement rocks of undetermined origin, the Columbia River basalt group with intercalated sediments of the Ellensburg Formation, the Ringold Formation, and the Hanford formation (Figure B.2). The basalt stratigraphy consists of the Grande Ronde, Wanapum, and Saddle Mountains. The focus of this report is on the potential intercommunication between water bearing units in the Saddle Mountain basalt and the overlying unconfined aquifer. The Saddle Mountains basalt within 
the Hanford Site area consists of four basalt flows separated by interbedded sediments (stratigraphically assigned to the Ellensburg Formation). The flows, in ascending order are the Umatilla, Esquatzel, Pomona, and Elephant Mountain. The major interbedded sediments are the Mabton, Cold Creek, Selah, and Rattlesnake Ridge.

The main water-bearing zones in the Saddle Mountains basalt are the sedimentary interbeds and the interflow zones between basalt flows associated with the interconnecting vesicles and fractures of basalt flow tops and bottoms as illustrated in Figure B.3. Collectively, these interbedded sediments and the Saddle Mountain basalt interflow zones form an extensive confined aquifer system, which in this report is referred to as the uppermost confined aquifer. Note however, that alternative nomenclature has been used in the Site literature. For example, several of the water bearing units in the Saddle Mountains basalt were designated as separate aquifers in PNL-6313 (e.g., Rattlesnake Ridge aquifer and Elephant Mountain aquifer).

The saturated zone within the unconsolidated glacio-fluvial sediments of the Hanford formation (an informal designation) and the semiconsolidated silts, sands, and gravels of the Ringold Formation form the unconfined aquifer. The bottom of the unconfined aquifer throughout most of the Hanford Site is the top of the Elephant Mountain basalt flow interior where it is present and, in some areas, the basal Ringold where it occurs as a low permeability silt and clay. There are locations where the Elephant Mountain basalt has been fully eroded and is absent, specifically between Gable Butte and Gable Mountain in the vicinity of West Lake. Figure B.4 is an isopach map of the Elephant Mountain basalt on the Hanford Site. Figure B.5 is an isopach map of the Elephant Mountain basalt in the West Lake and B Pond area showing the area where the basalt is absent between Gable Butte and Gable Mountain and two other localized pockets to the southeast. The Rattlesnake Ridge interbed which lies directly beneath the Elephant Mountain basalt has also been removed by erosion in the gap area as illustrated in Figure B.6, which is an isopach map of the Rattlesnake Ridge interbed in the vicinity of B Pond and West Lake. Likewise, a smaller area of Pomona basalt has been removed by erosion in the gap area as illustrated in the isopach map of the Pomona basalt (Figure B.7). The unconfined aquifer is generally well understood and has been conceptualized and numerically modeled as a part of the Hanford Groundwater/Vadose Zone Project. The focus of this report is potential intercommunication between the unconfined aquifer and the uppermost confined aquifer.

\section{B.2.2 Alternative Conceptual Models}

Alternative conceptualizations of the intercommunication between the unconfined and confined aquifer within the Hanford Site are provided in the following paragraph. The alternative conceptualizations start with the highest degree of separation between the two aquifers.

Alternative Conceptualization 1. The unconfined aquifer is assumed to be hydraulically separated from the underlying uppermost confined aquifer by silts and clay units within the Ringold Formation and dense, low hydraulic conductivity interior of the Elephant Mountain basalt flow. There would be no discernable intercommunication between the unconfined aquifer and the uppermost confined aquifer within the Saddle Mountains basalt. 
Alternative Conceptualization 2. Similar to conceptualization 1 in that the Elephant Mountain basalts provides for hydraulic separation between the unconfined and uppermost aquifers over most to the Hanford Site, except where the Elephant Mountain Member is absent, as shown in Figure B.5. The area where the Elephant Mountain basalt is absent, located between Gable Butte and Gable Mountain and southeast of the gap, is assumed to provide for direct hydraulic connection between the unconfined and uppermost confined aquifers. The unconfined aquifer would be assumed to be in direct hydraulic connection with the Rattlesnake Ridge interbed within this zone. Outside of the zone of intercommunication, there would be no other discernable intercommunication between the unconfined aquifer and the confined aquifers within the Saddle Mountains basalt.

Alternative Conceptualization 3. This alternative conceptualization includes those features assumed for alternative conceptualization 2, plus the assumption of areally distributed flow across the Elephant Mountain basalt. Over most of the Site, the Elephant Mountain basalt is relatively thick (see Figure B.4) and of low hydraulic conductivity resulting in a relatively small flow per unit area. There is an area where it has been thinned by erosion (see Figure B.5). Some local fracture zones and joints in the flow interior (see Figure B.3), which are otherwise generally vertically discontinuous, may provide flow paths between the unconfined and uppermost confined aquifer where the basalt has been eroded. This is a small area compared to the rest of the Site, but may have a significantly larger vertical hydraulic conductivity. The enhanced vertical hydraulic conductivity, combined with the thinner section, could result in greater hydraulic connection, compared to areas of the Site where the Elephant Mountain basalt has not been eroded.

Alternative Conceptualization 4. This alternative conceptualization includes those features assumed for alternative conceptualization 3, plus the assumption of enhanced vertical conductivity along the anticlinal structures (see Figure B.1). This enhanced vertical conductivity would allow for localized greater (compared to flow through the intact basalt) flow across the Elephant Mountain basalt. In the antclinal zones, the local fractured zone and flow hinge (see Figure B.3) may be open due to the tension stress of the fold and allow for some enhanced hydraulic connection between the unconfined and uppermost confined aquifers.

\section{B.3 Review of the Literature}

Several past investigations have provided important geologic, geophysical, hydrologic, and hydrochemical data relative to potential intercommunication between the unconfined aquifer and the uppermost confined aquifer. Contamination in the unconfined aquifer in the 200 areas was documented as early as 1950 (HW-17088) and investigation of contaminants in the Mabton interbed was published in 1976 (ARH-SA-253) in which it was hypothesized that there may be an area of intercommunication in the vicinity of Gable Mountain Pond. The most focused and relevant information and data on potential intercommunication between the unconfined and uppermost confined aquifers are found in the following studies:

Geohydrology of the Rattlesnake Ridge Interbed in the Gable Mountain Pond Area (RHO-ST-38). This report addresses potential aquifer intercommunication between the unconfined aquifer and the uppermost confined aquifer in an area north of the 200 East Area and south of Gable Mountain Pond. It calls 
upon geophysical data, geologic data (e.g., formation thickness from drilling logs), hydrogeologic data (e.g., hydraulic heads, hydraulic conductivity, and barometric efficiencies) contaminant data (e.g., nitrate ion concentrations), isotopic data (natural and man-made), and hydrochemical data (e.g., concentration of major cations and anions) in an effort to assess aquifer intercommunication.

An Assessment of Aquifer Intercommunication in the B Pond-Gable Mountain Pond Area of the Hanford Site (RHO-RE-ST-12P). This report addresses potential aquifer intercommunication between the unconfined aquifer and uppermost confined aquifer surrounding two former waste disposal ponds, B Pond and Gable Mountain Pond, and encompassing the 200 East Area where other liquid-waste disposal facilities are located. It calls upon geophysical data, geologic data (e.g., formation thickness from drilling logs), hydrogeologic data (e.g., hydraulic heads, hydraulic conductivity, and barometric efficiencies) contaminant data (e.g., nitrate ion concentrations), isotopic data (natural and man-made), and hydrochemical data (e.g., concentration of major cations and anions) in an effort to assess aquifer intercommunication.

Consultation Draft, Site Characterization Plan, Reference Repository Location, Hanford Site, Washington (DOE/RW-0164). This report is very comprehensive in its coverage of a broad range of Site characteristics and properties and although the focus of this report is on data and information associated with the candidate repository in the Grande Ronde, it does including data that supports the evaluation of aquifer intercommunication.

An Evaluation of Aquifer Intercommunication Between the Unconfined and Rattlesnake Ridge Aquifers on the Hanford Site (PNL-6313). This report addresses potential aquifer intercommunication between the unconfined aquifer and uppermost confined aquifer in the vicinity of two former waste disposal ponds, B Pond and Gable Mountain Pond. It relies on hydraulic head data and radionuclide concentration data from the uppermost confined aquifer to address aquifer intercommunication in this area.

Hydrologic Model for the 200 East Groundwater Aggregate Area (WHC-SD-TI-019, Rev. 0). This report contains a section on potential aquifer intercommunication that draws on the work from RHO-REST-12P and PNL-6313 with additional data collected for a the 200-BP-1 Remedial Investigation (WHCSD-EN-TI-037).

\section{Hydrochemistry and Hydrogeologic Conditions Within the Hanford Site Upper Basalt Confined} Aquifer System (PNL-10817). This report integrates hydogeologic and hydrochemical information on a Hanford Site-wide basis for the upper basalt confined aquifer system and address potential intercommunication with the overlying unconfined aquifer system.

\section{B.3.1 Geohydrology of the Rattlesnake Ridge Interbed in the Gable Mountain Pond Area (RHO-ST-38)}

In 1982, one of the earliest, focused evaluations of potential intercommunication between the unconfined and uppermost confined aquifers was published (RHO-ST-38). A series of seven wells were drilled for groundwater chemical, hydrostratigraphic, and hydraulic data. Also, several geophysical 
investigations were conducted. The Elephant Mountain basalt was encountered in all of the wells, the seven wells drilled for the investigation plus one existing well with thickness ranging from 14 to $35 \mathrm{~m}$ (45 to $117 \mathrm{ft}$ ). However, based on the geophysical and hydrochemical data, it was concluded that there was an area of erosion in the uppermost basalt flow (Elephant Mountain) that allowed intercommunication between the unconfined and the uppermost confined aquifer as illustrated in Figure B.8.

Relevant hyrdogeologic and hydrochemical parameter values and information provided in the published report (RHO-ST-38) include the following:

- The Elephant Mountain has an average thickness of $33.5 \mathrm{~m}$ (110 ft) around Gable Mountain Pond (p. 9).

- The Rattlesnake Ridge interbed has and average thickness of $14 \mathrm{~m}$ (46 ft) around Gable Mountain Pond and thins to between 1.5 to $5.5 \mathrm{~m}$ (5 and $18 \mathrm{ft}$ ) over Gable Mountain (p. 9).

- Hydraulic conductivity ranges from $3 \mathrm{E}-2$ to $30 \mathrm{~m} / \mathrm{d}(0.1$ to $100 \mathrm{ft} / \mathrm{d})$ for the sedimentary interbeds (p. 19).

- Hydraulic conductivity ranges from 30 to $610 \mathrm{~m} / \mathrm{d}$ (100 to $2,000 \mathrm{ft} / \mathrm{d}$ ) for the basalt interflow zones (p. 19).

- The confined aquifer system is of a sodium-bicarbonate chemical water type (p. 19).

- Magnetic profiling at the West Lake indicates that the Elephant Mountain basalt is eroded fully, exposing the Pomona basalt to the unconfined aquifer (p. 43).

- Geophysical data suggest that the erosional surfaces are isolated and discontinuous as opposed to an earlier assumption presented in RHO-BWI-LD-5 that had the erosional feature more as a continuous channel at Gable Gap.

- Hydraulic conductivity ranges from 0.2 to $5.2 \mathrm{~m} / \mathrm{d}(0.75$ to $17 \mathrm{ft} / \mathrm{d})$ for the Rattlesnake Ridge interbed in the Gable Mountain Pond area (Table 11, p. 45).

- Barometric efficiency of the Rattlesnake Ridge interbed ranges from 12 to 62 percent (Table 12, p. 46).

- The unconfined aquifer is of a calcium-bicarbonate chemical water type and water-bearing zones below the Rattlesnake interbed are of sodium-bicarbonate type (p. 48).

- There is a similarity in the water chemistry between the unconfined aquifer and Rattlesnake interbed that indicates intercommunication in the Gable Mountain Pond area (p. 51). 


\section{B.3.2 An Assessment of Aquifer Intercommunication in the B Pond-Gable Mountain Pond Area of the Hanford Site (RHO-RE-ST-12P)}

The next focused effort on aquifer intercommunication assessment was published in 1984 (RHO-REST-12P). As with RHO-ST-38, the area of interest was in the vicinity of B Pond and Gable Mountain Pond. Wells were drilled, deepened, or modified for this investigation and aquifer testing, sampling, and analytical analysis were performed. Four mechanisms that could results in intercommunication between the unconfined and uppermost confined aquifers were identified. These mechanisms are:

1. The lithologic framework of the Elephant Mountain basalt - the numerous vertical and horizontal cooling joints, if not sealed with secondary mineralization, could form a network of interconnecting pathways between the aquifers but is considered to be negligible except in areas where the Elephant Mountain is very thin.

2. Stratigraphic unconformities - where confining layers (e.g., the lower Ringold, Elephant Mountain flow interior, and Pomona flow interior) are absent, principally due to erosion. Two erosion areas are identified from borehole logs and two others are postulated based on abnormally thin Elephant Mountain basalt encountered in boreholes and low barometric efficiencies in wells 699-47-50 and 299-E26-8 (Figure B.9).

3. Structural deformation - anticlinal deformation of the Elephant Mountain basalt, particularly in the hinge zone, may act to enhance the aperture of cooling joints, providing a vertical pathway. Faulting could also provide a vertical pathway between aquifers. However, faulting does not appear to be a significant mechanism based on interpretation of the data

4. Human intrusion - improperly constructed wells and boreholes can provide a pathway between the two aquifers (e.g., well 299-E33-12) (see Figure B.9).

It was concluded that there are two areas of complete erosion of the confining bed (i.e., Elephant Mountain basalt) and two areas where erosion is inferred based on geologic and hyrdrogeologic data. An isopach of the Elephant Mountain basalt (see Figure B.5) shows the areas where the basalt has been completely eroded. Also, RHO-RE-ST-12P evaluated geochemical data from the unconfined aquifer and Rattlesnake Ridge interbed (part of the uppermost confined aquifer). Stiff diagrams based on these evaluations are provided on Figures B.10 and B.11. Groundwater samples from well 699-60-57, completed in the unconfined aquifer northwest of West Lake in the gap area was of a sodium bicarbonate chemical type which is typical of the uppermost confined aquifer, indicating discharge from the uppermost confined aquifer to the unconfined aquifer in this area.

Relevant hyrdogeologic and hydrochemical parameter values and information provided in this report (RHO-RE-ST-12P) include the following:

- Barometric efficiency of 14 wells completed in the uppermost confined aquifer ranges from 13 to $44 \%$ (Table 6, p. 33). 
- Transmissivities (average from drawdown, recovery, and slug testing) of selected water-bearing units in the uppermost confined aquifer range from 0.6 to $108 \mathrm{~m}^{2} / \mathrm{d}$ (6 to $1162 \mathrm{ft}^{2} / \mathrm{d}$ ) (Table 7, p. 34).

- The Pomona Member has an average thickness of $56 \mathrm{~m}(184 \mathrm{ft})$ in the study area and behaves as an aquiclude (p. 44).

- The hydraulic conductivity of Columbia River basalt flow interiors ranges from 1E-8 to $1 \mathrm{E}-6 \mathrm{~m} / \mathrm{d}$ (3.3E-8 to $3.3 \mathrm{E}-6 \mathrm{ft} / \mathrm{d})(\mathrm{p} .44)$.

- The hydraulic conductivity of Saddle Mountains basalt flow tops ranges from $1 \mathrm{E}-2$ to $1 \mathrm{E}+2 \mathrm{~m} / \mathrm{d}$ $(3.3 \mathrm{E}-2$ to $330 \mathrm{ft} / \mathrm{d})(\mathrm{p} .44)$.

- The Rattlesnake Ridge interbed is the most significant geologic unit in the present study and its lithologies control the storativity and the movement of groundwater within the aquifer (p. 44).

- The Rattlesnake Ridge interbed has an average thickness of $25 \mathrm{~m}(82 \mathrm{ft})$ southwest of the 200 East Area, thins to the northeast of Gable Mountain to between $1.5 \mathrm{~m}(0.5 \mathrm{ft})$, with an average thickness of $15.6 \mathrm{~m}$ in the study area (p. 44).

- Portions of the Pomona basalt in the West Lake area have been removed by erosion (see Figure 7) (p. 45).

- West of Gable Mountain Pond at borehole 53-55A, the Elephant Mountain basalt has been completely removed by erosion along with a portion of the Rattlesnake Ridge interbed (see Figure 5) (p. 46).

- The hydraulic conductivity of Rattlesnake Ridge interbed ranges from $1 \mathrm{E}-2$ to $1 \mathrm{E}+1 \mathrm{~m} / \mathrm{d}(3.3 \mathrm{E}-2$ to $33 \mathrm{ft} / \mathrm{d})(\mathrm{p} .47)$.

- The Elephant Mountain Member consists of two flows: 1) the lowermost flow is termed the Elephant Mountain I, and is continuous over most of the study area, with thickness ranges from 11.5 to $35 \mathrm{~m}$ (37.7 to $115 \mathrm{ft}$ ); and 2) the upper flow is termed the Elephant Mountain II, is absent in the northwest

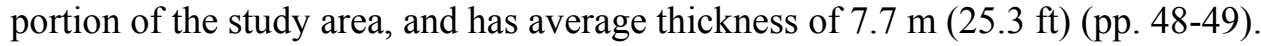

- The interflow zone between the Elephant Mountain I and II is water bearing and the hydraulic conductivity is approximately $622 \mathrm{~m} / \mathrm{d}(2040 \mathrm{ft} / \mathrm{d})(\mathrm{p} .49)$.

- The hydraulic conductivity of the Elephant Mountain II flow top ranges from $1 \mathrm{E}-2$ to $100 \mathrm{~m} / \mathrm{d}$ (3.3E-2 to $330 \mathrm{ft} / \mathrm{d}$ ) per personnel communication with BWIP staff (p. 49).

- The Ringold Formation is present over most of the Site, except over Gable Mountain (apparently not deposited there) and in the area north of the 200 East Area where erosion associated with late Pleistocene flooding has removed it (p. 49). 
- The Rattlesnake Ridge aquifer is discharging to the unconfined aquifer in the area of West Lake where the Rattlesnake Ridge basalt has been completely eroded (p. 66).

- The Elephant Mountain aquifer (i.e., the interflow zone between Elephant Mountain flows I and II probably discharges to the unconfined aquifer in the vicinity of B Pond where the upper flow has been eroded (pp. 66-67).

- The barometric efficiencies for the Rattlesnake Ridge aquifer along with areas of erosion for comparison are plotted on Figure 12. Wells located near known or suspected areas of erosion (699-47-50, 699-54-57, and 699-56-53) have relatively low barometric efficiency (13.3 to 25.4\%). The barometric efficiencies calculated for wells 699-E26-8 and 699-51-46 (24.5 to 28.5\%) are low for a confined aquifer and may indicate some enhanced intercommunication (e.g., erosion of the Elephant Mountain basalt) in the general area of these wells (p. 67).

- Groundwater samples from well 699-60-57 (completed in the unconfined aquifer in the area where the Elephant Mountain basalt has been removed by erosion) were of a sodium bicarbonate type, similar to that from the Mabton interbed, which is an indication of discharge from the uppermost confined aquifer to the unconfined aquifer (p. 70).

\section{B.3.3 Consultation Draft, Site Characterization Plan, Reference Repository Location, Hanford, Site, Washington (DOE/RW-0164)}

The Consultation Draft, Site Characterization Plan, Reference Repository Location, Hanford, Site, Washington (DOE/RW-0164) (SCP) was published in 1988. This report is very comprehensive in its coverage of a broad range of Site characteristics and properties including data that support the evaluation of aquifer intercommunication. However, its focus is on the underlying Grande Ronde basalts in which the candidate repository would have been built. The area that includes Gable Mountain, West Lake, and B Pond is identified as suspect to aquifer intercommunication between the unconfined and uppermost confined aquifers. This report also notes that interchange of groundwaters between the unconfined and confined flow systems takes place pervasively and areally whenever vertical hydraulic gradient conditions exist.

The notion of pervasive areal interchange of groundwater between the two aquifers is supported by vertical hydraulic head information (i.e., there is a difference in hydraulic head between the two aquifers indicating a potential for flow) from selected well pairs or multilevel piezometers across the site. Figure B.13 is a location map for wells and piezometers on the Hanford Site used in (DOE/RW-0164). Figure B.14 shows the vertical head distribution in boreholes DC-14 (located near the Columbia River several kilometers north of Gable Mountain) and DC-15 (located just south of Gable Mountain near B Pond). In DC-14, there is a $35 \mathrm{~m}(115 \mathrm{ft})$ head increase from the top to the bottom of the Saddle Mountains basalt indicating the potential for upward flow from the Saddle Mountains basalt to the overlying unconfined aquifer. At DC-15, which was located to examine the influence of the Gable Mountain structure on confined aquifer intercommunication (DOE/RW-0164, pp. 3.9 - 3.49), the vertical 
head is relatively uniform across the Saddle Mountains basalts indicating potential equilibrium between the unconfined and uppermost confined aquifers. This is consistent with geochemical data from wells in this area that show mixing of the groundwaters from the two aquifers.

Vertical hydraulic heads from three other boreholes are presented in (DOE/RW-0164, p. 3.9-3.52). The locations of these boreholes are south and southwest of the Gable Butte Gable Mountain gap area. The vertical head pattern in the Saddle Mountains basalt is similar for all three boreholes (Figure B.15), which is a decline in hydraulic head with depth, indicating a downward potential for flow. The presence of these hydraulic head differences is also interpreted as an indication of a confining layer(s) between the unconfined and uppermost confined aquifer in this region of the site. The areas of predominately upward and downward hydraulic gradient are shown in Figure B.16. For the period in which these data (hydraulic head) where collected, around 1982, the gradient between the unconfined and uppermost confined aquifers was downward on the eastern portion of the Site and upward on the western portion. This is consistent with the geochemical information discussed above that indicated groundwater from the uppermost confined aquifer was discharging to the unconfined aquifer in the West Lake area.

The Site Characterization Plan (SCP) contains a summary of potential aquifer intercommunication based on the state of the knowledge in the mid-1980s. The following is an excerpt from this summary (references to some figures have not been included and bolding added for emphasis):

In the West Lake and Gable Mountain Pond area,....the basalts were uplifted along the eastern extension of the Umtanum Ridge-Gable Mountain anticline. These basalts were then eroded by postglacial floodwaters and the ancestral Columbia River. Hydraulic communication between the unconfined and confined aquifers now appears to exist in this area. Hydraulic head information displayed in Figures 3.9-49 (see Figure B.16 of this appendix) implies that this area has a downward hydraulic gradient between the unconfined aquifer and Rattlesnake Ridge interbed. In addition, an examination of borehole hydrographs (Figure 3.9-78) (Figure B.17 of this appendix) in the vicinity of Gable Mountain and Gable Butte suggests that rather uniform head responses take place between the suprabasalt sediments, Rattlesnake Ridge interbed, and Mabton interbed. The period and amplitude of these responses appear quite similar. This suggests that these Stratigraphic horizons are hydraulically interconnected.

The SCP offers two alternative conceptual flow models for the Grande Ronde basalt, a point source model and a stagnation model (DOE/RW-0164, p. 3.9-168). The point source model would have vertical flux of deep groundwater occur everywhere under the Hanford Site and possibly enhanced along anticlinal structures. For the stagnation model, lateral flow in the various hydrostratigraphic units would dominate over vertical flow. The SCP evaluated the groundwater composition as a function of depth in an attempt to resolve differences between the two conceptual models. The $\mathrm{pH}$ and concentration of sodium and fluoride as a function of depth are plotted on Figure B.18 from groundwater samples collected from borehole DC-14 which is located about 8 miles northeast of the Gable Butte-Gable Mountain gap, along the Columbia River. As groundwater residence time increases, rock and water reactions (e.g., hydrolysis and ion exchange) lead to higher $\mathrm{pH}$ values and higher concentrations of sodium. The SCP indicates that based on hydrochemical inferences, the Selah/Cold Creek interbeds and 
Priest Rapids and Roza Members of the Wanapum basalt are relatively transmissive because the groundwater from these units have lower $\mathrm{pH}$ and sodium and fluoride concentrations compared to the other zones that were sampled. The rock intervals sampled in the lower Saddle Mountains basalt and Frenchman Springs Member of the Wanapum basalt are inferred to be less transmissive with longer groundwater residence times. The SCP continues to assert that the general property of geochemical irregularities of the depth profiles argues in favor of dominant lateral rather than vertical groundwater flow (DOE/RW-0164, pp. 3.9-170). If vertical flow dominated, it would not be possible to maintain the stratigraphic controlled geochemical irregularities observed. The areal extent of dominant lateral flow probably is not restricted just to the vicinity of borehole DC-14 but rather extends along the groundwater flow paths for each geohydrologic unit.

The SCP indicates (DOE/RW-0164, pp. 3.9-3.173) that while a flux of deep groundwaters through the areally distributed fractures in not likely, localized vertical conduits such as faults and fracture zones should be considered. Figure B.19 shows several structural features on or near the site that could be associated with vertical groundwater flow.

Relevant hyrdogeologic and hydrochemical parameter values and information provided in this report include the following:

- The hydraulic conductivity of the Saddle Mountains basalt flow tops range over five orders of magnitude, from $8.6 \mathrm{E}-4$ to $86 \mathrm{~m} / \mathrm{d}(1 \mathrm{E}-2$ to $1 \mathrm{E}+3 \mathrm{ft} / \mathrm{d})$, and the geometric mean is between 0.86 and $8.6 \mathrm{~m} / \mathrm{d}(1 \mathrm{E}+1$ and $1 \mathrm{E}+2 \mathrm{ft} / \mathrm{d})(\mathrm{pp} .3 .9-3.93)$.

- The hydraulic conductivity of the interbeds in the Saddle Mountains basalt ranges from 8.6E-2 to $0.86 \mathrm{~m} / \mathrm{d}(1 \mathrm{E} 0$ to $1 \mathrm{E}+1 \mathrm{ft} / \mathrm{d})(\mathrm{pp} .3 .9-3.99)$.

- The hydraulic conductivity of the flow interiors of the Wanapum and Grande Ronde basalts (the hydraulic conductivity of the Saddle Mountains basalt interiors is not reported) ranges 8.6E-5 to $8.6 \mathrm{E}-11 \mathrm{~m} / \mathrm{s}(1 \mathrm{E}-9$ to $1 \mathrm{E}-3 \mathrm{ft} / \mathrm{d})$ for $95 \%$ of the tests, with a mean between $8.6 \mathrm{E}-9$ and $8.6 \mathrm{E}-8 \mathrm{~m} / \mathrm{s}$ (1E-7 and 1E-6 ft/d) (pp. 3.9-3.101).

- The thicknesses of the Elephant Mountain Member of the Saddle Mountains basalt, and the Pomona Member are shown in Figures B.20 through B.22.

\section{B.3.4 An Evaluation of Aquifer Intercommunication Between the Unconfined and Rattlesnake Ridge Aquifers on the Hanford Site (PNL-6313)}

This report focuses on the area in the vicinity of B Pond and Gable Mountain Pond. Hydraulic head and chemical data were evaluated. Based on these data, it was concluded that communication between the unconfined and uppermost confined aquifers had occurred in the areas where the Elephant Mountain is absent, northwest of B Pond (PNL-6313, p. 11). It is also speculated that "leakage between the aquifers may also occur through fractures in the Elephant Mountain basalt" (PNL-6313, p. 11). Hydraulic head data for the uppermost confined and unconfined aquifer and results of chemical analysis for selected 
contaminants (e.g., tritium, nitrate, cobalt-60, cesium-137, and ruthenium-106) from the Rattlesnake Ridge interbed are provided. These data are consistent with the literature as discussed above and do not provide additional insight into aquifer intercommunication.

\section{B.3.5 Hydrologic Model for the 200 East Groundwater Aggregate Area (WHC-SD-EN-TI-019)}

This report provides an evaluation of the hyrdrogeologic and hydrochemical characteristics of the 200 East Area and vicinity. It concludes that aquifer intercommunication between the unconfined and uppermost confined aquifers has been demonstrated to be an active process in parts of the 200 East Area (WHC-SD-EN-TI-019, pp. 3-22). The area where the Elephant Mountain basalt is assumed absent, barometric efficiencies, and estimated area of direct hydraulic intercommunication between the unconfined and uppermost confined aquifer are shown in Figure B.23. Although tritium had been increasing in well 699-42-40C, a Rattlesnake Ridge interbed well located near B Pond, the barometric efficiency is high (i.e., 44\%) indicating that the well is installed in a location where the Rattlesnake Ridge interbed is relatively well confined. The actual location of the tritium source in the Rattlesnake Ridge interbed may be some distance away from 699-42-40C.

\section{B.3.6 Hydrochemistry and Hydrogeologic Conditions Within the Hanford Site Upper Basalt Confined Aquifer System (PNL-10817)}

This report, PNL-10817, is the most recent report to focus on potential intercommunication between the unconfined and uppermost confined aquifers. Further, it addresses potential intercommunication from more of a site-wide perspective than most of the previous work, in addition to the well-documented intercommunication in the Gable Butte Gable Mountain gap area. The report concludes that the principal area of intercommunication occurs in the area immediately north of the 200 East Area because of the absence of upper confining units, structural deformation, and/or presence of erosional paleostream channels. In this same region, direct evidence of contamination from the overlying unconfined aquifer exists based on elevated levels of tritium $\left({ }^{3} \mathrm{H}\right)$, nitrate ion $\left(\mathrm{NO}_{3}{ }^{-}\right)$, and $\mathrm{C}-14$. Also, the groundwater chemical type changes from $\mathrm{Na}$ or $\mathrm{Na}-\mathrm{Ca}-\mathrm{HCO}_{3}$ (in the uppermost confined aquifer) to $\mathrm{Ca}-\mathrm{Mg}-\mathrm{HCO}$ which is more characteristic of the unconfined aquifer chemical type.

This change is explained in PNL-10817 noting that the hydochemical characteristics of groundwater within the upper basalt confined aquifer system reflects the evolution and interaction of the groundwater with various processes (Figure B.24). Typically, juvenile waters (i.e., less-chemically evolved) are represented by calcium, magnesium-bicarbonate $\left(\mathrm{Ca}, \mathrm{Mg}-\mathrm{HCO}_{3}\right)$ chemical water type. As these waters mature with increased aquifer residence time and longer flow paths, the groundwater has and opportunity for chemical interaction with the aquifer matrix and the cation composition of the groundwater evolves to a predominantly sodium $(\mathrm{Na})$ character. The evolution of the upper basalt aquifer groundwater to an $\mathrm{Na}-$ $\mathrm{HCO}_{3}$ chemical type is attributed to a number of geochemical processes including calcite $\left(\mathrm{CaCO}_{3}\right)$ precipitation, cation exchange processes, and $\mathrm{Na}$ increases as a result of volcanic glass hydrolysis/ dissolution reactions. 
It is also noted that the Rattlesnake Ridge interbed is absent within much of this area (see Figure B.6). The absence of the Rattlesnake Ridge interbed in the area immediately north of the 200 East Area is of particular hydrogeologic importance - the perimeter region of this area represents a region of potential interchange of groundwater between the upper confined and the overlying unconfined aquifer systems.

Relevant hyrdogeologic and hydrochemical parameter values and information provided in PNL-10817 include the following:

- The presence of $\mathrm{NO}_{3}{ }^{-}$has been used by some as an indication of aquifer intercommunication. Ninety-two percent of the onsite wells with $\mathrm{NO}_{3}>1.0 \mathrm{mg} / \mathrm{L}$ are located in the vicinity of the 200 East Area and Gable Mountain.

- Offsite upper basalt wells located immediately south of the Yakima River have hydrochemical parameters indicative of recent recharge of Yakima River water associated with agricultural/irrigation practices. There should also be a strong hydraulic connection between the Yakima River and the unconfined aquifer.

- Upper basalt confined wells located along the eastern Hanford Site boundary show increase in head, in response to irrigation-related recharge within the area east of the Columbia River. This could imply intercommunication between the unconfined and confined aquifers because the irrigation water is first recharging the unconfined aquifer. Also, the fact that hydraulic head continues to rise in this region indicates 1) that equilibrium conditions have not yet been established for the level of recharge occurring in the area east of the river and 2) the Columbia River does not form a dominant line-sink discharge area for the groundwater within the upper basalt aquifer, along its entire reach within the Pasco Basin.

- Upper basalt confined aquifer wells not located along the eastern Hanford Site boundary had a decreasing head pattern (between 0.08 and $0.24 \mathrm{~m} / \mathrm{yr}$ ) with the greatest decline occurring in the vicinity of the 200 Areas. Most importantly, the declining head patterns are similar to those reported for the overlying unconfined aquifer.

- Recharge of the upper basalt confined aquifer occurs through direct recharge to the aquifer from precipitation and surface runoff (occurring primarily in the Rattlesnake Hills region and Saddle Mountains along the southwest and northern boundaries of the Site), pervasive recharge from the overlying unconfined aquifer in the western part of the Site where the vertical downward gradient is prevalent (Figure B.25), artificial recharge from past wastewater disposal practices, and artificial recharge from agricultural irrigation practices (located primarily east of the Site).

- Groundwater discharges from the upper basalt confined aquifer to the Columbia River (along the eastern-southeastern boundary of the Site), through direct pathways provided by geologic structure and erosional paleostream channels in the Gable Mountain-Gable Butte structural area north of the 200 East Area, and pervasive discharge to the overlying unconfined aquifer where the vertical upward head gradients occur between the two aquifer systems. 


\section{B.3.7 Conclusions and Data from Other Documents}

The Summary of the Geology of the 200-BP-1 Operable Unit (WHC-SD-EN-TI-037) includes a brief discussion of the erosional window north of the 200-BP-1 Operable Unit. The extent of the area where the Elephant Mountain Member is interpreted as being completely absent is shown in Figure B.26. Cross sections illustrating the absence of the Elephant Mountain Member were also presented in WHC-SD-ENTI-037. The cross section locations are shown in Figure B.27. Cross sections in a north-south and northwest-southeast orientation through area are shown in Figures B.28 and B.29, respectively.

The effects of discharging wastewater into the unconfined aquifer on the underlying confined aquifer were studied for the Basalt Waste Isolation Project and documented in Effects of Surface Waste Disposal Activity on Ground-water Levels in the Saddle Mountain basalt (Nevulis et al. 1987). The goal of this study was to obtain an estimate of basalt flow interior vertical hydraulic conductivity by taking advantage of hydraulic head responses in the confined aquifer. The vertical hydraulic conductivity of the Elephant Mountain Member is estimated to be 2.6E-4 m/d (8.5E-4 ft/d) (Nevulis et al. 1987, p. 23). The Nevulis et al. (1987) report has not been peer reviewed or formally published and is therefore included as Exhibit 1 to this appendix because it may have direct application to some of the future flow model refinements.

Another Basalt Waste Isolation Project document focused on predicting the hydraulic responses in basalt flow tops and flow interiors. This document, Draft, Plan for Multiple-Well Hydraulic Testing of Selected Hydrogeologic Units at the RRL-2 Site, Basalt Waste Isolation Project, Reference Repository Location (RHO-BWI-TP-040, 1986), assumed the vertical hydraulic conductivity and specific storage of the Grande Ronde basalt interiors are 3E-6 m/d (9.8E-6 ft/d) and 3.6E-7 per m (1.1E-7 per ft), respectively.

The Hanford Site 1998 Environmental Report (PNNL-12088) presents the results of environmental monitoring on the Hanford Site for Fiscal Year 1998. This includes a discussion of contaminants in confined aquifers within the Saddle Mountains basalt. Figure B.30 provides the concentration of tritium, technetium-99, cobalt-60, and nitrate found in monitoring selected confined aquifer wells in 1998 . The contaminants in the confined aquifer detected in well 299-E33-12 are attributed to poor well construction. There was a period of time in which the borehole was open to both the unconfined and confined aquifers, allowing the migration of high-salt waste in the unconfined aquifer to migrate to the confined aquifer. PNNL-12088 states that contaminants in the confined aquifer detected in well 699-42-40C are "believed to have originated from downward migration from the overlying, unconfined aquifer."

\section{B.4 Conclusions}

Four principal intercommunication mechanisms are identified in the literature:

1. Direct hydraulic connection between the unconfined and uppermost confined aquifer where the confining layer (i.e., Elephant Mountain basalt) is absent. 
2. Pervasive areally distributed flow across the confining layer(s) that separate the unconfined from the uppermost confined aquifer.

3. Localized communicative geologic structure associated anticlinal structures such as Umtanum Ridge and Yakima Ridge structural elements.

4. Human intrusion associated with improperly constructed wells and boreholes.

The four alternative conceptual models described in Section 2.0 address the first three mechanisms. The fourth mechanism, human intrusion, has been documented but is not considered herein because it has generally been eliminated where known to occur.

There are strong lines of evidence to indicate that hydraulic intercommunication is present in the Gable Butte-Gable Mountain Gap area. Hydraulic intercommunication between the unconfined and uppermost confined aquifers in this area is evidenced by the following:

- chemical composition of groundwater indicating mixing

- presence of chemical species (i.e., nitrate ion) and radioisotopes (e.g., tritium and I-129) that are associated with near-surface wastewater disposal in the uppermost confined aquifer

- similarity of hydraulic heads in the unconfined and uppermost confined aquifers in the vicinity of the Gable Butte-Gable Mountain Gap where the Elephant Mountain basalt is absent

- geologic information from borehole logs and geophysical information indicating an area where the Elephant Mountain basalt (confining layer) is absent and within this area, locations where the underlying Rattlesnake Ridge interbed (water-bearing unit) and portions of the Pomona basalt (confining layer) are absent.

The area where the Elephant Mountain basalt is absent (see Figure B.4) represents the area where aquifer intercommunication occurs, unimpeded by a confining layer. There are likely other localized areas to the southeast of the Gable Butte-Gable Mountain gap where the Elephant Mountain basalt has been fully or partially eroded, based on barometric efficiency information (see Figure B.23). Elsewhere on the Hanford Site, the Elephant Mountain basalt provides a significant impediment to vertical intercommunication between the aquifers owing to its thickness (Figure B.5) and low vertical hydraulic conductivity which may range from $1 \mathrm{E}-8 \mathrm{~m} / \mathrm{d}(3.3 \mathrm{E}-8 \mathrm{ft} / \mathrm{d})(\mathrm{RHO}-\mathrm{RE}-\mathrm{ST}-12 \mathrm{P})$ to $2.6 \mathrm{E}-4 \mathrm{~m} / \mathrm{d}$ (8.5E-4 ft/d) (Nevulis et al. 1987). The effectiveness of the Elephant Mountain basalt as a confining layer and impediment to vertical communication between the unconfined and uppermost confined aquifers is evidenced by the hydraulic head difference between the two aquifers and difference in groundwater chemistry.

A qualitative assessment of the alternative conceptual models described in Section A.2 is provided in Table B.1. 
Table B.1. Assessment of Alternative Conceptual Models

\begin{tabular}{|c|c|c|c|c|}
\hline $\begin{array}{c}\text { Alternative } \\
\text { Conceptualization }\end{array}$ & $\begin{array}{c}\text { Potential } \\
\text { Mathematical } \\
\text { Description of } \\
\text { Aquifer Inter- } \\
\text { communication }\end{array}$ & $\begin{array}{l}\text { Qualitative } \\
\text { Uncertainty }\end{array}$ & Data Needs & Comments \\
\hline $\begin{array}{l}\text { ACM1 }{ }^{(a)} \text { Complete } \\
\text { hydraulic separation }\end{array}$ & $\mathrm{N} / \mathrm{A}$ & $\begin{array}{l}\text { High in gap area, } \\
\text { moderate } \\
\text { elsewhere }\end{array}$ & None & $\begin{array}{l}\text { Several lines of evidence } \\
\text { point to inter- } \\
\text { communication in the gap } \\
\text { area. Elsewhere, data } \\
\text { suggest flow is small on a } \\
\text { unit area basis }\end{array}$ \\
\hline $\begin{array}{l}\text { ACM2- } \\
\text { Intercommunication } \\
\text { in gap area where } \\
\text { Elephant Mountain is } \\
\text { completely absent }\end{array}$ & $\begin{array}{l}\text { Analytical solution } \\
\text { for large diameter } \\
\text { well }\end{array}$ & $\begin{array}{l}\text { Low in gap area, } \\
\text { moderate } \\
\text { elsewhere }\end{array}$ & $\begin{array}{l}\text { Area of erosional } \\
\text { window, average } \\
\text { Rattlesnake Ridge } \\
\text { interbed thickness and } \\
\text { horizontal hydraulic } \\
\text { conductivity and } \\
\text { hydraulic head } \\
\text { difference }\end{array}$ & $\begin{array}{l}\text { Honors evidence of } \\
\text { intercommunication in area } \\
\text { of the gap }\end{array}$ \\
\hline $\begin{array}{l}\text { ACM3- same as } \\
\text { ACM2 plus areally } \\
\text { distributed } \\
\text { intercommunication. }\end{array}$ & $\begin{array}{l}\text { Analytical solution } \\
\text { for large diameter } \\
\text { well, with a head- } \\
\text { dependent flux } \\
\text { boundary to } \\
\text { represent areally } \\
\text { distributed flow }\end{array}$ & $\begin{array}{l}\text { Low in gap, low } \\
\text { elsewhere }\end{array}$ & $\begin{array}{l}\text { Same as ACM2 plus } \\
\text { Site-wide thickness } \\
\text { and vertical hydraulic } \\
\text { conductivity of } \\
\text { Elephant Mountain } \\
\text { basalt and hydraulic } \\
\text { head difference } \\
\text { between unconfined } \\
\text { and confined aquifers }\end{array}$ & $\begin{array}{l}\text { Honors evidence of } \\
\text { intercommunication in area } \\
\text { of the gap. Addresses } \\
\text { areally distributed flow } \\
\text { across the Elephant } \\
\text { Mountain basalt }\end{array}$ \\
\hline $\begin{array}{l}\text { ACM4- same as } \\
\text { ACM3 plus enhanced } \\
\text { intercommunication } \\
\text { at anticlinal features }\end{array}$ & $\begin{array}{l}\text { Analytical solution } \\
\text { for large diameter } \\
\text { well, with a head- } \\
\text { dependent flux } \\
\text { boundary to } \\
\text { represent areally } \\
\text { distributed flow } \\
\text { (with larger } \mathrm{K}_{\mathrm{v}} \\
\text { associated with } \\
\text { anticlinal features) }\end{array}$ & $\begin{array}{l}\text { Low in gap area, } \\
\text { low elsewhere } \\
\text { except for } \\
\text { moderate at } \\
\text { anticlinal } \\
\text { features }\end{array}$ & $\begin{array}{l}\text { Same as ACM3 plus } \\
\text { must make assumption } \\
\text { on vertical hydraulic } \\
\text { conductivity } \\
\text { associated with } \\
\text { anticlinal features }\end{array}$ & $\begin{array}{l}\text { Honors evidence of } \\
\text { intercommunication in area } \\
\text { of the gap. Addresses } \\
\text { areally distributed flow } \\
\text { across the Elephant } \\
\text { Mountain basalt. Enhanced } \\
\text { vertical flow at anticlinal } \\
\text { features not well supported } \\
\text { by data }\end{array}$ \\
\hline
\end{tabular}




\section{B.5 Bibliography}

Bauer HH, and JJ Vaccaro. Estimates of ground-water recharge to the Columbia Plateau regional aquifer system, Washington, Oregon, and Idaho, for predevelopment and current land-use conditions. US Geological Survey Water-Resources Investigation Report 88-4108, Tacoma, Washington.

Brocoum S. 1987. Minutes results from the hydrology task group meeting of July 21-23, 1987, Transmittal to DH Alexander from S Brocoum, Weston, Washington, D.C.

Brocoum S. 1987. Minutes results from the hydrology task group meeting of June 2-4, 1987, Transmittal to DH Alexander from S Brocoum, Weston, Washington, D.C.

Brown RE, and HG Ruppert. 1950. The underground disposal of liquid waste at the Hanford Works, Washington. HW-17088, General Electric Hanford Company, Richland, Washington.

Connelly MP, JV Borhese, CD Delaney, BH Ford, JW Lindberg, and SJ Trent. 1992. Hydrogeologic model for the 200 East Groundwater Aggregate Area. WHC-SD-EN-TI-012, Rev. 0, Westinghouse Hanford Company, Richland, Washington.

Cross RW. 1987. Intraflow Structure Study Plan, RHO-SD-BWI-SP-036, Rockwell Hanford Operations, Richland, Washington.

Delaney CD, and KA Lindsey. 1991. Geology and hydrology of the Hanford Site: A standardized text for use in Westinghouse Hanford Company documents and reports. WHC-SD-ER-TI-003, Westinghouse Hanford Company, Richland, Washington.

Dirkes RL, RW Hanf, and TM Poston. 1999. Hanford Site 1998 Environmental Report. PNNL-12088, Pacific Northwest National Laboratory, Richland, Washington.

Dove FH, CR Cole, MG Foley, FW Brown, WJ Deutsch, MD Freshley, SK Gupta, PJ Gutnecht, WL Kuhn, JW Lindberg, WA Rice, R Schalla, JF Washburn, and JT Zellmer. 1982. Assessment of effectiveness of geologic isolation systems, aegis technology demonstration for a nuclear waste repository in basalt. PNL-3632, Pacific Northwest Laboratory, Richland, Washington.

Drost BW, KJ Whiteman, and JB Gonthier. 1990. Geologic framework of the Columbia Plateau aquifer system, Washington, Oregon, and Idaho. US Geological Survey Water-Resources Investigation Report 87-4238, Portland, Oregon.

Fecht KR. 1978. Geology of Gable Mountain - Gable Butte Area. RHO-BWI-LD-5, Rockwell Hanford Operations, Richland, Washington.

Gephart RE, RC Arnett, RG Baca, LS Leonhart, and FA Spane, Jr. 1979. Hydrologic studies within the Columbia Plateau, Washington: An integration of current knowledge. RHO-BWI-ST-5, Rockwell Hanford Operations, Richland, Washington. 
Graham MJ, GV Last, and KR Fecht. 1984. An assessment of aquifer intercommunication in the B Pond-Gable Mountain Pond Area of the Hanford Site. RHO-RE-ST-12 P, Rockwell Hanford Operations, Richland, Washington.

Graham MJ, MD Hall, SR Strait, and WR Brown. 1981. Hydrology of the Separations Area. RHO-ST-42, Rockwell Hanford Operations, Richland, Washington.

Hansen AJ, JJ Vaccaro, and HH Bauer. Ground-water flow simulation of the Columbia Plateau regional aquifer system, Washington, Oregon, and Idaho. US Geological Survey Water-Resources Investigation Report 91-4187, Tacoma, Washington.

Hoffman KM, SJ Trent, KA Lindsey, and BW Bjornstad. 1992. Summary of the geology of the 200-BP-1 operable unit. WHC-SD-EN-TI-037, Rev. 0, Westinghouse Hanford Company, Richland, Washington.

Jensen EJ. 1987. An evaluation of aquifer intercommunication between the unconfined and Rattlesnake Ridge aquifers on the Hanford Site. PNL-6313, Pacific Northwest Laboratory, Richland, Washington.

Last GV. 1984. Technical support to the BWIP for monitoring the unconfined aquifer on the Hanford Site, groundwater level monitoring task: Status report. Pacific Northwest Laboratory, Richland, Washington.

Ledgerwood RK, and RA Deju. 1976. Hydrogeology of the uppermost confined aquifers underlying the Hanford Reservation. ARH-SA-253, Atlantic Richfield Hanford Company, Richland, Washington.

Lindsey KA, BW Bjornstad, JW Lindberg, and KM Hoffmann. 1992. Geologic setting of the 200 East Area: An update., Westinghouse Hanford Company, Richland, Washington.

Livesay DM. 1986. The hydrogeology of the upper Wanapum Basalt, upper Cold Creek Valley. Washington, Masters Thesis, Washington State University.

Lum WE II, and DR Cline. 1985. Test wells in Central Washington, 1977 to 1979: Description and results. US Geological Survey Water-Resources Investigation Report 83-4259, Tacoma, Washington.

Luzier JE, and JA Skrivan. Digital-simulation and projection of water-level declines in basalt aquifers of the Odessa_Lind Area, East-Central Washington. US Geological Survey Water-Supply Paper 20, Washington, D.C.

Mac Nish RD, and RA Barker. 1976. Digital simulation of a basalt aquifer system, Walla Walla River Basin, Washington and Oregon. US Geological Survey Water-Supply Bulletin 44.

McGhan VL, PJ Mitchell, and RS Argo. 1985. Hanford wells. PNL-5397, Pacific Northwest Laboratory, Richland, Washington. 
Nevulis R, S Sorooshian, and DR Davis. 1987. Effects of surface waste disposal activity on groundwater levels in the Saddle Mountain Basalt. Department of Hydrology and Water Resources, University of Arizona, Tucson, Arizona.

NUREG-0960. 1983. Draft site characterization report for the Basalt Waste Isolation Project. US Nuclear Regulatory Commission, Office of Nuclear Material Safety and Safeguards, Washington, D.C.

Spane FA, Jr., and WD Webber. 1995. Hydrochemistry and hydrogeologic conditions within the Hanford Site upper basalt confined aquifer system. PNL-10817, Pacific Northwest Laboratory, Richland, Washington.

Spane FA. 1986. Preliminary evaluation of piezometer responses at DC-19, DC-20, and DC-22, during construction of DC-23W. RHO-SD-BWI-TI-313, Rockwell Hanford Operations, Richland, Washington.

Stone R, PM Rogers, AH Lu, RW Bryce. 1986. Draft, plan for multiple-well hydraulic testing of selected hydrogeologic units at the RRL-2 Site, Basalt Waste Isolation Project, reference repository location. RHO-BWI-TP-040, Rockwell Hanford Operations, Richland, Washington.

Strait SR, and RB Mercer. 1986. Hydraulic property data from selected test zones on the Hanford Site. RHO-BWI-DP-051, Rockwell Hanford Operations, Richland, Washington.

Swanson LC, and BA Leventhal. 1984. Water-level data and borehole descriptions for monitoring wells used by the Basalt Waste Isolation Project. RHO-BWI-DP-042, Rockwell Hanford Operations, Richland, Washington.

US Department of Energy (DOE). 1982. Site characterization report for the Basalt Waste Isolation Project. DOE/RL 82-3, Richland, Washington.

US Department of Energy (DOE). 1984. Draft environmental assessment, reference repository location Hanford Site, Washington. DOE/RW-0017, Office of Civilian Radioactive Waste Management, Washington, D.C.

US Department of Energy (DOE). 1988. Consultation Draft, Site Characterization Plan, Reference Repository Location, Hanford Site, Washington. DOE/RW-0164, Washington, D.C.

Wintczak TM. 1984. Principal borehole report Borehole RRL-2. RHO-BWI-TI-113, Rockwell Hanford Operations, Richland, Washington.

Zimmerman DA, AE Reisenauer, GD Black, and MA Young. 1986. Hanford Site water table changes 1950 through 1980 data observations and evaluation. PNL-5506, Pacific Northwest Laboratory, Richland, Washington. 
Bibliography of the geology of the Columbia Plateau and adjacent areas of Washington.

RHO-BWI-C-82. Rockwell Hanford Operations, Richland, Washington.

1982. Geohydrology of the Rattlesnake Ridge Interbed in the Gable Mountain Pond Area. RHO-ST-38, Rockwell Hanford Operations, Richland, Washington.

Changes in ground-water levels and ground-water budgets, from predevelopment to 1986 in parts of the Pasco Basin, Washington. US Geological Survey Water-Resources Investigation Report 96-4086. 


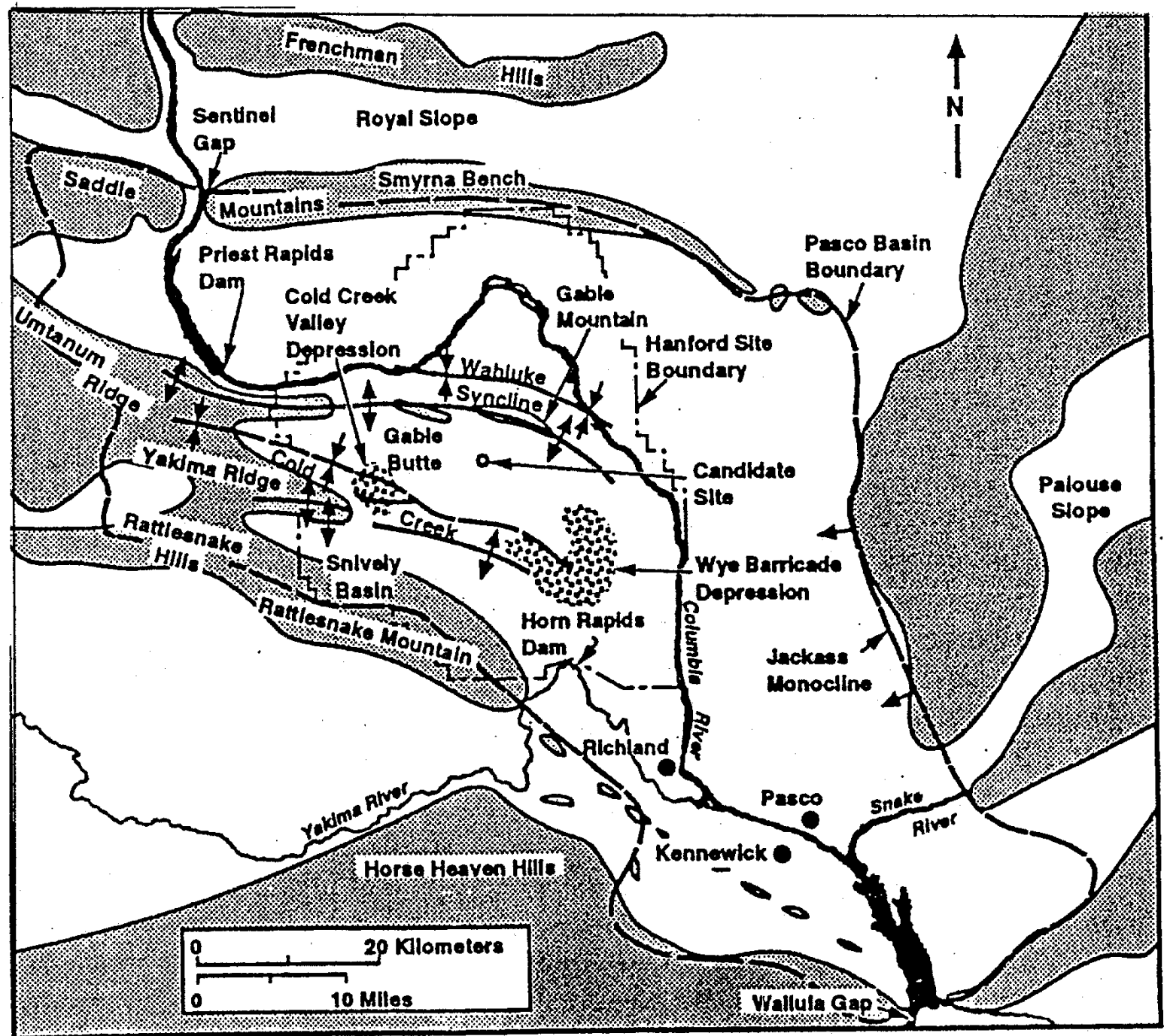

Hetitorat

Figure B.1. Geologic Structures of the Pasco Basin and the Hanford Site. 


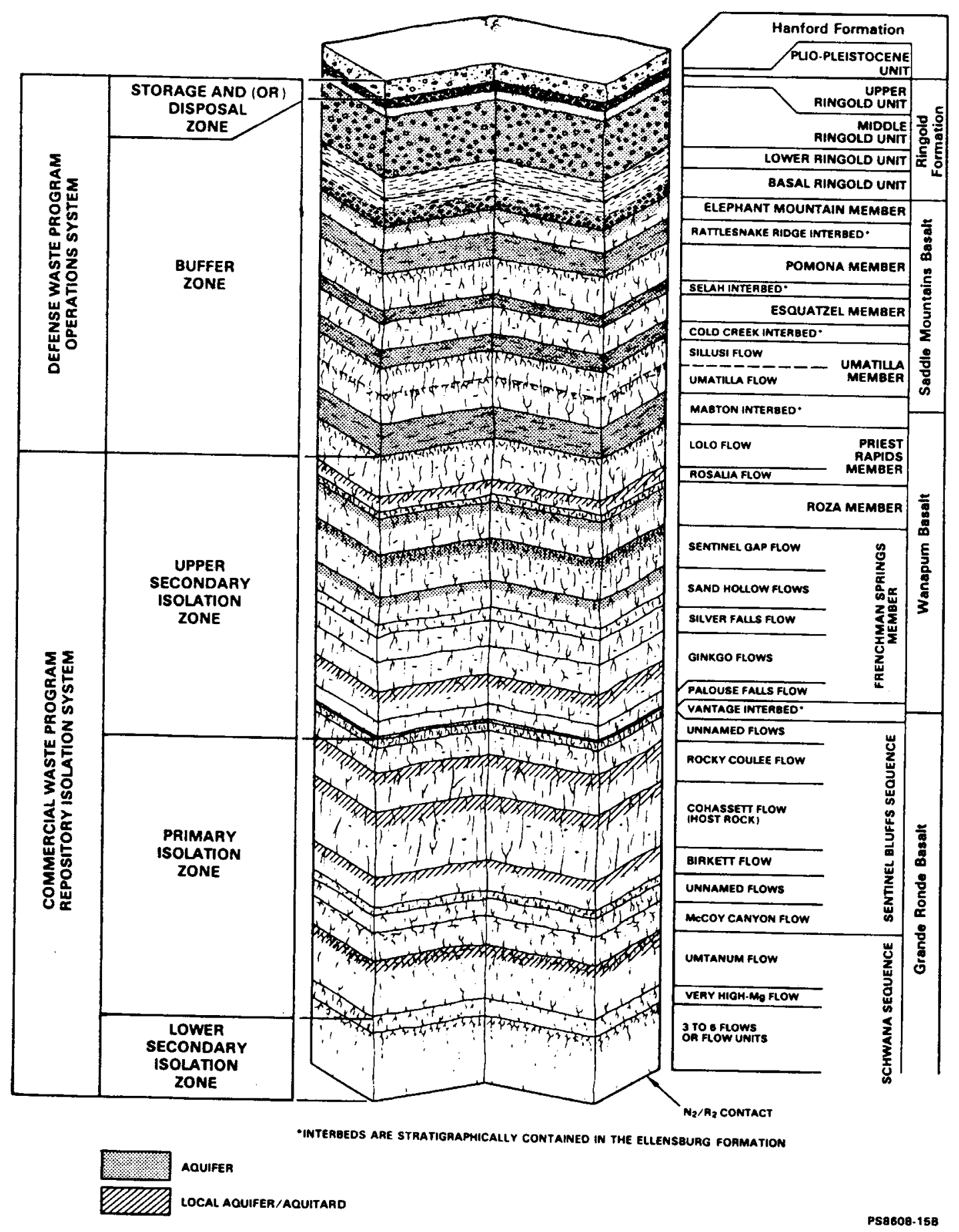

Figure B.2. Stratigraphic Location of Principal Aquifers at the Hanford Site. 


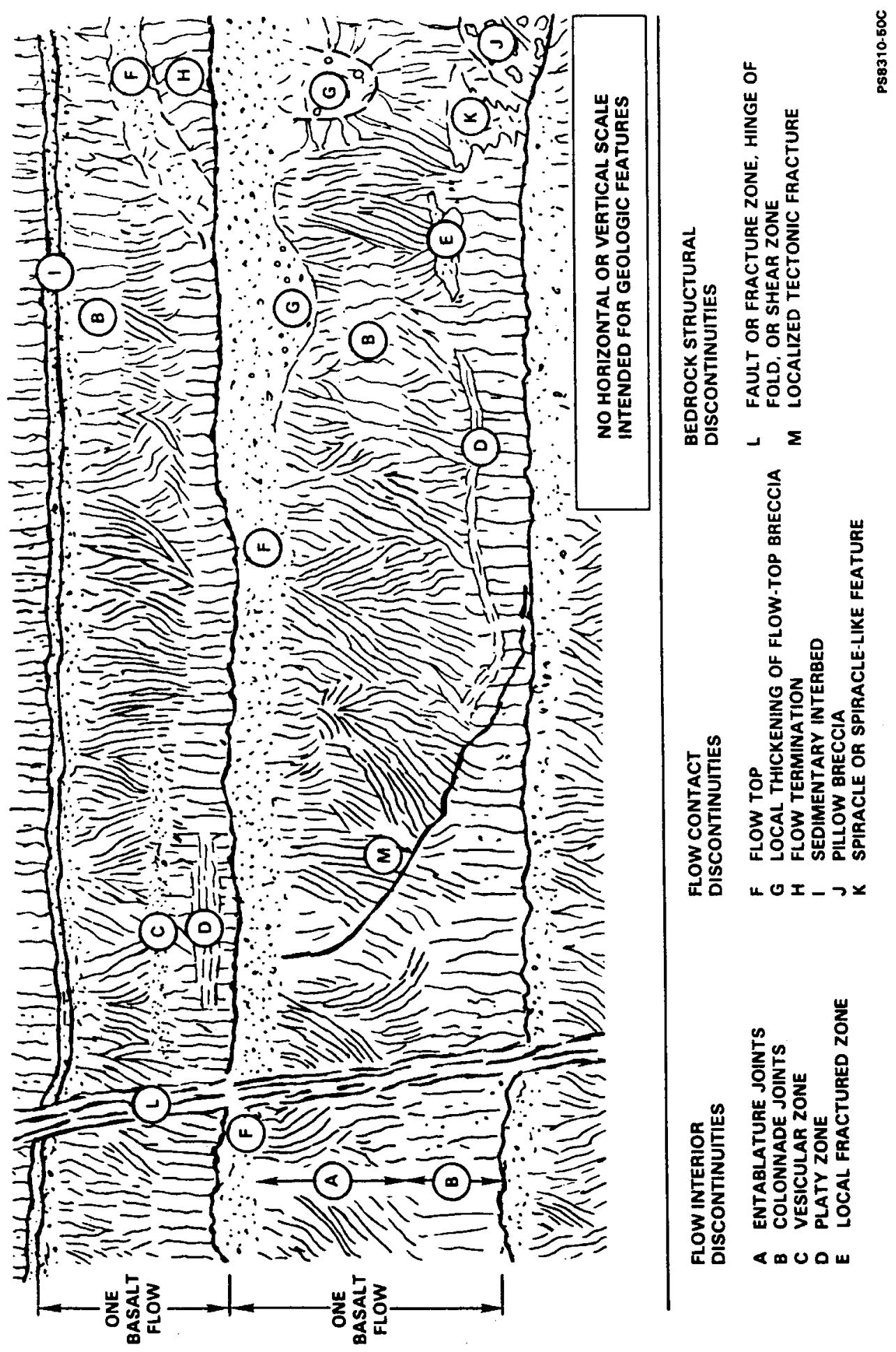

Figure B.3. Hypothetical Composite Cross Section of Possible Geologic Features in the Layered Basalt Sequence. 


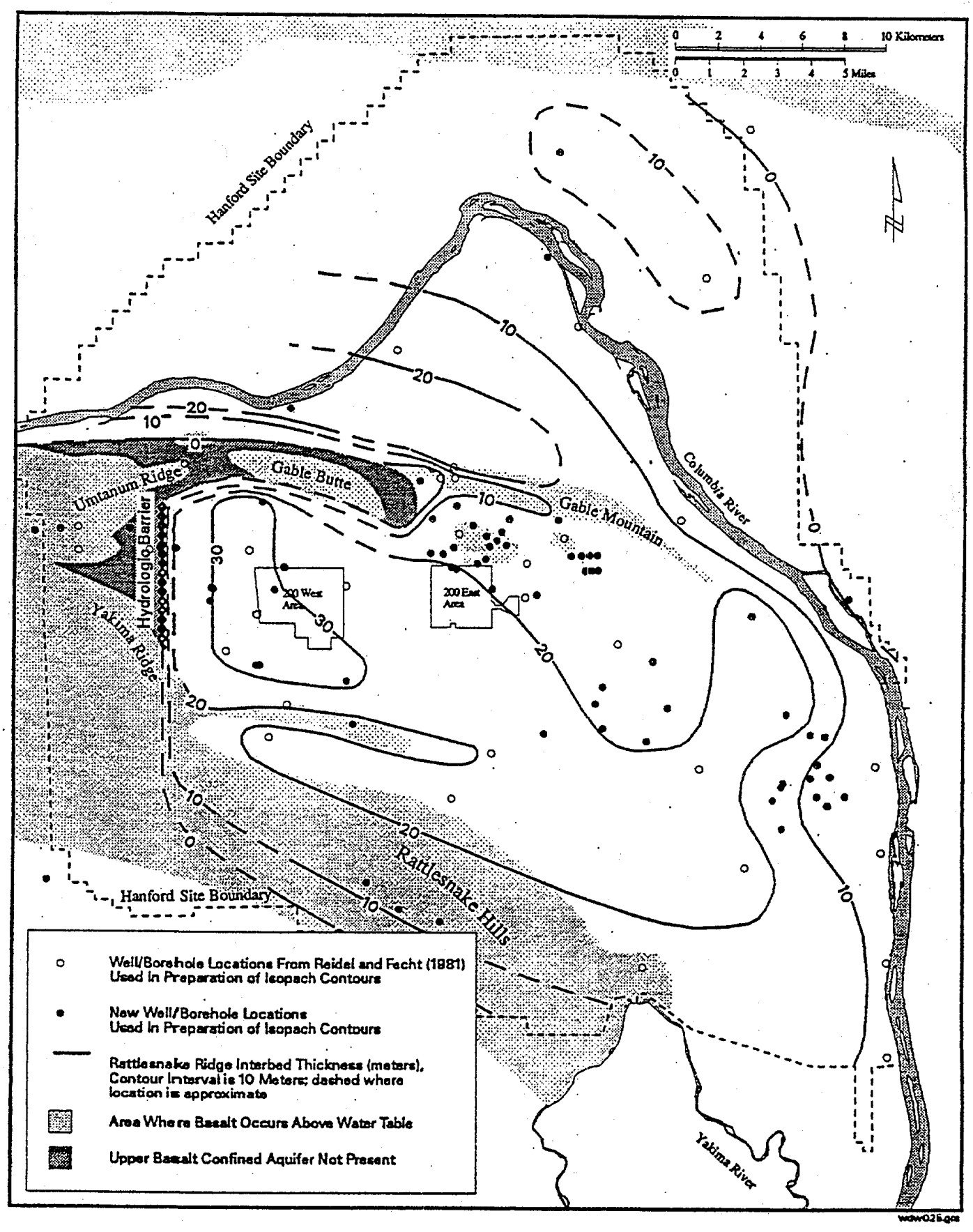

Source: PNL-10817.

Figure B.4. Isopach Map of Rattlesnake Ridge Interbed on the Hanford Site. 


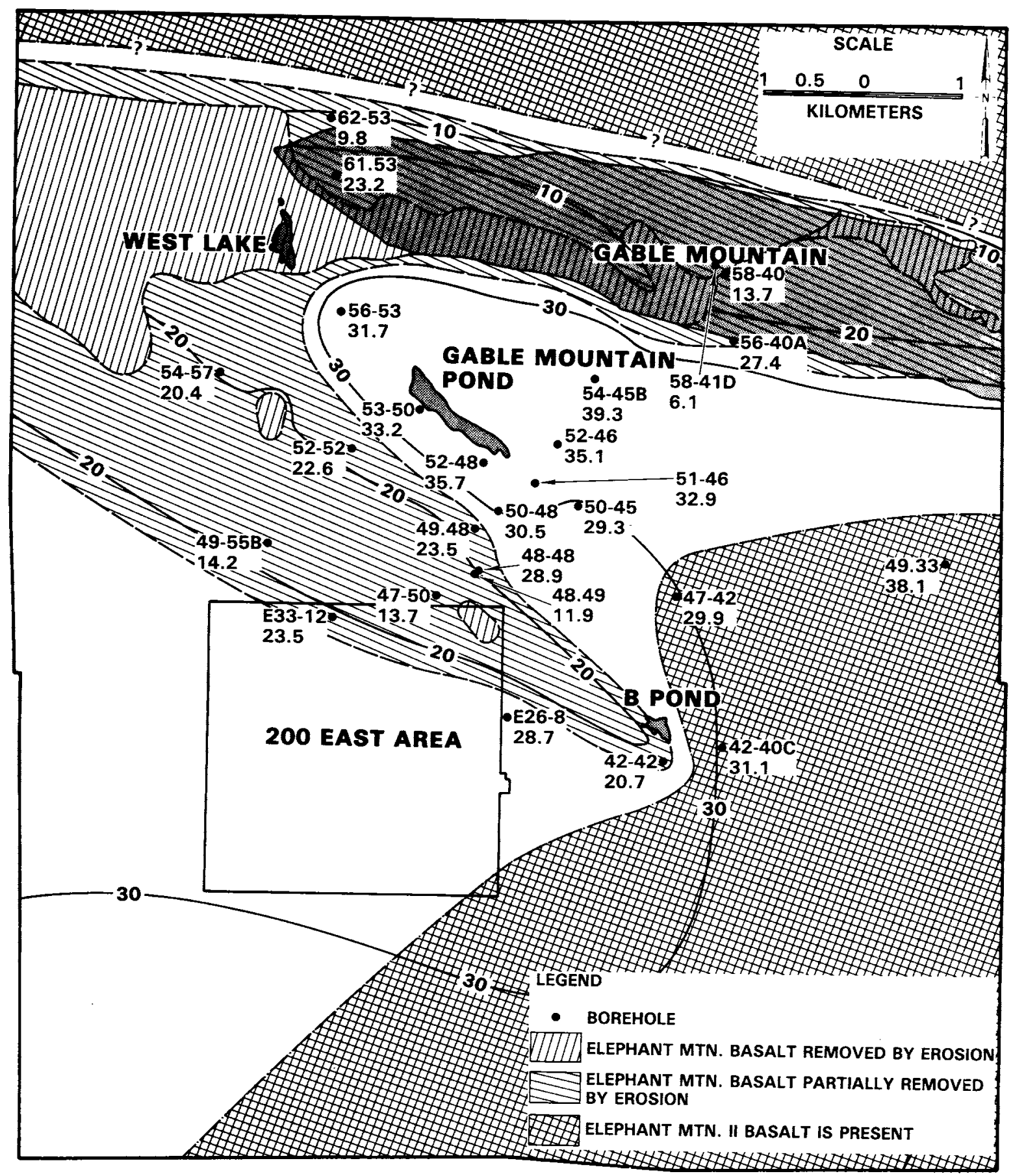

Figure B.5. Isopach Map of the Elephant Mountain Member in Vicinity of 200 East Area. 


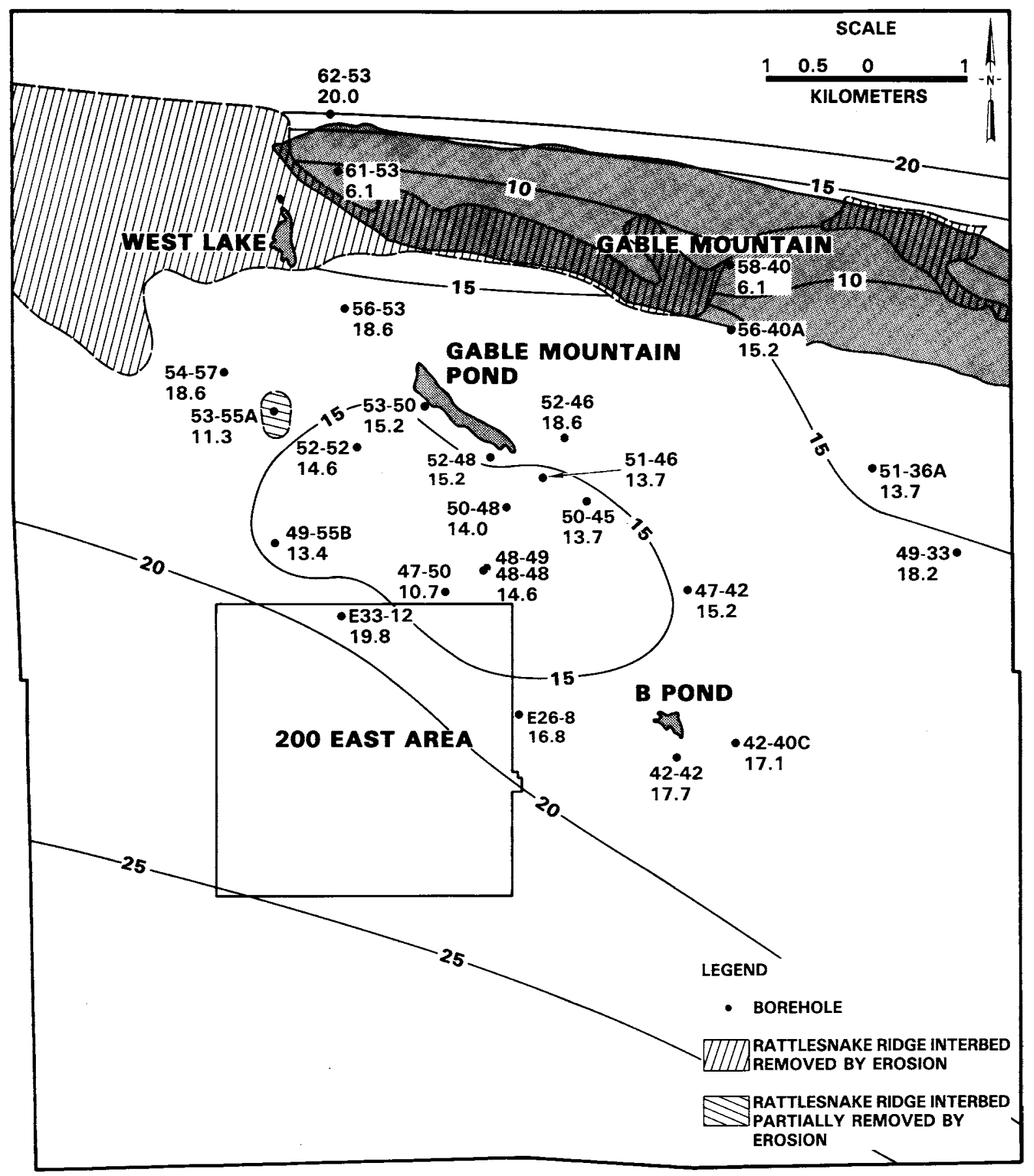

Figure B.6. Isopach Map of the Rattlesnake Ridge Interbed in Vicinity of 200 East Area. 


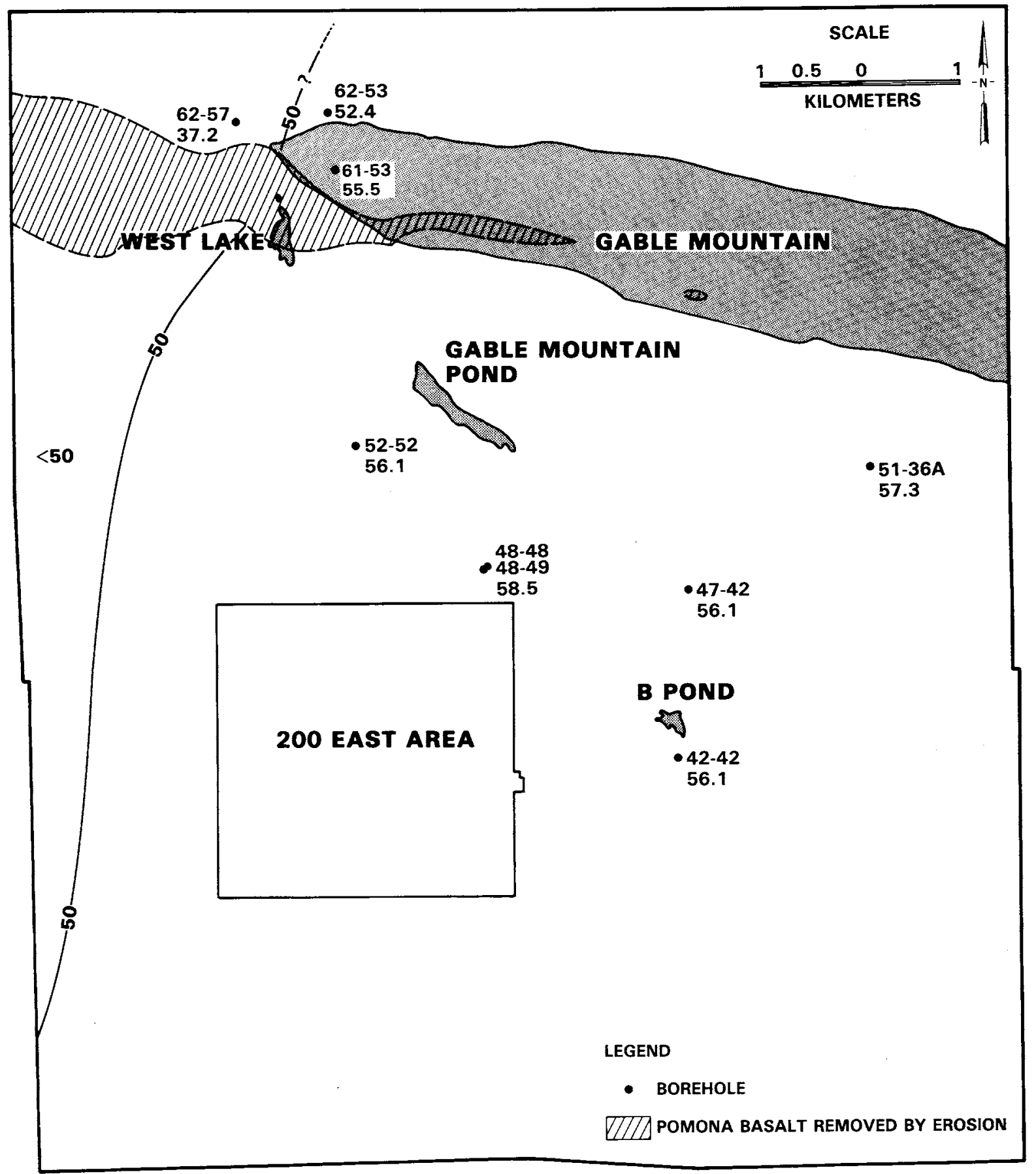

Figure B.7. Isopach Map of the Pomona Member in Vicinity of 200 East Area. 


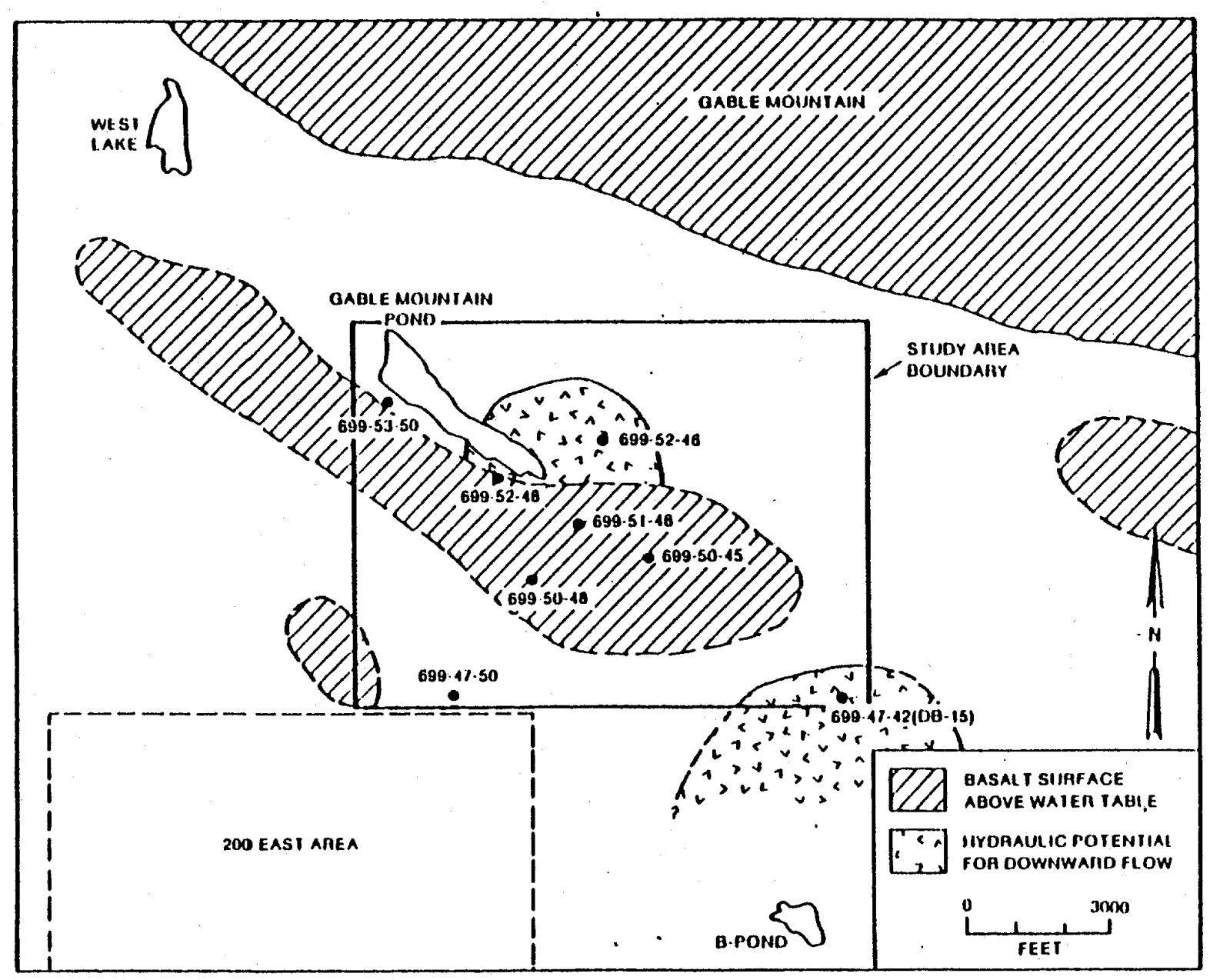

Source: RHO-ST-38

Figure B.8. Areas of Hydraulic Potential for Aquifer Communication Under Hydraulic Head Conditions as they Existed in the Early 1980s. 


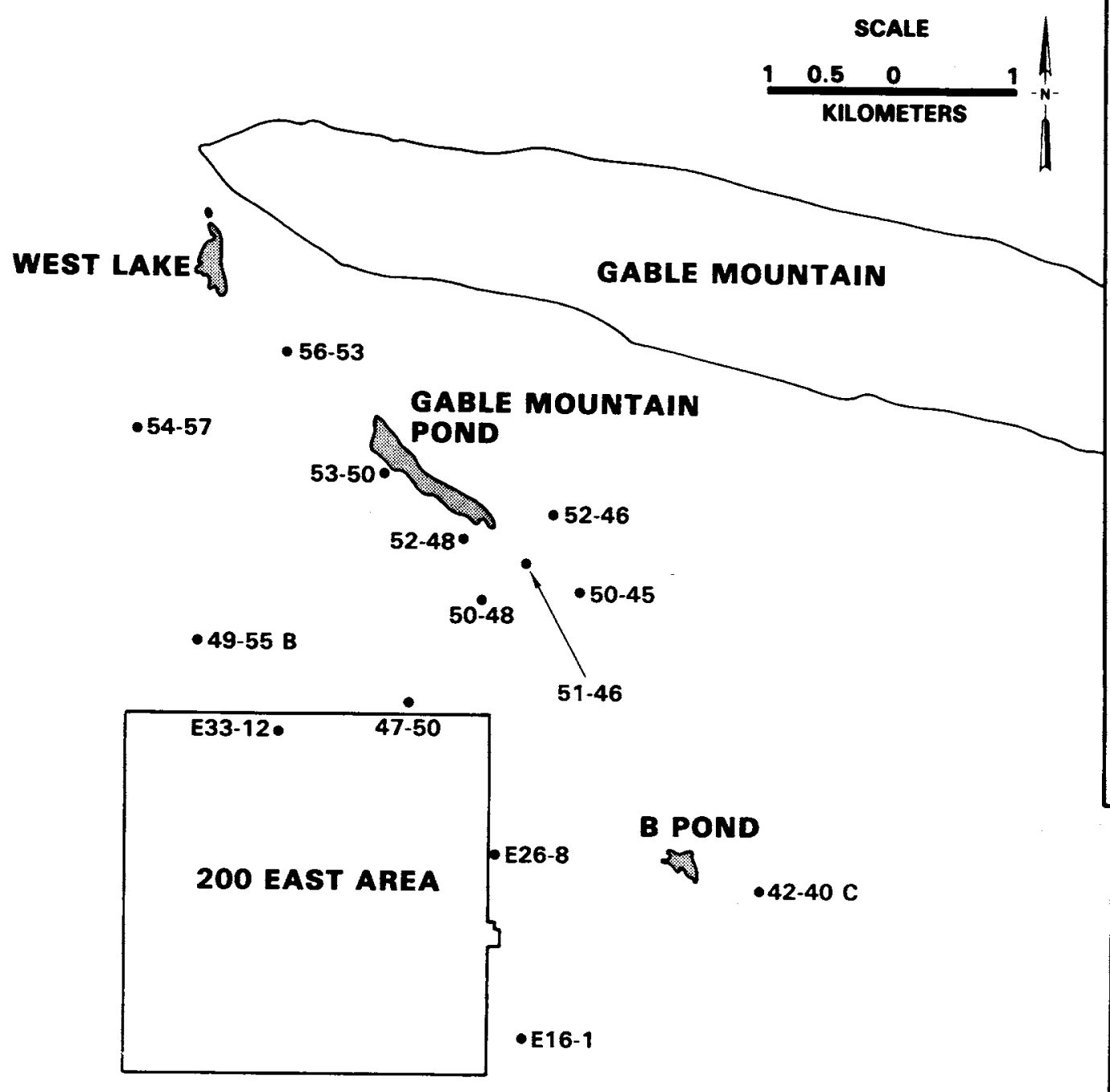

Figure B.9. Location of 14 Confined Aquifer Test Wells Used in the RHO-RE-ST-12P Study. 


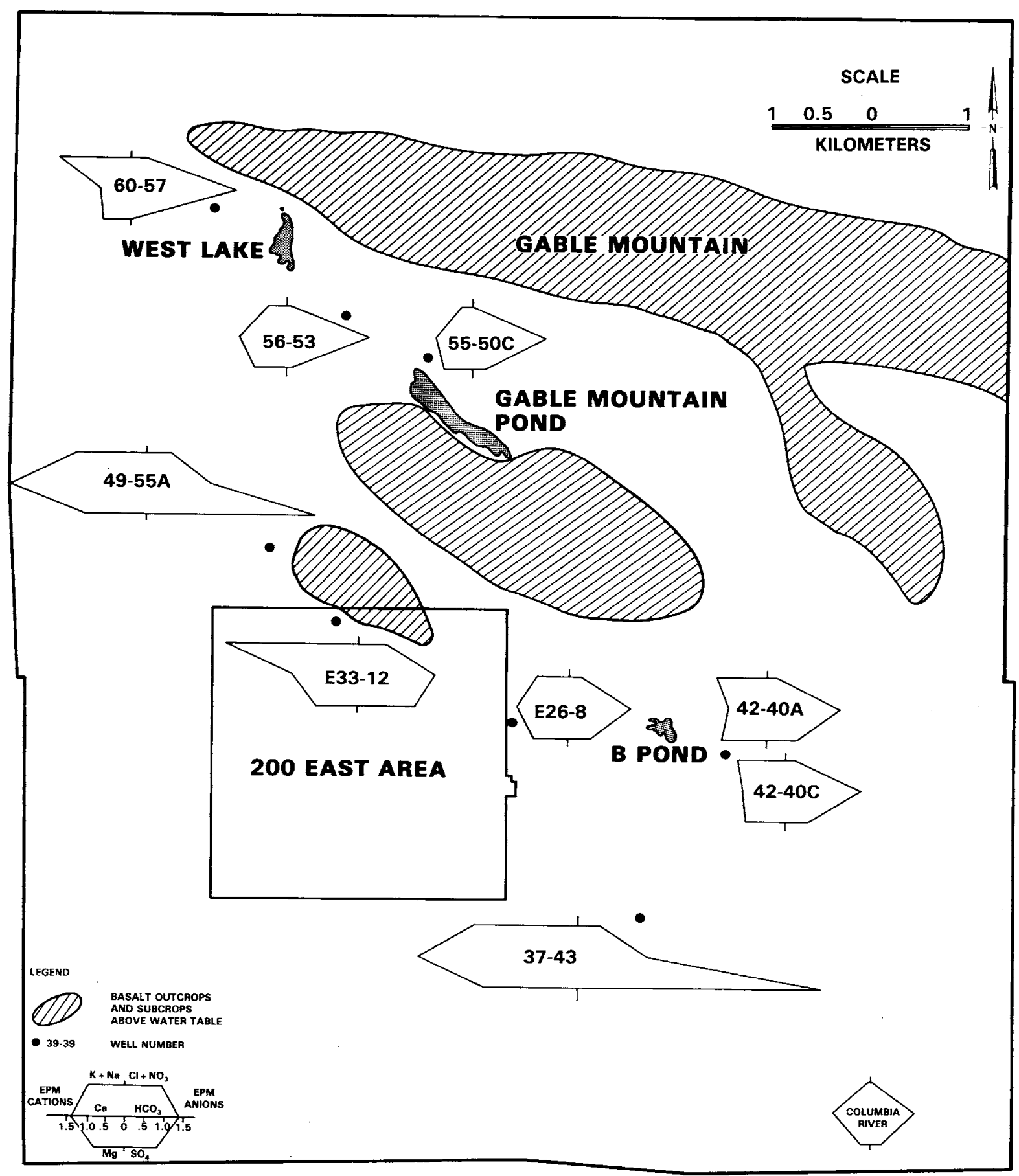

Figure B.10. Stiff Diagrams from Samples in the Unconfined Aquifer. 


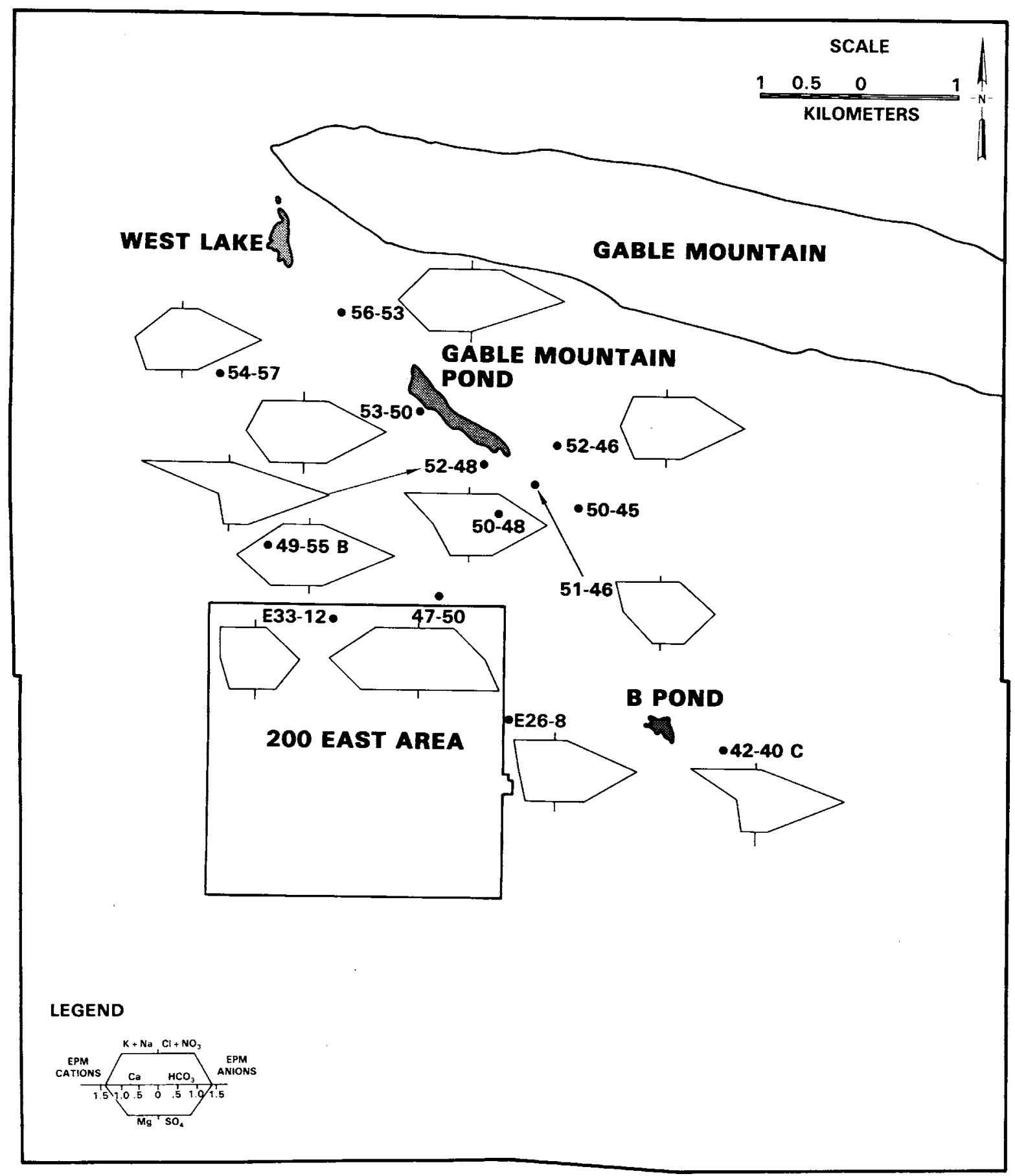

Figure B.11. Stiff Diagrams from Samples in the Rattlesnake Ridge Interbed (part of the uppermost confined aquifer). 


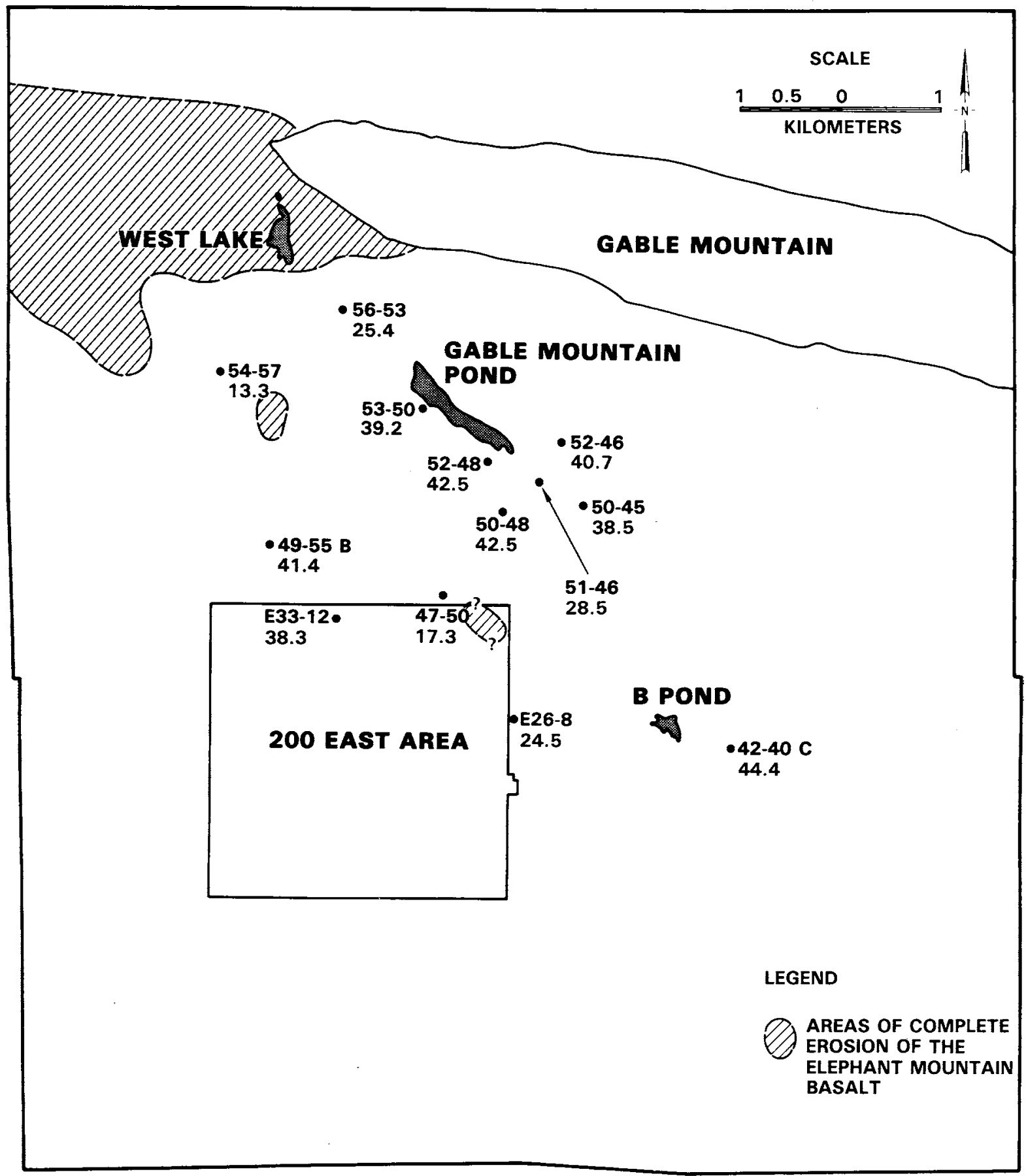

Figure B.12. Barometric Efficiencies for the Uppermost Confined Aquifer as Estimated in RHO-RE-ST-12P Study. 


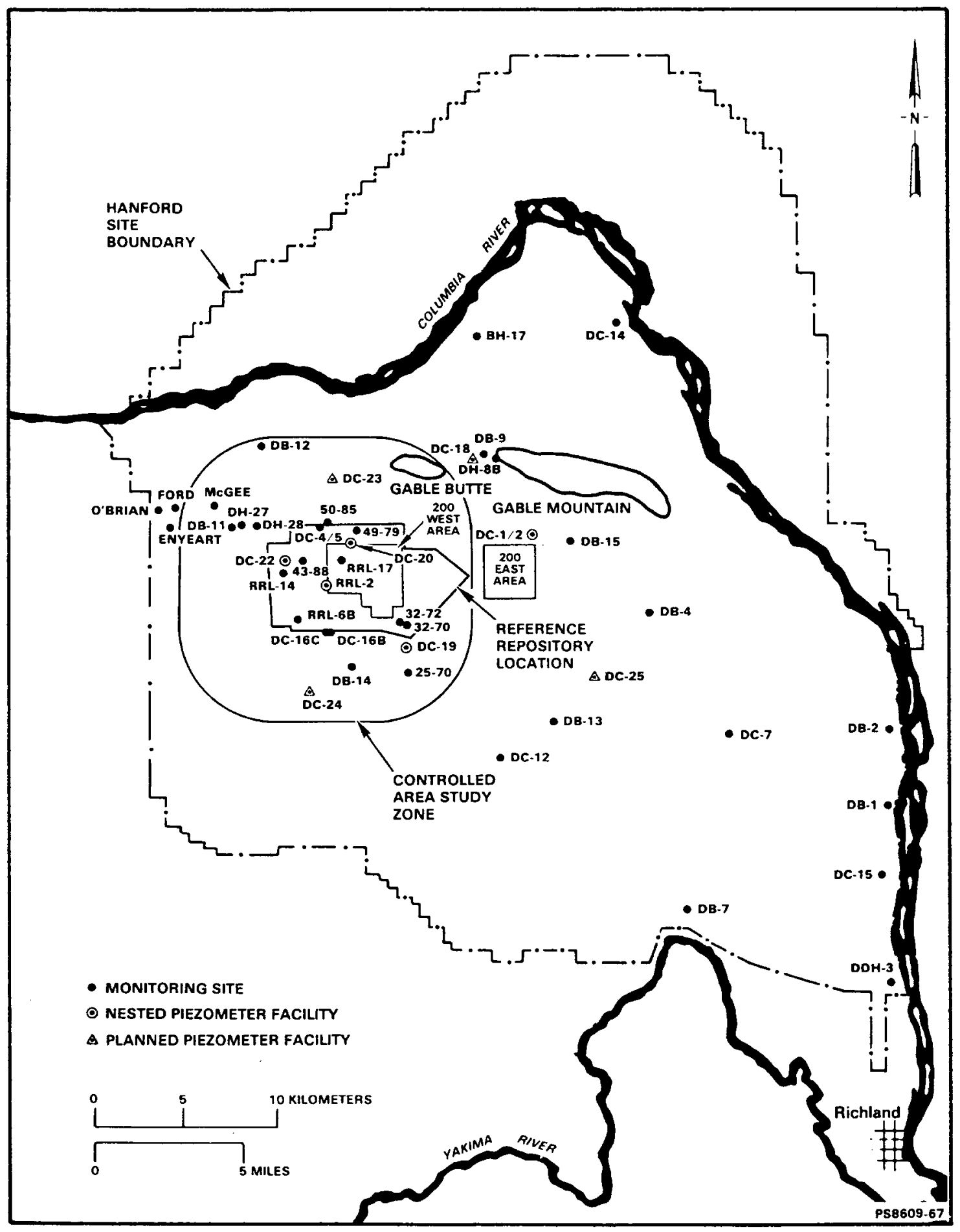

Figure B.13. Location of Wells and Boreholes Where Groundwater Levels were Measured for the Basalt Waste Isolation Project. 

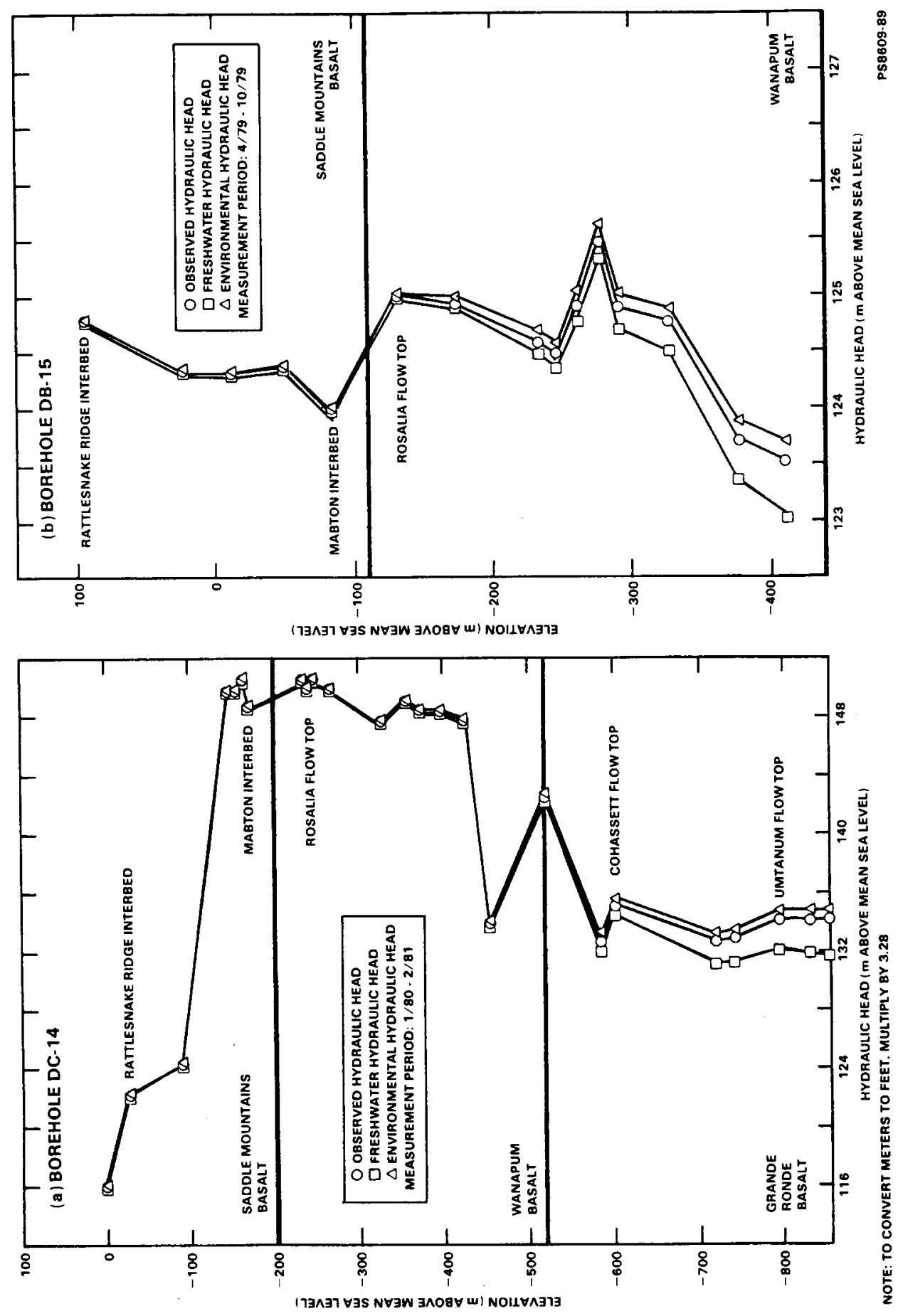

Figure B.14. Vertical Head Distributions Within the Columbia River Basalt from Boreholes DC-14 and DC-15 (see Figure B.13 for well locations). 

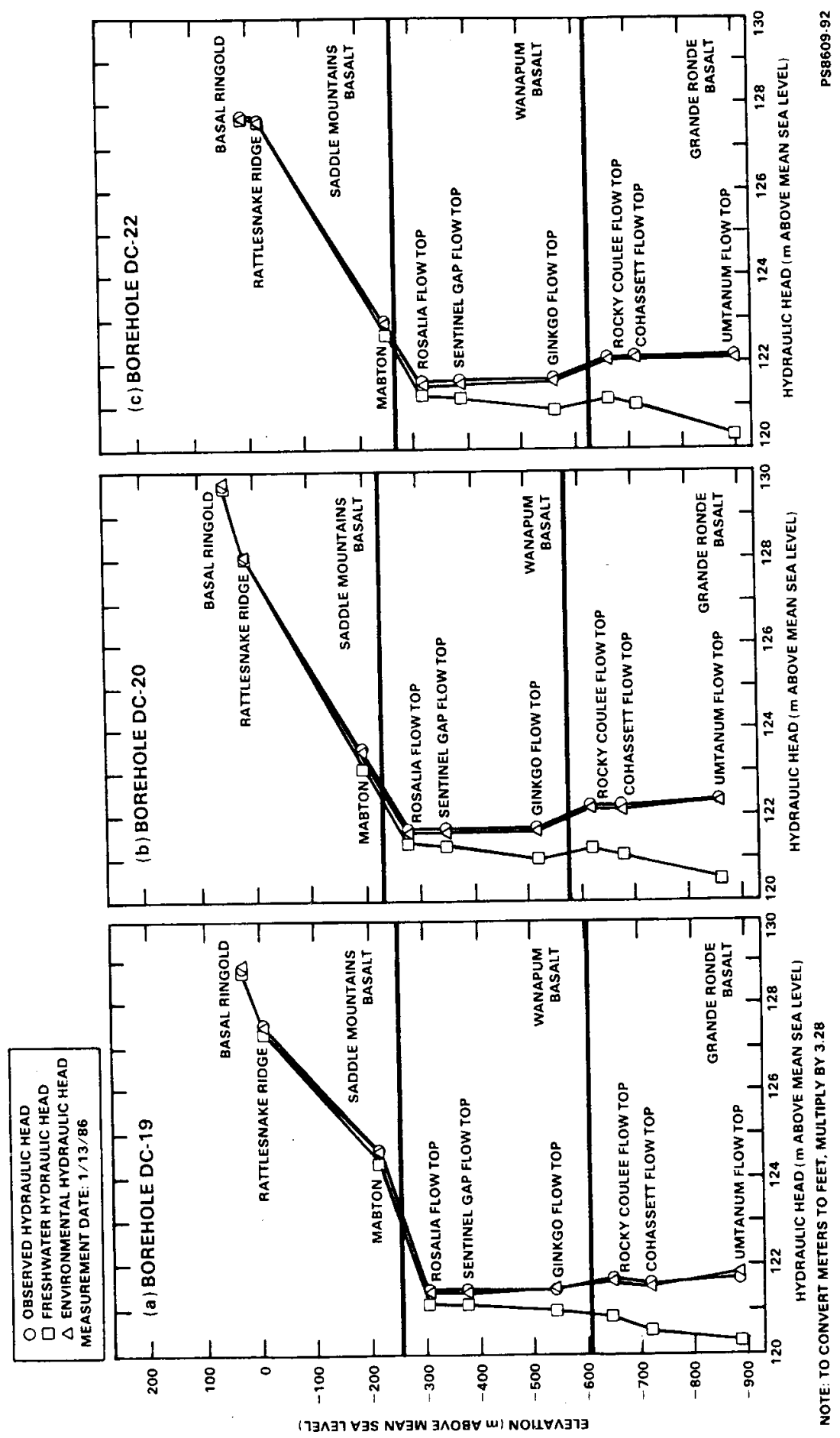

Figure B.15. Vertical Head Distributions Within the Columbia River Basalt from Boreholes DC-1419, DC-20, and DC-22 (see Figure B.13 for well locations). 


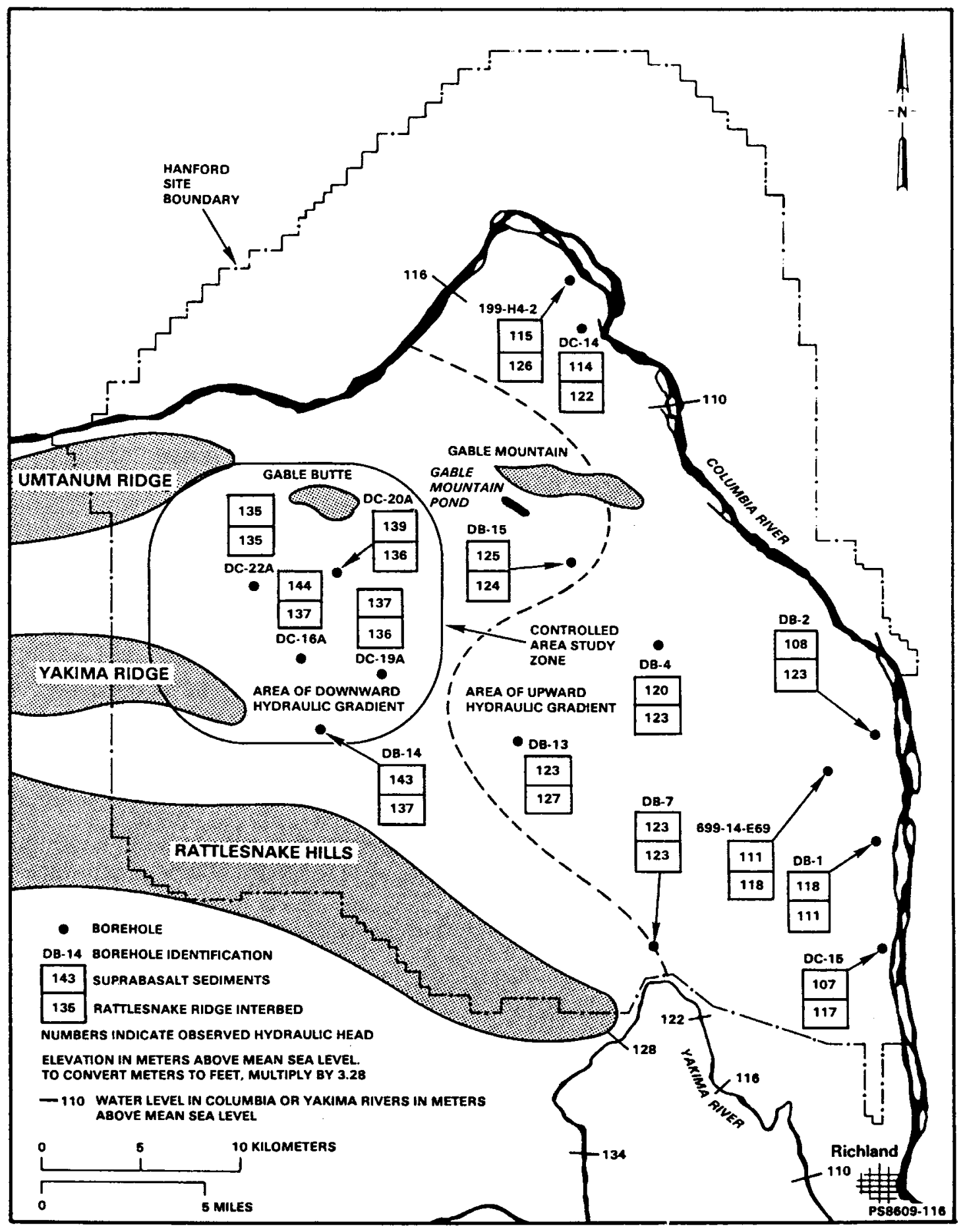

Figure B.16. Comparison of Hydraulic Heads Between the Unconfined Aquifer and the Rattlesnake Ridge Interbed (part of the uppermost confined aquifer) for Conditions in the Early 1980s. 


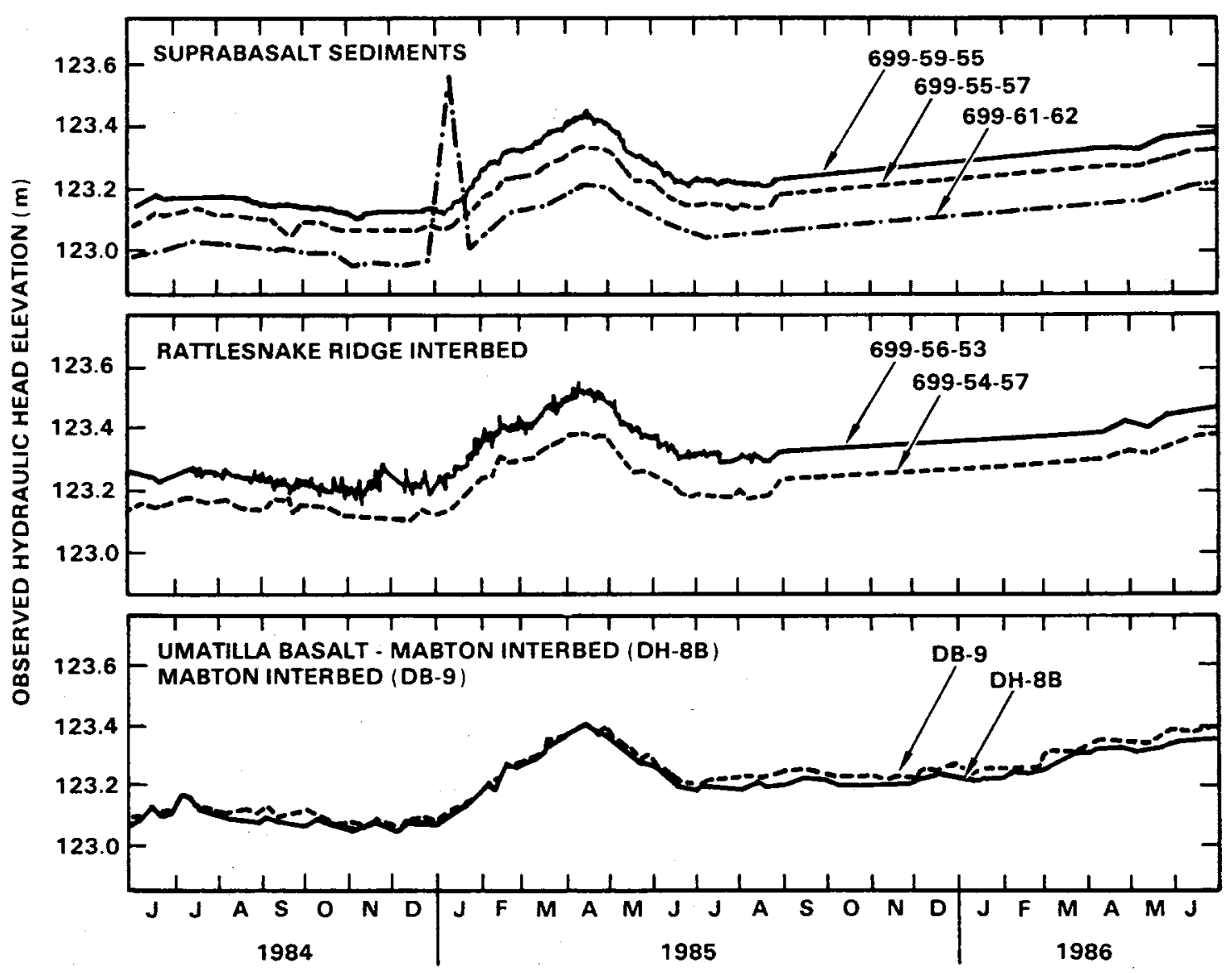

NOTE: WATER LEVELS ARE UNADJUSTED FOR ATMOSPHERIC PRESSURE

ISOLATED ZONES IN BOREHOLES

O UNCONFINED AQUIFER

D RATTLESNAKE RIDGE INTEABED

- mabton INTERBED

ESTIMATED BASALT OUTCROPS ABOVE WATER TABLE

TO CONVERT METERS TO FEET,

MULTILY BY 3.28

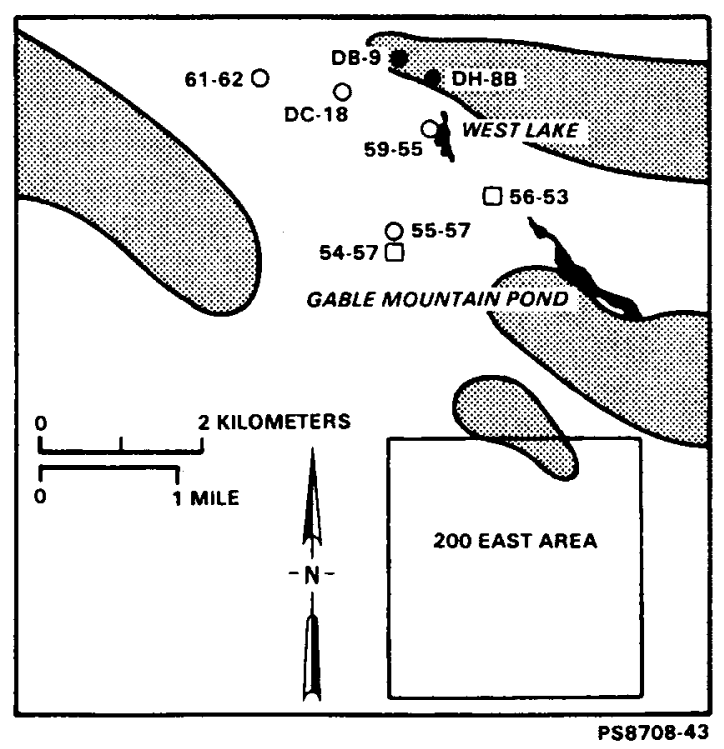

Figure B.17. Hydrographs for Selected Boreholes in the Vicinity of Gable Mountain and Gable Butte. 


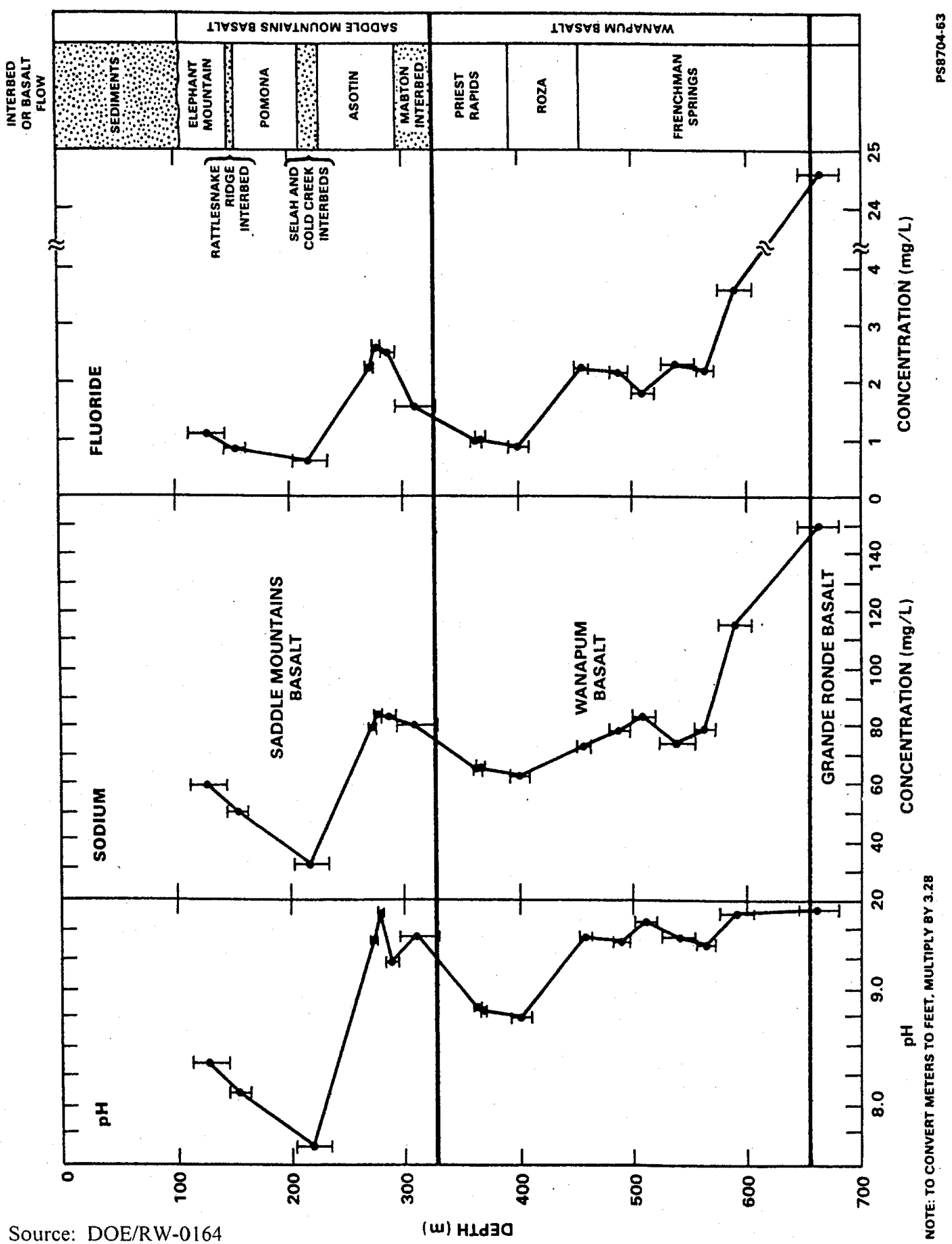

Figure B.18. Correlation of Geochemical Depth Profiles for Saddle Mountains and Wanapum Basalt Groundwaters in Borehole DC-14. 

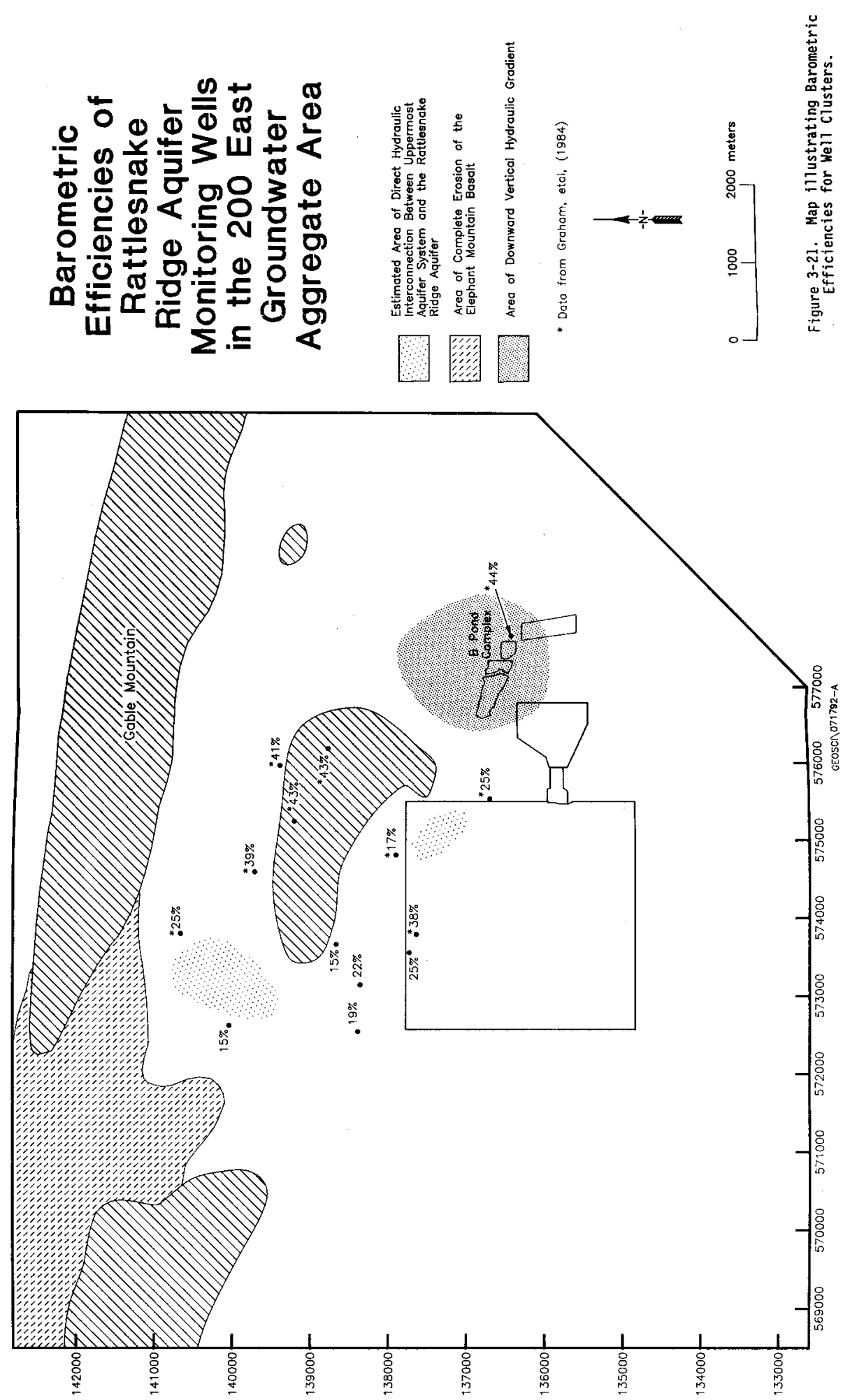

Figure B.19. Structural Features on or Near the Hanford Site that Could be Associated with Vertical Groundwater Flow. 


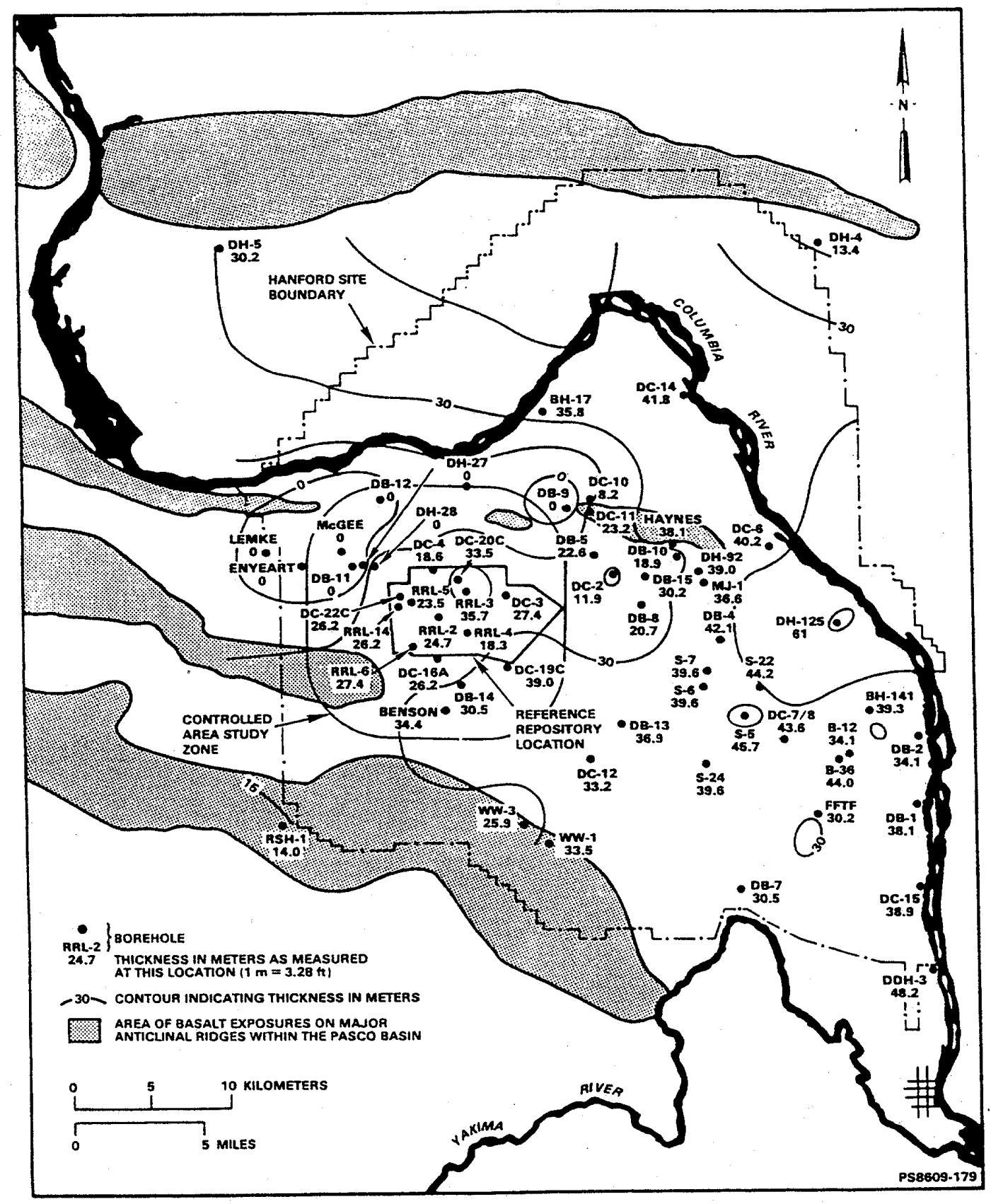

Source: DOE/RW-0164

Figure B.20. Isopach Map for the Elephant Mountain Member. 


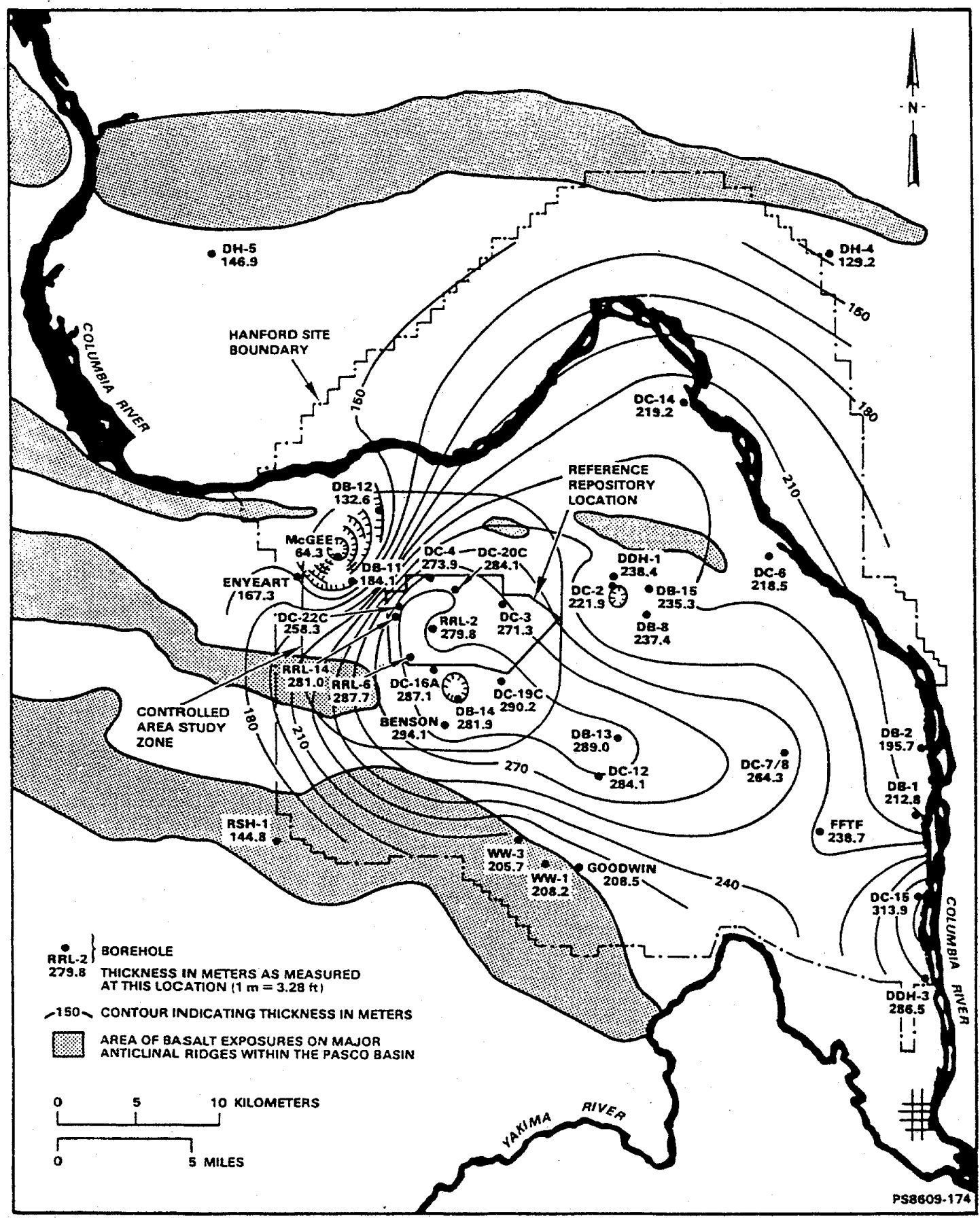

Source: DOE/RW-0 164

Figure B.21. Isopach Map of the Saddle Mountains Basalt. 


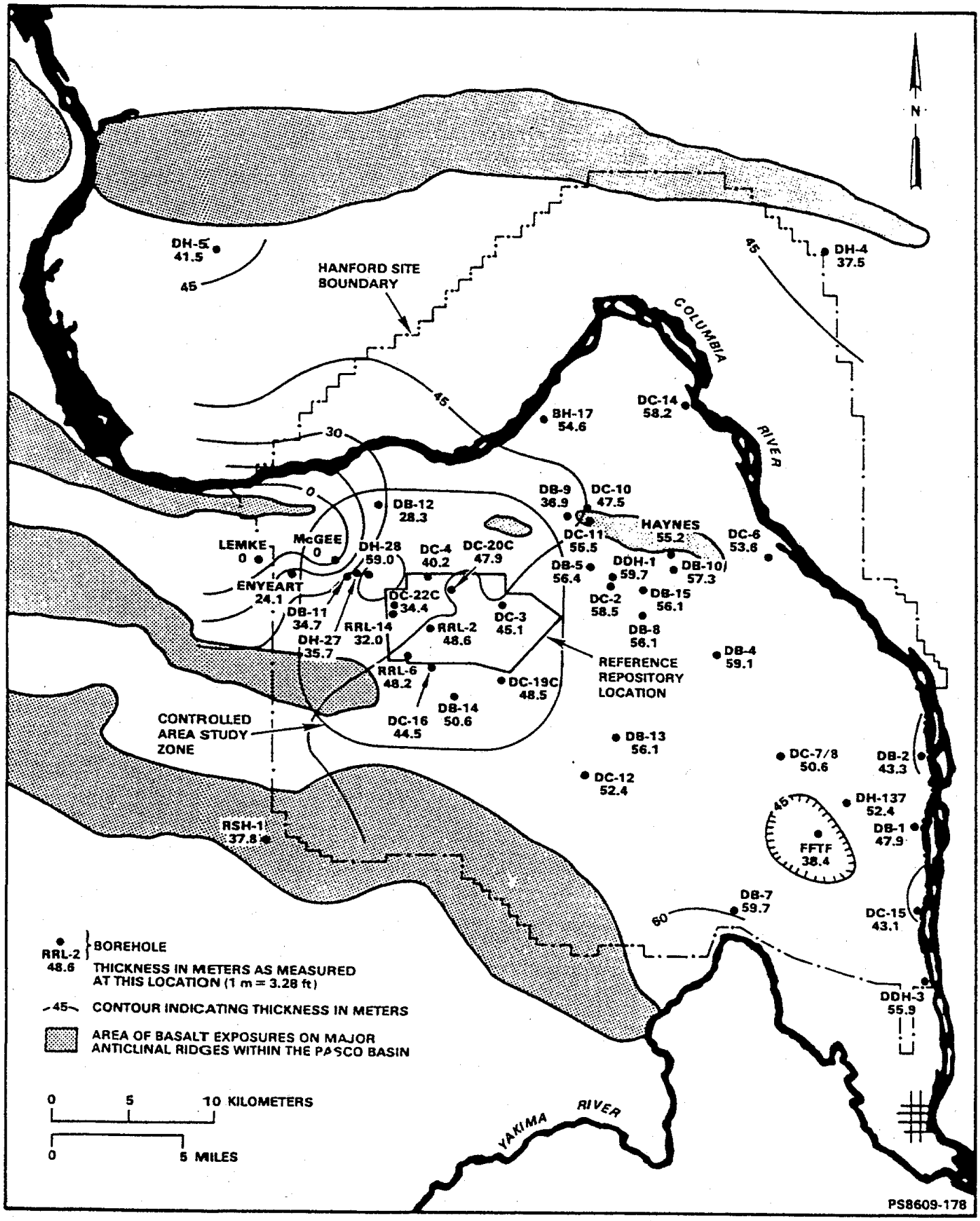

Source: DOE/RW-0 164

Figure B.22. Isopach Map of the Pomona Member. 

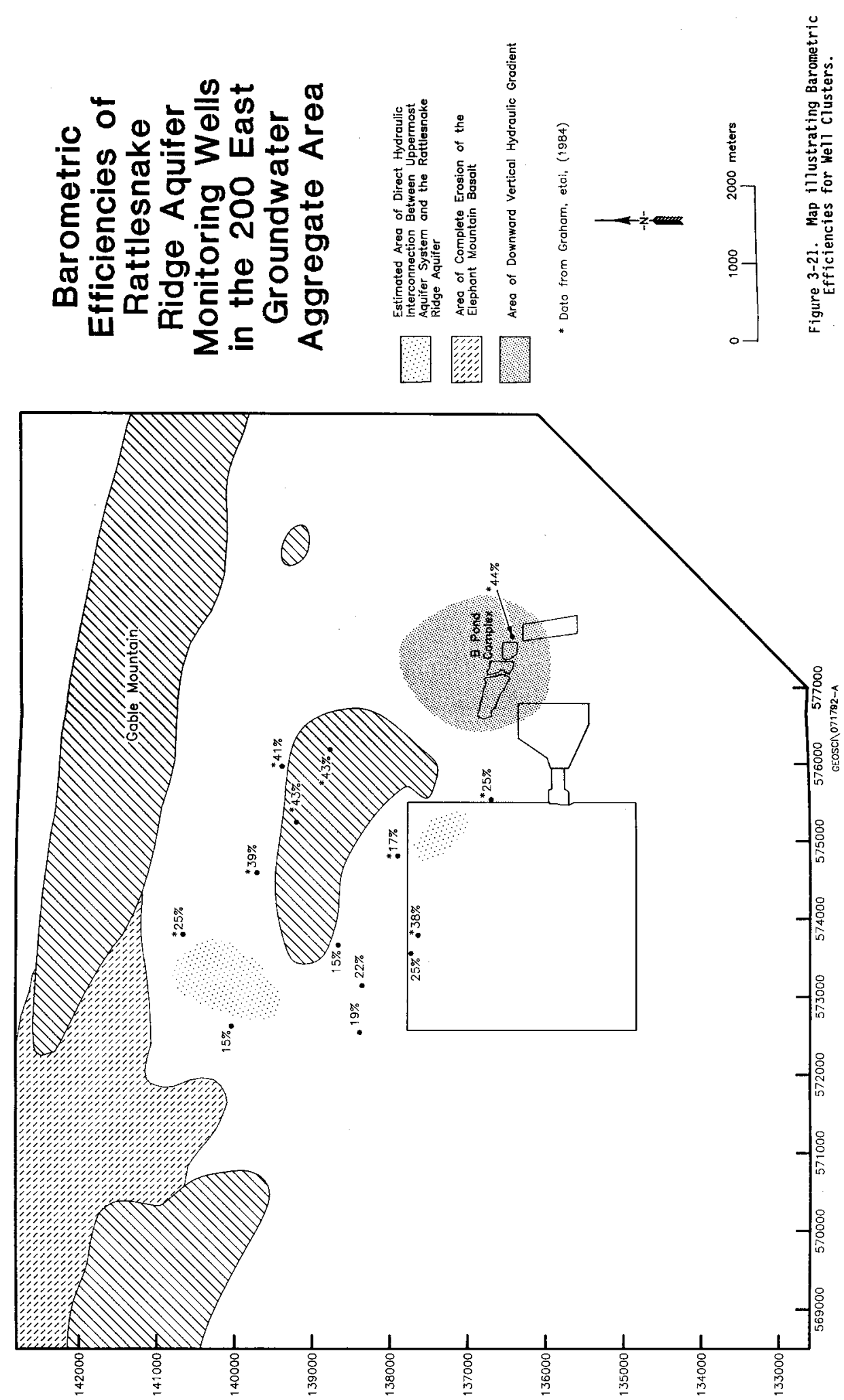

Figure B.23. Locations of Aquifer Intercommunication as Estimated in WHC-SD-EN-TI-019, Rev. 0. 


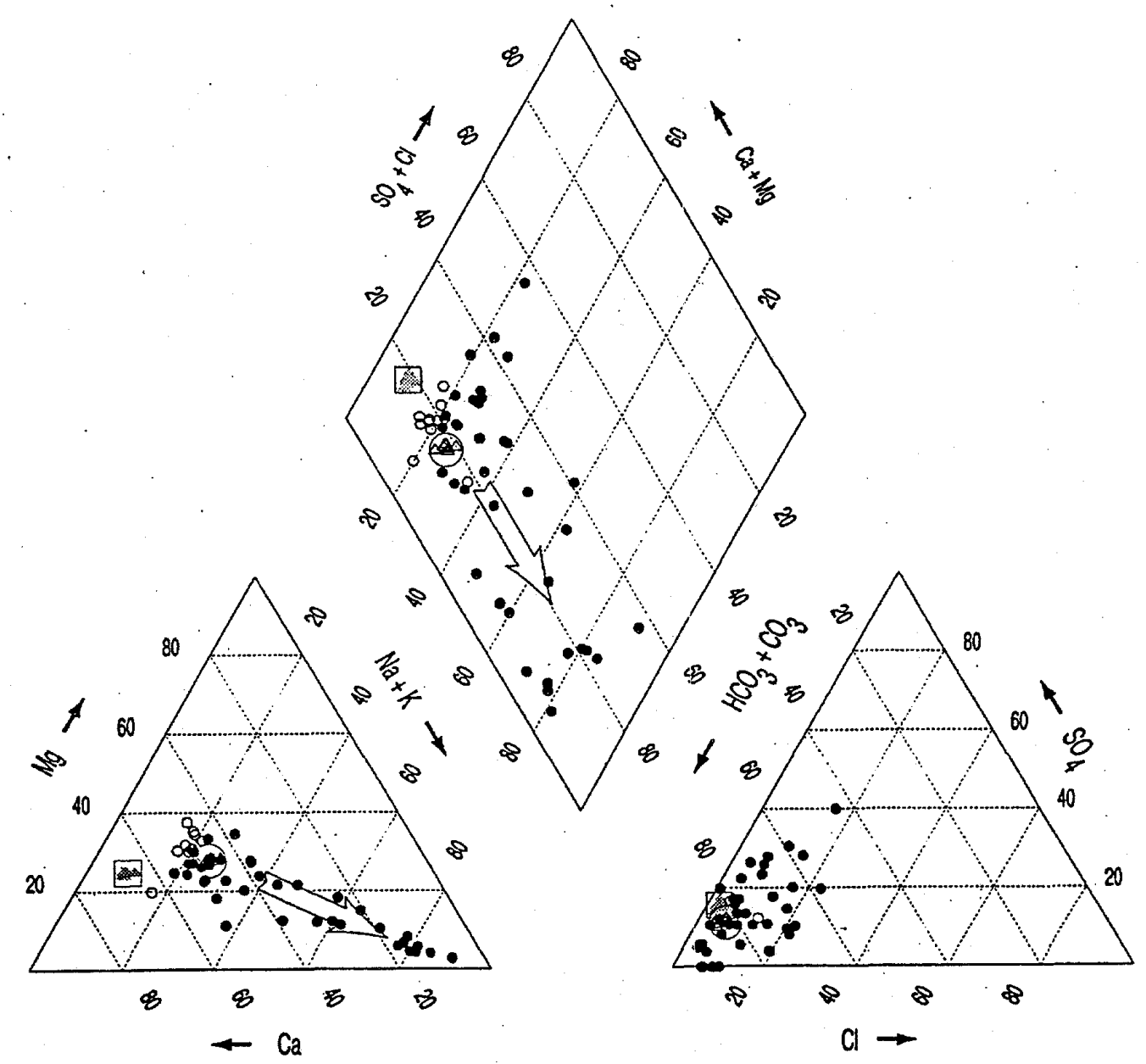

- Individual Columbia River Waters

Combined Columbia River Waters

$\Delta$ Individual Yakima River Waters

Combined Yakima River Waters
- Individual Rattlesnake Hills Spring Waters

- Upper Basalt Aquifer Water from Wells

$\longrightarrow$ Hydrochemical Evolutionary Path

Source: PNL-10817

Figure B.24. Tri-Linear Diagrams Showing Hydro-chemical Evolution of Groundwater Within the Upper Basalt Confined Aquifer System. 


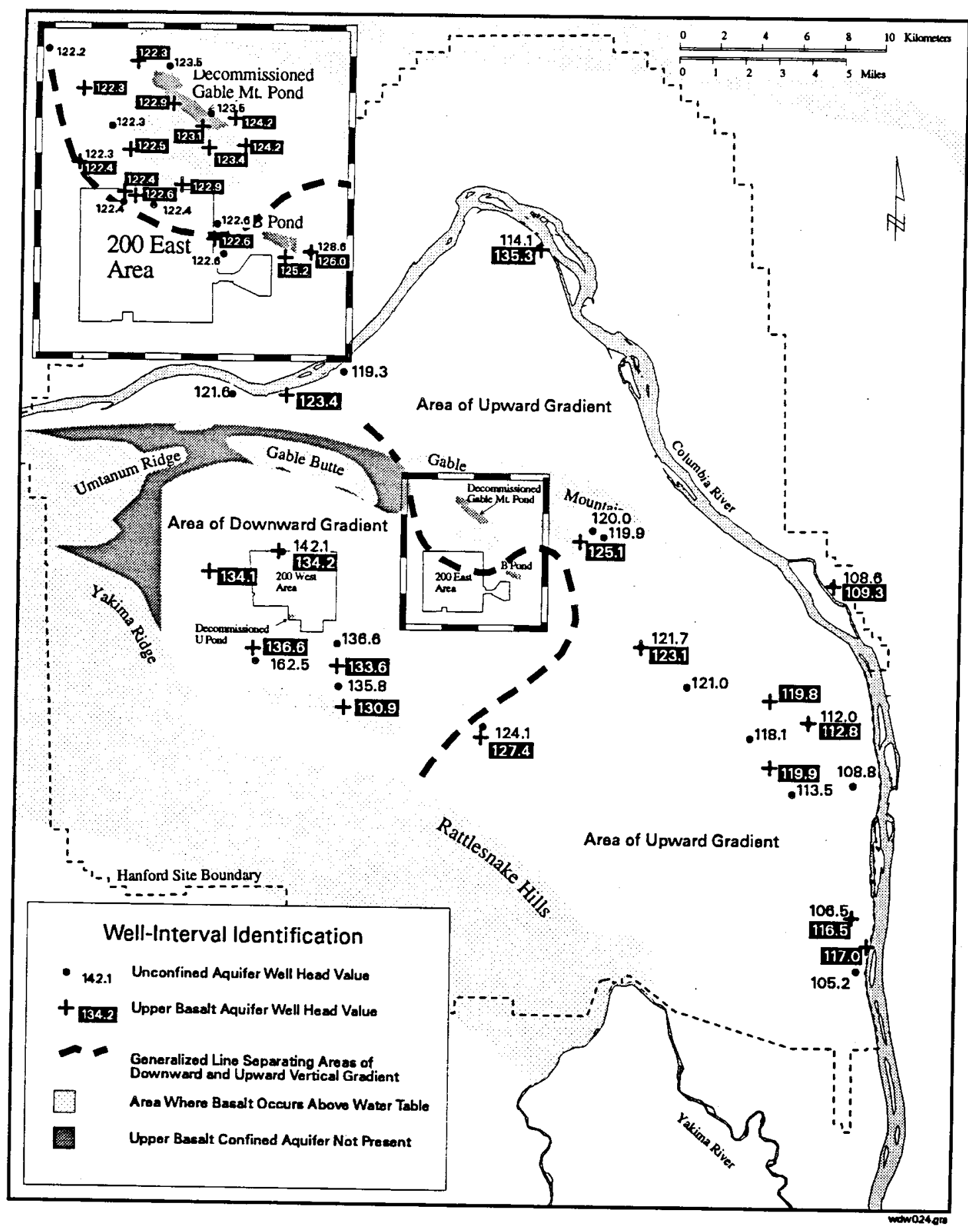

Figure B.25. Comparison of Observed Hydraulic Heads for the Upper Basalt and Overlying Unconfined Aquifer Systems. 


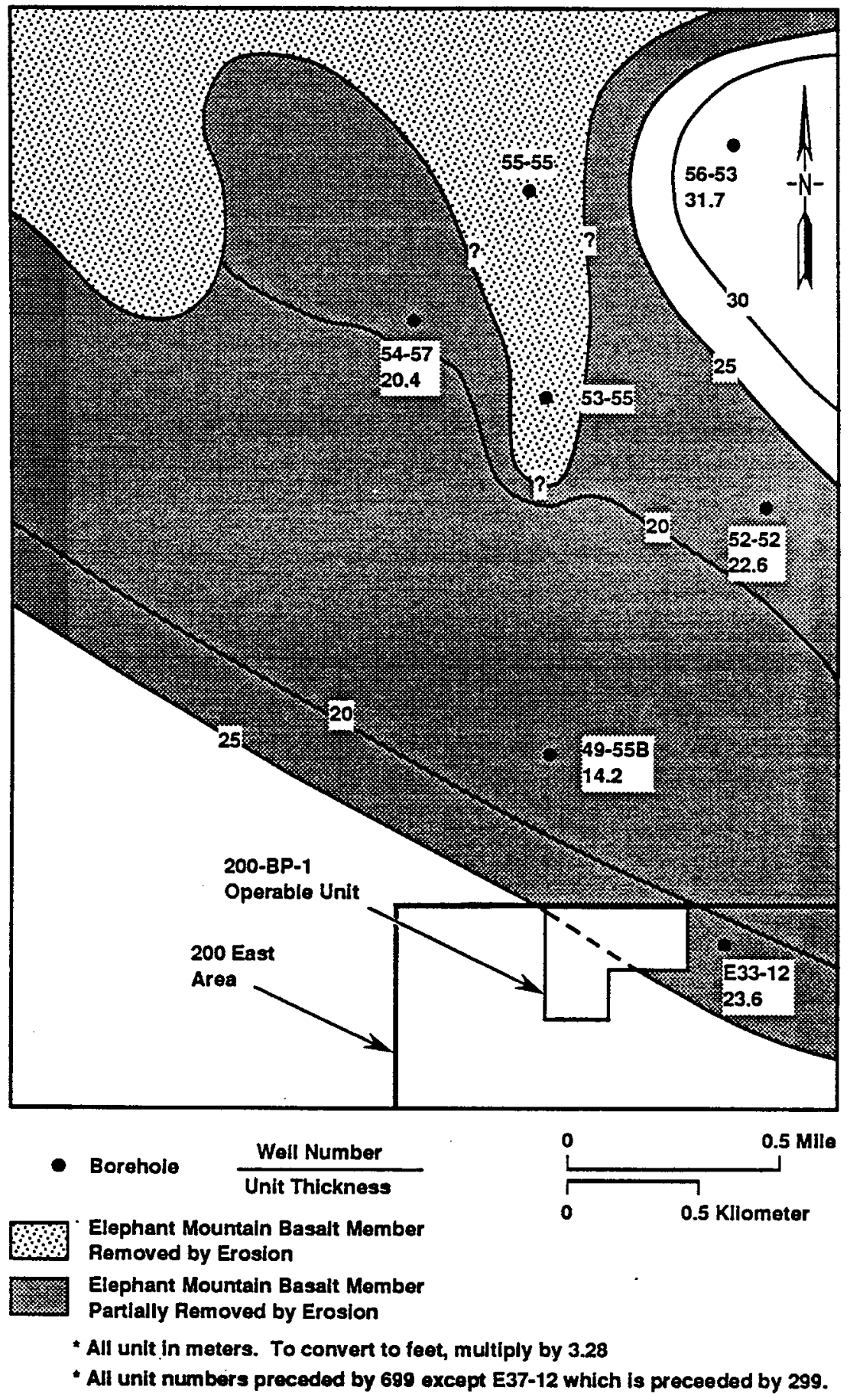

H9201028.1

Figure B.26. Isopach Map of the Elephant Mountain Member from 200 BP-1 Operable Unit. 


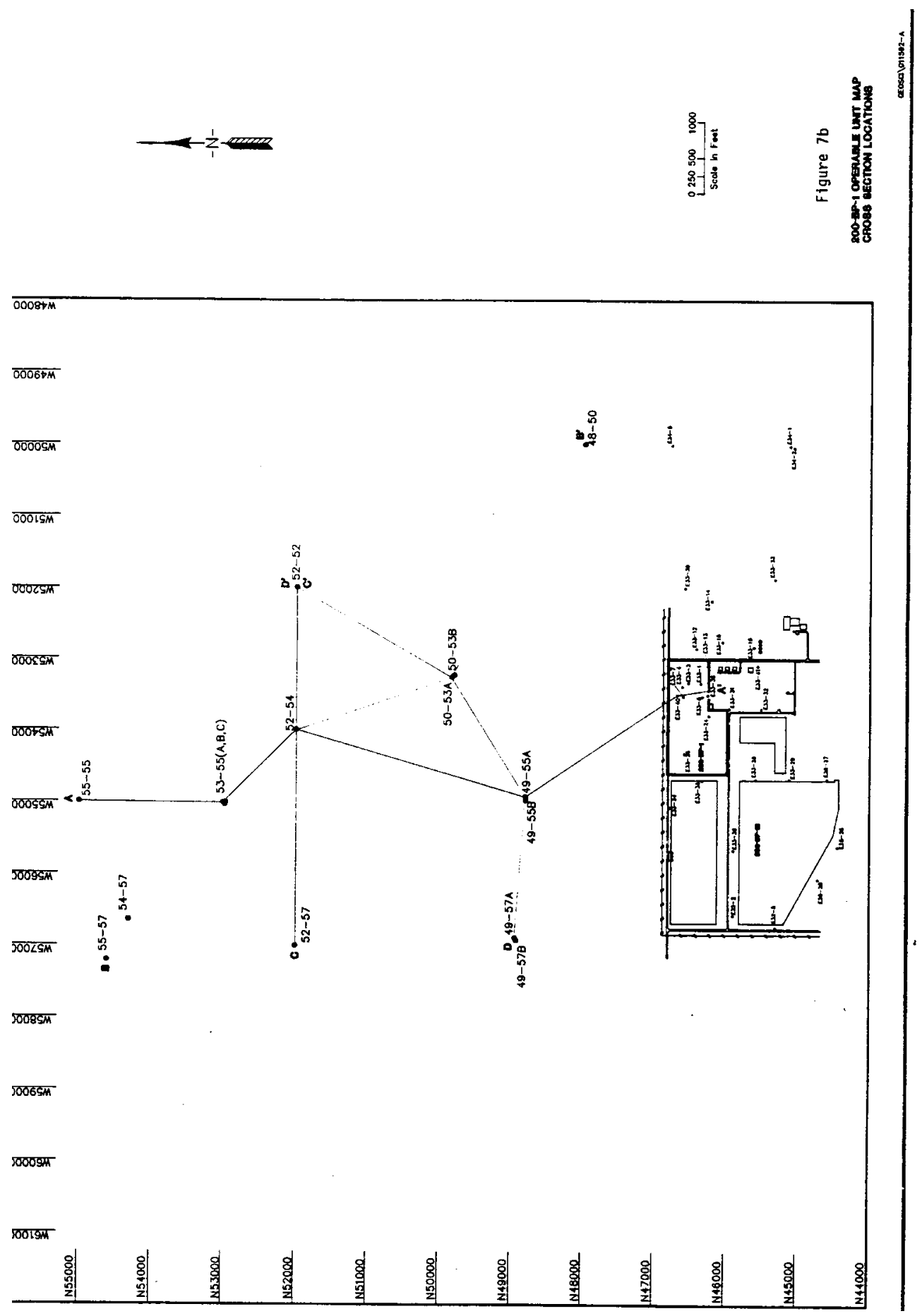

Figure B.27. 200-BP-1 Operable Unit Cross Section Locations. 


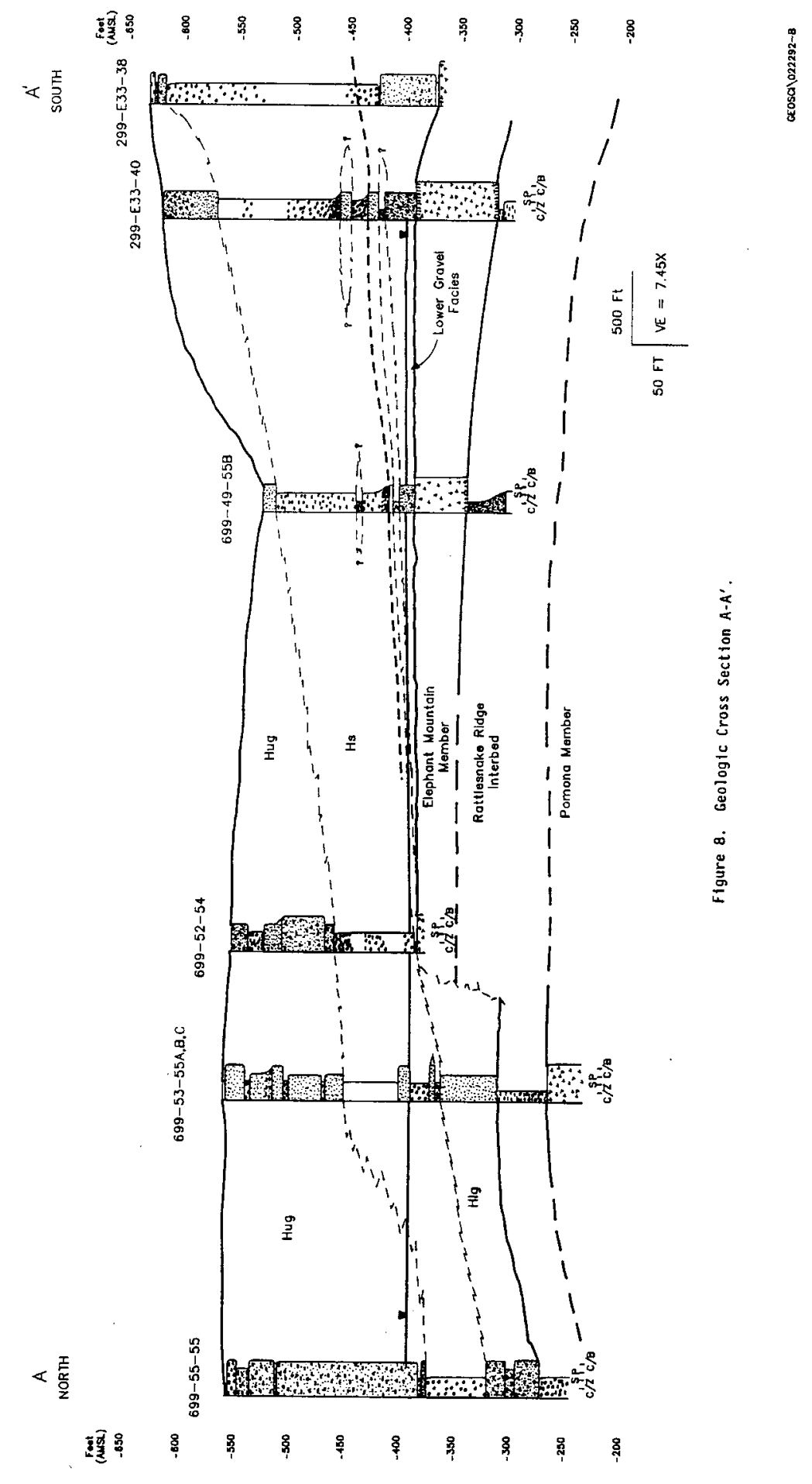

Figure B.28. Cross Section A-A’ (North-South) 200-BP-1 Operable Unit. 


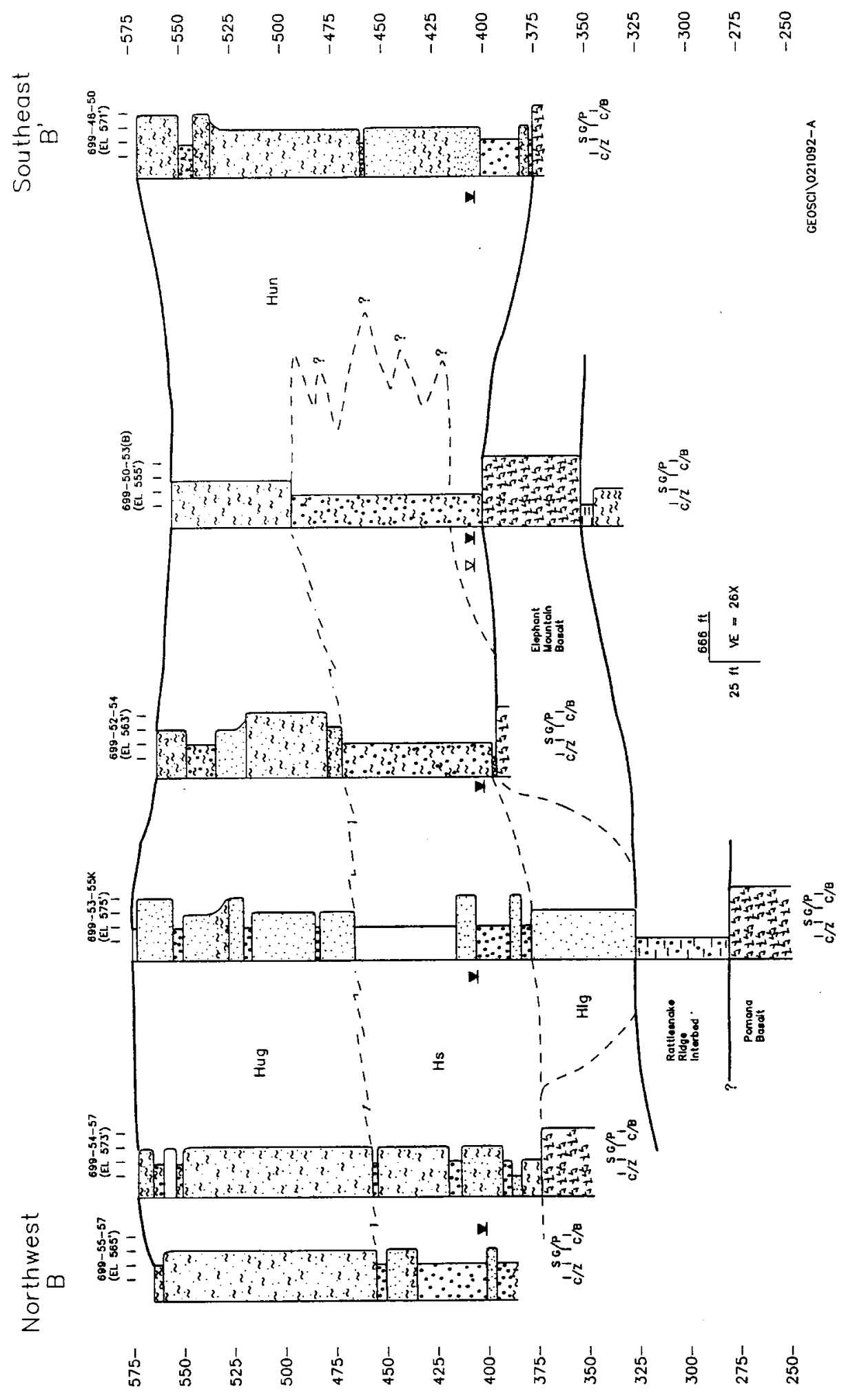

Figure B.29. Cross Section B-B' (Northwest-Southeast) 200-BP-1 Operable Unit. 


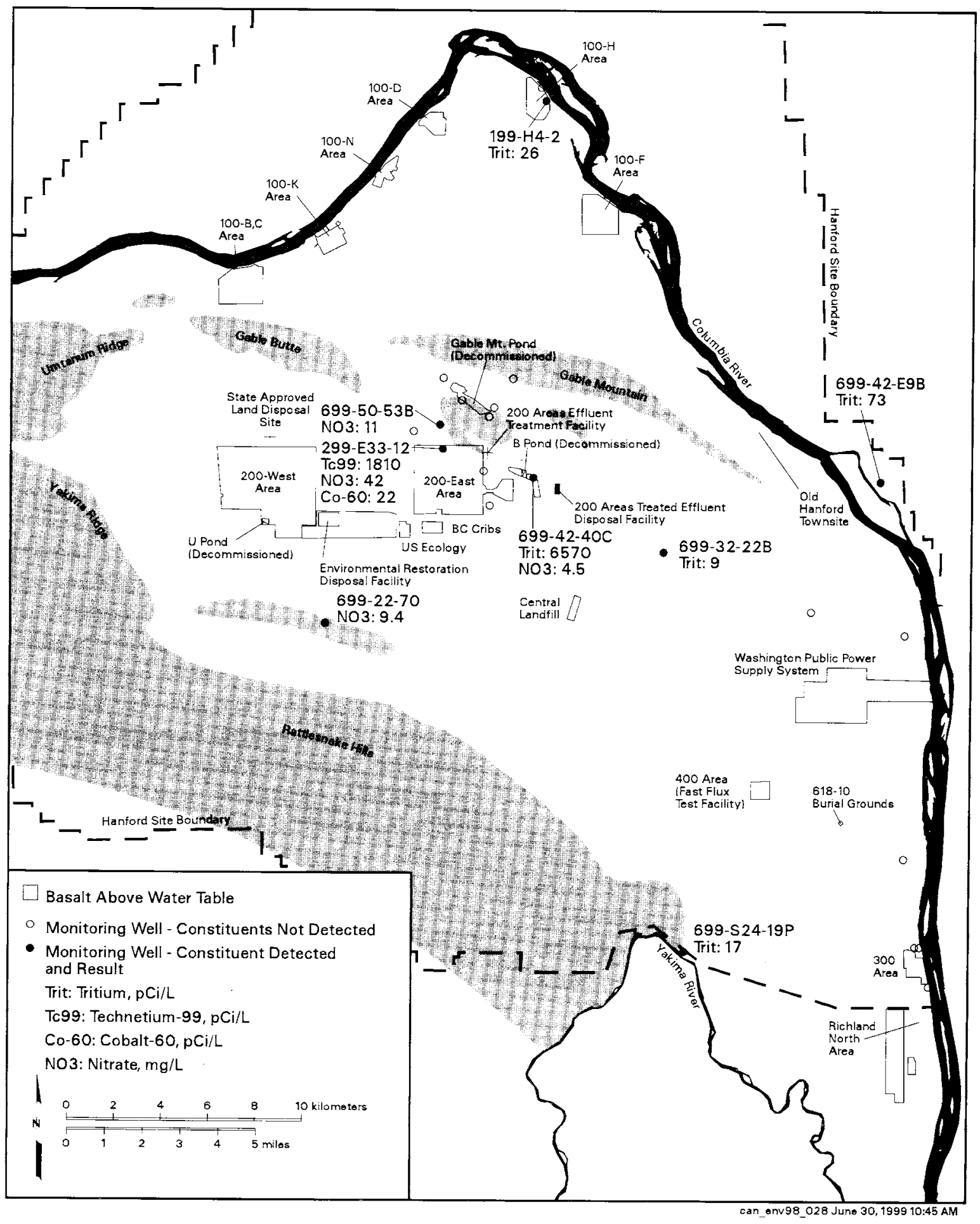

Figure B.30. Tritium, Nitrate, Technetium-99, and Cobalt-60 Detected in Confined Aquifer Wells, 1988. 


\title{
Appendix B, Exhibit 1
}

\section{Task 4.2: Effects of Surface Waste Disposal Activity on Ground-Water Levels in the Saddle Mountain Basalt}

\author{
Prepared By: \\ Richard Nevulis \\ Principal Investigators of the Project: \\ Soroosh Sorooshian \\ Donald R. Davis \\ Department of Hydrology and Water Resources \\ University of Arizona, Tucson, AZ 85721
}

August 20, 1987

B.51 


\section{Effects of Surface Waste Disposal Activity on Ground-Water Levels in the Saddle Mountain Basalt}

The effects of discharging wastewater into surface ponds at the Hanford site have been recognized for many years. Previous discussion and research; however, have concentrated on the effects within the unconfined aquifer (Bierschenk 1959; Newcomb 1973), but did not consider the effects in the underlying confined units (Figure B.31). Surface waste water disposal began in 1944. Figure B.32 is an estimate of the water levels in the unconfined aquifer prior to this activity. The effect of the disposal activity was observed in the steady growth of a water table mound. Forty years later in 1984, the mound's highest point reached a height of 480 feet above mean sea level (Figure B.33).

An examination of the groundwater levels within the Rosalia flow top, Mabton interbed, and Rattlesnake Ridge interbed indicated that there may be a "peizometric mound" beneath the water table mound (Figures B.34, B.35, and B.36). The boreholes that were used to create the contour maps and their respective water levels are also shown.

The following assumptions are necessary to continue the study of the effects of the water table mound on the confined units below:

1. A piezometric mound exists in the confined hydrostratigraphic units under the water table mound.

2. The water table mound in the unconfined aquifer is the cause of the piezometric mounds observed in the Rosalia flow top, Mabton interbed, and Rattlesnake Ridge interbed.

The first assumption is made because of the lack of monitoring wells to delineate the actual piezometric surface in the confined units. The second assumption is needed because there are no data for the piezometric levels in the confined units prior to the disturbance to the water table in 1944.

Accepting these assumptions, the presence of the piezometric mounds could be the result of two physical processes:

1. Loading of the confined aquifer due to the additional weight of water an the overlying unconfined aquifer as a result of the disposal activity.

2. Hydraulic communication between the unconfined and confined aquifer. 


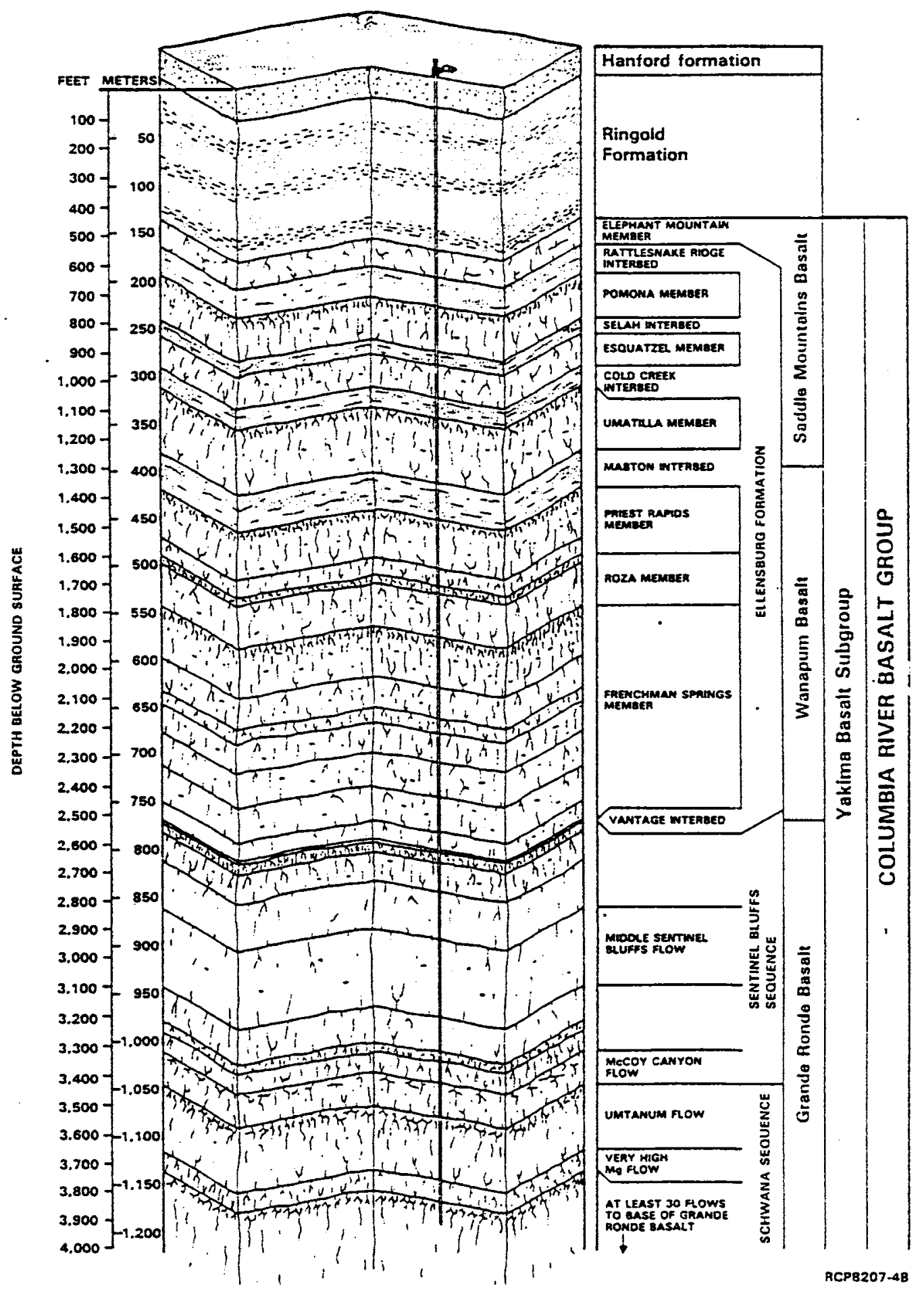

Figure B.31. Stratigraphy of the Columbia River Basalt. 


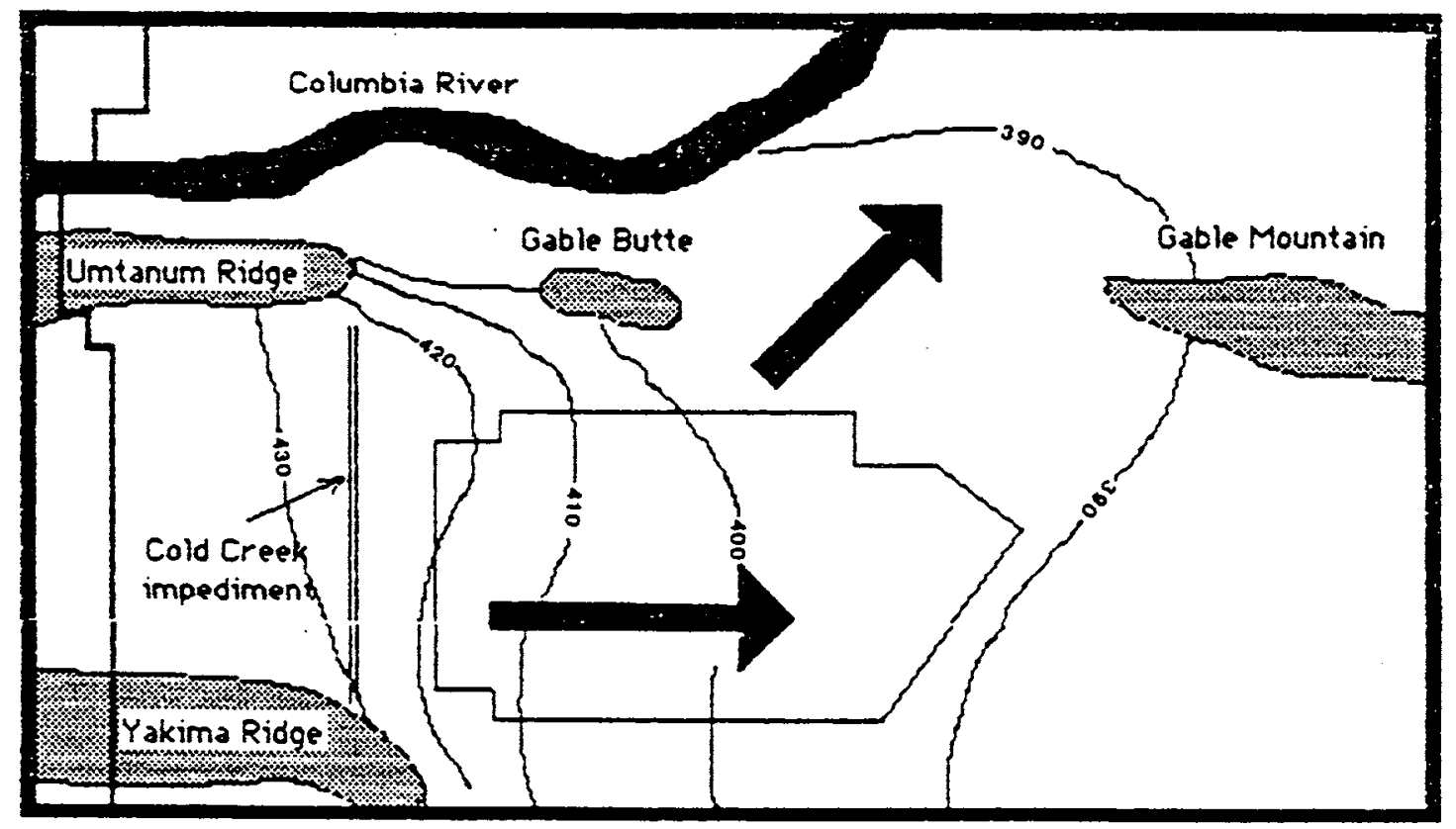

\section{Estimated 1944 Hater-Table Map}

Figure B.32. Estimated Water Table Elevation, 1944 (ft above mean sea level).

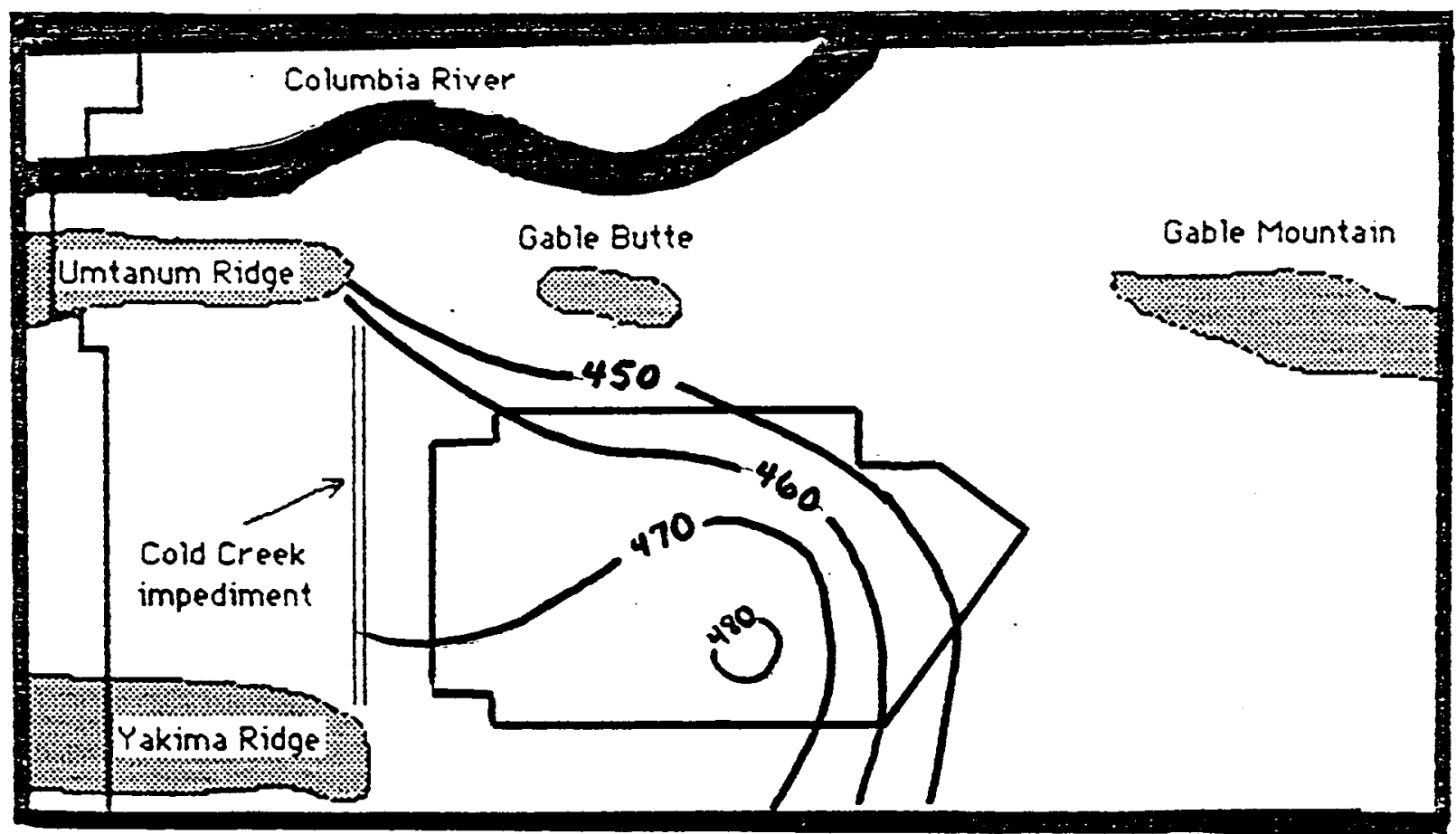

Figure B.33. Water Table Elevation Map in December 1984 (ft above mean sea level). 


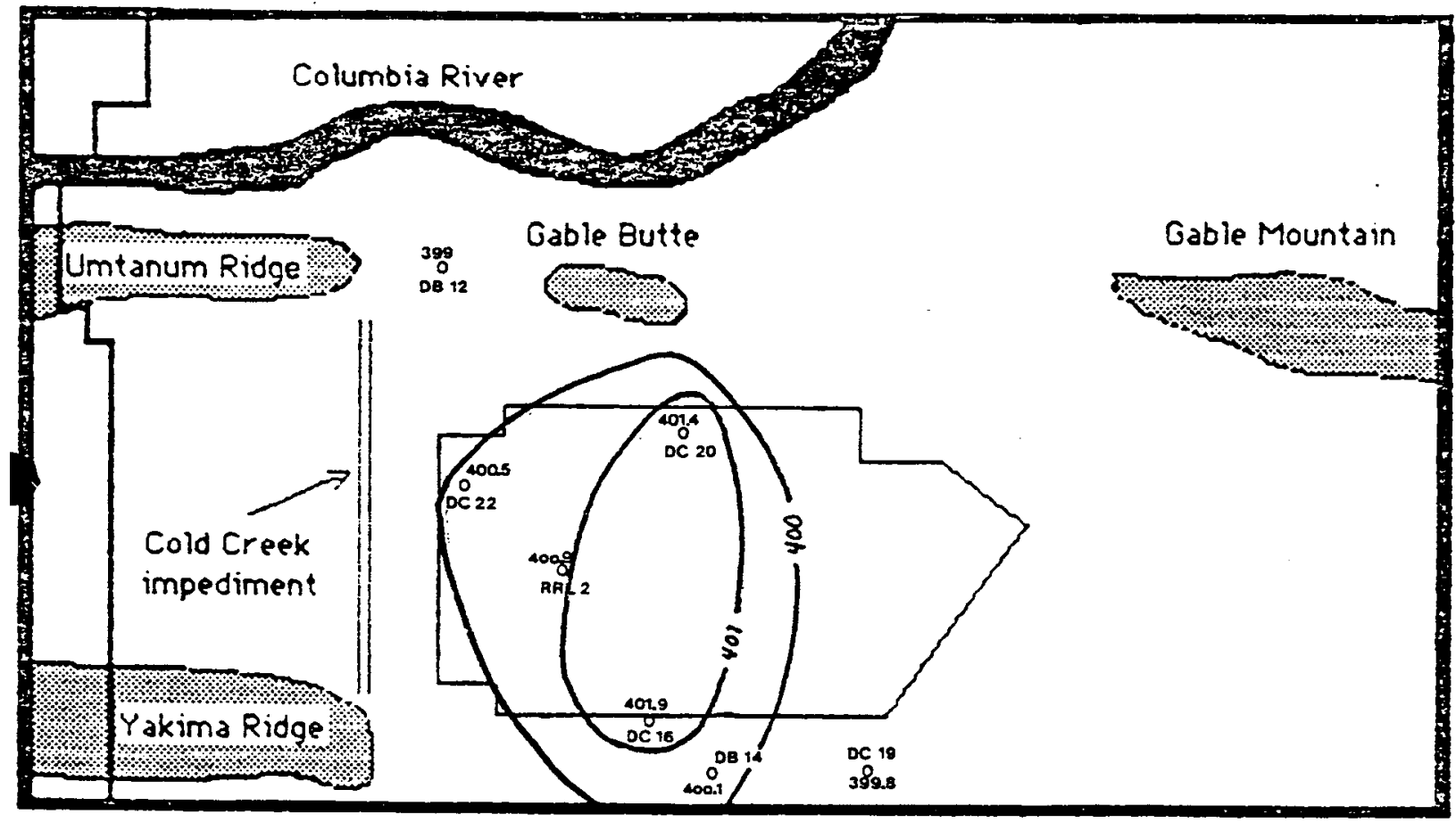

Figure B.34. Contour Map of the Piezometric Levels in the Rosalia Flow Top (ft above mean sea level).

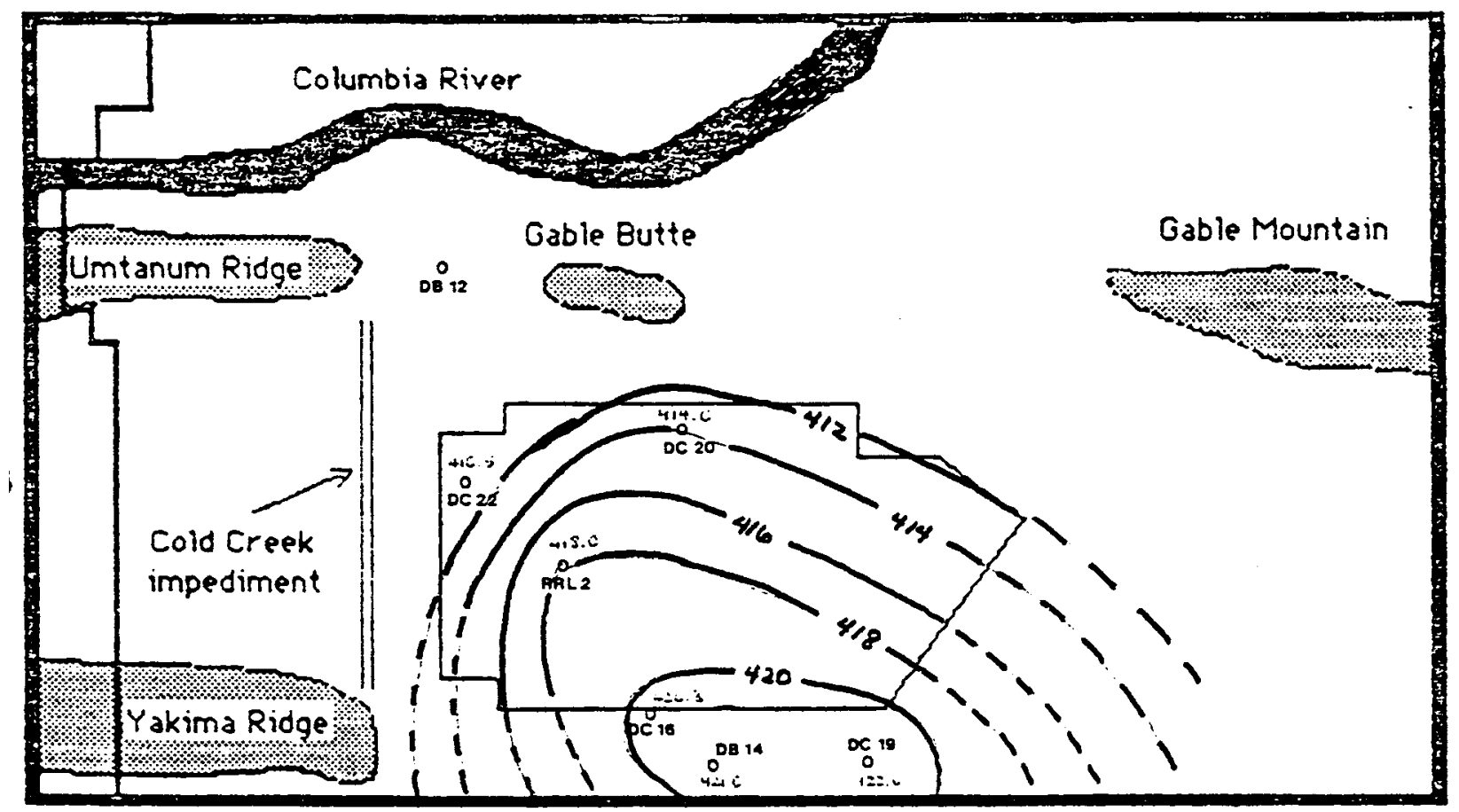

Figure B.35. Contour Map of the Piezometric Levels in the Mabton Interbed (ft above mean sea level). 


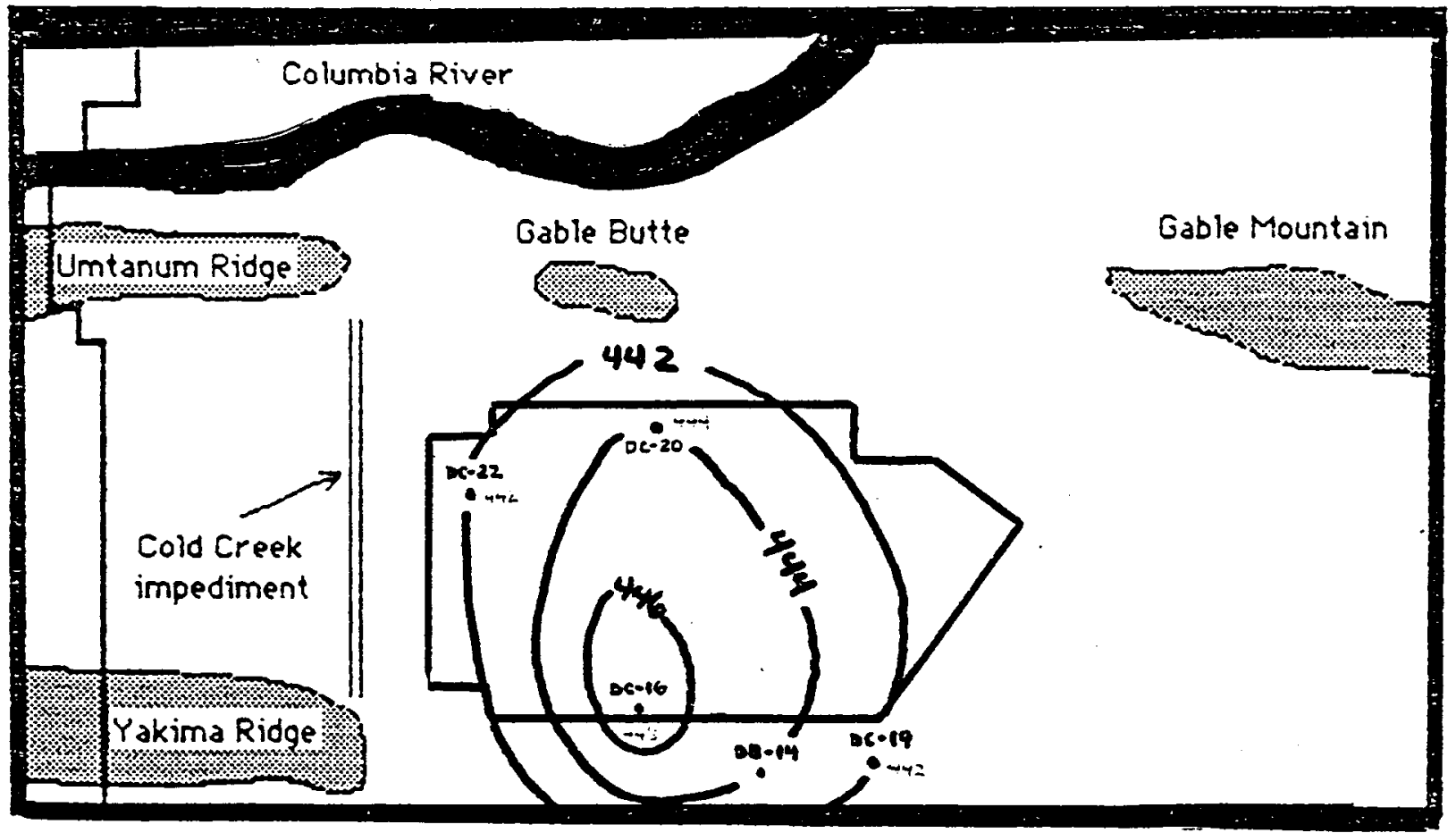

Figure B.36. Contour Map of the Piezometric Levels in the Rattlesnake Ridge Interbed (ft above mean sea level).

\section{Loading of the Confined Aquifer}

A change in the overlying weight on a confined aquifer will cause a change in the total stress within that aquifer. The change in stress will be borne by the skeletal mass of the aquifer and the pore fluid. The fraction of the stress that is transferred to the pore fluid will be reflected by a change in the piezometric level at that point. Because the loading response is proportional to the change in weight of the water table mound, a similar change in the piezometric surface would result in the underlying confined units.

To determine if loading is the cause of the mound within the Rosalia flow top, Mabton interbed, and Rattlesnake Ridge interbed, the next steps are to estimate

1. The growth or decay of the water table mound in time.

2. The expected loading response for a given weight applied a certain distance above the confined aquifer.

3. The dissipation rate of the piezometric mound due to outward flow of groundwater. 


\section{Changes in the Water Table Mound with Time}

Characteristics of the water table mound were studied by various early investigators (Bierschenk 1959; Newcomb 1973) and later by the Rockwell Hanford Operations (Schatz 1986). Figure B.37a shows the best estimate of the changes in the water table mound with time. Figure B.37b is identical to Figure B.37a except that the height of the mound does not decrease in the last year of study (1985). Both estimates will be used to study the effect of loading.

\section{Estimation of Loading Response}

A solution for the vertical stress due to a point source load at a given depth was obtained using analytical methods published in the soil engineering literature. These methods allow the calculation of the change in stress within the Rosalia flow top due to the change in weight applied by the water table.

The water table mound was approximated by a rectangular $9 \mathrm{mi}^{2}$ area with a uniformly distributed pressure applied on its surface. Depth from the base of the suprabasalts to the Rosalia flow top is approximately 0.2 miles. For a unit increase of pressure on the water table mound, the change in stress 0.2 miles vertically downward equaled approximately $95 \%$ of that increase. This high response is because the depth to the aquifer is a small fraction of the lengths of the area over which the pressure was applied.

Piezometric levels within the Rosalia flow top will reflect the portion of the increase in stress that is carried by the pore fluid. To estimate this fraction, the tidal efficiency of the flow top was used. Tidal efficiency, C, can be computed by

$$
\mathrm{C}=1-\mathrm{B}
$$

where $\mathrm{B}$ is the barometric efficiency. Previous studies have shown that the barometric efficiency for the Rosalia flow top is 0.70 ; therefore, the tidal efficiency, $\mathrm{C}=0.30$. The piezometric response in the Rosalia flow top, $\mathrm{P}_{\mathrm{r}}$, for a unit increase in pressure at the water table mound, $\mathrm{P}_{\mathrm{m}}$, is

$$
\mathrm{P}_{\mathrm{r}}=(0.95) \times(0.3) \times \mathrm{P}_{\mathrm{m}}=0.3 \times \mathrm{P}_{\mathrm{m}}
$$

This relationship was used to generate an estimate for the change in piezometric levels within the Rosalia flow top due to the change in the water table mound (Figure B.38). 


\section{Temporal Changes in the water Table Mound}
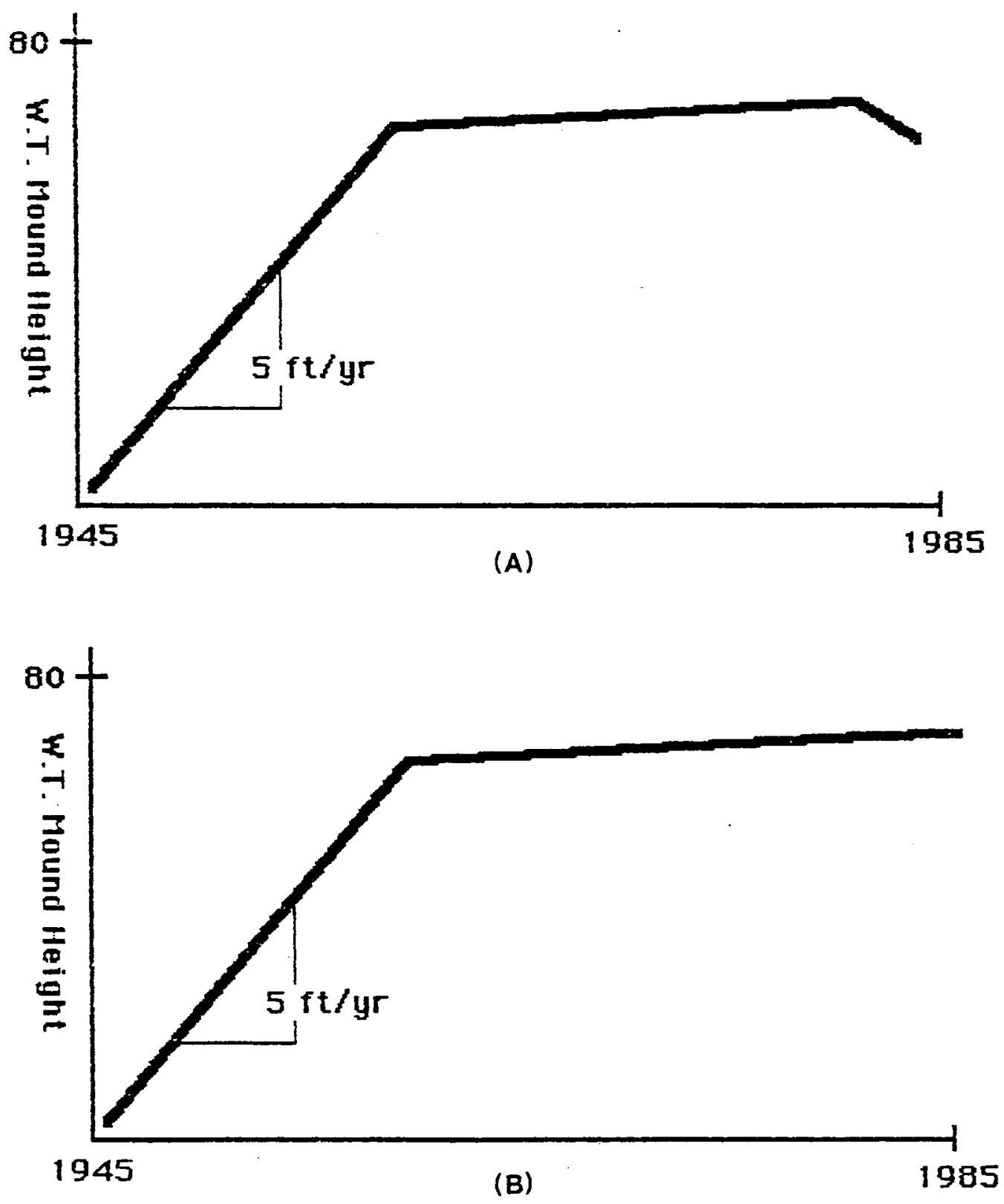

Figure B.37. Estimation of the Change in the Maximum Height of the Water Table with Time: A) Height Decreasing in 1985, and B) Height Increasing Through 1985. 


\section{Loading Response}

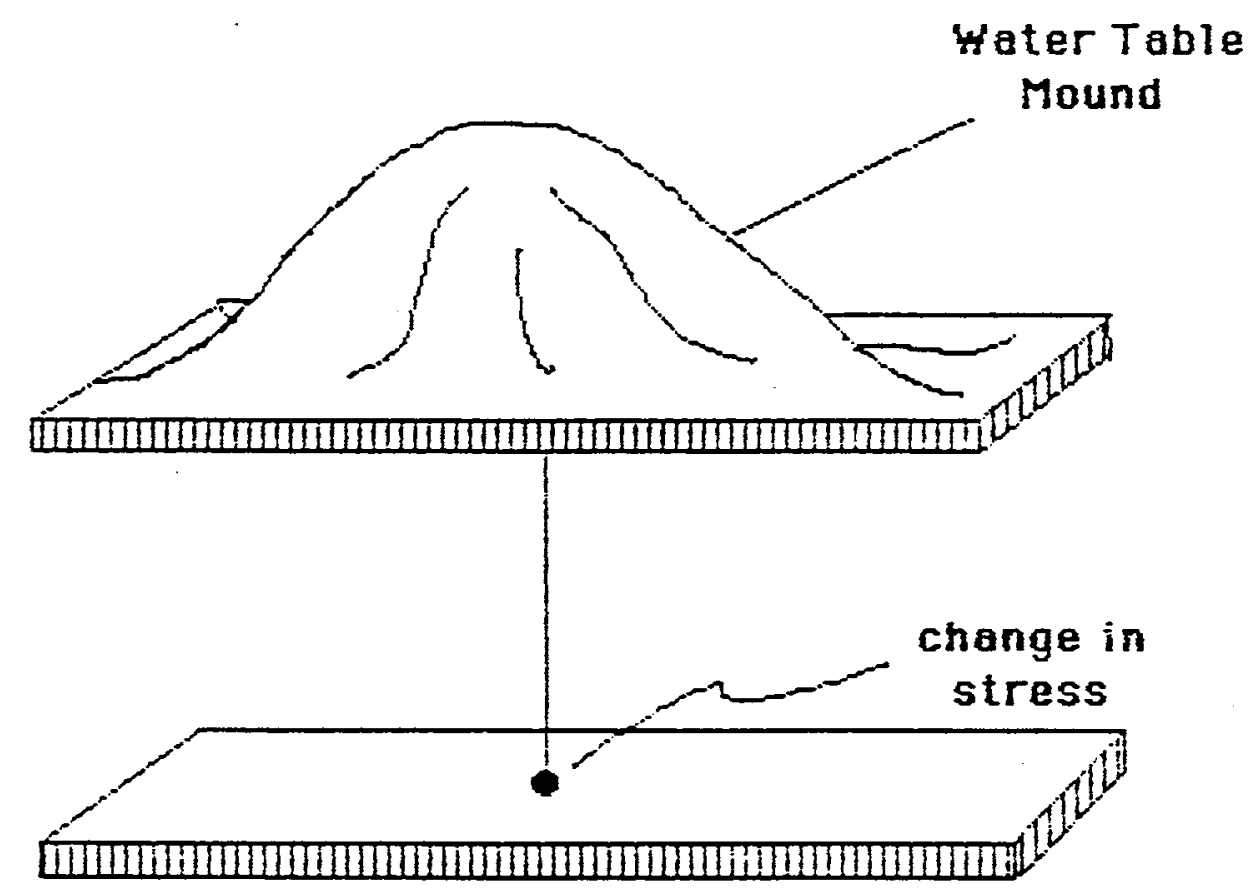

Change in stress is distributed to:

(1) rock

(2) pore fluid

Figure B.38. Load Response at Depth Due to a Growth in the Water Table Mound. 


\section{Piezometric Mound Dissipation}

Changes in the piezometric levels induced by loading will cause a hydraulic gradient in the flow top as seen in Figure B.39a. The gradient will cause radial, outward flow that the lateral of the flow top which is beneath the center of the water table mound. Assuming that the lateral extent of the Rosalia flow top is infinite and that no barriers interfere with the flow, radial flow will occur outward from this area as a function of

1. $\mathrm{dH}$ (gradient)

2. transmissivity

3. storativity.

To obtain an estimate of the dissipation rate of the piezometric mound, a one-dimensional-D finite difference model was used (Figure B.39b). A two-dimensional radial flow model would be more accurate for this situation; however, the simpler one-dimensional model was used. The fact that a one-dimensional model gives a slower dissipation rate than a radial model will be used to justify the conclusion at the end of this section.

\section{Results}

Loading can be discounted as the cause of the piezometric mounds by choosing the factors from the steps above, which would cause the effects of a loading phenomenon to be greatest then showing that the observed results are too small. This was accomplished by

- allowing the water table mound to grow through 1985 (Figure B.37b)

- assuming that $100 \%$ of the change in stress at the surface was propagated to the underlying confined units

- choosing flow properties for the confined units which would deter the dissipation of the piezeometric mound (transmissivity $=10 \mathrm{ft}^{2} /$ day; storativity $=10^{-4}$ )

- using a one-dimensional flow model instead of a two-dimensional radial flow model.

The result of this procedure is that the crest of the piezometric mound within the Rosalia flow top, $\mathrm{h}_{\mathrm{pr}}$, was calculated to be

$$
\mathrm{h}_{\mathrm{pr}}=0.06 \text { feet }
$$

\section{Conclusions}

The observed height of piezometric mound in the Rosalia flow top compared to the areas away from the disposal activity is approximately 2 feet. The previous result showed that the expected maximum 


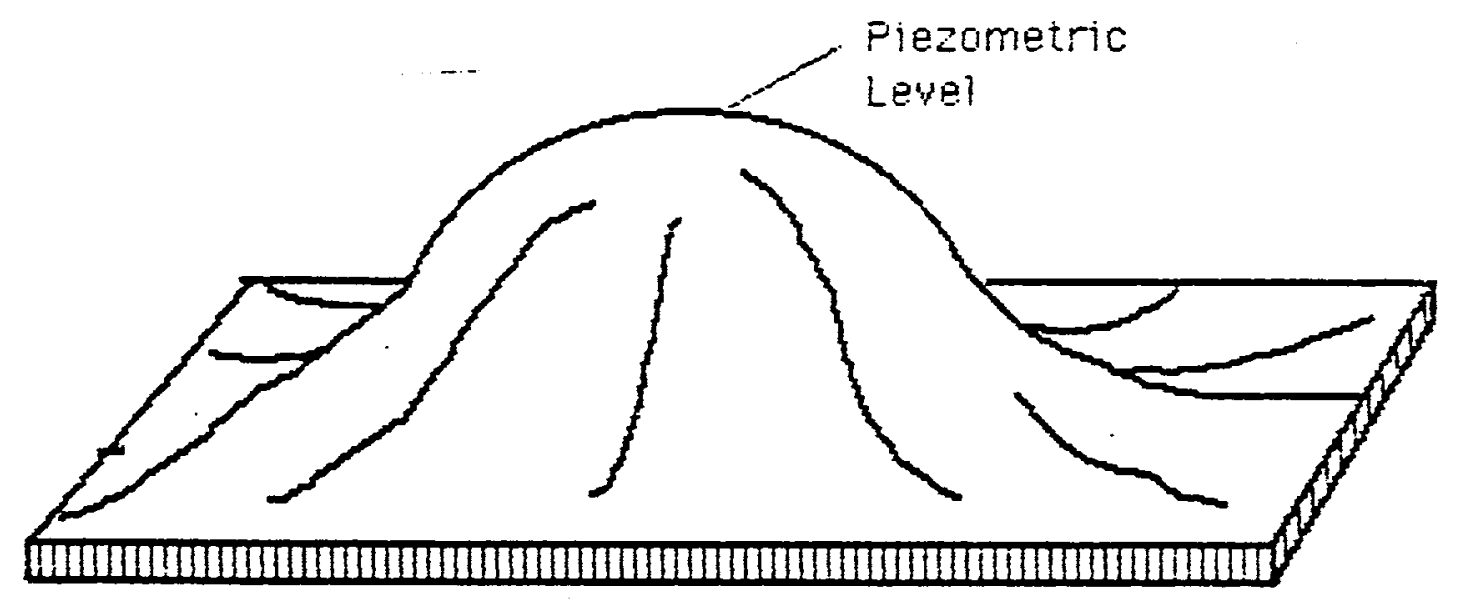

(A)

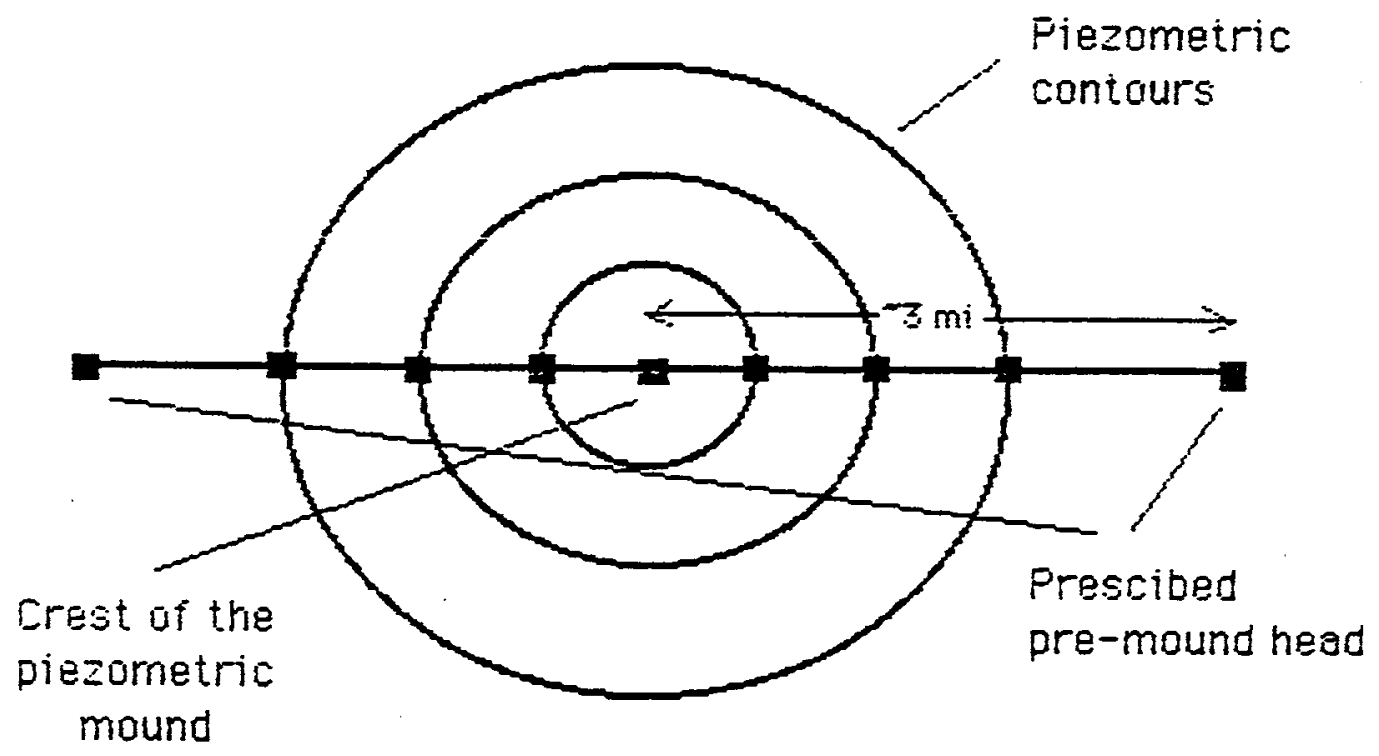

(B)

Figure B.39. Piezometric Mound and the Finite Difference Grid Used to Model the Dissipation. 
height of the piezometric mound in the Rosalia flow top is approximately 0.06 feet. If the best estimate for the changes in the water table mound with time are used (Figure B.37a), a rebound might be expected to occur which would cause a depression in the piezometric surface.

Based on the results of this analysis, loading of the Rosalia flow top by the water table mound is not the cause of the piezometric mound. This conclusion may also be made for the Mabton and Rattlesnake interbeds because the apparent height of their mounds exceeds the maximum change that could be caused by loading.

\section{Vertical Flow}

The remaining possibility that could explain the piezometric mounds is hydraulic communication from the water table mound to the underlying confined units. The remainder of this study will involve analyzing this possibility and hopefully learning more about the properties of the basalt formations in the area.

Hydraulic communication between the unconfined and confined units is controlled by the properties of the rock through which a disturbance must travel. More specifically, the vertical conductivity, K, and specific storage, $\mathrm{S}_{\mathrm{s}}$ of the rock dictate the degree of communication. The lithology of the Saddle Mountains basalt entails a series of basalt flows and sedimentary interbeds. The flow properties ( $\mathrm{K}$ and $\mathrm{S}_{\mathrm{s}}$ ) are fairly well documented for the basalt flow tops and sedimentary interbeds. The properties of the basalt interiors; however, are more uncertain.

The significant difference in the flow properties of the flow tops and interbeds compared with the flow interiors is important to the conceptualization of the problem. Documented values for the horizontal conductivities of the flow tops/interbeds $\left(10^{-6} \mathrm{~m} / \mathrm{s}\right.$ to $\left.10^{-4} \mathrm{~m} / \mathrm{s}\right)$ are orders of magnitude greater than estimated values for the flow interiors $\left(10^{-12} \mathrm{~m} / \mathrm{s}\right.$ to $10^{-10} \mathrm{~m} / \mathrm{s}$ in the Wanapum basalt). A significant component of horizontal flow; therefore, is expected through the flow tops and interbeds of the Saddle Mountains basalt.

Continuing with the conceptualization of the flow, Figure B.40 shows that a downward, vertical gradient exists at piezometers DC-19, DC-20, and DC-22. Assuming that ground-water levels in the Saddle Mountains basalt were fairly uniform before the start of the waste disposal activity, the linear change in the groundwater levels as indicated in Figure B.40 may correspond to steady state, downward flow. A simple analysis of the problem; therefore, may be accomplished under the pretense that steadystate, Darcian flow is representative of the actual situation.

\section{Procedure}

Conceptualization of the flow in the Saddle Mountains basalt under the water table mound leads to the conclusion that darcian, steady-state flow may exist. The equation, which governs flow for those conditions is

$$
\mathrm{Q}=\mathrm{K} * \mathrm{~A} / \mathrm{dH}
$$




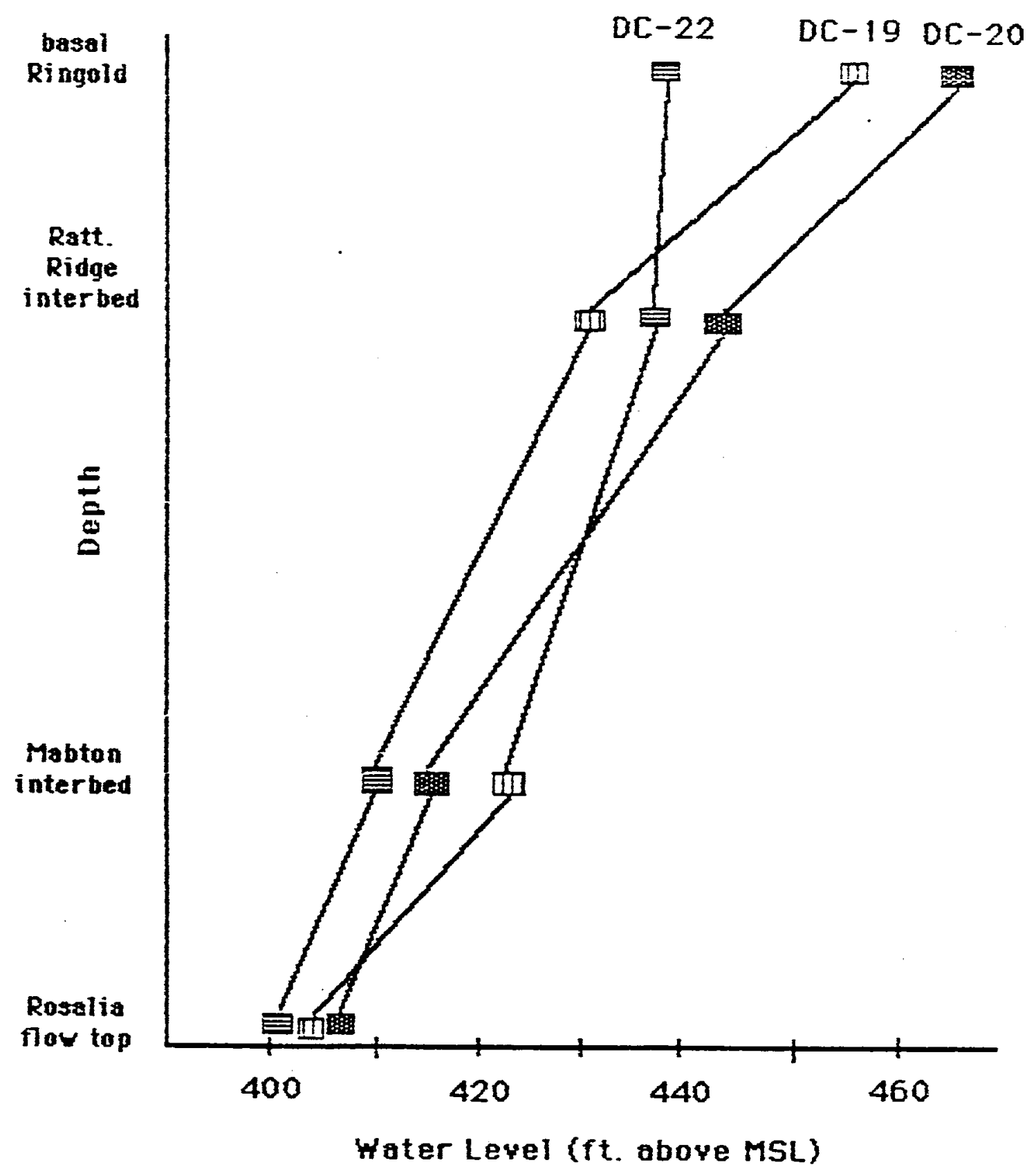

Figure B.40. Vertical Hydraulic Gradient through the Saddle Mountain Basalt at DC-19, DC-20, and DC-22. 
In this study, the vertical component of flow and the properties that control it are important. The ultimate goal is to estimate the vertical hydraulic conductivity, $\mathrm{K}_{\mathrm{e}}$, of the rock. The solution for the effective vertical conductivity is given by

$$
\mathrm{K}_{\mathrm{e}}=\mathrm{Q} / \mathrm{A} * \mathrm{CH}
$$

where,
A - area through which flow is occurring
Q - vertical flow rate though the given area
$\mathrm{dH}$ - vertical hydraulic gradient

Monitored groundwater levels at piezometer nests DC-19, DC-20, and DC-22 for the basal Ringold unit, Rattlesnake Ridge interbed, Mabton interbed, and Rosalia flow top give a reasonable estimate of the vertical hydraulic gradient at a radius of approximately $3.2 \mathrm{~km}$ from the center of the water table mound. There are difficulties; however, choosing the appropriate area and estimating the flow rate to be used in actuation 1.

Disregarding the possibility of isolated highly permeable zones (i.e., Fractures) or more gradual changes in the properties of the rock, the vertical conductivity of the rock mass should be independent of the area that is chosen. The area should be less than or equal to the area of influence of the water table mound (radius $=5 \mathrm{~km}$ ) and also optimize the quality of the data for the hydrostratigraphic units. Because the piesometer nests at DC-19, DC-20, and DC-22 provide the best vertical data in the area at a radius of 3.2 kilometers from the of center of the water table mound, this radius was chosen.

Estimation for the flow rate through the hypothetical cylinder is the next step in the procedure. Figure B.41 illustrates the process. The flow $\mathrm{Q}_{\text {total }}$, into the uppermost confined unit, the Rattlesnake Ridge interbed, is approximated by calculating the flow rate out of the interbed by horizontal flow, $\mathrm{Q}_{\mathrm{h}}$, plus the vertical leakage, $Q_{v}$, through the interbed to the underlying units

$$
\mathrm{Q}_{\mathrm{total}}=\mathrm{Q}_{\mathrm{h}}+\mathrm{Q}_{\mathrm{v}}
$$

The horizontal flow rate was calculated using the expression

$$
\mathrm{Q}_{\mathrm{h}}=\mathrm{K} * \mathrm{~A} * \mathrm{dH}
$$

where

$\mathrm{K}$ - geometric mean of the horizontal conductivities for the Rattlesnake Ridge interbed in the CASZ $(10-5 \mathrm{~m} / \mathrm{s})$

A - area of the hypothetical cylinder through which horizontal flow is occurring; thickness $\mathrm{x}$ circumference ( 30 meters $\mathrm{x} 2 \pi \mathrm{r}$ )

$\mathrm{dH}$ - horizontal hydraulic gradient in the Rattlesnake Ridge interbed as indicated by piezometers DC-19, DC-20, and DC-22 (3.8 x 10-4). 
Calculation of the vertical leakage, $\mathrm{Q}_{\mathrm{v}}$, was based on the assumption that the total flow out of the hypothetical cylinder below the Rattlesnake Ridge interbed is equal to the leakage through the interbed. This is illustrated in Figure B.41. The calculation gives the summation of horizontal flows out of the underlying flow tops and interbeds from the Rattlesnake Ridge interbed down to the Rosalia flow top. Vertical gradients at DC-19, DC-20, and DC-22 indicate that units below the Rosalia flow top do not contribute significantly to vertical flow.

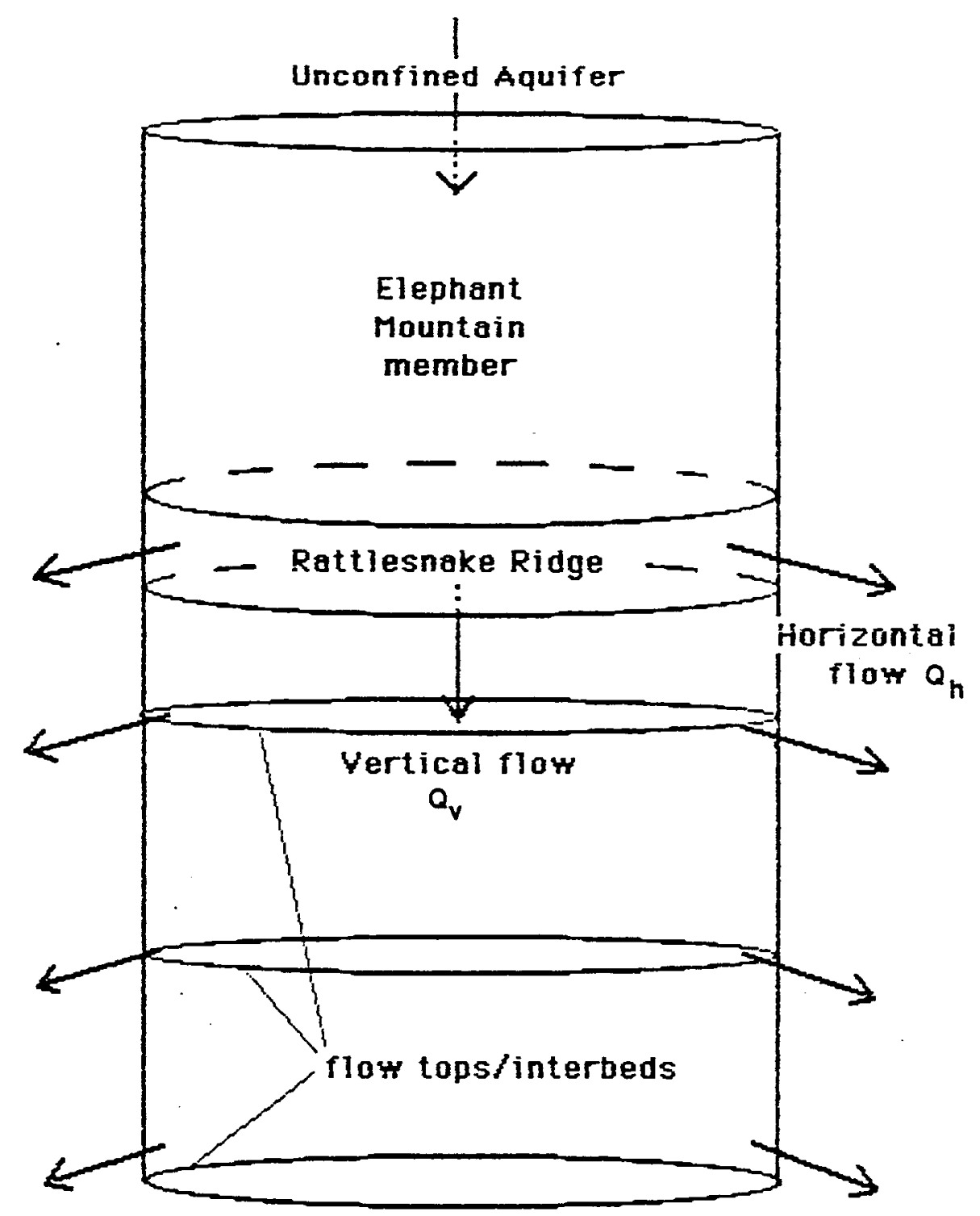

Figure B.41. Conceptualization of the Flow through a Volume of the Saddle Mountain Basalt. Primarily horizontal flow in the flow tops and interbeds and vertical flow through the flow interiors. 


$$
\begin{gathered}
\mathrm{Q}_{\mathrm{v}},=\mathrm{Q}_{\mathrm{n}} \\
\mathrm{Q}_{\mathrm{v}}=\Sigma \mathrm{K}_{\mathrm{I}} * \mathrm{~A} * \mathrm{dH}_{\mathrm{i}}
\end{gathered}
$$

where

$\mathrm{K}_{\mathrm{I}} \quad$ - are the respective horizontal conductivities of the flow tops or interbeds (Table 1)

A - is the area through which horizontal flow occurs (Table 1)

dHi - is the horizontal hydraulic gradient taken from the piezometric contour maps (Figures B.33, B.34, and B.35) at a radius of 3.2 kilometers (Table 1).

The total flow onto the Rattlesnake Ridge interbed was calculated using Equations 2 and 3 the substituted into Equation 1. The area in Equation 1 was assumed to be a circle with a radius of 3.2 kilometers and a vertical gradient equal to 0.05 .

Because we are looking at the flow from the unconfined aquifer to the Rattlesnake Ridge interbed, the calculated effective vertical conductivity is for the Elephant Mountain member which is situated between those two units. Vertical conductivities for deeper membranes in the Saddle Mountains basalt can be approximated using a similar procedure; however, recognizing that the procedure involves approximations from suspect sources such as the piezometric contour maps, a more qualitative approach for determining the properties of the deeper rock may be appropriate.

One qualitative observation which may aid in the understanding of the flow properties of the Saddle Mountains basalt was shown in Figure B.40. The vertical hydraulic gradient was shown to be nearly uniform at piezometers DC-19, DC-20, and DC-22. If we look again at the hypothetical cylinder we can conclude that

$$
A * \mathrm{dH}=\text { constant }
$$

The conceptualization of the flow through the cylinder; however, indicates that the flow rate is decreasing with depth due to horizontal flow out of the cylinder through the flow tops and interbeds.

$$
\mathrm{Q} \propto \mathrm{K}_{\mathrm{e}}
$$

This indicates that if the flow is decreasing with depth, then the effective vertical conductivity is decreasing to the "loss" of flow. An estimation of the decrease in the flow rate with depth is dependent on the properties used for the flow tops and interbeds. A reasonable estimate for the "loss" of flow from the shallow units to the deeper units is $90 \%$. This simple analysis indicates that the vertical hydraulic conductivities of the deeper units may be an order of magnitude less than the vertical conductivities of the shallow units. 


\section{Results}

The effective vertical conductivity of the rock mass between the unconfined aquifer and the Rattlesnake Ridge interbed was calculated using Equation 1.

$$
\mathrm{K}_{\mathrm{e}}=3 \times 10^{-9} \mathrm{~m} / \mathrm{s}
$$

Further speculation concluded that the deeper members of the Saddle Mountains basalt may have an effective vertical conductivity equal to $10 \%$ of the result above.

$$
\mathrm{K}_{\mathrm{e}}(\text { deeper })=3 \times 10^{-10} \mathrm{~m} / \mathrm{s}
$$

The ultimate goal of this analysis was to obtain an estimate for the vertical conductivity of the flow interiors. Because the effective conductivity is a harmonic average of the individual conductivities of the flow tops, interbeds, and flow interiors, the low permeability flow interiors will dominate the effective vertical conductivity. Assuming thicknesses for the flow tops, interbeds, and flow interiors.

$$
1 \times 10^{-9} \mathrm{~m} / \mathrm{s}<\mathrm{K}_{\text {in terior }}<1 \times \text { I } 0^{-10} \mathrm{~m} / \mathrm{s}
$$

\section{Summary and Conclusions}

The most important assumption in this study was that piezometric mounds in the confined units exist and are caused by the overlying water table mound. With these assumptions, two processes that may cause this relationship were analyzed. Loading of the confined units by the additional weight of the water table mound was eliminated as a possible explanation to determine whether useful information about the rock mass could be obtained. A simplistic analysis of the problem gave estimations for the vertical conductivities of the basalt flow interiors.

Results from this study are only as good as the assumptions and estimations that are made. The lack of data on the vertical conductivities of the basalt flow interiors; however, makes a simple analysis such as this one more useful. Numerical models, which are being used to determine the effects of disturbances through the Saddle Mountain basalt, might benefit from a better understanding of the properties of the untested flow interiors of the Saddle Mountain basalt.

\section{References}

Bierschenk WH. 1959. "Aquifer characteristics and groundwater movement at Hanford." Hanford Atomic Products Operations, June 9, 1959, p. 81, HW-6060I, Richland, Washington. 
Newcomb RC, JR Strand, and FJ Frank. 1973. "Geology and ground-water characteristics of the Hanford Reservation of the US Atomic Energy Commission Washington." US Geol. Survey Prof. Paper 717, p. 78.

Schatz AL, and TR Green. December 1986. "Unconfined aquifer and Rattlesnake Ridge aquifer waterlevel measurements data maps.” SD-WM-TI-273, Revision 1. 


\section{Distribution}

No. of

Copies

\section{OFFSITE}

2 Wanapum Indian Band

ATTN: R. Buck, Jr.

L. Seelatsee

PO Box 878

Ephrata, WA 98823

3 Washington State Department of Ecology

Nuclear Waste Program

ATTN: S. Dahl

1315 West $4^{\text {th }}$

D. N. Goswami (2)

Kennewick, WA 99336-6018

2 Yakima Indian Nation

ATTN: R. Jim

W. Rigsbee

PO Box 151

Toppenish, WA 98948

2 S. Harris

Confederated Tribes of the

Umatilla Indian Reservation

PO Box 638

Pendleton, OR 97801

2 Nez Perce Indian Tribe

ATTN: D. L. Powaukee

J. G. Heins

PO Box 365

Lapwai, ID 83540-0365
No. of

Copies

2 Oregon Department of Energy

ATTN: R. Bennett

K. Niles

625 Marion St. N.E.

Salem, OR 97310

S. Bede

U.S. Ecology

PO Box 638

Richland, WA 99352

J. Orban

U.S. Department of Energy

Albuquerque Operations Office

Pennsylvania and $\mathrm{H}$ Street

Kirtland Air Force Base

Albuquerque, NM 87116

3 U.S. Department of Energy

Savannah River Operations Office

ATTN: V. Sauls

W. Smith

H. M. Crapse

Road 1

Aiken, SC 29801

3 U.S. Department of Energy, Headquarters

Forrestal Building

ATTN: J. T. Bachmaier

E. Regnier

A. Wallo

1000 Independence Avenue, S.W.

Washington D.C. 20585

Distr.1 
No. of

Copies

J. T. Case

U.S. Department of Energy

Idaho Operations Office

765 Lindsay Blvd.

Idaho Falls, ID 83401

U.S. Department of Energy, Headquarters

Germantown

ATTN: M. K. Harmon

K. T. Lang

R. Bradley

J. Telerico

A. Williams

J. E. Rhoderick

W. Murphie

19901 Germantown Road

Germantown, MD 20874-1290

R.V. Curl

Idaho National Engineering Laboratory

850 Energy Drive

Idaho Falls, ID 83401

R. C. Janke

U.S. Department of Energy

Fernald Field Office

7400 Wiley Road

Cincinnati, OH 45030

F. DiSansa

U.S. Department of Energy

Nevada Operations Office

PO Box 98518

Las Vegas, NV 89193-8518

E. Wilhite

Westinghouse Savannah River Company

Savannah River Site

Aiken, SC 29808
No. of

Copies

D. Hollis

Los Alamos National Laboratory

PO Box 1663, MS-J595

Los Alamos, NM 87545

W. G. McMillan

U.S. Department of Energy

Oak Ridge Operations Office

PO Box 2001

Oak Ridge, TN 37831

R. H. French

Water Resources Center

Desert Research Institute

755 E. Flamingo Road

PO Box 1940

Las Vegas, NV 89132-0040

Sandia National Laboratories

ATTN: P. Vaugh

M. Marrietta

PO Box 5800

Albuquerque, MN 87185-1345

D. Metzler

U. S. Department of Energy

Grand Junction Project Office

25978314 Road

Grand Junction, CO 81503

D. Smith

2305 North Filmore St.

Arlington, VA 22201

Maxine Dunkleman

Department of Health

Division of Radiation Protection

Waste Management Section

PO Box 47827

Olympia, WA 98504-7827

Distr.2 
No. of

Copies

M. MacDonell

Argonne National Laboratory

9700 S. Cass Avenue

Argonne, IL 60439

Dr. Steven Gorelick

Braun Hall, Bldg 320

450 Serra Mall

Stanford University

Stanford, CA 94305-2115

Dr. James Mercer

46050 Manekin Plaza, Suite 100

HIS-Geotrans, Inc.

Sterling, VA 20166

Dr. Charles Andrews

S. S. Papadopulos and Assoc., Inc

7944 Wisconsin Avenue

Bethesda, MD 20814-3620

Dr. Peter Wierenga

Department of Hydrology and Water

Resources

Building 11, Rm 122

PO Box 210011

Tucson AZ 85721-0011

Dr. Shlomo Neuman

Department of Hydrology and Water

Resources

Building 11, Rm 122

PO Box 210011

Tucson AZ 85721-0011

Dr. Jacob Bear

Dept. of Civil Engineering

Technion-Israel Institute of Technology

Haifa 32000, Israel
No. of

Copies

Dr. Eileen Poeter

Poeter Engineering

1500 Illinois Avenue

Golden, CO 80401

Dr. Evan Andermann

Calibra Concsulting LLC

1776 Lincoln St. Suite 500

Denver Colorado 80203-1018

Dr. Mary Hill

U. S. Geological Survey

Water Resources Division

Lakewood, CO

Dr. Jesus Carrera

School of Civil Engineering

Technical University of Catalonia, UPC

Barcelona, Spain

\section{ONSITE}

\section{DOE Richland Operations Office}

B. Bilson

$\mathrm{H} 0-12$

P. F. Dunigan

A5-58

B. L. Foley

H0-12

J. D. Goodenough

$\mathrm{H} 0-12$

J. B. Hall

A2-15

R. D. Hildebrand (5)

A5-13

R. A. Holten

A5-16

M. F. Jarvis

A5-58

P. M. Knollmeyer

A5-11

K. Leary

$\mathrm{H} 0-12$

O. Robertson

$\mathrm{H} 0-12$

J. Sands

H6-60

G. H. Sanders

$\mathrm{H} 0-12$

T. Schroeder

$\mathrm{H} 8-24$

S. S. Seth

Distr.3 
No. of

Copies

G. L. Sinten

M. Thompson

A. Tortoso

A. K. Wright

6 DOE Office of River Protection

C. Babel

H. Boston

L. Erickson

P. Lamont

R. Lober

S. Wiegman

\section{CH2M Hill Group}

W. J. McMahon

V. J. Rohay

C. G. Swanson

\section{CH2M Hill Group}
A. J. Knepp
F. M. Mann
D Myers
R. W. Root
J. A. Voogd

\section{Fluor Federal Services}

M. C. Brown

R. Khaleel

R. J. Puigh

1 YAHSGS LLC

ATTN: W. Hewitt

PO Box 667

Richland, WA 99352
No. of

Copies

H0-12

A5-13

H0-12

T5-58

H6-60

H6-60

H6-60

H6-60

H6-60

H6-60

H9-03

H0-19

H9-02

$\mathrm{H} 0-22$

$\mathrm{H} 0-22$

$\mathrm{H} 0-22$

R2-58

R2-50

H6-60

B4-43

B4-43

\section{Fluor Hanford, Inc.}

M. M. Coony

H6-19

M. I. Wood

H8-44

1 Jacobs Engineering

$\begin{array}{ll}\text { P. M. Rogers B1-40 } & \text { B }\end{array}$

9 Bechtel Hanford

L. R Curry X3-61

B. Ford K1-55

M. J. Graham H0-09

M. N. Jarrayssi H0-19

J. J. McGuire S3-20

G. B. Mitchem H0-19

F. V. Roeck H0-17

C. D. Wittreich H9-03

T. M. Wintczak H0-09

1 U.S. Environmental Protection Agency
D. R. Sherwood
B5-01

62 Pacific Northwest Laboratory

D. B. Barnett K6-81

M. P. Bergeron (5) K9-36

B. W. Bryce K6-75

C. R. Cole (10) K9-36

D. Dauble K6-84

J. L. Devary K6-96

P. E. Dresel K6-96

P. Eslinger K3-54

M. J. Fayer K9-33

E. Freeman K9-36

M. D. Freshley H0-21

J. Fruchter K6-96 
No. of

Copies

G. W. Gee

V. G. Johnson

C. T. Kincaid

K. M. Krupka

P. E. Long

S. P. Luttrell

G. W. McNair

P. D. Meyer

C. J. Murray (5)

B. A. Napier

W. E. Nichols

S. Orr
No. of

Copies

K9-33

K6-96

K9-33

K6-81

K9-33

K6-96

H0-21

K9-33

K6-81

K3-54

K9-33

K9-33
T. L Page

M. J. Quadrel

T. D. Scheibe

R. J. Serne

R. M. Smith

D. L. Strenge

P. D. Thorne (5)

L. W. Vail

V. R. Vermeul

G. Whelan

S. K. Wurstner (5)
K9-18

K7-97

K9-33

K6-81

K6-96

K3-54

K9-33

K9-33

K9-36

K9-36

K9-36

Distr.5 